Cochrane Database of Systematic Reviews

\title{
Prophylactic vaccination against human papillomaviruses to prevent cervical cancer and its precursors (Review)
}

Arbyn M, Xu L, Simoens C, Martin-Hirsch PPL

Arbyn M, Xu L, Simoens C, Martin-Hirsch PPL.

Prophylactic vaccination against human papillomaviruses to prevent cervical cancer and its precursors.

Cochrane Database of Systematic Reviews 2018, Issue 5. Art. No.: CD009069.

DOI: 10.1002/14651858.CD009069.pub3.

www.cochranelibrary.com 
TABLE OF CONTENTS

HEADER

ABSTRACT

PLAIN LANGUAGE SUMMARY

SUMMARY OF FINDINGS

BACKGROUND

OBJECTIVES

METHODS

RESULTS

Figure 1.

Figure 2.

Figure 3.

Figure 4.

Figure 5.

Figure 6.

Figure 7.

Figure 8.

Figure 9.

Figure 10.

Figure 11.

Figure 12.

DISCUSSION

AUTHORS' CONCLUSIONS

ACKNOWLEDGEMENTS

REFERENCES

CHARACTERISTICS OF STUDIES

DATA AND ANALYSES

Analysis 1.1. Comparison $1 \mathrm{High}$-grade cervical lesions in hrHPV DNA negative women at baseline, Outcome 1 CIN2+ associated with HPV16/18, at least 1 dose.

Analysis 1.2. Comparison 1 High-grade cervical lesions in hrHPV DNA negative women at baseline, Outcome 2 CIN2+ associated with HPV6/11/16/18, at least 1 dose.

Analysis 1.3. Comparison $1 \mathrm{High}$-grade cervical lesions in hrHPV DNA negative women at baseline, Outcome 3 CIN3+ associated with HPV16/18, at least 1 dose.

Analysis 1.4. Comparison 1 High-grade cervical lesions in hrHPV DNA negative women at baseline, Outcome 4 CIN3+ associated with HPV6/11/16/18, at least 1 dose.

Analysis 1.5. Comparison 1 High-grade cervical lesions in hrHPV DNA negative women at baseline, Outcome 5 AIS associated with HPV16/18, at least 1 dose.

Analysis 1.6. Comparison 1 High-grade cervical lesions in hrHPV DNA negative women at baseline, Outcome 6 AIS associated with HPV6/11/16/18, at least 1 dose.

Analysis 1.7. Comparison 1 High-grade cervical lesions in hrHPV DNA negative women at baseline, Outcome 7 Any CIN2+ irrespective of HPV types, at least 1 dose.

Analysis 1.8. Comparison 1 High-grade cervical lesions in hrHPV DNA negative women at baseline, Outcome 8 Any CIN3+ irrespective of HPV types, at least 1 dose.

Analysis 1.9. Comparison 1 High-grade cervical lesions in hrHPV DNA negative women at baseline, Outcome 9 Any AIS irrespective of HPV types, at least 1 dose.

Analysis 2.1. Comparison 2 High-grade cervical lesions in HPV16/18 DNA negative women at baseline, Outcome 1 CIN2+ associated with HPV16/(18), 3 doses.

Analysis 2.2. Comparison 2 High-grade cervical lesions in HPV16/18 DNA negative women at baseline, Outcome 2 CIN2+ associated with HPV16/(18), at least 1 dose.

Analysis 2.3. Comparison 2 High-grade cervical lesions in HPV16/18 DNA negative women at baseline, Outcome 3 CIN2+ associated with HPV16/(18), 1 or 2 doses (post hoc analysis).

Analysis 2.4. Comparison 2 High-grade cervical lesions in HPV16/18 DNA negative women at baseline, Outcome 4 CIN2+ associated with HPV6/11/16/18, 3 doses. 
Analysis 2.5. Comparison 2 High-grade cervical lesions in HPV16/18 DNA negative women at baseline, Outcome 5 CIN2+ associated with HPV6/11/16/18, at least 1 dose.

Analysis 2.6. Comparison 2 High-grade cervical lesions in HPV16/18 DNA negative women at baseline, Outcome 6 CIN2+ associated with HPV6/11/16/18, 1 or 2 doses (post hoc analysis).

Analysis 2.7. Comparison 2 High-grade cervical lesions in HPV16/18 DNA negative women at baseline, Outcome 7 CIN3+ associated with HPV16/18 or HPV6/11/16/18, 3 doses.

Analysis 2.8. Comparison 2 High-grade cervical lesions in HPV16/18 DNA negative women at baseline, Outcome 8 CIN3+ associated with HPV 16/18 or HPV6/11/16/18, at least 1 dose.

Analysis 2.9. Comparison 2 High-grade cervical lesions in HPV16/18 DNA negative women at baseline, Outcome 9 CIN3+ associated with HPV16/18 or HPV6/11/16/18, 1 or 2 doses (post hoc analysis).

Analysis 2.10. Comparison 2 High-grade cervical lesions in HPV16/18 DNA negative women at baseline, Outcome 10 AIS associated with HPV16/18 or HPV6/11/16/18, 3 doses.

Analysis 2.11. Comparison 2 High-grade cervical lesions in HPV16/18 DNA negative women at baseline, Outcome 11 AIS associated with HPV16/18 or 6/11/16/18, at least 1 dose.

Analysis 2.12. Comparison 2 High-grade cervical lesions in HPV16/18 DNA negative women at baseline, Outcome 12 AIS associated with HPV16/18 or HPV6/11/16/18, 1 or 2 doses (post hoc analysis).

Analysis 2.13. Comparison 2 High-grade cervical lesions in HPV16/18 DNA negative women at baseline, Outcome 13 Any CIN2+ irrespective of HPV types, 3 doses.

Analysis 2.14. Comparison 2 High-grade cervical lesions in HPV16/18 DNA negative women at baseline, Outcome 14 Any CIN2+ irrespective of HPV types, at least 1 dose.

Analysis 2.15. Comparison 2 High-grade cervical lesions in HPV16/18 DNA negative women at baseline, Outcome 15 Any CIN2+ irrespective of HPV types, 1 or 2 doses (post hoc analysis).

Analysis 3.1. Comparison 3 High-grade cervical lesions in women regardless of baseline HPV DNA status, Outcome 1 CIN2+ associated with HPV16/18, at least 1 dose.

Analysis 3.2. Comparison 3 High-grade cervical lesions in women regardless of baseline HPV DNA status, Outcome 2 CIN2+ associated with HPV6/11/16/18, at least 1 dose.

Analysis 3.3. Comparison 3 High-grade cervical lesions in women regardless of baseline HPV DNA status, Outcome 3 CIN3+ associated with HPV16/18, at least 1 dose.

Analysis 3.4. Comparison 3 High-grade cervical lesions in women regardless of baseline HPV DNA status, Outcome 4 CIN3+ associated with HPV6/11/16/18, at least 1 dose.

Analysis 3.5. Comparison 3 High-grade cervical lesions in women regardless of baseline HPV DNA status, Outcome 5 AIS associated with HPV16/18, at least 1 dose.

Analysis 3.6. Comparison 3 High-grade cervical lesions in women regardless of baseline HPV DNA status, Outcome 6 AIS associated with HPV6/11/16/18, at least 1 dose.

Analysis 3.7. Comparison 3 High-grade cervical lesions in women regardless of baseline HPV DNA status, Outcome 7 Any CIN2+ irrespective of HPV types, at least 1 dose.

Analysis 3.8. Comparison 3 High-grade cervical lesions in women regardless of baseline HPV DNA status, Outcome 8 Any CIN3+ HPV type, at least 1 dose.

Analysis 3.9. Comparison 3 High-grade cervical lesions in women regardless of baseline HPV DNA status, Outcome 9 Any AIS irrespective of HPV types, at least 1 dose.

Analysis 4.1. Comparison 4 Infection with HPV vaccine types in hrHPV DNA negative women at baseline, Outcome 1 Incident HPV16/18 infection, 3 doses.

Analysis 4.2. Comparison 4 Infection with HPV vaccine types in hrHPV DNA negative women at baseline, Outcome 2 Persistent HPV16/18 infection (6M), 3 doses.

Analysis 4.3. Comparison 4 Infection with HPV vaccine types in hrHPV DNA negative women at baseline, Outcome 3 Persistent HPV16/18 infection (6M), at least 1 dose.

Analysis 4.4. Comparison 4 Infection with HPV vaccine types in hrHPV DNA negative women at baseline, Outcome 4 Persistent HPV16/18 infection(12M), 3 doses.

Analysis 4.5. Comparison 4 Infection with HPV vaccine types in hrHPV DNA negative women at baseline, Outcome 5 Persistent HPV16/18 infection (12M), at least 1 dose.

Analysis 5.1. Comparison 5 HPV16/18 infection in HPV16/18 DNA negative women at baseline, Outcome 1 Incident HPV16/18 infection, 3 doses.

Analysis 5.2. Comparison 5 HPV16/18 infection in HPV16/18 DNA negative women at baseline, Outcome 2 Incident HPV16/18 infection, at least 1 dose.

Analysis 5.3. Comparison 5 HPV16/18 infection in HPV16/18 DNA negative women at baseline, Outcome 3 Incident HPV16/18 infection, 1 or 2 doses (post hoc analysis). 
Analysis 5.4. Comparison 5 HPV16/18 infection in HPV16/18 DNA negative women at baseline, Outcome 4 Persistent HPV16/18 infection (6M), 3 doses.

Analysis 5.5. Comparison 5 HPV16/18 infection in HPV16/18 DNA negative women at baseline, Outcome 5 Persistent HPV16/18 infection (6M), at least 1 dose.

Analysis 5.6. Comparison 5 HPV16/18 infection in HPV16/18 DNA negative women at baseline, Outcome 6 Persistent HPV16/18 infection (6M), 1 or 2 doses (post hoc analysis).

Analysis 5.7. Comparison 5 HPV16/18 infection in HPV16/18 DNA negative women at baseline, Outcome 7 Persistent HPV6/11/16/18 infection (6M), 3 doses.

Analysis 5.8. Comparison 5 HPV16/18 infection in HPV16/18 DNA negative women at baseline, Outcome 8 Persistent HPV6/11/16/18 infection (6M), at least 1 dose.

Analysis 5.9. Comparison 5 HPV16/18 infection in HPV16/18 DNA negative women at baseline, Outcome 9 Persistent HPV16/18 infection (12M), 3 doses.

Analysis 5.10. Comparison 5 HPV16/18 infection in HPV16/18 DNA negative women at baseline, Outcome 10 Persistent HPV16/18 infection (12M), at least 1 dose.

Analysis 5.11. Comparison 5 HPV16/18 infection in HPV16/18 DNA negative women at baseline, Outcome 11 Persistent HPV16/18 infection (12M), 1 or 2 doses (post hoc analysis).

Analysis 6.1. Comparison 6 Infection with HPV types included in the vaccine in women regardless of HPV DNA status at baseline, Outcome 1 Incident HPV16/18 infection, at least 1 dose.

Analysis 6.2. Comparison 6 Infection with HPV types included in the vaccine in women regardless of HPV DNA status at baseline, Outcome 2 Persistent HPV16/18 infection (6M), at least 1 dose.

Analysis 6.3. Comparison 6 Infection with HPV types included in the vaccine in women regardless of HPV DNA status at baseline, Outcome 3 Persistent HPV6/11/16/18 infection (6M), at least 1 dose.

Analysis 6.4. Comparison 6 Infection with HPV types included in the vaccine in women regardless of HPV DNA status at baseline, Outcome 4 Persistent HPV16/18 infection (12M), at least 1 dose.

Analysis 6.5. Comparison 6 Infection with HPV types included in the vaccine in women regardless of HPV DNA status at baseline, Outcome 5 Persistent HPV16/18 infection (12M) by dose (post hoc analysis).

Analysis 7.1. Comparison 7 Adverse events, Outcome 1 Overall local/injection site adverse events. ........................................

Analysis 7.2. Comparison 7 Adverse events, Outcome 2 Pain at injection site.

Analysis 7.3. Comparison 7 Adverse events, Outcome 3 Swelling at injection site.

Analysis 7.4. Comparison 7 Adverse events, Outcome 4 Redness at injection site.

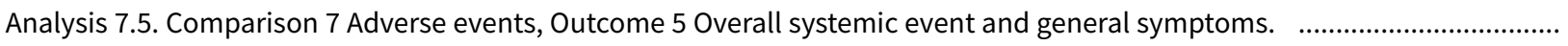
Analysis 7.6. Comparison 7 Adverse events, Outcome 6 Serious adverse events.

Analysis 7.7. Comparison 7 Adverse events, Outcome 7 Deaths.

Analysis 8.1. Comparison 8 Pregnancy outcomes, Outcome 1 Normal infant.

Analysis 8.2. Comparison 8 Pregnancy outcomes, Outcome 2 Spontaneous abortion/miscarriage.

Analysis 8.3. Comparison 8 Pregnancy outcomes, Outcome 3 Elective termination/induced abortion.

Analysis 8.4. Comparison 8 Pregnancy outcomes, Outcome 4 Stillbirth.

Analysis 8.5. Comparison 8 Pregnancy outcomes, Outcome 5 Abnormal infant. ADDITIONAL TABLES 
[Intervention Review]

\section{Prophylactic vaccination against human papillomaviruses to prevent cervical cancer and its precursors}

Marc Arbyn ${ }^{1 a}$, Lan Xu1b, Cindy Simoens ${ }^{2}$, Pierre PL Martin-Hirsch ${ }^{3}$

1Unit of Cancer Epidemiology, Belgian Cancer Centre, Sciensano, Brussels, Belgium. 2Laboratory of Cell Biology and Histology, University of Antwerp, Antwerp, Belgium. ${ }^{3}$ Gynaecological Oncology Unit, Royal Preston Hospital, Lancashire Teaching Hospital NHS Trust, Preston, UK

a Joint first author. $b$ Joint first author

Contact address: Marc Arbyn, Unit of Cancer Epidemiology, Belgian Cancer Centre, Sciensano, Juliette Wytsmanstreet 14, Brussels, B-1050, Belgium. marc.arbyn@sciensano.be.

Editorial group: Cochrane Gynaecological, Neuro-oncology and Orphan Cancer Group.

Publication status and date: Edited (no change to conclusions), published in Issue 3, 2020.

Citation: Arbyn M, Xu L, Simoens C, Martin-Hirsch PPL. Prophylactic vaccination against human papillomaviruses to prevent cervical cancer and its precursors. Cochrane Database of Systematic Reviews 2018, Issue 5. Art. No.: CD009069. DOI: 10.1002/14651858.CD009069.pub3.

Copyright ( 2020 The Cochrane Collaboration. Published by John Wiley \& Sons, Ltd.

\section{A B S T R A C T}

\section{Background}

Persistent infection with high-risk human papillomaviruses (hrHPV) types is causally linked with the development of cervical precancer and cancer. HPV types 16 and 18 cause approximately $70 \%$ of cervical cancers worldwide.

\section{Objectives}

To evaluate the harms and protection of prophylactic human papillomaviruses (HPV) vaccines against cervical precancer and HPV16/18 infection in adolescent girls and women.

\section{Search methods}

We searched MEDLINE, Cochrane Central Register of Controlled Trials (CENTRAL) and Embase (June 2017) for reports on effects from trials. We searched trial registries and company results' registers to identify unpublished data for mortality and serious adverse events.

\section{Selection criteria}

Randomised controlled trials comparing efficacy and safety in females offered HPV vaccines with placebo (vaccine adjuvants or another control vaccine).

\section{Data collection and analysis}

We used Cochrane methodology and GRADE to rate the certainty of evidence for protection against cervical precancer (cervical intraepithelial neoplasia grade 2 and above [CIN2+], CIN grade 3 and above [CIN3+], and adenocarcinoma-in-situ [AIS]), and for harms. We distinguished between the effects of vaccines by participants' baseline HPV DNA status. The outcomes were precancer associated with vaccine HPV types and precancer irrespective of HPV type. Results are presented as risks in control and vaccination groups and risk ratios (RR) with 95\% confidence intervals in brackets.

\section{Main results}

We included 26 trials (73,428 participants). Ten trials, with follow-up of 1.3 to 8 years, addressed protection against CIN/AIS. Vaccine safety was evaluated over a period of 6 months to 7 years in 23 studies. Studies were not large enough or of sufficient duration to evaluate cervical cancer outcomes. All but one of the trials was funded by the vaccine manufacturers. We judged most included trials to be at low risk of 
bias. Studies involved monovalent $(N=1)$, bivalent $(N=18)$, and quadrivalent vaccines $(N=7)$. Most women were under 26 years of age. Three trials recruited women aged 25 and over. We summarize the effects of vaccines in participants who had at least one immunisation.

\section{Efficacy endpoints by initial HPV DNA status}

\section{hrHPV negative}

HPV vaccines reduce CIN2+, CIN3+, AIS associated with HPV16/18 compared with placebo in adolescent girls and women aged 15 to 26. There is high-certainty evidence that vaccines lower CIN2+ from 164 to 2/10,000 (RR 0.01 (0 to 0.05)) and CIN3+ from 70 to 0/10,000 (RR 0.01 (0.00 to 0.10$)$. There is moderate-certainty evidence that vaccines reduce the risk of AIS from 9 to $0 / 10,000$ (RR 0.10 (0.01 to 0.82 ).

HPV vaccines reduce the risk of any CIN2+ from 287 to 106/10,000 (RR 0.37 (0.25 to 0.55), high certainty) and probably reduce any AIS lesions from 10 to 0/10,000 (RR 0.1 (0.01 to 0.76), moderate certainty). The size of reduction in CIN3+ with vaccines differed between bivalent and quadrivalent vaccines (bivalent: RR 0.08 ( 0.03 to 0.23 ), high certainty; quadrivalent: RR 0.54 ( 0.36 to 0.82 ), moderate certainty). Data in older women were not available for this comparison.

\section{HPV16/18 negative}

In those aged 15 to 26 years, vaccines reduce CIN2+ associated with HPV16/18 from 113 to $6 / 10,000$ (RR 0.05 (0.03 to 0.10 ). In women 24 years or older the absolute and relative reduction in the risk of these lesions is smaller (from 45 to $14 / 10,000,(R R 0.30$ (0.11 to 0.81 ), moderate certainty). HPV vaccines reduce the risk of CIN3+ and AIS associated with HPV16/18 in younger women (RR 0.05 (0.02 to 0.14 ), high certainty and RR 0.09 ( 0.01 to 0.72 ), moderate certainty, respectively). No trials in older women have measured these outcomes.

Vaccines reduce any CIN2+ from 231 to 95/10,000, (RR 0.41 (0.32 to 0.52)) in younger women. No data are reported for more severe lesions.

\section{Regardless of HPV DNA status}

In younger women HPV vaccines reduce the risk of CIN2+ associated with HPV16/18 from 341 to 157/10,000 (RR 0.46 (0.37 to 0.57), high certainty). Similar reductions in risk were observed for CIN3+ associated with HPV16/18 (high certainty). The number of women with AIS associated with HPV16/18 is reduced from 14 to 5/10,000 with HPV vaccines (high certainty).

HPV vaccines reduce any CIN2+ from 559 to $391 / 10,000$ (RR 0.70 (0.58 to 0.85 , high certainty) and any AIS from 17 to $5 / 10,000$ (RR 0.32 ( 0.15 to 0.67 ), high certainty). The reduction in any CIN3+ differed by vaccine type (bivalent vaccine: RR 0.55 ( 0.43 to 0.71 ) and quadrivalent vaccine: RR 0.81 (0.69 to 0.96$)$ ).

In women vaccinated at 24 to 45 years of age, there is moderate-certainty evidence that the risks of CIN2+ associated with HPV16/18 and any CIN2+ are similar between vaccinated and unvaccinated women (RR 0.74 (0.52 to 1.05) and RR 1.04 (0.83 to 1.30) respectively). No data are reported in this age group for CIN3+ or AIS.

\section{Adverse effects}

The risk of serious adverse events is similar between control and HPV vaccines in women of all ages (669 versus 656/10,000, RR 0.98 (0.92 to 1.05 ), high certainty). Mortality was $11 / 10,000$ in control groups compared with $14 / 10,000$ (9 to 22) with HPV vaccine (RR 1.29 [0.85 to 1.98]; low certainty). The number of deaths was low overall but there is a higher number of deaths in older women. No pattern in the cause or timing of death has been established.

\section{Pregnancy outcomes}

Among those who became pregnant during the studies, we did not find an increased risk of miscarriage $(1618$ versus $1424 / 10,000, \mathrm{RR}$ 0.88 ( 0.68 to 1.14 ), high certainty) or termination ( 931 versus $838 / 10,000$ RR 0.90 ( 0.80 to 1.02 ), high certainty). The effects on congenital abnormalities and stillbirths are uncertain (RR 1.22 (0.88 to 1.69), moderate certainty and (RR 1.12 (0.68 to 1.83), moderate certainty, respectively).

\section{Authors' conclusions}

There is high-certainty evidence that HPV vaccines protect against cervical precancer in adolescent girls and young women aged 15 to 26. The effect is higher for lesions associated with HPV16/18 than for lesions irrespective of HPV type. The effect is greater in those who are negative for hrHPV or HPV16/18 DNA at enrolment than those unselected for HPV DNA status. There is moderate-certainty evidence that HPV vaccines reduce CIN2+ in older women who are HPV16/18 negative, but not when they are unselected by HPV DNA status.

We did not find an increased risk of serious adverse effects. Although the number of deaths is low overall, there were more deaths among women older than 25 years who received the vaccine. The deaths reported in the studies have been judged not to be related to the vaccine. Increased risk of adverse pregnancy outcomes after HPV vaccination cannot be excluded, although the risk of miscarriage and termination are similar between trial arms. Long-term of follow-up is needed to monitor the impact on cervical cancer, occurrence of rare harms and pregnancy outcomes. 


\section{PLAIN LANGUAGE SUMMARY}

\section{HPV vaccination to prevent cancer and pre-cancerous changes of the cervix}

\section{Background}

Human papillomaviruses (HPV) are sexually transmitted and are common in young people. Usually they are cleared by the immune system. However, when high-risk (hr) types persist, they can cause the development of abnormal cervical cells, which are referred to as cervical precancer if at least two thirds of the surface layer of the cervix is affected. Precancer can develop into cervical cancer after several years. Not everyone who has cervical precancer goes on to develop cervical cancer, but predicting who will is difficult. There are a number of different hrHPV types which can cause cervical precancer and cancer. HPV16 and 18 are the most important high-risk types, since they cause about $70 \%$ of cervical cancers worldwide. Preventive vaccination, by injection of HPV virus-like particles in the muscle, triggers the production of antibodies which protect against future HPV infections.

\section{Review question}

Does HPV vaccination prevent the development of cervical precancer or cancer and what are the harms?

\section{Main results}

We included 26 studies involving 73,428 adolescent girls and women. All trials evaluated vaccine safety over a period 0.5 to 7 years and ten trials, with follow-up 3.5 to 8 years, addressed protection against precancer. Cervical cancer outcomes are not available. Most participants enrolled were younger than 26 years of age. Three trials recruited women between 25 to 45 years. The studies compared HPV vaccine with a dummy vaccine.

We assessed protection against precancer in individuals who were free of hrHPV, free of HPV16/18 or those with or without HPV infection at the time of vaccination. We separately assessed precancer associated with HPV16/18 and any precancer.

\section{Protection against cervical precancer}

1) Women free of hrHPV

Outcomes were only measured in the younger age group for this comparison ( 15 to 25 years). HPV vaccines reduce the risk of cervical precancer associated with HPV16/18 from 164 to 2/10,000 women (high certainty). They reduce also any precancer from 287 to 106/10,000 (high certainty).

\section{2) Women free of HPV16/18}

The effect of HPV vaccines on risk of precancer differ by age group. In younger women, HPV vaccines reduce the risk of precancer associated with HPV16/18 from 113 to $6 / 10,000$ women (high certainty). HPV vaccines lower the number of women with any precancer from 231 to $95 / 10,000$ (high certainty). In women older than 25 , the vaccines reduce the number with precancer associated with HPV16/18 from 45 to $14 / 10,000$ (moderate certainty).

\section{3) All women with or without HPV infection}

In those vaccinated between 15 to 26 years of age, HPV vaccination reduces the risk of precancer associated with HPV16/18 from 341 to $157 / 10,000$ (high certainty) and any precancer from 559 to 391/10,000 (high certainty).

In older women, vaccinated between 25 to 45 years of age, the effects of HPV vaccine on precancer are smaller, which may be due to previous exposure to HPV. The risk of precancer associated with HPV16/18 is probably reduced from 145/10,000 in unvaccinated women to $107 / 10,000$ women following HPV vaccination (moderate certainty). The risk of any precancer is probably similar between unvaccinated and vaccinated women (343 versus $356 / 10,000$, moderate certainty).

\section{Adverse effects}

The risk of serious adverse events is similar in HPV and control vaccines (placebo or vaccine against another infection than HPV (high certainty). The rate of death is similar overall (11/10,000 in control group, 14/10,000 in HPV vaccine group) (low certainty). The number of deaths overall is low although a higher number of deaths in older women was observed. No pattern in the cause or timing of death has been established.

\section{Pregnancy outcomes}

HPV vaccines did not increase the risk of miscarriage or termination of pregnancy. We do not have enough data to be certain about the risk of stillbirths and babies born with malformations (moderate certainty).

\section{Conclusion}

There is high-certainty evidence that HPV vaccines protect against cervical precancer in adolescent girls and women who are vaccinated between 15 and 26 years of age. The protection is lower when a part of the population is already infected with HPV. Longer-term follow-up 
is needed to assess the impact on cervical cancer. The vaccines do not increase the risk of serious adverse events, miscarriage or pregnancy termination. There are limited data from trials on the effect of vaccines on deaths, stillbirth and babies born with malformations. 


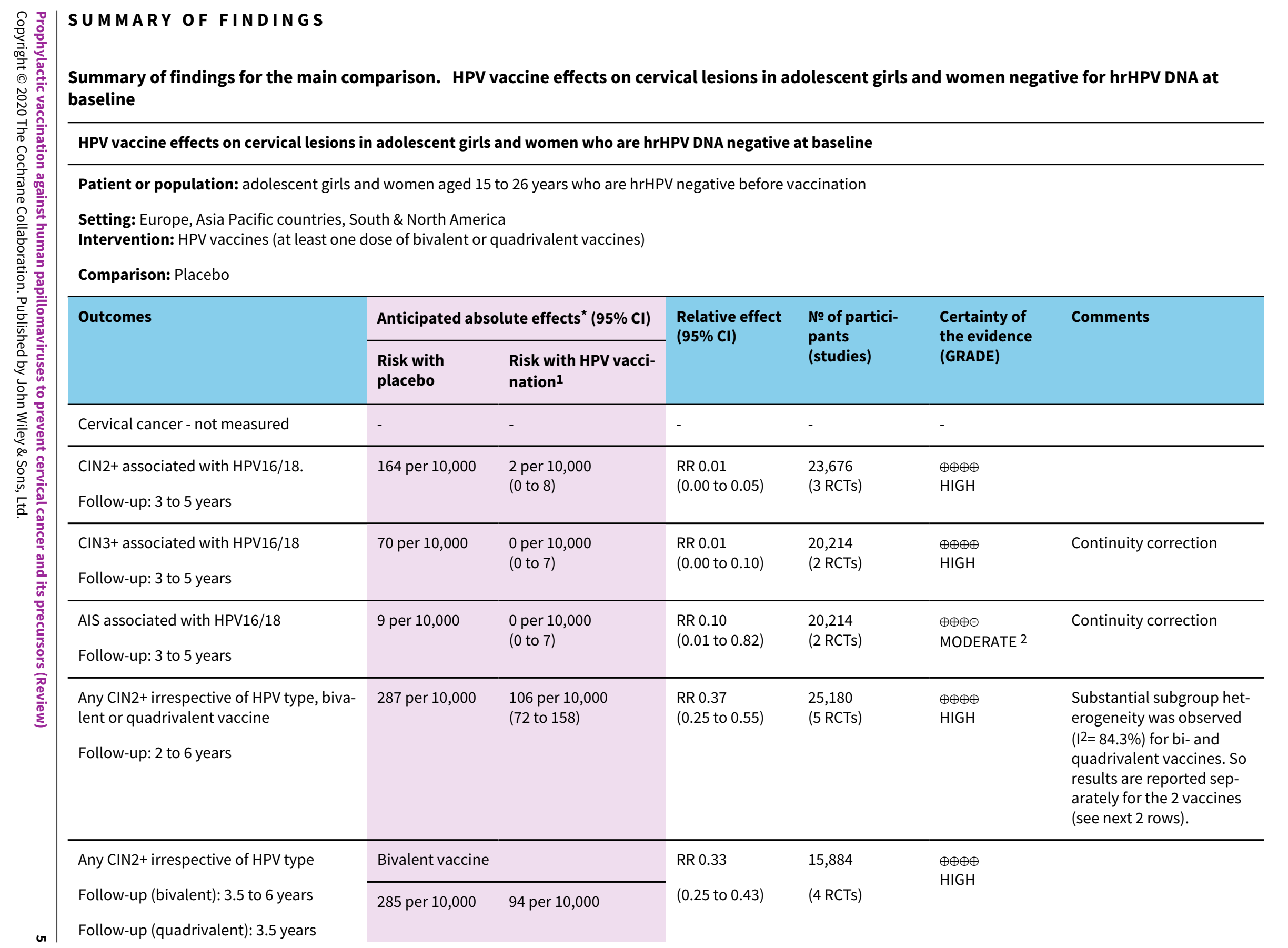

Setting: Europe, Asia Pacific countries, South \& North America

Comparison: Placebo 


\begin{tabular}{|c|c|c|c|c|c|c|c|}
\hline \multirow{3}{*}{\multicolumn{2}{|c|}{ 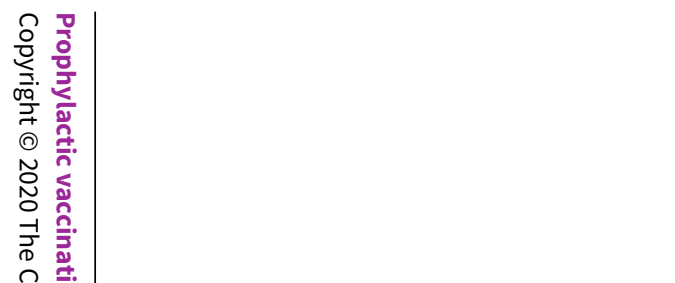 }} & \multicolumn{2}{|r|}{ (71 to 122 ) } & & & & \\
\hline & & \multicolumn{2}{|c|}{ Quadrivalent vaccine } & \multirow{2}{*}{$\begin{array}{l}\text { RR } 0.57 \\
(0.44 \text { to } 0.76)\end{array}$} & \multirow{2}{*}{$\begin{array}{l}9296 \\
(1 \mathrm{RCT})\end{array}$} & \multirow{2}{*}{$\begin{array}{l}\oplus \oplus \oplus \odot \\
\text { MODERATE } 3\end{array}$} & \multirow[b]{3}{*}{$\begin{array}{l}\text { Substantial subgroup het- } \\
\text { erogeneity was observed } \\
\left(I^{2}=84.3 \%\right) \text { for bi- and } \\
\text { quadrivalent vaccines. So } \\
\text { results are reported sep- } \\
\text { arately for the } 2 \text { vaccines } \\
\text { (see next } 2 \text { rows). }\end{array}$} \\
\hline & & 291 per 10,000 & $\begin{array}{l}166 \text { per } 10,000 \\
(128 \text { to } 221)\end{array}$ & & & & \\
\hline 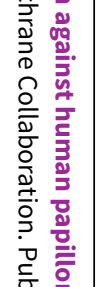 & $\begin{array}{l}\text { Any CIN3+ irrespective of HPV type, biva- } \\
\text { lent or quadrivalent vaccine } \\
\text { Follow-up: } 3.5 \text { to } 4 \text { years }\end{array}$ & 109 per 10,000 & $\begin{array}{l}23 \text { per } 10,000 \\
(4 \text { to } 120)\end{array}$ & $\begin{array}{l}\text { RR } 0.21 \\
(0.04 \text { to } 1.10)\end{array}$ & $\begin{array}{l}20,719 \\
(3 \mathrm{RCTs})\end{array}$ & $\begin{array}{l}\oplus \oplus \oplus \ominus \\
\text { MODERATE } 3\end{array}$ & \\
\hline 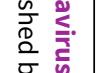 & Any $\mathrm{CIN} 3+$ irrespective of HPV type & \multicolumn{2}{|l|}{ Bivalent vaccine } & \multirow{2}{*}{$\begin{array}{l}\text { RR } 0.08 \\
\text { (0.03 to } 0.23)\end{array}$} & \multirow{2}{*}{$\begin{array}{l}11,423 \\
(2 \text { RCTs) }\end{array}$} & \multirow{2}{*}{$\begin{array}{l}\oplus \oplus \oplus \oplus \\
\mathrm{HIGH}\end{array}$} & \\
\hline 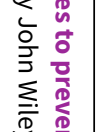 & \multirow[t]{3}{*}{$\begin{array}{l}\text { Follow-up (bivalent): } 4 \text { years } \\
\text { Follow-up (quadrivalent): } 3.5 \text { years }\end{array}$} & 81 per 10,000 & $\begin{array}{l}6 \text { per } 10,000 \\
(3 \text { to } 19)\end{array}$ & & & & \\
\hline 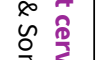 & & \multicolumn{2}{|c|}{ Quadrivalent vaccine } & \multirow{2}{*}{$\begin{array}{l}\text { RR } 0.54 \\
(0.36 \text { to } 0.82)\end{array}$} & \multirow{2}{*}{$\begin{array}{l}9296 \\
(1 \mathrm{RCT})\end{array}$} & \multirow{2}{*}{$\begin{array}{l}\oplus \oplus \oplus \ominus \\
\text { MODERATE }\end{array}$} & \\
\hline 高 & & 143 per 10,000 & $\begin{array}{l}77 \text { per } 10,000 \\
(51 \text { to } 117)\end{array}$ & & & & \\
\hline 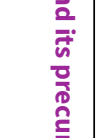 & $\begin{array}{l}\text { Any AIS irrespective of HPV type } \\
\text { Follow-up: } 3 \text { to } 5 \text { years }\end{array}$ & 10 per 10,000 & $\begin{array}{l}0 \text { per } 10,000 \\
\text { (0 to } 8)\end{array}$ & $\begin{array}{l}\text { RR } 0.10 \\
(0.01 \text { to } 0.76)\end{array}$ & $\begin{array}{l}20,214 \\
\text { (2 RCTs) }\end{array}$ & $\begin{array}{l}\oplus \oplus \oplus \ominus \\
\text { MODERATE } 2\end{array}$ & Continuity correction \\
\hline 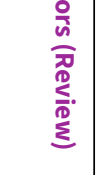 & \multicolumn{7}{|c|}{$\begin{array}{l}\text { 1The risk in the intervention group (and its } 95 \% \text { confidence interval) is based on the assumed risk in the comparison group and the relative effect of the intervention (and } \\
\text { its } 95 \% \mathrm{Cl} \text { ). When risk in vaccine group is zero, the } 95 \% \mathrm{Cl} \text { is computed using an exact binomial method. } \\
\text { AIS: adenocarcinoma in situ; Cl: Confidence interval; CIN: cervical intraepithelial neoplasia; RR: Risk ratio }\end{array}$} \\
\hline & $\begin{array}{l}\text { GRADE Working Group grades of eviden } \\
\text { High certainty: We are very confident tha } \\
\text { Moderate certainty: We are moderately } \\
\text { substantially different } \\
\text { Low certainty: Our confidence in the effe } \\
\text { Very low certainty: We have very little co }\end{array}$ & $\begin{array}{l}\text { onfident in the eff } \\
\text { the true effect lie } \\
\text { nfidence in the eff }\end{array}$ & $\begin{array}{l}\text { close to that of } \mathrm{tl} \\
\text { t estimate: The } t \\
\text { t The true effect } \\
\text { t estimate: The } t\end{array}$ & $\begin{array}{l}\text { ate of the effect } \\
\text { ct is likely to be } \\
\text { substantially di } \\
\text { ct is likely to be }\end{array}$ & $\begin{array}{l}\text { se to the es } \\
\text { ent from th } \\
\text { stantially }\end{array}$ & $\begin{array}{l}f \text { the effect, but } \\
\text { te of the effect } \\
\text { from the estima }\end{array}$ & $\begin{array}{l}\text { re is a possibility that it is } \\
\text { f effect }\end{array}$ \\
\hline
\end{tabular}




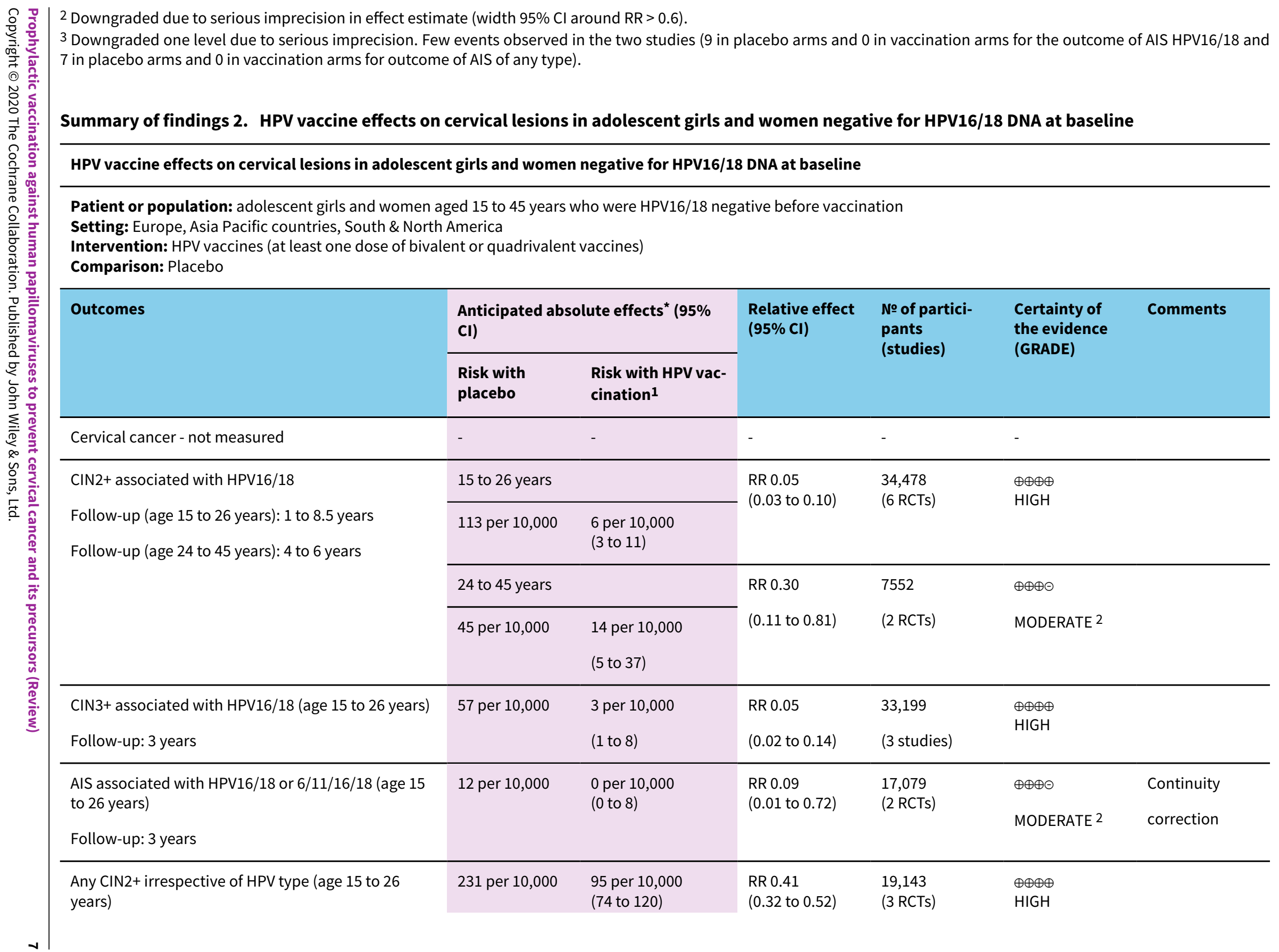

Patient or population: adolescent girls and women aged 15 to 45 years who were HPV16/18 negative before vaccination Setting: Europe, Asia Pacific countries, South \& North Americ

Intervention: HPV vaccines (at least one dose of bivalent or quadrivalent vaccines)

3 Downgraded one level due to serious imprecision. Few events observed in the two studies ( 9 in placebo arms and 0 in vaccination arms for the outcome of AIS HPV16/18 and

7 in placebo arms and 0 in vaccination arms for outcome of AIS of any type).

\section{Summary of findings 2 . HPV vaccine effects on cervical lesions in adolescent girls and women negative for HPV16/18 DNA at baseline}


Any CIN3+ irrespective of HPV type - not measured

Any AIS irrespective of HPV type - not measured

${ }^{1}$ The risk in the intervention group (and its 95\% confidence interval) is based on the assumed risk in the comparison group and the relative effect of the intervention (and its $95 \% \mathrm{Cl}$ ). Exception: when risk in vaccine group is zero, the $95 \% \mathrm{Cl}$ is computed using an exact binomial method.

AIS: adenocarcinoma in situ; Cl: Confidence interval; CIN: cervical intraepithelial neoplasia; RR: Risk ratio

\section{GRADE Working Group grades of evidence}

High certainty: We are very confident that the true effect lies close to that of the estimate of the effect

Moderate certainty: We are moderately confident in the effect estimate: The true effect is likely to be close to the estimate of the effect, but there is a possibility that it is substantially different

Low certainty: Our confidence in the effect estimate is limited: The true effect may be substantially different from the estimate of the effect

Very low certainty: We have very little confidence in the effect estimate: The true effect is likely to be substantially different from the estimate of effect

1 Assumed risk calculated from the sum of control group event rates.

2 Downgraded due to serious imprecision in effect estimate (width $95 \% \mathrm{Cl}$ around $\mathrm{RR}>0.6$ ).

\section{Summary of findings 3. HPV vaccine effects in adolescent girls and women regardless of HPV DNA status at baseline}

\section{HPV vaccine effects on cervical lesions in adolescent girls and women unselected for HPV DNA status at baseline}

Patient or population: adolescent girls and women aged 15 to 45 years regardless of HPV DNA status at baseline

Setting: Europe, Asia Pacific countries, South \& North America and Africa

Intervention: HPV vaccines (at least one dose of bivalent or quadrivalent vaccines)

Comparison: Placebo

\begin{tabular}{|c|c|c|c|c|c|c|}
\hline \multirow[t]{2}{*}{ Outcomes } & \multicolumn{2}{|c|}{ Anticipated absolute effects ${ }^{\star}(95 \% \mathrm{Cl})$} & \multirow{2}{*}{$\begin{array}{l}\text { Relative effect } \\
(95 \% \mathrm{CI})\end{array}$} & \multirow{2}{*}{$\begin{array}{l}\text { № of partici- } \\
\text { pants } \\
\text { (studies) }\end{array}$} & \multirow{2}{*}{$\begin{array}{l}\text { Certainty of } \\
\text { the evidence } \\
\text { (GRADE) }\end{array}$} & \multirow[t]{2}{*}{ Comments } \\
\hline & $\begin{array}{l}\text { Risk with } \\
\text { placebo }\end{array}$ & $\begin{array}{l}\text { Risk with HPV vacci- } \\
\text { nation } 1\end{array}$ & & & & \\
\hline Cervical cancer - not measured & - & - & - & - & - & \\
\hline CIN2+ associated with HPV16/18 & \multicolumn{2}{|l|}{15 to 26 years } & $\begin{array}{l}\text { RR } 0.46 \\
\text { (0.37 to } 0.57\end{array}$ & $\begin{array}{l}34,852 \\
\text { (3 RCTs) }\end{array}$ & $\begin{array}{l}\oplus \oplus \oplus \oplus \\
\mathrm{HIGH}\end{array}$ & \\
\hline
\end{tabular}




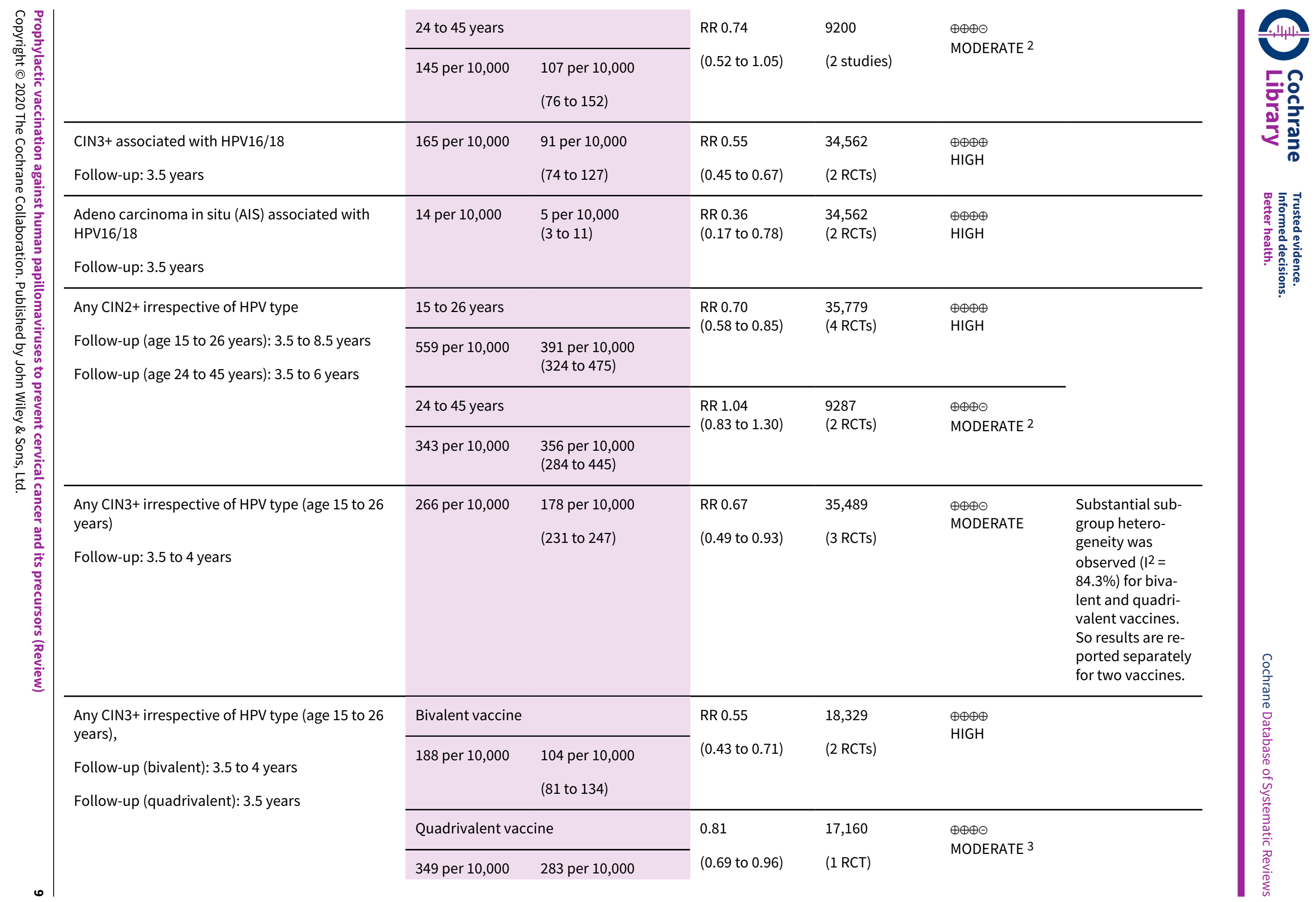




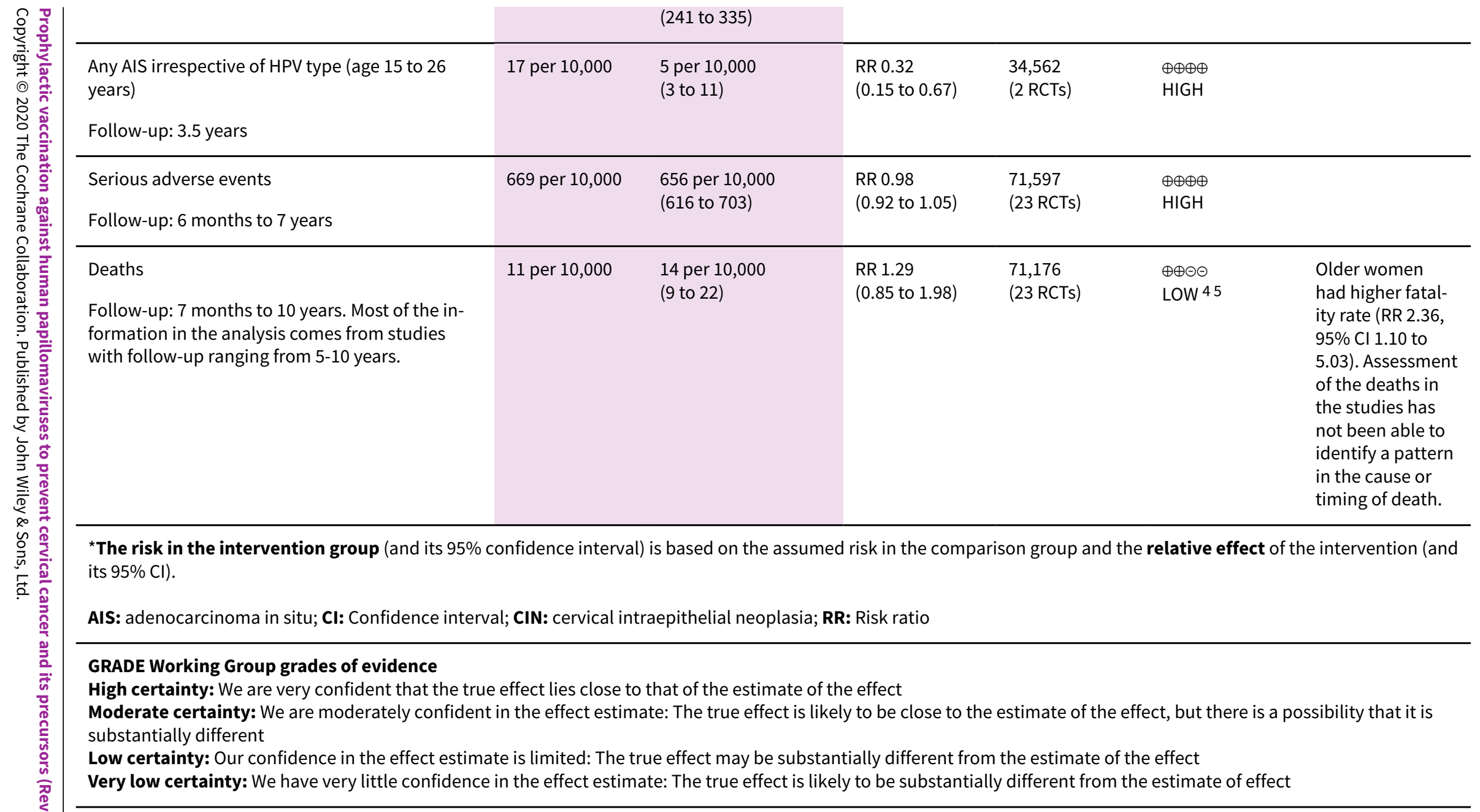

1 Assumed risk calculated from the sum of control group event rates for all outcomes unless otherwise stated.

2 Downgraded due to serious imprecision. Confidence interval is wide and includes large decrease and small increase in lesions with vaccination group in the older age group.

3 Downgraded one level due to serious inconsistency. Reduction in lesions was greater in younger women than in older women (RR 0.46 in 15 to 26 years versus RR 0.74 in 24 to 45 years; $P=0.02$ for interaction).

4 Downgraded one level due to serious imprecision. Confidence interval includes potentially meaningful increase in risk of mortality.

5 Downgraded one level due to serious inconsistency. Despite limited evidence of statistical variation, sub grouping studies by age showed higher fatality rate with vaccines in older age group. There is no clear pattern in causes or timing of deaths. 


\begin{tabular}{|c|c|c|c|c|c|}
\hline \multicolumn{6}{|c|}{ HPV vaccine adverse pregnancy outcomes (regardless of DNA status and age) } \\
\hline \multicolumn{6}{|c|}{$\begin{array}{l}\text { Patient or population: adolescent girls and women aged } 15 \text { to } 45 \text { years who became pregnant during the study } \\
\text { Setting: Europe, Asia Pacific, North, Central and South America } \\
\text { Intervention: HPV vaccines (bivalent or quadrivalent vaccines) } \\
\text { Comparison: Placebo }\end{array}$} \\
\hline \multirow[t]{2}{*}{ Outcomes } & \multicolumn{2}{|c|}{ Anticipated absolute effects ${ }^{\star}(95 \% \mathrm{Cl})$} & \multirow{2}{*}{$\begin{array}{l}\text { Relative effect } \\
(95 \% \mathrm{Cl})\end{array}$} & \multirow{2}{*}{$\begin{array}{l}\text { № of partici- } \\
\text { pants } \\
\text { (studies) }\end{array}$} & \multirow{2}{*}{$\begin{array}{l}\text { Certainty of } \\
\text { the evidence } \\
\text { (GRADE) }\end{array}$} \\
\hline & Risk with placebo & Risk with HPV vaccines & & & \\
\hline \multirow{2}{*}{$\begin{array}{l}\text { Spontaneous abortion/miscarriage } \\
\text { Follow-up: } 1 \text { to } 7 \text { years }\end{array}$} & \multicolumn{2}{|l|}{ Study population } & \multirow{2}{*}{$\begin{array}{l}\text { RR } 0.88 \\
\text { (0.68 to } 1.14)\end{array}$} & \multirow{2}{*}{$\begin{array}{l}8618 \\
\text { (9 RCTs) }\end{array}$} & \multirow{2}{*}{$\begin{array}{l}\oplus \oplus \oplus \oplus \\
\mathrm{HIGH}\end{array}$} \\
\hline & 1618 per 10,000 & $\begin{array}{l}1,424 \text { per } 10,000 \\
(1,100 \text { to } 1844)\end{array}$ & & & \\
\hline $\begin{array}{l}\text { Elective termination/induced abor- } \\
\text { tion }\end{array}$ & \multicolumn{2}{|l|}{ Study population } & \multirow{2}{*}{$\begin{array}{l}\text { RR } 0.90 \\
\text { (0.80 to } 1.02)\end{array}$} & \multirow{2}{*}{$\begin{array}{l}10,909 \\
\text { (9 RCTs) }\end{array}$} & \multirow{2}{*}{$\begin{array}{l}\oplus \oplus \oplus \oplus \\
\mathrm{HIGH}\end{array}$} \\
\hline Follow-up: 1 to 7 years & 931 per 10,000 & $\begin{array}{l}838 \text { per } 10,000 \\
(745 \text { to } 950)\end{array}$ & & & \\
\hline \multirow{2}{*}{$\begin{array}{l}\text { Stillbirth } \\
\text { Follow-up: } 1 \text { to } 3.5 \text { years }\end{array}$} & \multicolumn{2}{|l|}{ Study population } & \multirow{2}{*}{$\begin{array}{l}\text { RR } 1.12 \\
\text { (0.68 to } 1.83 \text { ) }\end{array}$} & \multirow{2}{*}{$\begin{array}{l}8754 \\
\text { (6 RCTs) }\end{array}$} & \multirow{2}{*}{$\begin{array}{l}\oplus \oplus \oplus \odot \\
\text { MODERATE } 2\end{array}$} \\
\hline & 70 per 10,000 & $\begin{array}{l}78 \text { per } 10,000 \\
(48 \text { to } 128)\end{array}$ & & & \\
\hline $\begin{array}{l}\text { Babies born with congenital mal- } \\
\text { formations }\end{array}$ & \multicolumn{2}{|l|}{ Study population } & \multirow{2}{*}{$\begin{array}{l}\text { RR } 1.22 \\
\text { (0.88 to } 1.69)\end{array}$} & \multirow{2}{*}{$\begin{array}{l}9252 \\
(5 \mathrm{RCTs})\end{array}$} & \multirow{2}{*}{$\begin{array}{l}\oplus \oplus \oplus \odot \\
\text { MODERATE } 2\end{array}$} \\
\hline Follow-up: 3 to 7 years & 205 per 10,000 & $\begin{array}{l}250 \text { per } 10,000 \\
(180 \text { to } 346)\end{array}$ & & & \\
\hline \multicolumn{6}{|c|}{$\begin{array}{l}{ }^{*} \text { The risk in the intervention group (and its } 95 \% \text { confidence interval) is based on the assumed risk in the comparison group and the relative effect of the intervention (anc } \\
\text { its } 95 \% \mathrm{Cl} \text { ). }\end{array}$} \\
\hline \multicolumn{6}{|c|}{$\begin{array}{l}\text { GRADE Working Group grades of evidence } \\
\text { High certainty: We are very confident that the true effect lies close to that of the estimate of the effect } \\
\text { Moderate certainty: We are moderately confident in the effect estimate: The true effect is likely to be close to the estimate of the effect, but there is a possibility that it is } \\
\text { substantially different } \\
\text { Low certainty: Our confidence in the effect estimate is limited: The true effect may be substantially different from the estimate of the effect } \\
\text { Very low certainty: We have very little confidence in the effect estimate: The true effect is likely to be substantially different from the estimate of effect }\end{array}$} \\
\hline
\end{tabular}




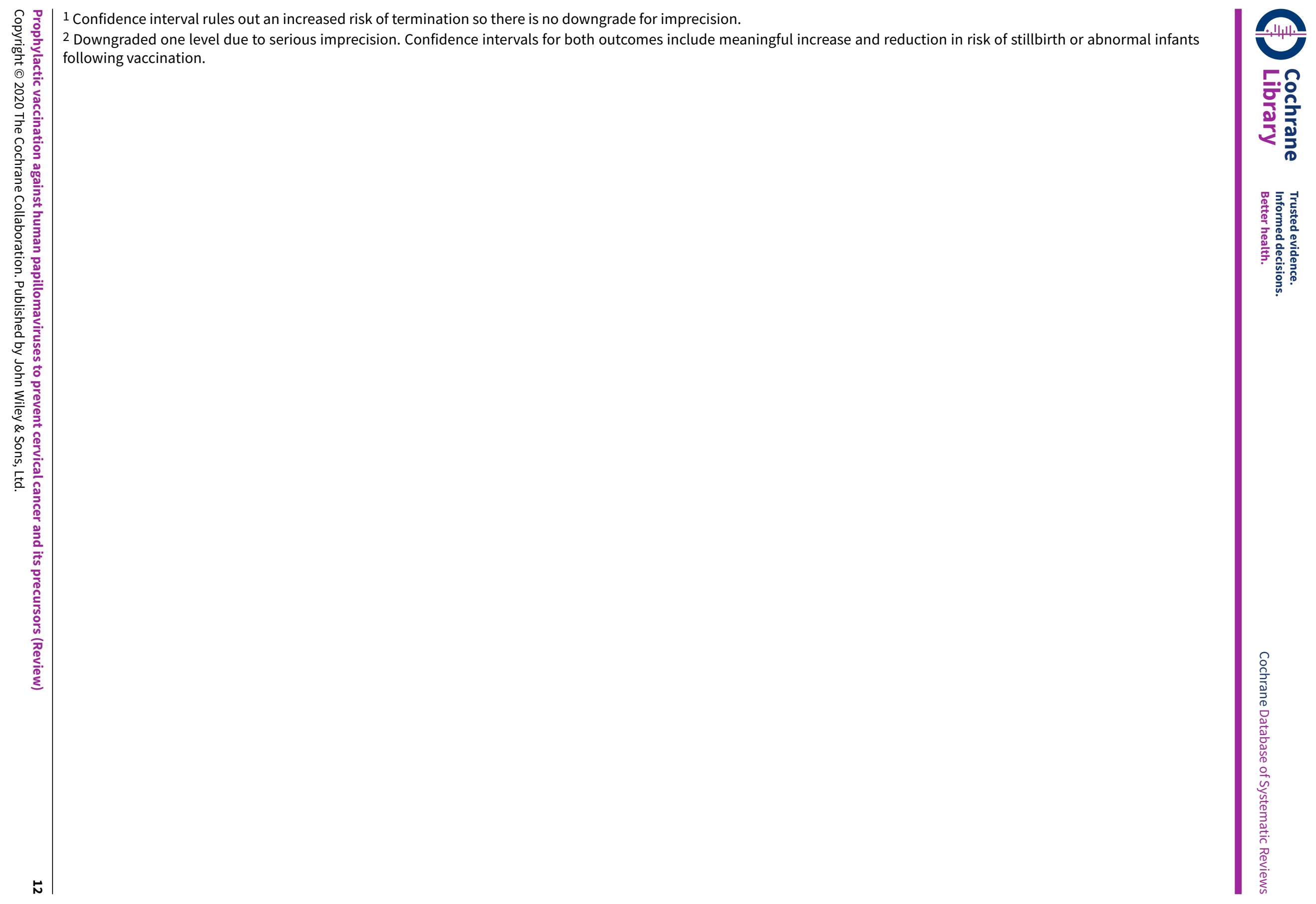




\section{B A C K G R O U N D}

\section{Description of the condition}

\section{Burden of cervical cancer}

Cervical cancer is the fourth most common cancer in women worldwide. It is estimated that in 2012, approximately 528,000 women developed cervical cancer and that 266,000 died from the disease (Ferlay 2015). Eighty-six per cent of cervical cancer cases occur in developing countries (Arbyn 2011). Cervical cancer is the predominant cancer in women in Eastern Africa, South-Central Asia and Melanesia, where a woman's risk of developing this disease by age 75 years ranges between $2.3 \%$ and $3.9 \%$. In many developed countries, the incidence of, and mortality from, squamous cervical cancer has dropped substantially over the last decades, as a consequence of population-based screening programmes (Arbyn 2009; Bray 2005a; Ferlay 2015; IARC 2005). However, approximately 54,000 and 11,000 cases are reported each year in Europe and the USA, respectively (Arbyn 2011; Ferlay 2013), and screening with cytology is less effective at preventing cervical adenocarcinoma (Bray 2005; Smith 2000). In contrast to many other malignancies, cervical cancer primarily affects younger women, with the peak age of incidence in the UK now between 25 and 29 years; between 2012 and $2014,52 \%$ of cancers occurred in those under 45 years of age (Cancer Research UK 2018). In the UK (2010 to 2011), despite a comprehensive screening programme, $37 \%$ of women with cervical cancer died from the disease within 10 years of diagnosis (Cancer Research UK 2018).

High-grade cervical intraepithelial neoplasia (CIN2+) is treated by local destruction (ablation) or excision of neoplastic tissue (Jordan 2009). Therapeutic procedures are similarly effective (Martin-Hirsch 2013), but are associated with an average risk of residual or recurrent $\mathrm{CIN} 2+$ of $7 \%$ (Arbyn 2017), and an increased risk of late miscarriage and premature labour (Kyrgiou 2017). Primary prevention of CIN lesions by prophylactic (an agent used to prevent disease) vaccination can therefore reduce the burden, costs and adverse effects associated with its treatment.

\section{Association between human papillomavirus (HPV) infection and cervical cancer and other HPV-related cancers and their precursors}

Papillomaviruses are small, icosahedral DNA viruses, that consist of one single double-stranded circular DNA molecule of approximately 8,000 base-pairs, contained within a protein capsid. The capsid is composed of two structural proteins, both are encoded by the viral genome: L1 and L2 (IARC 2007). The natural history of HPV infection towards cervical precancer and finally invasive cancer is well documented (Bosch 2002; Castellsagué 2006; IARC 2007). The development of cervical cancer passes through a number of phases: (a) infection of the cervical epithelium with highrisk human papillomaviruses (hrHPV); (b) persistence of the HPV infection; (c) progression to precancerous lesions with malignant transformation of infected cells; and (d) invasion of surrounding tissue. The steps prior to development of cancer, can regress spontaneously, although regression rates decrease with increasing severity of the precancerous lesion.

Twelve hrHPV types (HPV16, 18, 31, 33, 35, 39, 45, 51, 52, 56, 58, and 59) are causally linked with the development of cervical cancer (Bouvard 2009). HPV68 is considered as probably carcinogenic (Schiffman 2009). Some other HPV types may in rare occasions also cause cervical cancer (Arbyn 2014). HPV type 16, in particular, has a high potential for malignant transformation of infected cervical cells (Schiffman 2005). The HPV types 16 and 18 jointly cause seven out of 10 cervical cancers worldwide (Munoz 2004). The five next most important high-risk HPV types (HPV31, HPV33, HPV45, HPV52, and HPV58) together with HPV16/18 are causally linked with approximately $90 \%$ of cervical cancers (de Sanjose 2010). HPV16 is also linked with rarer types of cancer, such as cancer of the vulva and vagina in women, penile cancer in men and anal and oropharyngeal cancer in women and men (Cogliano 2005; IARC 2007).

The low-risk HPV types 6 and 11 cause approximately $90 \%$ of genital warts in women and men (Lacey 2006). They occur in low-grade dysplastic cervical lesions, but are not associated with developing cervical cancer (IARC 2007). HPV types 6 and 11 cause recurrent respiratory papillomatosis, a rare but very serious disease of the upper airways often requiring repetitive surgical interventions (Lacey 2006).

The main route of HPV transmission is sexual contact. Infection usually occurs soon after the onset of sexual activity (Winer 2003; Winer 2008). The prevalence of HPV infection in women generally peaks in late teenage or early twenties (de Sanjose 2007). HPV infection is usually cleared by the immune system (Ho 1998). HPV infection can result in cervical precancer (cervical intraepithelial neoplasia (CIN)), which can be detected by cervical cytological screening. By microscopic inspection of a cervical smear (also known as a Papanicolaou or 'Pap' test, cervical lesions can be detected (atypical squamous cells of undetermined significance (ASC-US), low-grade squamous intraepithelial lesion (LSIL), highgrade squamous intraepithelial lesion (HSIL),: atypical glandular cells (AGC); for a complete list of abbreviations used in the review, see Appendix 1), which can be confirmed histologically following a cervical biopsy at colposcopic examination (Jordan 2008). In some countries, cytological cervical cancer screening is being replaced by HPV-based screening, because the latter is more effective at preventing future CIN3 or invasive cancer (Arbyn 2012; Ronco 2014).

A World Health Organization (WHO) expert group accepted a reduction in the incidence of high-grade $\mathrm{CIN}(\mathrm{CIN} 2+)$ and cervical adenocarcinoma in situ (AIS) or worse as an acceptable surrogate outcome of HPV vaccination trials (Pagliusi 2004). This is because the reduction of the incidence of invasive cervical cancer would require large and very long-term studies, which are unlikely to be undertaken. The progression of HPV infection to invasive cancer is thought to take a minimum of 10 years (IARC 2007). Although CIN can regress, from historical data, it has been estimated that CIN3 has a probability of progressing to invasive cancer of $12 \%$ to $30 \%$, whereas for CIN2 this probability is substantially lower (McCredie 2008; Ostor 1993).

The recognition of the strong causal association between HPV infection and cervical cancer led to the development of molecular HPV assays to detect cervical cancer precursors (Iftner 2003), and of vaccines that prevent HPV infection (prophylactic vaccines) or that aim to treat present HPV infection or HPV-induced lesions (therapeutic vaccines) (Frazer 2004; Galloway 2003; Schneider 2003). Therapeutic vaccines are still in early experimental phases and are not further considered in this review.

Throughout this review, we will use the 2001 Bethesda System to define cytologically-defined neoplastic lesions of the cervical 
epithelium (Solomon 2002) and the CIN nomenclature to define histologically-confirmed CIN (Richart 1973).

\section{Description of the intervention}

The intervention evaluated in this review is prophylactic vaccination against the most carcinogenic HPV types. Prophylactic HPV vaccines are composed of virus-like particles (VLPs) of the L1 protein, which is the major protein of the capsid (shell) of the HPV virus. VLPs, do not contain viral DNA, and so are incapable of causing an active infection.

This review addresses evidence of three prophylactic HPV vaccines that have been clinically evaluated in randomised controlled trials (RCTs): a monovalent HPV16 vaccine (manufactured by Merck, Sharpe \& Dome (Merck), Whitehouse Station, NJ, USA); a quadrivalent vaccine, containing the L1 protein of HPV6, HPV11, HPV16 and HPV18 (Gardasil ${ }^{\circledR}$, produced by the same manufacturer as the monovalent vaccine); and a bivalent vaccine containing $L 1$ of HPV types 16 and 18 (Cervarix ${ }^{\circledR}$, produced by GlaxoSmithKline (GSK), Rixensart, Belgium). The vaccines produced by Merck contain amorphous aluminium hydroxyphosphate sulphate as an adjuvant, whereas the GSK vaccine contains aluminium salt and AS04 or monophosphoryl lipid A, which is an immunostimulating molecule (WHO 2009). Recently, a nona-valent vaccine targeting nine HPV types (HPV types 6, 11, 16, 18, 31, 33, 35, 45, 52 and 58) has been developed by Merck. We did not include the nona-valent vaccine in the current review, since the randomised trials assessing the efficacy of the nona-valent vaccine did not incorporate an arm with a non-HPV vaccine control, Nevertheless, data regarding the nona-valent vaccine are included in the Discussion. More details about the prophylactic HPV vaccines used are described in Appendix 2.

\section{How the intervention might work}

Animal experiments have shown that neutralising antibodies, elicited by vaccination with papillomavirus VLPs, prevent typespecific infection and subsequent development of lesions after viral challenge (Breitburd 1995; Ghim 2000; Stanley 2006). Vaccination by intramuscular injection of L1 VLPs in humans has been demonstrated to be highly immunogenic in phase I trials, which means that they induce high titres of anti-HPV antibodies in serum which are considerably higher than after natural infection. (Ault 2004; Brown 2001; Evans 2001; Harro 2001). Serum anti-L1 antibodies can transudate to the mucosa (cervical or other sites) where new HPV infection is impeded through virus-neutralisation (Stanley 2012). Prophylactic HPV vaccines may also induce specific memory B-lymphocytes which play a role in long-term humoral immunity (Giannini 2006). Anti-HPV antibodies do not trigger the elimination of an existing HPV infection. Cell-mediated immunity is required for viral clearance and regression of CIN lesions (Stanley 2012).

\section{Why it is important to do this review}

Several phase II and III studies have been conducted to date and numerous reviews have tried to summarise the results (Ault 2007; Arbyn 2007; Harper 2009; Initiative 2009; Kahn 2009; Kjaer 2009; Koutsky 2006; Lu 2011; Medeiros 2009; Rambout 2007; Szarewski 2010). However, none of the reviews combined information on all the available endpoints. Our purpose was to pool efficacy outcomes only when outcomes were similarly defined, taking the timing of follow-up into account. This review is also important since it provides a template for reporting future results of prophylactic vaccination trials according to the different outcomes (infections or cervical precancerous lesions, either associated with infection with vaccine types or irrespective of HPV infection) for different exposure groups (defined essentially by absence of hrHPV, absence of the HPV types included in the vaccine, or regardless of HPV infection at enrolment). Particular effort was undertaken to assess severe adverse effects in order to inform health professionals, stakeholders, adolescent girls and women, not only about the potential beneficial effects of HPV vaccines but also about possible harms.

\section{OBJECTIVES}

To evaluate the harms and protection of prophylactic human papillomaviruses (HPV) vaccines against cervical precancer and HPV16/18 infection in adolescent girls and women.

\section{METHODS}

\section{Criteria for considering studies for this review}

\section{Types of studies}

We considered only phase II and phase III randomised controlled trials (RCTs).

\section{Types of participants}

We included studies enrolling female participants, without any age restriction, distinguishing:

1. female participants with no evidence of baseline infection with high-risk human papillomaviruses (hrHPV) types (this group reflects the first target of basic vaccination programmes, i.e. girls before onset of sexual activity);

2. female participants with no evidence of baseline infection with HPV types included in the vaccines (per protocol population);

3. all female participants regardless of baseline infection with HPV (this group reflects the target of catch-up vaccination programs, adolescents or young adult women aged 15 to 26 years, where a considerable proportion may already have been exposed to HPV infection).

The distinction of different participant categories by HPV status at enrolment is essential, since the trial outcomes are expected to differ in women who are already infected with HPV types included in the vaccine and those who are not infected, Further distinction was made by:

1. broad age group (adolescents and young adult women, aged 15 to 26 years) and mid-adult women ( 25 to 45 years);

2. number of received doses: three doses in agreement with the trial protocol, at least one dose, and fewer than three doses (the latter analysis being a post-hoc assessment);

3. type of vaccine received (mono-, bi- or quadrivalent vaccine).

Studies with male participants or special target groups such as immunocompromised patients were not included. However, trials enrolling both female and male participants were potentially eligible under the condition that separate outcomes for female participants were reported or could be obtained from the authors. 


\section{Types of interventions}

\section{Intervention}

Vaccination with prophylactic HPV vaccines containing virus-like particles composed of the L1 capsid protein of HPV16 (monovalent vaccine), HPV16 and HPV18 (bivalent vaccine), or HPV6, HPV11, HPV16 and HPV18 (quadrivalent vaccine) (see Appendix 2). All vaccines were administered by intramuscular injection over a period of six months. The monovalent and quadrivalent vaccines were injected at zero, two and six months, whereas the bivalent vaccine was administered at zero, one and six months.

\section{Comparison}

Administration of placebo containing no active product or only the adjuvant of the HPV vaccine, without L1 VLP, or another non-HPV vaccine.

In head-to-head trials comparing directly the bivalent with the quadrivalent vaccine, participants who received the bivalent vaccine constituted the experimental group and participants who received the quadrivalent vaccine were considered as the comparison group.

\section{Types of outcome measures}

\section{Primary outcomes}

1. Histologically-confirmed high-grade cervical intraepithelial neoplasia (CIN2, CIN3 and adenocarcinoma in situ (AIS)) or worse, associated with the HPV types included in the vaccine or any lesions irrespective of HPV type. Association between HPV types and a diagnosed lesion means that the particular type or types have been detected in that lesion. These primary outcomes were judged by WHO to be adequate endpoints (Pagliusi 2004).

2. Invasive cervical cancer.

3. Safety/occurrence of adverse effects:

i. local adverse effects (redness, swelling, pain, itching at the injection site);

ii. mild systemic effects;

iii. serious systemic effects;

iv. mortality;

v. pregnancy outcomes observed during the trials, in particular occurrence of congenital anomalies.

\section{Secondary outcomes}

1. Incident infection with vaccine HPV types (HPV16 and HPV18, jointly; and HPV6, HPV11, HPV16 and HPV18 jointly).

2. Persistent infection (persisting during at least six months or at least 12 months) with vaccine HPV types.

\section{Search methods for identification of studies}

We searched for papers in all languages and translations were undertaken, if necessary.

\section{Electronic searches}

We retrieved published studies from the Cochrane Central Register of Controlled Trials (CENTRAL the Cochrane Library), MEDLINE and Embase.
Cochrane Central Register of Controlled Trials (CENTRAL 2002 to 2017, Issue 5).

MEDLINE (2002 to June Week 12017 ).

Embase (2002 to 2017 week 24).

The search strategies for MEDLINE, CENTRAL and Embase are listed in Appendix 3, Appendix 4 and Appendix 5.

The search string for MEDLINE was saved in $M y N C B I$, an electronic search tool developed by the National Center for Biotechnology Information (NCBI) at the National Library of Medicine, which saves searches and automatically retrieves newer references not pickedup at previous searches. An auto-alert was set up in Embase.

The 'related articles' feature in PubMed was used, departing from the original included studies; similarly, Scopus was used to retrieve articles which cite the originally included studies.

We searched databases were searched from 2002 (the year of publication of the results of the first phase II trial) until June 2017.

\section{Searching other resources}

\section{Registries of randomised trials}

We searched the following registries to identify unpublished or ongoing trials: www.clinicaltrials.gov, www.isrctn.com, and www.cancer.gov/clinicaltrials.

Data on adverse effects published in the peer-reviewed literature were complemented by searches in wwww.clinicaltrials.gov for the quadrivalent vaccine and on http://www.gskclinicalstudyregister.com/ for the bivalent vaccine. We collected data for the outcomes of serious adverse events, all-cause mortality and pregnancy outcomes from these sources and compared them with data extracted from the primary trial publications.

\section{International public health organisations}

We contacted international public health organisations that have investigated questions on HPV vaccine efficacy and safety or that have formulated recommendations on the use of HPV vaccines, to retrieve key documents. Concerned organisations included: the World Health Organization (WHO, Geneva), the US Centers for Disease Control and Prevention (CDC, Atlanta), the European Centre for Disease Prevention and Control (ECDC, Stockholm), and the International Agency for Research on Cancer (IARC, Lyon).

\section{Handsearching}

We handsearched the citation lists of included studies.

In addition, we searched the abstracts of the latest conferences of relevant scientific societies related to vaccination, virology (in particular the International Papillomavirus Society), paediatrics, and gynaecology for new or pending information not yet published in peer-reviewed journals.

\section{Correspondence}

We contacted study authors to request results on effects separated by gender, if the reports only contained data combined for both genders. 


\section{Data collection and analysis}

\section{Selection of studies}

We downloaded all titles and abstracts retrieved by electronic searching to a bibliographic database stored in Reference Manager We added any references obtained by handsearching and removed any duplicates.

We (MA, CS and LX) independently verified inclusion and exclusion of eligible studies and discussed any disagreements. In case of doubt, the full-text report was read. If no consensus could be reached, review author $\mathrm{PMH}$ was consulted. We documented reasons for exclusion.

\section{Data extraction and management}

For included studies, we extracted the following study characteristics and outcome data.

1. Study identification: first author, year of publication, journal, trial number.

2. Geographical area where the study was conducted.

3. Period when study was conducted.

4. Inclusion and exclusion criteria.

5. Characteristics of included participants (total number enrolled, age, number of previous sexual partners).

6. Initial HPV status (presence or absence of hrHPV DNA; presence or absence of DNA of the vaccine HPV types; serological status (presence of antibodies against vaccine HPV types) at enrolment). Differences in efficacy outcomes by initial HPV status will reflect protection in women or girls previously exposed, or not exposed to prior HPV infection.

7. Study design:

a. phase of the randomised trial (II or III);

b. type of vaccine evaluated (monovalent, bivalent, or quadrivalent);

c. control group: type of placebo or other vaccine administered;

d. time points (mean duration of follow-up after first dose) at which outcomes were collected and reported;

e. study size at enrolment and at subsequent time points of follow-up;

f. number of doses received;

g. scheduling of screening tests (HPV tests, cytology);

h. diagnostic algorithms used to confirm outcomes;

i. definition of study groups on which per-protocol and intention-to-treat analyses were applied;

j. risk of bias in study design (see below: Assessment of risk of bias in included studies).

8. Outcomes, subdivided by (i) the association with vaccine HPV types and (ii) irrespective of HPV types:

a. outcome definition (including diagnostic criteria and assays);

b. results: number of participants allocated to each intervention group; number of missing values and absolute values required to compute effect measures (see Types of outcome measures);

c. data for the efficacy outcomes and short-term adverse events relating to the injection procedures were collected from primary trial publications. For outcomes relating to serious adverse events, all-cause mortality and pregnancy outcomes, data were cross-checked between trial registries, study results websites, correspondence with investigators and the primary trial reports. The primary analysis used the data derived from the reports with the longest followup time. A sensitivity analysis on serious adverse effects and mortality was restricted to data derived from reports published in peer-reviewed journals.

9. Involvement of manufacturers.

We extracted data on outcomes as follows.

1. For dichotomous outcomes, we extracted the number of participants in each treatment arm who experienced the outcome of interest and the number of participants assessed at endpoint in order to estimate a risk ratio (RR) or risk difference (RD). Where possible, we also extracted the number of personyears at risk in order to compute incidence rates and incidence rate ratios or differences.

We (MA, CS until 2011 and LX from 2012) independently extracted data onto a data abstraction form specially designed for the review. Differences between review authors were resolved by discussion or by appeal to a third review author (PMH) if necessary.

\section{Assessment of risk of bias in included studies}

We assessed the risk of bias in included RCTs using Cochrane's 'Risk of bias' tool and the criteria specified in chapter 8 of the Cochrane Handbook for Systematic Reviews of Interventions (Higgins 2011a). This included assessment of:

1. the method used for randomisation to generate the sequence of participants allocated to the treatment arms;

2. allocation concealment;

3. blinding (of participants, healthcare providers;

4. blinding of outcome assessment;

5. reporting of incomplete outcome data for each outcome;

6. selective reporting of outcomes.

We (MA, CS and LX) independently applied the Cochrane 'Risk of bias' tool and differences were resolved by discussion or by appeal to a third review author (PMH). Results were presented in both a 'Risk of bias' graph and a 'Risk of bias' summary. We interpreted the results of meta-analyses in the light of the findings with respect to risk of bias.

\section{Measures of treatment effect}

We computed risk ratios (RR) from the ratio of proportions or rates of events among vaccine recipients versus placebo recipients. In the literature, protection against HPV infection or cervical precancer is usually presented as vaccine efficacy (VE), VE $=(1-R R)^{\star} 100$. However, pooling of VE is not supported by the Review Manager (RevMan) software (Review Manager 2014). Where perfect efficacy corresponds with $\mathrm{VE}=100 \%$, the corresponding $\mathrm{RR}=0$; VE $=$ $0 \%$ or RR $=1$ means absence of protection. Negative VE or RR exceeding unity reflect adverse protection (vaccinated participants are more at risk than non-vaccinated participants). When the $95 \%$ confidence interval contains unity, the protective effect is statistically insignificant. The number of participants needed to vaccinate (NNV) to avoid one outcome event was computed from the risk difference (NNV = 1/RD). 
To show vaccination effects at population level, Cates plots were drawn, showing effects in 1000 vaccinated and 1000 nonvaccinated women (Cates 2015).

\section{Dealing with missing data}

We contacted study authors or data owners to request data on the outcomes, separated by gender, if the reports only contained data combined for both genders in trials where both women and men were enrolled. We did not impute missing outcome data.

\section{Assessment of heterogeneity}

We assessed heterogeneity between studies by visual inspection of forest plots, by estimation of the percentage heterogeneity between trials that could not be ascribed to sampling variation (Higgins 2003), by a formal statistical test of the significance of the heterogeneity (Deeks 2001) and, if possible, by subgroup analyses. If there was evidence of substantial heterogeneity, we investigated and reported the possible reasons.

In order to avoid heterogeneity, we did not combine data series from participants with different baseline HPV status (presence of hrHPV DNA, presence of DNA of the HPV vaccine types). Age group and sexual history were investigated as potential sources that could explain possible heterogeneity.

\section{Assessment of reporting biases}

We planned to construct funnel plots and to perform regression tests to identify asymmetry in the meta-analysis (Harbord 2009). However, since each meta-analysis contained seven or fewer studies, this was not feasible. Instead, we performed metaregression to identify possible small-study effects grouping studies in two categories: large $(>=3000$ participants) and small $(<3000$ participants).

\section{Data synthesis}

Random-effects models with inverse variance weighting were applied using the RevMan 5 (DerSimonian 1986). From the pooled RR, VE was computed (VE $=1-R R)$. Pooled risk differences were computed also for the 'Summary of findings' tables.

\section{Subgroup analysis and investigation of heterogeneity}

We performed separate analyses determined by the participant's HPV status as defined by the result of an HPV DNA tests at enrolment. Three groups were distinguished: a) initially hrHPV DNA negative, b) initially HPV16/18 DNA negative, and c) regardless of initial HPV DNA test results. Subgroup meta-analyses were performed, if possible, using vaccine type and age group as a stratifying variable. We distinguished younger (15 to 26 years) from mid-adult women (24 to 45 years), which were the two age groups assessed in the available randomised trials. If efficacy estimates were not significantly different by vaccine type, jointly pooled estimates were retained. Only when significant heterogeneity by vaccine types was noted, were separate efficacy estimates by vaccine type pooled. We used meta-regression to investigate sources of heterogeneity such as serological status, study design items, study size and sexual history (Sharp 1998; Thompson 1999). The log relative risk (vaccinated versus placebo recipients) was used as the dependent variable. The antilog of coefficients of the meta-regression yielded relative risk ratios (RRR). 95\% confidence intervals around the RRR excluding unity indicated a statistically influential factor.

We assessed the influence of covariates, which were not defined uniformly throughout the trials, by Poisson regression in each trial concerned, separately and using person-years at risk as an offset.

A posteriori analysis was performed to investigate vaccine efficacy in women who had received fewer than three doses of vaccine, by subtraction of the number of events and total number of participants who had received three doses from those who had received at least one dose. This was the only possible approach, since outcomes stratified by number of doses received, were not usually reported in the published papers.

\section{Sensitivity analysis}

We assessed the robustness of data collected for serious adverse events, all-cause mortality and pregnancy outcomes based on the source of data. The primary analysis for these outcomes included data that we considered to represent the most complete follow-up. As a sensitivity analysis we used data for these same outcomes that had only been reported in the published trial reports.

\section{'Summary of findings' tables}

We created 'Summary of findings' tables for three populations of interest.

1. Adolescent girls and women who were negative for hrHPV DNA at baseline

2. Adolescent girls and women who were negative for HPV16/18 DNA at baseline

3. Adolescent girls and women regardless of HPV DNA status at baseline

We included findings for the following outcomes for each population.

1. Cervical cancer

2. CIN2+ or CIN3+ associated with HPV16/18; any CIN2+ or CIN3+, irrespective of HPV types

3. AIS associated with HPV/16/18; any AIS irrespective of HPV types

4. All-cause mortality (in all enrolled women)

5. Serious adverse events (in all enrolled women)

We included a fourth table summarising pregnancy outcomes as follows.
1. Spontaneous abortion/miscarriage
2. Elective termination/induced abortion
3. Spontaneous miscarriage
4. Babies born with congenital malformations

Since only randomised clinical trials were included in the review, the rating for each outcome started as high-quality evidence and was downgraded for the following considerations according to GRADE guidance (Higgins 2011b).

\section{Risk of bias}

2. Inconsistency (both quantitative and qualitative)

3. Imprecision (relating to the width of the $95 \%$ confidence interval and number of participants in the analysis) 
4. Indirectness

5. Publication bias

\section{RESULTS}

\section{Description of studies}

\section{Results of the search}

The search in MEDLINE, Embase and CENTRAL, conducted after the publication of the updated protocol (Arbyn 2013), was updated up on 15 June 2017, which resulted in 1685 records and was completed with 112 citations of previously published reviews retrieved from Scopus and reports from the personal CERVIX bibliographic database, yielding 1797 records. After eliminating 34 duplicates, 1763 references were considered from which 1617 could be excluded based on title or objectives described in the abstract. Full reading of the abstracts and materials of 146 papers allowed exclusion of 90 reports. Finally, 56 relevant references describing characteristics and results of 26 randomised trials were selected for this review (Characteristics of included studies). The retrieval and selection of studies is summarised in the PRISMA flow chart in Figure 1 . In addition, we included two reports of pooled analyses of included randomised controlled trials (RCTs) with original data (FUT I/II trials (ph3,4v); PATRICIA \& CVT (ph3,2v)). 
Figure 1. Flow diagram summarising the retrieval, inclusion and exclusion of relevant reports of randomised trials assessing the safety and effects of prophylactic HPV vaccines.

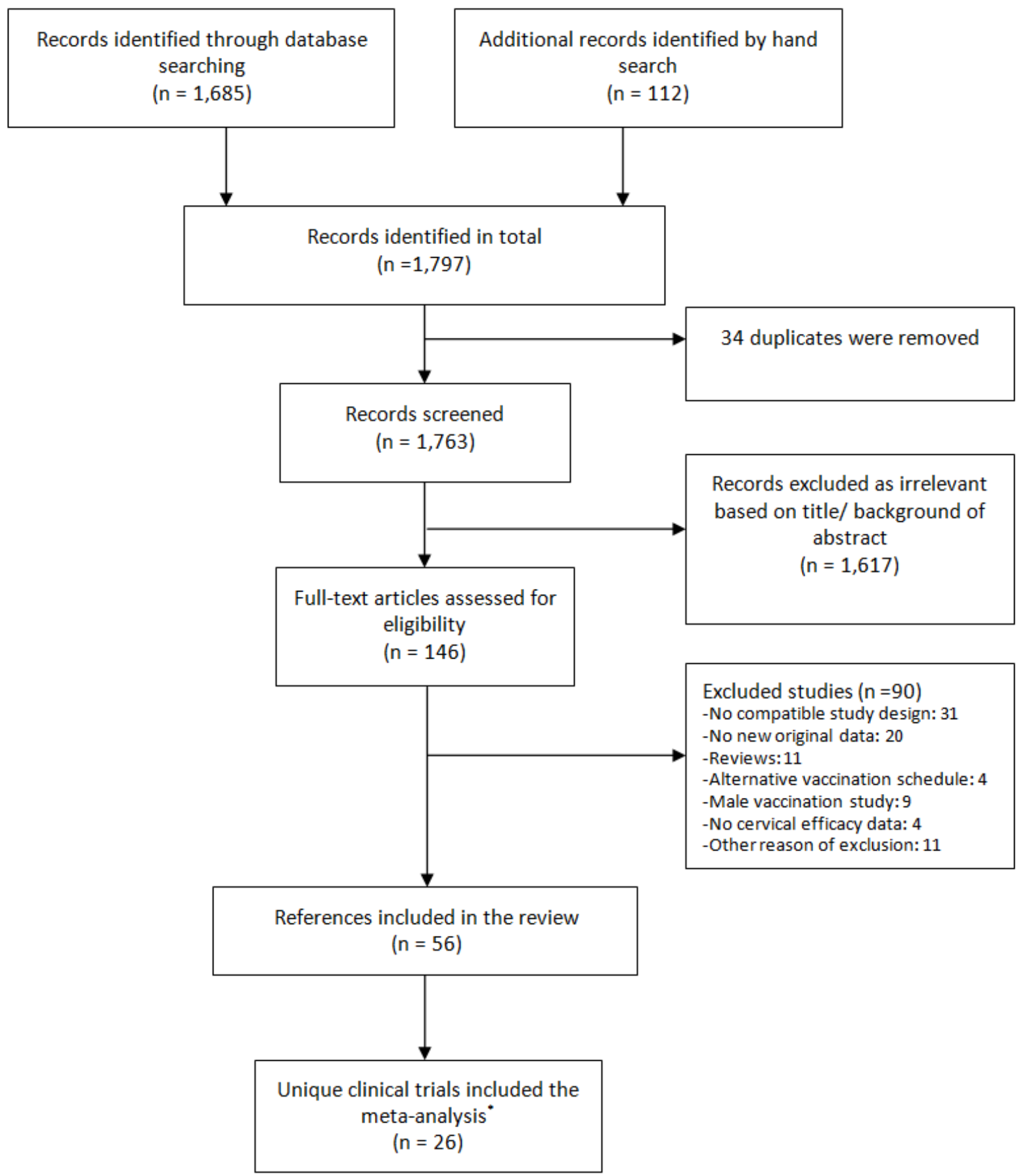

* Certain trials have multiple reports containing extractable data for the review

Details about the completeness of publication of HPV vaccination trials, registered in www.clinicaltrials.gov, can be found in Appendix 6 . No additional studies could be retrieved from www.isrctn.com or www.cancer.gov/clinicaltrials.

\section{Included studies}

Twenty-six randomised trials were identified that contained data on vaccine efficacy and/or safety, which all together enrolled 73,428 women. One trial (Phase2 trial (ph2,1v)) evaluated effects of a monovalent HPV 16 vaccine, 18 trials evaluated the bivalent vaccine 
(African_2 country trial (ph3,2v); Chinese trial (ph3,2v)_adolescent; Chinese trial (ph3,2v)_mid-adult; Chinese trial (ph3,2v)_young; Co-vaccination_dTpa_IPV trial (ph3,2v); Co-vaccination_HAB trial (Ph3, 2v); Co-vaccination_HepB trial (ph3, 2v); CVT (ph3,2v); Hong Kong trial (ph3,2v); Immunobridging(ph3,2v); Indian trial (ph3,2v); Japanese trial (ph2,2v); Korean trial (ph3,2v); Korean trial (ph3b,2v); Malaysian trial (ph3,2v); PATRICIA trial (ph3,2v); Phase2 trial (ph2,2v); VIVIANE trial $(p h 3,2 v))$ and seven others the quadrivalent vaccine (African_3 country trial (ph3,4v); FUTURE III trial (ph3,4v); FUTURE II trial (ph3,4v); FUTURE I trial (ph3,4v); Japanese trial (ph2,4v); Korean trial (ph2,4v); Phase2 trial (ph2,4v)). Six studies were phase II trials and 20 others were phase III trials. No phase I trials were included.

All trials were funded by the respective manufacturers of the vaccines, except one trial, which was financed by the National Cancer Instituite (CVT (ph3,2v)). The study size varied between 98 (African_3 country trial (ph3,4v)) and 18,644 (PATRICIA trial $(\mathrm{ph} 3,2 \mathrm{v}))$. The smaller studies $(<1000)$ essentially assessed safety and immunogenicity (not assessed in this review) or only addressed protection against infection with the HPV vaccine types, whereas the larger phase III trials assessed also protection against cervical precancer (CIN2+, $\mathrm{CIN} 3+$ and AIS+). A listing of the 26 studies ranked by valency of the vaccine, phase (II or III) and alphabetic order is provided in Table 1.
Other characteristics which are not described in Characteristics of included studies, are presented in Appendix 7 and Appendix 8.

\section{Excluded studies}

A list of 90 excluded studies and reasons for exclusion can be found below (Characteristics of excluded studies). We excluded a Chinese study (Li 2012) and an immuno-bridging study (Reisinger 2007), which contained safety and immunogenicity data reported jointly for men and women. We sent a request to the authors for separate data for women but we did not receive a reply from the former and an answer that gender-separated data were not available from the latter.

\section{Risk of bias in included studies}

The assessment of the risk of possible bias present in the selected studies according to the six criteria incorporated in Cochrane's tool for assessing risk of bias in randomised trials (Higgins 2011b) is shown in Characteristics of included studies.

We judged the risk of bias related to the six Cochrane criteria as low in most of the included trials (Figure 2, Figure 3 and Figure 4). We judged the generation of a random sequence as adequate in $24 / 26$ trials ( = 92\%). In two studies, the system used for randomisation was insufficiently described (unclear risk of bias) (Japanese trial (ph2,4v); Japanese trial (ph2,2v)). 
Figure 2. 'Risk of bias' summary: review authors' judgements about each 'Risk of bias' item for each included study.

V1 = Random sequence generation; V2 = Allocation concealment; V3 = Blinding participants \& personnel; V4 = Blinding of outcome assessment; V5 = Incomplete outcomes; V6 = Selective reporting.

\begin{tabular}{|c|c|c|c|c|c|c|c|c|c|c|c|c|c|c|c|}
\hline Valency & Phase & Number & Trial & V1 & V2 & V3 & V4 & v5 & v6 & \#Low & \#Unclear & \#High & $\%$ Low & \%Unclear & $\% \mathrm{High}$ \\
\hline Monovalent & $\|$ & 1 & Phase2 trial (ph2,1v) & $\mathrm{N}$ & $\mathrm{N}$ & $\mathrm{N}$ & $\mathrm{N}$ & $\mathrm{N}$ & $\mathrm{N}$ & 6 & 0 & 0 & $86 \%$ & $0 \%$ & $0 \%$ \\
\hline \multirow[t]{18}{*}{ Bivalent } & \multirow[t]{2}{*}{ ॥ } & \multirow[t]{2}{*}{2} & Japanese trial (ph2, 2v) & $\mathrm{U}$ & $\mathrm{u}$ & $\mathrm{N}$ & $\mathrm{N}$ & $\mathrm{N}$ & $\mathrm{N}$ & 4 & 2 & 0 & $57 \%$ & $29 \%$ & $0 \%$ \\
\hline & & & Phase2 trial (ph2,2v) & $\mathrm{N}$ & $\mathrm{N}$ & $\mathrm{N}$ & $\mathrm{N}$ & $\mathrm{N}$ & $\mathrm{N}$ & 6 & 0 & 0 & $86 \%$ & $0 \%$ & $0 \%$ \\
\hline & \multirow[t]{16}{*}{ III } & \multirow[t]{16}{*}{16} & African_2 country trial (ph3,2v) & $\mathrm{N}$ & $\mathrm{N}$ & $\mathrm{N}$ & $\mathrm{N}$ & $\mathrm{N}$ & $\mathrm{N}$ & 6 & 0 & 0 & $86 \%$ & $0 \%$ & $0 \%$ \\
\hline & & & Chinese trial $(\mathrm{ph} 3, \mathrm{v} 2) \_$young & $\mathrm{N}$ & $\mathrm{N}$ & $\mathrm{N}$ & $\mathrm{N}$ & $\mathrm{N}$ & $\mathrm{N}$ & 6 & 0 & 0 & $86 \%$ & $0 \%$ & $0 \%$ \\
\hline & & & Chinese trial (ph3,v2)_adolescent & $\mathrm{N}$ & $\mathrm{u}$ & u & $\mathrm{u}$ & $\mathrm{N}$ & $\mathrm{N}$ & 3 & 3 & 0 & $43 \%$ & $43 \%$ & $0 \%$ \\
\hline & & & Chinese trial (ph3,v2)_mid_adult & $\mathrm{N}$ & $\mathrm{U}$ & $\mathrm{u}$ & $\mathrm{u}$ & $\mathrm{N}$ & $\mathrm{N}$ & 3 & 3 & 0 & $43 \%$ & $43 \%$ & $0 \%$ \\
\hline & & & Co-vaccination $\mathrm{dT}$ pa IPV trial $(\mathrm{ph} 3,2 \mathrm{v})$ & $\mathrm{N}$ & Y & $\mathrm{U}$ & $\mathrm{u}$ & $\mathrm{N}$ & $\mathrm{N}$ & 3 & 2 & 1 & $43 \%$ & $29 \%$ & $14 \%$ \\
\hline & & & Co-vaccination_HAB trial $(\mathrm{Ph} 3,2 \mathrm{v})$ & $\mathrm{N}$ & $\mathrm{U}$ & $\mathrm{u}$ & $\mathrm{u}$ & $\mathrm{N}$ & $\mathrm{N}$ & 3 & 3 & 0 & $43 \%$ & $43 \%$ & $0 \%$ \\
\hline & & & Co-vaccination_HepB trial $(\mathrm{ph} 3,4 \mathrm{v})$ & $\mathrm{N}$ & $\mathrm{Y}$ & Y & $Y$ & $\mathrm{~N}$ & $\mathrm{~N}$ & 3 & 0 & 3 & $43 \%$ & $0 \%$ & $43 \%$ \\
\hline & & & $\operatorname{cVT}(\mathrm{ph} 3,2 \mathrm{v})$ & $\mathrm{N}$ & $\mathrm{N}$ & $\mathrm{N}$ & $\mathrm{N}$ & $\mathrm{N}$ & $\mathrm{u}$ & 5 & 1 & 0 & $71 \%$ & $14 \%$ & $0 \%$ \\
\hline & & & Hong Kong trial (ph3,2v) & $\mathrm{N}$ & $\mathrm{N}$ & $\mathrm{u}$ & $\mathrm{u}$ & $\mathrm{N}$ & $\mathrm{N}$ & 4 & 2 & 0 & $57 \%$ & $29 \%$ & $0 \%$ \\
\hline & & & Immunobridging (ph3,2v) & $\mathrm{N}$ & $\mathrm{Y}$ & $\mathrm{N}$ & $\mathrm{N}$ & $\mathrm{N}$ & $\mathrm{N}$ & 5 & 0 & 1 & $71 \%$ & $0 \%$ & $14 \%$ \\
\hline & & & Malaysian trial (ph3, 2v) & $\mathrm{N}$ & $\mathrm{N}$ & $\mathrm{U}$ & $\mathrm{u}$ & $\mathrm{N}$ & $\mathrm{N}$ & 4 & 2 & 0 & $57 \%$ & $29 \%$ & $0 \%$ \\
\hline & & & Indian Trial (ph3,2v) & $\mathrm{N}$ & $\mathrm{N}$ & $u$ & $u$ & $\mathrm{~N}$ & $\mathrm{~N}$ & 4 & 2 & 0 & $57 \%$ & $29 \%$ & $0 \%$ \\
\hline & & & Korean trial $(\mathrm{ph} 3,2 \mathrm{v})$ & $\mathrm{N}$ & $\mathrm{N}$ & $\mathrm{N}$ & $\mathrm{N}$ & $\mathrm{N}$ & $\mathrm{N}$ & 6 & 0 & 0 & $86 \%$ & $0 \%$ & $0 \%$ \\
\hline & & & Korean trial (ph3b,2v) & $\mathrm{N}$ & $\mathrm{N}$ & $\mathrm{N}$ & $\mathrm{N}$ & $\mathrm{N}$ & $\mathrm{N}$ & 6 & 0 & 0 & $86 \%$ & $0 \%$ & $0 \%$ \\
\hline & & & PATRICIA trial (ph3,2v) & $\mathrm{N}$ & $\mathrm{N}$ & $\mathrm{N}$ & $\mathrm{N}$ & $\mathrm{N}$ & $\mathrm{N}$ & 6 & 0 & 0 & $86 \%$ & $0 \%$ & $0 \%$ \\
\hline & & & VIVIANE trial (ph3,2v) & $\mathrm{N}$ & $\mathrm{N}$ & $\mathrm{N}$ & $\mathrm{N}$ & $\mathrm{N}$ & $\mathrm{N}$ & 6 & 0 & 0 & $86 \%$ & $0 \%$ & $0 \%$ \\
\hline \multirow[t]{7}{*}{ Quadrivalent } & \multirow[t]{3}{*}{$\|$} & \multirow[t]{3}{*}{3} & Japanese trial (ph2,4v) & $\mathrm{u}$ & $\mathrm{N}$ & $\mathrm{N}$ & $\mathrm{N}$ & $\mathrm{U}$ & $\mathrm{N}$ & 4 & 2 & 0 & $57 \%$ & $29 \%$ & $0 \%$ \\
\hline & & & Korean trial $(\mathrm{ph} 2,4 \mathrm{v})$ & $\mathrm{N}$ & $\mathrm{U}$ & $u$ & $u$ & $\mathrm{~N}$ & $\mathrm{~N}$ & 3 & 3 & 0 & $43 \%$ & $43 \%$ & $0 \%$ \\
\hline & & & Phase2 trial (ph2,4v) & $\mathrm{N}$ & $\mathrm{U}$ & $\mathrm{N}$ & $\mathrm{N}$ & $\mathrm{N}$ & $\mathrm{N}$ & 5 & 1 & 0 & $71 \%$ & $14 \%$ & $0 \%$ \\
\hline & \multirow{4}{*}{ III } & \multirow{4}{*}{4} & African_3 country trial $(\mathrm{ph} 3,4 \mathrm{v})$ & $\mathrm{N}$ & $U$ & $u$ & $u$ & $\mathrm{~N}$ & $\mathrm{~N}$ & 3 & 3 & 0 & $43 \%$ & $43 \%$ & $0 \%$ \\
\hline & & & FUTURE I trial $(\mathrm{ph} 3,4 \mathrm{v})$ & $\mathrm{N}$ & $\mathrm{N}$ & $\mathrm{N}$ & $\mathrm{N}$ & $\mathrm{N}$ & $\mathrm{N}$ & 6 & 0 & 0 & $86 \%$ & $0 \%$ & $0 \%$ \\
\hline & & & FUTURE II trial (ph3,4v) & $\mathrm{N}$ & $\mathrm{N}$ & $\mathrm{N}$ & $\mathrm{N}$ & $\mathrm{N}$ & $\mathrm{N}$ & 6 & 0 & 0 & $86 \%$ & $0 \%$ & $0 \%$ \\
\hline & & & FUTURE III trial $(\mathrm{ph} 3,4 \mathrm{v})$ & $\mathrm{N}$ & $\mathrm{N}$ & $\mathrm{N}$ & $\mathrm{N}$ & $\mathrm{N}$ & $\mathrm{N}$ & 6 & 0 & 0 & $86 \%$ & $0 \%$ & $0 \%$ \\
\hline \multirow[t]{6}{*}{ Total } & & \multirow[t]{6}{*}{26} & Nb of Low risk & 24 & 16 & 16 & 16 & 25 & 25 & & & & & & \\
\hline & & & Nb of Unclear risk & 2 & 7 & 9 & 9 & 1 & 1 & & & & & & \\
\hline & & & Nb of High risk & 0 & 3 & 1 & 1 & 0 & 0 & & & & & & \\
\hline & & & $\%$ of Low risk & $92 \%$ & $62 \%$ & \begin{tabular}{|l|l}
$62 \%$ & \\
\end{tabular} & $62 \%$ & $96 \%$ & $96 \%$ & & & & & & \\
\hline & & & $\%$ of Unclear risk & $8 \%$ & $27 \%$ & $35 \%$ & $35 \%$ & $4 \%$ & $4 \%$ & & & & & & \\
\hline & & & $\%$ of High risk & $0 \%$ & $12 \%$ & $4 \%$ & $4 \%$ & $0 \%$ & $0 \%$ & & & & & & \\
\hline
\end{tabular}


Figure 3. 'Risk of bias' graph: review authors' judgements about each 'Risk of bias' item presented as percentages across all included studies.

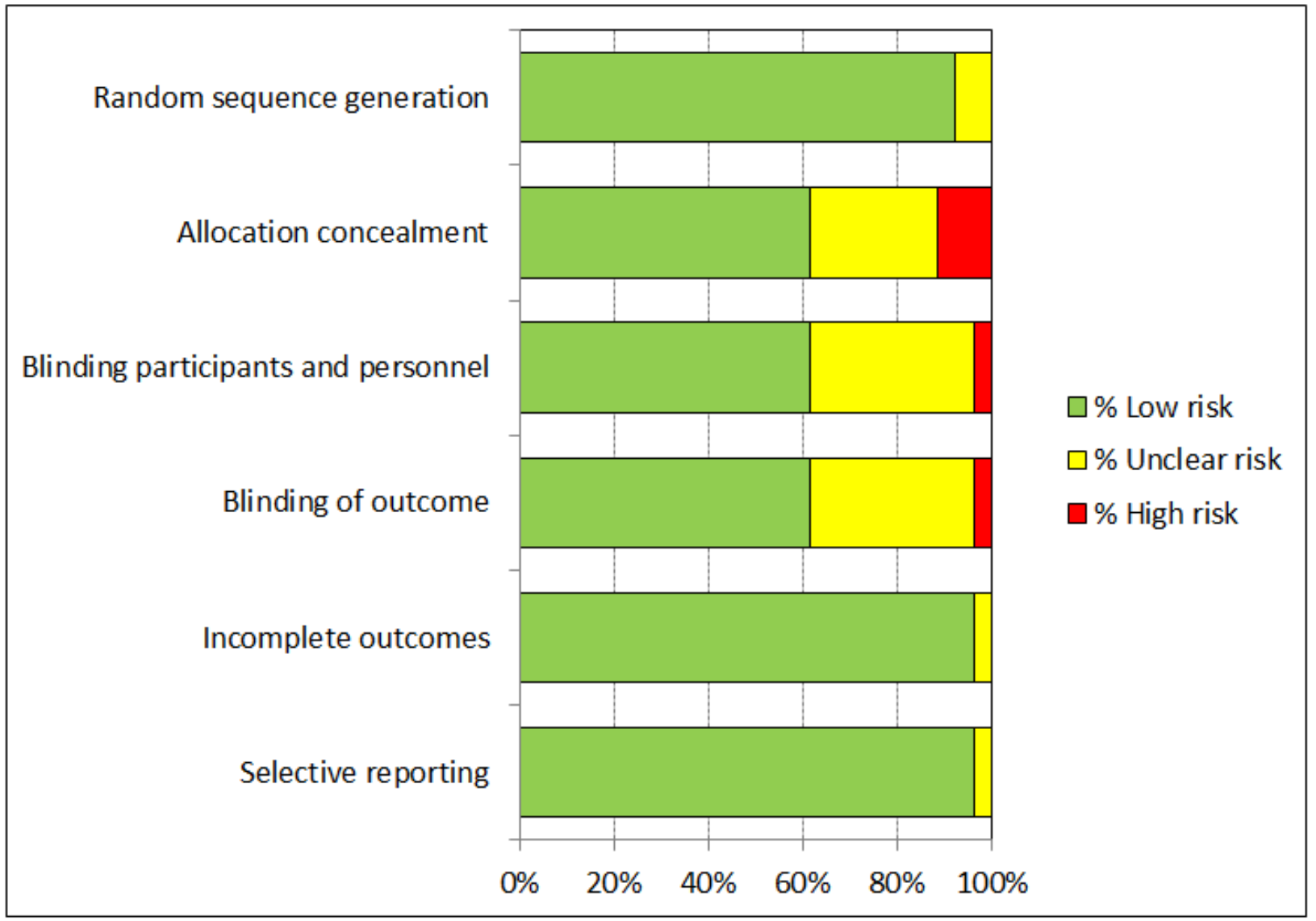


Figure 4. 'Risk of bias' summary: review authors' judgements about each 'Risk of bias' item for each included study.

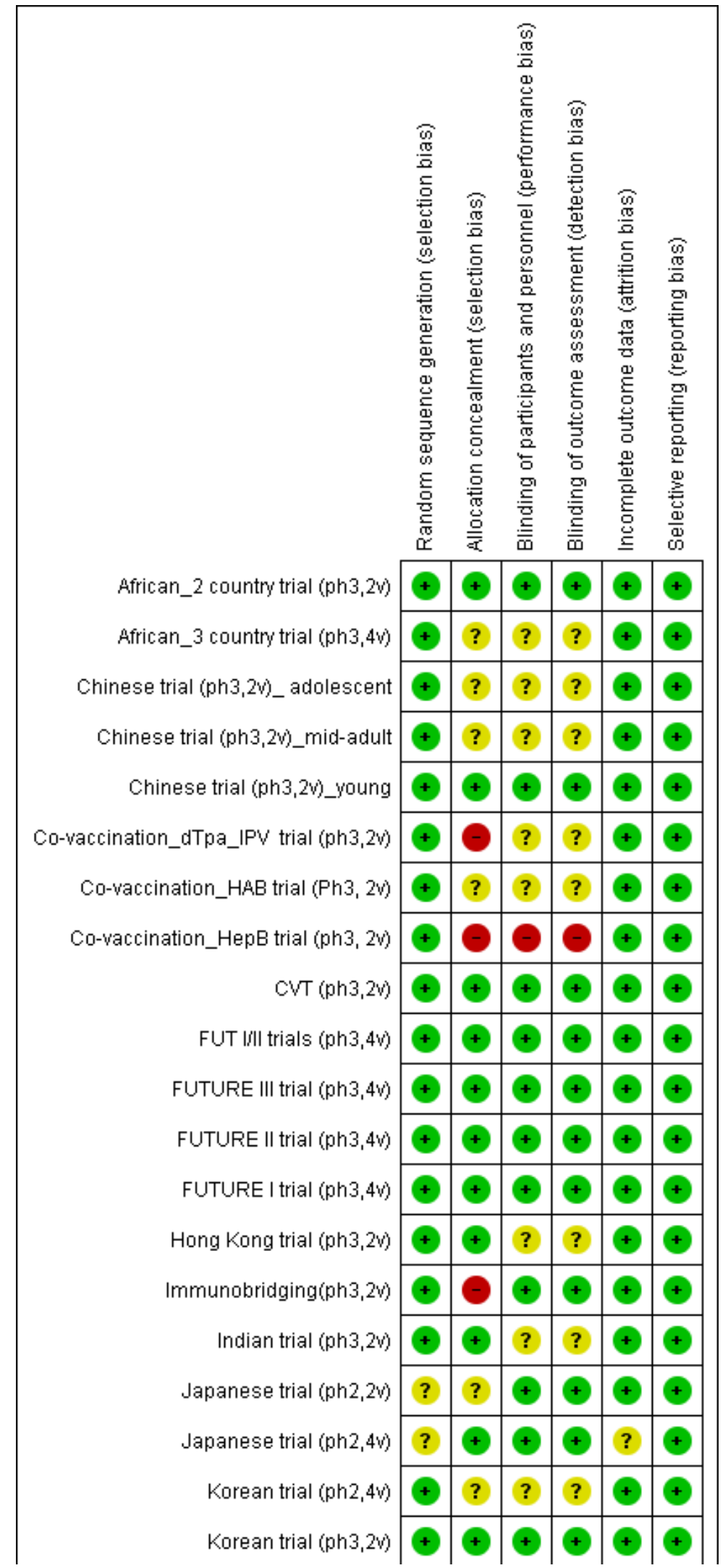


Figure 4. (Continued)

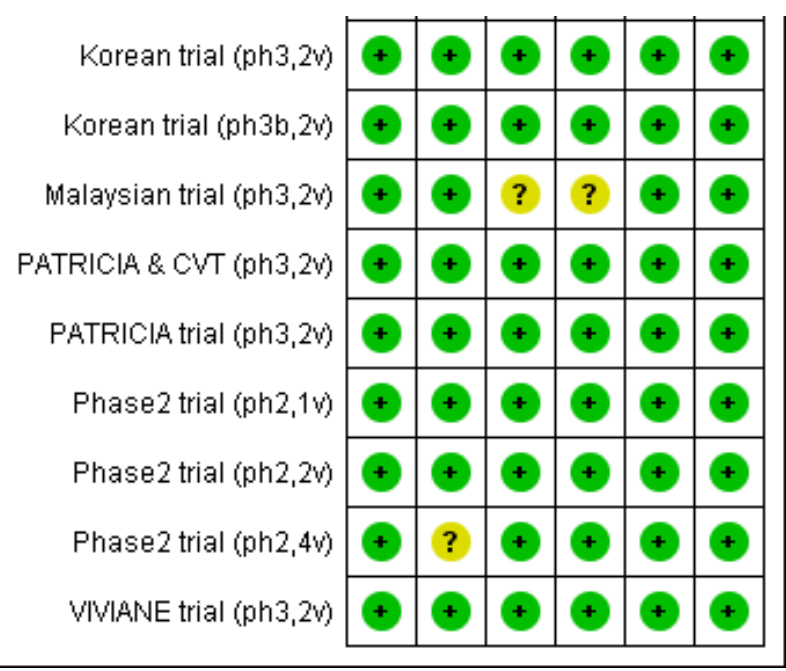

The allocation of participants to the vaccine or placebo arm was clearly concealed in 16/26 (62\%) trials. In three studies, randomisation was by design not concealed (Covaccination_dTpa_IPV trial (ph3,2v)); Co-vaccination_HepB trial (ph3, 2v); Immunobridging(ph3,2v)). These studies did not assess efficacy but compared immunogenicity and safety of HPV vaccines with other vaccines or combination of HPV vaccine and other vaccines that were visually distinguishable. Concealment of allocation was unclear in seven studies (African_3 country trial (ph3,4v); Chinese trial (ph3,2v)_ adolescent; Chinese trial (ph3,2v)_mid-adult; Japanese trial (ph2,2v); Korean trial (ph2,4v); Phase2 trial (ph2,4v);Co-vaccination_HAB trial (Ph3, 2v)).

Blinding of study participants and medical personnel and blind assessment of outcomes were assured in 16 trials but were not clearly documented in nine trials ( $9 / 26=35 \%$ unclear risk). One trial assessing immunogenicity and safety of HPV vaccine only versus vaccination of HPV vaccine with other vaccines was by design unblinded (Co-vaccination_HepB trial (ph3, 2v)). Drop out of enrolled participants, who did not follow the foreseen vaccination schedule, occurred in all trials, but the reasons for exclusion were well described in 25/26 (96\%) and outcomes were presented separately for restricted per-protocol groups and larger intentionto-treat groups in nearly all trials with one exception. In the Japanese trial $(\mathrm{ph} 2,4 \mathrm{v})$ only per-protocol results were presented. All intended outcomes were reported according to pre-published registered protocols in all included trials.

We did not judge any of the included trials assessing efficacy outcomes as having a high risk of bias. In eight of the included efficacy trials were considered to be at low risk of bias.

Only one study (CVT (ph3,2v)) was funded and conducted by an independent research institution.

Whether involvement of industry or other quality criteria influenced study outcomes will be explored below (Results, section 9.3).

\section{Effects of interventions}

See: Summary of findings for the main comparison HPV vaccine effects on cervical lesions in adolescent girls and women negative for hrHPV DNA at baseline; Summary of findings 2 HPV vaccine effects on cervical lesions in adolescent girls and women negative for HPV16/18 DNA at baseline; Summary of findings 3 HPV vaccine effects in adolescent girls and women regardless of HPV DNA status at baseline; Summary of findings 4 HPV vaccine effects on pregnancy outcomes

The duration of follow-up post vaccination in the studies was too short to show effects on cervical cancer outcomes. The presentation of the results on vaccine efficacy focuses on protection against precancerous cervical lesions and of HPV16/18 infection. They are organised according to the following features (see Summary of findings for the main comparison; Summary of findings 2; Summary of findings 3; Table 2):

1. three types of exposure groups:

a. women with hrHPV DNA negative status at baseline

b. women with HPV16/18 DNA negative status at baseline

c. all women regardless of HPV DNA status at baseline

2. two main types of outcomes:

a. precancerous lesions (CIN2+, $\mathrm{CIN} 3+$ and AIS+)

i. associated with HPV vaccine types (HPV16/18 or HPV6/11/16/18)

ii. irrespective of HPV type

b. infection by the HPV types included in the vaccine:

i. incident infection

ii. persistent infection (observed over six months or over 12 months)

3. number of doses received: one, two, three, at least one, one or two ( = difference between the number of women having received at least one dose and three-dose recipients which was the majority of enrolled women. All trials were designed as three-dose trials; women who received fewer than three were those who did not complete the planned schedule. We could not assess risk of bias due to differences in fewer than three-dose recipients and control groups in our analysis)

4. age group: 15 to 26 years; 24 to 45 years

Prophylactic vaccination against human papillomaviruses to prevent cervical cancer and its precursors (Review) 


\section{Protection against high-grade cervical lesions in women negative for hrHPV DNA at baseline}

Data on the protection against $\mathrm{CIN} 2+, \mathrm{CIN} 3+$ and AIS + associated with two HPV types included in the vaccines (HPV16 or 18) in adolescent girls and women aged 15 to 26 years could be extracted from three large phase III trials (CVT (ph3,2v); FUT I/II trials (ph3,4v); PATRICIA trial $(p h 3,2 v))$ for women having received at least one dose. The risk of $\mathrm{CIN} 2+, \mathrm{CIN} 3+$ and $\mathrm{AIS}+$ was lower following both vaccines: for CIN2+ (risk ratio $(\mathrm{RR})=0.01,95 \%$ confidence interval (Cl) 0.00 to 0.05 ; participants $=23,676$; studies $=3 ; 1^{2}=0 \%$; Analysis 1.1), for $\mathrm{CIN} 3+(\mathrm{RR}=0.01,95 \% \mathrm{Cl} 0.00$ to 0.10 ; participants $=20,214$; studies $=2 ; 1^{2}=0 \%$; Analysis 1.3$)$, and for AIS + (RR=0.10,95\% Cl 0.01 to 0.82 ; participants $=20,214$; studies $=2 ; 1^{2}=0 \%$; Analysis 1.5). We graded the evidence as high quality for the outcomes $\mathrm{CIN} 2+$ and $\mathrm{CIN} 3+$ and as moderate quality for AIS+ (downgraded for imprecision due to the rarity of the AIS+ outcome). The efficacy of the quadrivalent vaccine was similar when lesions associated with four vaccine types (HPV6/11/16/18) were considered (Analysis 1.2; Analysis 1.4 and Analysis 1.6).

Protection against any high-grade cervical lesions, irrespective of HPV type, was substantially lower than for lesions caused by the HPV types included in the vaccine. The test for subgroup differences suggested that efficacy differed significantly according to valency of the vaccine $(P$ values $=0.004$ and 0.001 , for CIN2+ and CIN3+, respectively). The bivalent vaccine showed higher efficacy than the quadrivalent vaccine for protection against $\mathrm{CIN} 2+(\mathrm{RR}=0.33$, $95 \% \mathrm{Cl} 0.25$ to 0.43 ; participants $=15,884$; studies $=4 ; \mathrm{I}^{2}=0 \%$ ) versus $\mathrm{RR}=0.57(95 \% \mathrm{Cl} 0.44$ to 0.76 ; participants $=9296$; studies $=1$ ) see (Analysis 1.7), and against CIN3+ $(\mathrm{RR}=0.08,95 \% \mathrm{Cl} 0.03$ to 0.23 ; participants $=11,423$; studies $=2 ; 12=0 \%$ ) versus $R$ 0.54, ( $95 \% \mathrm{Cl} 0.36$ to 0.82; participants $=9296$; studies $=1$; Analysis 1.8). We graded the quality of evidence regarding vaccine efficacy against any high-grade $\mathrm{CIN}$, irrespective of HPV type, as high for bivalent and moderate for the quadrivalent vaccine. Both vaccines were similarly efficacious regarding protection against any AIS, irrespective of HPV type (RR $0.10,95 \% \mathrm{Cl} 0.01$ to 0.76 ), $\mathrm{P}$ value for inter-vaccine heterogeneity $=0.71$, Analysis 1.9$)$. We graded this evidence as moderate quality (downgraded for imprecision due to the rarity of the AIS+ outcome).

\section{Protection against high-grade cervical lesions in women negative for HPV16/18 DNA at baseline}

Outcomes for women negative for HPV16/18 are more often reported as a per protocol analysis in vaccination trials. Some trials reported outcomes for women who received all three doses and for women who received at least one dose. This allows computation (by subtraction) of outcomes for women who receive one or two doses (see also Results section 9.4).

\subsection{CIN2+ associated with HPV types included in the vaccine}

In adolescent girls and women aged 15 to 26 years who received three vaccine doses, protection against $\mathrm{CIN2}+$ associated with HPV16/18 was consistently high with a RR pooled from six trials, including the mono-, bi- and quadrivalent vaccine of $0.07,(95 \% \mathrm{Cl}$ 0.03 to 0.15 ; participants $=36,579$; studies $=6 ; 1^{2}=0 \%$, Analysis 2.1.1). We judged this to be high-quality evidence.

Among mid-adult women, aged 24 to 45 years, vaccination with the bivalent or quadrivalent vaccine also showed protection (RR
$0.16,95 \% \mathrm{Cl} 0.04$ to 0.74 ; participants $=6797$; studies $=2 ;\left.\right|^{2}=0 \%$; moderate-quality evidence; Analysis 2.1.2). We downgraded this evidence due to the imprecision of the estimate. Protection in the mid-adult age groups was not significantly lower than that in the younger groups ( $P$ value for inter-group heterogeneity of $0.31,12=$ $3.8 \%)$.

In women who received at least one dose, protection was also consistently high in adolescent girls and women aged 15 to 26 years: RR pooled from six trials of $0.05(95 \% \mathrm{Cl} 0.03$ to 0.10 ; participants $=34,478$; studies $=6 ; 1^{2}=0 \%$; high-quality evidence; Analysis 2.2). In women aged 24 to 45 years, protection was lower than in the younger group (RR $0.30,95 \% \mathrm{Cl} 0.11$ to 0.81 ; participants $=7552$; studies = 2; $1^{2}=0 \%$; moderate-quality evidence; Analysis 2.2.2). For efficacy from at least one dose, the difference in $R R$ between age-groups was significant $(P$ value $=$ for inter-group heterogeneity of $0.005, \mathrm{I}^{2}=87.1 \%$ ).

Considering women who received just one or two doses, the risk of $\mathrm{CIN} 2+$ was lower after vaccination in the younger age groups (RR 0.10, 95\% Cl 0.04 to 0.26; participants = 2958; studies = 5; $1^{2}=0 \%$, low-quality evidence). The effect in mid-adult women was uncertain (RR $0.61,95 \% \mathrm{Cl} 0.14$ to 2.67 ; participants = 755; studies = 2; $1^{2}=0 \%$; low-quality evidence) (Analysis 2.3). The quadrivalent vaccine conferred similar degrees of protection against $\mathrm{CIN} 2+$ associated with the four types HPV16/18/6/11 as to those associated with HPV16/18 (Analysis 2.4; Analysis 2.5; Analysis 2.6).

\subsection{CIN3+ associated with HPV types included in the vaccine}

Efficacy of vaccination against occurrence of $\mathrm{CIN} 3+$ associated with HPV16/18 or HPV16/18/6/11 was reported in three large phase III trials assessing the bivalent vaccine (PATRICIA trial $(\mathrm{ph} 3,2 \mathrm{v})$ ) or quadrivalent vaccine (FUTURE I trial (ph3,4v); FUTURE II trial $(\mathrm{ph} 3,4 \mathrm{v}))$. Data were pooled in one outcome, given the similarity in direction and magnitude of effects. Protection with HPV vaccination was similarly high in women who received all three doses, (RR 0.07, 95\% Cl 0.02 to 0.29; participants = 29,720; studies $=3 ; 1^{2}=28 \%$; high-quality evidence; Analysis 2.7); in those who received at least one dose ( $\mathrm{RR} 0.05,95 \% \mathrm{Cl} 0.02$ to 0.14 ; participants $=33,199 ;$ studies = 3; $1^{2}=0 \%$; high-quality evidence; Analysis 2.8); and in women who received only one or two doses (RR $0.06,95 \%$ $\mathrm{Cl} 0.01$ to 0.24 ; participants $=3479$; studies $=3 ; 1^{2}=0 \%$; low-quality evidence; Analysis 2.9).

\subsection{AIS+ associated with HPV types included in the vaccine}

Data were pooled for AIS+ associated with HPV16/18 or associated with HPV16/18/6/11 in one group, given the similarity in magnitude of protection and insignificance of heterogeneity.

In women receiving three doses of bivalent or quadrivalent vaccine, at least one dose of quadrivalent, or one or two doses of bivalent or quadrivalent vaccine, the pooled protective effect was $100 \%$ (zero excluded from the $95 \% \mathrm{Cl}$ ). Applying a continuity correction gave pooled RRs of 0.12 for three doses $(95 \% \mathrm{Cl} 0.02$ to 0.70 ; participants $=29,707 ;$ studies $=3 ; 1^{2}=0 \%$; moderate-quality evidence; Analysis 2.10); 0.09 for at least one dose (95\% $\mathrm{Cl} 0.01$ to 0.72 ; participants $=17,079$; studies $=2 ; 1^{2}=0 \%$; moderate-quality evidence; Analysis 2.11); and 0.15 for one or two doses ( $95 \% \mathrm{Cl} 0.01$ to 2.97 ; participants $=2015$; studies $=2 ; 1^{2}=0 \%$; very low-quality evidence; Analysis 2.12). 


\subsection{Any CIN2+ irrespective of HPV type}

Protection against CIN2+ irrespective of HPV types was reported in five trials (CVT (ph3,2v); Japanese trial (ph2,2v); PATRICIA trial (ph3,2v); Phase2 trial (ph2,1v); Phase2 trial (ph2,2v)). Vaccination with three doses reduced the risk of $\mathrm{CIN} 2+$ by $60 \%$ on average (RR $0.40,95 \% \mathrm{Cl} 0.25$ to 0.64 ; participants $=7,320$; studies $=3$; $1^{2}=0 \%$; high-quality evidence Analysis 2.13). Vaccination with at least one dose of the bivalent vaccine produced similar effects (RR $0.41,95 \% \mathrm{Cl} 0.32$ to 0.52 ; participants $=19,143$; studies $=3 ; \mathrm{I}^{2}=$ $0 \%$; Analysis 2.14). We judged this to be high-quality evidence. Protection generated from the vaccine of one or two doses was unclear since the quality of evidence was very low (RR $0.71,95 \%$ $\mathrm{Cl} 0.15$ to 3.38; participants $=34$; studies $=1$ ), as this could only be assessed for one small trial (Analysis 2.15; Japanese trial (ph2,2v)).

No results were found for the outcomes any $\mathrm{CIN} 3+$ or AIS+ irrespective of HPV type.

\section{Protection against high-grade lesions in women regardless of baseline HPV DNA status}

Data on the protection induced by HPV vaccination against highgrade lesions in all enrolled women regardless of HPV DNA status at enrolment are reported only for those who received at least one dose.

\subsection{CIN2+ associated with the vaccine HPV types}

In adolescent girls and women aged 15 to 26 , the reduction of risk of $\mathrm{CIN2}+$ associated with the HPV types included in the vaccine was lower than in the hrHPV-naive or HPV16/18 negative groups (discussed in results sections 1 and 2), but was still significant with limited variation between mono-, bi- and quadrivalent vaccines
(RR 0.46, 95\% Cl 0.37 to 0.57 ; participants = 34,852; studies $=3 ; \mathrm{I}^{2}$ $=38 \%$; high-quality evidence) (Analysis 3.1 .1 and Analysis 3.2.1). However, in mid-adult women (24 to 45 years), the protection of the bi- and quadrivalent vaccine was not significant (RR 0.74, 95\% $\mathrm{Cl} 0.52$ to 1.05 ; participants $=9200$; studies $=2 ; \mathrm{I}^{2}=0 \%$; moderatequality evidence) (Analysis 3.1.2). Similar findings were observed for the protection induced by the quadrivalent vaccine against CIN2+ associated with HPV16/18/6/11 (Analysis 3.2.2).

\subsection{CIN3+ associated with HPV types included in the vaccine}

Both the bivalent and quadrivalent vaccines protected against CIN3+ associated with HPV16/18 (RR $0.55,95 \% \mathrm{Cl} 0.45$ to 0.67 ; participants $=34,562 ;$ studies $=2 ; 1^{2}=0 \%$ ) Analysis 3.3$)$ with similar protection against CIN3+ associated with HPV16/18/6/11 (Analysis 3.4).

\subsection{AIS + associated with HPV types included in the vaccine}

Both the bi- and quadrivalent vaccines offered protection against AIS+ associated with HPV16/18 (RR $0.36,95 \% \mathrm{Cl} 0.17$ to 0.78 ; participants $=34,562 ;$ studies $=2 ; 1^{2}=0 \%$; moderate-quality evidence; Analysis 3.5) and associated with HPV16/18/6/11 ((RR $0.40,95 \% \mathrm{Cl} 0.16$ to 0.98 ; participants $=20,830$; studies $=2 ;\left.\right|^{2}=0 \%$; moderate-quality evidence; Analysis 3.6).

\subsection{Any CIN2+ irrespective of HPV type}

Limited protection against CIN2+ irrespective of HPV type was shown for the mono-, bi-, and quadrivalent vaccines in younger women aged 15 to 26 years (RR $0.70,95 \% \mathrm{Cl} 0.58$ to 0.85 ; participants $=35,779$; studies $=4$; high-quality evidence; $1^{2}=31 \%$, see Figure 5; Analysis 3.7.1), the efficacy did not vary by the valency of the vaccine ( $P$ value for subgroup difference $=0.24$ ). 
Figure 5. Protection against CIN2+ irrespective of presence of HPV types in women, aged 15-26 years, regardless of their HPV DNA status at baseline, who received at least one dose.

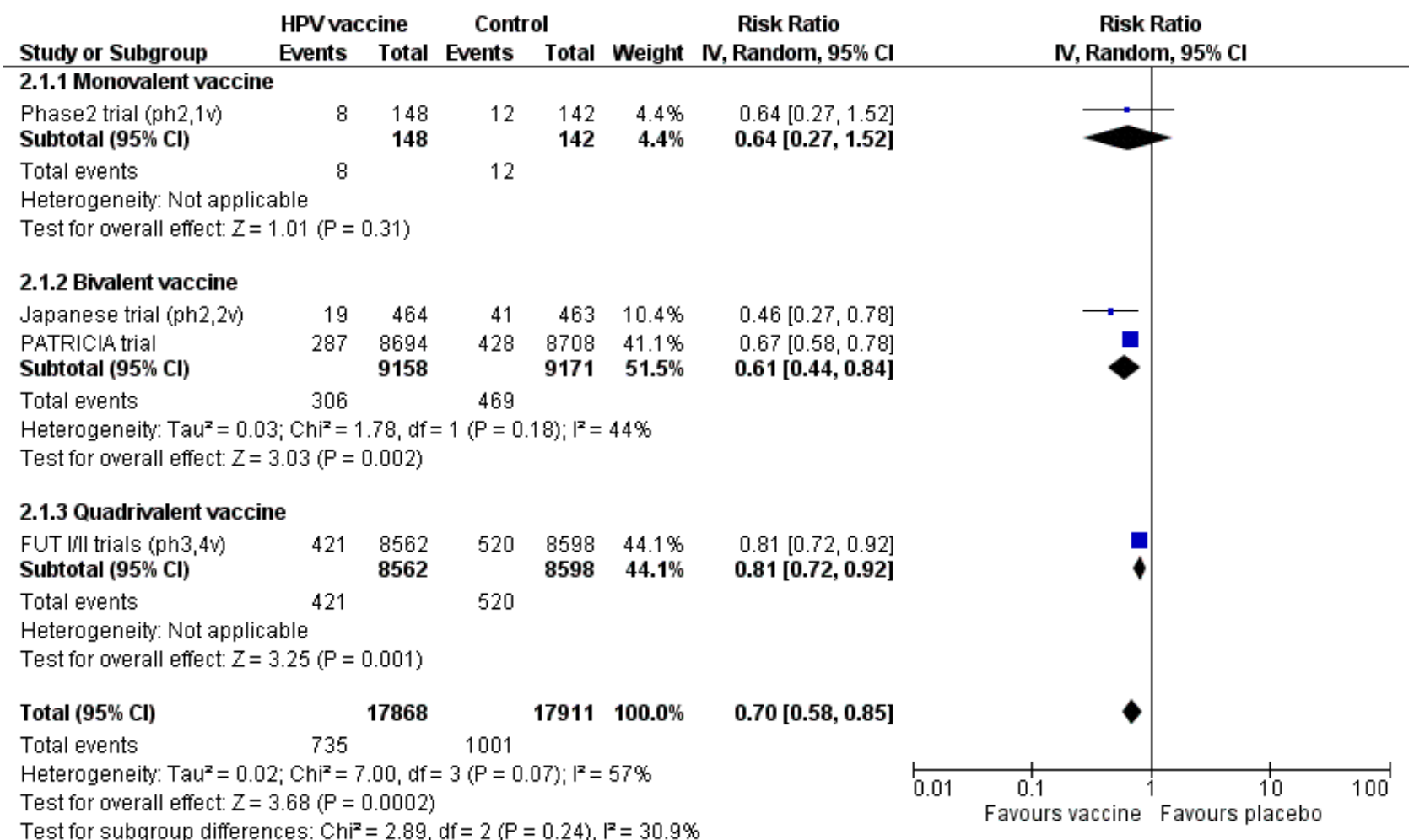

In the mid-adult group (24 to 45 years), HPV vaccination with the bior quadrivalent vaccine was not protective (RR $1.04,95 \% \mathrm{Cl} 0.83$ to 1.30; participants $=9287$; studies $=2 ; 1^{2}=8 \%$; Analysis 3.7.2).

\subsection{Any CIN3+ irrespective of HPV type}

The vaccines showed different results in protection against any CIN3+ irrespective of HPV type. Among young women (16 to 26 year) bivalent vaccines reduced the risk of CIN3+ (RR $0.55(95 \% \mathrm{Cl}$ 0.43 to 0.71 ; participants $=18,329$; studies $\left.=2 ; 1^{2}=0 \%\right)$, and the quadrivalent vaccine gave a smaller degree of protection (RR 0.81 $(95 \% \mathrm{Cl} 0.69$ to 0.96 , participants $=17,160$, studies $=1)$ (Analysis 3.8$)$. The interaction test for the subgroup differences gave a $P$ value of 0.01 .

No data were reported for mid-adult women.

\subsection{Any AIS+ irrespective of HPV type}

The two vaccines reduced the risk of any AIS+ irrespective of hrHPV types in young women (age 16 to 26 years) (RR $0.32,95 \% \mathrm{Cl} 0.15$ to 0.67 ; participants $=34,562$; studies $=2 ; 1^{2}=0 \%$; high-quality evidence; Analysis 3.9).

No data were reported for mid-adult women.

\section{Protection against infection with HPV types included in the vaccine in women negative for hrHPV DNA at baseline}

Protection against HPV16/18 infection among women negative for hrHPV DNA at baseline, aged 15 to 26 years, was documented only for the bivalent vaccine. One phase II trial (Phase2 trial (ph2,2v) provided data for the outcome of incident infection ((RR 0.06, 95\% $\mathrm{Cl} 0.02$ to 0.20 ; participants $=368$; studies $=1$ ) Analysis 4.1 ) and six- and 12-month-persisting infections among women who received three doses ( $(\mathrm{RR} 0.02,95 \% \mathrm{Cl} 0.00$ to 0.35 ; participants $=368$; studies $=1)$, Analysis 4.2); (RR $0.04,95 \% \mathrm{Cl} 0.00$ to 0.73 ; participants $=368$; studies $=1 ;$ moderate-quality evidence) (Analysis 4.4), respectively).

One large phase III trial (PATRICIA trial (ph3,2v)) assessed protection against 6 - and 12-month persisting infection among women who received at least one dose (RR $0.07,95 \% \mathrm{Cl} 0.05$ to 0.09 ; participants $=10,826$; studies $=1$; moderate-quality evidence; Analysis 4.3); (RR $0.08,95 \% \mathrm{Cl} 0.05$ to 0.12 ; participants $=14,153$; studies $=2 ;\left.\right|^{2}=0 \%$; moderate-quality evidence;(Analysis 4.5), respectively).

\section{Protection against HPV16/18 infection in women negative for HPV16/18 DNA at baseline}

In women who were initially HPV16/18 DNA negative at enrolment and who received three doses, protection against incident infection with HPV16/18 was consistently high in three trials assessing different vaccines and age groups: $\mathrm{RR}=0.17,95 \% \mathrm{Cl} 0.10$ to 0.31 ; participants $=8034 ;$ studies $=4 ; 12=52 \%$, high-quality evidence; Analysis 5.1). HPV vaccines also reduced the risk of incident infection in those who received at least one dose (RR $0.23,95 \% \mathrm{Cl}$ 0.14 to 0.37 ; participants $=23,872$; studies $=5 ; 1^{2}=79 \%$; high-quality evidence; Analysis 5.2) or just one or two doses ((RR $0.47,95 \% \mathrm{Cl}$ 0.26 to 0.84 ; participants $=331$; studies $=3 ; 1^{2}=14 \%$; moderatequality evidence; Analysis 5.3).

The reduction in risk of persistent HPV16/18 infection (lasting for at least six months) in women who received three doses was consistently high in younger women for all types of vaccine (RR 0.06 , $95 \% \mathrm{Cl} 0.05$ to 0.08 ; participants $=27,385$; studies $=6 ;\left.\right|^{2}=0 \%$; high- 
quality evidence) and was also high in mid-adult women aged 24 to 45 years who received three doses ( $(\mathrm{RR} 0.11,95 \% \mathrm{Cl} 0.06$ to 0.20 ; participants $=6728$; studies $\left.=2 ; 1^{2}=0 \%\right)$; high-quality evidence; Analysis 5.4). Similar protection was seen for the larger group of women who received at least one dose of bivalent or quadrivalent vaccine ( $\mathrm{RR} 0.10,95 \% \mathrm{Cl} 0.08$ to 0.12 ; participants $=22,803$; studies $=4 ;\left.\right|^{2}=0 \%$; for younger women aged 15 to 26 years, and RR 0.17 , $95 \% \mathrm{Cl} 0.10$ to 0.29 ; participants $=7520$; studies $=2 ; 1^{2}=43 \%$ ) or mid-adult women; high-quality evidence; Analysis 5.5). In young and mid-adult women, the reduction in risk following vaccination with one or two doses was $0.12(95 \% \mathrm{Cl} 0.03$ to 0.42; participants = 437 ; studies $=2 ;\left.\right|^{2}=0 \%$; moderate-quality evidence) and $0.31(95 \%$ $\mathrm{Cl} 0.18$ to 0.54 ; participants $=792$; studies $=2 ; \mathrm{I}^{2}=0 \%$; high-quality evidence), respectively. We did not have sufficient data to confirm the lower degree of protection associated with one or two doses in mid-adult compared to young women. The number of events and participants is small and the difference was not statistically significant ( $P$ value for interaction test 0.18 ; Analysis 5.6).

Protection induced by the quadrivalent vaccine against six-month persistent HPV infection with one of the four HPV types included in the vaccine was comparable with the protection against the two oncogenic types (Analysis 5.7 (after reception of three doses); Analysis 5.8 (after reception of at least one dose)).

Protection against 12-month persistent HPV16/18 infection was only reported from trials assessing the bivalent vaccine. The efficacy was high if three doses were given (RR 0.09, 95\% Cl 0.06 to 0.13 ; participants $=22,267$; studies $=4 ; 1^{2}=0 \%$ ); high-quality evidence; Analysis 5.9), and slightly lower with at least one dose (RR $0.16,95 \% \mathrm{Cl} 0.12$ to 0.20 ; participants $=29,464$; studies $=5 ;\left.\right|^{2}=0 \%$; high-quality evidence; Analysis 5.10), or only one or two doses ((RR $0.13,95 \% \mathrm{Cl} 0.06$ to 0.33 ; participants $=3912$; studies $\left.=3 ; \mathrm{I}^{2}=0 \%\right)$ high-quality evidence; Analysis 5.11).

\section{Protection against infection with HPV types included in the vaccine in women regardless of HPV DNA status at baseline}

Women aged 15 to 26 years, regardless of hrHPV DNA status, who received at least one dose of the bivalent vaccine were protected against incident HPV16/18 infection (RR 0.24, 95\% Cl 0.17 to 0.33 ; participants $=4210$; studies $=1$; moderate-quality evidence; Analysis 6.1). In these women, bivalent and quadrivalent vaccines also protected against persistent HPV16/18 infection lasting for six months: $\mathrm{RR}=0.44(95 \% \mathrm{Cl} 0.38$ to 0.51 ; participants $=25,199 ;$ studies $=2 ; 1^{2}=62 \%$; high-quality evidence). In mid- adult women (24 to 45 years), the vaccine also provided significant protection (RR $0.57,95 \% \mathrm{Cl} 0.47$ to 0.69 ; participants $=8648$; studies $=2 ; 1^{2}=0 \%$; high-quality evidence), but the protection was significantly lower compared to the younger women ( $P$ value for inter-group heterogeneity $=0.03$; Analysis 6.2 ). The protection against persistent HPV16/18/6/11 infection lasting for six months was similar: $\mathrm{RR}=0.52(95 \% \mathrm{Cl} 0.42$ to 0.65 ; participants $=3713$; studies $=1$ ) (Analysis 6.3).

The bivalent vaccine significantly reduced the occurrence of 12 month persistent HPV16/18 infection after administration of at least one dose: RR 0.46 ( $95 \% \mathrm{Cl} 0.40$ to 0.54; participants $=24,785$; studies $=2 ; 1^{2}=42 \%$; high-quality evidence; Analysis 6.4).

In a post hoc analysis, the Costa-Rica Vaccination Trial (CVT $(\mathrm{ph} 3,2 \mathrm{v}))$ demonstrated that protection was not significantly different in women who had received one ( $\mathrm{RR}=0.05,95 \% \mathrm{Cl}: 0$ to 0.77 ), two ( $R R=0.16,95 \% \mathrm{Cl}: 0.05$ to 0.54 ) or three doses ( $R R=0.19$, $95 \% \mathrm{Cl}: 0.13$ to 0.29 ) of the bivalent vaccine ( $\mathrm{P}$ value $=0.60, \mathrm{l} 2=0 \%)$ (Analysis 6.5), however, it should be noted that these women were not randomised to one, two or three doses.

\section{Summary of vaccine efficacy estimates}

Before assessing the adverse effects, we summarise the main efficacy outcomes described in previous sections. In Figure 6, we present the pooled effects observed in women who received at least one dose of vaccine according to HPV DNA status at enrolment, i.e. hrHPV DNA negative (column A), HPV16/18 DNA negative (column B), and all enrolled regardless of baseline HPV DNA status (column C), separated by age group (15 to 26 years, 25 to 45 years). In each age group, we distinguish high grade lesions (CIN2+, CIN3+, AIS+) associated with HPV16/18 and all lesions irrespective of HPV type and six-month persistent HPV16/18 infection. In each cell of the table, we provide the pooled RR and its $95 \% \mathrm{Cl}$, a shading corresponding with the degree of protection, the level of evidence, the number of trials that contributed data and the reference to the respective analysis. Figure 7 provides a synthesis of the same outcomes in women who were HPV16/18 negative at enrolment according the number of vaccine doses received: three doses (column A), at least one dose (column B) and one or two doses (column C). A complete list of all outcomes can be found in Table 2. This table contains the absolute risks in the placebo and vaccination arms, the relative risks (risk vaccinated/risk placebo), the vaccine efficacy $(V E=R R-1)$ and the risk differences $(R D=$ risk placebo -risk vaccinated, in \%) and the level of evidence. 
Figure 6. Summary of vaccine efficacy estimates, by age group, outcome and HPV DNA status at enrolment (for women who received at least one dose). [REFS BETWEEN SQUARE BRACKETS MUST BE ADAPTED][

A

Relative risks according to enrolment status among women who received $\geq 1$ dose

\begin{tabular}{|l|c|c|c|}
\hline & hr HPV DNA- & HPV16/18 DNA- & Regardless of HPV \\
Outcome & $\geq 1$ dose & $\geq 1$ dose & $\geq 1$ dose \\
\hline
\end{tabular}

Age group 15-26

High-grade intraepithelial neoplasia associated with HPV16/18

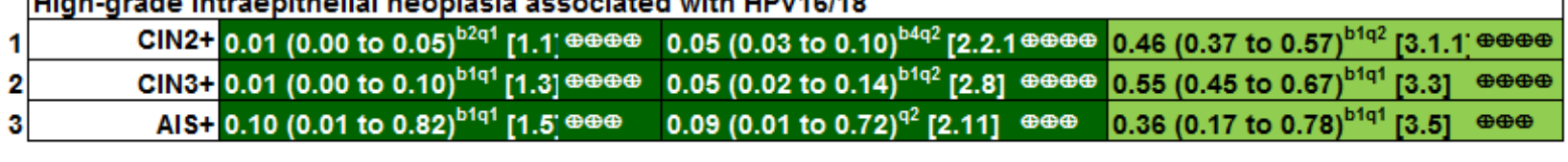

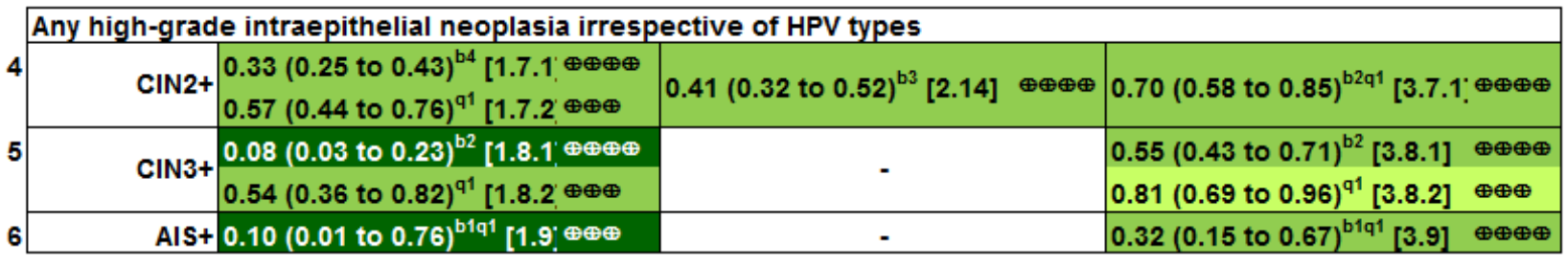

Persistent HPV16/18 infection

$76 \mathrm{M}$ persisting $0.07(0.05 \text { to } 0.90)^{\mathrm{b} 1}[4.3] \oplus \oplus \oplus \mid 0.10(0.08 \text { to } 0.12)^{\mathrm{b} 4}[5.5 .1] \oplus \oplus \oplus \oplus \mid 0.44(0.38 \text { to } 0.51)^{\mathrm{b} 2}[6.2 .1] \oplus \oplus \oplus$

\begin{tabular}{|c|c|c|c|c|}
\hline \multicolumn{5}{|c|}{ High-grade intraepithelial neoplasia associated with HPV16/18 } \\
\hline 8 & CIN2+ & - & $0.30(0.11 \text { to } 0.81)^{\mathrm{b} 1 \mathrm{q} 1}[2.2 .8 \oplus \oplus \oplus$ & $0.74(0.52 \text { to } 1.05)^{\mathrm{b} 1 \mathrm{q} 1}[3.1 .2 \oplus \oplus \oplus$ \\
\hline$\theta$ & CIN3+ & - & - & - \\
\hline 0 & AIS+ & - & - & - \\
\hline
\end{tabular}

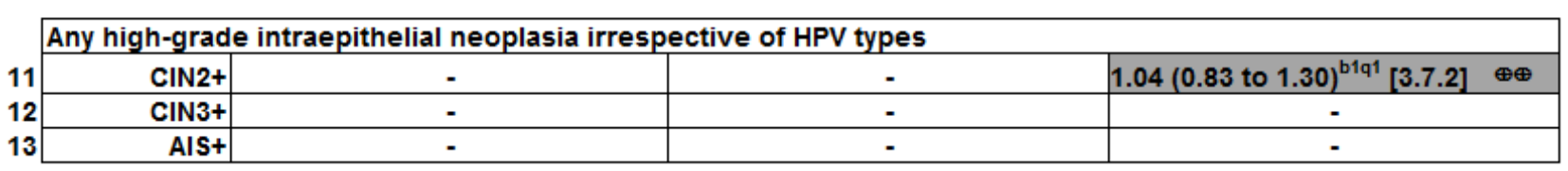

\begin{tabular}{|c|c|c|c|}
\hline \multicolumn{4}{|c|}{ Persistent HPV16/18 infection } \\
\hline $6 \mathrm{M}$ persisting & - & $0.17(0.10 \text { to } 0.29)^{\mathrm{b} 1 \mathrm{q} 1}[5.5 .2 \oplus \oplus \oplus \oplus$ & $0.57(0.47 \text { to } 0.69)^{\mathrm{b} 1 \mathrm{q} 1}\left[6.2 .2 \mathbf{p}^{\oplus \oplus \oplus \oplus}\right.$ \\
\hline
\end{tabular}

\begin{tabular}{|l|}
\hline Level of protection (RR) \\
$\leq 0.10,1$ excluded from $\mathrm{Cl}$ \\
$>0.10 \& \leq 0.20,1$ excluded from $\mathrm{Cl}$ \\
\hline$>0.20 \& \leq 0.80,1$ excluded from $\mathrm{Cl}$ \\
\hline$>0.80 \&<1,1$ excluded from $\mathrm{Cl}$ \\
\hline 1 included in $\mathrm{Cl}$ \\
\hline$>1$ and 1 excluded from $\mathrm{Cl}$ \\
\hline
\end{tabular}

\begin{tabular}{|l|}
\hline \multicolumn{1}{|c|}{ Quality of evidence } \\
\hline High $\oplus \oplus \oplus \oplus$ \\
\hline Moderate $\oplus \oplus \oplus$ \\
\hline Low $\oplus \oplus$ \\
\hline Very low $\oplus$ \\
\hline
\end{tabular}

index in superscript after the $95 \% \mathrm{Cl}$ corresponds with the number of trials where the bivalent (b) or quadrivalent (q) vaccines were assessed (for instance ${ }^{\mathrm{b} 1 \mathrm{q} 2}$. meta-analysis of 3 trials, one with the bivalent and two with the quadrivalent vaccine) [Ref]: the number between square brackets indicates the reference to the forest plot in Analyses and Tables 
Figure 7. Summary of vaccine efficacy estimates by age group, outcome and number of received doses (for women who were HPV16/18 DNA negative at enrolment). [REFS BETWEEN SQUARE BRACKETS MUST BE ADAPTED][

A

B

C

Relative risks according to number of the doses received among women who were HPV16/18- at enrolment
\begin{tabular}{|l|c|c|c|}
\hline \multirow{3}{*}{ Outcome } & HPV16/18 DNA- & 1 to 2 doses \\
\cline { 2 - 4 } & 3 doses & $\geq 1$ dose & \\
\hline
\end{tabular}

Age group 15-26

High-grade intraepithelial neoplasia due to HPV16/18

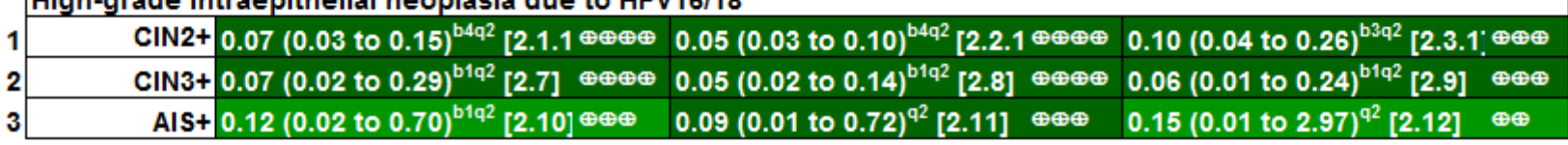

\begin{tabular}{|c|c|c|c|c|c|c|}
\hline \multicolumn{7}{|c|}{ High-grade intraepithelial neoplasia irrespective of HPV or due to whatever HPV type } \\
\hline & CIN2+ & $0.40(0.25 \text { to } 0.64)^{\mathrm{b} 2 \mathrm{q} 1}[2.13] \oplus \oplus \oplus \oplus$ & $0.41(0.32 \text { to } 0.52)^{\mathrm{b} 3}[2.14]$ & $\oplus \oplus \oplus \oplus$ & $0.71(0.15 \text { to } 3.38)^{\mathrm{b} 1}[2.15]$ & $\oplus \oplus$ \\
\hline 5 & \multirow{2}{*}{ CIN3+ } & - & - & & - & \\
\hline & & - & - & & - & \\
\hline 6 & AIS+ & - & - & & - & \\
\hline
\end{tabular}

Persistent HPV16/18 infection

$76 \mathrm{M}$ persisting $0.06(0.05 \text { to } 0.08)^{\mathrm{b} 4}[5.4 .1] \oplus \oplus \oplus \oplus \mid 0.10(0.08 \text { to } 0.12)^{\mathrm{b} 4}[5.5 .1] \oplus \oplus \oplus \oplus \mid 0.12(0.03 \text { to } 0.42)^{\mathrm{b} 2}[5.6 .1] \oplus \oplus$

Age group 24-45

High-grade intraepithelial neoplasia due to HPV16/18

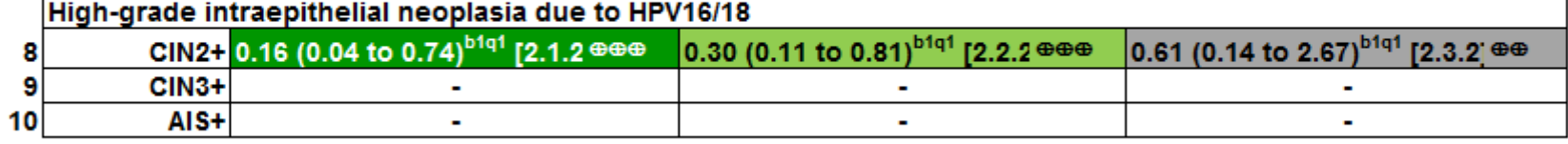

\begin{tabular}{|r|r|r|r|}
\hline \multicolumn{6}{|c|}{ High-grade intraepithelial neoplasia irrespective of HPV or due to whatever HPV type } \\
\hline 11 & - & - & - \\
\hline 12 & - & - & - \\
\hline CIN2+ & - & - & - \\
\hline CIN3+ & AIS+ & & \\
\hline
\end{tabular}

Persistent HPV16/18 infection

\begin{tabular}{ll|l|l|l|}
\hline $6 \mathrm{M}$ persisting $0.11(0.06 \text { to } 0.20)^{\mathrm{b} 1 \mathrm{q} 1}[5.4 .2 \oplus \oplus \oplus \oplus)$ & $0.17(0.10 \text { to } 0.29)^{\mathrm{b} 1 \mathrm{q} 1}[5.5 .2 \oplus \oplus \oplus \oplus)$ & $0.31(0.18 \text { to } 0.54)^{\mathrm{b} 1 \mathrm{q} 1}$ & {$[5.6 .2)^{\oplus \oplus \oplus}$} \\
\hline
\end{tabular}

\begin{tabular}{|l|}
\hline Level of protection (RR) \\
\hline$\leq 0.10,1$ excluded from $\mathrm{Cl}$ \\
\hline$>0.10 \& \leq 0.20,1$ excluded from $\mathrm{Cl}$ \\
\hline$>0.20 \& \leq 0.80,1$ excluded from $\mathrm{Cl}$ \\
\hline$>0.80 \&<1,1$ excluded from $\mathrm{Cl}$ \\
\hline 1 included in $\mathrm{Cl}$ \\
\hline$>1$ and 1 excluded from $\mathrm{Cl}$ \\
\hline
\end{tabular}

\begin{tabular}{|l|}
\hline \multicolumn{1}{|c|}{ Quality of evidence } \\
\hline High $\oplus \oplus \oplus \oplus$ \\
\hline Moderate $\oplus \oplus \oplus$ \\
\hline Low $\oplus \oplus$ \\
\hline Very low $\oplus$ \\
\hline
\end{tabular}

index in superscript after the $95 \% \mathrm{Cl}$ corresponds with the number of trials where the bivalent (b) or quadrivalent (q) vaccines were assessed (for instance ${ }^{\text {b1q2 }}$ : meta-analysis of 3 trials, one with the bivalent and two with the quadrivalent vaccine)

[Ref]: the number between square brackets indicates the reference to the forest plot in Analyses and Tables

From a public health point of view, the two most relevant groups are: 1) hrHPV negative participants who reflect the naive unexposed group and 2) all vaccinated participants regardless of initial HPV DNA status.

In young women (15 to 26 years) who were hrHPV DNA negative and who received at least one dose of vaccine, the risk of CIN2+ associated with HPV16/18 was reduced on average from 164 to 2 per 10,000 women, a reduction or risk difference (RD) of 162 per 10,000 women vaccinated (Cates plot in Figure 8 ). The reduction in any CIN2+ irrespective of HPV type was from 287 to 106 per 10,000 women (RD 181 per 10,000 women vaccinated, see (Figure 9). 
Figure 8. Modified Cates plot: Number of cases of CIN2+ associated with HPV16/18 occurring in women who were all hrHPV DNA negative at baseline. 16 out of 1000 non-vaccinated women developed the lesion (left) whereas fewer than one (0.2) out 1000 vaccinated women developed the lesion (right). Relative risk= 0.01 (95\% $\mathrm{Cl}: 0.01$ to 0.05$)$.

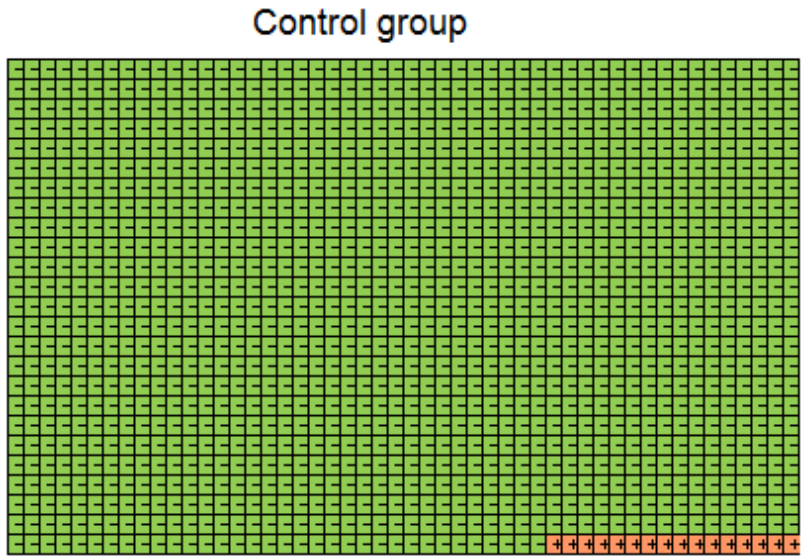

No lesions developed

Lesions developed
Vaccinated group

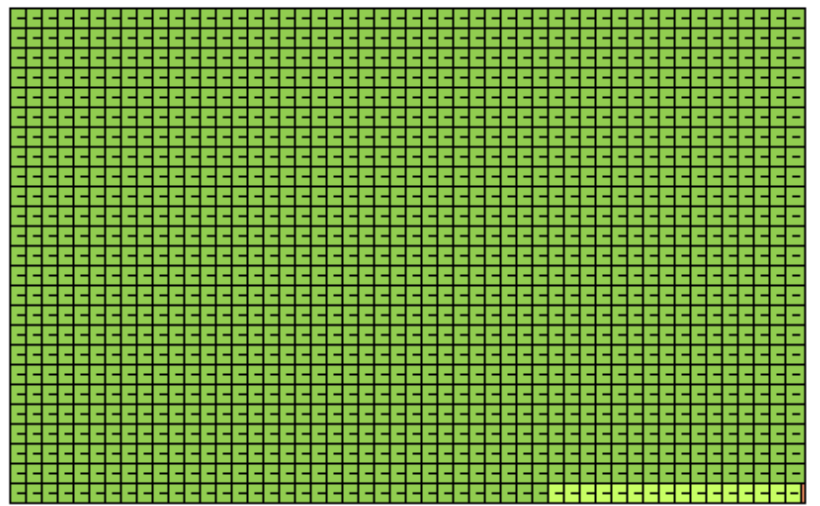

- No lesions developed

- Lesions avoided by vaccination

Lesions still developed in spite of vaccination

Figure 9. Modified Cates plot: Number of cases of CIN2+ irrespective of HPV types occurring in women who were all hrHPV DNA negative at baseline. 28 out of 1000 non-vaccinated women developed the lesion (left) whereas 11 out 1000 vaccinated women developed the lesion (right). Relative risk $=0.37$ (95\% $\mathrm{Cl}: 0.25$ to 0.55 ).

Control group

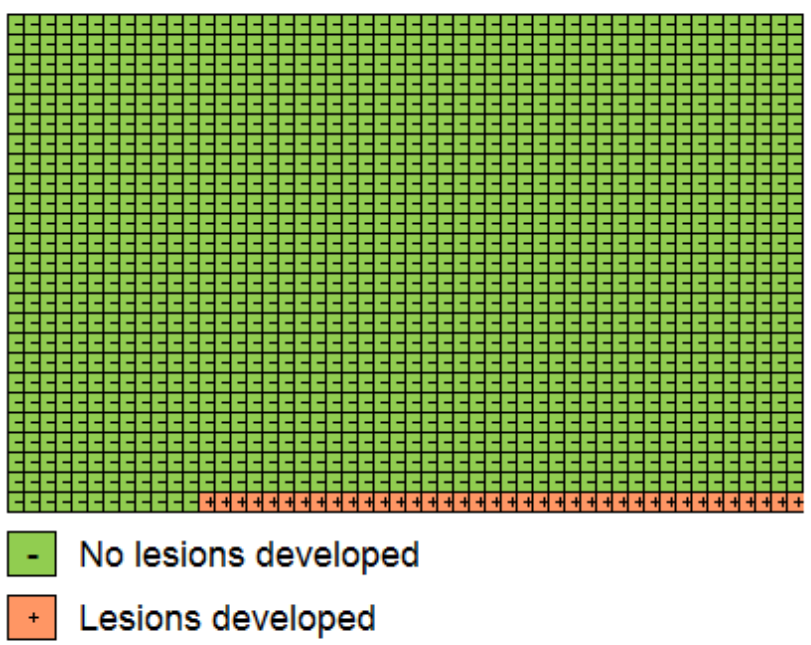

Among vaccinated women regardless of initial HPV DNA status, the risk reduction was from 341 to 157 per 10,000 women (RD 184 per 10,000 women vaccinated) and from 559 to 391 per 10,000 women (RD 168 per 10,000 women vaccinated), for CIN2+ associated with HPV16/18 or irrespective of HPV type, respectively (Summary of findings 3$)$.

The number needed to vaccinate was computed from the risk differences (NNV = 1/RD) (see Table 3). The number of women to be
Vaccinated group

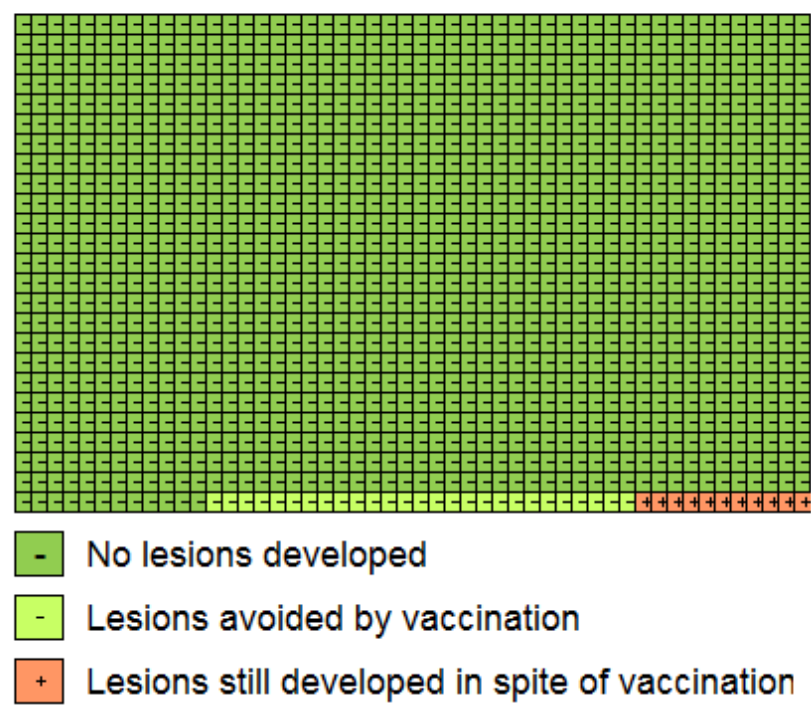

vaccinated to prevent one case of $\mathrm{CIN} 2+, \mathrm{CIN} 3+$ or AIS, associated with HPV16/18, in young women of age 15 to 26 years who were hrHPV negative at enrolment and who had received at least one dose of vaccine, was estimated to be 62, 204 and 1111, respectively. Although vaccine efficacy was lower in all participants regardless of initial HPV status, the NNVs were similar or slightly lower. Also, for lesions irrespective of HPV type, the NNVs were lower or similar. It must be noted that in populations where considerable exposure 
to HPV infection occurred prior to vaccination, the absolute risk of lesions in the vaccinated group is likely to be considerable.

\section{Adverse effects}

Safety issues are summarised in Table 4. All the vaccines were consistently associated with short-term local adverse effects (RR $1.18,95 \% \mathrm{Cl} 1.16$ to 1.20 ; participants $=18,113$; studies $=8 ; \mathrm{I}^{2}=$ $93 \%$; moderate-quality evidence; Analysis 7.1 ), such as pain at the injection site (RR 1.35, 95\% Cl 1.23 to 1.49 ; participants $=25,691$; studies $=13 ; 1^{2}=98 \% ;$ moderate-quality evidence; Analysis 7.2), local swelling (RR 1.73, 95\% Cl 1.32 to 2.27; participants = 22,106; studies = 9; $1^{2}=95 \%$; moderate-quality evidence; Analysis 7.3 ) and redness (RR 1.72, 95\% Cl 1.50 to 1.97; participants = 19,996; studies $=6 ; I^{2}=82 \%$; moderate-quality evidence; Analysis 7.4).
Systemic events with general mild symptoms were similarly frequent in vaccinated recipients and placebo or control vaccine recipients (RR 1.02, 95\% Cl 0.98 to 1.07 ; participants $=18,191$; studies $=8 ;\left.\right|^{2}=72 \%$; moderate-quality evidence; Analysis 7.5). The risk of serious adverse effects was similar in those vaccinated and those who received placebo or control vaccine (RR $0.98,95 \% \mathrm{Cl}$ 0.92 to 1.05 ; participants $=71,597$; studies $\left.=23 ; 1^{2}=6 \%\right)$ ) highquality evidence). There was little or no difference between the different vaccines $\left(P=0.19 ; 1^{2}=39.7 \%\right.$, Analysis 7.6$)$. Restriction to data extracted only from publications in peer-reviewed journals yielded very similar results: RR $1.01,95 \% \mathrm{Cl} 0.95$ to $1.06 ; 71,452$ participants; studies $=22,12=0 \%$, Figure 10$)$, with very minor differences between the vaccine types $(P=0.83,12=0 \%)$. 
Figure 10. Sensitivity analysis of Analysis 7.6 on severe adverse effects restricting to data extracted from publications in peer-reviewed journals.

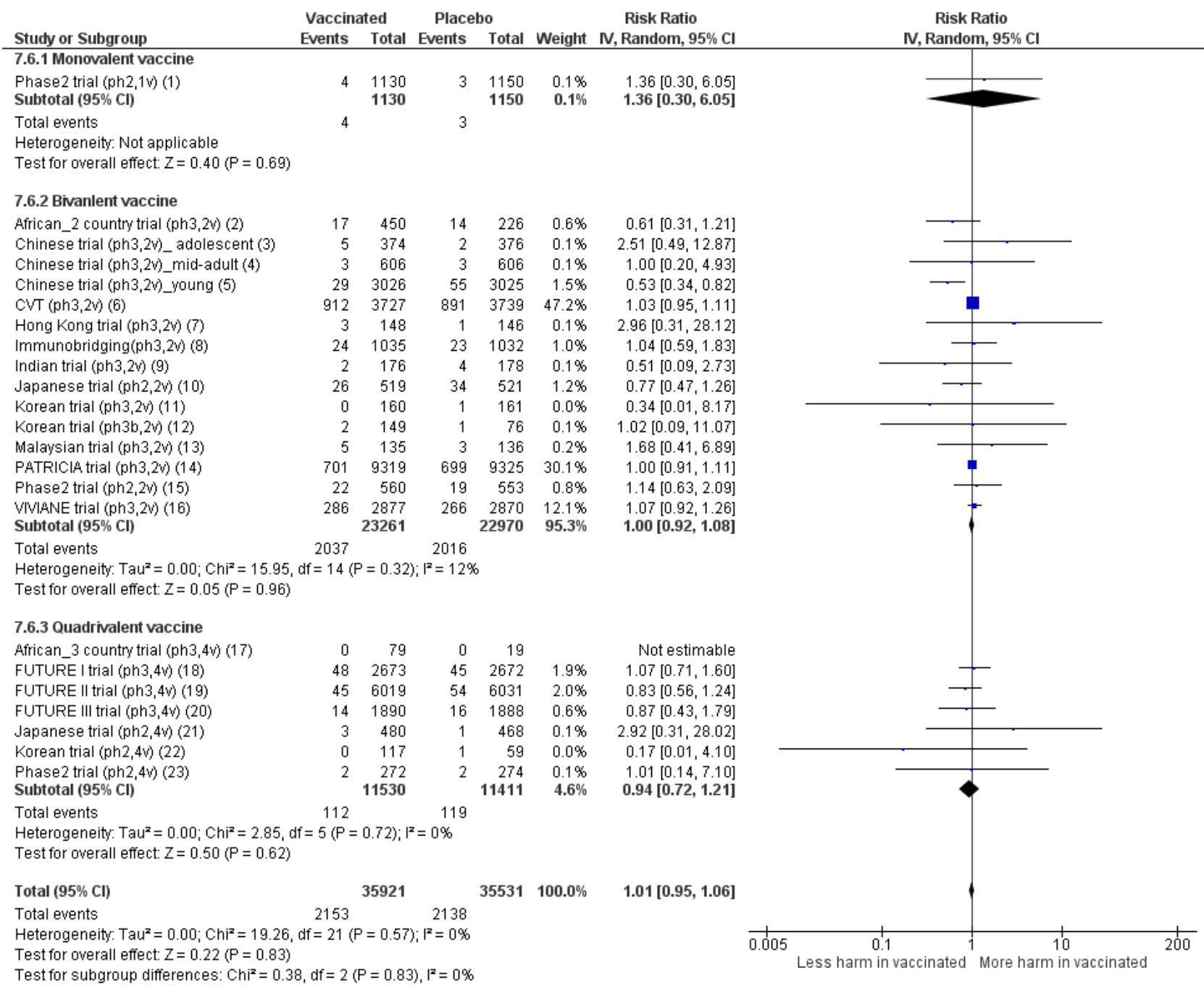

Footnotes

(1) Koutsky, New Engl J Med (2002). Follow-up: 14 days.

(2) Sow, J Infect Dis (2013). Follow-up: 12 months.

(3) Zhu, Hum Vaccin Immunother (2014). Follow-up: 12 months.

(4) Zhu, Hum Vaccin Immunother (2014). Follow-up: 12 months

(5) Zhu, Int J Cancer (2014). Follow-up: 21 months.

(6) Hildesheim, Vaccine (2014). Follow-up: 54 months.

(7) Ngan, Hong Kong Med J (2010). Follow-up: 7 months

(8) Medina, J Adolesc Health (2010). Follow-up; 12 months

(9) Bhatla, J Obstet Gynaecol Res (2010). Follow-up: 7 months.

(10) Konno, Hum Vaccine Immunother (2014), Follow-up: 48 months.

(11) Kim, J Kor Med Sci (2010). Follow-up; 7 months.

(12) Kim, J Gynecol Oncol (2011). Follow-up: 7 months.

(13) Lim, Med J Malaysia (2014). Follow-up; 7 months.

(14) Paavonen, Lancet (2009). Follow-up: 41 months.

(15) The GSK study group, Lancet (2009). Follow-up: 76 months.

(16) Wheeler, Lancet Infect Dis (2016). Follow-up: 48 months.

(17) Mugo, Hum Vacc Immunother (2014). Follow-up: 7 months.

(18) Garland, New Engl J Med (2007). Follow-up:36 months.

(19) The FUTURE II study group, New Engl J Med (2007). Follow-up: 36 months

(20) Castellsague, Br J Cancer (2011). Follow-up: median 48 months.

(21) Yoshikawa, Cancer Sc (2013). Follow-up: 15 days after any dose of vaccination.

(22) Kang, Int J Gynecol Cancer (2008). Follow-up: 7 months.

(23) Villa, Lancet Oncol (2005). Follow-up time was not clear for the safety outcomes.

Mortality during the study follow-up period in HPV vaccine recipients and control or placebo groups was reported in 23 trials (Analysis 7.7). We could not exclude an increased risk of

mortality among vaccinated women (RR $1.29,95 \% \mathrm{Cl} 0.85$ to 1.98 ; participants $=71,176$; studies $\left.=23 ;\left.\right|^{2}=0 \%\right)$. In absolute terms the rate of deaths in the control groups was 11 per 10,000 whereas in 
HPV vaccinated women the rate observed was between 9 and 22 women per 10,000. The difference between the bi-and quadrivalent vaccine was not significant $(P=0.62,12=0 \%)$. Again, results were very similar when data extraction was restricted to peer-reviewed published reports $(\mathrm{RR} 1.31,95 \% \mathrm{Cl} 0.84$ to 2.05 ; participants $=$
$71,452 ;$ studies $=23 ; 12=0 \%$, Figure 11$)$. We downgraded the quality of evidence for mortality to low. This was due to imprecision from the wide confidence interval and inconsistency due to a statistically different risk between the two age cohorts, with a higher risk of mortality in older women (Summary of findings 4). 
Figure 11. Sensitivity analysis of Analysis 7.7 on deaths restricting to data extracted from publications in peerreviewed journals.

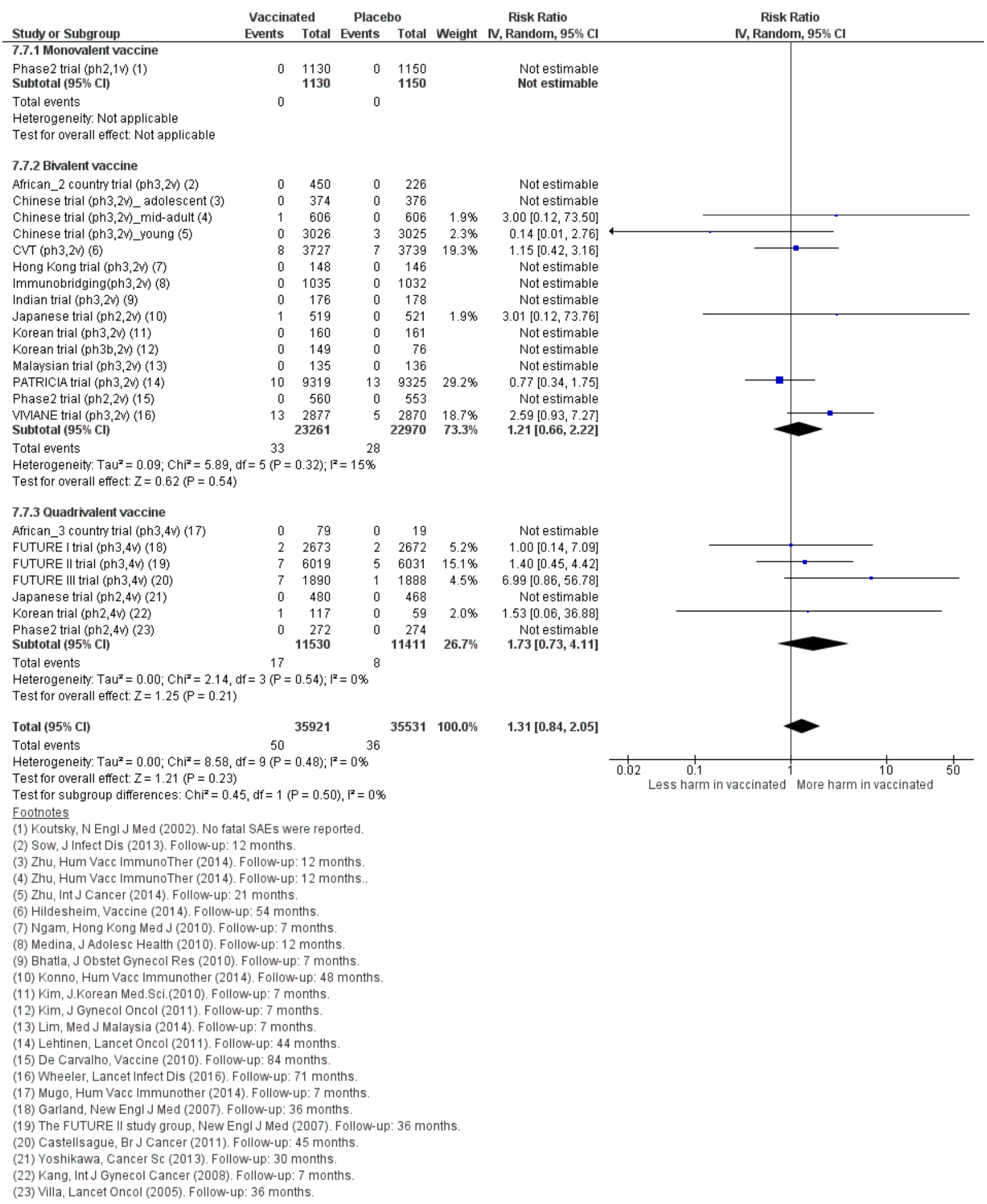

The higher number of deaths from both vaccination arms in the trials enrolling women older than 25 years may be expected due

eight deaths were observed within a period of 10 years of followto the longer periods of follow-up. In the FUTURE III trial (ph3,4v), up among women who received the quadrivalent vaccine versus four among women who received the placebo (RR $2.00,95 \% \mathrm{Cl} 0.60$ 
to 6.62, $\left.p_{\text {exact }}=0.25\right)$ (https://clinicaltrials.gov/ct2/show/results/ NCT00090220?sect=X30156\#evnt). In the VIVIANE trial (ph3,2v), 13 women died among women who received the bivalent vaccine compared with five among women in the placebo arm within six years of follow-up (RR $2.59,95 \% \mathrm{Cl} 0.93$ to 7.27 , $\mathrm{p}_{\text {exact }}=$ 0.09) (Wheeler 2016). In the smaller Chinese trial, where 606 midadult women were vaccinated with the bivalent vaccine, during 12 months of follow-up, one women died in the vaccine group whereas no deaths occurred in the control arm (Chinese trial (ph3,2v)_midadult; Zhu 2014a). When all the deaths among mid-adult women enrolled in the three trials are pooled, a higher case fatality rate was observed among those who received HPV vaccine compared to those who received placebo: (RR 2.36, $95 \% \mathrm{Cl} 1.10$ to 5.03; participants $=10,737$; studies $\left.=3 ; 1^{2}=0 \%\right)$, with no differences between different HPV vaccines $(P=0.73)$.

An overview of the causes of deaths observed after administration of HPV vaccine or control in the FUTURE III trial $(\mathrm{ph} 3,4 \mathrm{v})$ and VIVIANE trial $(\mathrm{ph} 3,2 \mathrm{v})$ is shown in Table 5 and Table 6 , respectively. In the smaller Chinese trial, one woman who was vaccinated died from intracranial haemorrhage (Zhu 2014a; https://www.gsk-clinicalstudyregister.com/files2/ d6eb0c75-b164-41e6-8295-f2718bce6adc). The higher number of deaths in the vaccine arms among mid-adult women may be a chance occurrence, since there was no pattern either in the causes of death, or in the timing of the occurrence of death (period between vaccine administration and date of death). In the study reports, none of the deaths were deemed to be related to vaccination (Castellsagué 2011; Skinner 2014; Wheeler 2016; https://www.gsk-clinicalstudyregister.com/files2/ d6eb0c75b164-41e6-8295-f2718bce6adc).

\section{Pregnancy outcomes}

Pregnancy outcomes were reported in a bivalent vaccine trial (VIVIANE trial $(\mathrm{ph} 3,2 \mathrm{v})$ ) and also through two pooled analyses of trials evaluating the bivalent (PATRICIA \& CVT (ph3,2v)) and quadrivalent vaccine (Pooled v4 trials), respectively (see Table 4).

Similar rates of normal term deliveries of a healthy infant were noted $(\mathrm{RR} 1.00,95 \% \mathrm{Cl} 0.97$ to 1.02 ; participants $=8782$; studies $=$ $8 ; 1^{2}=0 \%$; Analysis 8.1 ). The risk of miscarriage also was similar between HPV vaccinees and control vaccinees (RR 0.88, 95\% $\mathrm{Cl} 0.68$ to 1.14 ; participants $=8,618$; studies $=9 ; I^{2}=78 \%$; Analysis 8.2 ), as was elective termination of pregnancy (RR $0.90,95 \% \mathrm{Cl} 0.80$ to 1.02 ; participants $=10,909 ;$ studies $=9 ; 1^{2}=0 \%$; Analysis 8.3 ). Analyses of still births and abnormal infants lack sufficient power to rule out small increases or decreases in risk. The observed risk of stillbirth of 70 per 10,000 translates to a rate of 78 per 10,000 (48 to 128) based on the RR of 1.12 ( $95 \% \mathrm{Cl} 0.68$ to 1.83; Analysis 8.4). The observed risk of an abnormal infant in the control groups was 205 per 10,000 and in the vaccination arms was 250 per 10,000 (180 to 346) based on the RR of 1.22 ( 0.88 to 1.69 ) (Analysis 8.5). We downgraded the quality of evidence for both of these outcomes to moderate due to imprecision. See further in Summary of findings 4.

\section{Role of covariates}

\subsection{Age}

Most randomised trials assessing vaccine efficacy enrolled younger women, in the age range 15 to 26 years (Table 7). Only three randomised controlled trials (RCTs) evaluated the efficacy of the vaccines (FUTURE III trial (ph3,4v), VIVIANE trial (ph3,2v); Chinese trial (ph3,2v)_mid-adult) in mid-adult women (aged 24 to 45 years). A small overlap was noted ( 24 to 26 years) between the young and the mid-adult groups.

No difference in protection (difference in $\mathrm{RR}<=0.15$ and $\mathrm{P}$ value for age effect non significant) was found between younger and midadult women with respect to:

1. CIN2+ associated with HPV16/18 in women who were HPV16/18 negative at baseline and who received three doses (Analysis 2.1);

2. CIN2+ associated with HPV6/11/16/18 in women who were HPV16/18 negative at baseline and who received three doses (Analysis 2.4;.

3. six-month persistent HPV16/18 infection in women who were HPV16/18 negative at baseline and who receivedthree3 doses (Analysis 5.4) or at least one dose (Analysis 5.5).

Lower protection was found in mid-adult women compared to younger women with respect to:

1. CIN2+ associated with HPV16/18 in women who were HPV16/18 negative at baseline and who received one or two doses (Analysis 2.3) or at least one dose (Analysis 2.2);

2. CIN2+ associated with HPV6/11/16/18 in women who were HPV16/18 negative at baseline and who received at least one dose (Analysis 2.5);

3. CIN2+ associated with HPV16/18 in all women, regardless of their baseline hrHPV DNA status, who received at least one dose (Analysis 3.1);

4. six-month persistent HPV16/18 infection in all women, regardless of their baseline hrHPV DNA status, who received at least one dose (Analysis 6.2).

Lower protection (difference in RR $>0.15$ ) was found in mid-adult women compared to younger women (RR, but the difference was not significant for the following outcomes:

1. CIN2+ associated with HPV6/11/16/18 in women who were HPV16/18 negative at baseline who received one or two doses (Analysis 2.6);

2. CIN2+ associated with HPV6/11/16/18 in all women, regardless of their baseline hrHPV DNA status, who received at least one dose (Analysis 3.2);

3. Any CIN2+ irrespective of hrHPV types in all women, regardless of their baseline hrHPV DNA status, who received at least one dose (Analysis 3.7);

4. six-month persistent HPV16/18 infection in women who were HPV16/18 negative at baseline and who received one or two doses (Analysis 5.6).

For the bivalent vaccine, three trials (CVT (ph3,2v); PATRICIA trial (ph3,2v); VIVIANE trial (ph3,2v)) reported the efficacy within smaller age subgroups (Table 7 ). Since the age groups were not uniformly defined, age effects were assessed by Poisson regression for each trial separately. The $P$ value corresponding with the hypothesis of decreasing efficacy with increasing age is shown in the last column in Table 8 (PATRICIA trial (ph3,2v)), Table 9 (CVT (ph3,2v) and Table 10 (VIVIANE trial $(p h 3,2 v)$ ). This P value corresponds with checking the significance of the incorporation of the interaction term "vaccine*age" in the Poisson regression, treating age as a continuous variable. The protection against $\mathrm{CIN} 2+$ 
and $\mathrm{CIN} 3+$, associated with HPV16/18 or irrespective of hrHPV type, as well as the protection against six-month persistent HPV16/18 infection, decreased significantly by age in the intention-to-treat groups where women were enrolled regardless of baseline HPV DNA status. Within the per-protocol groups, enrolling women who were HPV16/18 DNA negative at baseline, no significant linear age effects were observed. Only for the outcome of persistent six-month HPV16/18 infection a slight decrease in protection was observed in the PATRICIA trial (PATRICIA trial $(p h 3,2 \mathrm{v}), \mathrm{P}$ value $=0.042)$, but not in the Costa Rica trial (CVT (ph3,2v), P value $=0.145)$, and not in the VIVIANE trial (VIVIANE trial (ph3,2v), P value $=0.532$ ).

\subsection{Serological status}

As described above, vaccine efficacy depends upon whether an hrHPV infection is present prior to vaccination, but could also potentially be influenced by prior hrHPV infection that cleared (as defined by being no longer detectable using a hrHPV DNA test), but with a positive hrHPV serology status. In Table 11, we pooled the relative risk of, and the vaccine efficacy against, CIN2+ associated with HPV16/18 stratified by initial hrHPV serology and DNA status from two phase III trials (FUTURE II trial (ph3,4v) and PATRICIA trial (ph3,2v)). In HPV16/18 DNA negative women, protection was strong, but varied by serology status: $\mathrm{RR}=0.03(95 \% \mathrm{Cl} 0.02$ to 0.09$)$ and $0.19(95 \% \mathrm{Cl} 0.09$ to 0.77$)$ for HPV16/18 in seronegative and seropositive women, respectively. No significant protection was observed in the HPV16/18 DNA-positive group, with RR being 0.79 $(95 \% \mathrm{Cl} 0.60$ to 1.05$)$ and 1.10 (95\% $\mathrm{Cl} 0.88$ to 1.36$)$ for HPV16/18 seronegative and seropositive women, respectively.

The effect of the serology status was computed by meta-regression as a relative risk ratio (RRR). This relative risk corresponds with

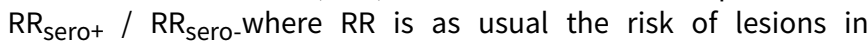
vaccinated versus unvaccinated women. The RRRs were 5.85 $(95 \% \mathrm{Cl} 0.53$ to 65.10$)$ for seropositive compared to seronegative women if HPV16/18 DNA-negative and 1.37 (95\% Cl 0.97 to 1.84 ) for seropositive compared to seronegative women if HPV16/18 DNApositive. Both RRRs were not statistically significantly different from unity. The RRRs were higher than unity, reflecting a tendency of higher risk of lesions or a lower vaccine efficacy in seropositive women. The effect of sero-positivity was more pronounced in HPV DNA-negative women, but even in this group, it was again not statistically significant. Whether the seropositivity effect is due to lower vaccine protection or presence of HPV virus prior to vaccination below the detection limit of the used HPV DNA test cannot be derived from the data.

\subsection{Study quality and involvement of vaccine manufacturers}

The impact of six study quality items (V1-V6) (see Assessment of risk of bias in included studies; Characteristics of included studies) on the protection against six- and 12-month persistent HPV16/18 infection was assessed by meta-regression. No significant effects were observed: $P$ values were all > 0.05 (see Table 12).
The impact of the involvement of the vaccine manufacture in the trials was also assessed by meta-regression. No significant effects were observed.

\subsection{Number of administered doses}

In a post hoc pooled analysis of the Costa-Rica Vaccination Trial (CVT $(p h 3,2 v)$ ), it was demonstrated that efficacy against 12-month incident persistent infection was no different $\left(P\right.$ value $=0.60,1^{2}=$ $0 \%)$ in women who had received one, $(\mathrm{RR}=0.05,95 \% \mathrm{Cl} 0.00$ to 0.77 ), two ( $R R=0.16,95 \% \mathrm{Cl} 0.05$ to 0.52 ), or three doses ( $R R=$ $0.19,95 \% \mathrm{Cl} 0.12$ to 0.29 ) of the bivalent vaccine (Analysis 6.5). More outcomes were assessed in a pooled analysis of the Costa Rica and PATRICIA trials (Kreimer 2015). Protection induced by the bivalent vaccine against incident and six- and 12-month persistent HPV16/18 infection in 15 to 26 years old women, initially HPV16/18 or hrHPV negative, did not differ by number of received doses (Table 13). It is planned to continue the follow-up in the Costa-Rica Vaccination Trial (CVT (ph3,2v)) for 10 years, to verify the durability of protection afforded by fewer than three doses of the bivalent vaccine (Kreimer 2015b). Results up to 6.9 years show that the cumulative incidence of HPV16/18 infections among women who received one dose, or two doses (received at months zero and six, or at months zero and one) are similarly low compared to those who received the three doses (see Table 14; Safaeian 2018).

For several trials, results were provided for the same outcome among women being initially HPV16/18 DNA negative and having received all three doses and at least one dose. This allowed us to compute, in a post-hoc analysis, by simple subtraction the number of events and women at risk having received only one or two doses (Table 15).

Significant protection was observed for women having received only one or two doses for the following outcomes:

1. CIN2+ associated with HPV16/18 in women aged 16 to 25 years (observation for mono-,bi- and quadrivalent vaccines, Analysis 2.3);

2. CIN3+ associated with HPV16/18 in women aged 16 to 25 years (observation for the bivalent and the quadrivalent vaccine, Analysis 2.9);

3. Incident HPV16/18 infection in women aged 15 to 26 years (observation only for the mono, and the bivalent vaccine, Analysis 5.3);

4. six-month persistent $16 / 18$ infection in women aged 15 to 26 and 25 to 45 years (observation for the bivalent and quadrivalent vaccine, respectively, Analysis 5.6).

No protection against CIN2+ associated with HPV16/18 was observed in women aged 24 to 45 who received only one or two doses. (Analysis 2.3).

Protection against $\mathrm{CIN} 2+$ associated with HPV16/18 in women aged 15 to 26 years and were baseline HPV DNA 16/18 negative, no subgroup difference was observed for women having received three doses or only one or two doses (Figure 12). 
Figure 12. Protection against CIN2+ associated with HPV16/18 in women, aged 15-26 years, who were HPV DNA $16 / 18$ negative at baseline, by number of doses.

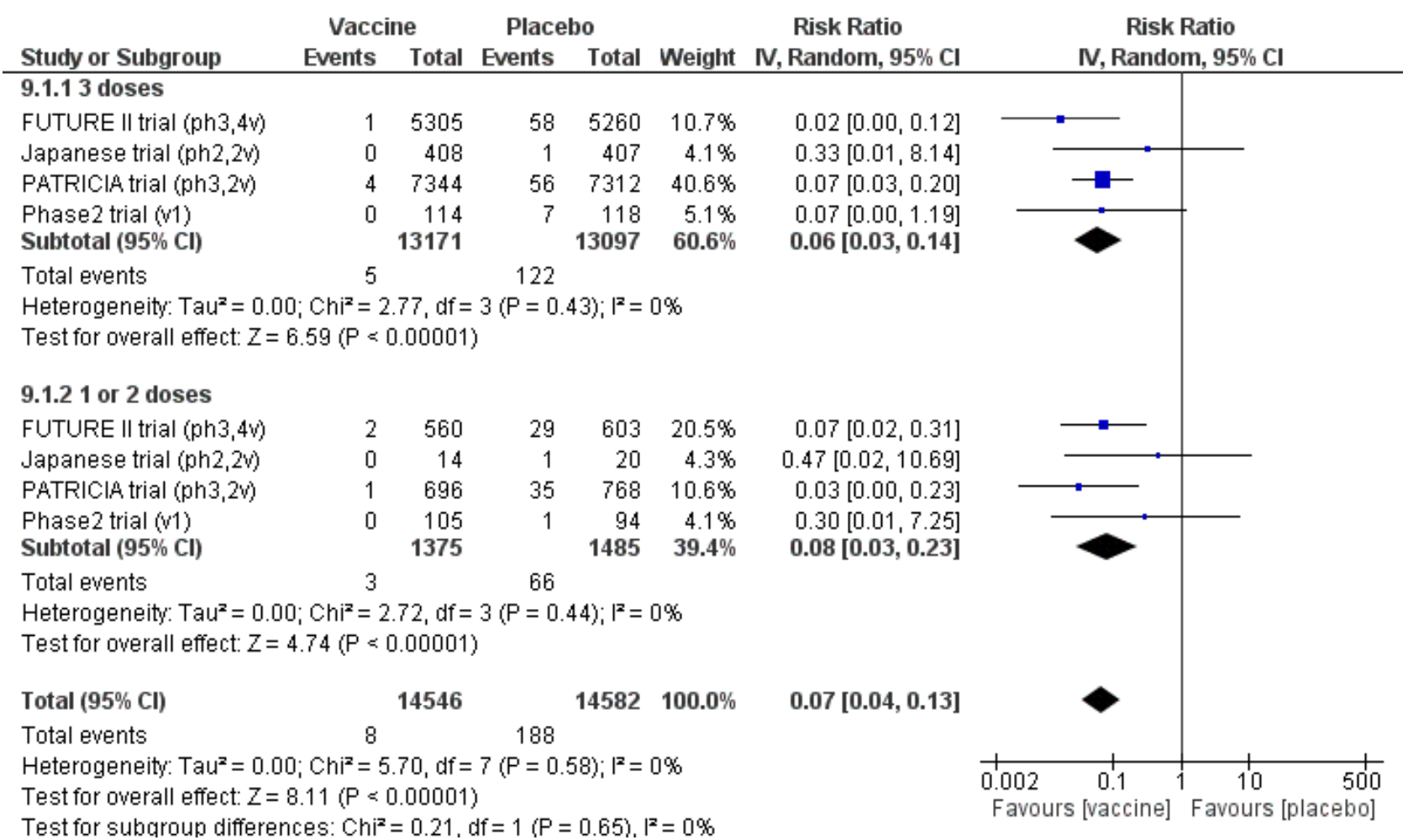

\subsection{Duration of follow-up}

The assessment of possible changes in vaccine efficacy over time was impeded due to uneven spacing of periodic reports. Efficacy was reported at several time points in two trials (FUTURE II trial (ph3,4v); PATRICIA trial (ph3,2v)), varying between 15 and 44 months on average. Protection against $\mathrm{CIN} 2+$ associated with HPV16/18 infection did not drop by longer follow-up time, either in women who were HPV16/18 DNA negative, or for those enrolled regardless of their HPV DNA status (Table 16).

\subsection{Sexual history}

The impact of sexual history on vaccine efficacy was assessed in only one trial (CVT (ph3,2v)) for the outcome protection against 12-month persistent HPV16/18 infection. The number of sexual partners had no effect in the analysis limited to participants who were HPV16/18 DNA negative at enrolment ( $P$ value $=0.7448$ ). However, in the group of women enrolled regardless of their baseline HPV DNA status, a very significant decrease in protection by increasing number of sexual partners was observed ( $P$ value < 0.00001) (see Table 17).

\subsection{Study size}

The vaccine efficacy did not vary between small or large trials (Table 18).

\section{DISCUSSION}

\section{Summary of main results}

\section{Comments on main results}

We included 26 randomised controlled trials (RCTs) involving 73,428 participants, ranging from 98 to 18,644 participants per trial. Studies involved monovalent (one trial), bivalent (18 trials), and quadrivalent vaccines (seven trials). Most trials recruited adolescent girls and women 15 to 26 years of age; three trials recruited women aged 24 to 45 years. We judged most included trials to be at a low risk of bias. All the trials, except one (CVT (ph3,2v), were funded by the vaccine manufacturers. However, vaccine efficacy and adverse effects were not different in trials funded by manufacturers and the one trial conducted with public resources.

\section{Protection against persistent human papillomavirus (HPV)16/18 infection and associated cervical precancer}

HPV vaccine efficacy was very high among young women (15 to 26 years) against six-month and 12-month persisting HPV16/18 infection (risk ratio $(R R) \leq 0.10)$ (high-quality evidence). It is also high against cervical intraepithelial neoplasia grade 2 and above $(\mathrm{CIN} 2+)$ and $\mathrm{CIN}$ grade 3 and above $(\mathrm{CIN} 3+)(\mathrm{RR} \leq 0.10)$ and against adenocarcinoma in situ (AIS+) (RR $\leq 0.12$ ) associated with these types when women were high-risk human papillomavirus (hrHPV) negative or HPV16/18 negative at enrolment (high-quality evidence for $\mathrm{CIN} 2+$ and $\mathrm{CIN} 3+$; moderate-quality evidence for AIS+). Absolute reductions in risk further illustrate the relative effects. HPV vaccines reduce the risk of CIN2+ from 164 per 10,000 people to 2 per 10,000, and AIS from 9 per 10,000 to 0 per 
10,000 in hrHPV negative women (Summary of findings for the main comparison). While all trials were designed as randomised trials of 3-dose schedules, we included also analyses of fewer than three doses. Protection against precancerous lesions and persistent infection was also strong $(R R \leq 0.15)$ when fewer than three doses were received. We were not able to determine possible bias in these analyses due to women who did not complete the three-dose schedule having different risk factors to those who completed the vaccination schedule as per protocol. Among all vaccinated women regardless of their initial HPV DNA test results, protection against persistent HPV16/18 infection and associated precancerous lesions was weaker. The RR varied between 0.36 and 0.55 , corresponding to differences in risk of $1.8 \%$ for CIN2+ and $0.09 \%$ for AIS associated with HPV16/18 (Summary of findings 3). Follow-up ranged between two and eight years, with most studies contributing data collected between three and five years post-vaccination, limiting the potential to measure cervical cancer outcomes, which would require very long follow-up periods.

Protection against persistent HPV16/18 infection and associated CIN2+ lesions ( $R R \leq 0.15$ ) was also observed in mid-adult women (24 to 45 years) when they were HPV16/18 negative at baseline and received three doses of vaccine. Fewer than three doses offered some protection in HPV16/18 DNA negative mid-adult women against persistent HPV16/18 infection (RR $=0.34)$, but not against $\mathrm{CIN} 2+$ associated with HPV16/18. When all vaccinated midadult women were considered, regardless of their baseline HPV DNA status, vaccination offered some protection against six-month persistent HPV16/18 infection (RR $=0.57$ ), but not against $\mathrm{CIN} 2+$ associated with HPV16/18.

The quality of evidence was moderate to high and there was little evidence of heterogeneity by valency of the vaccine for most outcomes.

\section{Protection against any cervical precancer, irrespective of HPV type}

The efficacy of HPV vaccines was generally lower when any high-grade squamous lesions, irrespective of HPV infection type, was considered compared to efficacy for HPV16/18-associated lesions. The protection in hrHPV negative women following bivalent vaccination against development of $\mathrm{CIN} 3+(\mathrm{RR}=0.08$; Analysis 1.8.1), was greater than that observed for the quadrivalent vaccine $(\mathrm{RR}=0.54$; Analysis 1.8.2). The three-dose efficacy against CIN2+ $(R R=0.40$; Analysis 2.13) was no different between the bi- and quadrivalent vaccines in women who were initially HPV16/18 negative. No significant difference in protection was observed when fewer than three doses were given (Analysis 2.14). The efficacy against $\mathrm{CIN} 3+$ of the bivalent vaccine $(\mathrm{RR}=0.55$; Analysis 3.8.1) was again greater than the quadrivalent vaccine among all enrolled women regardless of their initial HPV DNA status (RR = 0.81; Analysis 3.8.2). However, differences in the population HPV prevalence in the trial sites, or differences in study protocols and assays used, may explain the contrast in efficacy. The quality of evidence regarding protection against any precancer, irrespective of HPV type, among mid-adult women is low (Table 2).

\section{Vaccine safety}

Short-term local adverse events were more common in women who received the HPV vaccine compared to those in the control arms. The risk of mild or severe systemic adverse events were similar between intervention and control arms (high-quality evidence for serious adverse events, see Summary of findings 3 ). The deaths reported in the trials had an identified cause, and none were assessed to be due to vaccination. The risk in absolute terms was low in both trial arms. The rate of mortality was 11 per 10,000 in the control arms, and the confidence interval is wide enough to include a rate of between 9 and 22 per 10,000 following vaccination (moderate quality evidence). In trials enrolling mid-adult women, a higher mortality rate in the HPV arms was observed. The deaths occurred months to years after vaccination. However, no pattern in the series of death causes was identified and study investigators did not establish a causal role of the HPV vaccines for any of the deaths.

We have insufficient evidence available from RCTs to know how vaccination affects women who become pregnant during the vaccination period. Pregnancy outcomes indicated similar risks of miscarriage and elective termination between vaccination and control (high-quality evidence). Analysis of stillbirth and congenital abnormality outcomes do not yet have enough information to confidently exclude slightly higher or slightly lower risk with vaccination: stillbirths: 70 per 10,000 versus 78 (48 to 128) per 10,000 following HPV vaccination (moderate-quality evidence); abnormal infants: 205 per 10,000 versus 250 ( 180 to 346 ) per 10,000 following HPV vaccination (see Summary of findings 4).

\section{Other important comments}

\subsection{Duration of protection}

The longest duration of follow-up for which vaccine efficacy data are reported was 102 months for the monovalent HPV16 vaccine (Rowhani-Rahbar 2009), 113 months for the bivalent vaccine (Naud 2014), and 60 months for the quadrivalent vaccine (Villa 2006). For all the vaccines, continued protection was observed at the end of the follow-up period (Table 19).

\subsection{Differences in efficacy between the bivalent and quadrivalent vaccine}

Based on subgroup analysis by vaccine brands, licensed bivalent and quadrivalent vaccines confer similar protection against HPV16/18 infection and cervical lesions associated with HPV16/18. However, we did find some evidence that bivalent vaccine was more efficacious than the quadrivalent vaccine against any $\mathrm{CIN2+}$ and $\mathrm{CIN} 3+$ (irrespective of HPV type) among women who were hrHPV DNA negative (Analysis 1.7; Analysis 1.8) and against any CIN3+ regardless of HPV DNA status at baseline (Analysis 3.8). This difference may be due to differences in the populations enrolled in the trials, differences in serological or DNA methods used, or better cross-protection of the bivalent vaccine against other hrHPV types. Cross-protective vaccine efficacy was assessed in a recent metaanalysis including data from ; FUTURE I trial (ph3,4v); FUTURE II trial (ph3,4v); Malagon 2012; PATRICIA trial (ph3,2v); Phase2 trial (ph2,2v); Phase2 trial (ph2,4v). Better protection was found against six-month persistent infection with HPV31 and HPV45 and against CIN2+ related to HPV33 and HPV45 using the bivalent versus the quadrivalent vaccine among women who were hrHPV negative at enrolment (Malagon 2012). Also, CVT (ph3,2v) and VIVIANE trial (ph3,2v) provided significant cross-protective efficacy of the bivalent vaccine with respect to $\mathrm{CIN} 2+$ associated with non-vaccine hrHPV types (Hildesheim 2014) and persistent HPV31 and HPV45 infection (Skinner 2014), respectively (Table 20). Although there may be some evidence of waning cross-protection (Malagon 2012), efficacy of the bivalent vaccine lasting for more than nine years against incident HPV31, HPV33 and HPV45 (RR between 0.29 and 0.65) has been reported (Starkie Camejo 2016; Taylor 2016). 
Kuhs 2014 explored whether different serological testing methods and HPV DNA criteria, used to define the sub-cohort of HPV-naïve women in the trials, could have influenced efficacy estimates. Applying the less restrictive criteria used in the FUTURE I/II trials (FUTURE II trial (ph3,4v); FUTURE I trial (ph3,4v)) instead of those applied in the PATRICIA trial (PATRICIA trial (ph3,2v)) on the CVT decreased the estimated efficacy against any CIN2+ (irrespective of HPV type) from $81 \%$ to $69 \%$, suggesting this is part of the explanation of the differences observed between vaccines.

Note-worthy is the limited inter-vaccine difference in efficacy against any CIN2+ (irrespective of HPV type) (Analysis 1.7; Analysis 3.7), where the contribution of non-HPV16/18 hrHPV types is greater than for CIN3+ (Bzhalava 2013). Comparable significant reductions in the prevalence of HPV31, HPV33 and HPV45 have been observed in vaccinated versus unvaccinated young women attending screening in Australia (vaccinated mainly with the quadrivalent vaccine: Tabrizi 2014) and Scotland (vaccinated with the bivalent vaccine: Kavanagh 2014).

Differences in safety between the vaccine brands are discussed in section 2.4 .

\subsection{Adverse effects of HPV vaccines}

Local adverse events at the injection site (pain, redness, swelling) were more common in vaccinated participants than in placebo recipients. However, systemic mild symptoms and serious adverse effects reported after an administered dose were equally distributed between the trial arms.

A pooled analysis of safety data was conducted by the manufacturer of the AS04-adjuvanted bivalent HPV vaccine involving 31,173 adolescent girls and women who received the vaccine and 24,241 controls (Angelo 2014). Unsolicited adverse symptoms reported within 30 days after each dose were slightly more frequent in the vaccine group (30.8\% versus $29.7 \%$ ), whereas medically significant conditions $(25.0 \%$ versus $28.3 \%)$, serious adverse effects $(7.9 \%$ versus $9.3 \%)$ and potentially immunemediated diseases $(0.52 \%$ versus $0.55 \%)$, reported over the whole study period, were not more frequent in vaccinated participants versus controls (Angelo 2014).

Occurrence of autoimmune events, possibly associated with the use of the adjuvants ASO4 (3-O-desacyl-4' monophosphoryl lipid A and aluminium) included in several vaccines, including the bivalent HPV vaccine, was assessed in a pooled analysis of trials conducted by the manufacturer (Verstraeten 2008). More than 68,000 records were included, among which 39,160 participants received the HPV16/18 L1 vaccine. The mean follow-up time was 21 months. The overall rate of autoimmune-related conditions was approximately $0.5 \%$ and the relative risk versus control groups was $0.98(95 \% \mathrm{Cl}$ 0.80 to 1.21$)$ for all AS04-adjuvanted vaccines and $0.92(95 \% \mathrm{Cl}$ 0.70 to 1.22 ) for HPV16/18 vaccine. For the individual autoimmune events, relative risks always included unity (Verstraeten 2008).

All estimates of adverse effects in our review were restricted to those reported from randomised trials and therefore could not detect rare events, for which post-marketing surveillance, pharmacovigilance activities and linkage studies, joining vaccine and morbidity registries, are needed. The post-licensure safety surveillance in the USA confirmed the general safety profile of the quadrivalent vaccine, which was consistent with observations from the studies included in our review, but identified a disproportional reporting of syncope and venous thromboembolic events. However, no causal relation could be established (Slade 2009). Subsequent studies did not find an association with thromboembolic events (Naleway 2016). Two healthcare organisations in California (USA) assessed new-onset autoimmune conditions related to immunisation with the quadrivalent vaccine and did not identify significant associations, with the exception of Hashimoto thyroiditis ( $\mathrm{RR}=1.29,95 \% \mathrm{Cl} 1.08$ to 1.56). However, time-relation and biological plausibility did not reveal evidence of causality (Chao 2012). The Medicines and Healthcare products Regulatory Agency of the UK set up a comprehensive pharmacovigilance study assessing the temporal association between chronic fatigue syndromes and the administration of the bivalent HPV vaccine (Donegan 2013). Despite the high coverage in girls and young women (age 12 to 20), no increased incidence of fatigue syndromes was observed after the introduction of HPV vaccination (incidence rate ratio: $0.94,95 \% \mathrm{Cl}: 0.78$ to 1.14). Detailed analysis of self-controlled case series of 187 girls and young women did not reveal evidence that the HPV vaccine caused fatigue syndromes (ratio: $1.07,95 \% \mathrm{Cl}$ : 0.57 to 2.00). A large linkage study, joining hospital records with HPV vaccine registries in Sweden and Denmark, did not reveal associations between administration of the quadrivalent vaccine and most autoimmune, neurological or venous thromboembolic adverse events. However, three autoimmune conditions were more common (Behcet's disease, Raynaud's disease and type 1 diabetes), and two neurological conditions were less common (epilepsy and paralysis) in vaccinated compared to non-vaccinated cohorts. Authors considered that multiple comparisons may explain the significant findings (Arnheim-Dahlstrom 2013). No increased incidence of thromboembolism or multiple sclerosis or other demyelating neurologic diseases after administration of the quadrivalent vaccine was detected from the Danish-Swedish linkage studies (Scheller 2014; Scheller 2015).

In March 2014, the World Health Organization (WHO) Global Advisory Committee on Vaccine Safety (GACVS) reviewed the evidence base on safety of HPV vaccines and responded to questions related to reports on possible adverse effects (such as syncope, anaphylaxis, venous thromboembolism, adverse pregnancy outcomes, Guillain-Barré Syndrome (GBS), stroke, toxic effects of the aluminium adjuvant, multiple sclerosis, cerebral vasculitis, complex regional pain syndrome (CRPS) and/or other chronic pain conditions). The committee concluded that the riskbenefit profile of prophylactic HPV vaccines remains favourable and expressed its concerns about unjustified claims of harm, which lack biological and epidemiological evidence, and which may affect the confidence of the public (Larson 2011). At the same time, the Committee encouraged health authorities to continue surveillance and examination of potential adverse events (WHO 2014).

Seven large studies and one CDC review have investigated the association between HPV vaccination Guillain-Barré syndrome (GBS) and found no evidence of increased risk (Arnheim-Dahlstrom 2013; CDC 2015; Chao 2012; Gee 2017; Grimaldi-Bensouda 2014; Ojha 2014; Vichnin 2015). In contrast, a French linkage study, linking HPV vaccination and morbidity registries, comprising more than 2 million girls found an increased risk of GBS: 0.4 versus 1.4 per 100,000 for non-vaccinated and vaccinated girls and young women, respectively, adjusted hazard ratio (HR): $4.00,95 \% \mathrm{Cl} 1.84$ to 8.69) (ANSM/SANTE 2015). The association between GBS and 
HPV vaccination was strongest during the first three months after the last dose.

Upon request of the Danish health authorities, the Pharmacovigilance Risk Assessment Committee of the European Medicine Agency investigated complaints of complex regional pain syndrome (CRPS) and postural orthostatic tachycardia syndrome (POTS) among young women who received HPV vaccines (EMEA 2015). No causal relation could be established. Preliminary conclusions were confirmed by the EMA Committee for Medicinal Products for Human Use (CHMP) completed with representations from patient groups (EMA 2016). A Danish casecontrol study compared pre-vaccination health-seeking behaviour in HPV vaccinated girls who had reported adverse effects (cases) with matched cohorts of HPV vaccinated girls who had not reported adverse affects. Increased rates of health problems were reported in the case group (Molbak 2016). Increasing trends in the incidence of chronic fatigue syndrome, systemic exertion intolerance disease and POTS (assessed from hospital discharge records) were reported in girls aged 12 to 15 years in the decade preceding the introduction of HPV vaccination in Finland (Skufca 2017). The authors warned for pre-vaccination trends and variation in disease coding and healthcare-seeking behaviour, which may influence the interpretation of associations with HPV vaccination (Molbak 2016, Skufca 2017).

In its last statement, the GACVS confirmed previous conclusions on $H P V$ vaccine safety after revision of the recent signals on increased occurrence of GBS, POTS and CRPS (WHO 2016).

\subsection{Comparison of adverse effects of the bivalent versus quadrivalent vaccines}

Our review revealed a significantly higher rate of localised effects (Analysis 7.1), such as pain (Analysis 7.2) and swelling (Analysis 7.4) at the injection site for women who received the bivalent vaccine. However, in a meta-regression adjusting for age and type of adjuvants or other vaccine given to the control group, these differences became non-significant (Table 21). The meta-regression analysis suggested a higher rate of local adverse effects associated with bivalent compared with the quadrivalent vaccine (relative risk ratio $(\mathrm{RRR})=1.69,95 \% \mathrm{Cl} 0.96$ to $2.96, \mathrm{P}$ value $=0.61$ ) The non-significance might be due to the low power of metaregression. Higher rates of local adverse effects at the injection site with the bivalent vaccine were also observed in a head-to-head trial comparing immunogenicity and safety of the two licensed vaccines (Einstein 2011). A significantly higher rate of local injection site reactions was observed with the bivalent compared with the quadrivalent vaccine (RR for pain: 1.30 (95\% Cl 1.25 to 1.34); RR for swelling: 1.67 (95\% Cl 1.43 to 1.98); RR redness: 1.73 (95\% Cl 1.52 to 1.98). A marginally non-significant higher frequency of medical significant conditions was noted among women who received the bivalent vaccine: $\mathrm{RR}=1.15$ (95\% Cl: 0.99 to 1.34$)$. There were no statistically significant differences for the other adverse effects: new onset chronic diseases ( $\mathrm{RR}=0.95,95 \% \mathrm{Cl}: 0.52$ to 1.74 ), new onset autoimmune disease ( $\mathrm{RR}=0.60,95 \% \mathrm{Cl}: 0.22$ to 1.64$)$, serious adverse effects ( $R R=1.05,95 \% \mathrm{Cl}: 0.59$ to 1.85$)$ (Einstein 2011).

\subsection{Pregnancy and infant outcomes}

Pregnancy or sexual activity without contraception were exclusion criteria for enrolment in randomised HPV vaccination trials. However, if enrolled women became pregnant, pregnancy outcomes were surveyed carefully. In a pooled analysis of five phase III trials assessing the quadrivalent vaccine, miscarriage or congenital anomalies were not more common in the vaccine arm compared to the placebo arm (Garland 2009). No relation was found between the time from administration of the vaccine to conception and adverse pregnancy outcomes or occurrence of congenital anomalies. The rate of miscarriage was not higher for women who conceived within 30 days of any vaccination $(18.2 \%$ in the vaccine arm versus $21.0 \%$ in the placebo group ( $P$ values or $95 \% \mathrm{Cls}$ not computable by lack of denominator). Also in a pooled analysis of the PATRICIA and Costa Rica Vaccination trial (Wacholder 2010), miscarriage was not more frequent in women who received the bivalent HPV vaccine $(197 / 1709=11.5 \%)$ compared with those who received the hepatitis A vaccine in the control group (176/1727 $=10.2 \%): \mathrm{RR}=1.13,95 \% \mathrm{Cl} 0.93$ to 1.37 . However, for women who became pregnant within 90 days of administration of the bivalent vaccine, a significant increase in the rate of miscarriage was observed in women who received the HPV vaccine $(58 / 394=$ $14.7 \%)$ versus the control group $(34 / 374=9.1 \%)$ : $R R=1.62,95 \%$ Cl: 1.08 to 2.41) (Wacholder 2010). However, this finding was not confirmed in a larger study (Panagiotou 2015). After completion of the CVT trial (CVT (ph3,2v)), women in the placebo arm were offered the bivalent vaccine. Pregnancy outcomes were monitored for vaccinated women (from the vaccine arm + cross-over vaccination from the control arm) and for control women (from the placebo arm having received Hepatitis A vaccine only completed with an unvaccinated cohort). The miscarriage rate was 13.3\% (451 / 3394) among women who conceived at any time since bivalent HPV vaccination) versus $12.8 \%(414 / 3227)$ in pregnant women from the control group $\mathrm{RR}=1.04(95 \% 0.91$ to $1.17, \mathrm{P}$ value $=0.29)$ (Panagiotou 2015). There was no increased risk of miscarriage among women conceiving within 90 days of vaccination ( $P$ value $=0.436$ ) overall or in subgroups. However, among women who conceived at any time from vaccination, an increased rate was observed for miscarriage occurring at 13 to 20 weeks of gestation ( $\mathrm{RR}=1.35,95 \% \mathrm{Cl}$ 1.02-1.77) (Panagiotou 2015).

In a post-marketing surveillance study of 517 women who received the quadrivalent vaccine and became pregnant in the USA, Canada or France, the observed rates of miscarriage and birth defects were not higher than expected in the general population (Dana 2009). An updated analysis including 1752 pregnant women having received the quadrivalent vaccine confirmed previous conclusions (Goss 2015). No overall increased rate of adverse pregnancy outcomes was noted in British women who received the bivalent vaccine close to conception ( -30 to +45 or -30 to +90 days) versus women who became pregnant six to 18 months after the last dose. However, in one subgroup, who received two doses of bivalent vaccine around conception, an increased hazard of miscarriage was found (HR $2.55,95 \% \mathrm{Cl} 1.09$ to 5.93) (Baril 2015). A retrospective cohort study assessed pregnancy outcomes in women with live births vaccinated with the quadrivalent HPV vaccine, according the co-incident timing of vaccine administration and the pregnancy: a) 720 received the vaccine in the periconceptional period (two weeks before and after the last menstrual period); b) 638 during the pregnancy and c) 8196 four to 18 months before last menstrual period (Lipkind 2017). No increased risks neither in adverse obstetric events nor in birth outcomes were observed in the first two groups compared to the comparison group.

\subsection{Safety of HPV vaccines co-administered with other vaccines}

A systematic review comparing HPV vaccines administered alone versus co-administered with other vaccines (meningococcal 
conjugate, hepatitis $A$, hepatitis $B$, combined hepatitis $A$ and $B$, tetanus, diphtheria, acellular pertussis, and inactivated poliovirus vaccines) showed non-inferior seroconversion rates and similar rates of adverse effects (Noronha 2014).

\subsection{Efficacy of the nona-valent HPV vaccine}

A recent paper reported the effects up to 48 months of the new nona-valent vaccine which contains virus-like particles (VLP) of the L1 protein of the HPV types 31, 33, 45, 52 and 58 as well as the four types included in the quadrivalent vaccine in women aged 16 to 26 years (Joura 2015). The seven included high-risk types comprise the most prevalent types in cervical cancer and nearly $90 \%$ of all cervical cancer cases worldwide can be attributed to these types (Arbyn 2014; Bosch 2008). The randomised trial was not included in our review since it compared the nona-valent with the quadrivalent vaccine.

In women who were hrHPV DNA negative at baseline the relative risk (9- versus quadrivalent vaccinated) of persistent infection with HPV types 31, 33, 45, 52 and 58 as well as CIN2+ associated with these five types was $0.04(95 \% \mathrm{Cl} 0.03$ to 0.06$)$. In this group, the risk of any $\mathrm{CIN} 2+$ irrespective of HPV types was $0.60(95 \% \mathrm{Cl} 0.36$ to 0.98 ). In the modified intention-to-treat group, including women without cytological lesions regardless of baseline HPV DNA status, no protection was observed against any CIN2+ irrespective of HPV ( $R R=1.00,95 \% \mathrm{Cl} 0.96$ to 1.16 ) (Joura 2015). Three per cent more local adverse reactions were observed in women who received the nona-valent vaccine: $\mathrm{RR}=1.03(95 \% \mathrm{Cl} 1.02$ to 1.04$)$ but no significant differences in systemic of serious adverse events were noted. A more recent report confirmed efficacy findings over a follow-up period of six years (Huh 2017).

A similar immune response of the nona-valent vaccine compared to the quadrivalent vaccine against HPV6, HPV11, HPV16 and HPV18 was demonstrated for girls of age nine to 15 years (Vesikari 2015). Non-inferior immunogenicity of the nona-valent vaccine was shown in girls and boys aged nine to 15 years compared to young women aged 16 to 25 years (Van Damme 2015).

The efficacy and safety of the nona-valent vaccine will be assessed in a future update of this Cochrane review, when results of more trials are available. This update will include also inter-vaccine comparisons without a placebo arm.

\subsection{Post marketing surveillance of HPV vaccine effectiveness}

This review summarises efficacy estimated from randomised trials, which are not necessarily transposable to field conditions. However, trend analyses and linkage studies joining cervical cancer screening records and vaccination registries report a significant reduction in prevalence of HPV vaccine types, cervical cytological abnormalities and $\mathrm{CIN}$ in countries where HPV vaccination has been introduced and where a considerable HPV vaccination coverage has been achieved (Arbyn 2016; Baldur-Felskov 2014; Brotherton 2011; Kavanagh 2014; Kavanagh 2017; Leval 2013; Markowitz 2013; Merckx 2015; Tabrizi 2012). A recent meta-analysis assessed vaccination effects in the general population by comparing prevalence of HPV infection before and after introduction of HPV vaccination (Drolet 2015). Among girls and young women aged 13 to 19 years, a significant reduction was observed for infection with HPV16/18 infection (RR: $0.36, \mathrm{Cl}: 0.12$ to 0.89 ) and of also of infection with HPV31, HPV33 and HPV45 (RR: $0.72, \mathrm{Cl}: 0.54$ to 0.96$)$, suggesting cross-protection. No significant differences were observed in women of age 20 years and older (RR: $0.89, \mathrm{Cl}$ $0 \cdot 79$ to 1.02 ). The effects increased by vaccination coverage and years since vaccination. No differences by vaccine type (bi- or quadrivalent) were observed. These findings corroborate findings from the randomised trials. Women vaccinated at younger age reflect findings of young women who were free of HPV infection at enrolment in the RCTs. Herd immunity (protection of nonvaccinated women living in populations with high HPV vaccination coverage) was shown from recent surveillance studies, linking HPV vaccination studies and HPV genotyping of cervical specimen of young women entering the screening programme, in Scotland (Kavanagh 2017) and Australia (Tabrizi 2014).

The effect on the incidence of genital warts is the first clinical effect of HPV vaccination (with the quadrivalent vaccine) and may be an indicator of protection against cervical (pre-) cancer. Decreased incidence of genital warts in young (12 to 26 years) heterosexual, but not homosexual, males and decreased incidence of HPV vaccine types in non-vaccinated young women in Australia, indicate a certain level of herd immunity (Donovan 2011; Tabrizi 2014). However, in Sweden, higher (although not statistically significant) rates of genital warts were reported in vaccinated women older than 20 years (Leval 2013). This phenomenon is most plausibly explained by an association between the tendency of opportunistic vaccination and high-risk behaviour of adult sexually active women, who were vaccinated after exposure to HPV infection. The meta-analysis of Drolet 2015 suggests herd immunity by observing reduced prevalence of genital warts in males younger than 20 years (RR: $0.66, \mathrm{Cl} 0.47$ to 0.91 ) and in women in the age range 20 to $39(0.68,0.510 .89)$ in countries where vaccination coverage among young women exceeded $50 \%$.

To conclude, these real-life effectiveness data are in line with conclusions of our review regarding efficacy derived from the randomised trials.

\section{Overall completeness and applicability of evidence}

\section{Completeness of evidence}

Figure 12 summarises the main efficacy estimates. We can distinguish seven endpoints (CIN2+, CIN3+, AIS+ associated with HPV types covered by the vaccines or any lesions irrespective of HPV types and persistent HPV16/18 infection), five exposure groups (defined by initial HPV DNA status and number of doses received), and two major age groups ( 15 to 26 and 25 to 45 years). Altogether, 70 data cells could potentially be completed from the trial databases. However, for 32 cells no data could be extracted and for the other 38 only a limited number of trials contributed data. Nonetheless, for the most relevant endpoint-exposure group combinations, sufficient evidence could be derived allowing for evidence-based decision making.

\section{Endpoint of cervical cancer}

The purpose of prophylactic vaccination against HPV is to reduce the incidence of cervical cancer. However, this outcome could not be assessed in our review, since trials conducted were not powered and included insufficient follow-up time to demonstrate this end point. In agreement with World Health Organization (WHO) recommendation, reduction of histologically-classified cervical intraepithelial neoplasias (CIN) grade 2 or worse, associated with the HPV types targeted by the vaccine, was the proposed main endpoint of vaccination efficacy trials (Pagliusi 2004). Defining 
invasive cancer as an outcome of the trials was considered as an unethical and unfeasible endpoint and would require extremely expensive and lengthy observation periods and postpone the availability of vaccines for decades (Pagliusi 2004). The observation of a reduced incidence of cervical cancer (and other HPV-related cancer) in vaccinated cohorts will have to be obtained from population-based studies linking cancer and vaccination registries (Lehtinen 2006).

\section{Limited reported data for certain endpoints and exposure groups}

This Cochrane review primarily used efficacy data extractable from peer-reviewed published reports. Since, in principle all trials evaluated at baseline all enrolled women for presence of HPV genotypes and in addition cytology, and HPV serology, more efficacy data are available which would fit the defined analyses groups included in our Cochrane review. However, often only a restricted series of results were reported limiting the number of studies in each of the analyses (varying from one to eight), and gaps of non-reported outcomes (Figure 6 and Figure 7). Indeed, only the endpoints CIN2+ related to HPV16/18 (Analysis 2.2) and persistent infection of HPV16/18 at six months (Analysis 5.4) in women being negative for HPV16/18 DNA at enrolment have as many as eight trials in one forest plot. Originally, we planned requesting data from data owners, to fill in gaps with available unpublished data. However, due to constraints in time and other resources this was not possible. We do not believe that this has undermined the importance of our review. For each major outcome included in Summary of findings for the main comparison and Summary of findings 3 , we were able to obtain precise estimates of vaccine effects in the two main public health relevant groups: A) young women who were hrHPV negative at enrolment and received at least one dose of vaccine, who resemble the first target population of school-based HPV vaccination programs (adolescent girls aged 12 to 14 years) and B) young women regardless of HPV status at enrolment, who received at least one vaccine dose, reflecting a catch-up vaccination targeting older adolescents or young adult women. Among this latter category there is likely to be a considerable proportion who have already started sexual relations. In mid-adult women (aged 24 to 45 years), almost no data were reported with respect to protection against any high-grade $\mathrm{CIN}$, irrespective of HPV type.

\section{Non-published trials}

We consulted the trial registry https://clinicaltrials.gov/ to identify randomised trials which potentially could contain efficacy or safety data from women vaccinated with prophylactic HPV vaccines, but which were not published, or from which data could not be extracted (Appendix 6). A high level of reporting was noted for the safety outcome: results from $96 \%$ of women $197 \%$ and $95 \%$, for the bi- and quadrivalent vaccine, respectively) enrolled in registered trials were comprised in studies included in our review. From four small trials with the bivalent vaccine, we could not retrieve data. Three trials (one bi-bivalent (Denny 2013); two quadrivalent (Li 2012; Reisinger 2007)) were excluded. If the studies excluded from our Cochrane review were not taken into account, the inclusion coverage became $97.7 \%$ for the bivalent and $100 \%$ for the quadrivalent vaccine.

\section{Immunogenicity of HPV vaccines}

Intramuscular injection of L1-based HPV vaccines induce production of virus-specific antibodies in serum which exudate to epithelia and, by binding to HPV particles, impede new infection (Stanley 2006). The demonstration of serological responses in girls younger than 15 years of age, which were non-inferior to those in women aged 15 to 26 (where virological and clinical efficacy was demonstrated), was pivotal in accepting HPV vaccines for use before onset of sexual activity (Schiller 2009).

The trials of the bi- and quadrivalent HPV vaccine have used different assays to measure virus-specific antibody titres, making quantitative comparison of the serological data difficult. The chemiluminescence Immunoassay (CLIA), generally used to measure the serological response in quadrivalent vaccine trials, is known to be more specific for certain fractions of virusneutralising antibodies, whereas enzyme-linked immunosorbent assayS (ELISA) may also detect non-neutralising antibodies. Loss of detectable anti-HPV18 antibody by CLIA was not associated with waning of protection. Moreover, in trial reports, each company has used assay- and type-specific concentration measures. Recently, standardised international units have been proposed to quantify type-specific anti-HPV serological responses (Unger 2010). However, these international units have not yet been applied in vaccine trial reports. As yet, no immunological correlate for clinical efficacy has been identified.

Therefore, immunogenicity of prophylactic HPV vaccines was not assessed in the current version of our Cochrane review, as was originally foreseen in the protocol (Arbyn 2013).

There was one head-to-head trial, where women were randomised to receive the bi- or quadrivalent vaccines (Einstein 2009). Immunogenicity of both vaccines could be directly compared by measuring the antibody responses in serum and cervico-vaginal secretions with the same assays. Antibody titres and levels of memory B cells were significantly higher in all age groups for both HPV16 and HPV 18 with the bivalent, compared with the quadrivalent, vaccine. Differences were maintained 30 months after completion of vaccination. However, it was shown that adding VLP antigens from other HPV types to the AS04-adjuvanted bivalent vaccine resulted in lower anti-HPV16 and anti-HPV18 responses (Van Damme 2014).

As soon as an immunologically comparative framework for immunogenicity is agreed, this review will be updated and extended with serologically-defined endpoints.

\section{Quality of the evidence}

We rated the quality of evidence and present our findings in Summary of findings for the main comparison; Summary of findings 2; Summary of findings 3 for efficacy outcomes across the three populations as defined by HPV status at baseline. We present analyses of pregnancy outcomes in Summary of findings 4.

The studies providing data to this review are large and we have judged them to be at low risk of bias for efficacy endpoints for women who received three doses or at least one dose. For a few outcomes, we judged that the number of events to be low, even with large sample sizes, meaning that we cannot rule out different effect sizes to those we have found for adenocarcinoma in situ (AIS) associated with HPV16/18 and any AIS, irrespective of HPV type, in 
women who were hrHPV negative at baseline (Summary of findings for the main comparison). Although few trials could be identified for a given exposure group/endpoint combination, the results were generally consistent across the efficacy endpoints in women who are hrHPV negative and HPV16/18 negative at baseline (Summary of findings 2). For protection against precancer associated with HPV16/18, conferred by fewer than three doses of HPV vaccine, we downgraded the level of evidence to low or very low, since the risk in the placebo arms varied by number of doses received (Table 2: Analysis 2.3, Analysis 2.6, Analysis 2.9, Analysis 2.12; Analysis 2.15).

The quality of evidence was judged as high regarding absence of increased risk of severe systemic adverse effects associated with HPV vaccination. Regarding mortality associated with HPV vaccination, the quality of evidence is low. For the level of evidence regarding obstetrical safety, we judged the quality of evidence as moderate or high.

More than 70,000 women were included overall in the randomised trials and in the most important exposure group/efficacy endpoint combinations more than 10,000 women were enrolled, resulting in precise estimates. For certain post-hoc analyses with respect to effects in women having received only one or two doses, fewer than 1000 women were included, yielding pooled estimates, with wider confidence intervals.

The natural history of CIN and cervical cancer is strongly linked to persistent infection with hrHPV infection (Forman 2012; IARC 2007), and the contribution of HPV types 16 and 18 in the overall burden of cervical cancer is around $71 \%$ (Arbyn 2014). Given this strong link, we can accept a high level of directness between the observed prevention of persistent infection, CIN and the anticipated expected prevention of cervical cancer incurred by HPV vaccination. Nevertheless, it must be acknowledged that reduced incidence of invasive cervical cancer in HPV vaccinated women cannot be observed within the available trials (See Discussion 2.2).

Publication bias could not be assessed formally, given the small number of trials reporting clinical efficacy data. However, the level of completeness of reporting and absence of a correlation between study size and effects allow us to conclude that the risk of reporting bias may be small.

\section{Strict separation by type of endpoint and HPV DNA status at enrolment}

We have separated exposure groups in terms of age and enrolment status, mainly based on the presence or absence of hrHPV DNA or HPV16/18 DNA and the distinction of trial outcomes, such as cervical precancer associated with HPV vaccine types or irrespective of HPV type. This allowed us to pool comparable data which did not appear possible a priori because of the use of different definitions of exposure groups in the original trial reports, such as according-to-protocol, naive-vaccinated population, intention-to-treat, total-vaccinated-cohort, modified intention-to-treat. Other meta-analyses ignored this principle and pooled results from very heterogeneous groups. For instance, Rambout 2007 considered efficacy data from the FUTURE-1 and -2 trials (FUTURE I trial (ph3,4v), FUTURE II trial (ph3,4v), including women positive for HPV16/18 at enrolment for vaccination with the quadrivalent vaccine, and combined them with efficacy data from the Phase2 trial (ph2,2v) and PATRICIA trial (ph3,2v), excluding HPV16/18 positive women vaccinated with the bivalent vaccine.
Protection was higher in the latter group, but this may be due to the enrolment of more hr HPV-naïve women, rather than because of differences in the efficacy of the vaccine. By distinguishing exposure groups and outcomes in our review, homogenous data sets could be combined and significant protection could be demonstrated. We demonstrated protection against AIS associated with HPV16/18 in women vaccinated with the bi- or quadrivalent vaccines and who were initially hrHPV DNA negative or negative for the vaccine types (Analysis 1.5; Analysis 1.9; Analysis 2.10), or even regardless of initial HPV DNA status (Analysis 3.5). Without pooling trials with different vaccines, protection was not significant, since AIS is less common than high-grade CIN. We considered metaanalytical pooling as relevant only in the absence of heterogeneity. In contrast, we also found situations, where vaccine efficacy was significantly different between the two licensed vaccines. For instance, regarding protection against any $\mathrm{CIN2}+$ or $\mathrm{CIN} 3+$, irrespective of HPV type, in women who were hrHPV DNA negative at baseline, greater protection was found for the bivalent compared to the quadrivalent vaccine (RR: 0.33 versus $0.57, \mathrm{P}$ value $=0.0004$ (Analysis 1.7) or RR: 0.08 versus $0.54, \mathrm{P}$ value $=0.001$ (Analysis 1.8), respectively). Also in total vaccinated cohorts, whatever the initial HPV DNA status, women who received at least one dose of the bivalent compared to the quadrivalent vaccine had better protection against any CIN3+ (RR: 0.55 versus 0.81 , $P$ value $=0.01$ (Analysis 3.8]). See further discussion of potential methodologic reasons for this difference in Section 2.2 of the Discussion.

\section{Unreported estimated outcomes: vaccination effect when fewer than three doses were administered}

An original approach of this review was the estimation of the efficacy of fewer than three doses by subtracting the number of events in the populations that received all three doses from those who received at least one dose. By doing this subtraction, we found significant protection, in women initially negative for HPV16/18, against $\mathrm{CIN} 2+$ and $\mathrm{CIN} 3+$ associated with HPV16/18 in younger, but not in mid-adult, women (Analysis 2.3). It is important to remember that these were post hoc analyses and that the trials were not designed to assess the effects of fewer than three doses. Furthermore, we were not able to assess differences between threedose vaccine recipients and those who did not complete the series.

Recent randomised trials have demonstrated non-inferior antiHPV16 and anti-HPV18 antibody levels induced after a twodose schedule at months zero and six in girls aged nine to 14 years compared to the usual three-dose schedules of bivalent or quadrivalent vaccine in young women aged 15 to 26 years ( Dobson 2013; Lazcano-Ponce 2014; Romanowski 2011). These observations have convinced some regulatory agencies to allow a two-dose schedule for girls of nine to 14 years of age (EMEA 2014a; EMEA 2014b). Our findings suggest that two doses might provide protection in young women (aged 15 to 26 years), but not in mid-adult women ( 24 to 45 years). Some experts have expressed concerns that the two-dose schedule might affect the longevity of protection (Stanley 2014). Public health authorities should set up careful surveillance of the duration of protection by age and by the number and timing of received doses. A recent pooled post hoc analysis (PATRICIA \& CVT (ph3,2v)) showed a similar efficacy of the bivalent vaccine against incident HPV16/18 infection among women who were HPV16/18 DNA negative at baseline and who received one dose ( $\mathrm{RR}=0.16,95 \% \mathrm{Cl} 0.06$ to 0.29$)$, two doses ( $R R=0.24,95 \% \mathrm{Cl} 0.15$ to 0.38 ), or three doses ( $R R=0.23,95 \%$ $\mathrm{Cl} 0.21$ to 0.25 ) (Kreimer 2015), Protection appeared to be higher 
for those who received two doses when the interval between administration was six months compared to one month. No data on protection of fewer than three doses against cervical lesions were reported. Recent data show durability of protection against HPV16/18 afforded by fewer than three doses of the bivalent vaccine over at least seven years (Safaeian 2018).

Post licensure studies of the effectiveness of the quadrivalent vaccine in the USA (Hofstetter 2016) and Australia (Brotherton 2015; Crowe 2014; Gertig 2013) have shown decreased rates of high-grade and/or low-grade cervical lesions in partially vaccinated young women versus non-vaccinated young women who started cervical cancer screening. However. vaccine effectiveness was of lower magnitude than when three doses were given. A recent report from a suspended cluster-randomised trial, conducted in India, compared immunogenicity of the quadrivalent vaccine according to the actual number of doses administered to girls aged 10 to 18 years. The immune response (in terms of geometric mean antibody levels) in the group who received two doses at month zero and month six or later was not inferior to the group who received three doses at months zero, two and six or later. However, the immune response was inferior in the groups who received two doses at month zero and two or who received only one dose (Sankaranarayanan 2016).

A recent Scottish surveillance study of the effectiveness of the bivalent vaccine demonstrated significant protection against prevalent HPV16/18 infection conferred by two doses (RR of 0.45 , $95 \% \mathrm{Cl} 0.29$ to 0.69 ) or one dose (RR of $0.52,95 \% \mathrm{Cl} 0.31$ to 0.83 ) among those reached by catch-up vaccination targeting girls of age 14 to 18 years and who entered the screening programme (Cuschieri 2016). However, protection was lower than with three doses (RR of $0.27,95 \% \mathrm{Cl} 0.20$ to 0.36 ). No significant protection against cervical intra-epithelial neoplastic lesions irrespective of HPV types associated with fewer than three doses was observed (Pollock 2014).

It must be remarked that partially vaccinated women in published post-licensure studies were older than fully vaccinated women (so more likely to have been exposed to HPV prior to vaccination) and most women with two doses had a one to two month interval between vaccine administrations.

\section{Potential biases in the review process}

\section{Post hoc analysis of vaccine effects associated with one or two doses}

In this review, we computed efficacy estimates for women who received only one or two doses, by subtracting events and total number of women who received three doses from those who received at least one dose. We computed this for data presented within the same report for a given follow-up time. This is a post hoc analysis, which has limitations, since counting of events often started for the women who received at least one dose at day one, whereas for those who received all three doses counting started from the day of the last administration. Most of the women in the group that received at least one dose received three doses. We assumed that the possible protection, not accounted for in the group receiving three doses, induced by the vaccine in the period between $1^{\text {st }}$ and $2^{\text {nd }}$ dose, would be small. Reported observed data from the Costa Rica Trial, separated by groups receiving only one, two or three does, are in agreement with our estimates (Kreimer 2011).

An important finding with public health relevance, was that one or two doses of bi- or quadrivalent vaccine did not protect against CIN2+ associated with HPV16/18, in women older than 24 years, even if they were negative for HPV16/18 at enrolment (RR $=0.98$, $95 \% \mathrm{Cl} 0.20$ to 4.83 ), whereas women younger than 26 years experienced protection against HPV16/18 related $\mathrm{CIN} 2+, \mathrm{CIN} 3+$ and AIS+ if HPV16/18 DNA negative at baseline.

\section{Other potential biases}

As mentioned in Overall completeness and applicability of evidence, for several outcomes no information was available for the group of mid-adult women. We have focused efforts to obtain unpublished data from registered studies for adverse events. We tested the assumption that there is a difference between results obtained from published trial reports and trial registry and study results websites for serious adverse events and mortality by Sensitivity analysis. Journal-published trial reports provide data at fixed time points, whereas trial registry and study results websites can be updated over time as data are collected from more recent follow-up. Sensitivity analysis by source of data gives us some confidence that published and registry or website-sourced data are similar for the same study. However, data from regulatory sources and data from unregistered and unpublished studies were not consulted for efficacy endpoints and less than severe adverse events.

The comparison of the risks of adverse events was compromised by the use of different products administered to participants in the control group, varying from adjuvant (often aluminium hydroxide or other aluminium compound) or an alternative vaccine (often Hepatitis A or Hepatitis B). Therefore, the pooled risks of adverse effects associated with HPV vaccines and the assumed risks for control groups must be interpreted cautiously (Summary of findings 4).

\section{Agreements and disagreements with other studies or reviews}

A multitude of reviews and combined analyses have been conducted over recent years by regulatory agencies and institutions developing practice guidelines (Ault 2007; Haupt 2011; Harper 2009; Kjaer 2009; Lehtinen 2013; Romanowski 2011; Schiller 2012; Stanley 2014; WHO 2009) and systematic reviewers (Delere 2014; Lehtinen 2013; Lu 2011; Malagon 2012; McKeage 2011; Medeiros 2009; Rambout 2007). Our review is distinguished from previous reviews by its currency because of the inclusion of later reports of data from included trials. This includes the most recent results of the VIVIANE trial (Wheeler 2016) and the latest safety reports of the bivalent Chinese trial (July 2016). In general, the review corroborates findings from other major reviews. However, two findings were not previously reported: 1) the statistically significant protection against AIS both associated with HPV16/18 and irrespective of HPV type, and 2) the significant protection induced by fewer than three vaccine doses against CIN2+ associated with HPV16/18 in women who were HPV16/18 negative at baseline, although this was a post hoc analysis. 


\section{Future work}

The current review focused primarily on protection against cervical precancer related to the HPV types included in the vaccine or any cervical precancer irrespective of HPV type. In the future, sixmonth persistent infection with HPV types included in the vaccine probably will become the main assessed outcome, which is a more objectively measurable endpoint and highly correlated with clinical outcomes (IARC 2013). In future reviews, protection against persistent infection with the vaccine types may become the primary outcome.

We propose conducting additional Cochrane reviews on HPV vaccine efficacy against other HPV-related diseases such as genital warts, vaginal, vulvar, anal and penile intra-epithelial neoplasia and cancer, as well as HPV infection at these anatomical sites and in the oral cavity. These reviews may include study designs, in addition to randomised trials, such as cohort studies, registry linkage studies and trend analyses, The incidence of respiratory papillomatosis, which is a rare but very serious condition related to HPV6 and HPV11, could also be considered.

Reviews should assess effects in particular groups, such as men, immune-depressed populations, men-having-sex-with men (MSM) and women-having-sex-with-women (WSW), infants, and midadult age groups.

A particular important area for further research is the question of how to integrate primary protection against HPV-related disease with current and future cytology-based or HPV-based screening for cervical cancer. This research, and subsequent pooled analyses, should address how to screen vaccinated cohorts and whether nonvaccinated HPV-negative cohorts would benefit from vaccination at the time of screening (Bosch 2016).

Regulatory agencies (EMEA 2014a; EMEA 2014b) approved twodose schedules for L1 HPV vaccines in young girls, based on non-inferior seroconversion rates and anti-HPV antibody levels (Romanowski 2011; Stanley 2014). Our review provides some clinical efficacy evidence supporting this decision. Moreover, recent data suggest protection conferred by only one dose of HPV vaccine (Kreimer 2015; Safaeian 2018). However, it cannot be excluded that schemes with fewer than three doses would induce a protection of shorter duration. Comprehensive vaccine registries linked to screening, cytopathology, HPV virology, cancer registry data and linkable to cervical cytology and histology bio-banks will be extremely useful tools for epidemiological surveillance to answer questions on duration of protection, occurrence of cross-protection and type replacement (Arbyn 2010; Dillner 2011).

For reasons of statistical power and costs, trials often assess combined outcomes (persistent infection, cytological lesion, CIN1+, external ano-genital lesions). Although this is acceptable for clinical decision making, authors should be invited to report separate outcomes to facilitate future meta-analytical pooling. Editors of journals should also support publishing these detailed reports in appendices.

In later updates, we foresee inclusion of efficacy and safety data from trials which evaluate the nona-valent vaccine and possible other vaccines that do not involve comparisons with a placebo group but include comparisons with other HPV vaccines.

\section{AUTHORS' CONCLUSIONS}

\section{Implications for practice}

In studies designed to evaluate prevention of cervical precancer, an endpoint established by the World Health Organization (WHO) and regulatory agencies as a surrogate outcome for cervical cancer, high vaccine efficacy was demonstrated. The studies were not designed to evaluate cervical cancer and the duration of the studies was too short to determine the effects of human papillomaviruses (HPV) vaccination on cervical cancer outcomes. Although the trials were large and no safety concerns were established, vaccine safety requires evaluation in surveillance studies after introduction of vaccination programmes.

In young women aged 15 to 26 , who are high-risk human papillomavirus (hrHPV) negative or HPV16/18 negative at baseline, HPV vaccination reduces the risk of persistent HPV16/18 infection, high-grade cervical intraepithelial neoplasia or worse (CIN2+) and adenocarcinoma in situ (AIS) associated with the vaccine types. Average rates of CIN2+ reduced from 164 to 2 per 10,000 and CIN3+ from 70 per 10,000 to 0 per 10,000. The findings in these unexposed groups are relevant for adolescent girls prior to sexual debut. Our review suggests that fewer than three doses may offer protection against HPV16/18 endpoints in this age group. We found no evidence that one or two doses of bivalent or quadrivalent vaccine provide significant protection against any $\mathrm{CIN} 2+$, irrespective of HPV types, in young women (15 to 26 years)

Since prophylactic HPV vaccines do not clear existing HPV infection, protection is less effective in populations already exposed to HPV. However, protection is still moderate in young women (15 to 26 years) considered as an overall cohort regardless of baseline HPV infection status, which may be relevant for decision making in relation to 'catch-up' vaccination programmes.

Whereas the efficacy of the bivalent and quadrivalent vaccines against cervical precancer associated with HPV16 or 18 is similar, protection of the bivalent vaccine against any cervical precancer irrespective of HPV types seems to be higher.

Among mid-adult women (24 to 45 years), while evidence shows that three doses given to HPV negative women provides significant protection against $\mathrm{CIN} 2+$ associated with HPV16/18, evidence to date suggests that fewer than three doses of HPV vaccine do not provide protection against $\mathrm{CIN2}+$ associated with HPV16/18 or any $\mathrm{CIN} 2+$ irrespective of HPV type.

The HPV vaccines are responsible for local effects at the injection site, which are generally well tolerated. No increased incidence of serious adverse effects was noted in vaccinated participants. We did not find conclusive evidence of increases in the risk of congenital anomalies and adverse pregnancy outcomes in vaccinated women who became pregnant throughout the trials. However, more evidence is needed to determine long-term outcomes in pregnant women who received the vaccine.

While deaths occurred during follow-up of the trial participants, none were assessed to be due to vaccine and all occurred months to years after vaccination. More deaths occurred after vaccination of mid-adult women. These deaths were deemed by study investigators as not related to vaccination due to the absence 
of clustering of the causes of death and the lack of a temporal relation (Table 5 and Table 6).

Evidence on rare potential harms, such as autoimmune disorders, are difficult to capture in randomised controlled trials (RCTs). The findings of this review should be seen in the context of surveillance studies which have been conducted globally since the licensing of the vaccines and have demonstrated a consistently good safety profile in population usage as reviewed by the Global Advisory Committee on Vaccine Safety (GACVS) of the WHO on multiple occasions. A single French study found a small increase in GuillainBarré syndrome among HPV vaccinated girls but this was not confirmed in seven other studies.

\section{Implications for research}

Long-term surveillance and registry-based research (linking of vaccination databases with screening, cyto-histopathology, cancer registries and biobanks; and linking with morbidity, mortality and birth/maternity registries) are needed to establish vaccine efficacy and safety over time. This will help also to assess type replacement, cross-protection, duration of protection associated with three or fewer doses and vaccine safety in pregnant women.

Trials and registry-based research combined with mathematical modelling are needed to define new integrated strategies of cervical cancer prevention through a combination of vaccination and screening.

In mid-adult women (24 to 45 years), limited data were reported with respect to outcomes other than targeted type infections and disease. Studies are more difficult to undertake in this age group due to the lower incidence of new infections and incident disease and because of prevalent infection and disease. For this reason, we recommend monitoring of vaccinated cohorts over time to assess the overall effectiveness of vaccination over time on the burden of cervical disease in mid-adult women. Our review revealed the need to continuously update existing evidence and to complete gaps in the current accumulated knowledge with available, but unpublished data.

\section{ACKNOWLEDGEMENTS}

We would like to acknowledge the input of the following individuals in the development of the protocol for this review: L Markowitz, A Bryant, J Dillner, E. Paraskevaidis, P. Beutels, M Steben, A Schneider, A Kauffman, Z-H Zhao, Y-L. Qiao, and A Hildesheim. We thank Jo Morrison and Tess Lawrie for clinical advice, Jo Platt for designing the search strategy and Toby Lasserson, Gail Quinn and Clare Jess for their contribution to the editorial process.

We acknowledge Lauri Markowitz for her invaluable advice and contributions by reviewing the results and discussion sections.

This project was supported by the National Institute for Health Research, via Cochrane Programme Grant Funding to the Cochrane Gynaecological, Neuro-oncology and Orphan Cancer Group. The views and opinions expressed therein are those of the authors and do not necessarily reflect those of the Systematic Reviews Programme, NIHR, NHS or the Department of Health.

Koen de Visscher (KODEVIS, Lokeren, Belgium) is acknowledged for the production of high-quality graphical png files 


\section{R E F E R E N C E S}

\section{References to studies included in this review}

\section{African_2 country trial (ph3,2v) \{published data only\}}

Sow PS, Watson-Jones D, Kiviat N, Changalucha J, Mbaye KD, Brown J. Safety and immunogenicity of human papillomavirus-16/18 AS04-adjuvanted vaccine: a randomized trial in 10-25-year-old HIV-Seronegative African girls and young women. Journal of Infectious Diseases 2013;207(11):1753-63.

\section{African_3 country trial (ph3,4v) \{published data only\}}

Mugo N, Ansah NA, Marino D, Saah A, Garner E I. Evaluation of safety and immunogenicity of a quadrivalent human papillomavirus vaccine in healthy females between 9 and 26 years of age in Sub-Saharan Africa. Human Vaccines \& Immunotherapeutics 2015;11(6):1323-30.

\section{Chinese trial (ph3,2v)_ adolescent \{published data only\}}

Zhu F, Li J, Hu Y, Zhang X, Yang X, Zhao H, et al. Immunogenicity and safety of the HPV-16/18 AS04-adjuvanted vaccine in healthy Chinese girls and women aged 9 to 45 years. Human Vaccines \& Immunotherapeutics 2014;10(7):1795-806.

\section{Chinese trial (ph3,2v)_mid-adult \{published data only\}}

Zhu F, Li J, Hu Y, Zhang X, Yang X, Zhao H, et al. Immunogenicity and safety of the HPV-16/18 AS04-adjuvanted vaccine in healthy Chinese girls and women aged 9 to 45 years. Human Vaccines \& Immunotherapeutics 2014;10(7):1795-806.

\section{Chinese trial (ph3,2v)_young \{published data only\}}

* Zhu FC, Chen W, Hu YM, Hong Y, Li J, Zhang X, et al. Efficacy, immunogenicity and safety of the HPV-16/18 AS04-adjuvanted vaccine in healthy Chinese women aged $18-25$ years: results from a randomized controlled trial. International Journal of Cancer 2014;135(11):2612-22.

Zhu FC, Hu SY, Hong Y, Hu YM, Zhang X, Zhang YJ, et al. Efficacy, immunogenicity, and safety of the HPV-16/18 AS04-adjuvanted vaccine in Chinese women aged 18-25 years: event-triggered analysis of a randomized controlled trial. Cancer Medicine 2017;6(2045-7634 (Electronic), 2045-7634 (Linking), 1):12-25.

Co-vaccination_dTpa_IPV trial (ph3,2v) \{published data only\}

Garcia-Sicilia J, Schwarz TF, Carmona A, Peters K, Malkin JE, Tran PM. Immunogenicity and safety of human papillomavirus-16/18 AS04-adjuvanted cervical cancer vaccine coadministered with combined diphtheria-tetanus-acellular pertussis-inactivated poliovirus vaccine to girls and young women. Journal of Adolescent Health 2010;46(2):142-51.

\section{Co-vaccination_HAB trial $(\mathbf{P h} \mathbf{3}, \mathbf{2 v})$ \{published data only\}}

Pedersen C, Breindahl M, Aggarwal N, Berglund J, Oroszlan G, Silfverdal SA. Randomized trial: immunogenicity and safety of coadministered human papillomavirus-16/18AS04-adjuvanted vaccine and combined hepatitis $A$ and $B$ vaccine in girls. Journal of Adolescent Health 2012;50(1):38-46.

\section{Co-vaccination_HepB trial (ph3, 2v) \{published data only\}}

Schmeink CE, Bekkers RL, Josefsson A, Richardus JH, Berndtsson Blom K, et al. Co-administration of human papillomavirus-16/18 AS04-adjuvanted vaccine with hepatitis $B$ vaccine: randomized study in healthy girls. Vaccine 2011;29(49):9276-83.

\section{CVT (ph3,2v) \{published data only\}}

* Herrero R, Wacholder S, Rodriguez AC, Solomon D, Gonzalez P, Kreimer AR, et al. Prevention of persistent human papillomavirus infection by an HPV16/18 vaccine: A communitybased randomized clinical trial in Guanacaste, Costa Rica. Cancer Discovery 2011;1(5):408-19.

Hildesheim A, Wacholder S, Catteau G, Struyf F, Dubin G, Herrero R. Efficacy of the HPV-16/18 vaccine: Final according to protocol results from the blinded phase of the randomized Costa Rica HPV-16/18 vaccine trial. Vaccine 2014;32(39):5087-97.

Kreimer AR, Gonzalez P, Katki HA, Porras C, Schiffman M, Rodriguez AC. Efficacy of a bivalent HPV 16/18 vaccine against anal HPV 16/18 infection among young women: a nested analysis within the Costa Rica Vaccine Trial. Lancet Oncology 2011;12(9):862-70.

Kreimer AR, Rodriguez AC, Hildesheim A, Herrero R, Porras C, Schiffman $M$, et al. Proof-of-principle evaluation of the efficacy of fewer than three doses of a bivalent HPV16/18 vaccine. Journal of the National Cancer Institute 2011;103(19):1444-51.

Lang Kuhs KA, Porras C, Schiller JT, Rodriguez AC, Schiffman M, Gonzalez P. Effect of different human papillomavirus serological and DNA criteria on vaccine efficacy estimates. American Journal of Epidemiology 2014;180(6):599-607.

\section{FUT I/II trials (ph3,4v) \{published data only\}}

Brown DR, Kjaer SK, Sigurdsson K, Iversen OE, HernandezAvila M, Wheeler CM. The impact of quadrivalent human papillomavirus (HPV; types 6, 11, 16, and 18) L1 virus-like particle vaccine on infection and disease due to oncogenic nonvaccine HPV types in generally HPV-naive women aged 16 to 26 years. Journal of Infectious Diseases 2009;199(7):926-35.

Dillner J, Kjaer SK, Wheeler CM, Sigurdsson K, Iversen OE, Hernandez-Avila M. Four year efficacy of prophylactic human papillomavirus quadrivalent vaccine against low grade cervical, vulvar, and vaginal intraepithelial neoplasia and anogenital warts: randomised controlled trial. BMJ 2010;341:c3493.

Kjaer SK, Sigurdsson K, Iversen OE, Hernandez-Avila M, Wheeler CM, Perez G. A pooled analysis of continued prophylactic efficacy of quadrivalent human papillomavirus (Types 6/11/16/18) vaccine against high-grade cervical and external genital lesions. Cancer Prevention Research 2009;2(10):868-78

* Munoz N, Kjaer SK, Sigurdsson K, Iversen OE, HernandezAvila M, Wheeler CM. Impact of human papillomavirus (HPV)-6/11/16/18 vaccine on all HPV-associated genital diseases in young women. Journal of the National Cancer Institute 2010;102(5):325-39.

Olsson SE, Kjaer SK, Sigurdsson K, Iversen OE, HernandezAvila M, Wheeler CM. Evaluation of quadrivalent HPV 6/11/16/18 
vaccine efficacy against cervical and anogenital disease in subjects with serological evidence of prior vaccine type HPV infection. Human Vaccines 2009;5(10):696-704.

The FUTURE II study group. Prophylactic efficacy of a quadrivalent human papillomavirus (HPV) vaccine in women with virological evidence of HPV infection. Journal of Infectious Diseases 2007;196(10):1438-46.

Wheeler CM, Kjaer SK, Sigurdsson K, Iversen OE, HernandezAvila M, Perez G. The impact of quadrivalent human papillomavirus (HPV; types 6, 11, 16, and 18) L1 virus-like particle vaccine on infection and disease due to oncogenic nonvaccine HPV types in sexually active women aged 16 to 26 years. Journal of Infectious Diseases 2009;199(7):936-44.

\section{FUTURE III trial (ph3,4v) \{published data only\}}

* Castellsagué X, Munoz N, Pitisuttithum P, Ferris D, Monsonego J, Ault K. End-of-study safety, immunogenicity, and efficacy of quadrivalent HPV (types $6,11,16,18$ ) recombinant vaccine in adult women 24 to 45 years of age. British Journal of Cancer 2011;105(1):28-37.

Garland SM, Ault KA, Gall SA, Paavonen J, Sings HL, Ciprero KL, et al. Pregnancy and infant outcomes in the clinical trials of a human papillomavirus type $6 / 11 / 16 / 18$ vaccine: a combined analysis of five randomized controlled trials. Obstetrics and Gynecology 2009;114(6):1179-88.

Munoz N, Manalastas R Jr, Pitisuttithum P, Tresukosol D, Monsonego J, Ault K. Safety, immunogenicity, and efficacy of quadrivalent human papillomavirus (types $6,11,16,18$ ) recombinant vaccine in women aged $24-45$ years: a randomised, double-blind trial. Lancet 2009;373(9679):1949-57.

FUTURE II trial (ph3,4v) \{published data only\}

Garland SM, Ault KA, Gall SA, Paavonen J, Sings HL, Ciprero KL, et al. Pregnancy and infant outcomes in the clinical trials of a human papillomavirus type 6/11/16/18vaccine: a combined analysis of five randomized controlled trials. Obstetrics and Gynecology 2009;114(6):1179-88.

The FUTURE II study group. Quadrivalent vaccine against human papillomavirus to prevent high-grade cervical lesions. New England Journal of Medicine 2007;356(19):1915-27.

\section{FUTURE I trial (ph3,4v) \{published data only\}}

Garland SM, Ault KA, Gall SA, Paavonen J, Sings HL, Ciprero KL, et al. Pregnancy and infant outcomes in the clinical trials of a human papillomavirus type $6 / 11 / 16 / 18$ vaccine: a combined analysis of five randomized controlled trials. Obstetrics and Gynecology 2009;114(6):1179-88

Garland SM, Hernandez-Avila M, Wheeler CM, Perez G, Harper DM, Leodolter S. Quadrivalent vaccine against human papillomavirus to prevent anogenital diseases. New England Journal of Medicine 2007;356(19):1928-43.

\section{Hong Kong trial (ph3,2v) \{published data only\}}

Ngan HY, Cheung AN, Tam KF, Chan KK, Tang HW, Bi D, et al. Human papillomavirus-16/18 AS04-adjuvanted cervical cancer vaccine: immunogenicity and safety in healthy Chinese women from Hong Kong. Hong Kong Medical Journal 2010;16(1024-2708 (Print), 1024-2708 (Linking), 3):171-9.

Immunobridging(ph3,2v) \{published data only\}

Medina DM, Valencia A, de Velasquez A, Huang LM, Prymula R, Garcia-Sicilia J. Safety and immunogenicity of the HPV-16/18 AS04-adjuvanted vaccine: a randomized, controlled trial in adolescent girls. Journal of Adolescent Health 2010;46(5):414-21.

Indian trial (ph3,2v) \{published data only\}

Bhatla N, Suri V, Basu P, Shastri S, Datta SK, Bi D, et al. Immunogenicity and safety of human papillomavirus-16/18 AS04-adjuvanted cervical cancer vaccine in healthy Indian women. Journal of Obstetrics and Gynaecology Research 2010;36(1):123-32.

\section{Japanese trial (ph2,2v) \{published data only\}}

Konno R, Tamura S, Dobbelaere K, Yoshikawa H. Efficacy of human papillomavirus 16/18 AS04-adjuvanted vaccine in Japanese women aged 20 to 25 years: interim analysis of a phase 2 double-blind, randomized, controlled trial. International Journal of Gynecological Cancer 2010;20(3):404-10.

* Konno R, Tamura S, Dobbelaere K, Yoshikawa H. Efficacy of human papillomavirus type 16/18 AS04-adjuvanted vaccine in Japanese women aged 20 to 25 years: final analysis of a phase 2 double-blind, randomized controlled trial. International Journal of Gynecological Cancer 2010;20(5):847-55.

Konno R, Yoshikawa H, Okutani M, Quint W, Suryakiran V, Lin L, et al. Efficacy of the human papillomavirus (HPV)-16/18 AS04adjuvanted vaccine against cervical intraepithelial neoplasia and cervical infection in young Japanese women. Human Vaccines \& Immunotherapeutics 2014;10(7):1781-94.

\section{Japanese trial (ph2,4v) \{published data only\}}

Yoshikawa H, Ebihara K, Tanaka Y, Noda K. Efficacy of quadrivalent human papillomavirus (types 6, 11, 16 and 18) vaccine (GARDASIL) in Japanese women aged 18 to 26 years. Cancer Science 2013;104(4):465-72.

\section{Korean trial (ph2,4v) \{published data only\}}

Kang S, Kim KH, Kim YT, Kim YT, Kim JH, Song YS. Safety and immunogenicity of a vaccine targeting human papillomavirus types 6, 11, 16 and 18: a randomized, placebo-controlled trial in 176 Korean subjects. International Journal of Gynecological Cancer 2008;18(5):1013-9.

\section{Korean trial (ph3,2v) \{published data only\}}

Kim YJ, Kim KT, Kim JH, Cha SD, Kim JW, Bae DS, et al. Vaccination with a human papillomavirus (HPV)-16/18 AS04adjuvanted cervical cancer vaccine in Korean girls aged 10-14 years. Journal of Korean Medical Science 2010;25(8):1197-204.

Korean trial (ph3b,2v) \{published data only\}

Kim SC, Song YS, Kim YT, Kim YT, Ryu KS, Gunapalaiah B. Human papillomavirus 16/18 AS04-adjuvanted cervical cancer vaccine: immunogenicity and safety in 15 to 25 years old healthy Korean women. Journal of Gynecologic Oncology 2011;22(2):67-75. 
Malaysian trial (ph3,2v) \{published data only\}

Lim BK, Ng KY, Omar J, Omar SZ, Gunapalaiah B, Teoh YL, et al. Immunogenicity and safety of the AS04-adjuvanted human papillomavirus-16/18 cervical cancer vaccine in Malaysian women aged 18 to 35 years: a randomized controlled trial. Medical Journal of Malaysia 2014;69(0300-5283 (Print), 0300-5283 (Linking), 1):2-8.

\section{PATRICIA \& CVT (ph3,2v) \{published data only\}}

Wacholder S, Chen BE, Wilcox A, Macones G, Gonzalez P, Befano $B$. Risk of miscarriage with bivalent vaccine against human papillomavirus (HPV) types 16 and 18: pooled analysis of two randomised controlled trials. BMJ 2010;340:c712.

\section{PATRICIA trial (ph3,2v) \{published data only\}}

* Lehtinen M, Paavonen J, Wheeler CM, Jaisamrarn U, Garland S, Castellsagué X. Overall efficacy of HPV-16/18 ASO4-adjuvanted vaccine against grade 3 or greater cervical intraepithelial neoplasia: 4-year end-of-study analysis of the randomised, double-blind PATRICIA trial. Lancet Oncology 2012;13(1):89-99.

Paavonen J, Jenkins D, Bosch FX, Naud P, Salmeron J, Wheeler CM. Efficacy of a prophylactic adjuvanted bivalent $\mathrm{L} 1$ virus-like-particle vaccine against infection with human papillomavirus types 16 and 18 in young women: an interim analysis of a phase III double-blind, randomised controlled trial. Lancet 2007;369(9580):2161-70.

Paavonen J, Naud P, Salmeron J, Wheeler CM, Chow S-N, Apter D. Efficacy of human papillomavirus (HPV)-16/18 ASO4adjuvanted vaccine against cervical infection and precancer caused by oncogenic HPV types (PATRICIA): final analysis of a double-blind, randomised study in young women. Lancet 2009;374:301-14.

Szarewski A, Poppe WA, Skinner SR, Wheeler CM, Paavonen J, Naud P. Efficacy of the human papillomavirus (HPV)-16/18 AS04-adjuvanted vaccine in women aged 15 to 25 years with and without serological evidence of previous exposure to HPV-16/18. International Journal of Cancer 2011;131(1):106-16

Wheeler CM, Castellsagué X, Garland SM, Szarewski A, Paavonen J, Naud P. Cross-protective efficacy of HPV-16/18 ASO4-adjuvanted vaccine against cervical infection and precancer caused by non-vaccine oncogenic HPV-types: 4-year end-of-study analysis of the randomised, double-blind PATRICIA trial. Lancet Oncology 2011;13(1):100-10.

\section{Phase2 trial (ph2,1v) \{published data only\}}

* Koutsky LA, Ault KA, Wheeler CM, Brown DR, Barr E, Alvarez FB. A controlled trial of a human papillomavirus type 16 vaccine. New England Journal of Medicine 2002;347(21):1645-51.

Mao C, Koutsky LA, Ault KA, Wheeler CM, Brown DR, Wiley DJ. Efficacy of human papillomavirus-16 vaccine to prevent cervical intraepithelial neoplasia: a randomized controlled trial. Obstetrics and Gynecology 2006;107(1):18-27.

Rowhani-Rahbar A, Mao C, Hughes JP, Alvarez FB, Bryan JT, Hawes SE. Longer term efficacy of a prophylactic monovalent human papillomavirus type 16 vaccine. Vaccine 2009;27(41):5612-9.

\section{Phase2 trial (ph2,2v) \{published data only\}}

De Carvalho N, Teixeira J, Roteli-Martins CM, Naud P, De Borba P, Zahaf T. Sustained efficacy and immunogenicity of the HPV-16/18 AS04-adjuvanted vaccine up to 7.3 years in young adult women. Vaccine 2010;28(38):6247-55

Harper DM, Franco EL, Wheeler C, Ferris DG, Jenkins D, Schuind $A$. Efficacy of a bivalent $L 1$ virus-like particle vaccine in prevention of infection with human papillomavirus types 16 and 18 in young women: a randomised controlled trial. Lancet 2004;364(9447):1757-65.

Harper DM, Franco EL, Wheeler CM, Moscicki AB, Romanowski B, Roteli-Martins CM. Sustained efficacy up to 4.5 years of a bivalent $L 1$ virus-like particle vaccine against human papillomavirus types 16 and 18: follow-up from a randomised control trial. Lancet 2006;367(9518):1247-55.

Naud PS, Roteli-Martins CM, De Carvalho NS, Teixeira JC, de Borba PC, Sanchez N, et al. Sustained efficacy, immunogenicity, and safety of the HPV-16/18 AS04-adjuvanted vaccine: Final analysis of a long-term follow-up study up to 9.4 years post-vaccination. Human Vaccines \& Immunotherapeutics 2014;10(8):2147-62.

The GlaxoSmithKline Vaccine HPV-007 Study Group. Sustained efficacy and immunogenicity of the human papillomavirus (HPV)-16/18 ASO4-adjuvanted vaccine: analysis of a randomised placebo-controlled trial up to 6.4 years. Lancet 2009;374:1975-85.

Phase2 trial (ph2,4v) \{published data only\}

Olsson SE, Kjaer SK, Sigurdsson K, Iversen OE. Evaluation of quadrivalent HPV 6/11/16/18 vaccine efficacy against cervical and anogenital disease in subjects with serological evidence of prior vaccine type HPV infection. Human Vaccines 2009;5(10):696-704.

Villa LL, Ault KA, Giuliano AR, Costa RL, Petta CA, Andrade RP. Immunologic responses following administration of a vaccine targeting human papillomavirus Types $6,11,16$, and 18 . Vaccine 2006;24(27):5571-83.

Villa LL, Costa RL, Petta CA, Andrade RP, Ault KA, Giuliano AR. Prophylactic quadrivalent human papillomavirus (types 6,11 , 16 , and 18) L1 virus-like particle vaccine in young women: a randomised double-blind placebo-controlled multicentre phase Il efficacy trial. Lancet Oncology 2005;6(5):271-8.

Villa LL, Costa RL, Petta CA, Andrade RP, Paavonen J, Iversen OE. High sustained efficacy of a prophylactic quadrivalent human papillomavirus types $6 / 11 / 16 / 18 \mathrm{~L} 1$ virus-like particle vaccine through 5 years of follow-up. British Journal of Cancer 2006;95(11):1459-66.

\section{VIVIANE trial (ph3,2v) \{published data only\}}

* Skinner SR, Szarewski A, Romanowski B, Garland SM, Lazcano-Ponce E, Salmeron J. Efficacy, safety, and immunogenicity of the human papillomavirus 16/18 AS04adjuvanted vaccine in women older than 25 years: 4-year interim follow-up of the phase 3, double-blind, randomised controlled VIVIANE study. Lancet 2014;384(9961):2213-27.

Prophylactic vaccination against human papillomaviruses to prevent cervical cancer and its precursors (Review) 
Wheeler CM, Skinner SR, Del Rosario-Raymundo MR, Garland SM, Chatterjee A, Lazcano-Ponce E, et al. Efficacy, safety, and immunogenicity of the human papillomavirus 16/18 AS04-adjuvanted vaccine in women older than 25 years: 7-year interim follow-up of the phase 3, double-blind, randomised controlled VIVIANE study. Lancet Infectious Diseases 2016;16(10):1154-68.

\section{References to studies excluded from this review}

\section{Angelo 2014 \{published data only\}}

Angelo MG, David MP, Zima J, Baril L, Dubin G, Arellano F, et al. Pooled analysis of large and long-term safety data from the human papillomavirus-16/18-AS04-adjuvanted vaccine clinical trial programme. Pharmacoepidemiology and Drug Safety 2014;23(1099-557 (Electronic), 1053-8569 (Linking), 5):466-79.

\section{Arguedas 2010 \{published data only\}}

Arguedas A, Soley C, Loaiza C, Rincon G, Guevara S, Perez A, et al. Safety and immunogenicity of one dose of MenACWYCRM, an investigational quadrivalent meningococcal glycoconjugate vaccine, when administered to adolescents concomitantly or sequentially with Tdap and HPV vaccines. Vaccine 2010;28(1873-2518 (Electronic), 0264-410X (Linking), 18):3171-9.

\section{Ault 2004 \{published data only\}}

Ault KA, Giuliano AR, Edwards RP, Tamms G, Kim LL, Smith JF. A phase I study to evaluate a human papillomavirus (HPV) type 18 L1 VLP vaccine. Vaccine 2004;22(23-24):3004-7.

\section{Ault 2007 \{published data only\}}

Ault KA, Future II Study Group. Effect of prophylactic human papillomavirus $L 1$ virus-like-particle vaccine on risk of cervical intraepithelial neoplasia grade 2 , grade 3 , and adenocarcinoma in situ: a combined analysis of four randomised clinical trials. Lancet 2007;369(9576):1861-8.

\section{Basu 2013 \{published data only\}}

Basu P, Banerjee D, Singh P, Bhattacharya C, Biswas J. Efficacy and safety of human papillomavirus vaccine for primary prevention of cervical cancer: A review of evidence from phase III trials and national programs. South Asian Journal of Cancer 2013;2(4):187-92.

\section{Beachler 2016 \{published data only\}}

Beachler DC, Kreimer AR, Schiffman M, Herrero R, Wacholder S, Rodriguez AC, et al. Multisite HPV16/18 vaccine efficacy against cervical, anal, and oral HPV infection. Journal of the National Cancer Institute 2016;108(1):djv302.

\section{Brown 2004 \{published data only\}}

Brown DR, Fife KH, Wheeler CM, Koutsky LA, Lupinacci LM, Railkar R. Early assessment of the efficacy of a human papillomavirus type $16 \mathrm{~L} 1$ virus-like particle vaccine. Vaccine 2004;22(21-22):2936-42.

\section{Couto 2014 \{published data only\}}

Couto E, Saeterdal I, Juvet LK, Klemp M. HPV catch-up vaccination of young women: a systematic review and meta- analysis. BMC Public Health 2014;14(1471-2458 (Electronic), 1471-2458 (Linking)):867.

D'Addario 2017 \{published data only\}

D'Addario M, Redmond S, Scott P, Egli-Gany D, Riveros-Balta AX, Henao Restrepo AM, et al. Two-dose schedules for human papillomavirus vaccine: Systematic review and meta-analysis. Vaccine 2017;35(1873-2518 (Electronic), 0264-410X (Linking), 22):2892-901.

D'Souza 2013 \{published data only\}

D'Souza C, Mort GS, Zyngier S, Robinson P, Schlotterlein M. Preventive innovation: an Australian case study on HPV vaccination. Health Marketing Quartely 2013;30(3):206-20.

Delere 2013 \{published data only\} Delere Y, Bohmer MM, Walter D, Wichmann O. HPV vaccination coverage among women aged 18-20 years in Germany three years after recommendation of HPV vaccination for adolescent girls: results from a cross-sectional survey. Human Vaccines \& Immunotherapeutics 2013;9(8):1706-11.

Denny 2013 \{published data only\}

Denny L, Hendricks B, Gordon C, Thomas F, Hezareh M, Dobbelaere K. Safety and immunogenicity of the HPV-16/18 AS04-adjuvanted vaccine in HIV-positive women in South Africa: A partially-blind randomised placebo-controlled study. Vaccine 2013;31(48):5745-53.

\section{Descamps 2009 \{published data only\}}

Descamps D, Hardt K, Spiessens B, Izurieta P, Verstraeten T, Breuer T. Safety of human papillomavirus (HPV)-16/18 AS04-adjuvanted vaccine for cervical cancer prevention: a pooled analysis of 11 clinical trials. Human Vaccines \& Immunotherapeutics 2009;5(5):332-40.

\section{De Vincenzo 2014 \{published data only\}}

De Vincenzo R, Conte C, Ricci C, Scambia G, Capelli G. Longterm efficacy and safety of human papillomavirus vaccination. International Journal of Womens Health 2014;6(1179-411 (Electronic), 1179-411 (Linking)):999-1010.

\section{Dobson 2013 \{published data only\}}

Dobson SR, McNeil S, Dionne M, Dawar M, Ogilvie G, Krajden M. Immunogenicity of 2 doses of HPV vaccine in younger adolescents vs 3 doses in young women: a randomized clinical trial. JAMA 2013;309(17):1793-802.

\section{Draper 2011 \{published data only\}}

Draper E, Bissett SL, Howell-Jones R, Edwards D, Munslow G, Soldan K. Neutralization of non-vaccine human papillomavirus pseudoviruses from the $A 7$ and $A 9$ species groups by bivalent HPV vaccine sera. Vaccine 2011;29(47):8585-90.

Draper 2013 \{published data only\}

Draper E, Bissett SL, Howell-Jones R, Waight P, Soldan K, Jit M. A randomized, observer-blinded immunogenicity trial of cervarix((R)) and gardasil((R)) human papillomavirus vaccines in 12-15 year old girls. PLOS One 2013;8(5):e61825. 
Einstein 2009 \{published data only\}

Einstein MH, Baron M, Levin MJ, Chatterjee A, Edwards RP, Zepp F. Comparison of the immunogenicity and safety of Cervarix and Gardasil human papillomavirus (HPV) cervical cancer vaccines in healthy women aged 18 to 45 years. Human Vaccines \& Immunotherapeutics 2009;5(10):705-19.

\section{Einstein 2011 \{published data only\}}

Einstein MH, Baron M, Levin MJ, Chatterjee A, Fox B, Scholar S. Comparative immunogenicity and safety of human papillomavirus (HPV) 16/18 vaccine and HPV-6/11/16/18 vaccine: Follow-up from months 12 to 24 in a Phase III randomized study of healthy women aged 18 to 45 years. Human Vaccines \& Immunotherapeutics 2011;7(12):1343-58.

\section{Evans 2001 \{published data only\}}

Evans TG, Bonnez W, Rose RC, Koenig S, Demeter L, Suzich JA. A Phase 1 study of a recombinant viruslike particle vaccine against human papillomavirus type 11 in healthy adult volunteers. Journal of Infectious Diseases 2001;183(0022-1899, 10):1485-93.

\section{Forinash 2011 \{published data only\}}

Forinash AB, Yancey AM, Pitlick JM, Myles TD. Safety of the HPV bivalent and quadrivalent vaccines during pregnancy. Annals of Pharmacotherapy 2011;45(3):1-5.

\section{Garland 2016 \{published data only\}}

Garland SM, Paavonen J, Jaisamrarn U, Naud P, Salmeron J, Chow SN, et al. Prior human papillomavirus-16/18 AS04adjuvanted vaccination prevents recurrent high grade cervical intraepithelial neoplasia after definitive surgical therapy: Posthoc analysis from a randomized controlled trial. International Journal of Cancer 2016;139(1097-0215 (Electronic), 0020-7136 (Linking), 12):2812-26.

\section{Giuliano 2007 \{published data only\}}

Giuliano AR, Lazcano-Ponce E, Villa L, Nolan T, Marchant C, Radley D. Impact of baseline covariates on the immunogenicity of a quadrivalent (types $6,11,16$, and 18 ) human papillomavirus virus-like-particle vaccine. Journal of Infectious Diseases 2007;196(8):1153-62.

\section{Giuliano 2011 \{published data only\}}

Giuliano AR, Palefsky JM, Goldstone S, Moreira ED, Penny ME, Aranda C. Efficacy of quadrivalent HPV vaccine against HPV infection and disease in males. New England Journal of Medcine 2011;364(5):401-11.

\section{Giuliano 2015 \{published data only\}}

Giuliano AR, Isaacs-Soriano K, Torres BN, Abrahamsen M, Ingles DJ, Sirak BA, et al. Immunogenicity and safety of Gardasil among mid-adult aged men ( 27 to 45 years) The MAM Study. Vaccine 2015;33(1873-2518 (Electronic), 0264-410X (Linking), 42):5640-6

\section{Goldstone 2013 \{published data only\}}

Goldstone SE, Jessen H, Palefsky JM, Giuliano AR, Moreira ED Jr, Vardas $E$, et al. Quadrivalent HPV vaccine efficacy against disease related to vaccine and non-vaccine HPV types in males. Vaccine 2013;31(37):3849-55.

\section{Harro 2001 \{published data only\}}

Harro CD, Pang YY, Roden RB, Hildesheim A, Wang Z, Reynolds MJ. Safety and immunogenicity trial in adult volunteers of a human papillomavirus $16 \mathrm{~L} 1$ virus-like particle vaccine. Journal of the National Cancer Institute 2001;93(4):284-92.

\section{Haupt 2011 \{published data only\}}

Haupt RM, Wheeler CM, Brown DR, Garland SM, Ferris DG, Paavonen JA. Impact of an HPV6/11/16/18 L1 virus-like particle vaccine on progression to cervical intraepithelial neoplasia in seropositive women with HPV16/18 infection. International Journal of Cancer 2011;129(11):2632-42.

\section{Heijstek 2014 \{published data only\}}

Heijstek MW, Scherpenisse M, Groot N, Tacke C, Schepp RM, Buisman AM, et al. Immunogenicity and safety of the bivalent HPV vaccine in female patients with juvenile idiopathic arthritis: a prospective controlled observational cohort study. Annals of the RheumaticDiseases 2014;73(1468-2060 (Electronic), 0003-4967 (Linking), 8):1500-7.

\section{Hernandez-Avila 2016 \{published data only\}}

Hernandez-Avila M, Torres-Ibarra L, Stanley M, Salmeron J, CruzValdez A, Munoz N, et al. Evaluation of the immunogenicity of the quadrivalent $\mathrm{HPV}$ vaccine using 2 versus 3 doses at month 21: An epidemiological surveillance mechanism for alternate vaccination schemes. Human Vaccines \& Immunotherapeutics 2016;12 (2164-554X (Electronic), 2164-5515 (Linking), 1):30-8.

\section{Herrero 2013 \{published data only\}}

Herrero R, Quint W, Hildesheim A, Gonzalez P, Struijk L, Katki HA. Reduced prevalence of oral human papillomavirus (HPV) 4 years after bivalent HPV vaccination in a randomized clinical trial in Costa Rica. PLOS One 2013;8(7):e68329.

\section{Hildesheim 2007 \{published data only\}}

Hildesheim A, Herrero R, Wacholder S, Rodriguez AC, Solomon D, Bratti MC. Effect of human papillomavirus 16/18 L1 viruslike particle vaccine among young women with preexisting infection: a randomized trial. JAMA 2007;298(7):743-53.

\section{Hillman 2011 \{published data only\}}

Hillman RJ, Giuliano AR, Palefsky JM, Goldstone S, Moreira ED Jr, Vardas E. The immunogenicity of quadrivalent HPV (type 6/11/16/18) vaccine in males aged 16 to 26. Clinical and Vaccine Immunology 2011;19:261-7.

\section{Joura 2007 \{published data only\}}

Joura EA, Leodolter S, Hernandez-Avila M, Wheeler CM, Perez G, Koutsky LA. Efficacy of a quadrivalent prophylactic human papillomavirus (types 6, 11, 16, and 18) L1 virus-like-particle vaccine against high-grade vulval and vaginal lesions: a combined analysis of three randomised clinical trials. Lancet 2007;369(9574):1693-702.

\section{Kahn 2013 \{published data only\}}

Kahn JA, Xu J, Kapogiannis BG, Rudy B, Gonin R, Liu N, et al. Immunogenicity and safety of the human papillomavirus 6,11 , 16,18 vaccine in HIV-infected young women. Clinical Infectious Diseases 2013;57(5):735-44. 
Kang 2013 \{published data only\}

Kang WD, Choi HS, Kim SM. Is vaccination with quadrivalent HPV vaccine after loop electrosurgical excision procedure effective in preventing recurrence in patients with high-grade cervical intraepithelial neoplasia (CIN2-3)?. Gynecologic Oncology 2013;130(2):264-8.

\section{Khatun 2012 \{published data only\}}

Khatun S, Akram Hussain SM, Chowdhury S, Ferdous J, Hossain F, Begum SR. Safety and immunogenicity profile of human papillomavirus-16/18 AS04 adjuvant cervical cancer vaccine: a randomized controlled trial in healthy adolescent girls of Bangladesh. Japanese Journal of Clinical Oncology 2012;42(1):36-41.

\section{Kjaer 2009 \{published data only\}}

Kjaer SK, Sigurdsson K, Iversen OE, Hernandez-Avila M, Wheeler CM, Perez G, et al. A pooled analysis of continued prophylactic efficacy of quadrivalent human papillomavirus (Types 6/11/16/18) vaccine against high-grade cervical and external genital lesions. Cancer Prevention Research (Philadelphia, Pa.) 2009;2(1940-6215 (Electronic), 10):868-78.

\section{Kreimer 2015 \{published data only\}}

Kreimer AR, Sherman ME, Sahasrabuddhe VV, Safaeian M. The case for conducting a randomized clinical trial to assess the efficacy of a single dose of prophylactic HPV vaccines among adolescents. Journal of the National Cancer Institute 2015;107(3 1460-2105 (Electronic)):1-4 0027-8874 (Linking).

\section{Lamontagne 2013 \{published data only\}}

Lamontagne DS, Thiem VD, Huong VM, Tang Y, Neuzil KM. Immunogenicity of quadrivalent HPV vaccine among girls 11 to 13 Years of age vaccinated using alternative dosing schedules: results 29 to 32 months after third dose. Journal of Infectious Diseases 2013;208(8):1325-34.

\section{Lang 2014 \{published data only\}}

Lang Kuhs KA, Gonzalez P, Rodriguez AC, van Doorn LJ, Schiffman M, Struijk L, et al. Reduced prevalence of vulvar HPV16/18 infection among women who received the HPV16/18 bivalent vaccine: a nested analysis within the Costa Rica Vaccine Trial. Journal of Infectious Diseases 2014;210(12):1890-9.

\section{Lazcano-Ponce 2014 \{published data only\}}

Lazcano-Ponce E, Stanley M, Munoz N, Torres L, Cruz-Valdez A, Salmeron J, et al. Overcoming barriers to HPV vaccination: noninferiority of antibody response to human papillomavirus 16/18 vaccine in adolescents vaccinated with a two-dose vs. a threedose schedule at 21 months. Vaccine 2014;32(6):725-32.

\section{Lehtinen 2016 \{published data only\}}

Lehtinen M, Eriksson T, Apter D, Hokkanen M, Natunen K, Paavonen J, et al. Safety of the human papillomavirus (HPV)-16/18 AS04-adjuvanted vaccine in adolescents aged 12-15 years: Interim analysis of a large community-randomized controlled trial. Human Vaccines \& Immunotherapeutics 2016;12(2164-554X (Electronic), 2164-5515 (Linking), 12):3177-85.

\section{Leroux-Roels 2011 \{published data only\}}

Leroux-Roels G, Haelterman E, Maes C, Levy J, De Boever F, Licini L. Randomized trial of the immunogenicity and safety of the Hepatitis $B$ vaccine given in an accelerated schedule coadministered with the human papillomavirus type 16/18 AS04-adjuvanted cervical cancer vaccine. Clinical Vaccine Immunology 2011;18(9):1510-8.

\section{Leung 2015 \{published data only\}}

Leung TF, Liu AP, Lim FS, Thollot F, Oh HM, Lee BW, et al. Comparative immunogenicity and safety of human papillomavirus (HPV)-16/18 AS04-adjuvanted vaccine and HPV-6/11/16/18 vaccine administered according to 2 - and 3dose schedules in girls aged 9-14 years: Results to month 12 from a randomized trial. Human Vaccines \& Immunotherapeutics 2015;11(2164-554X (Electronic), 2164-5515 (Linking), 7):1689-702.

\section{Li 2012 \{published data only\}}

Li R, Li Y, Radley D, Liu Y, Huang T, Sings HL. Safety and immunogenicity of a vaccine targeting human papillomavirus types 6,11, 16 and 18: a randomized, double-blind, placebocontrolled trial in Chinese males and females. Vaccine 2012;30(28):4284-91.

\section{Lin 2014 \{published data only\}}

Lin CJ, Zimmerman RK, Nowalk MP, Huang HH, Raviotta JM. Randomized controlled trial of two dosing schedules for human papillomavirus vaccination among college age males. Vaccine 2014;32(6):693-9.

\section{Lu 2011 \{published data only\}}

Lu B, Kumar A, Castellsague X, Giuliano AR. Efficacy and safety of prophylactic vaccines against cervical HPV infection and diseases among women: a systematic review \& meta-analysis. BMC Infectious Disease 2011;11(1):13.

\section{Luna 2013 \{published data only\}}

Luna J, Plata M, Gonzalez M, Correa A, Maldonado I, Nossa C. Long-term follow-up observation of the safety, immunogenicity, and effectiveness of Gardasil in adult women. PLOS One 2013;8(12):e83431.

\section{Malagon 2012 \{published data only\}}

Malagon T, Drolet M, Boily MC, Franco EL, Jit M, Brisson J. Crossprotective efficacy of two human papillomavirus vaccines: a systematic review and meta-analysis. Lancet Infectious Diseases 2012;12(10):781-9.

\section{McCormack 2011 \{published data only\}}

McCormack PL, Joura EA. Spotlight on quadrivalent human papillomavirus (types $6,11,16,18$ ) recombinant vaccine $\left(\right.$ Gardasi $\left.^{\oplus}\right)$ in the prevention of premalignant genital lesions, genital cancer, and genital warts in women. BioDrugs 2011;25(5):339-43.

\section{McKeage 2011 \{published data only\}}

McKeage K, Romanowski B. Spotlight on AS04-adjuvanted human papillomavirus (HPV) types 16 and 18 vaccine (Cervarix ${ }^{\oplus}$ ). BioDrugs 2011;25(4):265-9. 


\section{Money 2016 \{published data only\}}

Money DM, Moses E, Blitz S, Vandriel SM, Lipsky N, Walmsley SL, et al. HIV viral suppression results in higher antibody responses in HIV-positive women vaccinated with the quadrivalent human papillomavirus vaccine. Vaccine 2016;34(40):4799-806.

\section{Moreira 2011 \{published data only\}}

Moreira ED, Palefsky JM, Giuliano AR, Goldstone S, Aranda C, Jessen $\mathrm{H}$. Safety and reactogenicity of a quadrivalent human papillomavirus (types $6,11,16,18$ ) L1 viral-like-particle vaccine in older adolescents and young adults. Human Vaccines \& Immunotherapeutics 2011;7(7):768-75.

\section{Nakalembe 2015 \{published data only\}}

Nakalembe M, Mirembe FM, Banura C. Vaccines against human papillomavirus in low and middle income countries: a review of safety, immunogenicity and efficacy. Infectious Agents and Cancer 2015;10(1750-9378 (Electronic), 1750-9378 (Linking)):17.

\section{Nelson 2013 \{published data only\}}

Nelson EA, Lam HS, Choi KC, Ho WCS, Fung LWE, Cheng FWT. A pilot randomized study to assess immunogenicity, reactogenicity, safety and tolerability of two human papillomavirus vaccines administered intramuscularly and intradermally to females aged $18-26$ years. Vaccine 2013;31(34):3452-60.

\section{Neuzil 2011 \{published data only\}}

Neuzil KM, Canh DG, Thiem VD, Janmohamed A, Huong VM, Tang Y. Immunogenicity and reactogenicity of alternative schedules of HPV vaccine in Vietnam: a cluster randomized noninferiority trial. JAMA 2011;305(14):1424-31.

\section{Olsson 2009 \{published data only\}}

Olsson SE, Kjaer SK, Sigurdsson K, Iversen OE, HernandezAvila M, Wheeler CM, et al. Evaluation of quadrivalent HPV $6 / 11 / 16 / 18$ vaccine efficacy against cervical and anogenital disease in subjects with serological evidence of prior vaccine type HPV infection. Human Vaccines 2009;5(1554-8619 (Electronic), 10):696-704.

\section{Palefsky 2011 \{published data only\}}

Palefsky JM, Giuliano AR, Goldstone SE, Moreira ED Jr, Aranda C, Jessen $\mathrm{H}$. HPV vaccine against anal HPV infection and anal intraepithelial neoplasia. New England Journal of Medicine 2011;365(17):1576-85.

\section{Pedersen 2007 \{published data only\}}

Pedersen C, Petaja T, Strauss G, Rumke HC, Poder A, Richardus JH, et al. Immunization of early adolescent females with human papillomavirus type 16 and $18 \mathrm{~L} 1$ virus-like particle vaccine containing AS04 adjuvant. Journal of Adolescent Health 2007;40(6):564-71.

\section{Perez 2008 \{published data only\}}

Perez G, Lazcano-Ponce E, Hernandez-Avila M, Garcia PJ, Munoz N, Villa LL. Safety, immunogenicity, and efficacy of quadrivalent human papillomavirus (types $6,11,16,18$ ) $\mathrm{L} 1$ virus-like-particle vaccine in Latin American women. International Journal of Cancer 2008;122(6):1311-8.

\section{Petaja 2009 \{published data only\}}

Petaja T, Keranen H, Karppa T, Kawa A, Lantela S, SiitariMattila M. Immunogenicity and safety of human papillomavirus (HPV)-16/18 AS04-adjuvanted vaccine in healthy boys aged 10-18 years. Journal of Adolescent Health 2009;44(1):33-40.

Petaja 2011 \{published data only\}

Petaja T, Pedersen C, Poder A, Strauss G, Catteau G, Thomas F, et al. Long-term persistence of systemic and mucosal immune response to HPV-16/18 AS04-adjuvanted vaccine in preteen/ adolescent girls and young women. International Journal of Cancer 2011;129(9):2147-57.

Poland 2005 \{published data only\}

Poland GA, Jacobson RM, Koutsky LA, Tamms GM, Railkar R, Smith JF. Immunogenicity and reactogenicity of a novel vaccine for human papillomavirus 16: a 2-year randomized controlled clinical trial. Mayo Clinic Proceedings 2005;80(5):601-10.

\section{Puthanakit 2016 \{published data only\}}

Puthanakit T, Huang LM, Chiu CH, Tang RB, Schwarz TF, Esposito S, et al. Randomized open trial comparing 2-dose regimens of the human papillomavirus 16/18 AS04-adjuvanted vaccine in girls aged $9-14$ years versus a 3 -dose regimen in women aged 15-25 years. Journal of Infectious Diseases 2016;214(4):525-36

\section{Ramanakumar 2016 \{published data only\}}

Ramanakumar AV, Naud P, Roteli-Martins CM, de Carvalho NS, de Borba PC, Teixeira JC, et al. Incidence and duration of typespecific human papillomavirus infection in high-risk HPV-naive women: results from the control arm of a phase II HPV-16/18 vaccine trial. BMJ Open. 2016;6(2044-6055 (Electronic), 2044-6055 (Linking), 8):e011371.

\section{Read 2011 \{published data only\}}

Read TR, Hocking JS, Chen MY, Donovan B, Bradshaw CS, Fairley CK. The near disappearance of genital warts in young women 4 years after commencing a national human papillomavirus (HPV) vaccination programme. Sexually Transmitted Infections 2011;87(7):544-7.

\section{Reisinger 2007 \{published data only\}}

Reisinger KS, Block SL, Lazcano-Ponce E, Samakoses R, Esser MT, Erick J, et al. Safety and persistent immunogenicity of a quadrivalent human papillomavirus types $6,11,16,18 \mathrm{~L} 1$ virus-like particle vaccine in preadolescents and adolescents: a randomized controlled trial. Pediatric Infectious Disease Journal 2007;26(3):201-9.

\section{Reisinger 2010 \{published data only\}}

Reisinger KS, Block SL, Collins-Ogle M, Marchant C, Catlett M, Radley D, et al. Safety, tolerability, and immunogenicity of gardasil given concomitantly with Menactra and Adacel. Pediatrics 2010;125(6):1142-51.

\section{Romanowski 2016 \{published data only\}}

Romanowski B, Schwarz TF, Ferguson L, Peters K, Dionne M, Behre U, et al. Sustained immunogenicity of the HPV-16/18 AS04-adjuvanted vaccine administered as a two-dose schedule in adolescent girls: Five-year clinical data and modeling 
predictions from a randomized study. Human Vaccines \& Immunotherapeutics 2016;12(2164-554X (Electronic), 2164-5515 (Linking), 1):20-9.

\section{Rowhani-Rahbar 2012 \{published data only\}}

Rowhani-Rahbar A, Alvarez FB, Bryan JT, Hughes JP, Hawes SE, Weiss NS. Evidence of immune memory 8.5 years following administration of a prophylactic human papillomavirus type 16 vaccine. Journal of Clinical Virology 2012;53(3):239-43.

Safaeian 2013 \{published data only\}

Safaeian M, Kemp TJ, Pan DY, Porras C, Rodriguez AC, Schiffman M, et al. Cross-protective vaccine efficacy of the bivalent HPV vaccine against HPV31 is associated with humoral immune responses: results from the Costa Rica Vaccine Trial. Human Vaccines \& Immunotherapeutics 2013;9(7):1399-406.

\section{Schwarz 2008 \{published data only\}}

Schwarz TF, Leo O. Immune response to human papillomavirus after prophylactic vaccination with AS04-adjuvanted HPV-16/18 vaccine: improving upon nature. Gynecologic Oncology 2008;110(3 Suppl 1):S1-10.

\section{Schwarz 2009 \{published data only\}}

Schwarz TF, Spaczynski M, Schneider A, Wysocki J, Galaj A, Perona P. Immunogenicity and tolerability of an HPV-16/18 AS04-adjuvanted prophylactic cervical cancer vaccine in women aged 15-55 years. Vaccine 2009;27(4):581-7.

\section{Schwarz 2010 \{published data only\}}

Schwarz TF, Kocken M, Petaja T, Einstein MH, Spaczynski M, Louwers JA. Correlation between levels of human papillomavirus (HPV) 16 and 18 antibodies in serum and cervicovaginal secretions in girls and women vaccinated with the HPV-16/18 AS04-adjuvanted vaccine. Human Vaccine \& Immunotherapeutics 2010;6(12):1054-61.

\section{Schwarz 2011 \{published data only\}}

Schwarz TF, Spaczynski M, Schneider A, Wysocki J, Galaj A, Schulze K. Persistence of immune response to HPV-16/18 AS04adjuvanted cervical cancer vaccine in women aged $15-55$ years. Human Vaccines \& Immunotherapeutics 2011;7(9):958-65.

\section{Schwarz 2014 \{published data only\}}

Schwarz TF, Huang LM, Lin TY, Wittermann C, Panzer F, Valencia $A$, et al. Long-term immunogenicity and safety of the HPV-16/18 AS04-adjuvanted vaccine in 10- to 14year-old girls: open 6-year follow-up of an initial observerblinded, randomized trial. Pediatric Infectious Disease Journal 2014;33(1532-0987 (Electronic), 0891-3668 (Linking), 12):1255-61.

\section{Sengupta 2011 \{published data only\}}

Sengupta A, Shenoi A, Sarojini NB, Madhavi Y. Human papillomavirus vaccine trials in India. Lancet 2011;377(9767):719.

\section{Singhal 2011 \{published data only\}}

Singhal SC. Human papilloma virus vaccines and current controversy. Indian Pediatrics 2011;48(3):248-9.

\section{Skinner 2016 \{published data only\}}

Skinner SR, Apter D, De Carvalho N, Harper DM, Konno R, Paavonen J, et al. Human papillomavirus (HPV)-16/18 AS04-adjuvanted vaccine for the prevention of cervical cancer and HPV-related diseases. Expert Review of Vaccines 2016;15(1744-8395 (Electronic), 1476-0584 (Linking), 3):367-87.

\section{Smith-McCune 2010 \{published data only\}}

Smith-McCune K. Quadrivalent HPV vaccine administered to women who became pregnant during trials did not appear to adversely affect pregnancy outcome; however, use during pregnancy is not recommended. Evidence Based Medicine 2010;15(3):80-1.

\section{Srinivasan 2011 \{published data only\}}

Srinivasan S. HPV vaccine trials and sleeping watchdogs. Indian Journal of Medical Ethics 2011;8(2):73-4.

\section{Toft 2014 \{published data only\}}

Toft L, Storgaard M, Muller M, Sehr P, Bonde J, Tolstrup M, et al. Comparison of the immunogenicity and reactogenicity of Cervarix and Gardasil human papillomavirus vaccines in HIV-infected adults: a randomized, double-blind clinical trial. Journal of Infectious Diseases 2014;209(8):1165-73.

\section{Van Klooster 2011 \{published data only\}}

Klooster TM, Kemmeren JM, van der Maas NA, de Melker HE. Reported adverse events in girls aged 13-16 years after vaccination with the human papillomavirus (HPV)-16/18 vaccine in the Netherlands. Vaccine 2011;29(28):4601-7.

\section{Vesikari 2010 \{published data only\}}

Vesikari T, Van Damme P, Lindblad N, Pfletschinger U, Radley D, Ryan D, et al. An open-label, randomized, multicenter study of the safety, tolerability, and immunogenicity of quadrivalent human papillomavirus (types 6/11/16/18) vaccine given concomitantly with diphtheria, tetanus, pertussis, and poliomyelitis vaccine in healthy adolescents 11 to 17 years of age. Pediatric Infectious Disease Journal 2010;29(4):314-8.

\section{Wheeler 2008 \{published data only\}}

Wheeler CM, Bautista OM, Tomassini JE, Nelson M, Sattler CA, Barr E. Safety and immunogenicity of co-administered quadrivalent human papillomavirus (HPV)-6/11/16/18 L1 viruslike particle (VLP) and hepatitis $B(\mathrm{HBV})$ vaccines. Vaccine 2008;26(0264-410X (Print), 5):686-96.

\section{Wheeler 2011 \{published data only\}}

Wheeler CM, Harvey BM, Pichichero ME, Simon MW, Combs SP, Blatter MM. Immunogenicity and safety of human papillomavirus-16/18 AS04-adjuvanted vaccine coadministered with tetanus toxoid, reduced diphtheria toxoid, and acellular pertussis vaccine and/or meningococcal conjugate vaccine to healthy girls 11 to 18 years of age: results from a randomized open trial. Pediatric Infectious Disease Journal 2011;30(12):e225-34.

\section{Yancey 2010 \{published data only\}}

Yancey AM, Pitlick JM, Forinash AB. The prophylactic role for the human papillomavirus quadrivalent vaccine in males. Annals of Pharmacotherapy 2010;44(7-8):1314-8. 
Zhu 2011 \{published data only\}

Zhu FC, Li CG, Pan HX, Zhang YJ, Bi D, Tang HW. Safety and immunogenicity of human papillomavirus-16/18 AS04-adjuvanted vaccine in healthy Chinese females aged 15 to 45 years: a phase I trial. Chinese Journal of Cancer 2011;30(8):559-64.

\section{Zimmerman 2010 \{published data only\}}

Zimmerman RK, Nowalk MP, Lin CJ, Fox DE, Ko FS, Wettick E. Randomized trial of an alternate human papillomavirus vaccine administration schedule in college-aged women. Journal of Women's Health 2010;19(8):1441-7.

\section{Additional references}

\section{Andrews 2017}

Andrews N, Stowe J, Miller E. No increased risk of GuillainBarré syndrome after human papilloma virus vaccine: A self-controlled case-series study in England. Vaccine 2017;35(1):1729-32.

\section{ANSM/SANTE 2015}

ANSM/SANTE. Risk of auto-immune diseases after vaccination against HPV: a pharmacoepidemiological study [Vaccins anti-HPV et risque de maladies auto-immunes : étude pharmacoépidémiologique]. http://ansm.sante.fr/S-informer/ Points-d-information-Points-d-information/Vaccination-contreles-infections-a-HPV-et-risque-de-maladies-auto-immunes-uneetude-Cnamts-ANSM-rassurante-Point-d-information September 2015;Website accessed 10-12-15:1-91.

\section{Arbyn 2007}

Arbyn M, Dillner J. Review of current knowledge on HPV vaccination: an appendix to the European Guidelines for Quality Assurance in Cervical Cancer Screening. Journal of Clinical Virology 2007;38(3):189-97.

\section{Arbyn 2009}

Arbyn M, Rebolj M, de Kok IM, Becker N, O'Reilly M, Andrae B. The challenges for organising cervical screening programmes in the 15 old member states of the European Union. European Journal of Cancer 2009;45(15):2671-8.

\section{Arbyn 2010}

Arbyn M, Van Veen EB, Andersson K, Bogers J, Boulet G, Bergeron $\mathrm{C}$, et al. Cervical cytology biobanking in Europe. Internatonal Journal of Biological Markers 2010;25(3):117-25.

\section{Arbyn 2011}

Arbyn M, Castellsagué X, de Sanjosé S, Bruni L, Saraiya M, Bray F, et al. Worldwide burden of cervical cancer in 2008. Annals of Oncology 2011;22:2675-86.

\section{Arbyn 2012}

Arbyn M, Ronco G, Anttila A, Meijer CJ, Poljak M, Ogilvie G, et al. Evidence regarding HPV testing in secondary prevention of cervical cancer. Vaccine 2012;30(Suppl 5):F88-F99.

\section{Arbyn 2014}

Arbyn M, Tommasino M, Depuydt C, Dillner J. Are 20 human papillomavirus types causing cervical cancer?. Journal of Pathology 2014;234(4):431-5.

\section{Arbyn 2016}

Arbyn M, Vanden Broeck D, Benoy I, Bogers J, Depuydt C, Praet M, et al. Surveillance of effects of HPV vaccination in Belgium. Cancer Epidemiology 2016;41:152-8.

\section{Arbyn 2017}

Arbyn M, Redman CW, Verdoodt F, Kyrgiou M, Tzafetas M, Ghaem-Maghami S, et al. Incomplete excision of cervical precancer as predictor of treatment failure: a systematic review and meta-analysis. Lancet Oncology 2017;18(12):1665-79.

\section{Arnheim-Dahlstrom 2013}

Arnheim-Dahlstrom L, Pasternak B, Svanstrom H, Sparen P, Hviid A. Autoimmune, neurological, and venous thromboembolic adverse events after immunisation of adolescent girls with quadrivalent human papillomavirus vaccine in Denmark and Sweden: cohort study. BMJ 2013;347:f5906.

\section{Baldur-Felskov 2014}

Baldur-Felskov B, Dehlendorff C, Munk C, Kjaer SK. Early impact of human papillomavirus vaccination on cervical neoplasianationwide follow-up of young Danish women. Journal of the National Cancer Institute 2014;106(3):dtj460.

\section{Baril 2015}

Baril L, Rosillon D, Willame C, Angelo MG, Zima J, van den Bosch JH, et al. Risk of spontaneous abortion and other pregnancy outcomes in 15-25 year old women exposed to human papillomavirus-16/18 AS04-adjuvanted vaccine in the United Kingdom. Vaccine 2015;33:6884-91.

\section{Bhatla 2010}

Bhatla N, Suri V, Basu P, Shastri S, Datta SK, Bi D, et al. Immunogenicity and safety of human papillomavirus-16/18 AS04-adjuvanted cervical cancer vaccine in healthy Indian women. Journal of Obstetrics and Gynaecology Research 2010;36(1):123-32.

\section{Bosch 2002}

Bosch FX, Lorincz A, Munoz N, Meijer CJ, Shah KV. The causal relation between human papillomavirus and cervical cancer. Journal of Clinical Pathology 2002;55:244-65.

\section{Bosch 2008}

Bosch FX, Burchell AN, Schiffman M, Giuliano AR, de Sanjose S, Bruni L, et al. Epidemiology and natural history of human papillomavirus infections and type-specific implications in cervical neoplasia. Vaccine 2008;26(Suppl 10):K1-K16.

\section{Bosch 2016}

Bosch FX, Robles C, Diaz M, Arbyn M, Baussano I, Clavel C, et al. HPV-FASTER: broadening the scope for prevention of HPV-related cancer. Nature Reviews, Clinical Oncology 2016;13:119-32. 


\section{Bouvard 2009}

Bouvard V, Baan R, Straif K, Grosse Y, Secretan B, El Ghissassi F. A review of human carcinogens - Part B: biological agents. Lancet Oncology 2009;10(4):321-2.

\section{Bray 2005}

Bray F, Carstensen B, Moller H, Zappa M, Zakelj MP, Lawrence G. Incidence trends of adenocarcinoma of the cervix in 13 European countries. Cancer Epidemiology, Biomarkers and Prevention 2005;14(9):2191-9.

\section{Bray 2005a}

Bray F, Loos AH, McCarron P, Weiderpass E, Arbyn M, Moller H. Trends in cervical squamous cell carcinoma incidence in 13 European countries: changing risk and the effects of screening. Cancer Epidemiology, Biomarkers and Prevention 2005;14(3):677-86.

\section{Breitburd 1995}

Breitburd F, Kirnbauer R, Hubbert NL, Nonnenmacher B, TrinDinh-Desmarquet C, Orth $\mathrm{G}$. Immunization with virus like particles from cottontail rabbit papillomavirus (CRPV) can protect against experimental CRPV infection. Journal of Virology 1995;69(6):3959-63.

\section{Brotherton 2011}

Brotherton JM, Fridman M, May CL, Chappell G, Saville AM, Gertig DM. Early effect of the HPV vaccination programme on cervical abnormalities in Victoria, Australia: an ecological study. Lancet 2011;377(1474-547X (Electronic), 0140-6736 (Linking), 9783):2085-92.

\section{Brotherton 2015}

Brotherton JML, Malloy M, Budd AC, Saville M, Drennan KT, Gertig DM. Effectiveness of less than three doses of quadrivalent human papillomavirus vaccine against cervical intraepithelial neoplasia when administered using a standard dose spacing schedule: Observational cohort of young women in Australia. Papillomavirus Research 2015;1(1):59-73.

\section{Brown 2001}

Brown DR, Bryan JT, Schroeder JM, Robinson TS, Fife KH, Wheeler CM. Neutralization of human papillomavirus type 11 (HPV-11) by serum from women vaccinated with yeast-derived HPV-11 L1 virus-like particles: correlation with competitive radioimmunoassay titer. Journal of Infectious Diseases 2001;184(9):1183-6.

\section{Bzhalava 2013}

Bzhalava D, Guan P, Franceschi S, Dillner J, Clifford G. A systematic review of the prevalence of mucosal and cutaneous human papillomavirus types. Virology 2013;445(1-2):224-31.

\section{Cancer Research UK 2018}

Cancer Researck UK. Cervical cancer incidence statistics. http://www.cancerresearchuk.org/health-professional/cancerstatistics/statistics-by-cancer-type/cervical-cancer/incidence Accessed Jan 2018.

\section{Castellsagué 2006}

Castellsagué X, Diaz M, De Sanjose S, Munoz N, Herrero R, Franceschi S. Worldwide human papillomavirus etiology of cervical adenocarcinoma and its cofactors: implications for screening and prevention. Journal of the National Cancer Institute 2006;98(1460-2105 (Electronic), 5):303-15.

\section{Castellsagué 2011}

Castellsagué X, Munoz N, Pitisuttithum P, Ferris D, Monsonego J, Ault K. End-of-study safety, immunogenicity, and efficacy of quadrivalent HPV (types $6,11,16,18$ ) recombinant vaccine in adult women 24-45 years of age. British Journal of Cancer 2011;105(1):28-37.

\section{Cates 2015}

Cates M, Karner C. Clinical importance cannot be ruled out using mean difference alone. BMJ 2015;351:h5496.

\section{CDC 2015}

Sukumaran L, Advisory Committee on Immunization Practices - Immunization Safety Office - Centers for Disease Control and Prevention (CDC, Atlanta). Human Papillomavirus Vaccine Safety Update. http://www.cdc.gov/vaccines/acip/meetings/ downloads/slides-2015-10/hpv-04-sukumaran.pdf 21/10/2015.

\section{Chao 2012}

Chao C, Klein NP, Velicer CM, Sy LS, Slezak JM, Takhar H, et al. Surveillance of autoimmune conditions following routine use of quadrivalent human papillomavirus vaccine. Journal of Internal Medicine 2012;271(2):193-203.

\section{Cogliano 2005}

Cogliano V, Baan R, Straif K, Grosse Y, Secretan B, El Ghissassi F. Carcinogenicity of human papillomaviruses. Lancet Oncology 2005;6(1470-2045, 4):204.

\section{Crowe 2014}

Crowe E, Pandeya N, Brotherton JM, Dobson AJ, Kisely S, Lambert SB, et al. Effectiveness of quadrivalent human papillomavirus vaccine for the prevention of cervical abnormalities: case-control study nested within a population based screening programme in Australia. BMJ 2014;348(g1458):1-10.

\section{Cuschieri 2016}

Cuschieri K, Kavanagh K, Moore C, Bhatia R, Love J, Pollock KG. mpact of partial bivalent HPV vaccination on vaccine-type infection: a population-based analysis. British Journal of Cancer 2016;114(11):1261-4.

\section{Dana 2009}

Dana A, Buchanan KM, Goss MA, Seminack MM, Shields KE, Korn S. Pregnancy outcomes from the pregnancy registry of a human papillomavirus type 6/11/16/18 vaccine. Obstetrics and Gynaecology 2009;114(1873-233X (Electronic), 6):1170-8.

\section{De Carvalho 2010}

De Carvalho N, Teixeira J, Roteli-Martins CM, Naud P, De Borba P, Zahaf T. Sustained efficacy and immunogenicity of the HPV-16/18 AS04-adjuvanted vaccine up to 7.3 years in young adult women. Vaccine 2010;28(38):6247-55. 


\section{de Sanjose 2007}

de Sanjose S, Diaz M, Castellsagué X, Clifford G, Bruni L, Munoz N. Worldwide prevalence and genotype distribution of cervical human papillomavirus DNA in women with normal cytology: a meta-analysis. Lancet Infectious Diseases 2007;7(7):453-9.

\section{de Sanjose 2010}

de Sanjose S, Quint WG, Alemany L, Geraets DT, Klaustermeier JE, Lloveras B, et al. Human papillomavirus genotype attribution in invasive cervical cancer: a retrospective cross-sectional worldwide study. Lancet Oncology 2010;11(11):1048-56.

\section{Deeks 2001}

Deeks JJ, Altman DG, Bradburn MJ. Statistical methods for examining heterogeneity and combining results from several studies in meta-analysis. In: Egger M, Davey Smith G, Altman DG (eds). Systematic Reviews in Health Care: Meta-Analysis in Context (2nd edition). London: BMJ Publication Group, 2001.

\section{Delere 2014}

Delere Y, Wichmann O, Klug SJ, van der Sande M, Terhardt M, Zepp F. The efficacy and duration of vaccine protection against human papillomavirus: a systematic review and meta-analysis. Deutsches Arzteblatt International 2014;111(35-36):584-91.

\section{DerSimonian 1986}

DerSimonian R, Laird N. Meta-analysis in clinical trials. Controlled Clinical Trials 1986;7:177-88.

\section{Dillner 2011}

Dillner J, Arbyn M, Unger E, Dillner L. Monitoring of human papillomavirus vaccination. Clinical \& Experimental Immunology 2011;163(1):17-25.

\section{Donegan 2013}

Donegan K, Beau-Lejdstrom R, King B, Seabroke S, Thomson A, Bryan P. Bivalent human papillomavirus vaccine and the risk of fatigue syndromes in girls in the UK. Vaccine 2013;31(43):4961-7.

\section{Donovan 2011}

Donovan B, Franklin N, Guy R, Grulich AE, Regan DG, Ali H, et al. Quadrivalent human papillomavirus vaccination and trends in genital warts in Australia: analysis of national sentinel surveillance data. Lancet Infectious Diseases 2011;11(1474-4457 (Electronic), 1473-3099 (Linking), 1):39-44.

\section{Drolet 2015}

Drolet M, Benard E, Boily MC, Ali H, Baandrup L, Bauer H, et al. Population-level impact and herd effects following human papillomavirus vaccination programmes: a systematic review and meta-analysis. Lancet Infectious Diseases 2015;15(5):565-80.

\section{EMA 2016}

European Medicines Agency. HPV vaccines: EMA confirms evidence does not support that they cause CRPS or POTS. http://www.ema.europa.eu/docs/en_GB/document_ library/Referrals_document/HPV_vaccines_20/European_ Commission_final_decision/WC500196773.pdf 16/01/2016.

\section{EMEA 2014a}

European Medicines Agency. EMEA/H/C/000721-Cervarix-EPAR summary for the public 2014. http://www.ema.europa.eu/docs/ en_GB/document_library/EPAR_-_Summary_for_the_public/ human/000721/WC500024634.pdf. Februrary 2014;Accessed 10-12-15:1-3.

\section{EMEA 2014b}

European Medicines Agency. EMEA/H/C/000703-Gardasil-EPAR summary for the public 2014. http://www.ema.europa.eu/ docs/en_GB/document_library/EPAR_-_Summary_for_the_ public/human/000703/WC500021146.pdf August 2014;Accessed 10-12-15:1-4.

\section{EMEA 2015}

EMEA. Review concludes evidence does not support that HPV vaccines cause CRPS or POTS. http://www.ema.europa.eu/ema/ index.jsp?curl=pages/news_and_events/news/2015/11/news_ detail_002429.jsp\&mid=WC0b01ac058004d5c1 05/11/2015.

\section{Ferlay 2013}

Ferlay J, Steliarova-Foucher E, Lortet-Tieulent J, Rosso S, Coebergh JW, Comber H, et al. Cancer incidence and mortality patterns in Europe: Estimates for 40 countries in 2012. European Journal of Cancer 2013;49(6):1374-403.

\section{Ferlay 2015}

Ferlay J, Soerjomataram I, Dikshit R, Eser S, Mathers C, Rebelo $\mathrm{M}$, et al. Cancer incidence and mortality worldwide: sources, methods and major patterns in GLOBOCAN 2012. International Journal of Cancer 2015;136(5):E359-96.

\section{Forman 2012}

Forman D, de Martel C, Lacey CJ, Soerjomataram I, Lortet-Tieulent J, Bruni L, et al. Global burden of human papillomavirus and related diseases. Vaccine 2012;30 Suppl 5:F12-23.

\section{Frazer 2004}

Frazer IH. Prevention of cervical cancer through papillomavirus vaccination. Nature Reviews Immunology 2004;4:46-55.

\section{FUTURE-II 2007}

The FUTURE II study group. Quadrivalent vaccine against human papillomavirus to prevent high-grade cervical lesions. New England Journal of Medicine 2007;356(19):1915-27.

\section{Galloway 2003}

Galloway DA. Papillomavirus vaccines in clinical trials. Lancet 2003;3:469-75.

\section{Garcia-Sicilia 2010}

Garcia-Sicilia J, Schwarz TF, Carmona A, Peters K, Malkin JE, Tran PM. Immunogenicity and safety of human papillomavirus-16/18 AS04-adjuvanted cervical cancer vaccine coadministered with combined diphtheria-tetanus-acellular pertussis-inactivated poliovirus vaccine to girls and young women. Journal of Adolescent Health 2010;46(2):142-51. 


\section{Garland 2007}

Garland SM, Hernandez-Avila M, Wheeler CM, Perez G, Harper DM, Leodolter S. Quadrivalent vaccine against human papillomavirus to prevent anogenital diseases. New England Journal of Medicine 2007;356(19):1928-43.

\section{Garland 2007a}

Garland SM, Steben M, Hernandez-Avila M, Koutsky LA, Wheeler CM, Perez G, et al. An evaluation of non-inferiority in antibody response to human papillomavirus (HPV) 16 in subjects vaccinated with monovalent (HPV 16) and quadrivalent (HPV 6, 11, 16, 18) L1 virus like particle vaccines. Clinical and Vaccine Immunology 2007;10(1128):1-17.

\section{Garland 2009}

Garland SM, Ault KA, Gall SA, Paavonen J, Sings HL, Ciprero KL, et al. Pregnancy and infant outcomes in the clinical trials of a human papillomavirus type $6 / 11 / 16 / 18$ Vaccine: a combined analysis of five randomized controlled trials. Obstetrics and Gynecology 2009;114(6):1179-88.

\section{Gee 2017}

Gee J, Sukumaran L, Weintraub E. Risk of Guillain-Barré syndrome following quadrivalent human papillomavirus vaccine in the Vaccine Safety Datalink. Vaccine 2017;35(43):5756-8

\section{Gertig 2013}

Gertig DM, Brotherton JM, Budd AC, Drennan K, Chappell G, Saville AM. mpact of a population-based HPV vaccination program on cervical abnormalities: a data linkage study. BMC Medicine 2013;11:227.

\section{Ghim 2000}

Ghim S, Newsome J, Bell J, Sundberg JP, Schlegel R, Jenson AB. Spontaneously regressing oral papillomas induce systemic antibodies that neutralize canine oral papillomavirus. Experimental and Molecular Pathology 2000;68(3):147-51.

\section{Giannini 2006}

Giannini SL, Hanon E, Moris P, Van Mechelen M, Morel S, Dessy F, et al. Enhanced humoral and memory B cellular immunity using HPV16/18 L1 VLP vaccine formulated with the MPL/aluminium salt combination (AS04) compared to aluminium salt only. Vaccine 2006;24:5937-49.

\section{Goss 2015}

Goss MA, Lievano F, Buchanan KM, Seminack MM, Cunningham ML, Dana A. Final report on exposure during pregnancy from a pregnancy registry for quadrivalent human papillomavirus vaccine. Vaccine 2015;33:3422-8.

\section{Grimaldi-Bensouda 2014}

Grimaldi-Bensouda L, Guillemot D, Godeau B, Benichou J, Lebrun-Frenay C, Papeix C, et al. Autoimmune disorders and quadrivalent human papillomavirus vaccination of young female subjects. Journal of Internal Medicine 2014;275(4):398-408.

\section{Grimaldi-Bensouda 2017}

Grimaldi-Bensouda L, Rossignol M, Kone-Paut I, Krivitzky A, Lebrun-Frenay $\mathrm{C}$, Clet J, et al. Risk of autoimmune diseases and human papilloma virus (HPV) vaccines: Six years of casereferent surveillance. Journal of Autoimmunity 2017;79:84-90.

\section{Guyatt 2008}

Guyatt GH, Oxman AD, Vist GE, Kunz R, Falck-Ytter Y, AlonsoCoello $P$, et al. GRADE: an emerging consensus on rating quality of evidence and strength of recommendations. $B M J$ 2008;336(7650):924-6.

\section{Harbord 2009}

Harbord RM, Harris RJ, Sterne JAC. Updated tests for smallstudy effects in meta-analyses. Stata Journal 2009;9(2):197-210.

\section{Harper 2004}

Harper DM, Franco EL, Wheeler C, Ferris DG, Jenkins D, Schuind A. Efficacy of a bivalent L1 virus-like particle vaccine in prevention of infection with human papillomavirus types 16 and 18 in young women: a randomised controlled trial. Lancet 2004;364(9447):1757-65.

\section{Harper 2006}

Harper DM, Franco EL, Wheeler CM, Moscicki AB, Romanowski B, Roteli-Martins CM. Sustained efficacy up to 4.5 years of a bivalent $L 1$ virus-like particle vaccine against human papillomavirus types 16 and 18: follow-up from a randomised control trial. Lancet 2006;367(9518):1247-55.

\section{Harper 2009}

Harper DM. Currently approved prophylactic HPV vaccines. Expert Review of Vaccines 2009;8(12):1663-79.

\section{Herrero 2011}

Herrero R, Wacholder S, Rodriguez AC, Solomon D, Gonzalez P, Kreimer AR. Prevention of persistent human papillomavirus infection by an HPV16/18 vaccine: A community-based randomized clinical trial in guanacaste, Costa Rica. Cancer Discovery 2011;1(5):408-19.

\section{Higgins 2003}

Higgins JPT, Thompson SG, Deeks JJ, Altman DG. Measuring inconsistency in meta-analyses. BMJ 2003;327:557-60.

\section{Higgins 2011a}

Higgins JPT, Green S (editors). Cochrane Handbook for Systematic Reviews of Interventions Version 5.1.0 (updated March 2011). The Cochrane Collaboration, 2011. Available from handbook.cochrane.org.

\section{Higgins 2011b}

Higgins JP, Altman DG, Gøtzsche PC, Jüni P, Moher D, Oxman AD, et al. Cochrane Bias Methods Group, Cochrane Statistical Methods Group. The Cochrane Collaboration's tool for assessing risk of bias in randomised trials. BMJ 2011;343:d5928.

\section{Hildesheim 2014}

Hildesheim A, Wacholder S, Catteau G, Struyf F, Dubin G, Herrero R. Efficacy of the HPV-16/18 vaccine: Final according 
to protocol results from the blinded phase of the randomized Costa Rica HPV-16/18 vaccine trial. Vaccine 2014;32(39):5087-97.

\section{Ho 1998}

Ho GYF, Bierman R, Beardsley I, Chang CJ, Burk RD. Natural history of cervicovaginal papillomavirus infection in young women. New England Journal of Medicine 1998;338:423-8.

\section{Hofstetter 2016}

Hofstetter AM, Ompad DC, Stockwell MS, Rosenthal SL, Soren K. Human papillomavirus vaccination and cervical cytology outcomes among urban low-income minority females. JAMA Pediatrics 2016;170(5):445-52.

\section{Huh 2017}

Huh WK, Joura EA, Giuliano AR, Iversen OE, de Andrade RP, Ault KA, et al. Final efficacy, immunogenicity, and safety analyses of a nine-valent human papillomavirus vaccine in women aged $16-26$ years: a randomised, double-blind trial. Lancet 2017;390(10108):2043-59.

\section{IARC 2005}

IARC Expert team cervical cancer screening, (Day N, Hakama M, Arbyn M). Cervix Cancer Screening. IARC Handbooks of Cancer Prevention. Vol. 10, Lyon: IARC Press, 2005:1-302.

\section{IARC 2007}

IARC Monograph Working Group on Carcinogenesis (Coglinano V, Baan R, Straif K, Secretan B, El Ghissasi F, Zur Hausen H, et al) . In: Cogliano V, Baan R, Straif K, Grosse Y, Secretan B, El Ghissassi F editor(s). IARC Monographs on the Evaluation of Carcinogenic Risks to Humans. Vol 90: Human Papillomaviruses. Vol. 90, Lyon: IARC Press, 2007:1-670.

\section{IARC 2013}

IARC HPV Working Group. Primary end-points for prophylactic HPV vaccine trials. IARC Working Group Reports. Vol. 7, Lyon, France: WHO Press, 2013:1-120.

\section{Iftner 2003}

Iftner T, Villa LL. Chapter 12: Human papillomavirus technologies. Journal of National Cancer Institute Monographs 2003;31:80-8.

\section{Initiative 2009}

Initiative for Vaccine Research of the Department of Immunization VaB. Human Papillomavirus (HPV) Vaccine Background Paper. World Health Organization (WHO). Geneva, 2009:1-249.

\section{Jordan 2008}

Jordan J, Arbyn M, Martin-Hirsch P, Schenck U, Baldauf J-J, Da Silva $D$, et al. European guidelines for quality assurance in cervical cancer screening: recommendations for clinical management of abnormal cervical cytology, part 1. Cytopathology 2008;19(6):342-54.

\section{Jordan 2009}

Jordan J, Martin-Hirsch P, Arbyn M, Schenck U, Baldauf J$J$, Da Silva D, et al. European guidelines for management of abnormal cervical cytology, Part 2. Cytopathology 2009;20(1):5-16.

\section{Joura 2015}

Joura EA, Giuliano AR, Iversen OE, Bouchard C, Mao C, Mehlsen J, et al. A 9-Valent HPV Vaccine against Infection and Intraepithelial Neoplasia in Women. New England Journal of Medicine 2015;372(8):711-23.

\section{Kahn 2009}

Kahn JA. HPV vaccination for the prevention of cervical intraepithelial neoplasia. New England Journal of Medicine 2009;361(3):271-8.

\section{Kang 2008}

Kang S, Kim KH, Kim YT, Kim YT, Kim JH, Song YS. Safety and immunogenicity of a vaccine targeting human papillomavirus types 6, 11, 16 and 18: a randomized, placebo-controlled trial in 176 Korean subjects. International Journal of Gynecological Cancer 2008;18(5):1013-9.

\section{Kavanagh 2014}

Kavanagh K, Pollock KG, Potts A, Love J, Cuschieri K, Cubie H, et al. Introduction and sustained high coverage of the HPV bivalent vaccine leads to a reduction in prevalence of HPV $16 / 18$ and closely related HPV types. British Journal of Cancer 2014;110(11):2804-11.

\section{Kavanagh 2017}

Kavanagh K, Pollock KG, Cuschieri K, Palmer T, Cameron RL, Watt $\mathrm{C}$, et al. Changes in the prevalence of human papillomavirus following a national bivalent human papillomavirus vaccination programme in Scotland: a 7-year cross-sectional study. Lancet Infectious Diseases 2017;17(12):1293-302

\section{$\operatorname{Kim} 2010$}

Kim YJ, Kim KT, Kim JH, Cha SD, Kim JW, Bae DS, et al. Vaccination with a human papillomavirus (HPV)-16/18 AS04adjuvanted cervical cancer vaccine in Korean girls aged 10-14 years. Journal of Korean Medical Science 2010;25(8):1197-204.

\section{Kim 2011}

Kim SC, Song YS, Kim YT, Kim YT, Ryu KS, Gunapalaiah B. Human papillomavirus 16/18 AS04-adjuvanted cervical cancer vaccine: immunogenicity and safety in 15-25 years old healthy Korean women. Journal of Gynecologic Oncology 2011;22(2):67-75.

\section{Konno 2010}

Konno R, Tamura S, Dobbelaere K, Yoshikawa H. Efficacy of human papillomavirus 16/18 AS04-adjuvanted vaccine in Japanese women aged 20 to 25 years: interim analysis of a phase 2 double-blind, randomized, controlled trial. International Journal of Gynecological Cancer 2010;20(3):404-10.

\section{Konno 2010a}

Konno R, Tamura S, Dobbelaere K, Yoshikawa H. Efficacy of human papillomavirus type 16/18 AS04-adjuvanted vaccine in Japanese women aged 20 to 25 years: final analysis of a phase 2 double-blind, randomized controlled trial. International Journal of Gynecological Cancer 2010;20(5):847-55. 


\section{Konno 2014}

Konno R, Yoshikawa H, Okutani M, Quint W, Suryakiran V, Lin L, et al. Efficacy of the human papillomavirus (HPV)-16/18 AS04adjuvanted vaccine against cervical intraepithelial neoplasia and cervical infection in young Japanese women. Human Vaccines \& Immunotherapeutics 2014;10(7):1781-94.

\section{Koutsky 2002}

Koutsky LA, Ault KA, Wheeler CM, Brown DR, Barr E, Alvarez FB. A controlled trial of a human papillomavirus type 16 vaccine. New England Journal of Medicine 2002;347:1645-51.

\section{Koutsky 2006}

Koutsky LA, Harper DM. Chapter 13: Current findings from prophylactic HPV vaccine trials. Vaccine 2006;24(Suppl 3):114-21

\section{Kreimer 2011}

Kreimer AR, Rodriguez AC, Hildesheim A, Herrero R, Porras C, Schiffman M, et al. Proof-of-principle evaluation of the efficacy of fewer than three doses of a bivalent HPV16/18 vaccine. Journal of the National Cancer Institute 2011;103(19):1444-51.

\section{Kreimer 2015b}

Kreimer AR, Sherman ME, Sahasrabuddhe VV, Safaeian M. The case for conducting a randomized clinical trial to assess the efficacy of a single dose of prophylactic HPV vaccines among adolescents. Journal of the National Cancer Institute 2015;107(3):dju436.

\section{Kuhs 2014}

Kuhs KA, Porras C, Schiller JT, Rodriguez AC, Schiffman M, Gonzalez P. Effect of different human papillomavirus serological and DNA criteria on vaccine efficacy estimates. American Journal of Epidemiology 2014;180(6):599-607.

\section{Kyrgiou 2017}

Kyrgiou M, Athanasiou A, Kalliala IEJ, Paraskevaidi M, Mitra A, Martin-Hirsch PPL, et al. Obstetric outcomes after conservative treatment for cervical intraepithelial lesions and early invasive disease. Cochrane Database of Systematic Reviews 2017, Issue 11. [DOI: 10.1002/14651858.CD012847]

\section{Lacey 2006}

Lacey CJ, Lowndes CM, Shah KV. Chapter 4: Burden and management of non-cancerous HPV-related conditions: HPV-6/11 disease. Vaccine 2006;24(0264-410X (Print), Suppl 3):35-41.

\section{Larson 2011}

Larson HJ, Cooper LZ, Eskola J, Katz SL, Ratzan S. Addressing the vaccine confidence gap. Lancet 2011;378(9790):526-35.

\section{Lehtinen 2006}

Lehtinen M, Apter D, Dubin G, Kosunen E, Isaksson R, Korpivaara EL. Enrolment of 22,000 adolescent women to cancer registry follow-up for long-term human papillomavirus vaccine efficacy: guarding against guessing. International Journal of STD \& AIDS 2006;17(0956-4624 (Print), 8):517-21.

\section{Lehtinen 2012}

Lehtinen M, Paavonen J, Wheeler CM, Jaisamrarn U, Garland S, Castellsagué X. Overall efficacy of HPV-16/18 ASO4-adjuvanted vaccine against grade 3 or greater cervical intraepithelial neoplasia: 4-year end-of-study analysis of the randomised, double-blind PATRICIA trial. Lancet Oncology 2012;13(1):89-99.

\section{Lehtinen 2013}

Lehtinen M, Dillner J. Clinical trials of human papillomavirus vaccines and beyond. Nature Reviews Clinical Oncology 2013;10(7):400-10.

\section{Leval 2013}

Leval A, Herweijer E, Ploner A, Eloranta S, Fridman Simard J, Dillner J, et al. Quadrivalent human papillomavirus vaccine effectiveness: a Swedish national cohort study. Journal of the National Cancer Institute 2013;105(7):469-74.

\section{Lim 2014}

Lim BK, Ng KY, Omar J, Omar SZ, Gunapalaiah B, Teoh YL, et al. Immunogenicity and safety of the AS04-adjuvanted human papillomavirus-16/18 cervical cancer vaccine in Malaysian women aged 18-35 years: a randomized controlled trial. Medical Journal of Malaysia 2014;69(0300-5283 (Print), 0300-5283 (Linking), 1):2-8.

\section{Lipkind 2017}

Lipkind HS, Vazquez-Benitez G, Nordin JD, Romitti PA, Naleway AL, Klein NP, et al. Maternal and infant outcomes after human papillomavirus vaccination in the periconceptional period or during pregnancy. Obstetrics and Gynecology 2017;130(3):599-608.

\section{Luxembourg 2015}

Luxembourg A, Brown D, Bouchard C, Giuliano AR, Iversen OE, Joura EA, et al. Phase II studies to select the formulation of a multivalent HPV L1 virus-like particle (VLP) vaccine. Human Vaccines \& Immunotherapeutics 2015;11(6):1313-22.

\section{Mao 2006}

Mao C, Koutsky LA, Ault KA, Wheeler CM, Brown DR, Wiley DJ. Efficacy of human papillomavirus-16 vaccine to prevent cervical intraepithelial neoplasia: a randomized controlled trial. Obstetrics and Gynecology 2006;107(1):18-27.

\section{Markowitz 2013}

Markowitz LE, Hariri S, Lin C, Dunne EF, Steinau M, McQuillan G, et al. Reduction in in human papillomavirus (HPV) prevalence among young women following HPVvaccine introduction in the United States, National Health and Nutrition Examination Surveys, 2003-2010. Journal of Infectious Diseases 2013;208(3):385-93.

\section{Martin-Hirsch 2013}

Martin-Hirsch PP, Paraskevaidis E, Bryant A, Dickinson HO. Surgery for cervical intraepithelial neoplasia. Cochrane Database of Systematic Reviews 2013, Issue 12. [DOI: 10.1002/14651858.CD001318.pub3] 


\section{McCredie 2008}

McCredie MR, Sharples KJ, Paul C, Baranyai J, Medley G, Jones RW. Natural history of cervical neoplasia and risk of invasive cancer in women with cervical intraepithelial neoplasia 3: a retrospective cohort study. Lancet Oncology 2008;9(5):425-34.

\section{Medeiros 2009}

Medeiros LR, Rosa DD, Da Rosa MI, Bozzetti MC, Zanini RR. Efficacy of human papillomavirus vaccines: a systematic quantitative review. International Journal of Gynecological Cancer 2009;19(7):1166-76.

\section{Medina 2010}

Medina DM, Valencia A, de Velasquez A, Huang LM, Prymula R, Garcia-Sicilia J. Safety and immunogenicity of the HPV-16/18 AS04-adjuvanted vaccine: a randomized, controlled trial in adolescent girls. Journal of Adolescent Health 2010;46(1879-1972 (Electronic), 1054-139X (Linking), 5):414-21.

\section{Merckx 2015}

Merckx M, Vanden Broeck D, Benoy I, Depuydt C, Weyers S, Arbyn M. Early effects of human papillomavirus vaccination in Belgium. European Journal of Cancer Prevention 2015;24(4):340-2.

\section{Molbak 2016}

Molbak K, Hansen ND, Valentiner-Branth P. Pre-vaccination care-seeking in females reporting severe adverse reactions to HPV vaccine. A registry based case-control study. PLOS One 2016;11(9):e0162520.

\section{Mugo 2015}

Mugo N, Ansah NA, Marino D, Saah A, Garner ElO. Evaluation of safety and immunogenicity of a quadrivalent human papillomavirus vaccine in healthy females between 9 and 26 years of age in Sub-Saharan Africa. Human Vaccines \& Immunotherapeutics 2015;11(6):1323-30.

\section{Munoz 2004}

Munoz N, Bosch FX, Castellsague X, Diaz M, De Sanjose S, Hammouda D. Against which human papillomavirus types shall we vaccinate and screen? The international perspective. International Journal of Cancer 2004;111:278-85.

\section{Munoz 2009}

Munoz N, Manalastas R Jr, Pitisuttithum P, Tresukosol D, Monsonego J, Ault K. Safety, immunogenicity, and efficacy of quadrivalent human papillomavirus (types $6,11,16,18$ ) recombinant vaccine in women aged $24-45$ years: a randomised, double-blind trial. Lancet 2009;373(9679):1949-57.

\section{Munoz 2010}

Munoz N, Kjaer SK, Sigurdsson K, Iversen OE, HernandezAvila M, Wheeler CM. Impact of human papillomavirus (HPV)-6/11/16/18 vaccine on all HPV-associated genital diseases in young women. Journal of the National Cancer Institute 2010;102(5):325-39.

\section{Naleway 2016}

Naleway AL, Crane B, Smith N, Daley MF, Donahue J, Gee J, et al. Absence of venous thromboembolism risk following quadrivalent human papillomavirus vaccination, Vaccine Safety Datalink, 2008-2011. Vaccine 2016;34(1):167-71.

\section{Naud 2014}

Naud PS, Roteli-Martins CM, De Carvalho NS, Teixeira JC, de Borba PC, Sanchez N, et al. Sustained efficacy, immunogenicity, and safety of the HPV-16/18 AS04-adjuvanted vaccine: Final analysis of a long-term follow-up study up to 9.4 years post-vaccination. Human Vaccines \& Immunotherapeutics 2014;10(8):2147-62.

\section{Ngan 2010}

Ngan HY, Cheung AN, Tam KF, Chan KK, Tang HW, Bi D, et al. Human papillomavirus-16/18 AS04-adjuvanted cervical cancer vaccine: immunogenicity and safety in healthy Chinese women from Hong Kong. Hong Kong Medical Journal 2010;16(1024-2708 (Print), 1024-2708 (Linking), 3):171-9.

\section{Noronha 2014}

Noronha AS, Markowitz LE, Dunne EF. Systematic review of human papillomavirus vaccine coadministration. Vaccine 2014;32(23):2670-4.

\section{Ojha 2014}

Ojha RP, Jackson BE, Tota JE, Offutt-Powell TN, Singh KP, Bae S. Guillain-Barré syndrome following quadrivalent human papillomavirus vaccination among vaccine-eligible individuals in the United States. Human Vaccines \& Immunotherapeutics 2014;10(1):232-7.

\section{Ostor 1993}

Ostor AG. Natural history of cervical intraepithelial neoplasia: a critical review. International Journal of Gynegological Pathology 1993;12(2):186-92.

\section{Paavonen 2007}

Paavonen J, Jenkins D, Bosch FX, Naud P, Salmeron J, Wheeler CM. Efficacy of a prophylactic adjuvanted bivalent $L 1$ virus-like-particle vaccine against infection with human papillomavirus types 16 and 18 in young women: an interim analysis of a phase III double-blind, randomised controlled trial. Lancet 2007;369(9580):2161-70.

\section{Paavonen 2009}

Paavonen J, Naud P, Salmeron J, Wheeler CM, Chow S-N, Apter D. Efficacy of human papillomavirus (HPV)-16/18 ASO4adjuvanted vaccine against cervical infection and precancer caused by oncogenic HPV types (PATRICIA): final analysis of a double-blind, randomised study in young women. Lancet 2009;374:301-14.

\section{Pagliusi 2004}

Pagliusi SR, Teresa Aguado M. Efficacy and other milestones for human papillomavirus vaccine introduction. Vaccine 2004;23(0264-410X, 5):569-78. 


\section{Panagiotou 2015}

Panagiotou OA, Befano BL, Gonzalez P, Rodriguez AC, Herrero R, Schiller JT, et al. Effect of bivalent human papillomavirus vaccination on pregnancy outcomes: long term observational follow-up in the Costa Rica HPV Vaccine Trial. BMJ 2015;351:h4358.

\section{Pedersen 2012}

Pedersen C, Breindahl M, Aggarwal N, Berglund J, Oroszlan G, Silfverdal SA. Randomized trial: immunogenicity and safety of coadministered human papillomavirus-16/18AS04-adjuvanted vaccine and combined hepatitis $A$ and $B$ vaccine in girls. Journal of Adolescent Health 2012;50(1):38-46.

\section{Pollock 2014}

Pollock KG, Kavanagh K, Potts A, Love J, Cuschieri K, Cubie H, et al. Reduction of low- and high-grade cervical abnormalities associated with high uptake of the HPV bivalent vaccine in Scotland. British Journal of Cancer 2014;111(9):1261-4.

\section{Rambout 2007}

Rambout L, Hopkins L, Hutton B, Fergusson D. Prophylactic vaccination against human papillomavirus infection and disease in women: a systematic review of randomized controlled trials. Canadian Medical Association Journal 2007;177(5):469-79.

\section{Review Manager 2014 [Computer program]}

Nordic Cochrane Centre, The Cochrane Collaboration. Review Manager 5 (RevMan 5). Version 5.3. Copenhagen: Nordic Cochrane Centre, The Cochrane Collaboration, 2014.

\section{Richart 1973}

Richart RM. Cervical intraepithelial neoplasia. Pathology Annual 1973;8:301-23.

\section{Romanowski 2009}

Romanowski B, de Borba PC, Naud PS, Roteli-Martins CM, De Carvalho NS, Teixeira JC, et al The GlaxoSmithKline Vaccine HPV-007 Study Group. Sustained efficacy and immunogenicity of the human papillomavirus (HPV)-16/18 AS04-adjuvanted vaccine: analysis of a randomised placebo-controlled trial up to 6.4 years. Lancet 2009;374(9706):1975-85.

\section{Romanowski 2011}

Romanowski B. Long term protection against cervical infection with the human papillomavirus: Review of currently available vaccines. Human Vaccines 2011;7(2):161-9.

\section{Ronco 2014}

Ronco G, Dillner J, Elfstrom KM, Tunesi S, Snijders PJ, Arbyn M, et al. Efficacy of HPV-based screening for prevention of invasive cervical cancer: follow-up of four European randomised controlled trials. Lancet 2014;383(9916):524-32.

\section{Rowhani-Rahbar 2009}

Rowhani-Rahbar A, Mao C, Hughes JP, Alvarez FB, Bryan JT, Hawes SE. Longer term efficacy of a prophylactic monovalent human papillomavirus type 16 vaccine. Vaccine 2009;27(41):5612-9.

\section{Safaeian 2018}

Safaeian M, Sampson JN, Pan Y, Porras C, Kemp TJ, Herrero R, et al. Durability of protection afforded by fewer doses of the HPV16/18 vaccine: The CVT Trial. Journal of the National Cancer Institute 2018;110(2):djx158.

\section{Sankaranarayanan 2016}

Sankaranarayanan R, Prabhu PR, Pawlita M, Gheit T, Bhatla N, Muwonge R, et al. Immunogenicity and HPV infection after one, two, and three doses of quadrivalent HPV vaccine in girls in India: a multicentre prospective cohort study. Lancet Oncology 2016;17(1):67-77.

\section{Scheller 2014}

Scheller NM, Pasternak B, Svanstrom H, Hviid A. Quadrivalent human papillomavirus vaccine and the risk of venous thromboembolism. JAMA 2014;312(2):187-8.

\section{Scheller 2015}

Scheller NM, Svanstrom H, Pasternak B, Arnheim-Dahlstrom L, Sundstrom K, Fink K, et al. Quadrivalent HPV vaccination and risk of multiple sclerosis and other demyelinating diseases of the central nervous system. JAMA 2015;313:54-61.

\section{Schiffman 2005}

Schiffman MA, Herrero R, Desalle R, Hildesheim A, Wacholder S, Rodriguez AC, et al. The carcinogenicity of human papillomavirus types reflects viral evolution. Virology 2005;337(1):76-84.

\section{Schiffman 2009}

Schiffman M, Clifford G, Buonaguro FM. Classification of weakly carcinogenic human papillomavirus types: addressing the limits of epidemiology at the borderline. Infectious Agents and Cancer 2009; Vol. 4, issue 1:1-8.

\section{Schiller 2009}

Schiller JT, Lowy DR. Immunogenicity testing in human papillomavirus virus-like-particle vaccine trials. Journal of Infectious Diseases 2009;200(2):166-71.

\section{Schiller 2012}

Schiller JT, Castellsague X, Garland SM. A review of clinical trials of human papillomavirus prophylactic vaccines. Vaccine 2012;30 Suppl 5:F123-38.

\section{Schmeink 2011}

Schmeink CE, Bekkers RL, Josefsson A, Richardus JH, Berndtsson Blom K, David MP, et al. Co-administration of human papillomavirus-16/18 AS04-adjuvanted vaccine with hepatitis $B$ vaccine: randomized study in healthy girls. Vaccine 2011;29(1873-2518):9276-83.

\section{Schneider 2003}

Schneider A, Gissmann L. Cervical cancer. The potential role of human papillomavirus (HPV)-specific vaccines in prevention and treatment. American Journal of Cancer 2003;2:1-253.

\section{Schwarz 2012}

Schwarz TF, Huang LM, Medina DM, Valencia A, Lin TY, Behre U, et al. Four-year follow-up of the immunogenicity and safety of 
the HPV-16/18 AS04-adjuvanted vaccine when administered to adolescent girls aged 10-14 years. Journal of Adolescent Health 2012;50(2):187-94.

\section{Sharp 1998}

Sharp S. Meta-analysis regression. Statistics Technical Bulletin 1998;7(42):148-55. [MEDLINE: 11989]

\section{Skinner 2014}

Skinner SR, Szarewski A, Romanowski B, Garland SM, LazcanoPonce E, Salmeron J. Efficacy, safety, and immunogenicity of the human papillomavirus $16 / 18$ AS04-adjuvanted vaccine in women older than 25 years: 4-year interim follow-up of the phase 3, double-blind, randomised controlled VIVIANE study. Lancet 2014;384(9961):2213-27.

\section{Skufca 2017}

Skufca J, Ollgren J, Ruokokoski E, Lyytikäinen O, Nohynek H. Incidence rates of Guillain-Barré, (GBS), chronic fatigue/ systemic exertion intolerance disease (CFS/SEID) and postural orthostatic tachycardia syndrome (POTS) prior to introduction of human papilloma virus (HPV) vaccination among adolescent girls in Finland, 2002-2012. Papillomavirus Research 2017;3:91-6.

\section{Slade 2009}

Slade BA, Leidel L, Vellozzi C, Woo EJ, Hua W, Sutherland A, et al. Postlicensure safety surveillance for quadrivalent human papillomavirus recombinant vaccine. JAMA 2009;302(7):750-7.

\section{Smith 2000}

Smith HO, Tiffany MF, Qualls CR, Key CR. The rising incidence of adenocarcinoma relative to squamous cell carcinoma of the uterine cervix in the United States-a 24-year population-based study. Gynecologic Oncology 2000; Vol. 78, issue 2:97-105.

\section{Solomon 2002}

Solomon D, Davey D, Kurman R, Moriarty A, O'Connor D, Prey M. The 2001 Bethesda System: terminology for reporting results of cervical cytology. JAMA 2002;287:2114-9.

\section{Sow 2013}

Sow PS, Watson-Jones D, Kiviat N, Changalucha J, Mbaye KD, Brown J. Safety and immunogenicity of human papillomavirus-16/18 AS04-adjuvanted vaccine: a randomized trial in 10-25-year-old HIV-seronegative African girls and young women. Journal of Infectious Diseases 2013;207(11):1753-63.

\section{Stanley 2006}

Stanley MA. Human papillomavirus vaccines. Reviews in Medical Virology 2006;16(1052-9276 (Print), 3):139-49.

\section{Stanley 2012}

Stanley M, Pinto LA, Trimble C. Human papillomavirus vaccines immune responses. Vaccine 2012;30 (Suppl 5):F83-7.

\section{Stanley 2014}

Stanley MA, Sudenga SL, Giuliano AR. Alternative dosage schedules with HPV virus-like particle vaccines. Expert Review of Vaccines 2014;13(8):1027-38.

\section{Starkie Camejo 2016}

Starkie Camejo H, Li X, Van Kriekinge G, Ryser M. Response letter regarding the letter to the editors by Butt et al. Does it matter Discounting and its role in the cost-effectiveness of preventative interventions. The case of HPV vaccination. Public Health 2016;132:110-2.

\section{Szarewski 2010}

Szarewski A. HPV vaccine: Cervarix. Expert Opinion on Biological Therapy 2010;10(3):477-87.

\section{Szarewski 2011}

Szarewski A, Poppe WA, Skinner SR, Wheeler CM, Paavonen J, Naud P. Efficacy of the human papillomavirus (HPV)-16/18 AS04-adjuvanted vaccine in women aged $15-25$ years with and without serological evidence of previous exposure to HPV-16/18. Internaitonal Journal of Cancer 2011;131(1):106-16.

\section{Tabrizi 2012}

Tabrizi SN, Brotherton JM, Kaldor JM, Skinner SR, Cummins E, Liu B, et al. Fall in human papillomavirus prevalence following a national vaccination program. Journal of Infectious Diseases 2012;206(11):1645-51.

\section{Tabrizi 2014}

Tabrizi SN, Brotherton JM, Kaldor JM, Skinner SR, Liu B, Bateson $D$, et al. Assessment of herd immunity and crossprotection after a human papillomavirus vaccination programme in Australia: a repeat cross-sectional study. Lancet Infectious Diseases 2014;14(10):958-66.

\section{Taylor 2016}

Taylor S, Ryser M, Mihalyi A, van Effelterre T. Response letter regarding the letter to the editors by Brown et al. Human Vaccinnes and Immunotherapeutics 2016; Vol. 7:1943-6.

\section{The GSK Study Group 2009}

The GlaxoSmithKline Vaccine HPV-007 Study Group. Sustained efficacy and immunogenicity of the human papillomavirus (HPV)-16/18 ASO4-adjuvanted vaccine: analysis of a randomised placebo-controlled trial up to 6.4 years. Lancet 2009;374:1975-85.

\section{Thompson 1999}

Thompson SG, Sharp SJ. Explaining heterogeneity in metaanalysis: a comparison of methods. Statistics in Medicine 1999;18(20):2693-708. [MEDLINE: 11967]

\section{Unger 2010}

Unger E, Dillner J. Human Papillomavirus Laboratory Manual. 1st Edition. Geneva: WHO (Quality, Safety and Standards (QSS) team of the Department of Immunization, Vaccines and Biologicals), 2010.

\section{Van Damme 2014}

van Damme P, Leroux-Roels G, Simon P, Foidart JM, Donders G, Hoppenbrouwers $\mathrm{K}$, et al. Effects of varying antigens and adjuvant systems on the immunogenicity and safety of investigational tetravalent human oncogenic papillomavirus vaccines: results from two randomized trials. Vaccine 2014;32(29):3694-705. 


\section{Van Damme 2015}

Van Damme P, Olsson SE, Block S, Castellsague X, Gray GE, Herrera T, et al. Immunogenicity and Safety of a 9-Valent HPV Vaccine. Pediatrics 2015;136(1):e28-e39.

\section{Verstraeten 2008}

Verstraeten T, Descamps D, David MP, Zahaf T, Hardt K, Izurieta P, et al. Analysis of adverse events of potential autoimmune aetiology in a large integrated safety database of AS04 adjuvanted vaccines. Vaccine 2008;26(0264-410X (Print), 0264-410X (Linking), 51):6630-8.

\section{Vesikari 2015}

Vesikari T, Brodszki N, Van Damme P, Diez-Domingo J, Icardi G, Kjeld $\mathrm{PL}$, et al. A randomized, double-blind, phase III study of the immunogenicity and safety of a 9-valent human papillomavirus L1 virus-like particle vaccine (V503) versus Gardasil(R) in 9-15-year-old girls. Pediatric Infectious Disease Journal 2015;34(9):992-8.

\section{Vichnin 2015}

Vichnin M, Bonanni P, Klein NP, Garland SM, Block SL, Kjaer SK, et al. An overview of quadrivalent human papillomavirus vaccine safety: 2006 to 2015. Pediatric Infectious Disease Journal 2015;34(9):983-1.

\section{Villa 2005}

Villa LL, Costa RL, Petta CA, Andrade RP, Ault KA, Giuliano AR. Prophylactic quadrivalent human papillomavirus (types 6,11 , 16 , and 18) L1 virus-like particle vaccine in young women: a randomised double-blind placebo-controlled multicentre phase II efficacy trial. Lancet Oncology 2005;6(5):271-8.

\section{Villa 2006}

Villa LL, Ault KA, Giuliano AR, Costa RL, Petta CA, Andrade RP. Immunologic responses following administration of a vaccine targeting human papillomavirus Types $6,11,16$, and 18 . Vaccine 2006;24(27):5571-83.

\section{Villa 2006a}

Villa LL, Costa RL, Petta CA, Andrade RP, Paavonen J, Iversen OE. High sustained efficacy of a prophylactic quadrivalent human papillomavirus types $6 / 11 / 16 / 18 \mathrm{~L} 1$ virus-like particle vaccine through 5 years of follow-up. British Journal of Cancer 2006;95(11):1459-66.

\section{Wacholder 2010}

Wacholder S, Chen BE, Wilcox A, Macones G, Gonzalez P, Befano $B$. Risk of miscarriage with bivalent vaccine against human papillomavirus (HPV) types 16 and 18: pooled analysis of two randomised controlled trials. BMJ 2010;340:c712.

\section{Wheeler 2016}

Wheeler CM, Skinner SR, Del Rosario-Raymundo MR, Garland SM, Chatterjee A, Lazcano-Ponce E, et al. Efficacy, safety, and immunogenicity of the human papillomavirus $16 / 18$ AS04-adjuvanted vaccine in women older than 25 years: 7-year interim follow-up of the phase 3, double-blind, randomised controlled VIVIANE study. Lancet Infectious Diseases 2016;16 (10):1154-68.

\section{WHO 2009}

Initiative for Vaccine Research of the Department of Immunization Vaccines and Biologicals (WHO). Human Papillomavirus (HPV) Vaccine Background Paper. World Health Organization (WHO) 2009:1-249.

\section{WHO 2014}

WHO. Global Advisory Committee on Vaccine Safety Statement on the continued safety of HPV vaccination. http:// www.who.int/vaccine_safety/committee/topics/hpv/GACVS_ Statement_HPV_12_Mar_2014.pdf 2014.

\section{WHO 2016}

WHO. Safety of HPV vaccines (from meeting of 2-3 December, 2015). Weekly Epidemiological Record 2016;91(3):26-8.

\section{Winer 2003}

Winer RL, Lee SK, Hughes JP, Adam DE, Kiviat NB, Koutsky LA. Genital human papillomavirus infection: incidence and risk factors in a cohort of female university students. Journal of Epidemiology 2003;157:218-26.

\section{Winer 2008}

Winer RL, Feng Q, Hughes JP, O'Reilly S, Kiviat NB, Koutsky LA. Risk of female human papillomavirus acquisition associated with first male sex partner. Journal of Infectious Diseases 2008;197(0022-1899 (Print), 2):279-82.

\section{Yoshikawa 2013}

Yoshikawa H, Ebihara K, Tanaka Y, Noda K. Efficacy of quadrivalent human papillomavirus (types 6, 11, 16 and 18) vaccine (GARDASIL) in Japanese women aged 18-26 years. Cancer Science 2013;104(4):465-72.

\section{Zhu 2014}

Zhu FC, Chen W, Hu YM, Hong Y, Li J, Zhang X. Efficacy, immunogenicity and safety of the HPV-16/18 AS04-adjuvanted vaccine in healthy Chinese women aged $18-25$ years: results from a randomized controlled trial. International Journal of Cancer 2014;135(1097-0215 (Electronic), 0020-7136 (Linking), 11):2612-22.

\section{Zhu 2014a}

Zhu F, Li J, Hu Y, Zhang X, Yang X, Zhao H, et al. Immunogenicity and safety of the HPV-16/18 AS04-adjuvanted vaccine in healthy Chinese girls and women aged 9 to 45 years. Human Vaccines \& Immunotherapeutics 2014;10(7):1795-806.

\section{References to other published versions of this review}

\section{Arbyn 2011b}

Arbyn M, Bryant A, Beutels P, Martin-Hirsch PL, Paraskevaidis E, Van Hoof E, et al. Prophylactic vaccination against human papillomaviruses to prevent cervical cancer and its precursors. Cochrane Database of Systematic Reviews 2011, Issue 4. [DOI: 10.1002/14651858.CD009069]

\section{Arbyn 2013}

Arbyn M, Bryant A, Martin-Hirsch PL, Xu L, Simoens C, Markowitz L. Prophylactic vaccination against human 
papillomaviruses to prevent cervical cancer and its precursors. Cochrane Database of Systematic Reviews 2013, Issue 12. [DOI: 10.1002/14651858.CD009069.pub2]

* Indicates the major publication for the study

\section{CHARACTERISTICS OF STUDIES}

\section{Characteristics of included studies [author-defined order]}

\section{Phase2 trial (ph2,1v)}

\begin{tabular}{ll}
\hline Methods & Phase Ilb, randomised, double-blind, placebo-controlled trial \\
\hline Participants & 2392 women (1194 in the vaccine arm and 1198 in the placebo arm) from 16 centres in the USA \\
& Age range: 16 to 23 years \\
& $\begin{array}{l}\text { Inclusion criteria: young women who were HPV16 DNA negative at enrolment and month 7, were HPV16 } \\
\text { seronegative at enrolment, had had no other vaccination } \pm 1 \text { month around each dose. Virgins were en- } \\
\text { rolled if they were seeking contraception }\end{array}$ \\
& Exclusion criteria: pregnancy, history of abnormal Pap smears, more than 5 sexual partners \\
\hline Interventions & $\begin{array}{l}\text { Vaccine: monovalent HPV16 L1 virus-like particles } \\
\text { Placebo: visually indistinguishable aluminium adjuvant placebo }\end{array}$ \\
\hline Outcomes & $\begin{array}{l}\text { Safety, immunogenicity and efficacy (persistent HPV16 infection and histological lesions of CIN } 1+, 2+ \\
\text { and } 3+\text { ) }\end{array}$ \\
\hline Notes & Reports: Koutsky 2002; Mao 2006 and Rowhani-Rahbar 2009 \\
Last report average follow-up time: 8.5 years (Rowhani-Rahbar 2009)
\end{tabular}

\section{Risk of bias}

\begin{tabular}{lll}
\hline Bias & Authors' judgement & Support for judgement \\
\hline $\begin{array}{l}\text { Random sequence genera- } \\
\text { tion (selection bias) }\end{array}$ & Low risk & $\begin{array}{l}\text { Study participants were randomised in a 1:1 ratio to receive vaccine or place- } \\
\text { bo. Permuted blocks were used to ensure similar numbers of participants in } \\
\text { each arm }\end{array}$ \\
\hline
\end{tabular}

\begin{tabular}{|c|c|c|}
\hline $\begin{array}{l}\text { Allocation concealment } \\
\text { (selection bias) }\end{array}$ & Low risk & $\begin{array}{l}\text { Allocation sequence was generated by computer, allocation numbers were as- } \\
\text { signed at each centre. No further details were provided regarding the conceal- } \\
\text { ment of allocation }\end{array}$ \\
\hline
\end{tabular}

Blinding of participants Low risk Participants and study staff were blinded to the group assignments
and personnel (performance bias)

All outcomes

\begin{tabular}{lll}
\hline $\begin{array}{l}\text { Blinding of outcome as- } \\
\text { sessment (detection bias) } \\
\text { All outcomes }\end{array}$ & Low risk & $\begin{array}{l}\text { An independent masked group of } 4 \text { pathologists reviewed the slides without } \\
\text { knowledge of other clinical or laboratory data }\end{array}$ \\
\hline $\begin{array}{l}\text { Incomplete outcome data } \\
\text { (attrition bias) }\end{array}$ & Low risk & $\begin{array}{l}\text { Besides the per-protocol (PP) analysis (HPV16 DNA negative at enrolment and } \\
\text { during vaccination, HPV16 seronegative at enrolment, } 3 \text { doses received, no } \\
\text { protocol violations) also modified-intention-to-treat (MITT-1 [HPV16 DNA neg- } \\
\text { ative and seronegative at enrolment, at least } 1 \text { dose received], MITT-2 (includ- } \\
\text { ing also women being HPV16 DNA positive at enrolment) analyses were per- } \\
\text { formed. Unrestricted susceptible population and ITT analysis done. Exclusions }\end{array}$
\end{tabular}


Phase2 trial (ph2,1v) (Continued)

and reasons for exclusions were described and were balanced over the trial arms.

\begin{tabular}{l}
$\begin{array}{l}\text { Selective reporting (re- Low risk } \\
\text { porting bias) }\end{array}$ \\
\hline
\end{tabular}

Japanese trial (ph2,2v)

\begin{tabular}{ll}
\hline Methods & A phase II randomised, double-blind, controlled multicentre study in Janpan \\
\hline Participants & Participants: 1040 Japanese women (519 in the vaccine arm and 521 in the placebo arm) \\
& Age range: 20 to 25 years \\
& Inclusion criteria: women who were not pregnant, had an intact cervix and use adequate contraception \\
over the vaccination period & $\begin{array}{l}\text { Exclusion criteria: women who had a previous vaccination with HPV vaccine or hepatitis A vaccine, pre- } \\
\text { vious 3-O-desacy l-4'-monophosphoral lipid A administration, hepatitis A infection and various clinical- } \\
\text { ly significant diseases, previous colposcopic examination for cervical cytological abnormality }\end{array}$ \\
\hline Interventions & $\begin{array}{l}\text { Vaccine: bivalent HPV16/18 L1 VLP vaccine } \\
\text { Placebo: Hepatitis A vaccine }\end{array}$ \\
\hline Outcomes & $\begin{array}{l}\text { Safety, immunogenicity, incident \& persistent HPV16/18 infection, cytological (ASCUS+) \& histopatho- } \\
\text { logical abnormalities (CIN1+, CIN2+) associated with vaccine and non-vaccine oncogenic HPV types }\end{array}$ \\
\hline Notes & $\begin{array}{l}\text { Main reports: Konno 2010 and Konno 2010a } \\
\text { Maximum follow-up time: } 24 \text { months (Konno 2010a) } \\
\text { For the outcome high-grade CIN irrespective of types, the follow-up results up to 48 months were used } \\
\text { (Konno 2014) }\end{array}$
\end{tabular}

\section{Risk of bias}

\begin{tabular}{lll}
\hline Bias & Authors' judgement & Support for judgement \\
\hline $\begin{array}{l}\text { Random sequence genera- } \\
\text { tion (selection bias) }\end{array}$ & Unclear risk & $\begin{array}{l}\text { Women were randomised 1:1 to receive the vaccine or placebo. No further de- } \\
\text { tails given }\end{array}$ \\
\hline $\begin{array}{l}\text { Allocation concealment } \\
\text { (selection bias) }\end{array}$ & Unclear risk & Not described in the paper \\
\hline $\begin{array}{l}\text { Blinding of participants } \\
\begin{array}{l}\text { and personnel (perfor- } \\
\text { mance bias) }\end{array}\end{array}$ & Low risk & $\begin{array}{l}\text { Blinding was maintained for all personnel, investigators, study collaborators, } \\
\text { all outcomes }\end{array}$ \\
\hline
\end{tabular}

\begin{tabular}{|c|c|c|}
\hline $\begin{array}{l}\text { Blinding of outcome as- } \\
\text { sessment (detection bias) }\end{array}$ & Low risk & $\begin{array}{l}\text { Blinding can be assumed as covering also the outcome assessment since all in- } \\
\text { vestigators including the statisticians were blinded }\end{array}$ \\
\hline
\end{tabular}

All outcomes

Incomplete outcome data Low risk
(attrition bias)

All outcomes 
Japanese trial (ph2,2v) (Continued)

dose, were DNA negative for HPV vaccine types at month 0; had normal or LSIL cytology at month 0)

\begin{tabular}{l}
$\begin{array}{l}\text { Selective reporting (re- Low risk } \\
\text { porting bias) }\end{array}$ \\
\hline
\end{tabular}

Phase2 trial (ph2,2v)

\begin{tabular}{|c|c|}
\hline Methods & Phase II randomised, multicentre, double-blind placebo-controlled study \\
\hline \multirow[t]{3}{*}{ Participants } & $\begin{array}{l}1113 \text { women ( } 560 \text { in the vaccine arm and } 553 \text { in the placebo arm) from } 32 \text { study sites; } 433 \text { women were } \\
\text { from a Brazilian cohort with longer follow-up) }\end{array}$ \\
\hline & Age range: 15 to 25 years \\
\hline & $\begin{array}{l}\text { Inclusion criteria: healthy women who had had no more than } 6 \text { sexual partners, no history of an abnor- } \\
\text { mal Pap test, no ablative or excisional treatment of the cervix, and no ongoing treatment for external } \\
\text { condylomata; being, at enrolment, cytologically negative, seronegative for HPV16 and HPV18 antibod- } \\
\text { ies by ELISA, and HPV-DNA negative by PCR for } 14 \text { high-risk HPV types }\end{array}$ \\
\hline
\end{tabular}

\begin{tabular}{ll}
\hline Interventions & Vaccine: bivalent HPV16/18 L1 VLP vaccine \\
& Placebo: Hepatitis A vaccine \\
\hline Outcomes & Safety, tolerability, immunogenicity, incident \& persistent HPV infection, cytological (ASC-US+, LSIL+) \\
& \& histopathological abnormalities (CIN1+, CIN2+) associated with vaccine and non-vaccine oncogenic \\
& HPV types
\end{tabular}

Notes Main reports: Harper 2004; Harper 2006; The GSK Study Group 2009 and De Carvalho 2010

Last report average follow-up time: 7.3 years (De Carvalho 2010)

\section{Risk of bias}

\begin{tabular}{|c|c|c|}
\hline Bias & Authors' judgement & Support for judgement \\
\hline $\begin{array}{l}\text { Random sequence genera- } \\
\text { tion (selection bias) }\end{array}$ & Low risk & $\begin{array}{l}\text { Stratified, block randomisation according to validated algorithms was cen- } \\
\text { tralised with an Internet-based randomisation system }\end{array}$ \\
\hline $\begin{array}{l}\text { Allocation concealment } \\
\text { (selection bias) }\end{array}$ & Low risk & $\begin{array}{l}\text { Trial allocation remained concealed from investigators and the women partici- } \\
\text { pating throughout initial and follow-up studies }\end{array}$ \\
\hline $\begin{array}{l}\text { Blinding of participants } \\
\text { and personnel (perfor- } \\
\text { mance bias) } \\
\text { All outcomes }\end{array}$ & Low risk & Double-blinded: trial arms were masked for women and medical personal \\
\hline $\begin{array}{l}\text { Blinding of outcome as- } \\
\text { sessment (detection bias) } \\
\text { All outcomes }\end{array}$ & Low risk & $\begin{array}{l}\text { A central laboratory, reported cytology results...the central histology laborato- } \\
\text { ry made an initial diagnosis from the formalin-fixed tissue specimens for clini- } \\
\text { cal management }\end{array}$ \\
\hline $\begin{array}{l}\text { Incomplete outcome data } \\
\text { (attrition bias) } \\
\text { All outcomes }\end{array}$ & Low risk & $\begin{array}{l}\text { Outcomes are assessed both in the PP group ( } 3 \text { doses received, seronegative } \\
\text { for HPV16/18 at month } 0 \text { and negative for hrHPV DNA at month } 7 \text { ) and in the } \\
\text { ITT group (at least } 1 \text { dose,, seronegative for HPV16/18, negative for hrHPV DNA } \\
\text { at month } 7 \text {, accepting HPV16/18 positive at month } 0 \text {, including also protocol } \\
\text { violations) are shown and reasons for exclusion are presented }\end{array}$ \\
\hline
\end{tabular}


Phase2 trial (ph2,2v) (Continued)

Selective reporting (re- Low risk All outcomes (safety, immunogenicity and efficacy) are presented porting bias)

African_2 country trial (ph3,2v)

\begin{tabular}{ll} 
Methods & Phase IIIb, double-blind, randomised, placebo-controlled, multicentre trial \\
\hline Participants & $\begin{array}{l}\text { Participants: } 676 \text { females ( } 450 \text { in the vaccine arm and } 226 \text { in the placebo arm) enrolled in Senegal or } \\
\text { Tanzania. } \\
\text { Age range: } 10 \text { to } 25 \text { years } \\
\text { Inclusion criteria: healthy HIV-seronegative girls and young women } 10 \text { to } 25 \text { years old at first vaccina- } \\
\text { tion, who were not pregnant and had fewer than } 6 \text { lifetime sexual partners }\end{array}$ \\
\hline Interventions & Vaccine: HPV16/18 bivalent vaccine \\
& Placebo: Al(OH)3 placebo \\
\hline Outcomes & Immunogenicity and safety outcomes \\
\hline Notes & Main report: Sow 2013 \\
& Last report average follow-up time: 12 months
\end{tabular}

\section{Risk of bias}

\begin{tabular}{|c|c|c|}
\hline Bias & Authors' judgement & Support for judgement \\
\hline $\begin{array}{l}\text { Random sequence genera- } \\
\text { tion (selection bias) }\end{array}$ & Low risk & $\begin{array}{l}\text { The randomisation list was computer generated using an Internet-based ran- } \\
\text { domisation blocking scheme }\end{array}$ \\
\hline $\begin{array}{l}\text { Allocation concealment } \\
\text { (selection bias) }\end{array}$ & Low risk & Allocation was concealed until end of the study \\
\hline $\begin{array}{l}\text { Blinding of participants } \\
\text { and personnel (perfor- } \\
\text { mance bias) } \\
\text { All outcomes }\end{array}$ & Low risk & $\begin{array}{l}\text { Investigators, study staff, and participants in each country were blinded to vac- } \\
\text { cine assignment until all participants in that country had completed the } 12- \\
\text { month visit }\end{array}$ \\
\hline $\begin{array}{l}\text { Blinding of outcome as- } \\
\text { sessment (detection bias) } \\
\text { All outcomes }\end{array}$ & Low risk & Outcome assessment was blinded \\
\hline $\begin{array}{l}\text { Incomplete outcome data } \\
\text { (attrition bias) } \\
\text { All outcomes }\end{array}$ & Low risk & $\begin{array}{l}\text { Safety analyses were based on the total vaccinated cohort, with at least one } \\
\text { dose. Immunogenicity analyses were assessed in the PP cohort ( } 3 \text { doses re- } \\
\text { ceived, no protocol violations). The dropout rates were low and balanced be- } \\
\text { tween the vaccine and placebo group }\end{array}$ \\
\hline $\begin{array}{l}\text { Selective reporting (re- } \\
\text { porting bias) }\end{array}$ & Low risk & All intended outcomes reported \\
\hline
\end{tabular}


Chinese trial (ph3,2v)_young

\begin{tabular}{ll}
\hline Methods & Phase II/III randomised, double-blind, controlled trial \\
\hline Participants & $\begin{array}{l}\text { Participants: } 3819 \text { women (3026 in the vaccine arm and } 3025 \text { in the placebo arm) enrolled at four sites } \\
\text { in JiangSu Province } \\
\text { Age range: } 18 \text { to } 25 \text { years } \\
\text { Inclusion criteria: women were agreed to use contraceptive precautions } 30 \text { days before the 1st vaccine } \\
\text { dose and } 2 \text { months after completion of the vaccine series } \\
\text { Exclusion criteria: women who were pregnant or breastfeeding, had an immunosuppressive or immun- } \\
\text { odeficient condition, a history of colposcopy, an allergic disease likely to be exacerbated by any com- } \\
\text { ponent of the vaccine or previously received HPV vaccination or adjuvant were excluded }\end{array}$ \\
\hline Interventions & $\begin{array}{l}\text { Vaccine: bivalent vaccine } \\
\text { Placebo: aluminium hydroxide placebo }\end{array}$ \\
\hline Outcomes & Efficacy (incident and persistent HPV infection, CIN), safety and immunogenicity outcomes \\
\hline Notes & Follow-up of 15 months \\
\hline
\end{tabular}

\section{Risk of bias}

\begin{tabular}{|c|c|c|}
\hline Bias & Authors' judgement & Support for judgement \\
\hline $\begin{array}{l}\text { Random sequence genera- } \\
\text { tion (selection bias) }\end{array}$ & Low risk & $\begin{array}{l}\text { Women were randomised in a 1:1 ratio with an Internet-based centralised ran- } \\
\text { domisation system }\end{array}$ \\
\hline $\begin{array}{l}\text { Allocation concealment } \\
\text { (selection bias) }\end{array}$ & Low risk & $\begin{array}{l}\text { Treatment allocation at the investigation site were using an Internet-based } \\
\text { system }\end{array}$ \\
\hline $\begin{array}{l}\text { Blinding of participants } \\
\text { and personnel (perfor- } \\
\text { mance bias) } \\
\text { All outcomes }\end{array}$ & Low risk & $\begin{array}{l}\text { All participants, investigators and study staff were blinded to individual partic- } \\
\text { ipant treatment assignments and results }\end{array}$ \\
\hline $\begin{array}{l}\text { Blinding of outcome as- } \\
\text { sessment (detection bias) } \\
\text { All outcomes }\end{array}$ & Low risk & $\begin{array}{l}\text { All participants, investigators and study staff were blinded to individual partic- } \\
\text { ipants treatment assignments and results }\end{array}$ \\
\hline $\begin{array}{l}\text { Incomplete outcome data } \\
\text { (attrition bias) } \\
\text { All outcomes }\end{array}$ & Low risk & $\begin{array}{l}\text { All outcomes (safety and immunogenicity) were reported on the total vacci- } \\
\text { nated cohort. Reason for exclusion was noted and balanced between vaccine } \\
\text { group and placebo group }\end{array}$ \\
\hline $\begin{array}{l}\text { Selective reporting (re- } \\
\text { porting bias) }\end{array}$ & Low risk & All outcomes (safety and immunogenicity) were presented \\
\hline
\end{tabular}

Chinese trial (ph3,2v)_adolescent

\begin{tabular}{ll}
\hline Methods & Phase Illb randomised, double-blind, controlled trial \\
\hline Participants & Participants: 750 girls ( 374 in the vaccine arm and 376 in the placebo arm) enrolled in JiangSu Province \\
& Age range: 9 to 17 years
\end{tabular}


Chinese trial (ph3,2v)_ adolescent (Continued)

Inclusion criteria: healthy girls with non-childbearing potential or who were agreed to use contraceptive precautions 30 days before the 1 st vaccine dose and 2 months after completion of the vaccine series; must with written informed consent obtained from the parents

Exclusion criteria: girls who had an immunosuppressive or immunodeficient condition, concurrently participating in another clinical study, hypersensitivity to latex, had an allergic disease likely to be exacerbated by any component of the vaccine or previously received HPV vaccination or adjuvant were excluded

\begin{tabular}{ll}
\hline Interventions & Vaccine: bivalent vaccine \\
& Placebo: aluminium hydroxide placebo \\
\hline Outcomes & Safety and immunogenicity outcomes \\
\hline Notes & Report: Zhu 2014a \\
& Follow-up of 12 months \\
\hline
\end{tabular}

\section{Risk of bias}

\begin{tabular}{lll}
\hline Bias & Authors' judgement & Support for judgement \\
\hline $\begin{array}{l}\text { Random sequence genera- } \\
\text { tion (selection bias) }\end{array}$ & Low risk & $\begin{array}{l}\text { Women were randomised in a 1:1 ratio to receive HPV vaccine or control, using } \\
\text { a central Internet-based randomisation system (see Zhu 2014) }\end{array}$ \\
\hline $\begin{array}{l}\text { Allocation concealment } \\
\text { (selection bias) }\end{array}$ & Unclear risk & Not described in the paper \\
\hline $\begin{array}{l}\text { Blinding of participants } \\
\text { and personnel (perfor- } \\
\text { mance bias) } \\
\text { All outcomes }\end{array}$ & Unclear risk & Not described in the paper \\
\hline
\end{tabular}

\begin{tabular}{lll}
\hline $\begin{array}{l}\text { Blinding of outcome as- } \\
\text { sessment (detection bias) } \\
\text { All outcomes }\end{array}$ & Unclear risk & Not described in the paper \\
\hline $\begin{array}{l}\text { Incomplete outcome data } \\
\begin{array}{l}\text { (attrition bias) } \\
\text { All outcomes }\end{array}\end{array}$ & Low risk & $\begin{array}{l}\text { All outcomes (safety and immunogenicity) were reported on the total vacci- } \\
\text { nated cohort. Reason for exclusion was noted and balanced between vaccine } \\
\text { group and placebo group }\end{array}$
\end{tabular}

Selective reporting (re- Low risk All outcomes (safety and immunogenicity) were presented
porting bias)

Chinese trial (ph3,2v)_mid-adult

\begin{tabular}{|c|c|}
\hline Methods & Phase II/III randomised, double-blind, controlled trial \\
\hline \multirow[t]{3}{*}{ Participants } & $\begin{array}{l}\text { Participants: } 1212 \text { women (606 in the vaccine arm and } 606 \text { in the placebo arm) enrolled in JiangSu } \\
\text { Province }\end{array}$ \\
\hline & Age range: 26 to 45 years \\
\hline & $\begin{array}{l}\text { Inclusion criteria: women were agreed to use contraceptive precautions } 30 \text { days before the } 1 \text { st vaccine } \\
\text { dose and } 2 \text { months after completion of the vaccine series }\end{array}$ \\
\hline
\end{tabular}


Chinese trial (ph3,2v)_mid-adult (Continued)

Exclusion criteria: women who were pregnant or breastfeeding, had an immunosuppressive or immunodeficient condition, a history of colposcopy, an allergic disease likely to be exacerbated by any component of the vaccine or previously received HPV vaccination or adjuvant were excluded

\begin{tabular}{ll}
\hline Interventions & Vaccine: bivalent vaccine \\
& Placebo: HBV vaccine \\
\hline Outcomes & Safety and immunogenicity outcomes \\
\hline Notes & Report: Zhu 2014 a \\
& Follow-up of 12 months
\end{tabular}

\section{Risk of bias}

\begin{tabular}{lll}
\hline Bias & Authors' judgement & Support for judgement \\
\hline $\begin{array}{l}\text { Random sequence genera- } \\
\text { tion (selection bias) }\end{array}$ & Low risk & $\begin{array}{l}\text { Women were randomised in a 1:1 ratio to receive HPV vaccine or control, using } \\
\text { a central internet-based randomisation system (see Zhu 2014) }\end{array}$ \\
\hline $\begin{array}{l}\text { Allocation concealment } \\
\text { (selection bias) }\end{array}$ & Unclear risk & Not described in the paper \\
\hline $\begin{array}{l}\text { Blinding of participants } \\
\begin{array}{l}\text { and personnel (perfor- } \\
\text { mance bias) } \\
\text { All outcomes }\end{array}\end{array}$ & Unclear risk & Not described in the paper \\
\hline
\end{tabular}

\begin{tabular}{|c|c|c|}
\hline $\begin{array}{l}\text { Blinding of outcome as- } \\
\text { sessment (detection bias) } \\
\text { All outcomes }\end{array}$ & Unclear risk & Not described in the paper \\
\hline $\begin{array}{l}\text { Incomplete outcome data } \\
\text { (attrition bias) } \\
\text { All outcomes }\end{array}$ & Low risk & $\begin{array}{l}\text { All outcomes (safety and immunogenicity) were reported on the total vacci- } \\
\text { nated cohort. Reason for exclusion was noted and balanced between vaccine } \\
\text { group and placebo group }\end{array}$ \\
\hline $\begin{array}{l}\text { Selective reporting (re- } \\
\text { porting bias) }\end{array}$ & Low risk & All outcomes (safety and immunogenicity) were presented \\
\hline
\end{tabular}

\section{Co-vaccination_dTpa_IPV trial (ph3,2v)}

\begin{tabular}{|c|c|}
\hline Methods & Randomised, controlled, open, multicentre parallel group study \\
\hline Participants & $\begin{array}{l}\text { Participants: } 751 \text { healthy girls and young women were enrolled in France, Germany and Spain. Partici- } \\
\text { pants were randomised to receive a) HPV vaccine }(n=248) \text {, b) combined Diphtheria-Tetanus-Acellular } \\
\text { Pertussis-inactivated Poliovirus vaccine (dTpa-IPV) together with HPV vaccine at month } 0 \text { and the HPV } \\
\text { vaccine at months } 1 \text { and } 6(n=255) \text { or } c) \text { dTpa-IPV only at month } 0 \text { and HPV vaccine at months } 1,2 \text { and } \\
7(n=248)\end{array}$ \\
\hline
\end{tabular}

Age range: 10 to 18 years

Inclusion criteria: healthy girls and young women who had a negative pregnancy test at the time of each vaccination, not breastfeeding, and if of child-bearing potential, to be abstinent from sexual activity or using adequate contraceptive precautions, should have complete routine childhood vaccinations against diphtheria, tetanus, pertussis, and poliomyelitis 
Co-vaccination_dTpa_IPV trial (ph3,2v) (Continued)

Exclusion criteria: girls who had received diphtheria, tetanus, pertussis vaccine, diphtheria-tetanus booster or dTpa vaccine, and/or oral or inactivated poliovirus vaccine within the previous 5 years; had known exposure to diphtheria or household exposure to pertussis, or diphtheria, tetanus, pertussis, or polio diagnosed within 30 days before vaccination

\begin{tabular}{ll} 
Interventions & Vaccine: bivalent HPV vaccine \\
& Placebo: combined Diphtheria-Tetanus-Acellular Pertussis-inactivated Poliovirus vaccine (dTpa-IPV) \\
\hline Outcomes & Safety and immunogenicity outcomes \\
\hline Notes & Report: Garcia-Sicilia 2010
\end{tabular}

\section{Risk of bias}

\begin{tabular}{lll}
\hline Bias & Authors' judgement & Support for judgement \\
\hline $\begin{array}{l}\text { Random sequence genera- } \\
\text { tion (selection bias) }\end{array}$ & Low risk & $\begin{array}{l}\text { A randomisation list was computer generated using a standard SAS program } \\
\text { at GSK Biological, Rixensart, Belgium }\end{array}$ \\
\hline $\begin{array}{l}\text { Allocation concealment } \\
\text { (selection bias) }\end{array}$ & High risk & $\begin{array}{l}\text { Treatment allocation at the investigator site was performed using a central } \\
\text { randomisation system but not blinded }\end{array}$ \\
\hline $\begin{array}{l}\text { Blinding of participants } \\
\text { and personnel (perfor- } \\
\text { mance bias) } \\
\text { All outcomes }\end{array}$ & Unclear risk & Not described in the paper \\
\hline
\end{tabular}

\begin{tabular}{lll}
\hline $\begin{array}{l}\text { Blinding of outcome as- } \\
\text { sessment (detection bias) } \\
\text { All outcomes }\end{array}$ & Unclear risk & Not described in the paper \\
\hline $\begin{array}{l}\text { Incomplete outcome data } \\
\text { (attrition bias) }\end{array}$ & Low risk & $\begin{array}{l}\text { All safety outcomes were reported for the total vaccinated cohort. Immuno- } \\
\text { gll outcomes }\end{array}$ \\
& & $\begin{array}{l}\text { son for exclusion was noted and balanced between vaccine group and placebo } \\
\text { group }\end{array}$
\end{tabular}

Selective reporting (re- Low risk All outcomes (safety and immunogenicity) were presented
porting bias)

\section{Co-vaccination_HepB trial (ph3, 2v)}

\begin{tabular}{ll}
\hline Methods & Randomised, controlled, open, multicentre parallel group study \\
\hline Participants & $\begin{array}{l}\text { Participants: } 741 \text { girls enrolled at seven centres in the Netherlands and Sweden. Participants were ran- } \\
\text { domised to receive HPV vaccine }(n=247), \text { Hepatitis B vaccine }(n=247) \text { or HPV vaccine co-administrated } \\
\text { with Hepatitis B vaccine }(n=247)\end{array}$
\end{tabular}

Age range: 9 to 15 years

Inclusion criteria: healthy girls who had a negative pregnancy test at the time of each vaccination and if of child-bearing potential, to be abstinent from sexual activity or using adequate contraceptive precautions

Exclusion criteria: girls with a history of hepatitis B infection or with known exposure to hepatitis B within 6 weeks prior to vaccination, girls with previous vaccination against HPV or hepatitis $B$ 
Co-vaccination_HepB trial (ph3, 2v) (Continued)

Interventions Vaccine: bivalent HPV vaccine

Placebo: hepatitis $B$ vaccine

\begin{tabular}{ll}
\hline Outcomes & Safety and immunogenicity outcomes \\
\hline Notes & Report: Schmeink 2011
\end{tabular}

\section{Risk of bias}

\begin{tabular}{|c|c|c|}
\hline Bias & Authors' judgement & Support for judgement \\
\hline $\begin{array}{l}\text { Random sequence genera- } \\
\text { tion (selection bias) }\end{array}$ & Low risk & $\begin{array}{l}\text { A randomisation list was computer generated at GSK Biological, Rixensart, Bel- } \\
\text { gium }\end{array}$ \\
\hline $\begin{array}{l}\text { Allocation concealment } \\
\text { (selection bias) }\end{array}$ & High risk & $\begin{array}{l}\text { This was an open study, the participants and investigators were aware of the } \\
\text { group allocated and vaccines given }\end{array}$ \\
\hline $\begin{array}{l}\text { Blinding of participants } \\
\text { and personnel (perfor- } \\
\text { mance bias) } \\
\text { All outcomes }\end{array}$ & High risk & See above \\
\hline $\begin{array}{l}\text { Blinding of outcome as- } \\
\text { sessment (detection bias) } \\
\text { All outcomes }\end{array}$ & High risk & See above \\
\hline $\begin{array}{l}\text { Incomplete outcome data } \\
\text { (attrition bias) } \\
\text { All outcomes }\end{array}$ & Low risk & $\begin{array}{l}\text { All safety outcomes were reported for the total vaccinated cohort. Immuno- } \\
\text { genicity outcomes were reported on the according-to-protocol cohort. Rea- } \\
\text { son for exclusion was noted and balanced between vaccine group and placebo } \\
\text { group }\end{array}$ \\
\hline $\begin{array}{l}\text { Selective reporting (re- } \\
\text { porting bias) }\end{array}$ & Low risk & All outcomes (safety and immunogenicity) were presented \\
\hline
\end{tabular}

Co-vaccination_HAB trial $(\mathrm{Ph} 3,2 \mathrm{v})$

\begin{tabular}{|c|c|}
\hline Methods & Randomised, controlled, open, multicentre parallel group study \\
\hline \multirow[t]{4}{*}{ Participants } & $\begin{array}{l}\text { Participants: } 813 \text { girls enrolled in Canada, Denmark, Hungary and Sweden. Participants were ran- } \\
\text { domised to receive HPV vaccine }(n=270) \text {, Hepatitis A and B vaccine }(n=271) \text { or HPV vaccine co-admin- } \\
\text { istrated with Hepatitis A and B vaccine }(n=272)\end{array}$ \\
\hline & Age range: 9 to 15 years \\
\hline & $\begin{array}{l}\text { Inclusion criteria: healthy girls with a negative pregnancy test at the time of each vaccination and if of } \\
\text { child-bearing potential, to be abstinent from sexual activity or using adequate contraceptive precau- } \\
\text { tions }\end{array}$ \\
\hline & $\begin{array}{l}\text { Exclusion criteria: girls with a history of hepatitis and or B infection or with known exposure to hepatitis } \\
\text { A or B within } 6 \text { weeks prior to vaccination, girls with previous vaccination against HPV or hepatitis A or } \\
\text { B, or planned administration of HPV, hepatitis A or B or non routine vaccines not foreseen by the study } \\
\text { protocol were excluded }\end{array}$ \\
\hline
\end{tabular}

Interventions Vaccine: bivalent vaccine

Placebo: GSK combined hepatitis A and B vaccine 
Co-vaccination_HAB trial (Ph3, 2v) (Continued)

\begin{tabular}{ll} 
Outcomes & Safety and immunogenicity outcomes \\
\hline Notes & Report: Pedersen 2012
\end{tabular}

\section{Risk of bias}

\begin{tabular}{|c|c|c|}
\hline Bias & Authors' judgement & Support for judgement \\
\hline $\begin{array}{l}\text { Random sequence genera- } \\
\text { tion (selection bias) }\end{array}$ & Low risk & A randomisation list was computer generated at GSK Biological, Rixensart \\
\hline $\begin{array}{l}\text { Allocation concealment } \\
\text { (selection bias) }\end{array}$ & Unclear risk & Not described in the paper \\
\hline $\begin{array}{l}\text { Blinding of participants } \\
\text { and personnel (perfor- } \\
\text { mance bias) } \\
\text { All outcomes }\end{array}$ & Unclear risk & $\begin{array}{l}\text { Pesonnel performing serological assays were blinded to group assignment. } \\
\text { Not mentioned for safety outcome investigator. }\end{array}$ \\
\hline $\begin{array}{l}\text { Blinding of outcome as- } \\
\text { sessment (detection bias) } \\
\text { All outcomes }\end{array}$ & Unclear risk & See above \\
\hline $\begin{array}{l}\text { Incomplete outcome data } \\
\text { (attrition bias) } \\
\text { All outcomes }\end{array}$ & Low risk & $\begin{array}{l}\text { All safety outcomes were reported for the total vaccinated cohort. Immuno- } \\
\text { genicity outcomes were reported for the according-to protocol cohort. Rea- } \\
\text { son for exclusion was noted and balanced between vaccine group and placebo } \\
\text { group }\end{array}$ \\
\hline $\begin{array}{l}\text { Selective reporting (re- } \\
\text { porting bias) }\end{array}$ & Low risk & All safety outcomes were presented \\
\hline
\end{tabular}

\section{CVT (ph3,2v)}

Methods Phase III randomised, double-blind, controlled trial

Participants
Age: 18 to 25 years
Inlcusion criteria: healthy women who were not pregnant, not breastfeeding and using contraception
during the vaccine period. Women were enrolled regardless of past sexual behavior, HPV status, or cy-
tology.
Exclusion criteria: women were excluded if they had history of chronic diseases, history of reactions to
vaccines and history of hepatitis A vaccination

Placebo: Hepatitis A vaccine-licensed Havrix vaccine

\begin{tabular}{ll}
\hline Outcomes & Vaccine efficacy (persistent infection 6M \& 12M), cross-protection and pregnancy outcomes \\
\hline Notes & Main report: Herrero 2011. \\
& Last report average follow-up time: 50.4 months \\
\hline
\end{tabular}


CVT (ph3,2v) (Continued)

Risk of bias

\begin{tabular}{|c|c|c|}
\hline Bias & Authors' judgement & Support for judgement \\
\hline $\begin{array}{l}\text { Random sequence genera- } \\
\text { tion (selection bias) }\end{array}$ & Low risk & $\begin{array}{l}\text { HPV vaccines and placebo were assigned random vaccine identification num- } \\
\text { bers at the time of labelling by the manufacturer. These numbers were ran- } \\
\text { domised by the study Data Management Centre with a standard SAS program }\end{array}$ \\
\hline $\begin{array}{l}\text { Allocation concealment } \\
\text { (selection bias) }\end{array}$ & Low risk & $\begin{array}{l}\text { Codes were kept at the study's data management centre and GSK under con- } \\
\text { trolled and secured access }\end{array}$ \\
\hline $\begin{array}{l}\text { Blinding of participants } \\
\text { and personnel (perfor- } \\
\text { mance bias) } \\
\text { All outcomes }\end{array}$ & Low risk & $\begin{array}{l}\text { All field workers were blinded to group assignment; as well as investigators } \\
\text { from the USA and Costa Rica, participants, and medical monitors }\end{array}$ \\
\hline $\begin{array}{l}\text { Blinding of outcome as- } \\
\text { sessment (detection bias) } \\
\text { All outcomes }\end{array}$ & Low risk & $\begin{array}{l}\text { Analyses were conducted by an external group (Information Management Sys- } \\
\text { tems) under the direction of the investigators who remain masked to individu- } \\
\text { als' randomisation }\end{array}$ \\
\hline $\begin{array}{l}\text { Incomplete outcome data } \\
\text { (attrition bias) } \\
\text { All outcomes }\end{array}$ & Low risk & $\begin{array}{l}\text { Outcomes are assessed in the PP cohort ( } 3 \text { doses received, HPV16/18 DNA neg- } \\
\text { ative at enrolment, no biopsy or LEEP, no protocol violations) and in the ITT } \\
\text { cohort were assessed }\end{array}$ \\
\hline $\begin{array}{l}\text { Selective reporting (re- } \\
\text { porting bias) }\end{array}$ & Low risk & Efficacy, cross-protection pregnancy and other safety outcomes were reported \\
\hline
\end{tabular}

Hong Kong trial (ph3,2v)

\begin{tabular}{ll}
\hline Methods & Phase III, double-blind, randomised controlled trial \\
\hline Participants & 294 women (148 in the vaccine arm and 146 in the placebo arm) from Hong Kong \\
& Age range: women aged 18 to 35 years. \\
& Inclusion criteria: women who were healthy \\
& Exclusion criteria: women who were receiving any investigational or non-registered drug or vaccine, \\
those who had received AS04-adjuvant or HPV vaccine, those having a chronic disease or were preg- \\
nant, breastfeeding or planning to conceive were excluded
\end{tabular}

\begin{tabular}{|c|c|c|}
\hline Interventions & \multirow{2}{*}{\multicolumn{2}{|c|}{$\begin{array}{l}\text { Vaccine: HPV16/18 AS04-adjuvant bivalent vaccine } \\
\text { Placebo: visually indistinguishable aluminium-containing placebo }\end{array}$}} \\
\hline & & \\
\hline Outcomes & \multicolumn{2}{|c|}{ Safety and immunogenicity } \\
\hline Notes & \multicolumn{2}{|c|}{ Last report average follow-up time: 7 months (Hong Kong trial (ph3,2v)) } \\
\hline \multicolumn{3}{|l|}{ Risk of bias } \\
\hline Bias & Authors' judgement & Support for judgement \\
\hline $\begin{array}{l}\text { Random sequence genera- } \\
\text { tion (selection bias) }\end{array}$ & Low risk & $\begin{array}{l}\text { Women were randomised in a 1:1 ratio with an Internet-based centralised ran- } \\
\text { domisation system }\end{array}$ \\
\hline
\end{tabular}


Hong Kong trial (ph3,2v) (Continued)

\begin{tabular}{l}
$\begin{array}{l}\text { Allocation concealment } \\
\text { (selection bias) }\end{array} \quad \begin{array}{l}\text { A single treatment number was used for each patient uniquely identify the } \\
\text { doses administered to the participant }\end{array}$ \\
\hline
\end{tabular}

\begin{tabular}{|c|c|c|}
\hline $\begin{array}{l}\text { Blinding of participants } \\
\text { and personnel (perfor- } \\
\text { mance bias) } \\
\text { All outcomes }\end{array}$ & Unclear risk & Not described in the paper \\
\hline $\begin{array}{l}\text { Blinding of outcome as- } \\
\text { sessment (detection bias) } \\
\text { All outcomes }\end{array}$ & Unclear risk & Not described in the paper \\
\hline $\begin{array}{l}\text { Incomplete outcome data } \\
\text { (attrition bias) } \\
\text { All outcomes }\end{array}$ & Low risk & $\begin{array}{l}\text { All outcomes (safety and immunogenicity) were reported on the total vacci- } \\
\text { nated cohort. Reason for exclusion was noted and balanced between vaccine } \\
\text { group and placebo group }\end{array}$ \\
\hline
\end{tabular}

Selective reporting (re- Low risk All outcomes (safety and immunogenicity) were presented

porting bias)

Immunobridging(ph3,2v)

\begin{tabular}{ll}
\hline Methods & Phase III, observer-blind, randomised, controlled and multicentre trial \\
\hline Participants & 2067 women (1035 in the vaccine arm and 1032 in the placebo arm) recruited from Australia, Colombia, \\
the Czech Republic, France etc. \\
Age range: women aged 10 to 14 years \\
Inclusion criteria: girls who were healthy, were not excluded based on HPV status, Pap smear history or \\
history of sexual activity \\
Exclusion criteria: girls were excluded if they had immunodeficiency, history of allergic disease likely to \\
be exacerbated by a vaccine component, known acute or chronic clinically significant neurologic, he- \\
patic, or renal functional abnormality
\end{tabular}

Interventions Vaccine: HPV16/18 AS04-adjuvant bivalent vaccine

Placebo: Hepatitis A vaccine, appearance of the vaccine is different

\begin{tabular}{ll}
\hline Outcomes & Safety and immunogenicity \\
\hline Notes & Last report average follow-up time: 12 months (Medina 2010) \\
\hline
\end{tabular}

\section{Risk of bias}

\begin{tabular}{lll}
\hline Bias & Authors' judgement & Support for judgement \\
\hline $\begin{array}{l}\text { Random sequence genera- } \\
\text { tion (selection bias) }\end{array}$ & Low risk & $\begin{array}{l}\text { Girls were randomised in 1:1 ratio based on an algorithm accounted for study } \\
\text { centre and age strata }\end{array}$ \\
\hline $\begin{array}{l}\text { Allocation concealment } \\
\text { (selection bias) }\end{array}$ & High risk & $\begin{array}{l}\text { Allocation was not blinded since the HPV vaccine and the control vaccine (He- } \\
\text { patitis A) were different in appearance }\end{array}$ \\
\hline $\begin{array}{l}\text { Blinding of participants } \\
\text { and personnel (perfor- } \\
\text { mance bias) }\end{array}$ & Low risk & $\begin{array}{l}\text { Because of differences in vaccines appearance, study staff who administered } \\
\text { them were not otherwise involved in study conduct; } \\
\text { participants and staff involved in assessment remained blinded }\end{array}$ \\
\hline
\end{tabular}


Immunobridging(ph3,2v) (Continued)

All outcomes

\begin{tabular}{|c|c|c|}
\hline $\begin{array}{l}\text { Blinding of outcome as- } \\
\text { sessment (detection bias) }\end{array}$ & Low risk & $\begin{array}{l}\text { The study staff involved in the assessment of outcomes remained blinded to } \\
\text { the administered vaccine }\end{array}$ \\
\hline
\end{tabular}

All outcomes

\begin{tabular}{|c|c|c|}
\hline $\begin{array}{l}\text { Incomplete outcome data } \\
\text { (attrition bias) } \\
\text { All outcomes }\end{array}$ & Low risk & $\begin{array}{l}\text { All outcomes (safety and immunogenicity) were reported on the total vacci- } \\
\text { nated cohort. Reasons for exclusion were noted and balanced between the } \\
\text { vaccine group and placebo group }\end{array}$ \\
\hline
\end{tabular}

Selective reporting (re- Low risk $\quad$ All outcomes (safety and immunogenicity) were presented
porting bias)

porting bias)

Indian trial (ph3,2v)

\begin{tabular}{|c|c|}
\hline Methods & Phase III, double-blind, randomised, controlled and multicentre trial \\
\hline \multirow[t]{3}{*}{ Participants } & 354 women (176 in the vaccine arm and 178 in the placebo arm) from Hong Kong \\
\hline & Age range: women aged 18 to 35 years \\
\hline & $\begin{array}{l}\text { Inclusion criteria: healthy women not taking any other investigational products or steroids and not } \\
\text { pregnant or planning to become pregnant }\end{array}$ \\
\hline \multirow[t]{2}{*}{ Interventions } & Vaccine: HPV16/18 AS04-adjuvant bivalent vaccine \\
\hline & Placebo: visually indistinguishable aluminium-containing placebo \\
\hline Outcomes & Safety and immunogenicity \\
\hline Notes & Last report average follow-up time: 7 months (Indian trial (ph3,2v)) \\
\hline
\end{tabular}

\section{Risk of bias}

\begin{tabular}{|c|c|c|}
\hline Bias & Authors' judgement & Support for judgement \\
\hline $\begin{array}{l}\text { Random sequence genera- } \\
\text { tion (selection bias) }\end{array}$ & Low risk & Women were randomised in a 1:1 ratio with SAS analysis system \\
\hline $\begin{array}{l}\text { Allocation concealment } \\
\text { (selection bias) }\end{array}$ & Low risk & $\begin{array}{l}\text { Throughout the study, a single treatment number was used to uniquely identi- } \\
\text { fy the vaccine doses to be given to the same participant }\end{array}$ \\
\hline $\begin{array}{l}\text { Blinding of participants } \\
\text { and personnel (perfor- } \\
\text { mance bias) } \\
\text { All outcomes }\end{array}$ & Unclear risk & Not described in the paper \\
\hline $\begin{array}{l}\text { Blinding of outcome as- } \\
\text { sessment (detection bias) } \\
\text { All outcomes }\end{array}$ & Unclear risk & Not described in the paper \\
\hline $\begin{array}{l}\text { Incomplete outcome data } \\
\text { (attrition bias) } \\
\text { All outcomes }\end{array}$ & Low risk & $\begin{array}{l}\text { All outcomes (safety and immunogenicity) were reported on the total vacci- } \\
\text { nated cohort. Reason for exclusion was noted and balanced between vaccine } \\
\text { group and placebo group }\end{array}$ \\
\hline
\end{tabular}


Indian trial (ph3,2v) (Continued)

Selective reporting (re- Low risk $\quad$ All outcomes (safety and immunogenicity) were presented porting bias)

Korean trial $(\mathrm{ph} 3,2 \mathrm{v})$

\begin{tabular}{|c|c|}
\hline Methods & Phase III randomised, double-blind, placebo-controlled trial \\
\hline \multirow[t]{4}{*}{ Participants } & Participants: 321 females (160 in the vaccine arm and 161 in the placebo arm) \\
\hline & Age range: 10 to 14 years. \\
\hline & $\begin{array}{l}\text { Inclusion criteria: include healthy Korean women who were using no other investigational products or } \\
\text { immune-modifying drugs, not pregnant or planning to become pregnant, not breastfeeding during the } \\
\text { study. Use effective contraception or abstinent from sexual relations }\end{array}$ \\
\hline & Exclusion criteria: women who had received previous HPV vaccination \\
\hline \multirow[t]{2}{*}{ Interventions } & Vaccine: HPV16/18 bivalent vaccine \\
\hline & Placebo: hepatitis A vaccine \\
\hline Outcomes & Immunogenicity and safety outcomes \\
\hline \multirow[t]{2}{*}{ Notes } & Main report: Kim 2010; \\
\hline & Last report average follow-up time: 7 months \\
\hline
\end{tabular}

\section{Risk of bias}

\begin{tabular}{|c|c|c|}
\hline Bias & Authors' judgement & Support for judgement \\
\hline $\begin{array}{l}\text { Random sequence genera- } \\
\text { tion (selection bias) }\end{array}$ & Low risk & $\begin{array}{l}\text { Participants were randomly allocated to two groups in a 1:1 ratio using an In- } \\
\text { ternet-based randomisation system }\end{array}$ \\
\hline $\begin{array}{l}\text { Allocation concealment } \\
\text { (selection bias) }\end{array}$ & Low risk & $\begin{array}{l}\text { Syringes were prepared and administered by qualified medical personnel not } \\
\text { otherwise involved in the study }\end{array}$ \\
\hline $\begin{array}{l}\text { Blinding of participants } \\
\text { and personnel (perfor- } \\
\text { mance bias) } \\
\text { All outcomes }\end{array}$ & Low risk & See above \\
\hline $\begin{array}{l}\text { Blinding of outcome as- } \\
\text { sessment (detection bias) } \\
\text { All outcomes }\end{array}$ & Low risk & $\begin{array}{l}\text { The assessment of symptoms were conducted by personnel not involved in } \\
\text { study }\end{array}$ \\
\hline $\begin{array}{l}\text { Incomplete outcome data } \\
\text { (attrition bias) } \\
\text { All outcomes }\end{array}$ & Low risk & $\begin{array}{l}\text { All outcomes (safety and immunogenicity) were reported on the total vacci- } \\
\text { nated cohort. Reason for exclusion was noted and balanced between vaccine } \\
\text { group and placebo group }\end{array}$ \\
\hline $\begin{array}{l}\text { Selective reporting (re- } \\
\text { porting bias) }\end{array}$ & Low risk & All outcomes (safety and immunogenicity) were presented \\
\hline
\end{tabular}


Korean trial (ph3b,2v)

\begin{tabular}{|c|c|}
\hline Methods & Phase IIIb randomised, double-blind, placebo-controlled, multicentre trial \\
\hline \multirow[t]{4}{*}{ Participants } & Participants: 208 women (149 in the vaccine arm and 76 in the placebo arm) \\
\hline & Age range: 15 to 25 years \\
\hline & $\begin{array}{l}\text { Inclusion criteria: include healthy Korean women who were not pregnant and agreed to use effective } \\
\text { contraception during the vaccination period }\end{array}$ \\
\hline & $\begin{array}{l}\text { Exclusion criteria: women who were used investigational or non-registered drug or vaccines, who had a } \\
\text { history of HPV vaccination, a history of chronic diseases or cancer were also excluded from the study }\end{array}$ \\
\hline \multirow[t]{2}{*}{ Interventions } & Vaccine: HPV16/18 bivalent vaccine \\
\hline & Placebo: hepatitis A vaccine \\
\hline Outcomes & Immunogenicity and safety outcomes \\
\hline \multirow[t]{2}{*}{ Notes } & Main report: Kim 2011; \\
\hline & Last report average follow-up time: 7 months \\
\hline
\end{tabular}

\section{Risk of bias}

\begin{tabular}{|c|c|c|}
\hline Bias & Authors' judgement & Support for judgement \\
\hline $\begin{array}{l}\text { Random sequence genera- } \\
\text { tion (selection bias) }\end{array}$ & Low risk & $\begin{array}{l}\text { Women were randomised in a 2:1 ratio to vaccine or placebo. Random alloca- } \\
\text { tion was done with standard statistical analysis system program applying an } \\
\text { Internet-based 2:1 blocking scheme }\end{array}$ \\
\hline $\begin{array}{l}\text { Allocation concealment } \\
\text { (selection bias) }\end{array}$ & Low risk & $\begin{array}{l}\text { A single treatment number was utilised in the entire study to identify the doses } \\
\text { to be administered to the participant }\end{array}$ \\
\hline $\begin{array}{l}\text { Blinding of participants } \\
\text { and personnel (perfor- } \\
\text { mance bias) } \\
\text { All outcomes }\end{array}$ & Low risk & $\begin{array}{l}\text { All participants and study personnel involved in the study were blinded } \\
\text { throughout the study until the last participant and last visit and the database } \\
\text { was frozen }\end{array}$ \\
\hline $\begin{array}{l}\text { Blinding of outcome as- } \\
\text { sessment (detection bias) } \\
\text { All outcomes }\end{array}$ & Low risk & See above \\
\hline $\begin{array}{l}\text { Incomplete outcome data } \\
\text { (attrition bias) } \\
\text { All outcomes }\end{array}$ & Low risk & $\begin{array}{l}\text { Immunogenecity was assessed in the PP-cohort (initially seronegative women) } \\
\text { and in the TVC (at least one dose, all participants randomised); safety was as- } \\
\text { sessed in the TVC }\end{array}$ \\
\hline $\begin{array}{l}\text { Selective reporting (re- } \\
\text { porting bias) }\end{array}$ & Low risk & All intended outcomes were reported \\
\hline
\end{tabular}

\section{Malaysian trial (ph3,2v)}

\begin{tabular}{ll}
\hline Methods & Phase IIIb, double-blind, randomised controlled trial \\
\hline Participants & 271 women (135 in the vaccine arm and 136 in the placebo arm) from Malaysia \\
& Age range: women aged 18 to 35 years
\end{tabular}


Malaysian trial (ph3,2v) (Continued)

Inclusion criteria: women who were healthy

Exclusion criteria: women who had HPV vaccine, chronic use of immunosuppressants, history of allergy to vaccine compounds, history of chronic conditions of cancer and autoimmune disease, acute disease, pregnant

Interventions Vaccine: HPV16/18 AS04-adjuvant bivalent vaccine

Placebo: aluminium hydroxide as placebo

\begin{tabular}{ll}
\hline Outcomes & Safety and immunogenicity \\
\hline Notes & Report: Lim 2014 \\
& Last report average follow-up time: 7 months after first dose \\
\hline
\end{tabular}

\section{Risk of bias}

\begin{tabular}{|c|c|c|}
\hline Bias & Authors' judgement & Support for judgement \\
\hline $\begin{array}{l}\text { Random sequence genera- } \\
\text { tion (selection bias) }\end{array}$ & Low risk & $\begin{array}{l}\text { Women were randomised in a 1:1 ratio with an Internet-based centralised ran- } \\
\text { domisation system }\end{array}$ \\
\hline $\begin{array}{l}\text { Allocation concealment } \\
\text { (selection bias) }\end{array}$ & Low risk & $\begin{array}{l}\text { A single treatment number was used for each patient uniquely identify the } \\
\text { doses administered to the participant }\end{array}$ \\
\hline $\begin{array}{l}\text { Blinding of participants } \\
\text { and personnel (perfor- } \\
\text { mance bias) } \\
\text { All outcomes }\end{array}$ & Unclear risk & Not described in the paper \\
\hline $\begin{array}{l}\text { Blinding of outcome as- } \\
\text { sessment (detection bias) } \\
\text { All outcomes }\end{array}$ & Unclear risk & Not described in the paper \\
\hline $\begin{array}{l}\text { Incomplete outcome data } \\
\text { (attrition bias) } \\
\text { All outcomes }\end{array}$ & Low risk & $\begin{array}{l}\text { All outcomes (safety and immunogenicity) were reported on the total vacci- } \\
\text { nated cohort. Reason for exclusion was noted and balanced between vaccine } \\
\text { group and placebo group }\end{array}$ \\
\hline $\begin{array}{l}\text { Selective reporting (re- } \\
\text { porting bias) }\end{array}$ & Low risk & All outcomes (safety and immunogenicity) were presented \\
\hline
\end{tabular}

\section{PATRICIA trial (ph3,2v)}

\begin{tabular}{|c|c|}
\hline Methods & Phase III randomised, double-blind, controlled trial \\
\hline \multirow[t]{4}{*}{ Participants } & $\begin{array}{l}18,644 \text { women (9319 in the vaccine arm and } 9325 \text { in the placebo arm) enrolled for the study from } 135 \\
\text { centres in } 14 \text { countries in Asia, Pacific, Europe, Latin America and North America }\end{array}$ \\
\hline & Age range: 15 to 25 years \\
\hline & $\begin{array}{l}\text { Inclusion criteria: women who reported no more than six lifetime sexual partners before study enrol- } \\
\text { ment, agreed to using adequate contraception over the vaccination period, and had an intact cervix } \\
\text { were eligible. Enrolled irrespective of their HPV DNA status, HPV serostatus or cytology at baseline }\end{array}$ \\
\hline & $\begin{array}{l}\text { Exclusion criteria: women were excluded if they had a history of colposcopy, were pregnant or breast- } \\
\text { feeding, or had chronic or autoimmune disease or immunodeficiency }\end{array}$ \\
\hline
\end{tabular}


PATRICIA trial (ph3,2v) (Continued)
Interventions
Vaccine: HPV16/18 AS04-adjuvant bivalent vaccine
Placebo: Hepatitis A vaccine-licensed Havrix vaccine
Outcomes
Safety, immunogenicity, efficacy (incident infection, persistent infection, $\mathrm{CIN1+}$, CIN2+, CIN3+, AIS asso- ciated with HPV16, HPV18, HPV16/18, other oncogenic HPV types, irrespective of HPV DNA) and cross- protection

Notes

Main reports: Paavonen 2007; Paavonen 2009; Szarewski 2011 and Lehtinen 2012.

Last report average follow-up time: 34.9 months (Lehtinen 2012)

\section{Risk of bias}

\begin{tabular}{|c|c|c|}
\hline Bias & Authors' judgement & Support for judgement \\
\hline $\begin{array}{l}\text { Random sequence genera- } \\
\text { tion (selection bias) }\end{array}$ & Low risk & $\begin{array}{l}\text { Women were randomised in a 1:1 ratio with an Internet-based centralised ran- } \\
\text { domisation system }\end{array}$ \\
\hline $\begin{array}{l}\text { Allocation concealment } \\
\text { (selection bias) }\end{array}$ & Low risk & $\begin{array}{l}\text { Allocation of treatment numbers was stratified by study site and by age.The tri- } \\
\text { al remained double-blinded until all individuals had completed } 48 \text { months of } \\
\text { follow-up after the first immunisation }\end{array}$ \\
\hline $\begin{array}{l}\text { Blinding of participants } \\
\text { and personnel (perfor- } \\
\text { mance bias) } \\
\text { All outcomes }\end{array}$ & Low risk & Enrolled women and study investigators were masked to allocated vaccine \\
\hline $\begin{array}{l}\text { Blinding of outcome as- } \\
\text { sessment (detection bias) } \\
\text { All outcomes }\end{array}$ & Low risk & $\begin{array}{l}\text { All CIN cases were reviewed by a panel of three pathologists who were blind- } \\
\text { ed to vaccine allocation. Analysis was done by an independent statistician to } \\
\text { maintain the trial blinding }\end{array}$ \\
\hline $\begin{array}{l}\text { Incomplete outcome data } \\
\text { (attrition bias) } \\
\text { All outcomes }\end{array}$ & Low risk & $\begin{array}{l}\text { Outcomes are assessed in the PP cohort (received } 3 \text { doses, seronegative and } \\
\text { DNA negative for the corresponding vaccine type at month 0, normal or low- } \\
\text { grade cytology at month 0, no protocol violations) and in the TVC-naive cohort } \\
\text { (at least one vaccine dose, at baseline normal cytology, DNA negative for hrH- } \\
\text { PV, seronegative for HPV-16 and HPV-18) and in the total vaccinated cohort (all } \\
\text { women randomised). Reasons for exclusion were presented }\end{array}$ \\
\hline $\begin{array}{l}\text { Selective reporting (re- } \\
\text { porting bias) }\end{array}$ & Low risk & $\begin{array}{l}\text { All outcomes (safety, immunogenicity, efficacy and cross-protection) are pre- } \\
\text { sented }\end{array}$ \\
\hline
\end{tabular}

\section{PATRICIA \& CVT (ph3,2v)}

\begin{tabular}{ll}
\hline Methods & Pooled analysis of two phase III randomised, double-blind, controlled trials \\
\hline Participants & 26,130 women who enrolled for PATRICIA trial and Costa Rica trial \\
\hline Interventions & Vaccine: HPV16/18 AS04-adjuvant bivalent vaccine \\
& Placebo: Hepatitis A vaccine-licensed Havrix vaccine \\
\hline Outcomes & Pregnancy outcomes \\
\hline Notes & Main report: Wacholder 2010
\end{tabular}


PATRICIA \& CVT (ph3,2v) (Continued)

Risk of bias

\begin{tabular}{lll}
\hline Bias & Authors' judgement & Support for judgement \\
\hline $\begin{array}{l}\text { Random sequence genera- } \\
\text { tion (selection bias) }\end{array}$ & Low risk & see PATRICIA \& Costa Rica trials \\
\hline $\begin{array}{l}\text { Allocation concealment } \\
\text { (selection bias) }\end{array}$ & Low risk & see PATRICIA \& Costa Rica trials \\
\hline $\begin{array}{l}\text { Blinding of participants } \\
\text { and personnel (perfor- } \\
\text { mance bias) } \\
\text { All outcomes }\end{array}$ & Low risk & see PATRICIA \& Costa Rica trials \\
\hline
\end{tabular}

Blinding of outcome as- Low risk see PATRICIA \& Costa Rica trials

sessment (detection bias)

All outcomes

Incomplete outcome data Low risk see PATRICIA \& Costa Rica trials
(attrition bias)

All outcomes

Selective reporting (re- Low risk see PATRICIA \& Costa Rica trials
porting bias)

porting bias)

VIVIANE trial (ph3,2v)

\begin{tabular}{|c|c|}
\hline Methods & Phase III randomised, double-blind, controlled trial \\
\hline \multirow[t]{4}{*}{ Participants } & $\begin{array}{l}5752 \text { women ( } 2881 \text { in the vaccine arm and } 2871 \text { in the placebo arm) from Australia, Canada, Mexico, the } \\
\text { Netherlands, Peru, Philippines, Portugal, Russia, Singapore, Thailand, the UK and the USA }\end{array}$ \\
\hline & Age range: women older than 25 years old, age stratified in 26 to 35, 36 to 45 and older than 46 \\
\hline & $\begin{array}{l}\text { Inclusion criteria: Women who were older than } 25 \text { years old. Each age-stratum had } 15 \% \text { of women with } \\
\text { a history of HPV infection to represent a real-world setting; no limits on number of lifetime sexual part- } \\
\text { ners }\end{array}$ \\
\hline & $\begin{array}{l}\text { Exclusion criteria: women were excluded if they were pregnant, breastfeeding and who had chronic or } \\
\text { autoimmune disease or immunodeficiency }\end{array}$ \\
\hline \multirow[t]{2}{*}{ Interventions } & Vaccine: HPV16/18 AS04-adjuvant bivalent vaccine; \\
\hline & Placebo: visually indistinguishable aluminium-containing placebo \\
\hline Outcomes & Safety, immunogenicity, efficacy and cross-protection \\
\hline \multirow[t]{2}{*}{ Notes } & Main report: Skinner 2014 \\
\hline & Last report average follow-up time: 43.3 months (Skinner 2014) \\
\hline \multicolumn{2}{|l|}{ Risk of bias } \\
\hline Bias & Authors' judgement Support for judgement \\
\hline
\end{tabular}


VIVIANE trial (ph3,2v) (Continued)

$\begin{array}{ll}\begin{array}{l}\text { Random sequence genera- } \\ \text { tion (selection bias) }\end{array} & \text { Low risk } \\ \end{array}$

\begin{tabular}{|c|c|c|}
\hline $\begin{array}{l}\text { Allocation concealment } \\
\text { (selection bias) }\end{array}$ & Low risk & $\begin{array}{l}\text { The randomisation list was generated by GSK with an algorithm which used a } \\
\text { minimisation process that accounted for region, age stratum and HPV history }\end{array}$ \\
\hline
\end{tabular}

\begin{tabular}{|c|c|c|}
\hline $\begin{array}{l}\text { Blinding of participants } \\
\text { and personnel (perfor- } \\
\text { mance bias) }\end{array}$ & Low risk & $\begin{array}{l}\text { All participants, investigators and staff involved were masked to treatment al- } \\
\text { location and study results. The interim analysis was done by an external statis- } \\
\text { tician blinded to the allocation of vaccine versus placebo }\end{array}$ \\
\hline
\end{tabular}

All outcomes

tician blinded to the allocation of vaccine versus placebo

\begin{tabular}{|c|c|c|}
\hline $\begin{array}{l}\text { Blinding of outcome as- } \\
\text { sessment (detection bias) } \\
\text { All outcomes }\end{array}$ & Low risk & $\begin{array}{l}\text { All CIN cases were reviewed by a panel of three pathologists who were blind- } \\
\text { ed to vaccine allocation. Analysis was done by an independent statistician to } \\
\text { maintain the trial blinding }\end{array}$ \\
\hline
\end{tabular}

\begin{tabular}{ll}
\hline $\begin{array}{l}\text { Incomplete outcome data Low risk } \\
\text { (attrition bias) }\end{array}$ & Outcomes were assessed in the PP cohort (received 3 doses, seronegative and \\
All outcomes & DNA negative for the corresponding vaccine type at month 0, normal or low- \\
& grade cytology at month 0, no protocol violations) and in the TVC-naive cohort \\
& (at least one vaccine dose, at baseline normal cytology, DNA negative for hrH- \\
& PV, seronegative for HPV-16 and HPV-18) and in the total vaccinated cohort (all \\
& women randomised). Reasons for exclusion were presented
\end{tabular}

\begin{tabular}{|c|c|c|}
\hline $\begin{array}{l}\text { Selective reporting (re- } \\
\text { porting bias) }\end{array}$ & Low risk & $\begin{array}{l}\text { All outcomes (safety, immunogenicity, efficacy and cross-protection) are pre- } \\
\text { sented }\end{array}$ \\
\hline
\end{tabular}

Japanese trial (ph2,4v)

\begin{tabular}{ll}
\hline Methods & Phase Il randomised, double-blind, controlled trial in Japan \\
\hline Participants & Participants: 1021 Japanese women (509 in the vaccine arm and 512 in the placebo arm) \\
Age range: 18 to 26 years \\
Inclusion criteria: healthy women who were not pregnant, had no previous abnormal Pap smears and \\
reported a lifetime history of four or fewer male sex partners and agreed to use effective contraception \\
were eligible. Women with previous HPV infection were not excluded
\end{tabular}

Interventions
Vaccine: quadrivalent HPV 6/11/16/18 L1 VLP vaccine
Placebo: visually indistinguishable aluminium-containing placebo

\begin{tabular}{ll}
\hline Outcomes & $\begin{array}{l}\text { Efficacy (composite primary endpoint of persistent infection and external genital disease), immuno- } \\
\text { genicity and safety outcomes }\end{array}$
\end{tabular}

\begin{tabular}{ll}
\hline Notes & Main report: Yoshikawa 2013 \\
& Last report average follow-up time: 30 months
\end{tabular}

\section{Risk of bias}

\begin{tabular}{lll}
\hline Bias & Authors' judgement & Support for judgement \\
\hline $\begin{array}{l}\text { Random sequence genera- } \\
\text { tion (selection bias) }\end{array}$ & Unclear risk & Not mentioned in the paper \\
\hline
\end{tabular}


Japanese trial (ph2,4v) (Continued)
Allocation concealment Low risk
The prepared randomisation schedule was sealed with other corresponding (selection bias) randomisation listings and retained strictly until un-blinding by the Center for Patients Allocation

\begin{tabular}{lll}
$\begin{array}{l}\text { Blinding of participants } \\
\text { and personnel (perfor- } \\
\text { mance bias) }\end{array}$ & Low risk \\
All outcomes & \\
\hline $\begin{array}{l}\text { Blinding of outcome as- } \\
\begin{array}{l}\text { sessment (detection bias) } \\
\text { All outcomes }\end{array}\end{array}$ & Low risk & $\begin{array}{l}\text { Endpoint analysis was done by use of consensus diagnosis from a panel of } \\
\text { pathologists who were blinded to the central laboratory diagnosis, vaccination } \\
\text { group and HPV status }\end{array}$ \\
\hline
\end{tabular}

$\begin{array}{ll}\begin{array}{l}\text { Incomplete outcome data } \\ \text { (attrition bias) }\end{array} & \text { Unclear risk } \\ \text { All outcomes } & \begin{array}{l}\text { Efficacy result only reported in PP-cohort (received } 3 \text { doses, being naive for the } \\ \text { relevant HPV type at enrolment, remained free of infection with the same vac- } \\ \text { cine HPV type through completion of the vaccination regimen, did not violate } \\ \text { the protocol) }\end{array}\end{array}$

Selective reporting (re- Low risk All outcomes (efficacy, safety and immunogenicity) reported
porting bias)

Korean trial (ph2,4v)

\begin{tabular}{ll}
\hline Methods & Phase II randomised, double-blind, placebo-controlled trial \\
\hline Participants & Participant: 176 Korean participants (117 in the vaccine arm and 59 in the placebo arm) \\
Age range: 9 to 23 years \\
Inclusion criteria: women who were not pregnant, had no fever more than $37.8^{\star} C$ at vaccination, age \\
9-15 years must have had no sexual experience before and no plan to have sexual experience during \\
the study period. Participants aged 16 to 23 years must have had history of fewer than 4 sexual partners \\
and use effective contraception during the study period \\
Exclusion criteria: participants who were enrolled in studies of other investigation agents, history of \\
any HPV vaccination, history of allergy to vaccine compound, thrombocytopenia, history of vaccination \\
within 14 days from enrolment (previous 21 days for live vaccine), receipt of blood or blood-derived \\
products within the 6 months preceding injection, and immunosuppression. Age group 16 to 23 were \\
required to have not had a prior Pap test showing a squamous intraepithelial lesion or worse and/or a \\
biopsy indicating CIN or worse
\end{tabular}

Interventions Vaccine: quadrivalent HPV 6/11/16/18 L1 VLP vaccine

Placebo: visually indistinguishable aluminium-containing placebo

\begin{tabular}{ll}
\hline Outcomes & Immunogenicity and safety outcomes \\
\hline Notes & Main report: Kang 2008 \\
& Last report average follow-up time: 7 months \\
\hline
\end{tabular}

\section{Risk of bias}

\begin{tabular}{lll}
\hline Bias & Authors' judgement & Support for judgement \\
\hline $\begin{array}{l}\text { Random sequence genera- } \\
\text { tion (selection bias) }\end{array}$ & Low risk & $\begin{array}{l}\text { Randomisation was performed by the study centres using the block method } \\
\text { with decreasing block sizes }\end{array}$ \\
\hline \hline
\end{tabular}

Prophylactic vaccination against human papillomaviruses to prevent cervical cancer and its precursors (Review) 
Korean trial (ph2,4v) (Continued)

\begin{tabular}{lll}
$\begin{array}{l}\text { Allocation concealment } \\
\text { (selection bias) }\end{array}$ & Unclear risk & Not described in the paper \\
\hline $\begin{array}{l}\text { Blinding of participants } \\
\text { and personnel (perfor- } \\
\text { mance bias) } \\
\text { All outcomes }\end{array}$ & Unclear risk & The trial was described as double-blind but no further details are given \\
\hline $\begin{array}{l}\text { Blinding of outcome as- } \\
\text { sessment (detection bias) } \\
\text { All outcomes }\end{array}$ & Unclear risk & Not described in the paper \\
\hline $\begin{array}{l}\text { Incomplete outcome data } \\
\text { (attrition bias) } \\
\text { All outcomes }\end{array}$ & Low risk & $\begin{array}{l}\text { The loss to follow-up rate is low and well-balanced for both the vaccine and } \\
\text { the placebo groups. Participants being baseline seropositive for the concerned } \\
\text { HPV vaccine type were excluded for the immunogenicity outcome }\end{array}$
\end{tabular}

Selective reporting (re- Low risk Immunogenicity and safety outcomes reported
porting bias)

porting bias)

Phase2 trial (ph2,4v)

\begin{tabular}{ll}
\hline Methods & Phase II randomised, multicentre, double-blind, placebo-controlled trial \\
\hline Participants & $\begin{array}{l}1158 \text { women enrolled for the study, among them } 52 \text { participants were included in study part A which } \\
\text { was a dose-escalation study, and the } 1106 \text { remaining women were included study part B which was a } \\
\text { dose-ranging study. In study part B, } 554 \text { were in intermediate-/high-dose groups and } 552 \text { were in low- } \\
\text { dose groups; } 277 \text { in low-dose vaccine group and } 275 \text { in the placebo group }\end{array}$
\end{tabular}

Age range: 16 to 23 years

Inclusion criteria: healthy women who were not pregnant, had no previous abnormal Pap smear and reported a lifetime history of four or fewer male sex partners. The study did not exclude women with previous HPV infection.Virgins were restricted to women of 18 years or older and seeking contraception

\begin{tabular}{ll}
\hline Interventions & Vaccine: quadrivalent HPV 6/11/16/18 L1 VLP \\
& Placebo: visually indistinguishable aluminium-containing placebo \\
\hline
\end{tabular}

Outcomes Persistent infection ( $\geq 4 \mathrm{M}$ or at last visit) associated with HPV 6,11,16 or 18, cervical or external genital lesions (CIN 1-3, condylomata acuminata, vulvar intraepithelial neoplasia and vaginal intraepithelial neoplasia), immunogenicity, safety and tolerability

Main reports: Villa 2005; Villa 2006 and Villa 2006a
Last report average follow-up time: 36 months and 60 months for a subset of 241 participants (Villa
2006a)

\section{Risk of bias}

\begin{tabular}{lll}
\hline Bias & Authors' judgement & Support for judgement \\
\hline $\begin{array}{ll}\text { Random sequence genera- } \\
\text { tion (selection bias) }\end{array}$ & Low risk & $\begin{array}{l}\text { Randomisation to the placebo or vaccine arms was applied only on a part of } \\
\text { the enrolled women (those included in the low-dose group). The other women } \\
\text { were enrolled in a dose-escalating or dose-ranging studies. Only women from } \\
\text { the low-dose group were included were used for the Cochrane Review }\end{array}$ \\
\end{tabular}


African_3 country trial (ph3,4v) (Continued)

\begin{tabular}{lll}
$\begin{array}{l}\text { Blinding of participants } \\
\text { and personnel (perfor- } \\
\text { mance bias) } \\
\text { All outcomes }\end{array}$ & Unclear risk & Not described in the paper \\
\hline $\begin{array}{l}\text { Blinding of outcome as- } \\
\text { sessment (detection bias) } \\
\text { All outcomes }\end{array}$ & Unclear risk & Not described in the paper \\
\hline $\begin{array}{l}\text { Incomplete outcome data } \\
\text { (attrition bias) }\end{array}$ & Low risk & $\begin{array}{l}\text { All safety outcomes were reported for the total vaccinated cohort. Immuno- } \\
\text { genicity outcomes were reported for the according-to-protocol cohort. Rea- } \\
\text { son for exclusion was noted and balanced between vaccine group and placebo } \\
\text { group }\end{array}$
\end{tabular}

Selective reporting (re- Low risk All outcomes (immunogenicity and safety) were presented

porting bias)

\section{FUTURE I trial (ph3,4v)}

\begin{tabular}{ll}
\hline Methods & Phase III randomised, placebo-controlled, double-blind trial \\
\hline Participants & $\begin{array}{l}\text { Participants: } 5455 \text { women ( } 2723 \text { women in the vaccine arm and } 2732 \text { in the placebo arm) from } 62 \text { study } \\
\text { centres in } 16 \text { countries } \\
\text { Age range: } 16 \text { to } 24 \text { years } \\
\text { Inclusion criteria: healthy women who were not pregnant and had no history of genital warts or abnor- } \\
\text { mal results on cervical cytologic testing, had a lifetime number of no more than four sex partners and } \\
\text { agreed to use contraception during the vaccination period }\end{array}$
\end{tabular}

\begin{tabular}{ll}
\hline Interventions & Vaccine: quadrivalent HPV 6/11/16/18 vaccine \\
& Placebo: visually indistinguishable aluminium-containing placebo
\end{tabular}

Outcomes

Efficacy (CIN of any grade, AIS, cervical cancer, VIN, VaIN, GW, vulvar-vaginal cancer, Pap abnormalities), immunogenicity and safety

\begin{tabular}{ll}
\hline Notes & Main reports: Garland 2007; \\
& Last report average follow-up time: 4.9 years (Munoz 2010)
\end{tabular}

\section{Risk of bias}

\begin{tabular}{lll}
\hline Bias & Authors' judgement & Support for judgement \\
\hline $\begin{array}{l}\text { Random sequence genera- } \\
\text { tion (selection bias) }\end{array}$ & Low risk & $\begin{array}{l}\text { A computer-based randomised allocation schedule provided by the statisti- } \\
\text { cian was used for sequence allocation }\end{array}$ \\
\hline $\begin{array}{l}\text { Allocation concealment } \\
\text { (selection bias) }\end{array}$ & Low risk & $\begin{array}{l}\text { An interactive voice response system was used to randomise participants with- } \\
\text { in each study centre }\end{array}$ \\
\hline $\begin{array}{l}\text { Blinding of participants } \\
\begin{array}{l}\text { and personnel (perfor- } \\
\text { mance bias) }\end{array}\end{array}$ & Low risk & $\begin{array}{l}\text { The participants, investigator and sponsor were blinded to the allocated trial } \\
\text { all outcomes }\end{array}$ \\
\hline
\end{tabular}


FUTURE I trial (ph3,4v) (Continued)

$\begin{array}{lll}\begin{array}{l}\text { Blinding of outcome as- } \\ \text { sessment (detection bias) }\end{array} & \text { Low risk } & \begin{array}{l}\text { Central laboratory was unaware of treatment-group assignment and HPV sta- } \\ \text { tus. A panel of } 4 \text { pathologists was unaware of diagnosis made at the central }\end{array} \\ \text { All outcomes } & \text { laboratory, clinical findings, treatment group, and HPV status }\end{array}$

\begin{tabular}{|c|c|c|}
\hline $\begin{array}{l}\text { Incomplete outcome data } \\
\text { (attrition bias) } \\
\text { All outcomes }\end{array}$ & Low risk & $\begin{array}{l}\text { Outcomes are assessed in the PP cohort (received } 3 \text { doses without protocol vi- } \\
\text { olations, being HPV DNA negative for the relevant HPV vaccine type from en- } \\
\text { rolment to } 1 \text { month after dose 3), in the unrestricted susceptible group (all } \\
\text { women who were negative on HPV DNA and serology negative for the relevant } \\
\text { HPV vaccine type at enrolment) and in the ITT cohort (all participants who had } \\
\text { undergone randomisation, regardless of baseline HPV status or presence of } \\
\text { HPV-associated an lanogenital disease). Reasons for exclusion were presented }\end{array}$ \\
\hline
\end{tabular}

Selective reporting (re- Low risk All outcomes (efficacy, safety and immunogenicity) reported

porting bias)

FUTURE II trial (ph3,4v)

\begin{tabular}{ll}
\hline Methods & Phase III randomised, placebo-controlled, double-blind trial. \\
\hline Participants & $\begin{array}{l}\text { Participants: } 12167 \text { women ( } 6087 \text { women in vaccine arm and } 6080 \text { women in the placebo arm) from } 90 \\
\text { study centres in } 13 \text { countries. } \\
\text { Age range: } 15 \text { to } 26 \text { years. } \\
\text { Inclusion criteria: healthy women with an intact uterus, who were not pregnant and had no history of } \\
\text { genital warts or abnormal results on cervical cytologic testing, had a lifetime number of no more than } \\
\text { four sex partners and agreed to use contraception during the vaccination period }\end{array}$
\end{tabular}

\begin{tabular}{ll}
\hline Interventions & Vaccine: Quadrivalent HPV6/11/16/18 vaccine \\
& Placebo: visually indistinguishable aluminium-containing placebo
\end{tabular}

\begin{tabular}{ll}
\hline Outcomes & Efficacy (CIN of any grade, AIS, cervical cancer, VIN, VaIN, GW, vulvar-vaginal cancer, Pap abnormali- \\
ties), safety and immunogenicity
\end{tabular}

\begin{tabular}{ll}
\hline Notes & Main reports: FUTURE-II 2007 and Munoz 2010 \\
& Last report average follow-up time: 4.9 years (Munoz 2010) \\
\hline
\end{tabular}

\begin{tabular}{|c|c|c|}
\hline \multicolumn{3}{|l|}{ Risk of bias } \\
\hline Bias & Authors' judgement & Support for judgement \\
\hline $\begin{array}{l}\text { Random sequence genera- } \\
\text { tion (selection bias) }\end{array}$ & Low risk & $\begin{array}{l}\text { A computer-based randomised allocation schedule provided by the statisti- } \\
\text { cian.was used for sequence allocation }\end{array}$ \\
\hline $\begin{array}{l}\text { Allocation concealment } \\
\text { (selection bias) }\end{array}$ & Low risk & $\begin{array}{l}\text { An interactive voice response system was used to randomise participants with- } \\
\text { in each study centre }\end{array}$ \\
\hline $\begin{array}{l}\text { Blinding of participants } \\
\text { and personnel (perfor- } \\
\text { mance bias) } \\
\text { All outcomes }\end{array}$ & Low risk & $\begin{array}{l}\text { The participants, investigator and sponsor were blinded to the allocated trial } \\
\text { arm }\end{array}$ \\
\hline
\end{tabular}


FUTURE II trial (ph3,4v) (Continued)
Blinding of outcome as- Low risk
Central laboratory was unaware of treatment-group assignment and HPV sta- sessment (detection bias) tus. A panel of 4 pathologists was unaware of diagnosis made at the central All outcomes laboratory, clinical findings, treatment group, and HPV status

Incomplete outcome data Low risk
(attrition bias)
All outcomes

Outcomes were assessed in the PP cohort (received 3 doses without protocol violations, being HPV DNA negative for the relevant HPV vaccine type from enrolment to 1 month after dose 3), in the unrestricted susceptible group (all women who were negative on HPV DNA and serology negative for the relevant HPV vaccine type at enrolment) and in the ITT cohort (all participants who had undergone randomisation, regardless of baseline HPV status or presence of $\mathrm{HPV}$-associated anogenital disease). Reasons for exclusion were presented

Selective reporting (re- Low risk $\quad$ All outcomes (efficacy, safety and immunogenicity) reported
porting bias)

porting bias)

\section{FUT I/II trials (ph3,4v)}

\begin{tabular}{ll}
\hline Methods & Pooling of two phase III randomised, placebo-controlled, double-blind trials \\
\hline Participants & Participants: 17,622 women (see FUTURE I and FUTURE II trials for more details) \\
& Age range: 16 to 26 years \\
\hline Interventions & Vaccine: Quadrivalent HPV6/11/16/18 vaccine \\
& Placebo: visually indistinguishable aluminium-containing placebo \\
\hline Outcomes & Efficacy (CIN of any grade, AIS, cervical cancer, VIN, VaIN, GW, vulvar-vaginal cancer, Pap abnormali- \\
ties), safety and immunogenicity
\end{tabular}

\section{Risk of bias}

\begin{tabular}{lll}
\hline Bias & Authors' judgement & Support for judgement \\
\hline $\begin{array}{l}\text { Random sequence genera- } \\
\text { tion (selection bias) }\end{array}$ & Low risk & See FUTURE I \& II trials \\
\hline $\begin{array}{l}\text { Allocation concealment } \\
\text { (selection bias) }\end{array}$ & Low risk & See FUTURE I \& II trials \\
\hline $\begin{array}{l}\text { Blinding of participants } \\
\begin{array}{l}\text { and personnel (perfor- } \\
\text { mance bias) }\end{array}\end{array}$ & Low risk \\
$\begin{array}{ll}\text { All outcomes } \\
\text { Blinding of outcome as- } \\
\text { sessment (detection bias) } \\
\text { All outcomes }\end{array}$ & Low risk & See FUTURE I \& II trials \\
\hline $\begin{array}{l}\text { Incomplete outcome data } \\
\text { (attrition bias) } \\
\text { All outcomes }\end{array}$ & Low risk & See FUTURE I \& II trials \\
\hline
\end{tabular}


FUT I/II trials (ph3,4v) (Continued)
Selective reporting (re-
Low risk
All outcomes (efficacy, safety and immunogenicity) reported porting bias)

\section{FUTURE III trial (ph3,4v)}

\begin{tabular}{|c|c|}
\hline Methods & Phase III randomised, double-blind, controlled trial \\
\hline \multirow[t]{4}{*}{ Participants } & $\begin{array}{l}\text { Participants: } 3819 \text { women ( } 1911 \text { in the vaccine arm and } 1908 \text { in the placebo arm) enrolled in } 38 \text { interna- } \\
\text { tional study sites from } 7 \text { countries. }\end{array}$ \\
\hline & Age range: 24 to 45 years \\
\hline & $\begin{array}{l}\text { Inclusion criteria: women were not pregnant, who had not undergone hysterectomy and agreed to use } \\
\text { effective contraception until month } 7 \text { of the study }\end{array}$ \\
\hline & $\begin{array}{l}\text { Exclusion criteria: women were excluded if they have a history of surgical cervical procedure, had biop- } \\
\text { sy less than } 5 \text { years ago, had history of genital warts and cervical disease. Women infected with HIV and } \\
\text { those who were immunocompromised were not eligible for enrolment }\end{array}$ \\
\hline
\end{tabular}

\begin{tabular}{ll} 
Interventions & Vaccine: quadrivalent vaccine \\
& Placebo: visually indistinguishable aluminium-containing placebo \\
\hline Outcomes & Efficacy (persistent HPV infection, CIN, condyloma, VIN or ValN), safety and immunogenicity outcomes \\
\hline Notes & Main reports: Munoz 2009 and Castellsagué 2011 \\
& Last report average follow-up time: 48 months ( Castellsagué 2011)
\end{tabular}

\section{Risk of bias}

\begin{tabular}{|c|c|c|}
\hline Bias & Authors' judgement & Support for judgement \\
\hline $\begin{array}{l}\text { Random sequence genera- } \\
\text { tion (selection bias) }\end{array}$ & Low risk & $\begin{array}{l}\text { A computer-generated allocation schedule was generated by the sponsor's } \\
\text { Clinical Biostatistics department }\end{array}$ \\
\hline $\begin{array}{l}\text { Allocation concealment } \\
\text { (selection bias) }\end{array}$ & Low risk & $\begin{array}{l}\text { Randomised to a vaccination group using an interactive Voice Response Sys- } \\
\text { tem }\end{array}$ \\
\hline $\begin{array}{l}\text { Blinding of participants } \\
\text { and personnel (perfor- } \\
\text { mance bias) } \\
\text { All outcomes }\end{array}$ & Low risk & $\begin{array}{l}\text { All study-site investigators and personnel, study participants, monitors, and } \\
\text { central laboratory personnel were blinded to treatment allocation throughout } \\
\text { the study }\end{array}$ \\
\hline $\begin{array}{l}\text { Blinding of outcome as- } \\
\text { sessment (detection bias) } \\
\text { All outcomes }\end{array}$ & Low risk & $\begin{array}{l}\text { Biopsy material was first read for clinical management by pathologists at a } \\
\text { central laboratory, and then read for endpoint determination by a blinded } \\
\text { panel of } 4 \text { pathologists }\end{array}$ \\
\hline $\begin{array}{l}\text { Incomplete outcome data } \\
\text { (attrition bias) } \\
\text { All outcomes }\end{array}$ & Low risk & $\begin{array}{l}\text { Outcomes were assessed in the PP cohort (received } 3 \text { doses, seronegative at } \\
\text { day } 1 \text { and HPV DNA negative for the HPV vaccine types from day } 0 \text { until month } \\
7 \text {, no protocol violations), in the naive to the relevant type (NRT) cohort (at } \\
\text { least } 1 \text { dose, seronegative at day } 1 \text { and HPV DNA negative for the HPV vaccine } \\
\text { types on day } 1 \text { ) and in the ITT-cohort (at least } 1 \text { dose, irrespective of initial HPV } \\
\text { status, protocol violators included) }\end{array}$ \\
\hline
\end{tabular}

\begin{tabular}{l}
$\begin{array}{l}\text { Selective reporting (re- } \\
\text { porting bias) }\end{array} \quad$ Low risk outcomes (safety, immunogenicity and efficacy) are presented \\
\hline
\end{tabular}
porting bias) 
AIS: adenocarcinoma in situ

ASC: atypical squamous cells

ASC-US: atypical squamous cells of undetermined significance

$\mathrm{CIN}$ : cervical intraepithelial neoplasia

DNA: Desoxyribo-nucleic acid

ELISA: enzyme-linked immunosorbent assay

GSK: GlaxoSmithKline

GW: genital wart

HPV: human papillomavirus

ITT: intention-to-treat

LEEP: loop electrosurgical excision procedure

LSIL: low-grade squamous intraepithelial lesion

MITT: modified intention-to-treat

PATRICIA: PApiloma TRlal against Cancer In young Adults

PCR: polymerase chain reaction

PP: per-protocol

TVC: total vaccinated cohort

VAIN: Vaginal intra-epithelial neoplasia,

VIN: vaginal intraepithelial neoplasia

VLP: virus-like particles

Characteristics of excluded studies [ordered by study ID]

\begin{tabular}{|c|c|}
\hline Study & Reason for exclusion \\
\hline Angelo 2014 & $\begin{array}{l}\text { Post-licensure safety surveillance over more than } 4 \text { years of routine use of HPV bivalent vaccine. } \\
\text { Not a randomised controlled trial. }\end{array}$ \\
\hline Arguedas 2010 & $\begin{array}{l}\text { Randomised trial to evaluate Novartis vaccines co-administrated with Tdap vaccine and HPV vac- } \\
\text { cine. No HPV alone group. }\end{array}$ \\
\hline Ault 2004 & Phase I trial. \\
\hline Ault 2007 & $\begin{array}{l}\text { Pooled analysis of } 4 \text { RCTs on both bivalent and quadrivalent vaccine. No new original data were } \\
\text { presented. }\end{array}$ \\
\hline Basu 2013 & $\begin{array}{l}\text { A review of evidence from phase III trials and national immunisation programs regarding efficacy } \\
\text { and safety of HPV vaccines. }\end{array}$ \\
\hline Beachler 2016 & $\begin{array}{l}\text { Efficacy of the bivalent vaccine against cervical, anal and oral infection in a sub-cohort nested in } \\
\text { the CVT trial. Cervical outcomes already included. }\end{array}$ \\
\hline Brown 2004 & Post hoc analysis using combined data from two Phase I tolerability/immunogenicity trials. \\
\hline Couto 2014 & $\begin{array}{l}\text { Systemactic review and meta-analysis of protection of HPV vaccines against CIN, VIN, VAIN, and } \\
\text { genital warts in catch-up populations. No Original data, not a randomised controlled trial. }\end{array}$ \\
\hline D'Addario 2017 & $\begin{array}{l}\text { Systematic review and meta-analysis of the immunogenicity of the } 2 \text {-dose vaccination schedule } \\
\text { versus } 3 \text {-dose schedule. }\end{array}$ \\
\hline D'Souza 2013 & A case-study on HPV vaccination national programme in Australia for future innovation prevention. \\
\hline De Vincenzo 2014 & Review of the long-term efficacy and safety of HPV vaccines. No original data. \\
\hline Delere 2013 & $\begin{array}{l}\text { Assessment of HPV vaccine update and post-vaccination cervical cancer prevention in Germany. } \\
\text { Not a randomised controlled trial. }\end{array}$ \\
\hline
\end{tabular}




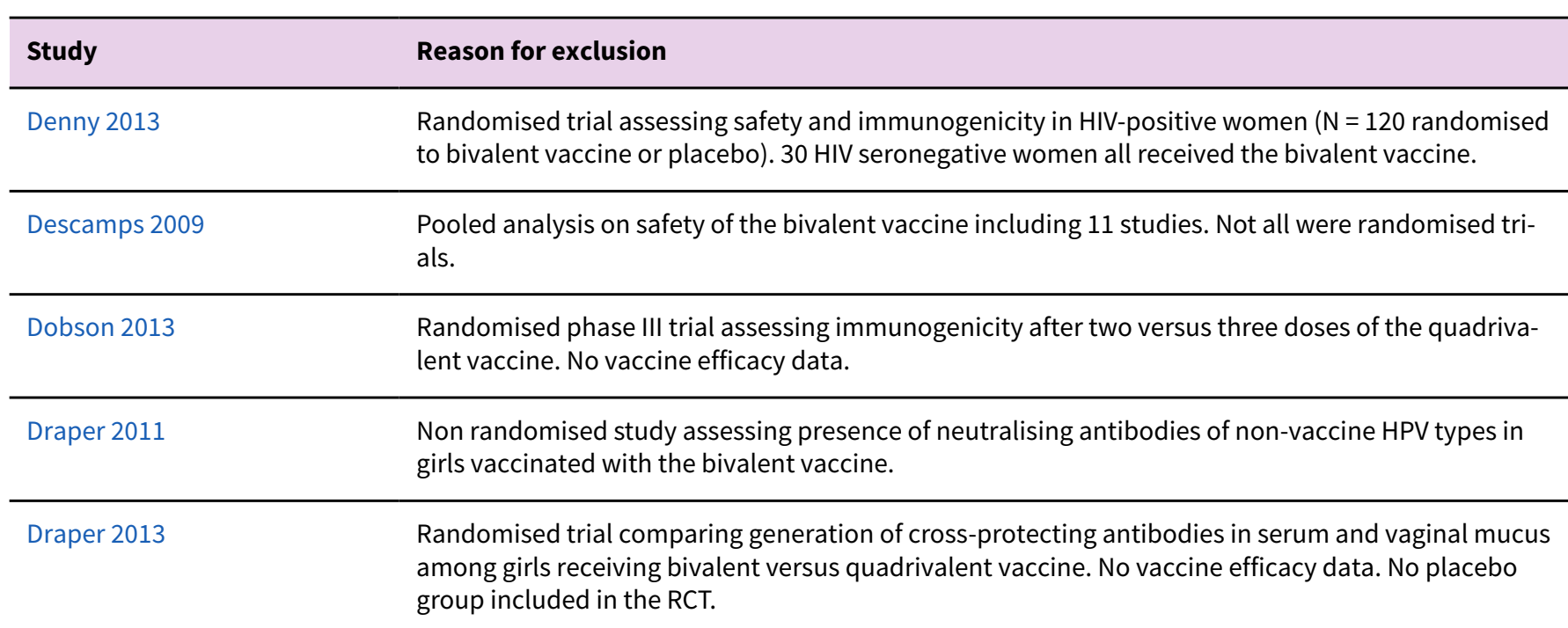

\section{Einstein 2009}

Randomised trial comparing safety and generation of antiHPV16/18 antibodies in serum and vaginal mucus among girls receiving bivalent versus quadrivalent vaccine ( 7 months after 3 rd dose). No vaccine efficacy data. No placebo group included in the RCT.

$\begin{array}{ll}\text { Einstein } 2011 & \text { Randomised trial comparing safety and generation of antiHPV16/18 antibodies in serum and vagi- } \\ \text { nal mucus among girls receiving bivalent versus quadrivalent vaccine (12 months after } 3 \text { rd dose). } \\ \text { No vaccine efficacy data. No placebo group included in the RCT. }\end{array}$

Evans 2001 Dose-escalation phase I trial addressing immunological response and safety after administration of an L1 HPV11 vaccine.

$\begin{array}{ll}\text { Forinash } 2011 & \begin{array}{l}\text { Systematic review on pregnancy outcomes of bi- and quadrivalent vaccines using data from RCTs } \\ \text { and post-marketing surveillance. }\end{array}\end{array}$

$\begin{array}{ll}\text { Garland } 2016 & \begin{array}{l}\text { Post-hoc analysis of the bivalent HPV vaccine against the recurrent of the high-grade CIN after sur- } \\ \text { gical therapy. }\end{array}\end{array}$

\begin{tabular}{ll}
\hline Giuliano 2007 & $\begin{array}{l}\text { Pooled analysis of phase II/III trials assessing immunogenicity according to baseline covariates. No } \\
\text { original data. No vaccine-efficacy or safety data. }\end{array}$
\end{tabular}

\begin{tabular}{ll}
\hline Giuliano 2011 & Phase III trial assessing safety and efficacy of vaccination with the quadrivalent vaccine in men. \\
\hline Giuliano 2015 & $\begin{array}{l}\text { Immunogenicity and safety of Gardasil vaccine among mid-adult aged men of } 27 \text { to } 45 \text { years. No } \\
\text { data on women. }\end{array}$ \\
\hline Goldstone 2013 & $\begin{array}{l}\text { Quadrivalent HPV vaccine efficacy against disease related to vaccine and non-vaccine HPV types in } \\
\text { men. }\end{array}$ \\
\hline Harro 2001 & $\begin{array}{l}\text { Phase I dose-escalation trial assessing immunogenicity and safety of a mono-valent HPV16 vac- } \\
\text { cine. }\end{array}$ \\
\hline Haupt 2011 & $\begin{array}{l}\text { Pooled analysis of } 2 \text { RCTs assessing the incidence of CIN2+/AIS+ related to HPV } 16 / 18 \text { in women } \\
\text { who received the quadrivalent vaccine or placebo and who were HPV16/18 DNA and seropositive. } \\
\text { The data of the separate studies are already included in the review. }\end{array}$ \\
\hline Heijstek 2014 & $\begin{array}{l}\text { Cohort study on immunogenicity and safety of the bivalent HPV vaccines in female patients with ju- } \\
\text { venile idiopathic arthritis. Not a randomised controlled trial. }\end{array}$ \\
\hline Hernandez-Avila 2016 & $\begin{array}{l}\text { Non randomised trial to evaluate the immunogenicity of the quadrivalent HPV vaccine using } 2 \text { ver- } \\
\text { sus } 3 \text { doses, An observational surveillance study to evaluate alternative vaccination schedules. }\end{array}$
\end{tabular}




\begin{tabular}{ll}
\hline Study & Reason for exclusion \\
\hline Herrero 2013 & $\begin{array}{l}\text { Report from the Costa Rica vaccination trial assessing effect of the bivalent vaccine on oral HPV in- } \\
\text { fection. }\end{array}$
\end{tabular}

\begin{tabular}{ll}
\hline Hildesheim 2007 & Report of the Costa Rica trial assessing the effect of the bivalent vaccine on clearance of existing \\
& HPV infection.
\end{tabular}
HPV infection.

\begin{tabular}{ll}
\hline Hillman 2011 & Phase III randomised trial assessing immunogenicity of the quadrivalent vaccine in men. \\
\hline Joura 2007 & $\begin{array}{l}\text { Pooled analyses of three randomised trials assessing protection of the quadrivalent vaccine } \\
\text { against vulval and vaginal intraepithelial lesions. Protection against cervical lesions was not ad- } \\
\text { dressed. }\end{array}$ \\
\hline Kahn 2013 & $\begin{array}{l}\text { Immunogenicity and safety of the human papillomavirus } 6,11,16,18 \text { vaccine in HIV-infected young } \\
\text { women. }\end{array}$
\end{tabular}

Kang 2013 Non randomised study assessing effect of the quadrivalent vaccine on the incidence of recurrence
of CIN in women treated by excision for high-grade CIN.

\begin{tabular}{ll}
\hline Khatun 2012 & Girls randomised to the experimental arm received the bivalent vaccine, those in the control arm \\
did not receive anything. The trial was not placebo-controlled. Observation of effects were restrict- \\
ed to participants in the experimental arm.
\end{tabular}

$\begin{array}{ll}\text { Kjaer } 2009 & \text { A pooled analysis of efficacy of quadrivalent HPV vaccines against cervical and genital lesions. No } \\ & \text { separate data on FUTURE I and FUTURE II trials. The data of the separate studies are already in- } \\ \text { cluded in the review. }\end{array}$

\begin{tabular}{ll}
\hline Kreimer 2015 & $\begin{array}{l}\text { Discussion about conducting a randomised clinical trial to assess the efficacy of a single dose of } \\
\text { prophylactic HPV vaccines among adolescent. No original data. }\end{array}$ \\
\hline Lamontagne 2013 & $\begin{array}{l}\text { Immunogenicity of quadrivalent HPV vaccine among girls aged } 11 \text { to } 13 \text { years of age vaccinated us- } \\
\text { ing alternative dosing schedules. }\end{array}$
\end{tabular}

\begin{tabular}{ll}
\hline Lang 2014 & $\begin{array}{l}\text { A nested analysis of CVT trial on vaccine efficacy against vulvar HPV infection. Cervical outcomes } \\
\text { from the trial have been included in the review. }\end{array}$ \\
\hline Lazcano-Ponce 2014 & $\begin{array}{l}\text { Non-inferiority of antibody response to human papillomavirus 16/18 vaccine in adolescents vacci- } \\
\text { nated with alternative dosing schedules. }\end{array}$ \\
\hline Lehtinen 2016 & $\begin{array}{l}\text { Phase IV RCT to evaluate the effectiveness, safety and immunogenicity of Cervarix in boys and girls } \\
\text { aged 12-15 years in Finland. }\end{array}$ \\
\hline Leroux-Roels 2011 & $\begin{array}{l}\text { Randomised trial assessing safety and immunogenicity of vaccination with the hepatitis-B vaccine } \\
\text { alone versus co-administration of the hepatitis-B vaccine with the bivalent HPV vaccine. No HPV } \\
\text { alone group. }\end{array}$ \\
\hline Leung 2015 & $\begin{array}{l}\text { Non RCT to compare immunogenicity and safety of 2-dose bivalent, 2-dose quadrivalent and 3- } \\
\text { dose quadrivalent vaccination schedule among girls aged 9-14 years. }\end{array}$ \\
\hline Li 2012 & $\begin{array}{l}\text { Randomised trial assessing safety and immunogenicity of the quadrivalent vaccine in a group of } \\
\text { Chinese women and men. Outcomes are presented jointly. Data separated by gender were request- } \\
\text { ed from the authors with no response. }\end{array}$ \\
\hline
\end{tabular}

Lin 2014 Randomised controlled trial of two dosing schedules for human papillomavirus vaccination among college-age men. 


\section{Study Reason for exclusion}

Luna 2013

Follow-up report (up to 6 years after dose 1 ) of the Columbian cohort of the FUTURE III trial, assessing the safety, immunogenicity and protection against the joint ocutome of CIN and extra-genital lesions combined of the quadrivalent vaccine. No separated data for protection against CIN2+ were reported.

\begin{tabular}{ll}
\hline Malagon 2012 & Systematic review and meta-analysis on cross-protection of the bi- and quadrivalent vaccines. \\
\hline McCormack 2011 & Review paper on the efficacy of the quadrivalent vaccine. \\
\hline McKeage 2011 & Review paper on the efficacy of the bivalent vaccine. \\
\hline Money 2016 & Not a randomised trial. Only HIV+ girls or women enrolled. \\
\hline Moreira 2011 & Randomised trial assessing the safety of vaccination with the quadrivalent vaccine in men \\
\hline Nakalembe 2015 & $\begin{array}{l}\text { Review of safety, immunogenicity and efficacy of HPV vaccines in low- and middle-income coun- } \\
\text { tries. No original data. }\end{array}$ \\
\hline Nelson 2013 & $\begin{array}{l}\text { Randomised comparison of safety and immunogenicity of the bi- and quadrivalent vaccine admin- } \\
\text { istered by intra-muscular versus intradermal injection. No placebo comparison group. }\end{array}$
\end{tabular}

Neuzil 2011

Randomised trial assessing safety and immunogenicity of four alternative schedules of administration of the quadrivalent vaccine in Vietnamese girls. No placebo group.

\section{Olsson 2009}

A pooled analysis of efficacy and safety of quadrivalent HPV vaccines on women with previous HPV infection. No separate data on FUTURE I and FUTURE II trials. The data of the separate studies are already included in the review.

\begin{tabular}{ll}
\hline Palefsky 2011 & $\begin{array}{l}\text { Randomised trial assessing the effect of vaccination with the quadrivalent vaccine on anal HPV in } \\
\text { fection and AIN in men }\end{array}$ \\
\hline Pedersen 2007 & $\begin{array}{l}\text { Immunobridging study assessing immunogenicity and safety of the bivalent HPV vaccines in } \\
\text { women aged } 15-25 \text { years and } 10-14 \text { years. Not a randomised controlled trial and all participants re- } \\
\text { ceived the bivalent vaccines. }\end{array}$ \\
\hline Perez 2008 & $\begin{array}{l}\text { Pooled analysis of RCTs of the efficacy of the quadrivalent vaccine regarding protection against } \\
\text { HPV-related lesions, restricted to the Latin-American cohorts included in the phase II and III trials } \\
\text { (FUTURE II trial (ph3,4v); FUTURE I trial (ph3,4v); Phase2 trial (ph2,4v)). The data of the separate } \\
\text { studies are already included in the review. }\end{array}$
\end{tabular}

\begin{tabular}{ll}
\hline Petaja 2009 & $\begin{array}{l}\text { Randomised trial assessing the immunogenicity and safety of vaccination with the bivalent vaccine } \\
\text { in boys. }\end{array}$
\end{tabular}
in boys.

Petaja 2011 Trial assessing long-term (at 48 months) safety and immunogenicity (antibodies in serum and cer-
vicovaginal secretions) of the bivalent vaccine. No placebo group.

Poland 2005 Dose-ranging study assessing safety and immunogenicity of a monovalent HPV16 vaccine.

Puthanakit $2016 \quad \begin{aligned} & \text { Randomised open trial to compare 2-dose versus 3-dose regimens of the bivalent vaccine in terms } \\ & \text { of immunogenicity and safety. No placebo group. }\end{aligned}$
of immunogenicity and safety. No placebo group.

Ramanakumar 2016

Read 2011
Incidence and duration of type-specific human papillomavirus infection in high-risk HPV-naive women. Post study results of Phase2 trial (ph2,2v) trial.

Surveillance of the incidence of genital warts before and after introduction of HPV vaccination in Australia. Not a randomised controlled trial. 


\section{Study Reason for exclusion}

Reisinger 2007

Randomised controlled trial assessing the safety and persistent immunogenicity of quadrivalent HPV vaccine in a group of boys and girls. Outcomes were presented jointly. Author was contacted to request data separated by gender. The author responded that separated data were not available.

\section{Reisinger 2010}

Romanowski 2016

Rowhani-Rahbar 2012

Randomised open-label study to assess the safety, tolerability and immunogenicity of quadrivalent vaccine co-administrated with enactra and Adacel vaccine. No quadrivalent only group and no separate data between girls and boys.

Five-year sustained immunogenicity of the bivalent vaccine administered as a 2-dose schedule in girls aged 9-14 years. No placebo group.

Trial demonstrating immune memory after administration of a dose of quadrivalent vaccine to women enrolled 8.5 years before in a phase II trial assessing the effects of the monovalent HPV16 vaccine.
Cross-protection efficacy against HPV 31 of bivalent vaccine, results from Costa Rica trials.

Review of the immunological response, including the induction of immune memory after vaccination with the bivalent vaccine. No original data.
Non randomised trial assessing immunogenicity and tolerability of the bivalent vaccine in female participants aged $15-55$ years from Germany and Poland.

Non randomised trial assessing presence of HPV antibodies in serum and cervicovaginal secretions of induced by the bivalent vaccine in female participants aged $15-55$ years from Germany and Poland.
Schwarz $2011 \quad$ Follow-up study to the Schwarz 2009 report.

Schwarz 2014

An open follow-up study of an RCT on safety and immunogenicity of bivalent vaccine in girls aged 10-13 years. Medina 2010 trial.
Correspondence about HPV vaccine trials in India. No extractable original data.

Correspondence about HPV vaccine trials in India. No extractable original data.

Systematic review on the efficacy of bivalent vaccine summarized from 6 RCTs. No original data.

Short review on pregnancy outcomes after vaccination against HPV. No original data.

Review article. No original data.

RCT to compare of the immunogenicity and reactogenicity of Cervarix and Gardasil human papillomavirus vaccines in HIV-infected adults.

Surveillance study assessing occurrence of adverse effects reported after HPV vaccination in the Netherlands.

Randomised open-label study to assess the safety, tolerability and immunogenicity of quadrivalent vaccine co-administrated with REPEVAX vaccine. No quadrivalent only group and no separate data between girls and boys.

Wheeler 2008

Randomised open-label study to assess the safety, tolerability and immunogenicity of quadrivalent vaccine co-administrated with Hepatitis $B$ vaccine. No quadrivalent only group. 


\begin{tabular}{ll}
\hline Study & Reason for exclusion \\
\hline Wheeler 2011 & $\begin{array}{l}\text { Trial assessing reactogenicity and immunogenicity of Tdap (tetanosdiphteria, pertussis) and MCV4 } \\
\text { (meningococcal polysaccharide \& diphtheria toxoid) vaccines when given alone or co-administrat- } \\
\text { ed with the bivalent HPV vaccine. Not a randomised controlled trial. }\end{array}$ \\
\hline Yancey 2010 & Systematic review on vaccine immunogenicity and efficacy in men. \\
\hline Zhu 2011 & $\begin{array}{l}\text { Non randomised phase I trial on safety and immunogenicity of the bivalent vaccine,conducted in } \\
\text { China (female participants aged 15-45 years). }\end{array}$ \\
\hline Zimmerman 2010 & $\begin{array}{l}\text { Randomised trial assessing the immunogenicity of the quadrivalent vaccine with two alternative } \\
\text { schedules (months } 0,2 \& 6, \text { versus months } 0,2 \text { \& 12). }\end{array}$ \\
\hline
\end{tabular}

AIS: adenocarcinoma in situ

CIN: cervical intraepithelial neoplasia

CVT: Costa Rica Vaccination Trial

HPV: human papillomavirus

$\mathrm{RCT}$ : randomised controlled trial

Tdap: tetanosdiphteria, pertussis

VAIN: Vaginal intra-epithelial neoplasia,

VIN: vaginal intraepithelial neoplasia

\section{DATA AND ANALYSES}

\section{Comparison 1. High-grade cervical lesions in hrHPV DNA negative women at baseline}

\begin{tabular}{|c|c|c|c|c|}
\hline Outcome or subgroup title & No. of studies & $\begin{array}{l}\text { No. of partici- } \\
\text { pants }\end{array}$ & Statistical method & Effect size \\
\hline $\begin{array}{l}1 \text { CIN2+ associated with HPV16/18, at } \\
\text { least } 1 \text { dose }\end{array}$ & 3 & 23676 & $\begin{array}{l}\text { Risk Ratio (IV, Random, 95\% } \\
\text { CI) }\end{array}$ & $0.01[0.00,0.05]$ \\
\hline $\begin{array}{l}2 \text { CIN2+ associated with } \\
\text { HPV6/11/16/18, at least } 1 \text { dose }\end{array}$ & 1 & 9296 & $\begin{array}{l}\text { Risk Ratio (IV, Random, 95\% } \\
\text { CI) }\end{array}$ & $0.01[0.00,0.09]$ \\
\hline $\begin{array}{l}3 \text { CIN3+ associated with HPV16/18, at } \\
\text { least } 1 \text { dose }\end{array}$ & 2 & 20214 & $\begin{array}{l}\text { Risk Ratio (IV, Random, 95\% } \\
\mathrm{CI} \text { ) }\end{array}$ & $0.01[0.00,0.10]$ \\
\hline $\begin{array}{l}4 \text { CIN3+ associated with } \\
\text { HPV6/11/16/18, at least } 1 \text { dose }\end{array}$ & 1 & 9296 & $\begin{array}{l}\text { Risk Ratio (IV, Random, 95\% } \\
\mathrm{Cl} \text { ) }\end{array}$ & $0.01[0.00,0.18]$ \\
\hline $\begin{array}{l}5 \text { AIS associated with HPV16/18, at } \\
\text { least } 1 \text { dose }\end{array}$ & 2 & 20214 & $\begin{array}{l}\text { Risk Ratio (IV, Random, 95\% } \\
\text { Cl) }\end{array}$ & $0.10[0.01,0.82]$ \\
\hline $\begin{array}{l}6 \text { AIS associated with HPV6/11/16/18, } \\
\text { at least } 1 \text { dose }\end{array}$ & 1 & 9296 & $\begin{array}{l}\text { Risk Ratio (IV, Random, 95\% } \\
\text { Cl) }\end{array}$ & $0.14[0.01,2.80]$ \\
\hline $\begin{array}{l}7 \text { Any } \mathrm{CIN} 2+\text { irrespective of HPV types, } \\
\text { at least } 1 \text { dose }\end{array}$ & 5 & 25180 & $\begin{array}{l}\text { Risk Ratio (IV, Random, 95\% } \\
\text { CI) }\end{array}$ & $0.37[0.25,0.55]$ \\
\hline 7.1 Bivalent vaccine & 4 & 15884 & $\begin{array}{l}\text { Risk Ratio (IV, Random, 95\% } \\
\text { CI) }\end{array}$ & $0.33[0.25,0.43]$ \\
\hline
\end{tabular}




\begin{tabular}{|c|c|c|c|c|}
\hline Outcome or subgroup title & No. of studies & $\begin{array}{l}\text { No. of partici- } \\
\text { pants }\end{array}$ & Statistical method & Effect size \\
\hline 7.2 Quadrivalent vaccine & 1 & 9296 & $\begin{array}{l}\text { Risk Ratio (IV, Random, 95\% } \\
\mathrm{CI} \text { ) }\end{array}$ & $0.57[0.44,0.76]$ \\
\hline $\begin{array}{l}8 \text { Any CIN3+ irrespective of HPV types, } \\
\text { at least } 1 \text { dose }\end{array}$ & 3 & 20719 & $\begin{array}{l}\text { Risk Ratio (IV, Random, 95\% } \\
\mathrm{CI} \text { ) }\end{array}$ & $0.21[0.04,1.10]$ \\
\hline 8.1 Bivalent vaccine & 2 & 11423 & $\begin{array}{l}\text { Risk Ratio (IV, Random, 95\% } \\
\mathrm{CI})\end{array}$ & $0.08[0.03,0.23]$ \\
\hline 8.2 Quadrivalent vaccine & 1 & 9296 & $\begin{array}{l}\text { Risk Ratio (IV, Random, 95\% } \\
\mathrm{Cl} \text { ) }\end{array}$ & $0.54[0.36,0.82]$ \\
\hline $\begin{array}{l}9 \text { Any AIS irrespective of HPV types, at } \\
\text { least } 1 \text { dose }\end{array}$ & 2 & 20214 & $\begin{array}{l}\text { Risk Ratio (IV, Random, 95\% } \\
\text { Cl) }\end{array}$ & $0.10[0.01,0.76]$ \\
\hline
\end{tabular}

Analysis 1.1. Comparison 1 High-grade cervical lesions in hrHPV DNA negative women at baseline, Outcome 1 CIN2+ associated with HPV16/18, at least 1 dose.

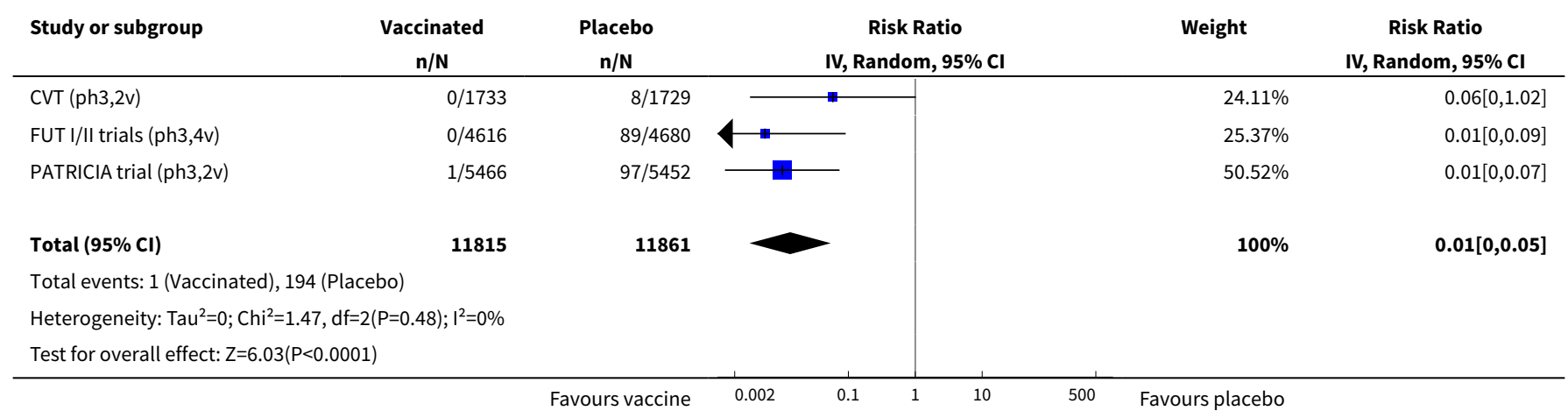

Analysis 1.2. Comparison 1 High-grade cervical lesions in hrHPV DNA negative women at baseline, Outcome 2 CIN2+ associated with HPV6/11/16/18, at least 1 dose.

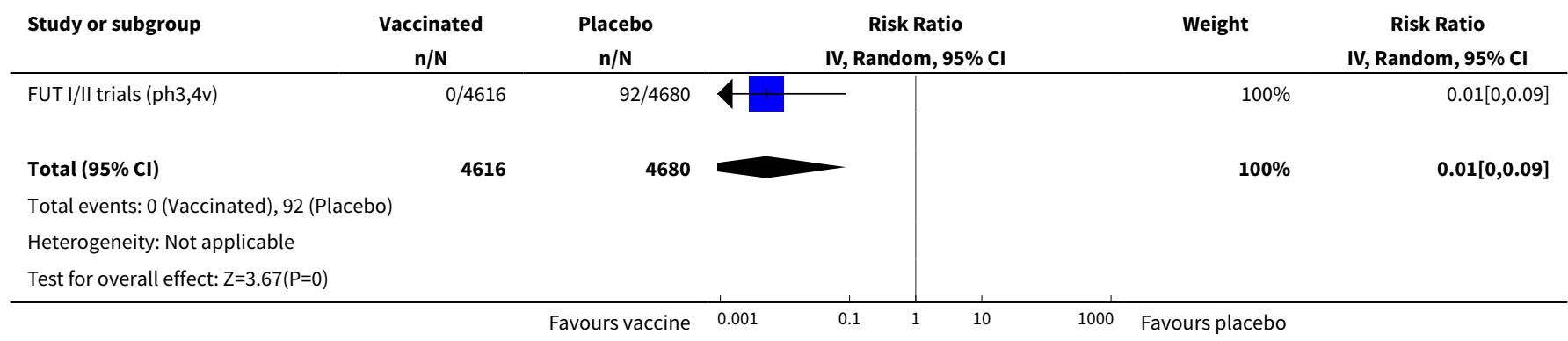


Analysis 1.3. Comparison 1 High-grade cervical lesions in hrHPV DNA negative women at baseline, Outcome 3 CIN3+ associated with HPV16/18, at least 1 dose.

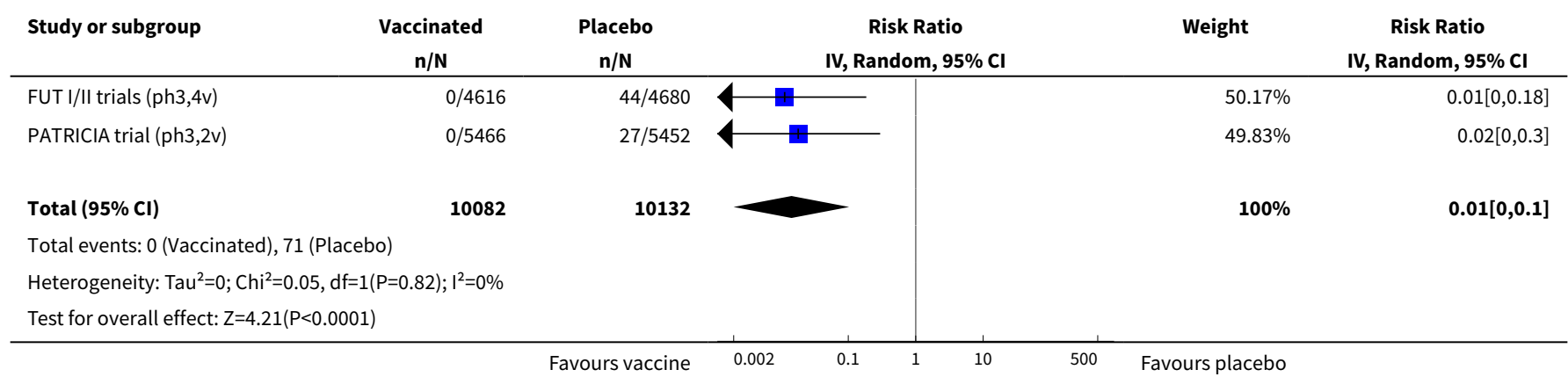

Analysis 1.4. Comparison 1 High-grade cervical lesions in hrHPV DNA negative women at baseline, Outcome 4 CIN3+ associated with HPV6/11/16/18, at least 1 dose.

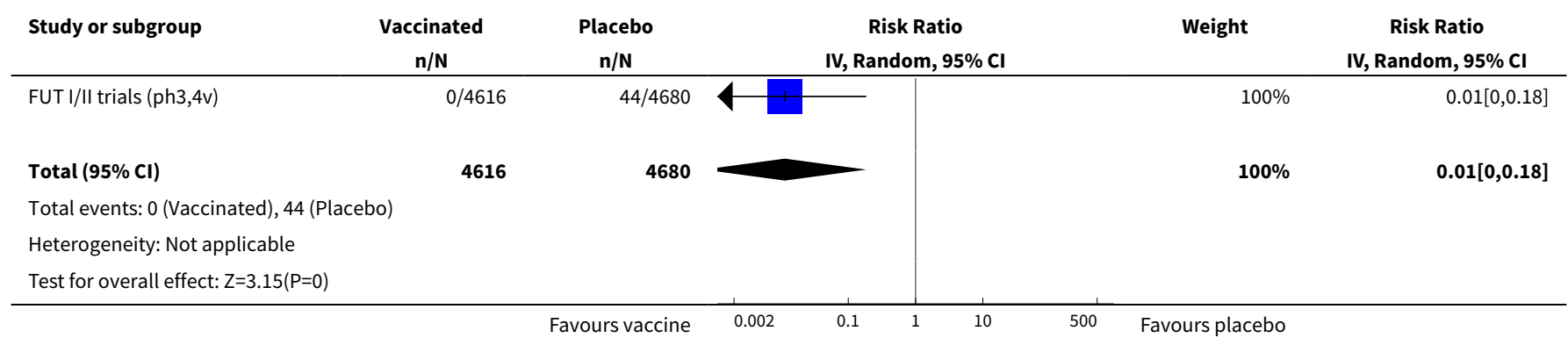

Analysis 1.5. Comparison 1 High-grade cervical lesions in hrHPV DNA negative women at baseline, Outcome 5 AIS associated with HPV16/18, at least 1 dose.

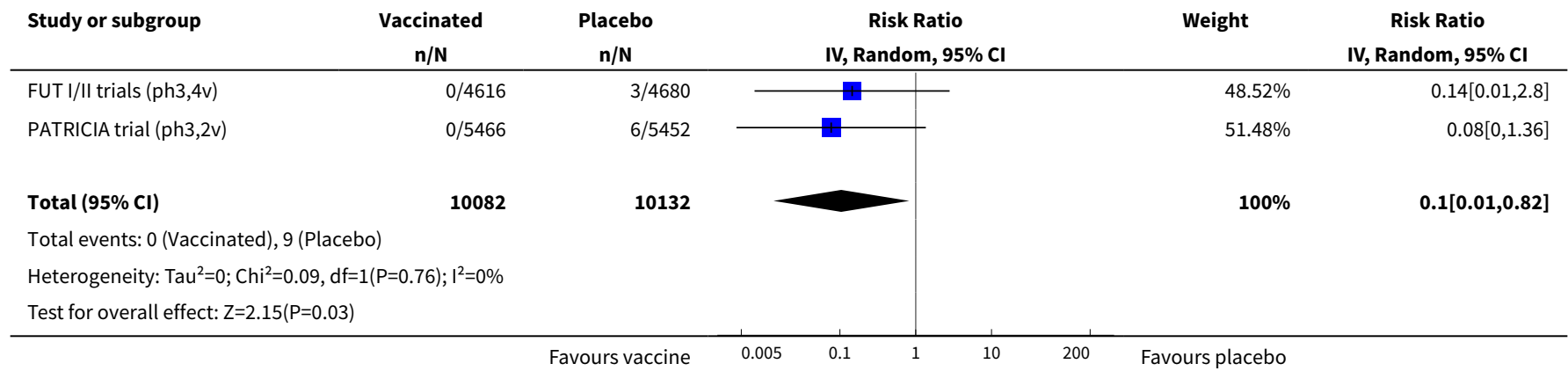

Analysis 1.6. Comparison 1 High-grade cervical lesions in hrHPV DNA negative women at baseline, Outcome 6 AIS associated with HPV6/11/16/18, at least 1 dose.

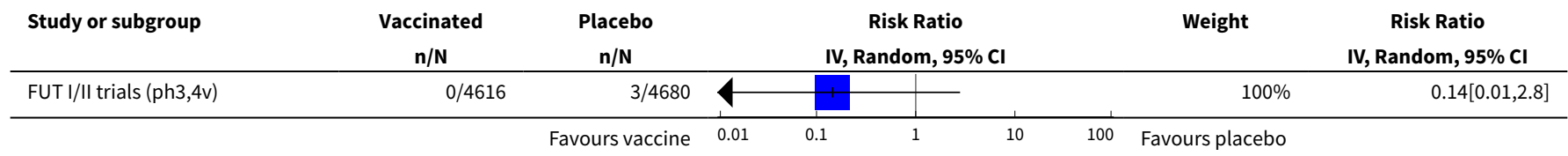




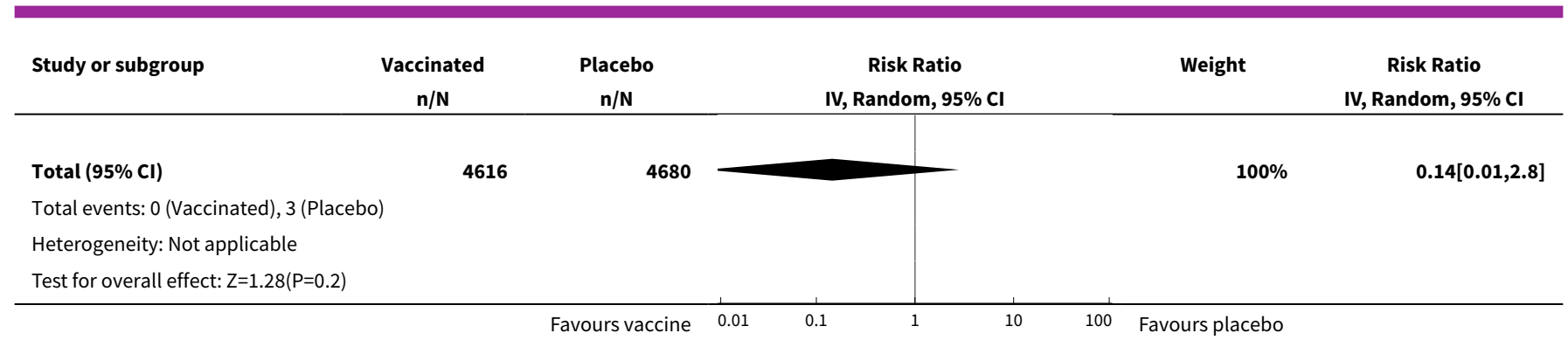

Analysis 1.7. Comparison 1 High-grade cervical lesions in hrHPV DNA negative women at baseline, Outcome 7 Any CIN2+ irrespective of HPV types, at least 1 dose.

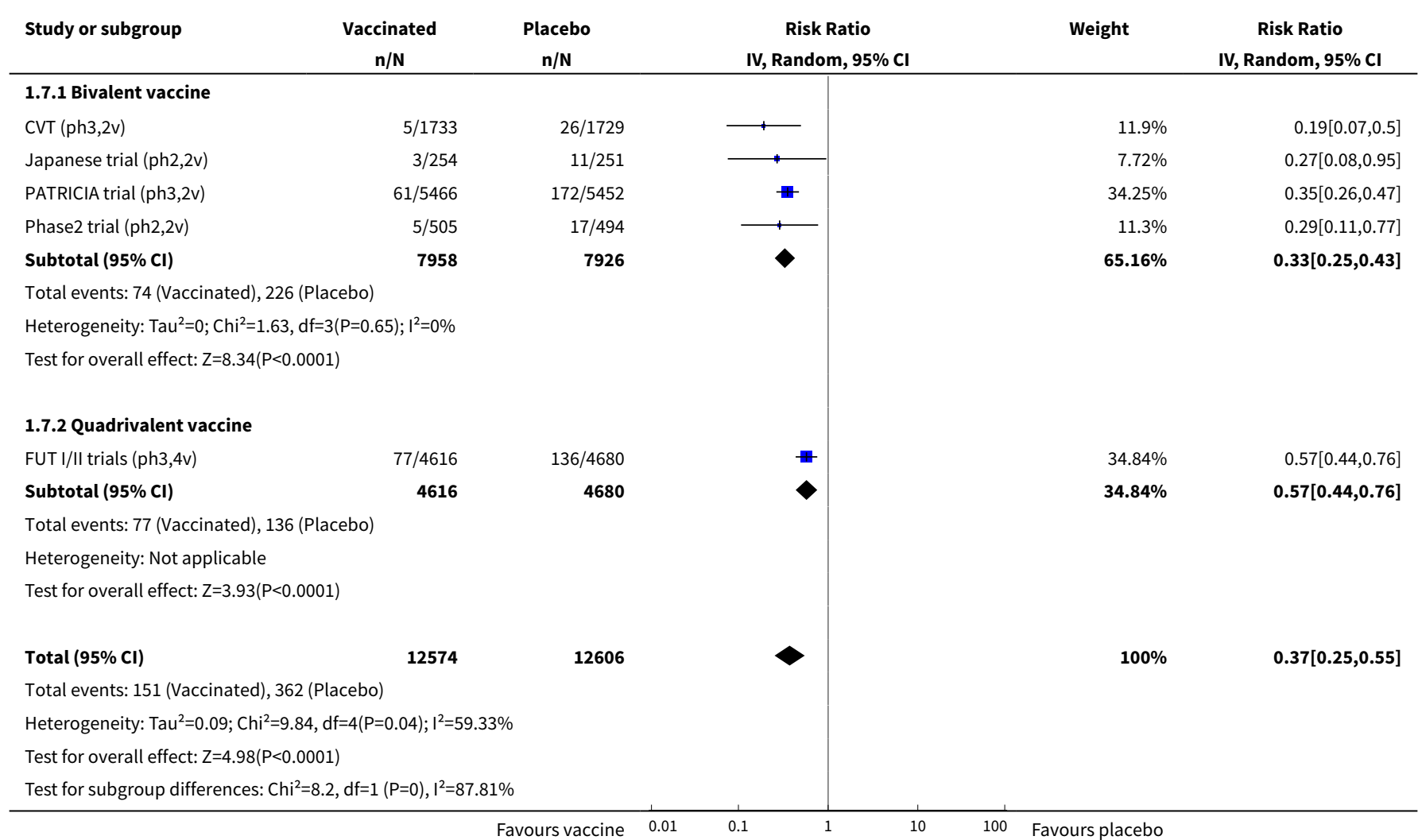

Analysis 1.8. Comparison 1 High-grade cervical lesions in hrHPV DNA negative women at baseline, Outcome 8 Any CIN3+ irrespective of HPV types, at least 1 dose.

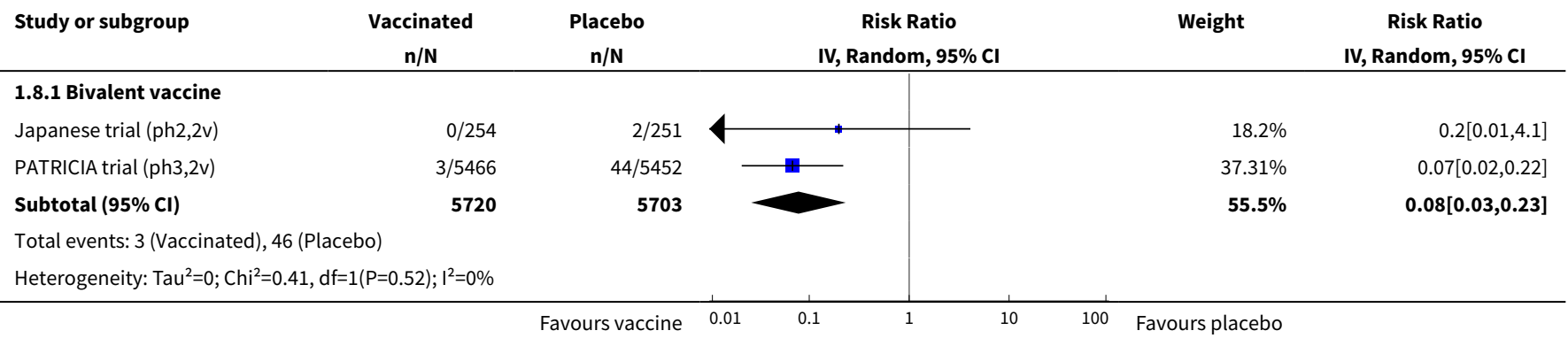




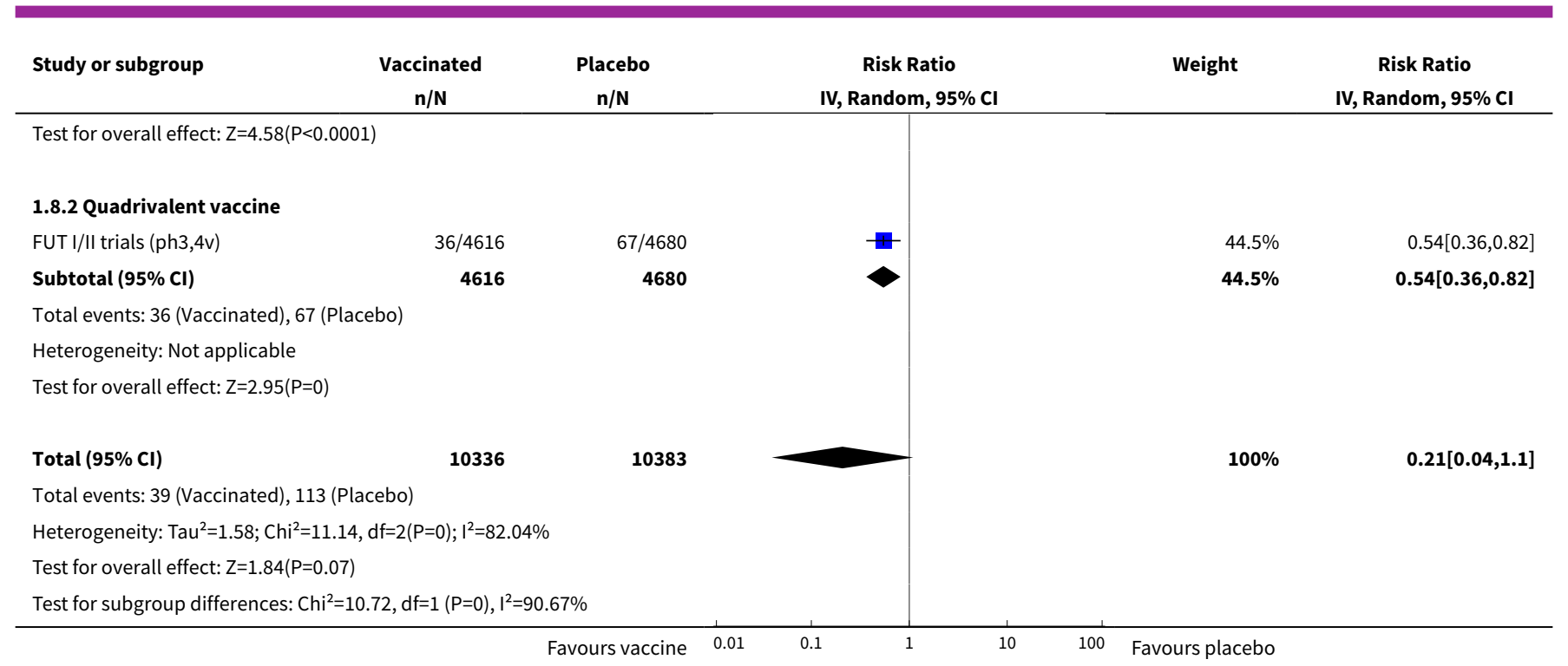

Analysis 1.9. Comparison 1 High-grade cervical lesions in hrHPV DNA negative women at baseline, Outcome 9 Any AIS irrespective of HPV types, at least 1 dose.

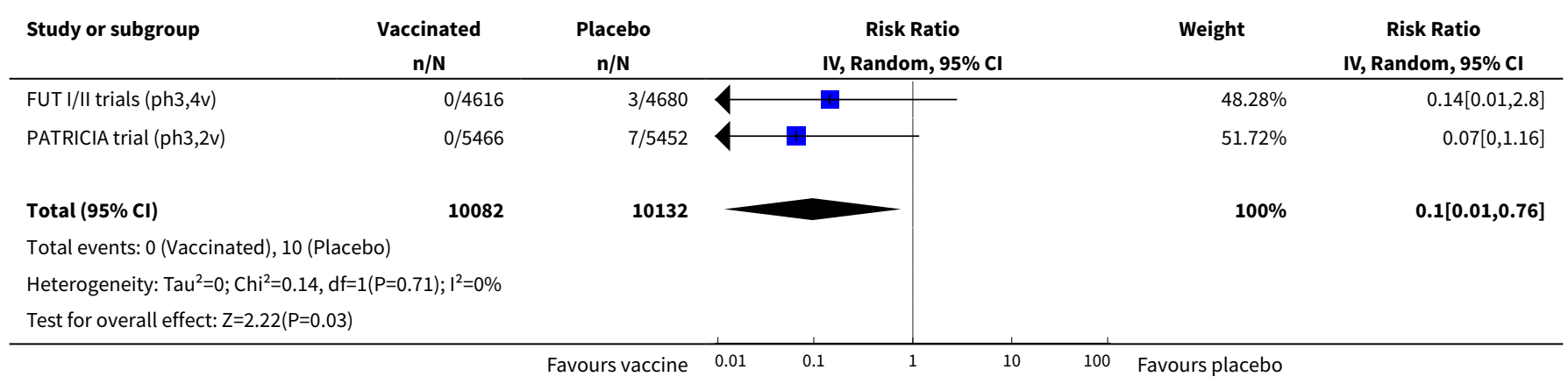

\section{Comparison 2. High-grade cervical lesions in HPV16/18 DNA negative women at baseline}

\begin{tabular}{|c|c|c|c|c|}
\hline Outcome or subgroup title & No. of studies & $\begin{array}{l}\text { No. of partici- } \\
\text { pants }\end{array}$ & Statistical method & Effect size \\
\hline $\begin{array}{l}1 \text { CIN2+ associated with HPV16/(18), } 3 \\
\text { doses }\end{array}$ & 8 & 43376 & $\begin{array}{l}\text { Risk Ratio (IV, Random, 95\% } \\
\mathrm{Cl} \text { ) }\end{array}$ & $0.08[0.04,0.16]$ \\
\hline 1.1 Age group $15-26$ years & 6 & 36579 & $\begin{array}{l}\text { Risk Ratio (IV, Random, 95\% } \\
\mathrm{CI} \text { ) }\end{array}$ & $0.07[0.03,0.15]$ \\
\hline 1.2 Age group $24-45$ years & 2 & 6797 & $\begin{array}{l}\text { Risk Ratio (IV, Random, 95\% } \\
\text { CI) }\end{array}$ & $0.16[0.04,0.74]$ \\
\hline $\begin{array}{l}2 \text { CIN2+ associated with HPV16/(18), at } \\
\text { least } 1 \text { dose }\end{array}$ & 8 & 42030 & $\begin{array}{l}\text { Risk Ratio (IV, Random, 95\% } \\
\text { Cl) }\end{array}$ & $0.10[0.05,0.20]$ \\
\hline 2.1 Age group $15-26$ years & 6 & 34478 & $\begin{array}{l}\text { Risk Ratio (IV, Random, 95\% } \\
\text { Cl) }\end{array}$ & $0.05[0.03,0.10]$ \\
\hline
\end{tabular}




\begin{tabular}{|c|c|c|c|c|}
\hline Outcome or subgroup title & No. of studies & $\begin{array}{l}\text { No. of partici- } \\
\text { pants }\end{array}$ & Statistical method & Effect size \\
\hline 2.2 Age group $24-45$ years & 2 & 7552 & $\begin{array}{l}\text { Risk Ratio (IV, Random, 95\% } \\
\mathrm{CI})\end{array}$ & $0.30[0.11,0.81]$ \\
\hline $\begin{array}{l}3 \text { CIN2+ associated with HPV16/(18), } 1 \\
\text { or } 2 \text { doses (post hoc analysis) }\end{array}$ & 7 & 3713 & $\begin{array}{l}\text { Risk Ratio (IV, Random, 95\% } \\
\mathrm{CI})\end{array}$ & $0.19[0.07,0.51]$ \\
\hline 3.1 women age $15-26$ years & 5 & 2958 & $\begin{array}{l}\text { Risk Ratio (IV, Random, 95\% } \\
\mathrm{CI})\end{array}$ & $0.10[0.04,0.26]$ \\
\hline 3.2 women age $24-45$ years & 2 & 755 & $\begin{array}{l}\text { Risk Ratio (IV, Random, 95\% } \\
\text { Cl) }\end{array}$ & $0.61[0.14,2.67]$ \\
\hline $\begin{array}{l}4 \text { CIN2+ associated with } \\
\text { HPV6/11/16/18, } 3 \text { doses }\end{array}$ & 2 & 7664 & $\begin{array}{l}\text { Risk Ratio (IV, Random, 95\% } \\
\mathrm{Cl} \text { ) }\end{array}$ & $0.06[0.01,0.61]$ \\
\hline 4.1 Age group $15-26$ years & 1 & 4499 & $\begin{array}{l}\text { Risk Ratio (IV, Random, 95\% } \\
\mathrm{Cl} \text { ) }\end{array}$ & $0.02[0.00,0.25]$ \\
\hline 4.2 Age group $24-45$ years & 1 & 3165 & $\begin{array}{l}\text { Risk Ratio (IV, Random, 95\% } \\
\mathrm{CI})\end{array}$ & $0.17[0.02,1.39]$ \\
\hline $\begin{array}{l}5 \text { CIN2+ associated with } \\
\text { HPV6/11/16/18, at least } 1 \text { dose }\end{array}$ & 2 & 8980 & $\begin{array}{l}\text { Risk Ratio (IV, Random, 95\% } \\
\text { Cl) }\end{array}$ & $0.08[0.00,2.41]$ \\
\hline 5.1 Age group $15-26$ years & 1 & 5351 & $\begin{array}{l}\text { Risk Ratio (IV, Random, 95\% } \\
\text { Cl) }\end{array}$ & $0.01[0.00,0.19]$ \\
\hline 5.2 Age group 24-45 years & 1 & 3629 & $\begin{array}{l}\text { Risk Ratio (IV, Random, 95\% } \\
\text { Cl) }\end{array}$ & $0.37[0.10,1.41]$ \\
\hline $\begin{array}{l}6 \text { CIN2+ associated with } \\
\text { HPV6/11/16/18, } 1 \text { or } 2 \text { doses (post hoc } \\
\text { analysis) }\end{array}$ & 2 & 1316 & $\begin{array}{l}\text { Risk Ratio (IV, Random, 95\% } \\
\mathrm{CI})\end{array}$ & $0.24[0.01,5.00]$ \\
\hline 6.1 Age group $15-26$ years & 1 & 852 & $\begin{array}{l}\text { Risk Ratio (IV, Random, 95\% } \\
\mathrm{Cl} \text { ) }\end{array}$ & $0.04[0.00,0.74]$ \\
\hline 6.2 Age group $24-45$ years & 1 & 464 & $\begin{array}{l}\text { Risk Ratio (IV, Random, 95\% } \\
\text { Cl) }\end{array}$ & $0.97[0.14,6.80]$ \\
\hline $\begin{array}{l}7 \text { CIN3+ associated with HPV16/18 or } \\
\text { HPV6/11/16/18, } 3 \text { doses }\end{array}$ & 3 & 29720 & $\begin{array}{l}\text { Risk Ratio (IV, Random, 95\% } \\
\text { Cl) }\end{array}$ & $0.07[0.02,0.29]$ \\
\hline $\begin{array}{l}8 \mathrm{CIN} 3+\text { associated with HPV } 16 / 18 \text { or } \\
\text { HPV6/11/16/18, at least } 1 \text { dose }\end{array}$ & 3 & 33199 & $\begin{array}{l}\text { Risk Ratio (IV, Random, 95\% } \\
\mathrm{Cl} \text { ) }\end{array}$ & $0.05[0.02,0.14]$ \\
\hline $\begin{array}{l}9 \text { CIN3+ associated with HPV } 16 / 18 \text { or } \\
\text { HPV6/11/16/18, } 1 \text { or } 2 \text { doses (post hoc } \\
\text { analysis) }\end{array}$ & 3 & 3479 & $\begin{array}{l}\text { Risk Ratio (IV, Random, 95\% } \\
\mathrm{CI} \text { ) }\end{array}$ & $0.06[0.01,0.24]$ \\
\hline $\begin{array}{l}10 \text { AIS associated with HPV16/18 or } \\
\text { HPV6/11/16/18, } 3 \text { doses }\end{array}$ & 3 & 29707 & $\begin{array}{l}\text { Risk Ratio (IV, Random, 95\% } \\
\mathrm{Cl} \text { ) }\end{array}$ & $0.12[0.02,0.70]$ \\
\hline $\begin{array}{l}11 \text { AIS associated with HPV } 16 / 18 \text { or } \\
6 / 11 / 16 / 18 \text {, at least } 1 \text { dose }\end{array}$ & 2 & 17079 & $\begin{array}{l}\text { Risk Ratio (IV, Random, 95\% } \\
\text { Cl) }\end{array}$ & $0.09[0.01,0.72]$ \\
\hline
\end{tabular}




\begin{tabular}{lllll}
\hline Outcome or subgroup title & No. of studies & $\begin{array}{l}\text { No. of partici- } \\
\text { pants }\end{array}$ & Statistical method & Effect size \\
\hline $\begin{array}{l}\text { 12 AIS associated with HPV16/18 or } \\
\text { HPV6/11/16/18, 1 or 2 doses (post hoc } \\
\text { analysis) }\end{array}$ & 2 & 2015 & $\begin{array}{l}\text { Risk Ratio (IV, Random, 95\% } \\
\text { Cl) }\end{array}$ & 0.15 [0.01, 2.97] \\
\hline $\begin{array}{l}13 \text { Any CIN2+ irrespective of HPV types, } \\
\text { 3 doses }\end{array}$ & 3 & 7320 & $\begin{array}{l}\text { Risk Ratio (IV, Random, 95\% } \\
\text { CI) }\end{array}$ & 0.40 [0.25, 0.64] \\
\hline $\begin{array}{l}14 \text { Any CIN2+ irrespective of HPV types, } \\
\text { at least 1 dose }\end{array}$ & 3 & 19143 & $\begin{array}{l}\text { Risk Ratio (IV, Random, 95\% } \\
\text { Cl) }\end{array}$ & $0.41[0.32,0.52]$ \\
\hline $\begin{array}{l}15 \text { Any CIN2+ irrespective of HPV types, } \\
1 \text { or 2 doses (post hoc analysis) }\end{array}$ & 1 & 34 & $\begin{array}{l}\text { Risk Ratio (IV, Random, 95\% } \\
\text { CI) }\end{array}$ & $0.71[0.15,3.38]$ \\
\hline
\end{tabular}

\section{Analysis 2.1. Comparison 2 High-grade cervical lesions in HPV16/18 DNA negative} women at baseline, Outcome 1 CIN2+ associated with HPV16/(18), 3 doses.

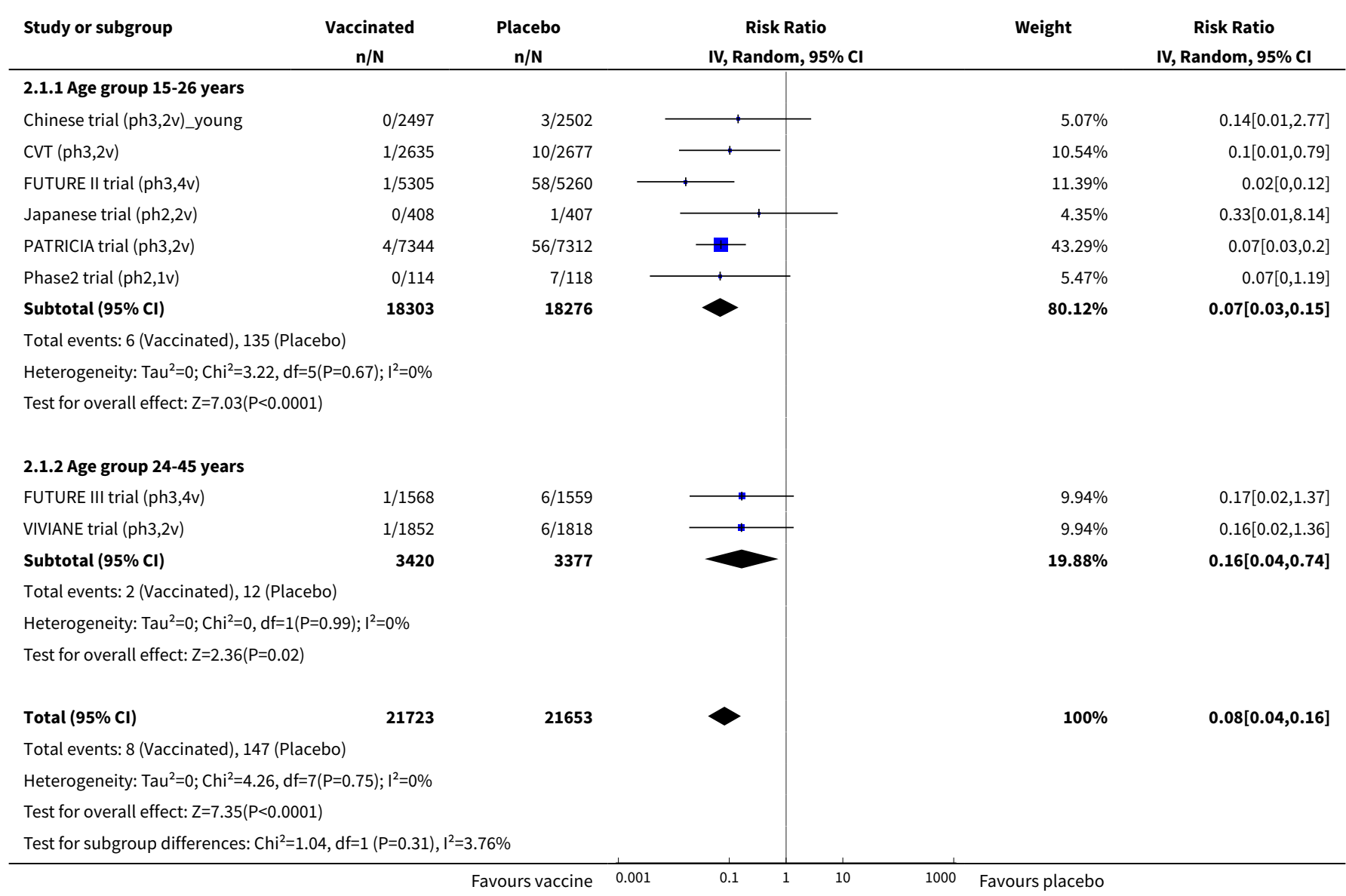


Analysis 2.2. Comparison 2 High-grade cervical lesions in HPV16/18 DNA negative women at baseline, Outcome 2 CIN2+ associated with HPV16/(18), at least 1 dose.

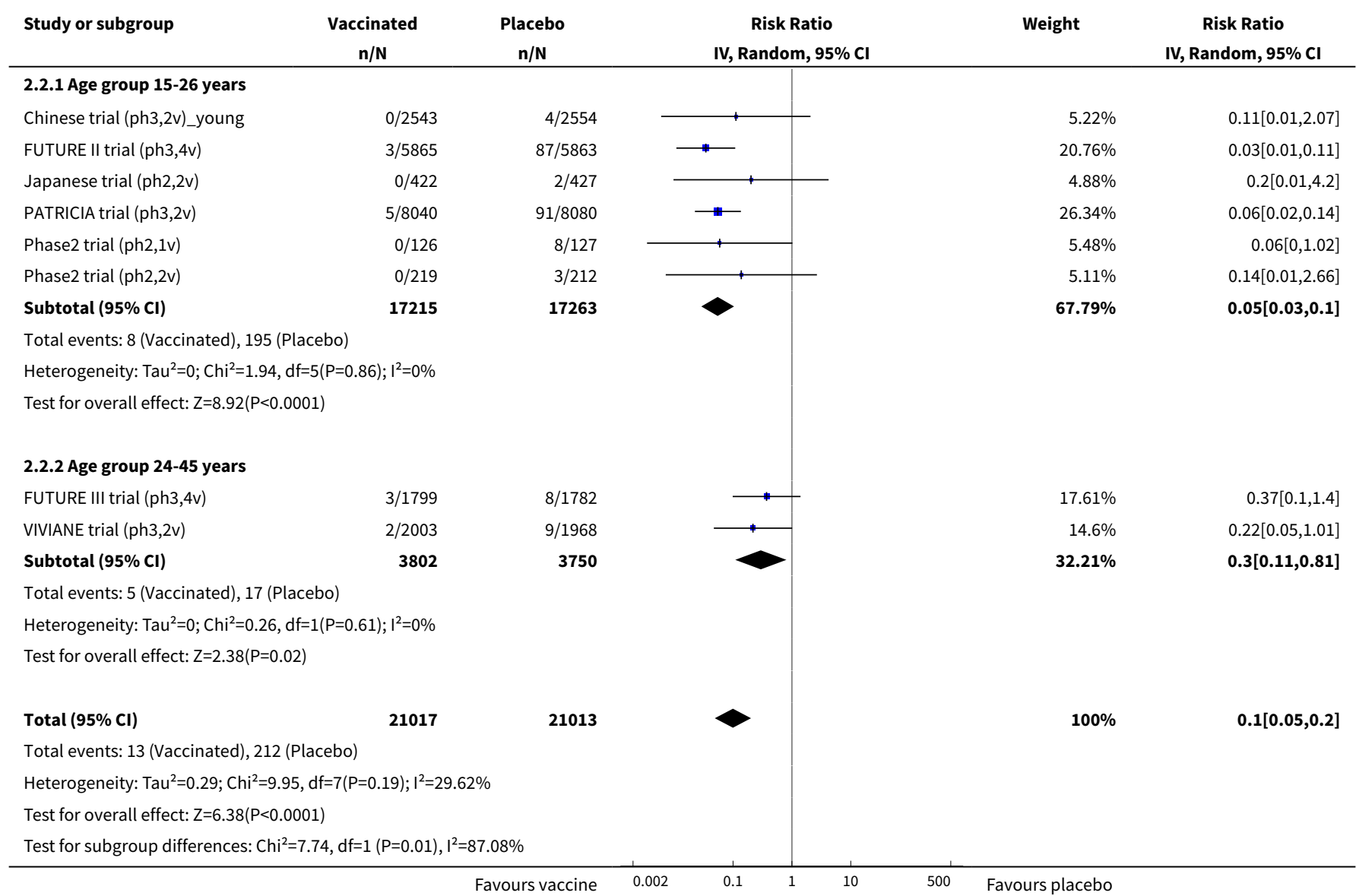

Analysis 2.3. Comparison 2 High-grade cervical lesions in HPV16/18 DNA negative women at baseline, Outcome 3 CIN2+ associated with HPV16/(18), 1 or 2 doses (post hoc analysis).

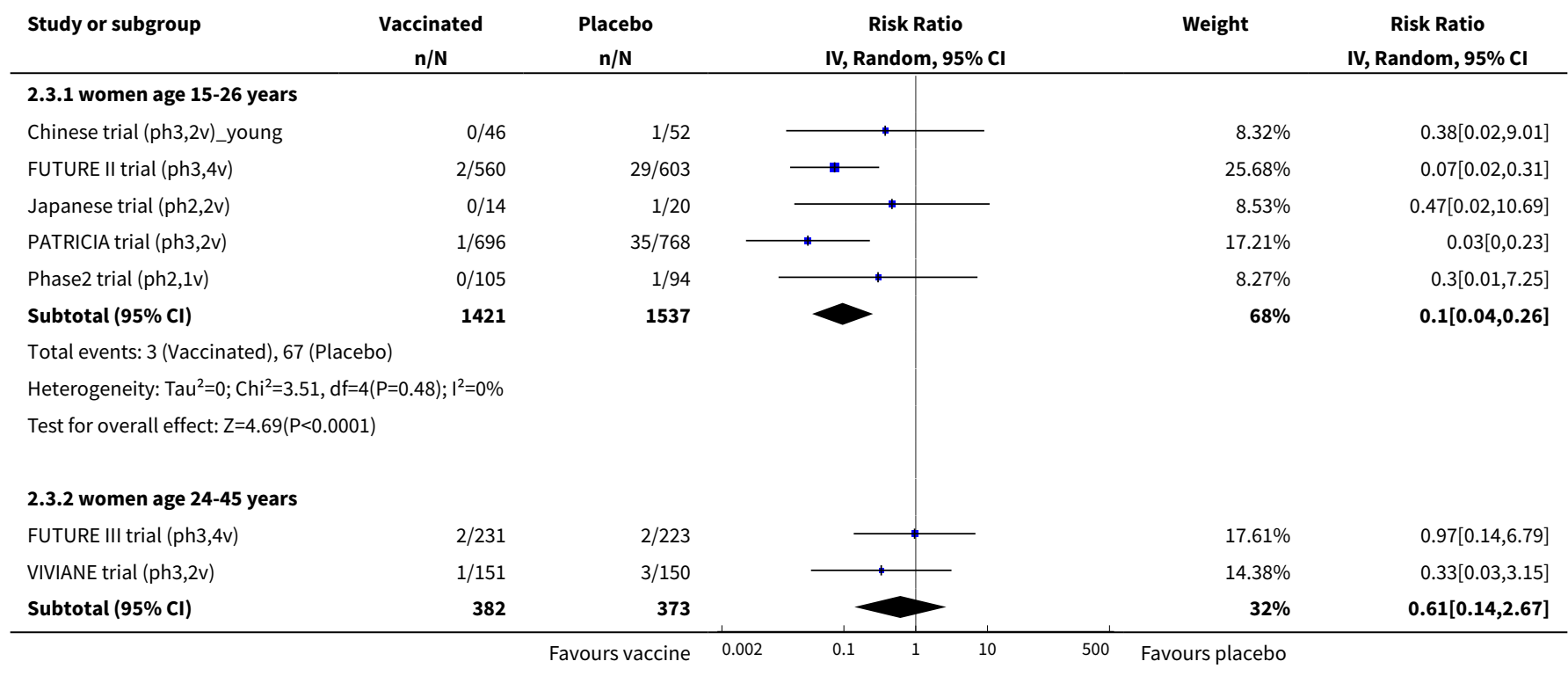




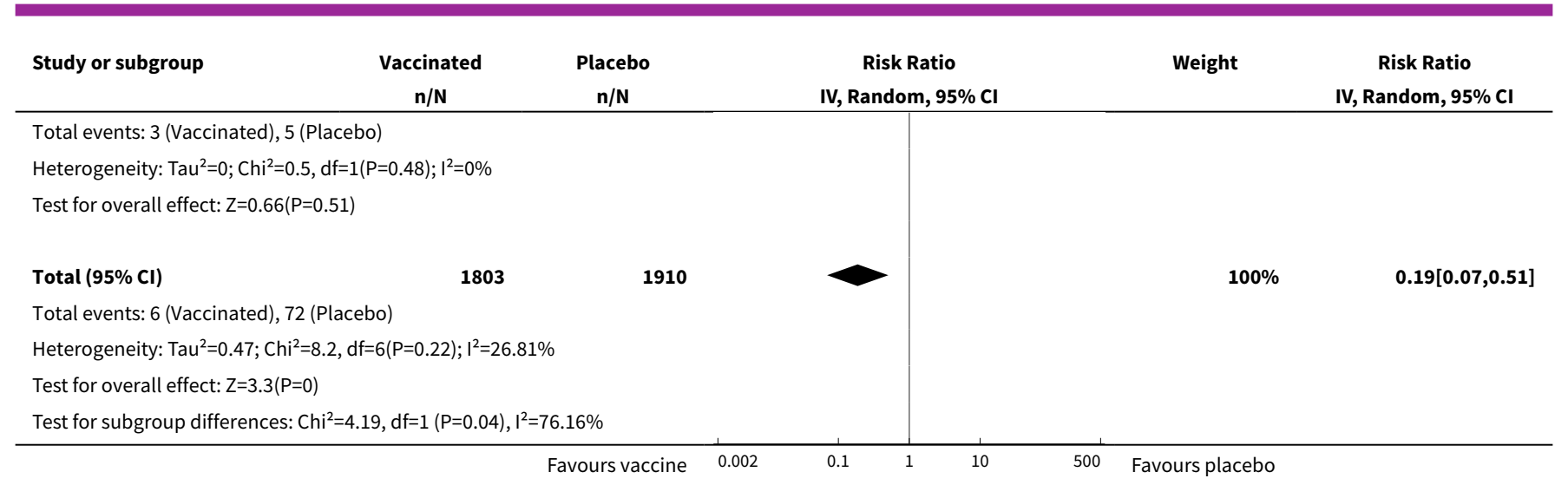

Analysis 2.4. Comparison 2 High-grade cervical lesions in HPV16/18 DNA negative women at baseline, Outcome 4 CIN2+ associated with HPV6/11/16/18, 3 doses.

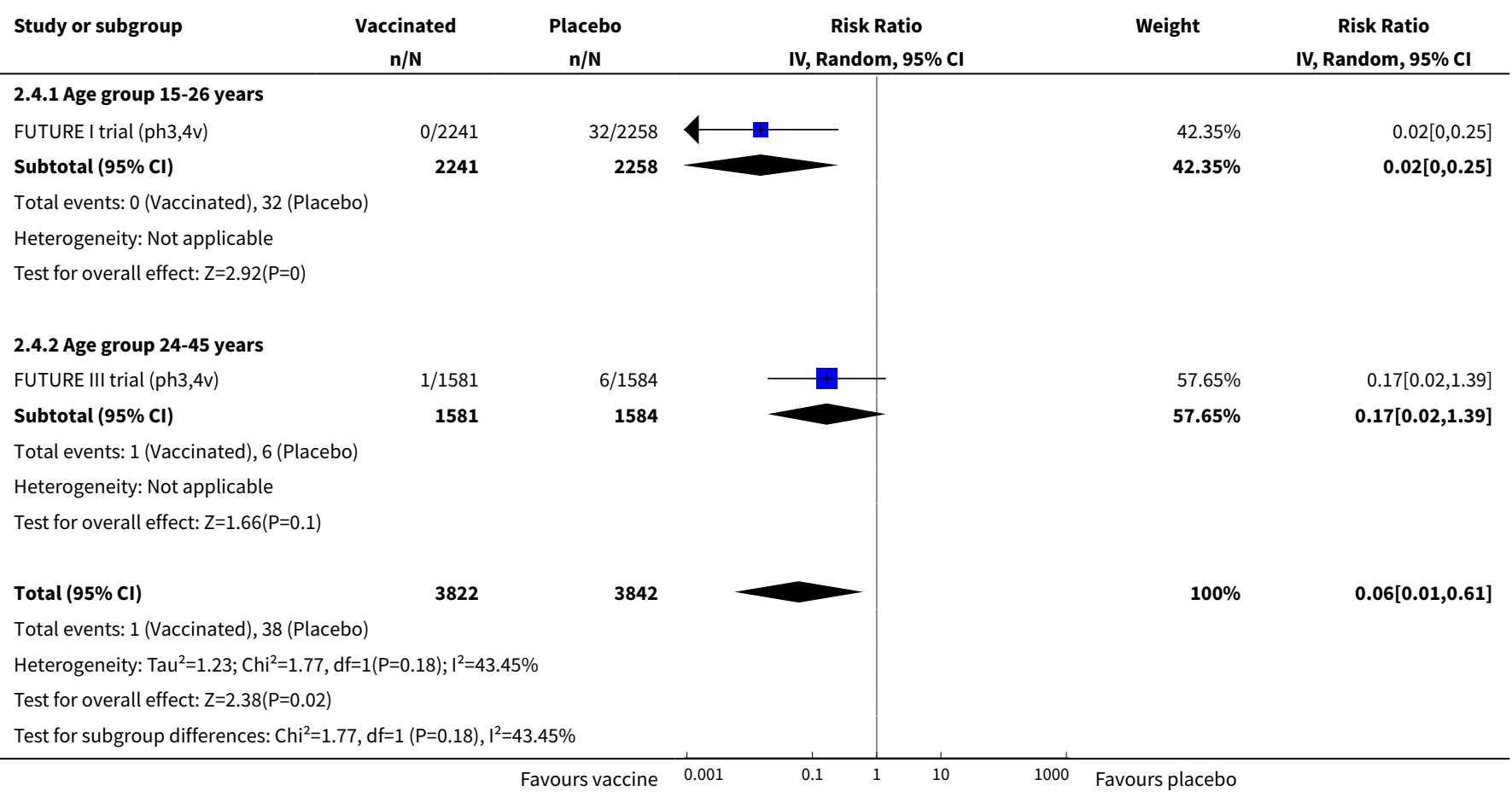

Analysis 2.5. Comparison 2 High-grade cervical lesions in HPV16/18 DNA negative women at baseline, Outcome 5 CIN2+ associated with HPV6/11/16/18, at least 1 dose.

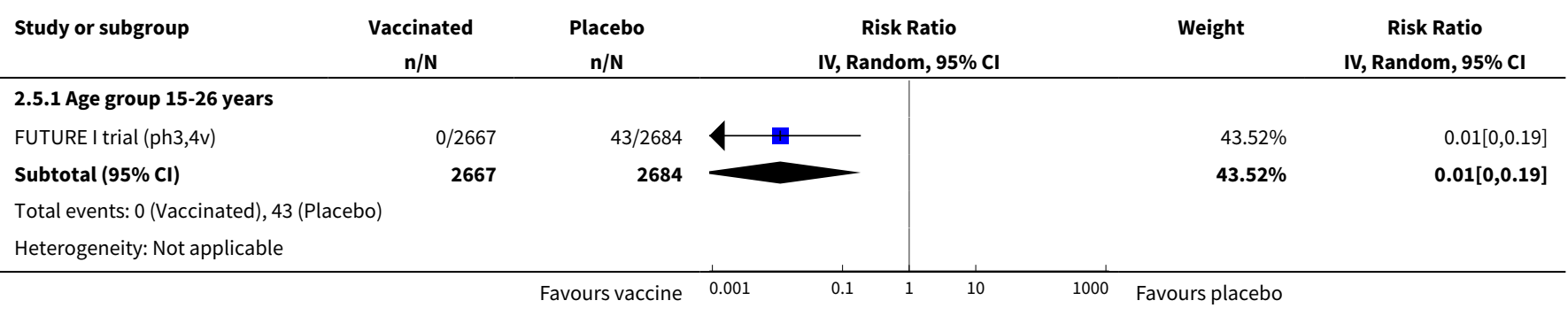




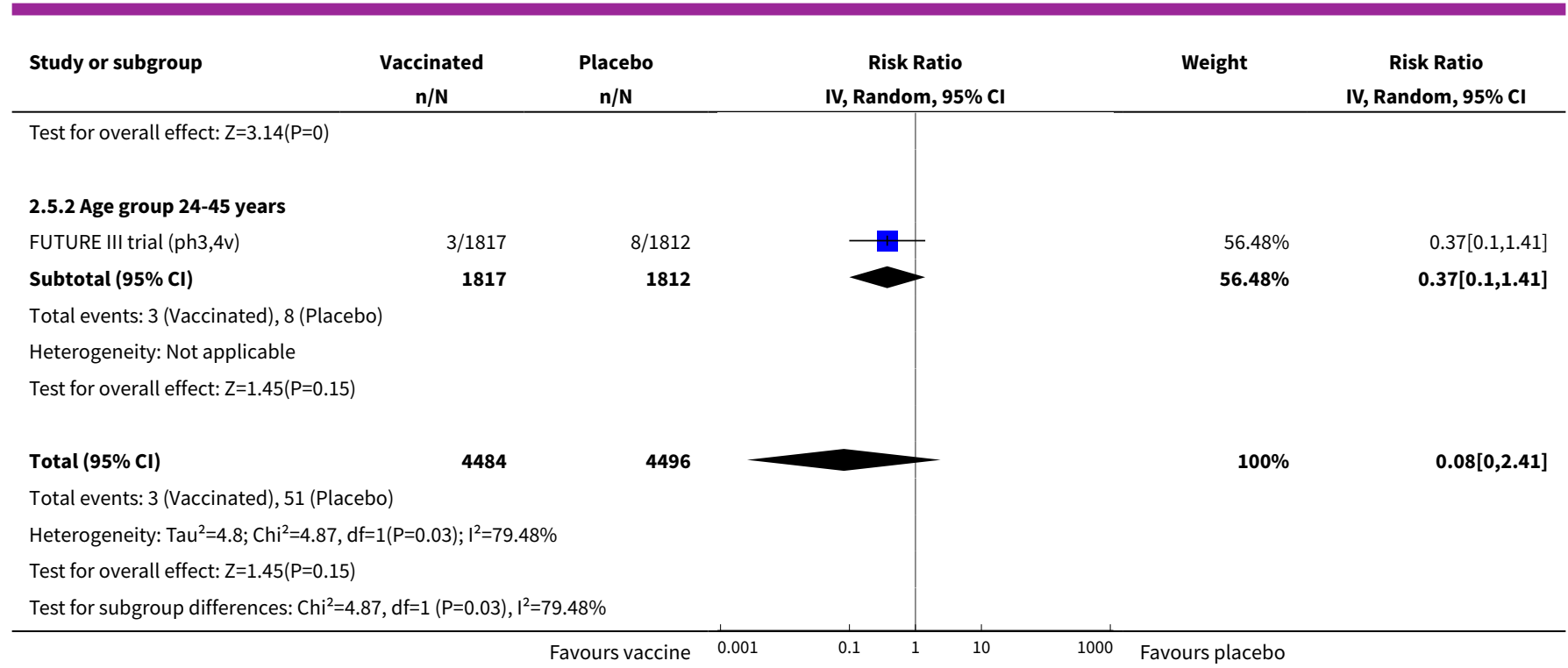

\section{Analysis 2.6. Comparison 2 High-grade cervical lesions in HPV16/18 DNA negative women at baseline, Outcome 6 CIN2+ associated with HPV6/11/16/18, 1 or 2 doses (post hoc analysis).}

\begin{tabular}{|c|c|c|c|c|c|}
\hline Study or subgroup & $\begin{array}{c}\text { Vaccinated } \\
n / N \\
\end{array}$ & $\begin{array}{c}\text { Placebo } \\
n / N\end{array}$ & $\begin{array}{c}\text { Risk Ratio } \\
\text { IV, Random, 95\% CI }\end{array}$ & Weight & $\begin{array}{c}\text { Risk Ratio } \\
\text { IV, Random, } 95 \% \text { CI }\end{array}$ \\
\hline \multicolumn{6}{|c|}{ 2.6.1 Age group $15-26$ years } \\
\hline FUTURE I trial (ph3,4v) & $0 / 426$ & $11 / 426$ & - & $44.33 \%$ & $0.04[0,0.74]$ \\
\hline Subtotal $(95 \% \mathrm{Cl})$ & 426 & 426 & & $44.33 \%$ & $0.04[0,0.74]$ \\
\hline \multicolumn{6}{|c|}{ Total events: 0 (Vaccinated), 11 (Placebo) } \\
\hline \multicolumn{6}{|c|}{ Heterogeneity: Not applicable } \\
\hline \multicolumn{6}{|c|}{ 2.6.2 Age group 24-45 years } \\
\hline FUTURE III trial (ph3,4v) & $2 / 236$ & $2 / 228$ & & $55.67 \%$ & $0.97[0.14,6.8]$ \\
\hline Subtotal $(95 \% \mathrm{Cl})$ & 236 & 228 & & $55.67 \%$ & $0.97[0.14,6.8]$ \\
\hline \multicolumn{6}{|c|}{ Total events: 2 (Vaccinated), 2 (Placebo) } \\
\hline \multicolumn{6}{|c|}{ Heterogeneity: Not applicable } \\
\hline Total $(95 \% \mathrm{Cl})$ & 662 & 654 & & $100 \%$ & $0.24[0.01,5]$ \\
\hline \multicolumn{6}{|c|}{ Total events: 2 (Vaccinated), 13 (Placebo) } \\
\hline \multicolumn{6}{|c|}{ Heterogeneity: $\operatorname{Tau}^{2}=3.27 ; \mathrm{Chi}^{2}=3.13, \mathrm{df}=1(\mathrm{P}=0.08) ; \mathrm{I}^{2}=68.04 \%$} \\
\hline \multicolumn{6}{|c|}{ Test for overall effect: $Z=0.91(P=0.36)$} \\
\hline Test for subgroup differen & $3, d f=1(P=0.08)$, & $04 \%$ & & & \\
\hline
\end{tabular}


Analysis 2.7. Comparison 2 High-grade cervical lesions in HPV16/18 DNA negative women at baseline, Outcome 7 CIN3+ associated with HPV16/18 or HPV6/11/16/18, 3 doses.

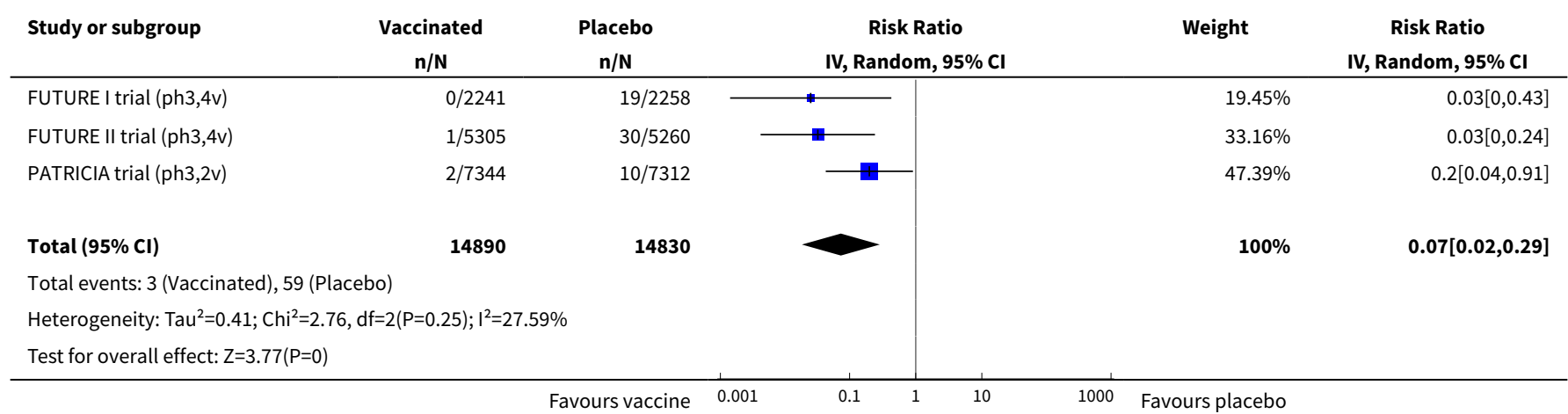

Analysis 2.8. Comparison 2 High-grade cervical lesions in HPV16/18 DNA negative women at baseline, Outcome $8 \mathrm{CIN3}+$ associated with HPV 16/18 or HPV6/11/16/18, at least 1 dose.

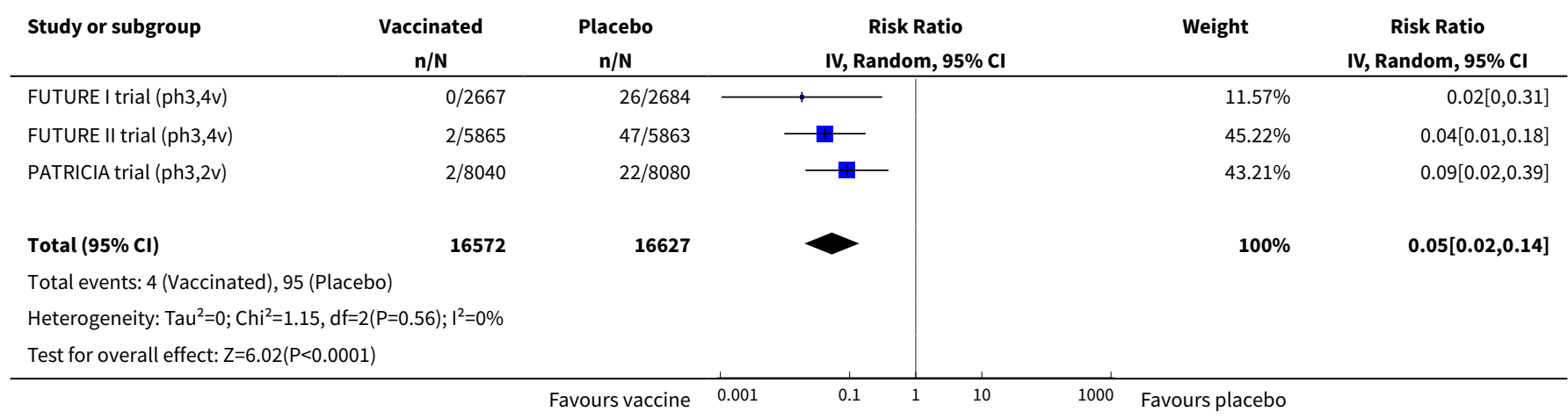

Analysis 2.9. Comparison 2 High-grade cervical lesions in HPV16/18 DNA negative women at baseline, Outcome 9 CIN3+ associated with HPV16/18 or HPV6/11/16/18, 1 or 2 doses (post hoc analysis).

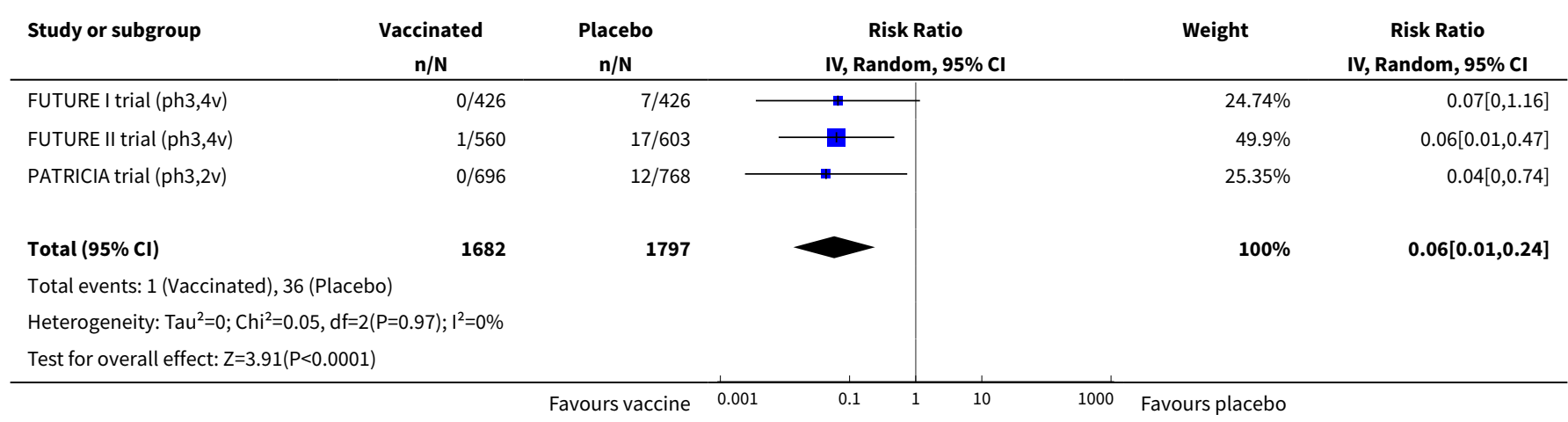


Analysis 2.10. Comparison 2 High-grade cervical lesions in HPV16/18 DNA negative women at baseline, Outcome 10 AIS associated with HPV16/18 or HPV6/11/16/18, 3 doses.

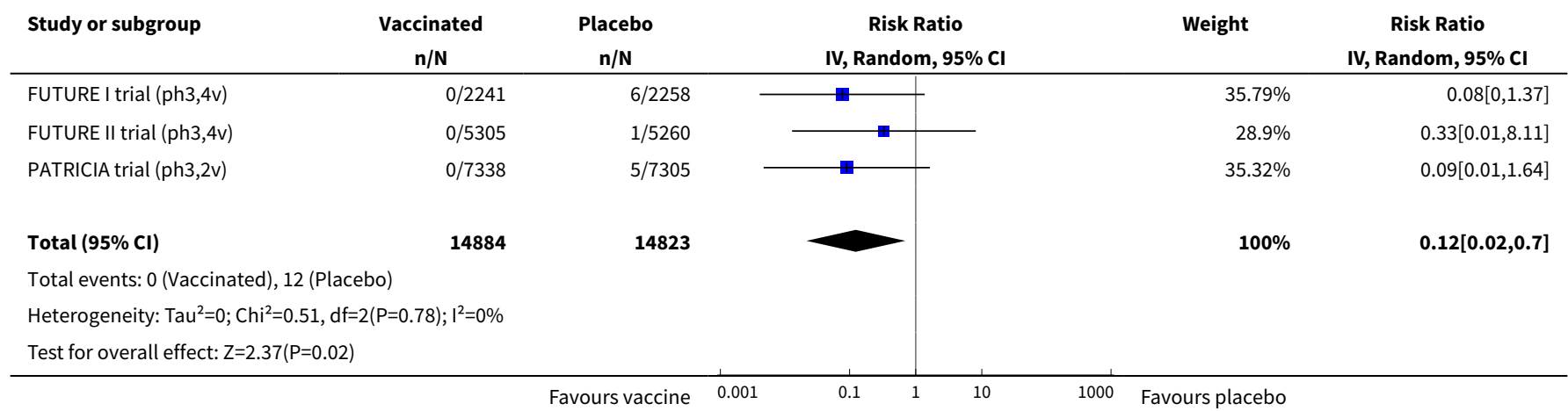

Analysis 2.11. Comparison 2 High-grade cervical lesions in HPV16/18 DNA negative women at baseline, Outcome 11 AIS associated with HPV16/18 or 6/11/16/18, at least 1 dose.

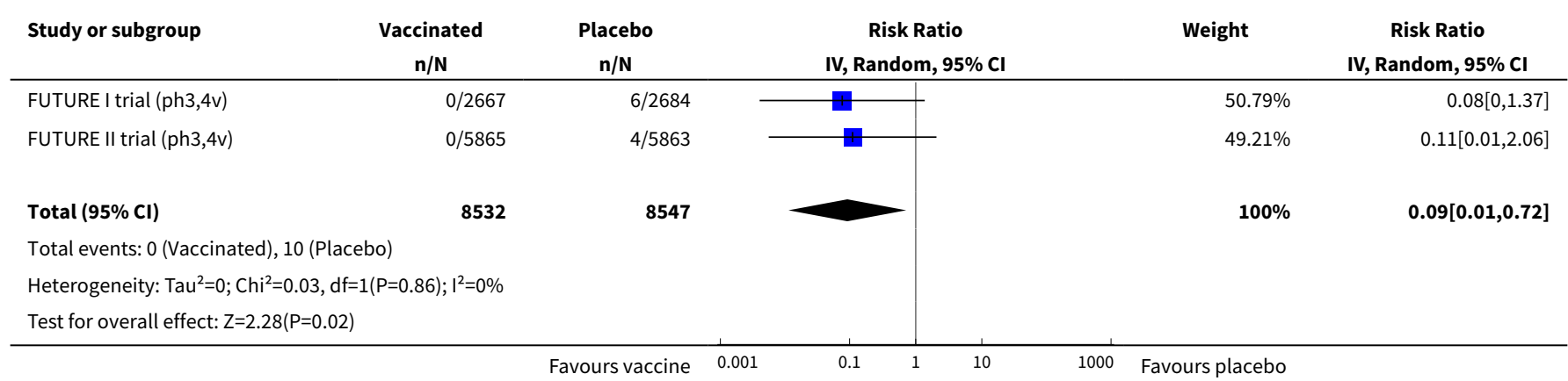

Analysis 2.12. Comparison 2 High-grade cervical lesions in HPV16/18 DNA negative women at baseline, Outcome 12 AIS associated with HPV16/18 or HPV6/11/16/18, 1 or 2 doses (post hoc analysis).

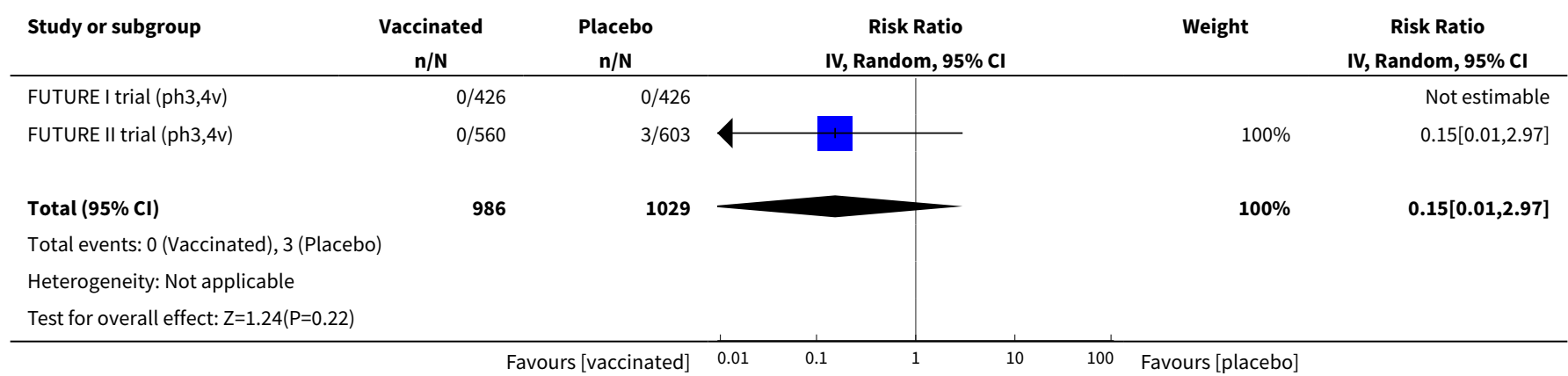


Analysis 2.13. Comparison 2 High-grade cervical lesions in HPV16/18 DNA negative women at baseline, Outcome 13 Any CIN2+ irrespective of HPV types, 3 doses.

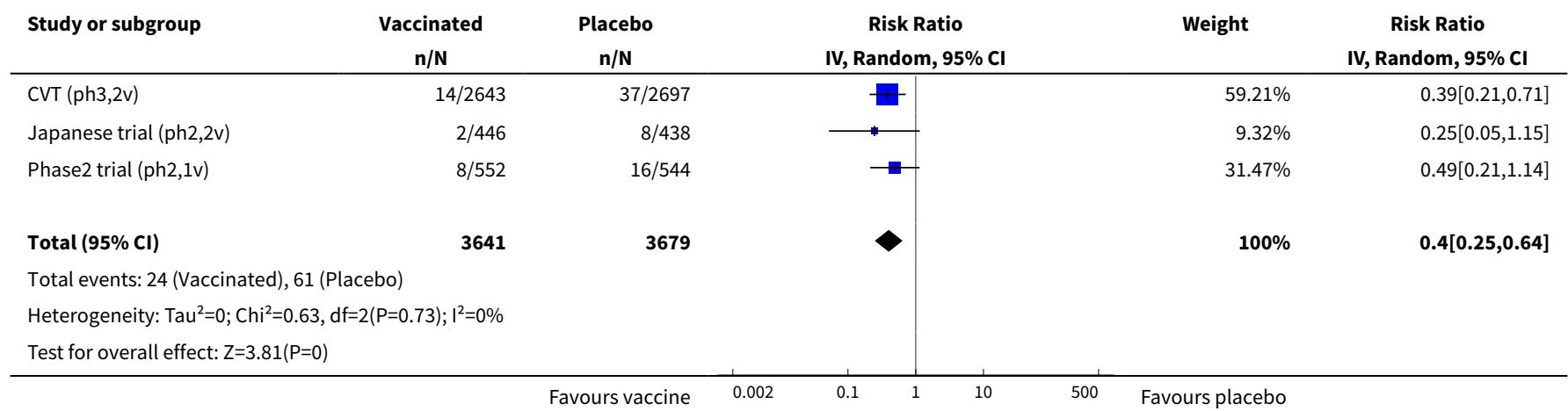

Analysis 2.14. Comparison 2 High-grade cervical lesions in HPV16/18 DNA negative women at baseline, Outcome 14 Any CIN2+ irrespective of HPV types, at least 1 dose.

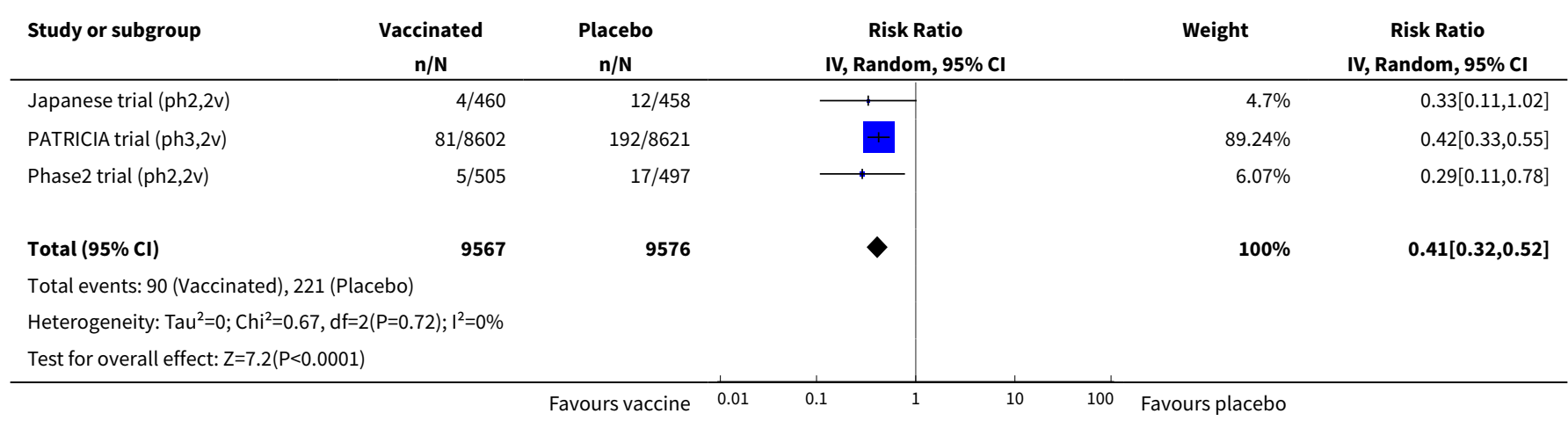

Analysis 2.15. Comparison 2 High-grade cervical lesions in HPV16/18 DNA negative women at baseline, Outcome 15 Any CIN2+ irrespective of HPV types, 1 or 2 doses (post hoc analysis).

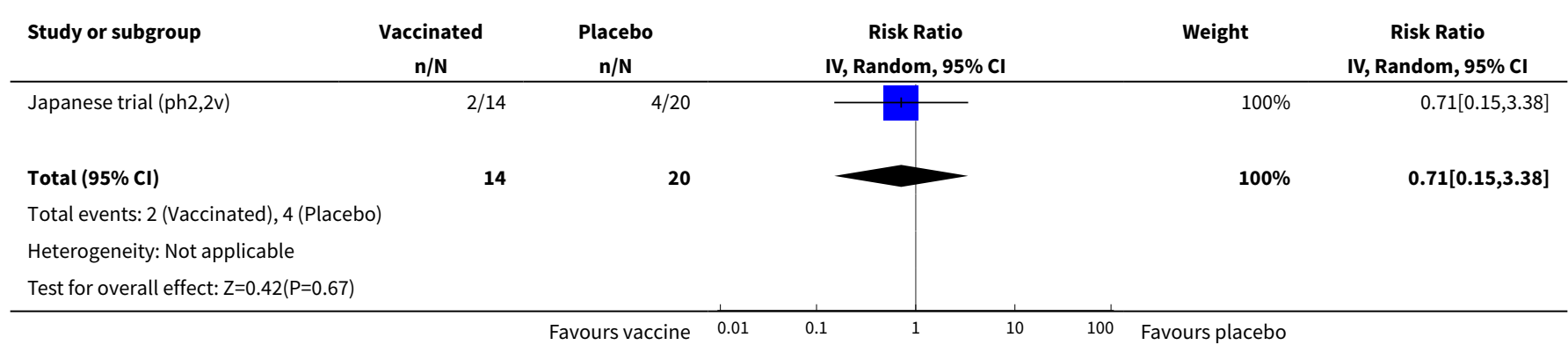


Comparison 3. High-grade cervical lesions in women regardless of baseline HPV DNA status

\begin{tabular}{|c|c|c|c|c|}
\hline Outcome or subgroup title & No. of studies & $\begin{array}{l}\text { No. of partici- } \\
\text { pants }\end{array}$ & Statistical method & Effect size \\
\hline $\begin{array}{l}1 \mathrm{CIN} 2+\text { associated with HPV16/18, at } \\
\text { least } 1 \text { dose }\end{array}$ & 5 & 44052 & $\begin{array}{l}\text { Risk Ratio (IV, Random, 95\% } \\
\mathrm{Cl} \text { ) }\end{array}$ & $0.52[0.41,0.67]$ \\
\hline 1.1 Age group $15-26$ years & 3 & 34852 & $\begin{array}{l}\text { Risk Ratio (IV, Random, 95\% } \\
\mathrm{CI})\end{array}$ & $0.46[0.37,0.57]$ \\
\hline 1.2 Age group $24-45$ years & 2 & 9200 & $\begin{array}{l}\text { Risk Ratio (IV, Random, 95\% } \\
\mathrm{Cl} \text { ) }\end{array}$ & $0.74[0.52,1.05]$ \\
\hline $\begin{array}{l}2 \text { CIN2+ associated with } \\
\text { HPV6/11/16/18, at least } 1 \text { dose }\end{array}$ & 2 & 20883 & $\begin{array}{l}\text { Risk Ratio (IV, Random, 95\% } \\
\mathrm{Cl} \text { ) }\end{array}$ & $0.57[0.38,0.86]$ \\
\hline 2.1 Age group $15-26$ years & 1 & 17160 & $\begin{array}{l}\text { Risk Ratio (IV, Random, 95\% } \\
\mathrm{Cl} \text { ) }\end{array}$ & $0.50[0.42,0.59]$ \\
\hline 2.2 Age group $24-45$ years & 1 & 3723 & $\begin{array}{l}\text { Risk Ratio (IV, Random, 95\% } \\
\mathrm{Cl} \text { ) }\end{array}$ & $0.78[0.44,1.37]$ \\
\hline $\begin{array}{l}3 \text { CIN3+ associated with HPV16/18, at } \\
\text { least } 1 \text { dose }\end{array}$ & 2 & 34562 & $\begin{array}{l}\text { Risk Ratio (IV, Random, 95\% } \\
\mathrm{Cl} \text { ) }\end{array}$ & $0.55[0.45,0.67]$ \\
\hline $\begin{array}{l}4 \text { CIN3+ associated with } \\
\text { HPV6/11/16/18, at least } 1 \text { dose }\end{array}$ & 1 & 17160 & $\begin{array}{l}\text { Risk Ratio (IV, Random, 95\% } \\
\mathrm{CI})\end{array}$ & $0.54[0.43,0.68]$ \\
\hline $\begin{array}{l}5 \text { AIS associated with HPV16/18, at } \\
\text { least } 1 \text { dose }\end{array}$ & 2 & 34562 & $\begin{array}{l}\text { Risk Ratio (IV, Random, 95\% } \\
\mathrm{CI} \text { ) }\end{array}$ & $0.36[0.17,0.78]$ \\
\hline $\begin{array}{l}6 \text { AIS associated with HPV6/11/16/18, } \\
\text { at least } 1 \text { dose }\end{array}$ & 2 & 20830 & $\begin{array}{l}\text { Risk Ratio (IV, Random, 95\% } \\
\mathrm{Cl} \text { ) }\end{array}$ & $0.40[0.16,0.98]$ \\
\hline $\begin{array}{l}7 \text { Any } \mathrm{CIN} 2+\text { irrespective of HPV types, } \\
\text { at least } 1 \text { dose }\end{array}$ & 6 & 45066 & $\begin{array}{l}\text { Risk Ratio (IV, Random, 95\% } \\
\mathrm{Cl} \text { ) }\end{array}$ & $0.79[0.65,0.97]$ \\
\hline 7.1 Age group $15-26$ years & 4 & 35779 & $\begin{array}{l}\text { Risk Ratio (IV, Random, 95\% } \\
\mathrm{Cl} \text { ) }\end{array}$ & $0.70[0.58,0.85]$ \\
\hline 7.2 Age group $24-45$ years & 2 & 9287 & $\begin{array}{l}\text { Risk Ratio (IV, Random, 95\% } \\
\mathrm{Cl} \text { ) }\end{array}$ & $1.04[0.83,1.30]$ \\
\hline 8 Any $\mathrm{CIN} 3+\mathrm{HPV}$ type, at least 1 dose & 3 & 35489 & $\begin{array}{l}\text { Risk Ratio (IV, Random, 95\% } \\
\mathrm{Cl} \text { ) }\end{array}$ & $0.67[0.49,0.93]$ \\
\hline 8.1 Bivalent vaccine & 2 & 18329 & $\begin{array}{l}\text { Risk Ratio (IV, Random, 95\% } \\
\mathrm{CI})\end{array}$ & $0.55[0.43,0.71]$ \\
\hline 8.2 Quadrivalent vaccine & 1 & 17160 & $\begin{array}{l}\text { Risk Ratio (IV, Random, 95\% } \\
\mathrm{Cl} \text { ) }\end{array}$ & $0.81[0.69,0.96]$ \\
\hline $\begin{array}{l}9 \text { Any AIS irrespective of HPV types, at } \\
\text { least } 1 \text { dose }\end{array}$ & 2 & 34562 & $\begin{array}{l}\text { Risk Ratio (IV, Random, 95\% } \\
\mathrm{Cl} \text { ) }\end{array}$ & $0.32[0.15,0.67]$ \\
\hline
\end{tabular}


Analysis 3.1. Comparison 3 High-grade cervical lesions in women regardless of baseline HPV DNA status, Outcome 1 CIN2+ associated with HPV16/18, at least 1 dose.

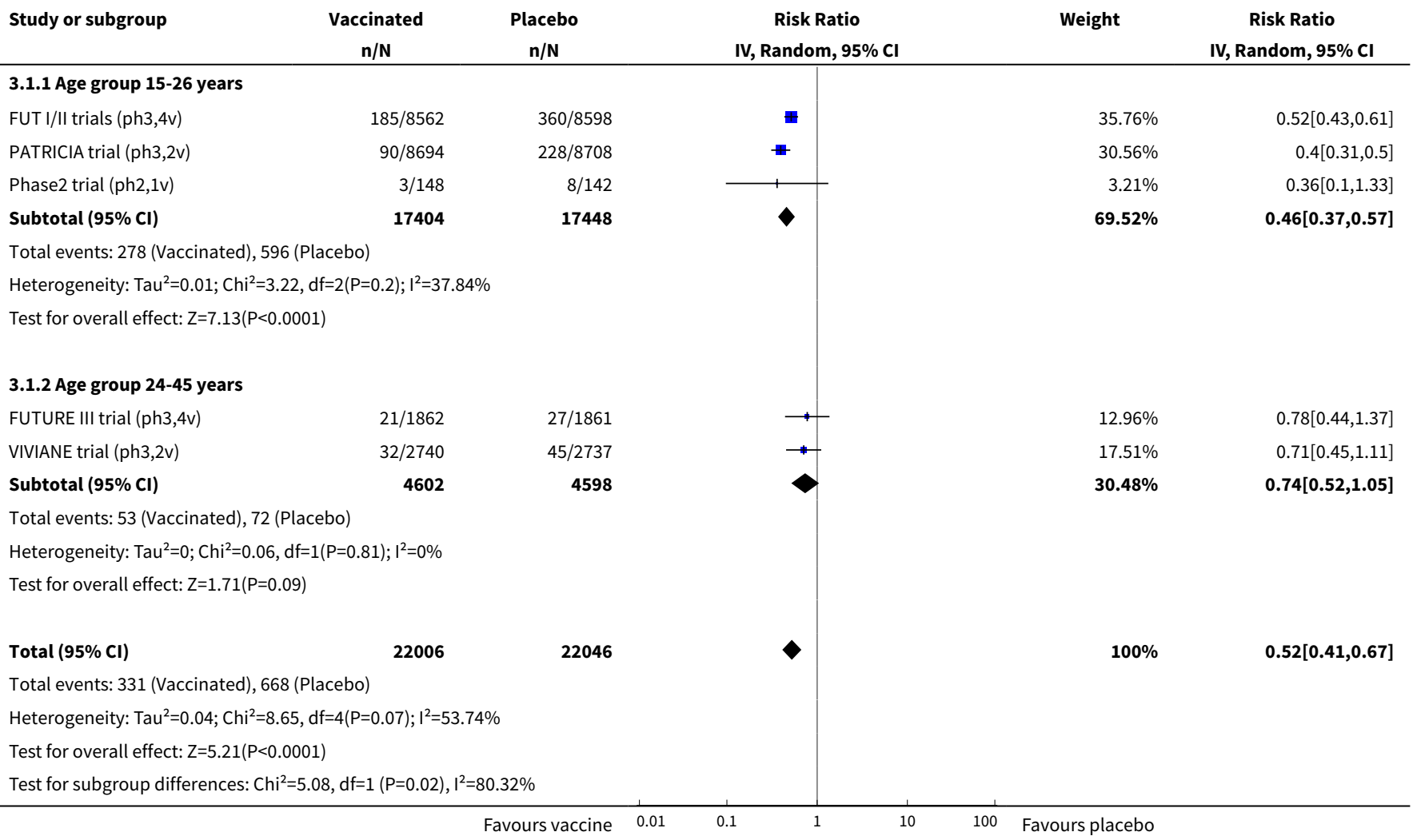

\section{Analysis 3.2. Comparison 3 High-grade cervical lesions in women regardless of baseline HPV DNA status, Outcome 2 CIN2+ associated with HPV6/11/16/18, at least 1 dose.}

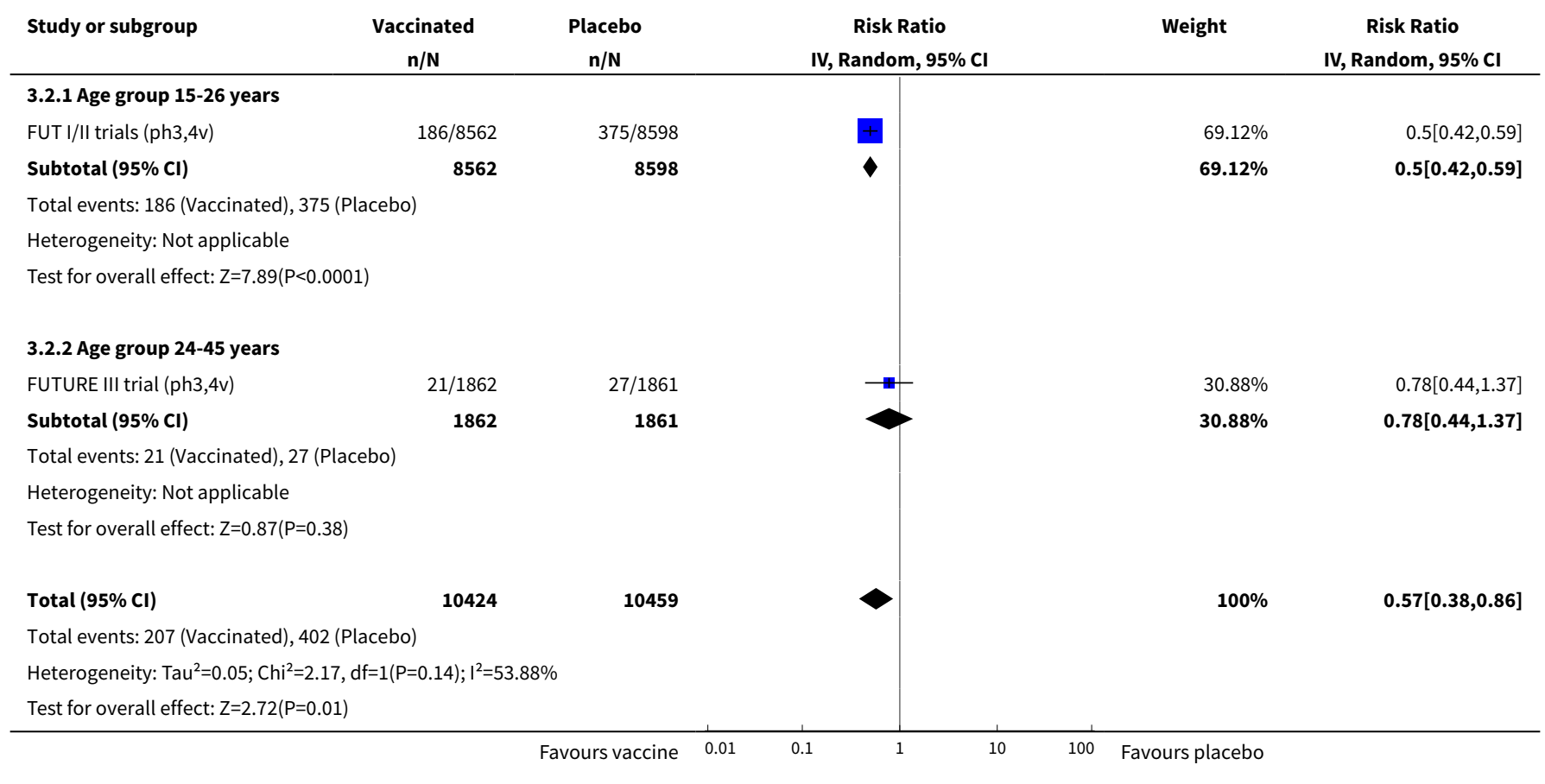




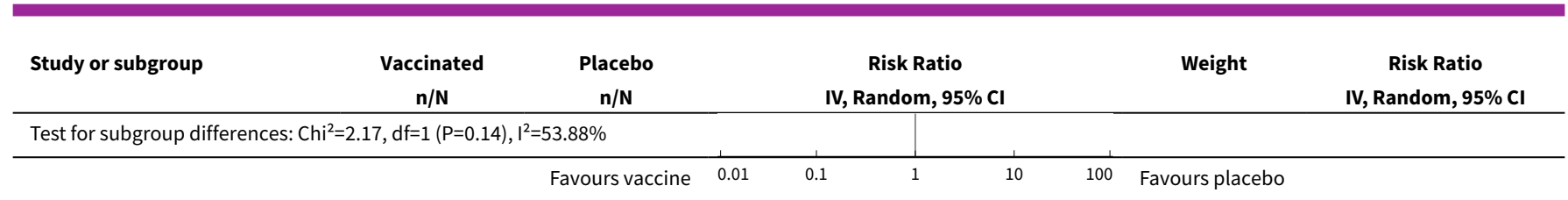

Analysis 3.3. Comparison 3 High-grade cervical lesions in women regardless of baseline HPV DNA status, Outcome 3 CIN3+ associated with HPV16/18, at least 1 dose.

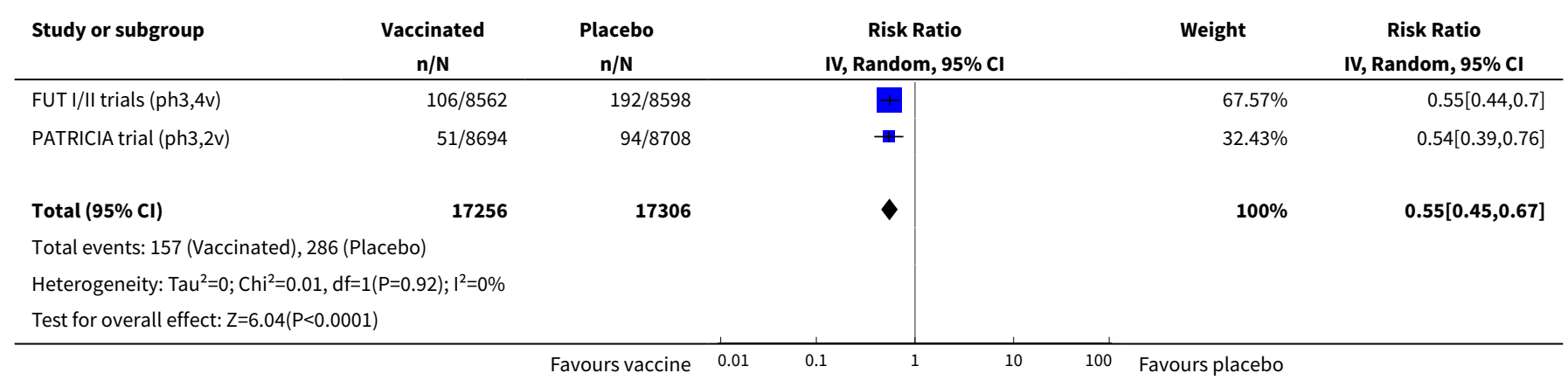

Analysis 3.4. Comparison 3 High-grade cervical lesions in women regardless of baseline HPV DNA status, Outcome 4 CIN3+ associated with HPV6/11/16/18, at least 1 dose.

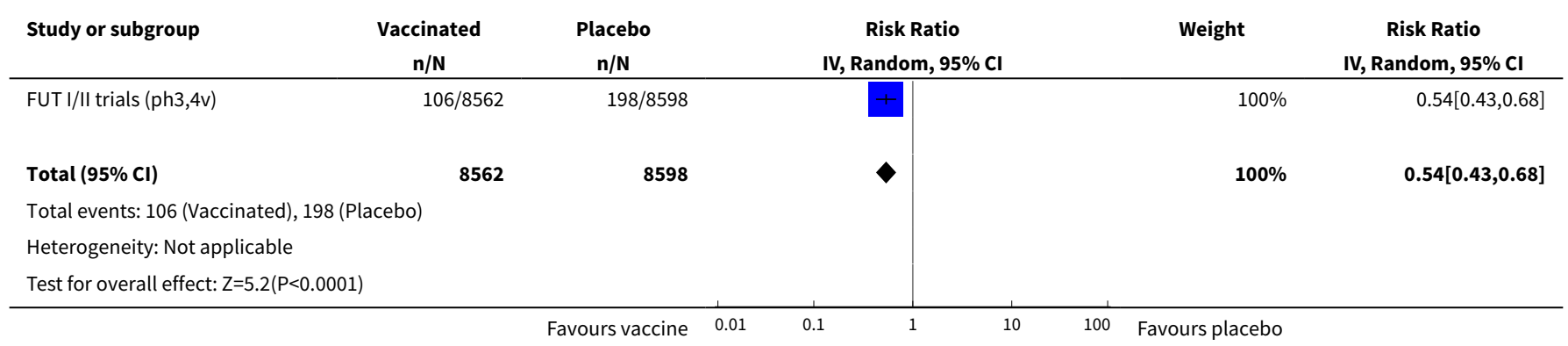

Analysis 3.5. Comparison 3 High-grade cervical lesions in women regardless of baseline HPV DNA status, Outcome 5 AIS associated with HPV16/18, at least 1 dose.

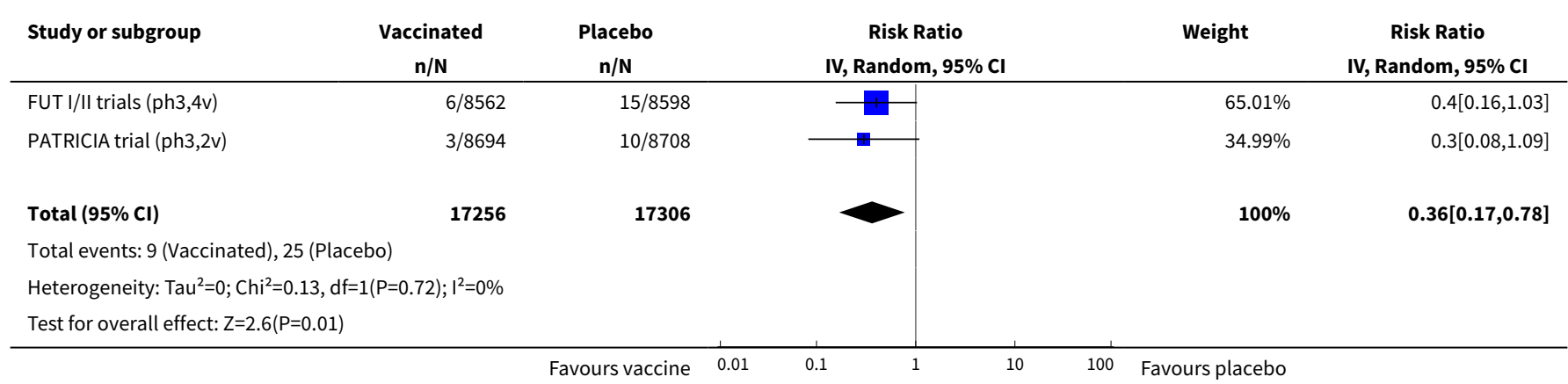


Analysis 3.6. Comparison 3 High-grade cervical lesions in women regardless of baseline HPV DNA status, Outcome 6 AIS associated with HPV6/11/16/18, at least 1 dose.

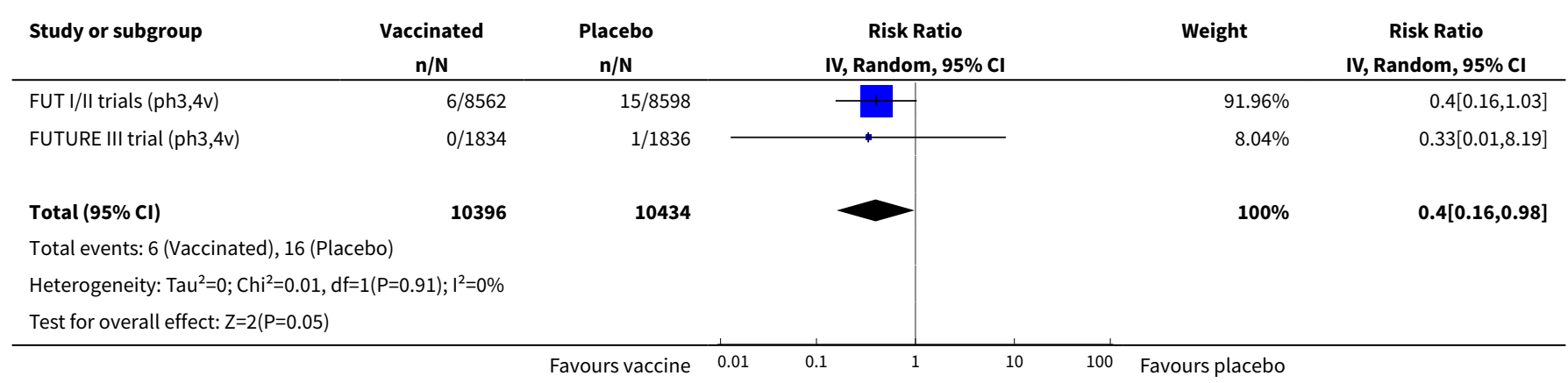

\section{Analysis 3.7. Comparison 3 High-grade cervical lesions in women regardless of baseline} HPV DNA status, Outcome 7 Any CIN2+ irrespective of HPV types, at least 1 dose.

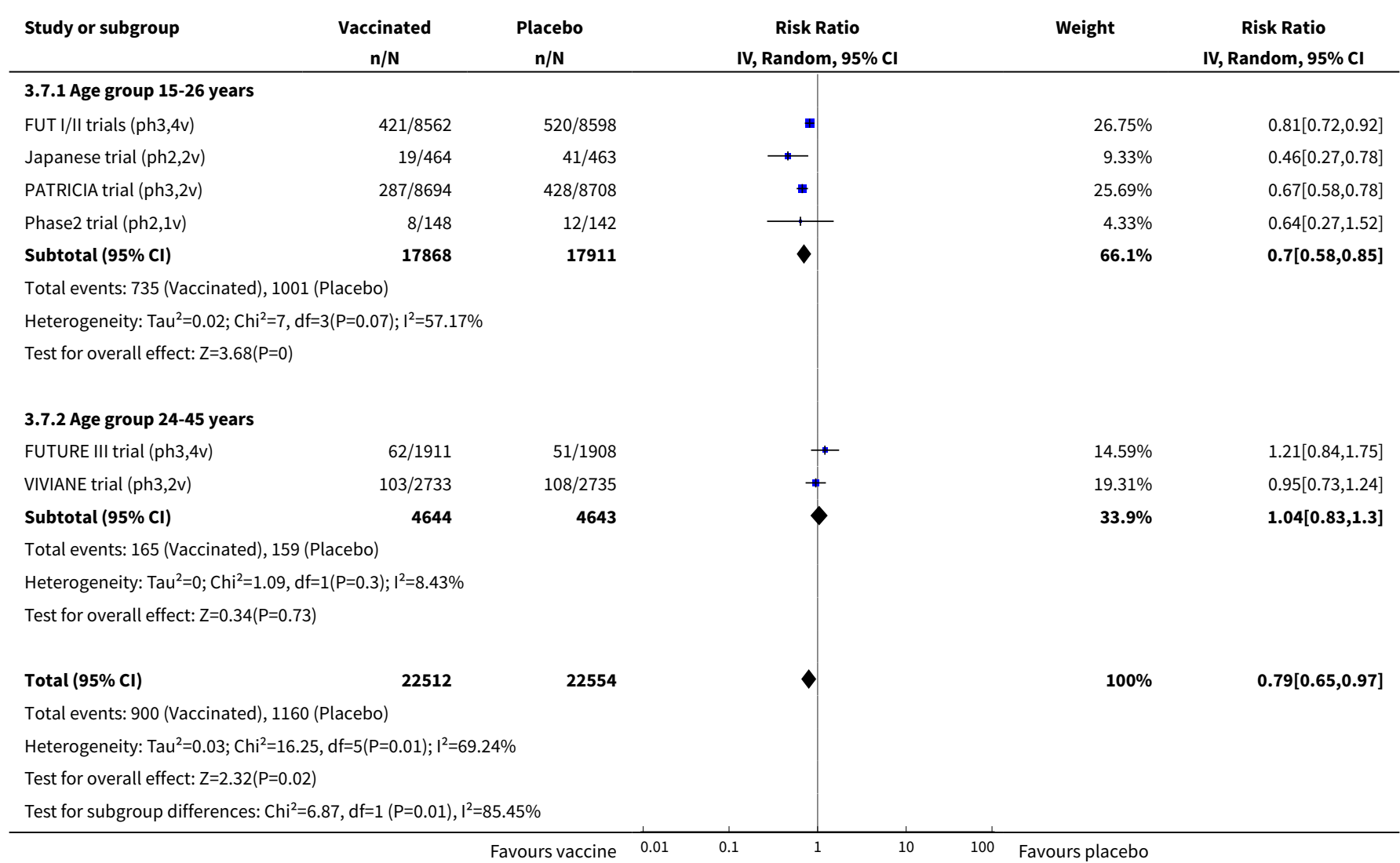


Analysis 3.8. Comparison 3 High-grade cervical lesions in women regardless of baseline HPV DNA status, Outcome 8 Any CIN3+ HPV type, at least 1 dose.

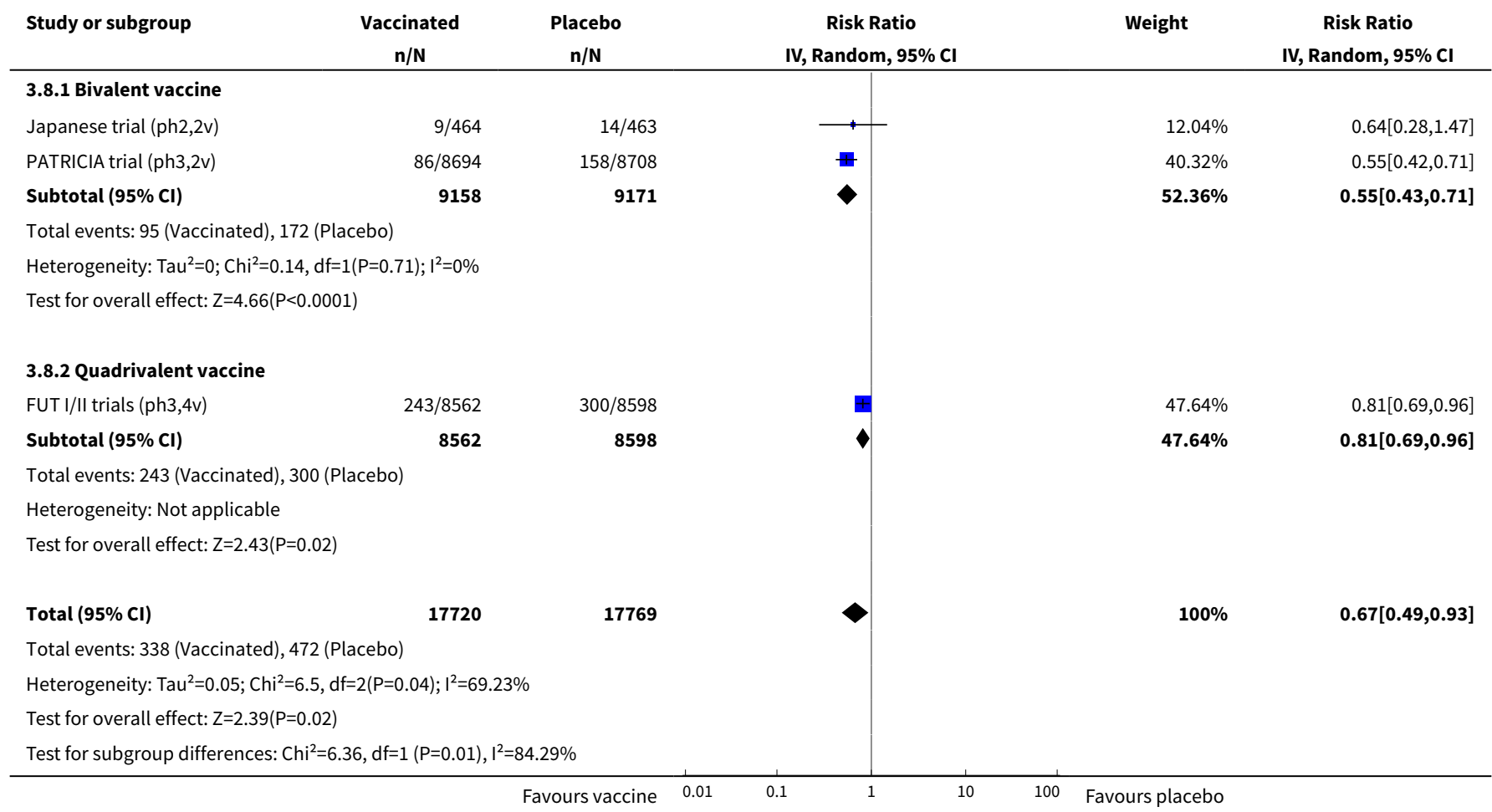

Analysis 3.9. Comparison 3 High-grade cervical lesions in women regardless of baseline HPV DNA status, Outcome 9 Any AIS irrespective of HPV types, at least 1 dose.

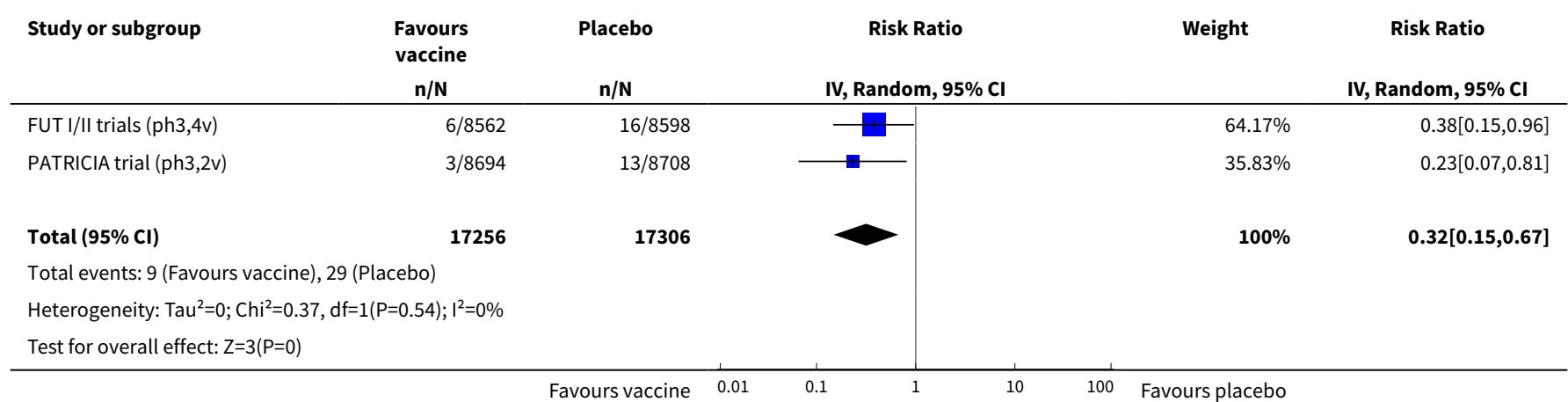

Comparison 4. Infection with HPV vaccine types in hrHPV DNA negative women at baseline

\begin{tabular}{lllll}
\hline Outcome or subgroup title & No. of studies & $\begin{array}{l}\text { No. of partici- } \\
\text { pants }\end{array}$ & Statistical method & Effect size \\
\hline 1 Incident HPV16/18 infection, 3 doses & 1 & 368 & $\begin{array}{l}\text { Risk Ratio (IV, Random, 95\% } \\
\text { Cl) }\end{array}$ & $0.06[0.02,0.20]$ \\
\hline
\end{tabular}




\begin{tabular}{lllll}
\hline Outcome or subgroup title & No. of studies & $\begin{array}{l}\text { No. of partici- } \\
\text { pants }\end{array}$ & Statistical method & Effect size \\
\hline $\begin{array}{l}2 \text { Persistent HPV16/18 infection (6M), } 3 \\
\text { doses }\end{array}$ & 1 & 368 & $\begin{array}{l}\text { Risk Ratio (IV, Random, 95\% } \\
\text { Cl) }\end{array}$ & 0.02 [0.00, 0.35] \\
\hline $\begin{array}{l}3 \text { Persistent HPV16/18 infection (6M), at } \\
\text { least 1 dose }\end{array}$ & 1 & 10826 & $\begin{array}{l}\text { Risk Ratio (IV, Random, 95\% } \\
\text { Cl) }\end{array}$ & 0.07 [0.05, 0.09] \\
\hline $\begin{array}{l}4 \text { Persistent HPV16/18 infection(12M), 3 } \\
\text { doses }\end{array}$ & 1 & 368 & $\begin{array}{l}\text { Risk Ratio (IV, Random, 95\% } \\
\text { Cl) }\end{array}$ & 0.04 [0.00, 0.73] \\
\hline $\begin{array}{l}5 \text { Persistent HPV16/18 infection (12M), } \\
\text { at least 1 dose }\end{array}$ & 2 & 14153 & $\begin{array}{l}\text { Risk Ratio (IV, Random, 95\% } \\
\text { CI) }\end{array}$ & 0.08 [0.05, 0.12] \\
\hline
\end{tabular}

Analysis 4.1. Comparison 4 Infection with HPV vaccine types in hrHPV DNA negative women at baseline, Outcome 1 Incident HPV16/18 infection, 3 doses.

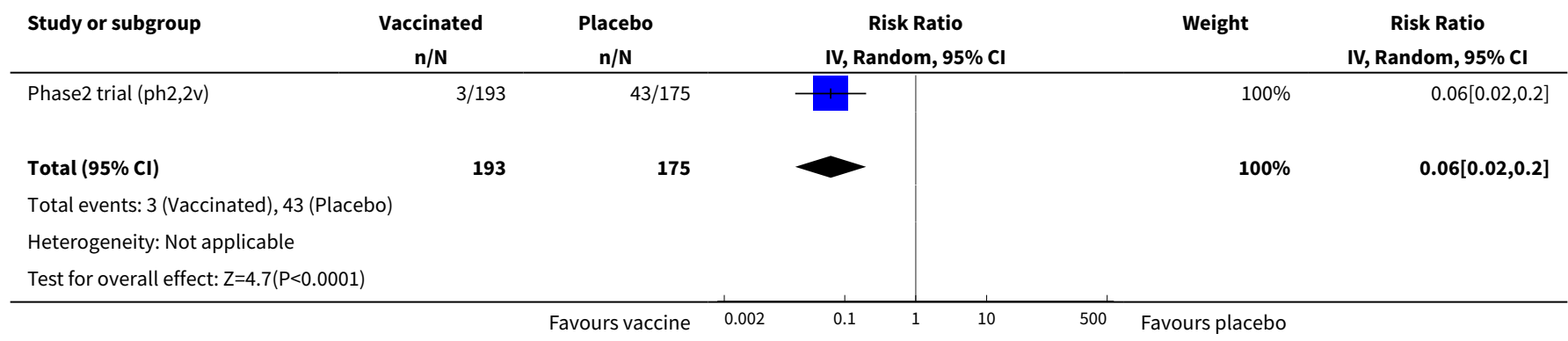

Analysis 4.2. Comparison 4 Infection with HPV vaccine types in hrHPV DNA negative women at baseline, Outcome 2 Persistent HPV16/18 infection (6M), 3 doses.

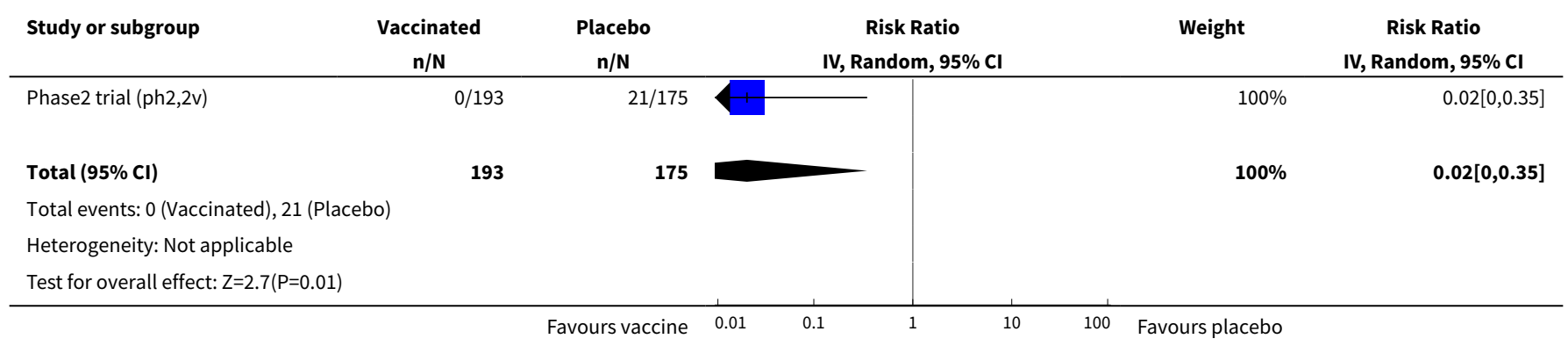

Analysis 4.3. Comparison 4 Infection with HPV vaccine types in hrHPV DNA negative women at baseline, Outcome 3 Persistent HPV16/18 infection (6M), at least 1 dose.

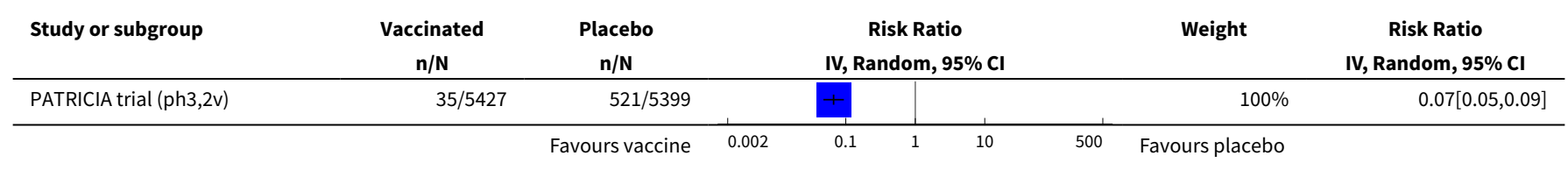




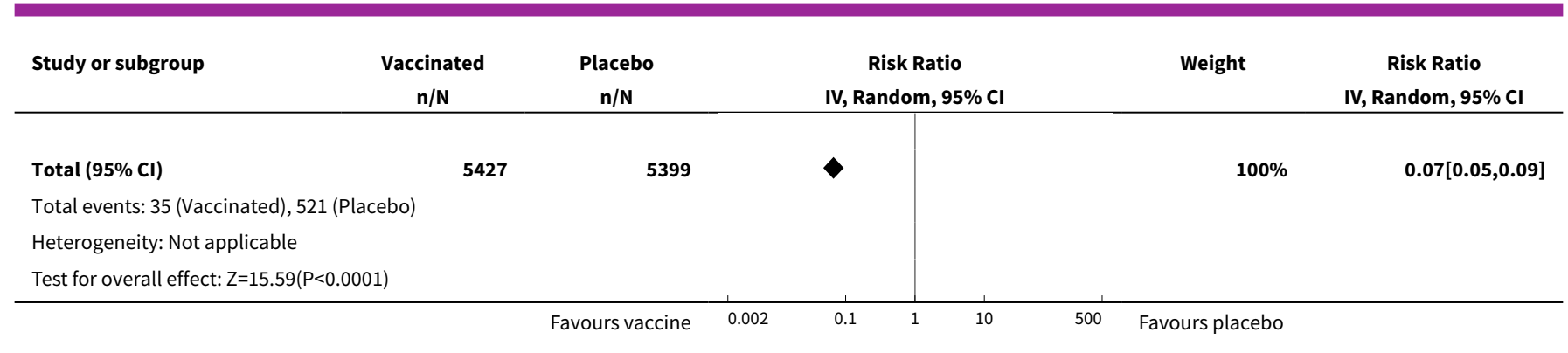

\section{Analysis 4.4. Comparison 4 Infection with HPV vaccine types in hrHPV DNA negative} women at baseline, Outcome 4 Persistent HPV16/18 infection(12M), 3 doses.

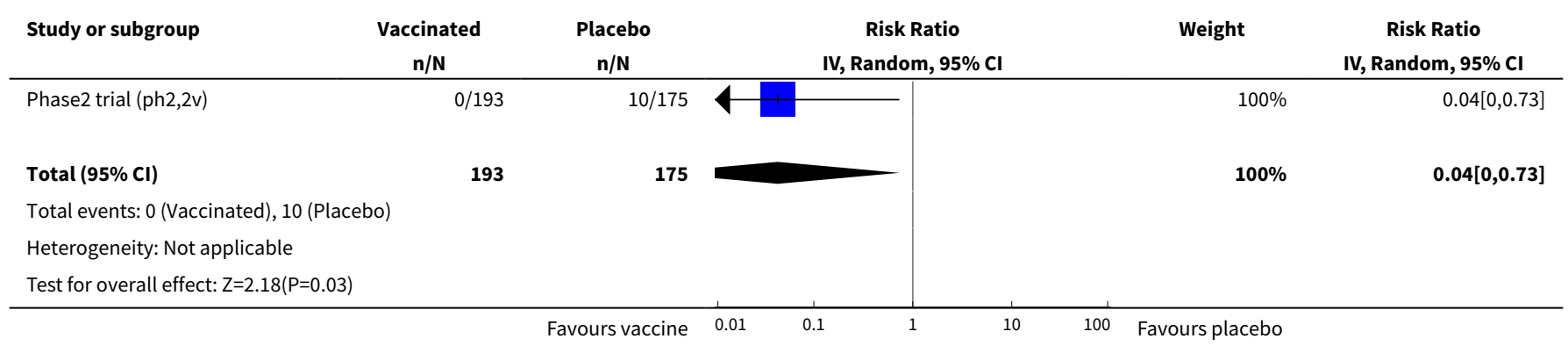

Analysis 4.5. Comparison 4 Infection with HPV vaccine types in hrHPV DNA negative women at baseline, Outcome 5 Persistent HPV16/18 infection (12M), at least 1 dose.

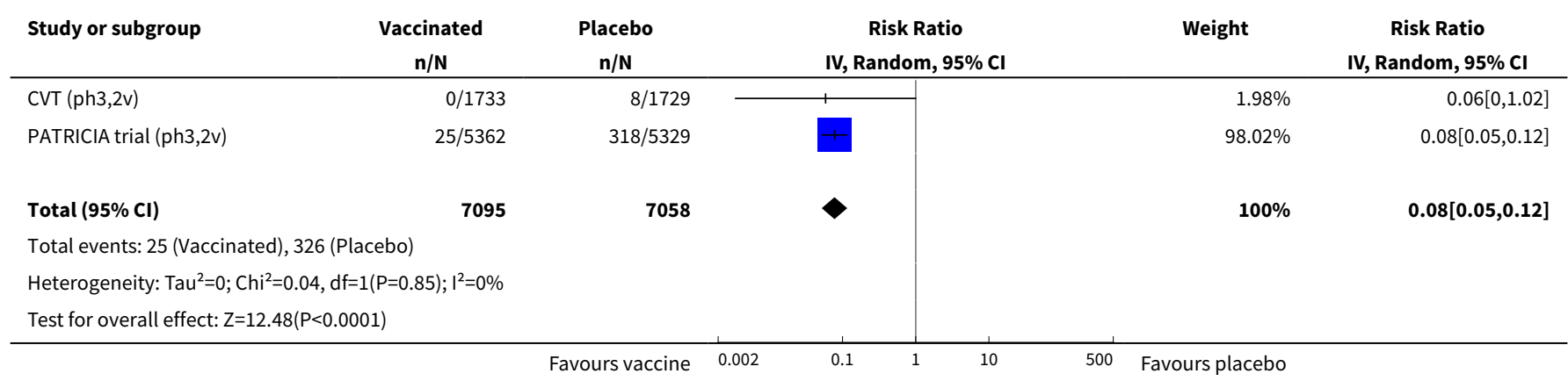

\section{Comparison 5. HPV16/18 infection in HPV16/18 DNA negative women at baseline}

\begin{tabular}{lllll}
\hline Outcome or subgroup title & No. of studies & $\begin{array}{l}\text { No. of partici- } \\
\text { pants }\end{array}$ & Statistical method & Effect size \\
\hline 1 Incident HPV16/18 infection, 3 doses & 4 & 8034 & $\begin{array}{l}\text { Risk Ratio (IV, Random, 95\% } \\
\text { Cl) }\end{array}$ & 0.17 [0.10, 0.31] \\
\hline $\begin{array}{l}2 \text { Incident HPV16/18 infection, at least } \\
1 \text { dose }\end{array}$ & 5 & 23872 & $\begin{array}{l}\text { Risk Ratio (IV, Random, 95\% } \\
\text { Cl) }\end{array}$ & $0.23[0.14,0.37]$ \\
\hline
\end{tabular}




\begin{tabular}{|c|c|c|c|c|}
\hline Outcome or subgroup title & No. of studies & $\begin{array}{l}\text { No. of partici- } \\
\text { pants }\end{array}$ & Statistical method & Effect size \\
\hline $\begin{array}{l}3 \text { Incident HPV16/18 infection, } 1 \text { or } 2 \\
\text { doses (post hoc analysis) }\end{array}$ & 3 & 331 & $\begin{array}{l}\text { Risk Ratio (IV, Random, 95\% } \\
\mathrm{CI} \text { ) }\end{array}$ & $0.47[0.26,0.84]$ \\
\hline $\begin{array}{l}4 \text { Persistent HPV16/18 infection (6M), } 3 \\
\text { doses }\end{array}$ & 8 & 34113 & $\begin{array}{l}\text { Risk Ratio (IV, Random, 95\% } \\
\mathrm{Cl} \text { ) }\end{array}$ & $0.07[0.06,0.09]$ \\
\hline 4.1 Age group $15-26$ years & 6 & 27385 & $\begin{array}{l}\text { Risk Ratio (IV, Random, 95\% } \\
\mathrm{CI})\end{array}$ & $0.06[0.05,0.08]$ \\
\hline 4.2 Age group 24-45 years & 2 & 6728 & $\begin{array}{l}\text { Risk Ratio (IV, Random, 95\% } \\
\mathrm{CI})\end{array}$ & $0.11[0.06,0.20]$ \\
\hline $\begin{array}{l}5 \text { Persistent HPV16/18 infection (6M), } \\
\text { at least } 1 \text { dose }\end{array}$ & 6 & 30323 & $\begin{array}{l}\text { Risk Ratio (IV, Random, 95\% } \\
\mathrm{CI} \text { ) }\end{array}$ & $0.12[0.08,0.17]$ \\
\hline 5.1 Age group $15-26$ years & 4 & 22803 & $\begin{array}{l}\text { Risk Ratio (IV, Random, 95\% } \\
\mathrm{CI})\end{array}$ & $0.10[0.08,0.12]$ \\
\hline 5.2 Age group $24-45$ years & 2 & 7520 & $\begin{array}{l}\text { Risk Ratio (IV, Random, 95\% } \\
\mathrm{CI})\end{array}$ & $0.17[0.10,0.29]$ \\
\hline $\begin{array}{l}6 \text { Persistent HPV16/18 infection (6M), } 1 \\
\text { or } 2 \text { doses (post hoc analysis) }\end{array}$ & 4 & 1229 & $\begin{array}{l}\text { Risk Ratio (IV, Random, 95\% } \\
\mathrm{Cl} \text { ) }\end{array}$ & $0.26[0.16,0.44]$ \\
\hline 6.1 Age group $15-26$ years & 2 & 437 & $\begin{array}{l}\text { Risk Ratio (IV, Random, 95\% } \\
\mathrm{CI})\end{array}$ & $0.12[0.03,0.42]$ \\
\hline 6.2 Age group $24-45$ years & 2 & 792 & $\begin{array}{l}\text { Risk Ratio (IV, Random, 95\% } \\
\mathrm{CI})\end{array}$ & $0.31[0.18,0.54]$ \\
\hline $\begin{array}{l}7 \text { Persistent HPV6/11/16/18 infection } \\
(6 \mathrm{M}), 3 \text { doses }\end{array}$ & 2 & 4008 & $\begin{array}{l}\text { Risk Ratio (IV, Random, 95\% } \\
\mathrm{CI} \text { ) }\end{array}$ & $0.12[0.06,0.21]$ \\
\hline $\begin{array}{l}8 \text { Persistent HPV6/11/16/18 infection } \\
(6 \mathrm{M}) \text {, at least } 1 \text { dose }\end{array}$ & 2 & 4129 & $\begin{array}{l}\text { Risk Ratio (IV, Random, 95\% } \\
\mathrm{Cl} \text { ) }\end{array}$ & $0.13[0.05,0.37]$ \\
\hline $\begin{array}{l}9 \text { Persistent HPV16/18 infection (12M), } \\
3 \text { doses }\end{array}$ & 4 & 22267 & $\begin{array}{l}\text { Risk Ratio (IV, Random, 95\% } \\
\mathrm{Cl} \text { ) }\end{array}$ & $0.09[0.06,0.13]$ \\
\hline $\begin{array}{l}10 \text { Persistent HPV } 16 / 18 \text { infection } \\
(12 \mathrm{M}) \text {, at least } 1 \text { dose }\end{array}$ & 5 & 29464 & $\begin{array}{l}\text { Risk Ratio (IV, Random, 95\% } \\
\mathrm{Cl} \text { ) }\end{array}$ & $0.16[0.12,0.20]$ \\
\hline $\begin{array}{l}11 \text { Persistent HPV } 16 / 18 \text { infection } \\
(12 \mathrm{M}), 1 \text { or } 2 \text { doses (post hoc analysis) }\end{array}$ & 3 & 3912 & $\begin{array}{l}\text { Risk Ratio (IV, Random, 95\% } \\
\mathrm{Cl} \text { ) }\end{array}$ & $0.13[0.06,0.33]$ \\
\hline
\end{tabular}

\section{Analysis 5.1. Comparison 5 HPV16/18 infection in HPV16/18 DNA negative women at baseline, Outcome 1 Incident HPV16/18 infection, 3 doses.}

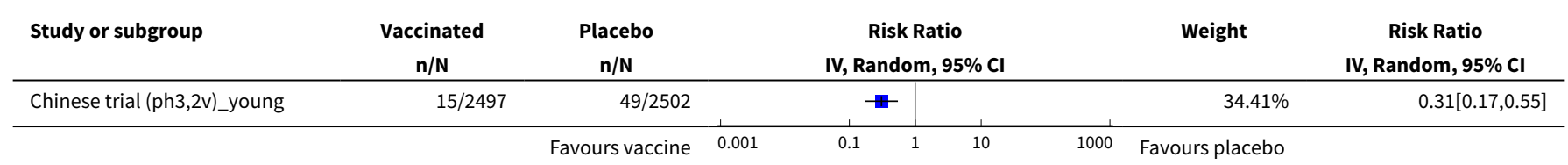




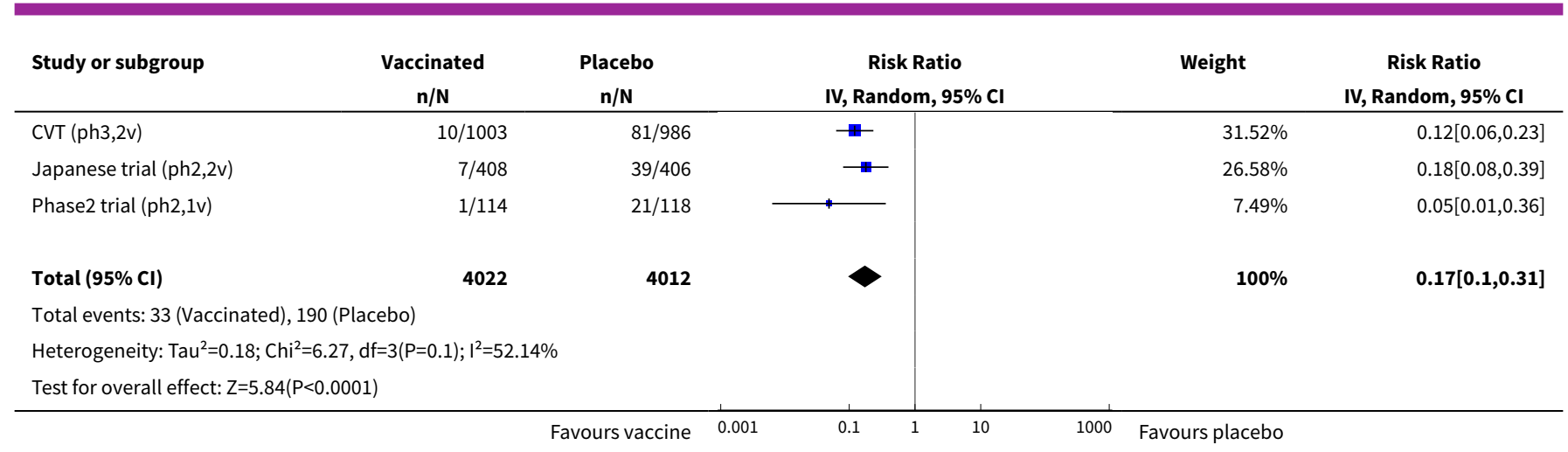

Analysis 5.2. Comparison 5 HPV16/18 infection in HPV16/18 DNA negative women at baseline, Outcome 2 Incident HPV16/18 infection, at least 1 dose.

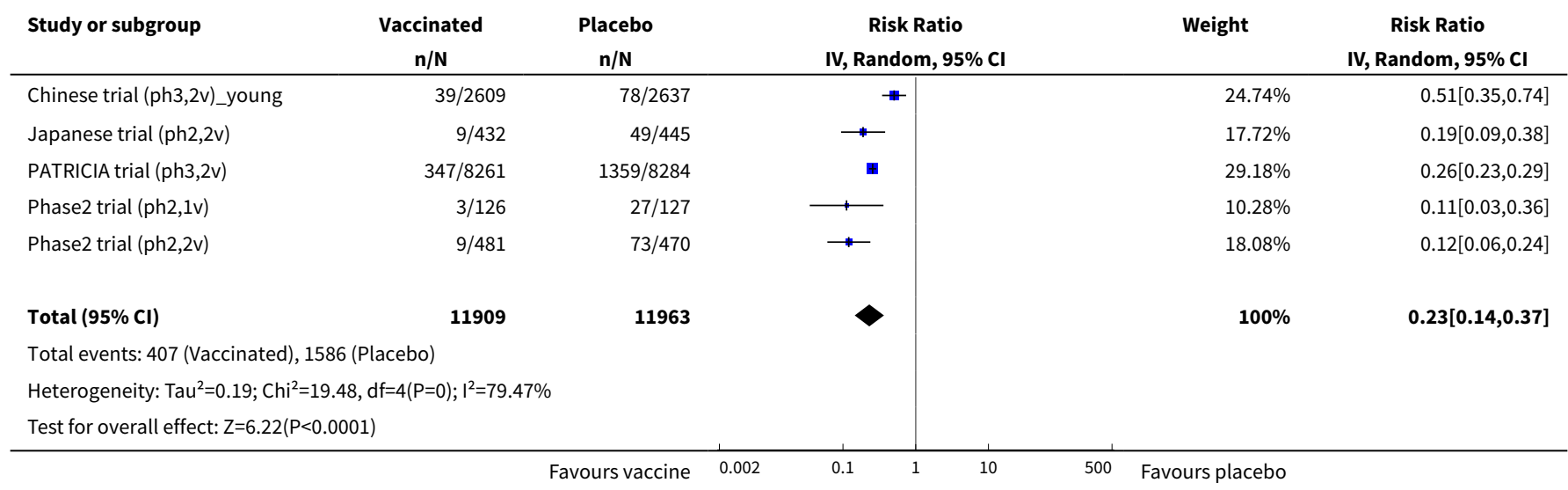

Analysis 5.3. Comparison 5 HPV16/18 infection in HPV16/18 DNA negative women at baseline, Outcome 3 Incident HPV16/18 infection, 1 or 2 doses (post hoc analysis).

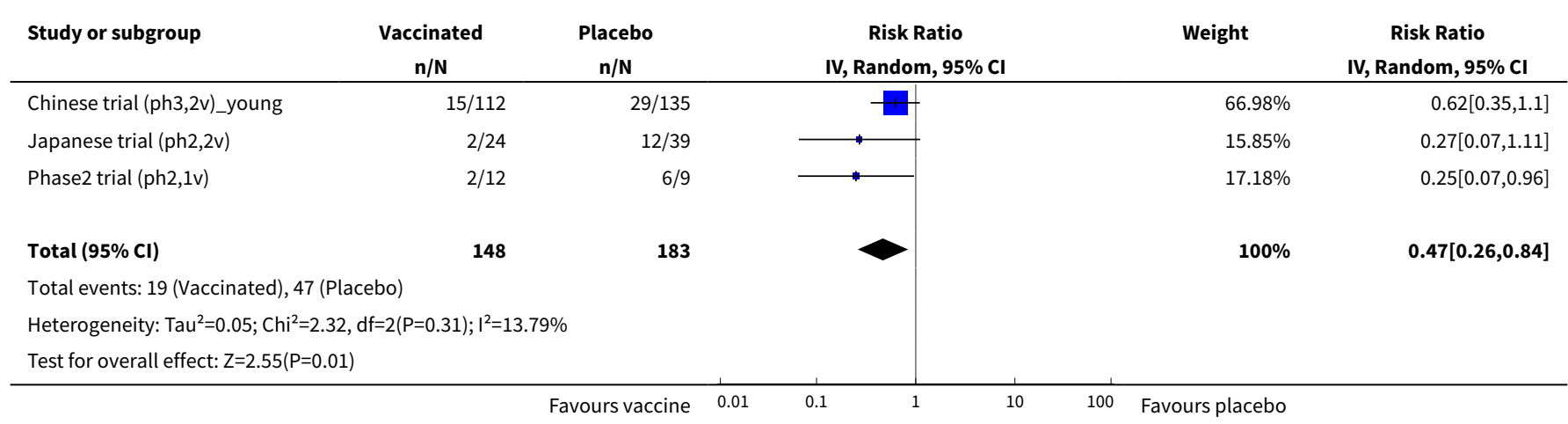


Analysis 5.4. Comparison 5 HPV16/18 infection in HPV16/18 DNA negative women at baseline, Outcome 4 Persistent HPV16/18 infection (6M), 3 doses.

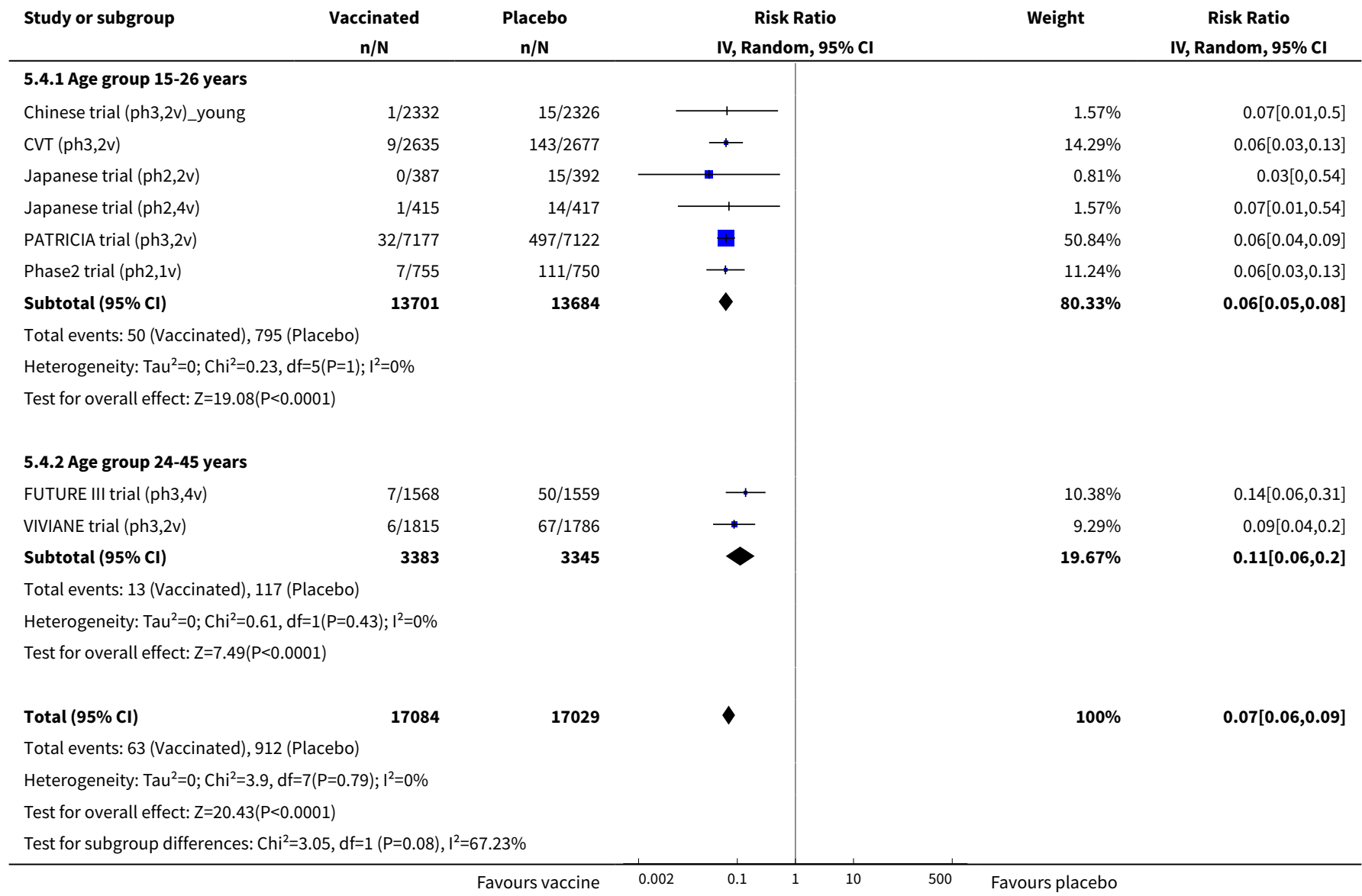

\section{Analysis 5.5. Comparison 5 HPV16/18 infection in HPV16/18 DNA negative women} at baseline, Outcome 5 Persistent HPV16/18 infection (6M), at least 1 dose.

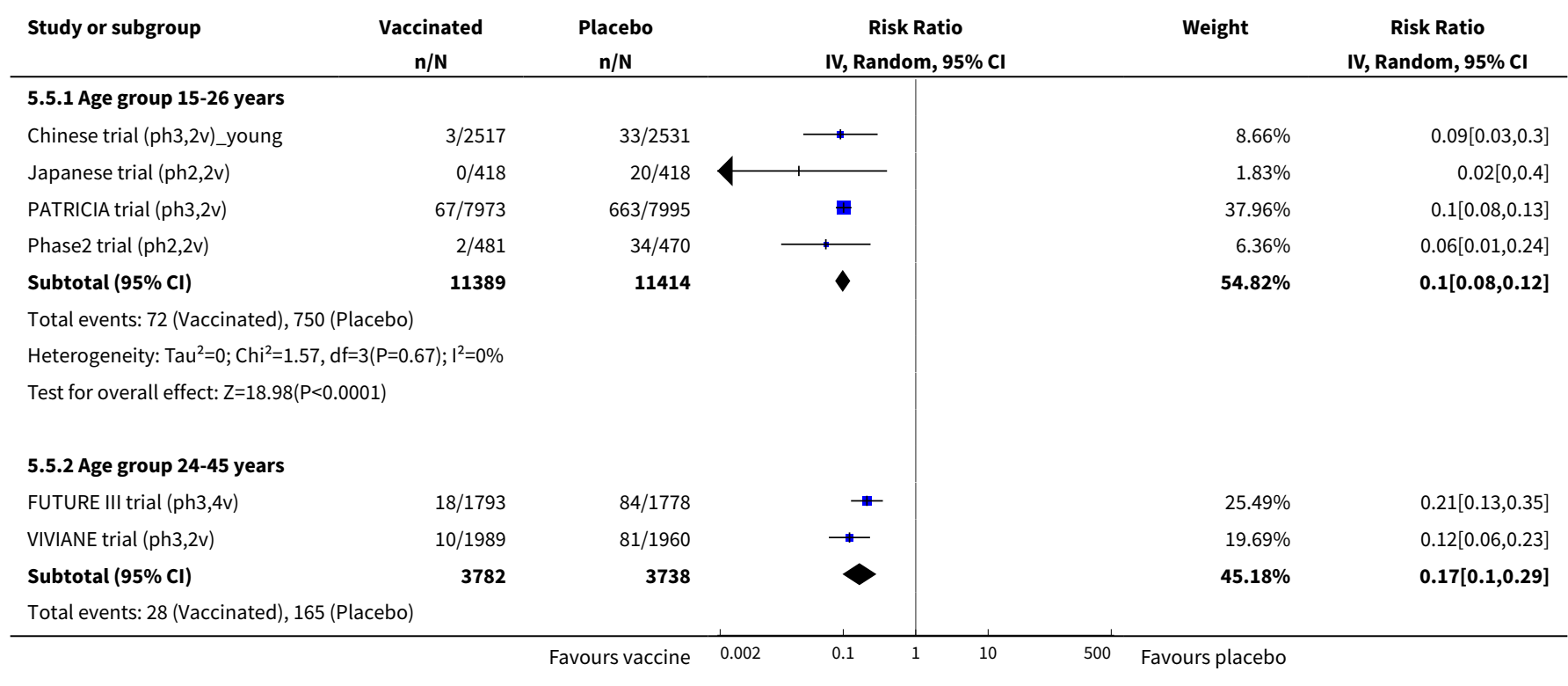




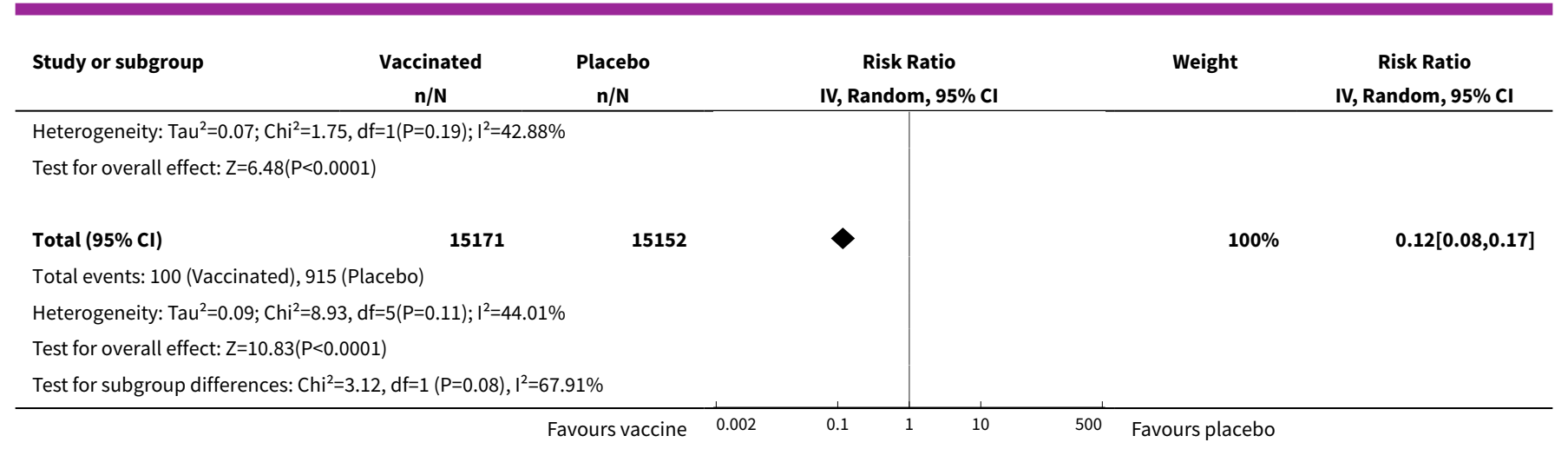

Analysis 5.6. Comparison 5 HPV16/18 infection in HPV16/18 DNA negative women at baseline, Outcome 6 Persistent HPV16/18 infection (6M), 1 or 2 doses (post hoc analysis).

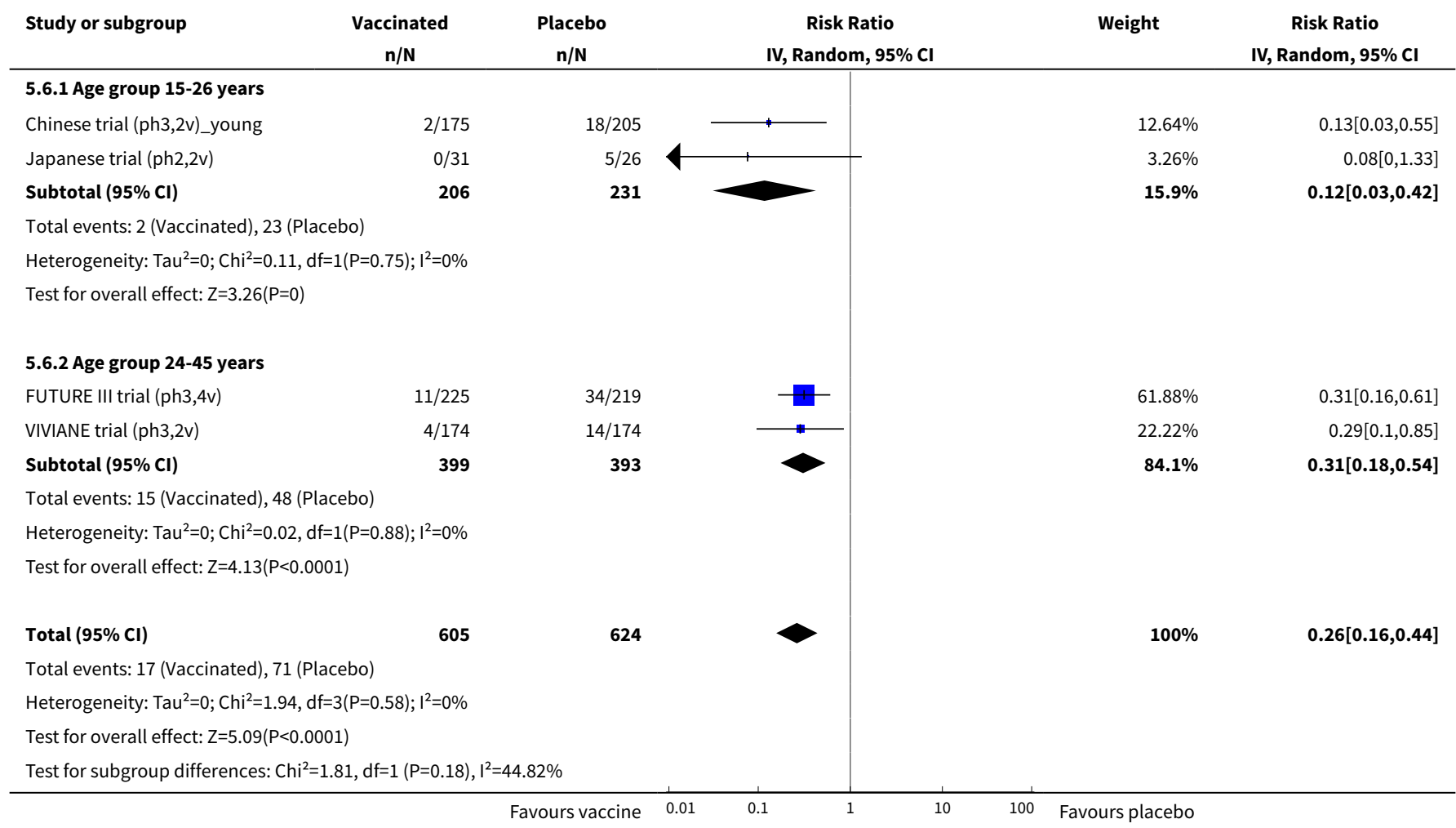

Analysis 5.7. Comparison 5 HPV16/18 infection in HPV16/18 DNA negative women at baseline, Outcome 7 Persistent HPV6/11/16/18 infection (6M), 3 doses.

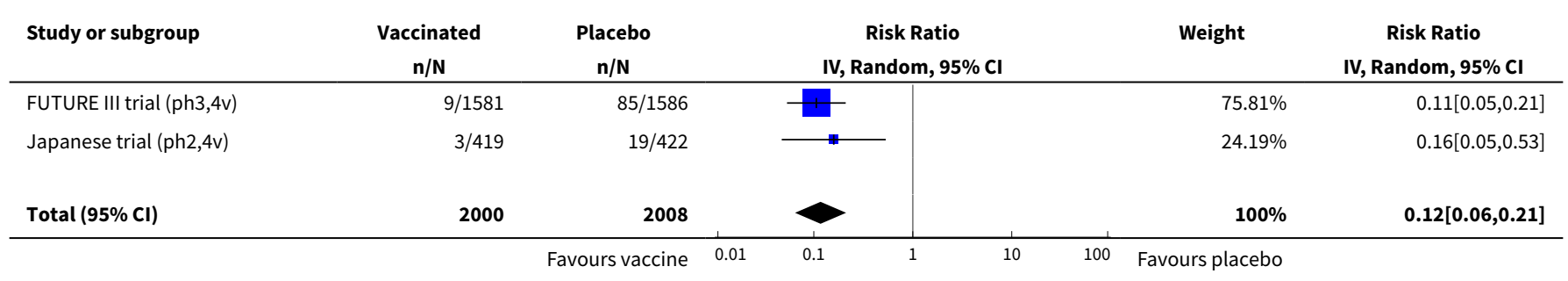




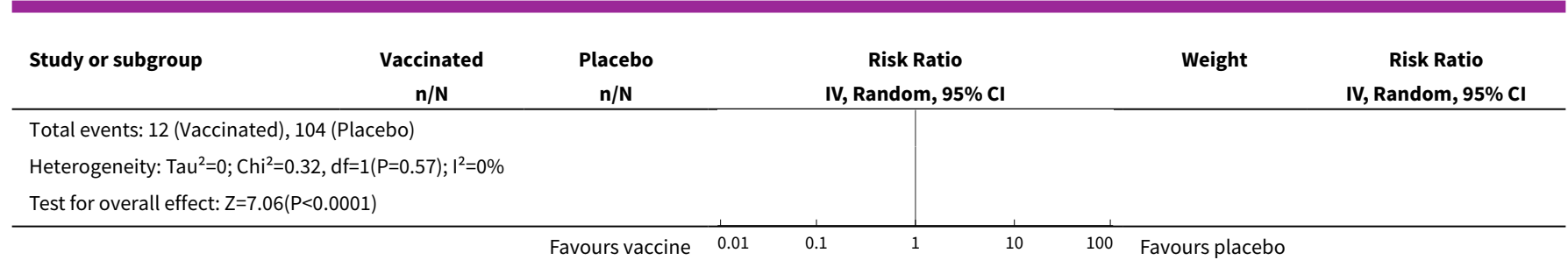

Analysis 5.8. Comparison 5 HPV16/18 infection in HPV16/18 DNA negative women at baseline, Outcome 8 Persistent HPV6/11/16/18 infection (6M), at least 1 dose.

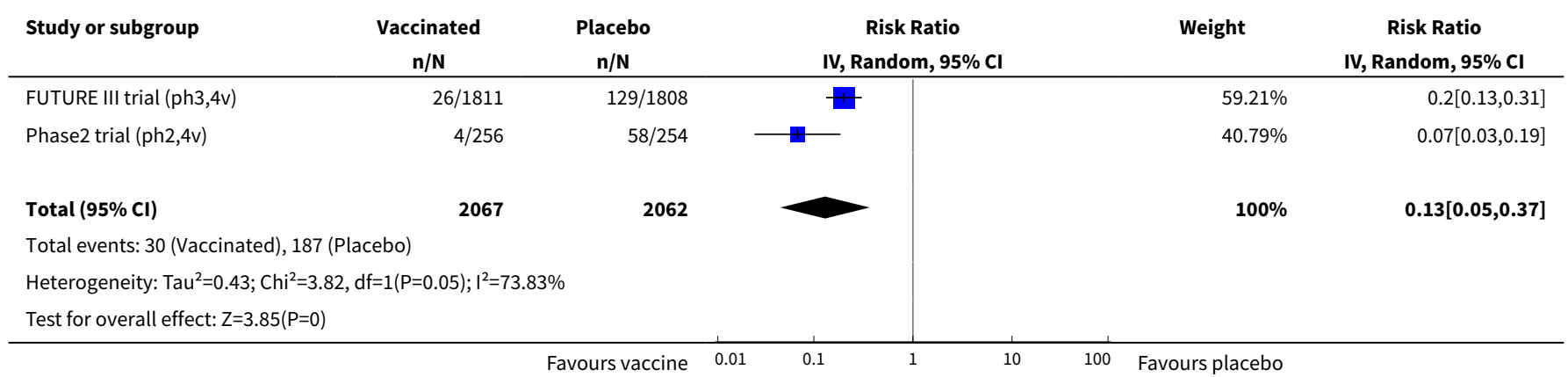

Analysis 5.9. Comparison 5 HPV16/18 infection in HPV16/18 DNA negative women at baseline, Outcome 9 Persistent HPV16/18 infection (12M), 3 doses.

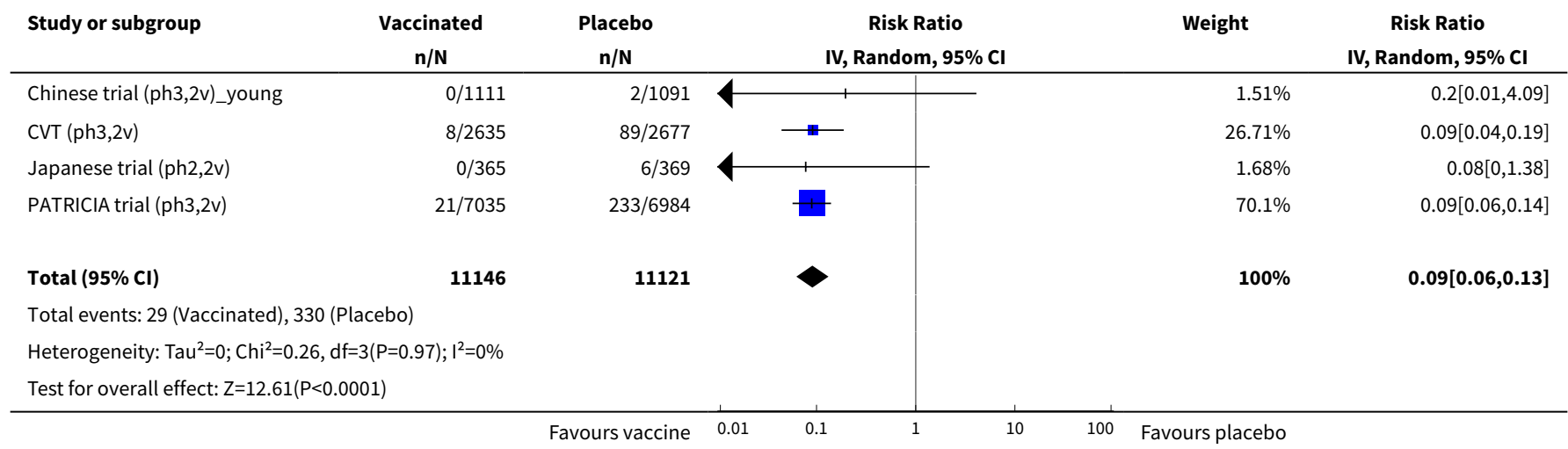

Analysis 5.10. Comparison 5 HPV16/18 infection in HPV16/18 DNA negative women at baseline, Outcome 10 Persistent HPV16/18 infection (12M), at least 1 dose.

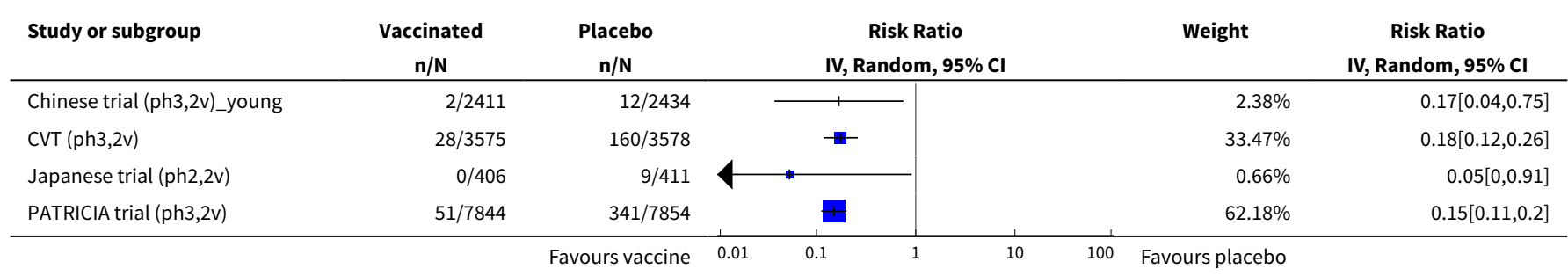




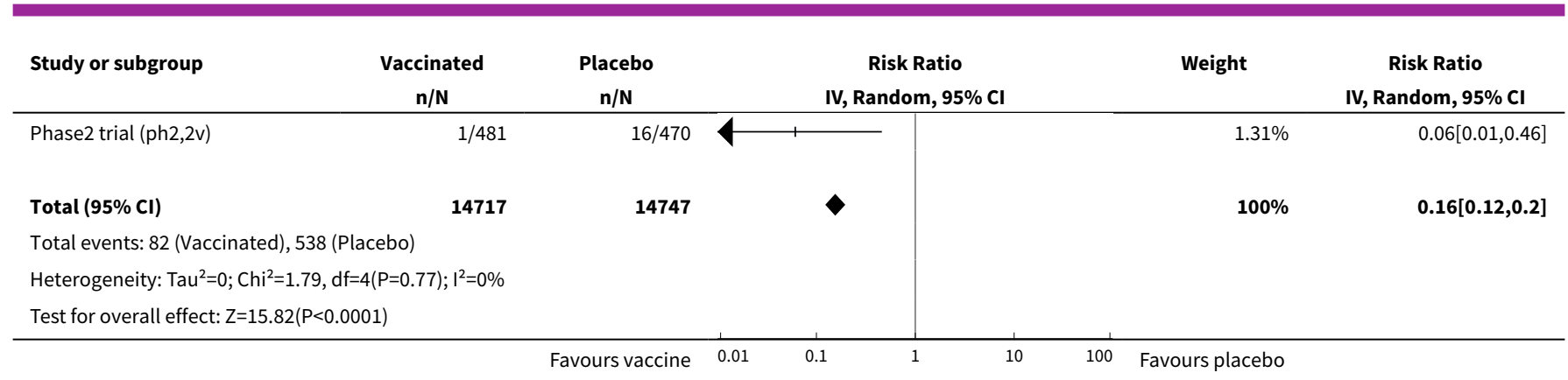

Analysis 5.11. Comparison 5 HPV16/18 infection in HPV16/18 DNA negative women at baseline, Outcome 11 Persistent HPV16/18 infection (12M), 1 or 2 doses (post hoc analysis).

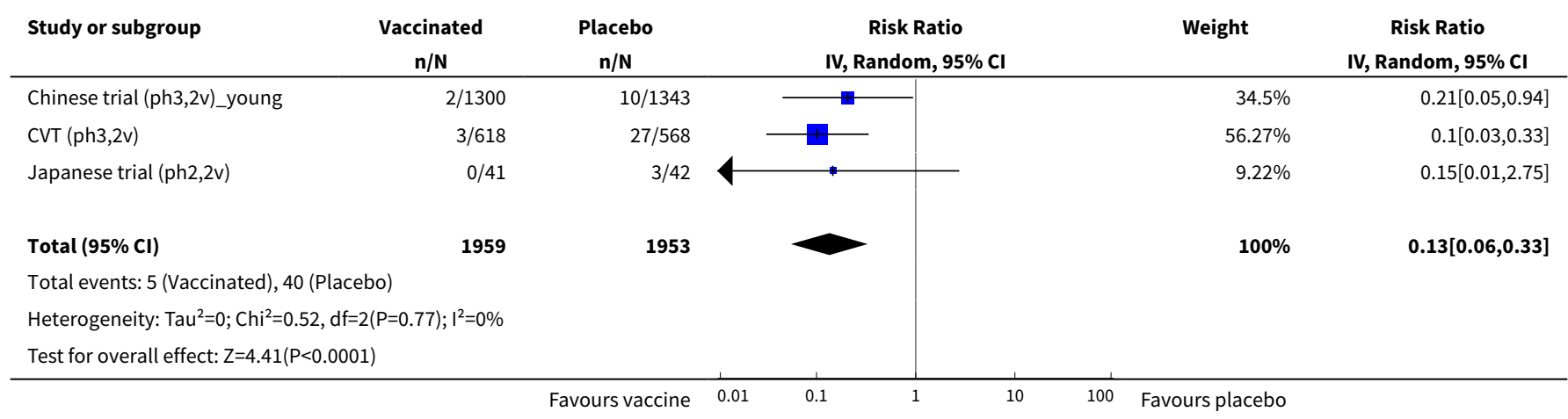

Comparison 6. Infection with HPV types included in the vaccine in women regardless of HPV DNA status at baseline

\begin{tabular}{|c|c|c|c|c|}
\hline Outcome or subgroup title & No. of studies & $\begin{array}{l}\text { No. of partici- } \\
\text { pants }\end{array}$ & Statistical method & Effect size \\
\hline $\begin{array}{l}1 \text { Incident HPV16/18 infection, at least } \\
1 \text { dose }\end{array}$ & 1 & 4210 & $\begin{array}{l}\text { Risk Ratio (IV, Random, 95\% } \\
\mathrm{CI})\end{array}$ & $0.24[0.17,0.33]$ \\
\hline $\begin{array}{l}2 \text { Persistent HPV16/18 infection (6M), } \\
\text { at least } 1 \text { dose }\end{array}$ & 4 & 33847 & $\begin{array}{l}\text { Risk Ratio (IV, Random, 95\% } \\
\text { Cl) }\end{array}$ & $0.48[0.41,0.57]$ \\
\hline 2.1 Age group $15-26$ years & 2 & 25199 & $\begin{array}{l}\text { Risk Ratio (IV, Random, 95\% } \\
\text { Cl) }\end{array}$ & $0.44[0.38,0.51]$ \\
\hline 2.2 Age group $24-45$ years & 2 & 8648 & $\begin{array}{l}\text { Risk Ratio (IV, Random, 95\% } \\
\mathrm{Cl} \text { ) }\end{array}$ & $0.57[0.47,0.69]$ \\
\hline $\begin{array}{l}3 \text { Persistent HPV6/11/16/18 infection } \\
(6 \mathrm{M}) \text {, at least } 1 \text { dose }\end{array}$ & 1 & 3713 & $\begin{array}{l}\text { Risk Ratio (IV, Random, 95\% } \\
\mathrm{CI} \text { ) }\end{array}$ & $0.52[0.42,0.65]$ \\
\hline $\begin{array}{l}4 \text { Persistent HPV16/18 infection (12M), } \\
\text { at least } 1 \text { dose }\end{array}$ & 2 & 24785 & $\begin{array}{l}\text { Risk Ratio (IV, Random, 95\% } \\
\mathrm{CI} \text { ) }\end{array}$ & $0.46[0.40,0.54]$ \\
\hline $\begin{array}{l}5 \text { Persistent HPV16/18 infection (12M) } \\
\text { by dose (post hoc analysis) }\end{array}$ & 1 & 7153 & $\begin{array}{l}\text { Risk Ratio (IV, Random, 95\% } \\
\mathrm{Cl} \text { ) }\end{array}$ & $0.18[0.12,0.27]$ \\
\hline
\end{tabular}


Analysis 6.1. Comparison 6 Infection with HPV types included in the vaccine in women regardless of HPV DNA status at baseline, Outcome 1 Incident HPV16/18 infection, at least 1 dose.

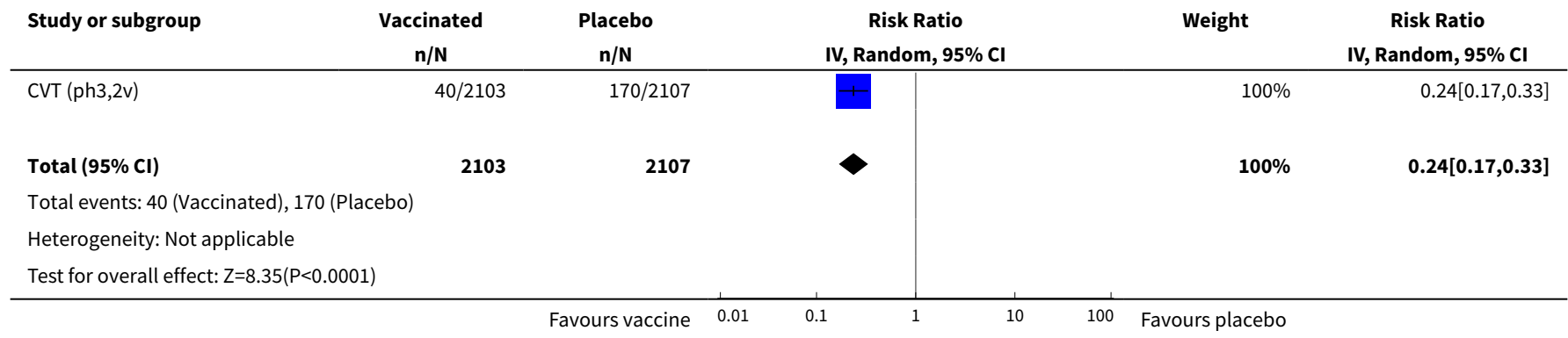

Analysis 6.2. Comparison 6 Infection with HPV types included in the vaccine in women regardless of HPV DNA status at baseline, Outcome 2 Persistent HPV16/18 infection (6M), at least 1 dose.

\begin{tabular}{|c|c|c|c|c|c|}
\hline \multirow{2}{*}{$\begin{array}{l}\text { Study or subgroup } \\
\text { 6.2.1 Age group 15-26 yea }\end{array}$} & \multirow[t]{2}{*}{$\begin{array}{c}\text { Vaccinated } \\
\mathbf{n} / \mathbf{N} \\
\end{array}$} & \multirow[t]{2}{*}{$\begin{array}{c}\text { Placebo } \\
\mathbf{n} / \mathbf{N}\end{array}$} & $\begin{array}{c}\text { Risk Ratio } \\
\text { IV, Random, } 95 \% \text { CI } \\
\end{array}$ & \multirow[t]{2}{*}{ Weight } & \multirow[t]{2}{*}{$\begin{array}{c}\text { Risk Ratio } \\
\text { IV, Random, } 95 \% \text { CI } \\
\end{array}$} \\
\hline & & & & & \\
\hline CVT (ph3,2v) & $231 / 3727$ & $486 / 3739$ & $=$ & $29.09 \%$ & $0.48[0.41,0.55]$ \\
\hline PATRICIA trial (ph3,2v) & $504 / 8863$ & $1227 / 8870$ & $\mathbf{m}$ & $33.9 \%$ & $0.41[0.37,0.45]$ \\
\hline Subtotal $(95 \% \mathrm{CI})$ & 12590 & 12609 & 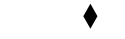 & $62.99 \%$ & $0.44[0.38,0.51]$ \\
\hline \multicolumn{6}{|c|}{ Total events: 735 (Vaccinated), 1713 (Placebo) } \\
\hline \multicolumn{6}{|c|}{ Heterogeneity: $\mathrm{Tau}^{2}=0.01 ; \mathrm{Chi}^{2}=2.61, \mathrm{df}=1(\mathrm{P}=0.11) ; \mathrm{I}^{2}=61.74 \%$} \\
\hline \multicolumn{6}{|c|}{ Test for overall effect: $Z=11.26(P<0.0001)$} \\
\hline \multicolumn{6}{|c|}{ 6.2.2 Age group $24-45$ years } \\
\hline FUTURE III trial $(\mathrm{ph} 3,4 \mathrm{v})$ & $91 / 1856$ & $157 / 1857$ & + & $20.05 \%$ & $0.58[0.45,0.74]$ \\
\hline VIVIANE trial (ph3,2v) & $67 / 2465$ & $121 / 2470$ & $\rightarrow$ & $16.96 \%$ & $0.55[0.41,0.74]$ \\
\hline Subtotal $(95 \% \mathrm{Cl})$ & 4321 & 4327 & $\diamond$ & $37.01 \%$ & $0.57[0.47,0.69]$ \\
\hline \multicolumn{6}{|c|}{ Heterogeneity: $\mathrm{Tau}^{2}=0 ; \mathrm{Chi}^{2}=0.05, \mathrm{df}=1(\mathrm{P}=0.82) ; \mathrm{I}^{2}=0 \%$} \\
\hline \multicolumn{6}{|c|}{ Test for overall effect: $Z=5.8(P<0.0001)$} \\
\hline Total $(95 \% \mathrm{Cl})$ & 16911 & 16936 & $\boldsymbol{\nabla}$ & $100 \%$ & $0.48[0.41,0.57]$ \\
\hline \multicolumn{6}{|c|}{ Total events: 893 (Vaccinated), 1991 (Placebo) } \\
\hline \multicolumn{6}{|c|}{ Heterogeneity: $\operatorname{Tau}^{2}=0.02 ; \mathrm{Chi}^{2}=9.66, \mathrm{df}=3(\mathrm{P}=0.02) ; \mathrm{I}^{2}=68.96 \%$} \\
\hline \multicolumn{6}{|c|}{ Test for overall effect: $Z=8.85(P<0.0001)$} \\
\hline Test for subgroup differe & $55, \mathrm{df}=1(\mathrm{P}=0.03)$, & $48 \%$ & & & \\
\hline
\end{tabular}

Analysis 6.3. Comparison 6 Infection with HPV types included in the vaccine in women regardless of HPV DNA status at baseline, Outcome 3 Persistent HPV6/11/16/18 infection (6M), at least 1 dose.

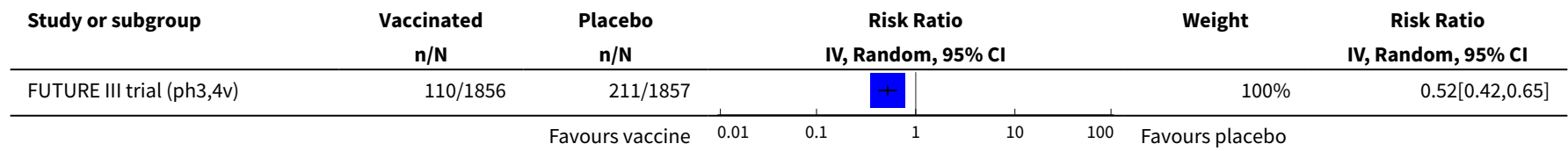




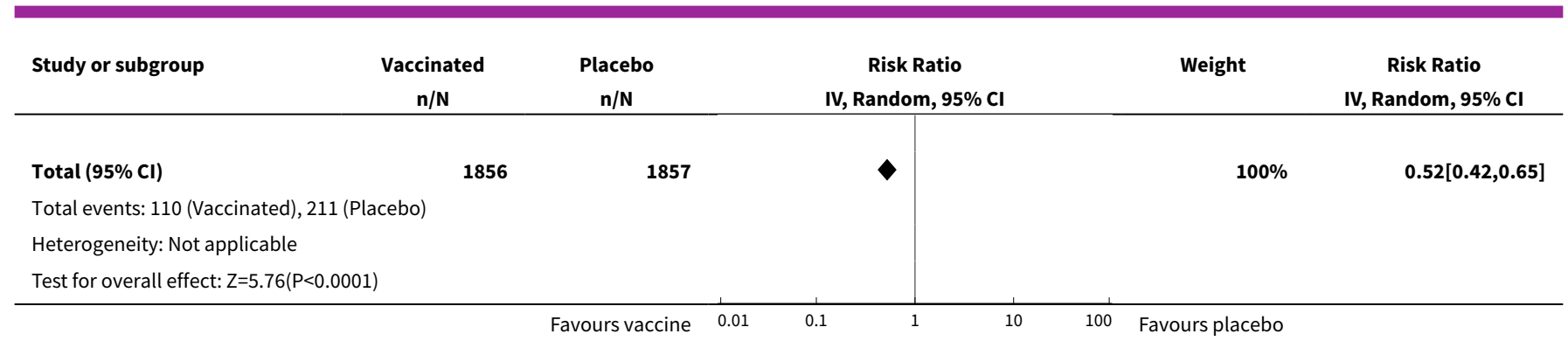

\section{Analysis 6.4. Comparison 6 Infection with HPV types included in the vaccine in women regardless} of HPV DNA status at baseline, Outcome 4 Persistent HPV16/18 infection (12M), at least 1 dose.

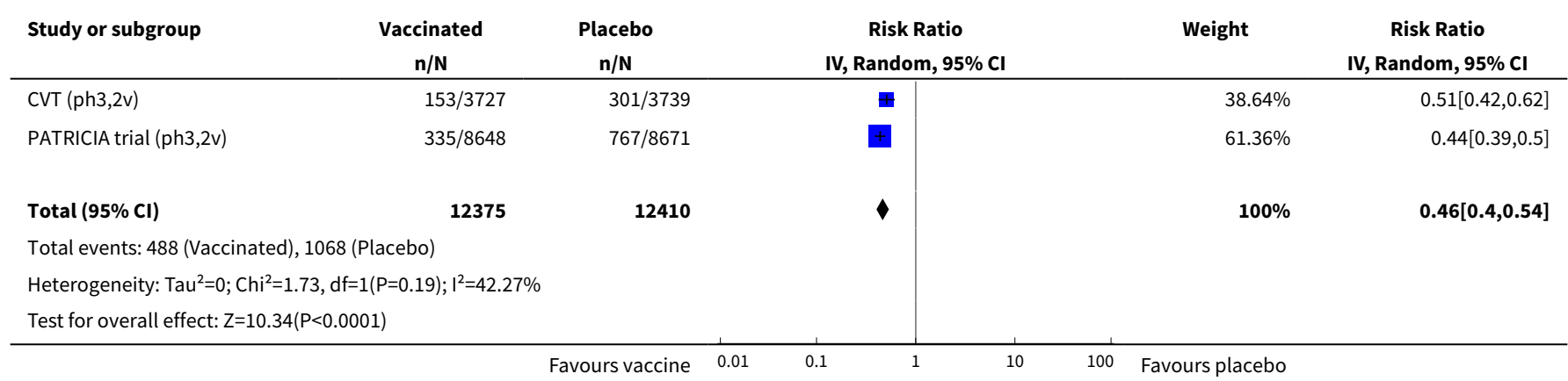

Analysis 6.5. Comparison 6 Infection with HPV types included in the vaccine in women regardless of HPV DNA status at baseline, Outcome 5 Persistent HPV16/18 infection (12M) by dose (post hoc analysis).

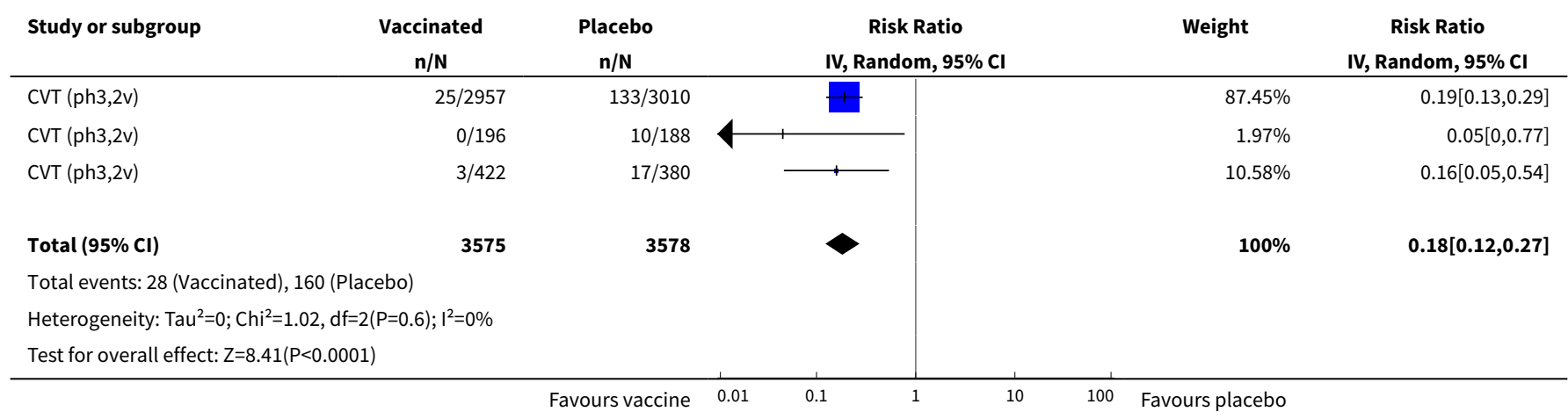

\section{Comparison 7. Adverse events}

\begin{tabular}{lllll}
\hline Outcome or subgroup title & No. of studies & $\begin{array}{l}\text { No. of partici- } \\
\text { pants }\end{array}$ & Statistical method & Effect size \\
\hline $\begin{array}{l}\text { 1 Overall local/injection site } \\
\text { adverse events }\end{array}$ & 8 & 18113 & Risk Ratio (IV, Fixed, 95\% Cl) & $1.18[1.16,1.20]$ \\
\hline \begin{tabular}{l}
1.1 Bivalent vaccine \\
\hline
\end{tabular} & 2 & 6503 & Risk Ratio (IV, Fixed, 95\% Cl) & $1.29[1.26,1.33]$ \\
\hline
\end{tabular}




\begin{tabular}{|c|c|c|c|c|}
\hline Outcome or subgroup title & No. of studies & $\begin{array}{l}\text { No. of partici- } \\
\text { pants }\end{array}$ & Statistical method & Effect size \\
\hline 1.2 Quadrivalent vaccine & 6 & 11610 & Risk Ratio (IV, Fixed, 95\% CI) & $1.14[1.12,1.16]$ \\
\hline 2 Pain at injection site & 13 & 25691 & Risk Ratio (IV, Random, 95\% CI) & $1.35[1.23,1.49]$ \\
\hline 2.1 Monovalent vaccine & 1 & 2280 & Risk Ratio (IV, Random, 95\% CI) & $1.05[1.01,1.09]$ \\
\hline 2.2 Bivalent vaccine & 8 & 16897 & Risk Ratio (IV, Random, 95\% CI) & $1.49[1.26,1.75]$ \\
\hline 2.3 Quadrivalent vaccine & 4 & 6514 & Risk Ratio (IV, Random, 95\% CI) & $1.13[1.07,1.19]$ \\
\hline 3 Swelling at injection site & 9 & 22106 & Risk Ratio (IV, Random, 95\% Cl) & $1.73[1.32,2.27]$ \\
\hline 3.1 Bivalent vaccine & 7 & 16603 & Risk Ratio (IV, Random, 95\% CI) & $1.62[1.15,2.29]$ \\
\hline 3.2 Quadrivalent vaccine & 2 & 5503 & Risk Ratio (IV, Random, 95\% CI) & $2.79[0.85,9.15]$ \\
\hline 4 Redness at injection site & 6 & 19996 & Risk Ratio (IV, Random, 95\% CI) & $1.72[1.50,1.97]$ \\
\hline 4.1 Quadrivalent vaccine & 1 & 5345 & Risk Ratio (IV, Random, 95\% Cl) & $1.46[1.32,1.63]$ \\
\hline 4.2 Bivalent vaccine & 5 & 14651 & Risk Ratio (IV, Random, 95\% CI) & $1.80[1.53,2.11]$ \\
\hline $\begin{array}{l}5 \text { Overall systemic event } \\
\text { and general symptoms }\end{array}$ & 8 & 18191 & Risk Ratio (IV, Random, 95\% CI) & $1.02[0.98,1.07]$ \\
\hline 5.1 Bivalent vaccine & 2 & 6503 & Risk Ratio (IV, Random, 95\% CI) & $1.07[0.97,1.19]$ \\
\hline 5.2 Quadrivalent vaccine & 6 & 11688 & Risk Ratio (IV, Random, 95\% CI) & $1.01[0.98,1.04]$ \\
\hline 6 Serious adverse events & 23 & 71597 & Risk Ratio (IV, Random, 95\% CI) & $0.98[0.92,1.05]$ \\
\hline 6.1 Monovalent vaccine & 1 & 2387 & Risk Ratio (IV, Random, 95\% CI) & $0.95[0.51,1.78]$ \\
\hline 6.2 Bivalent vaccine & 15 & 46231 & Risk Ratio (IV, Random, 95\% CI) & $1.01[0.96,1.07]$ \\
\hline 6.3 Quadrivalent vaccine & 7 & 22979 & Risk Ratio (IV, Random, 95\% CI) & $0.81[0.65,1.02]$ \\
\hline 7 Deaths & 23 & 71176 & Risk Ratio (IV, Random, 95\% CI) & $1.29[0.85,1.98]$ \\
\hline 7.1 Monovalent vaccine & 1 & 2280 & Risk Ratio (IV, Random, 95\% CI) & $0.0[0.0,0.0]$ \\
\hline 7.2 Bivalent vaccine & 15 & 46231 & Risk Ratio (IV, Random, 95\% CI) & $1.21[0.66,2.22]$ \\
\hline 7.3 Quadrivalent vaccine & 7 & 22665 & Risk Ratio (IV, Random, 95\% CI) & $1.54[0.73,3.23]$ \\
\hline
\end{tabular}

Analysis 7.1. Comparison 7 Adverse events, Outcome 1 Overall local/injection site adverse events.

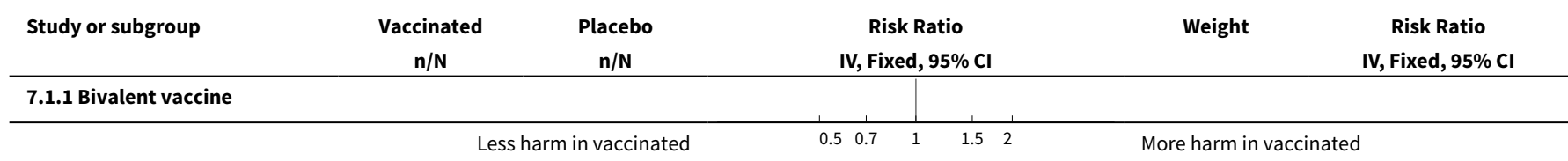




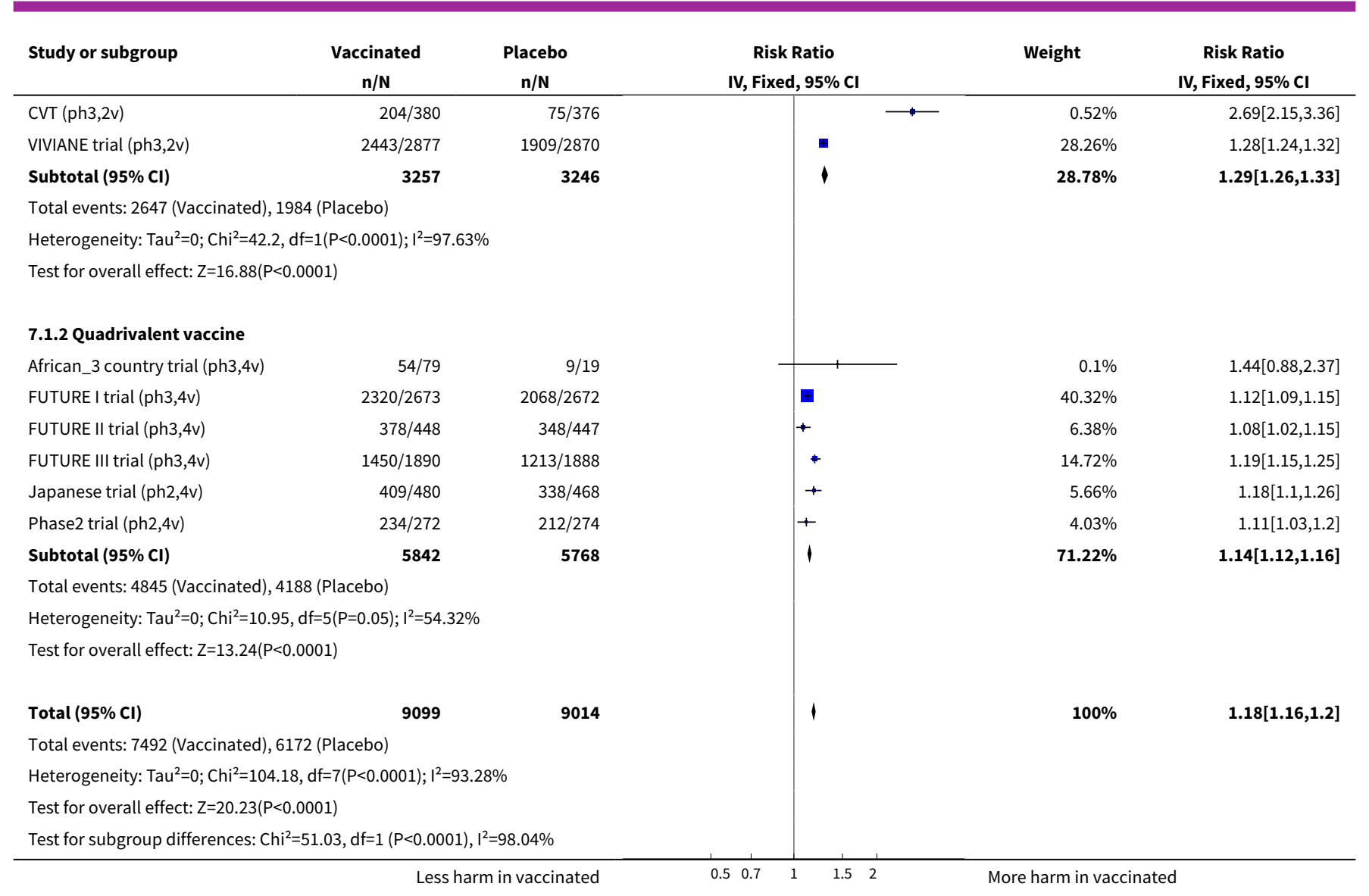

Analysis 7.2. Comparison 7 Adverse events, Outcome 2 Pain at injection site.

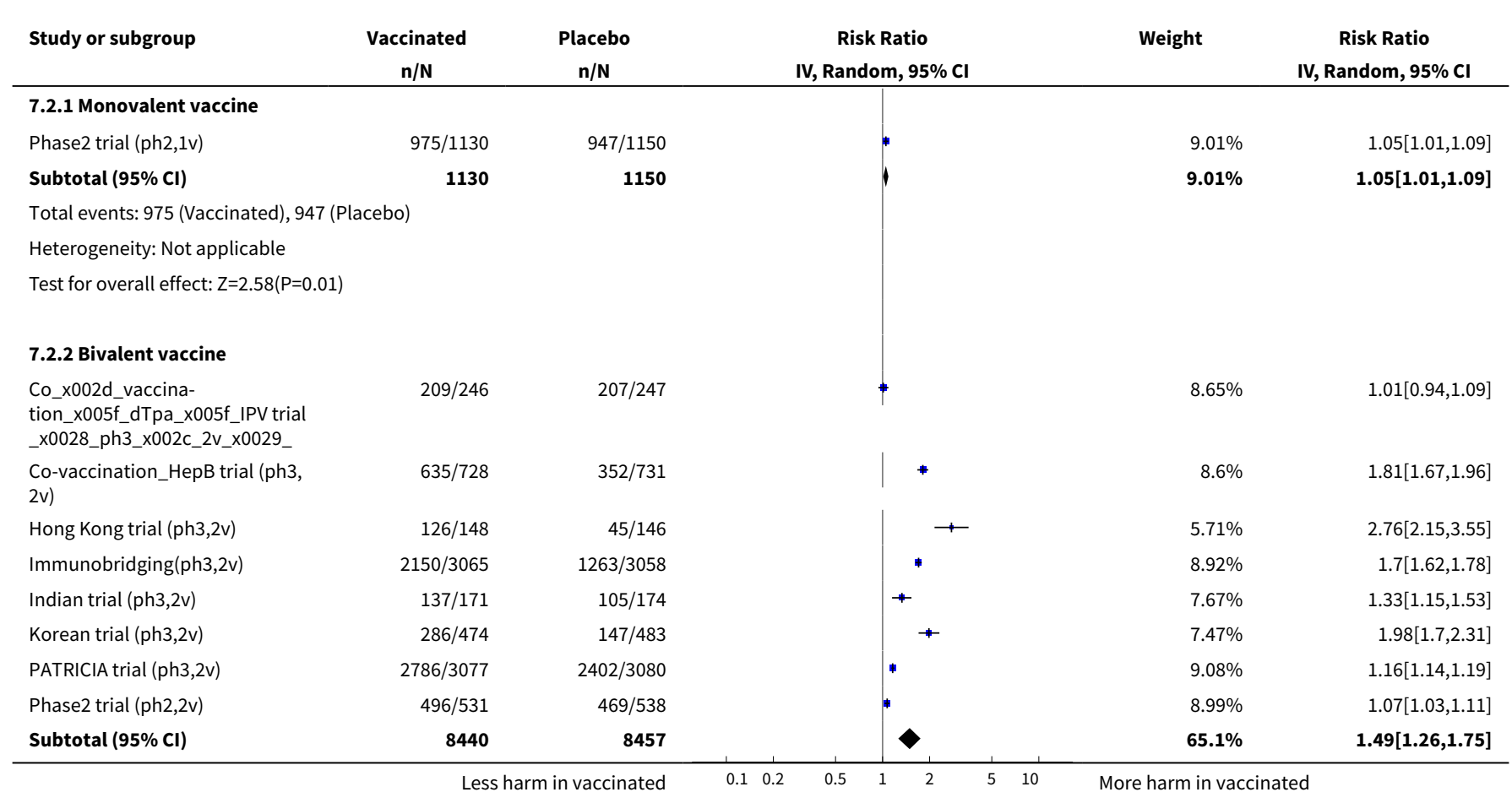




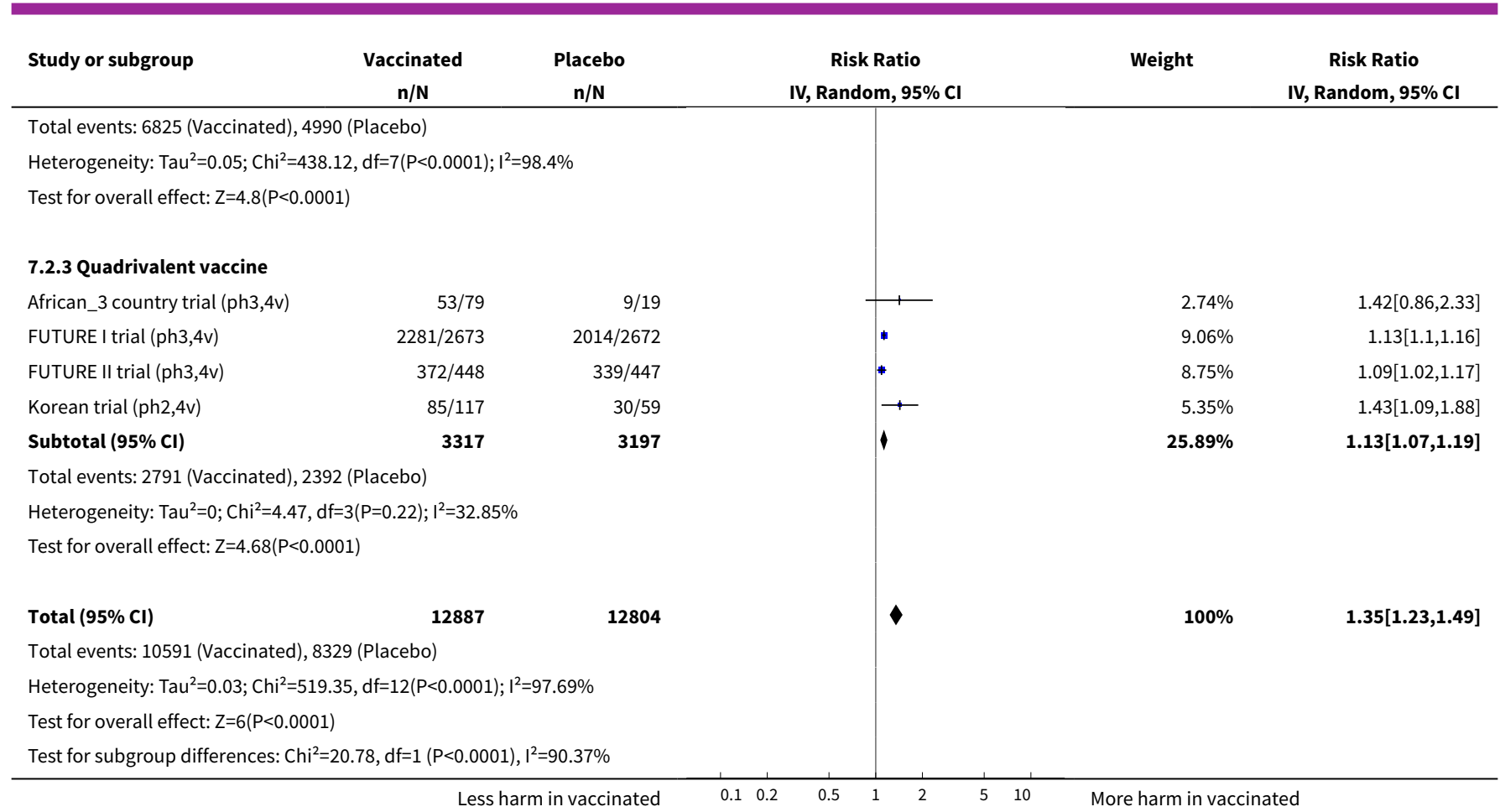

Analysis 7.3. Comparison 7 Adverse events, Outcome 3 Swelling at injection site.

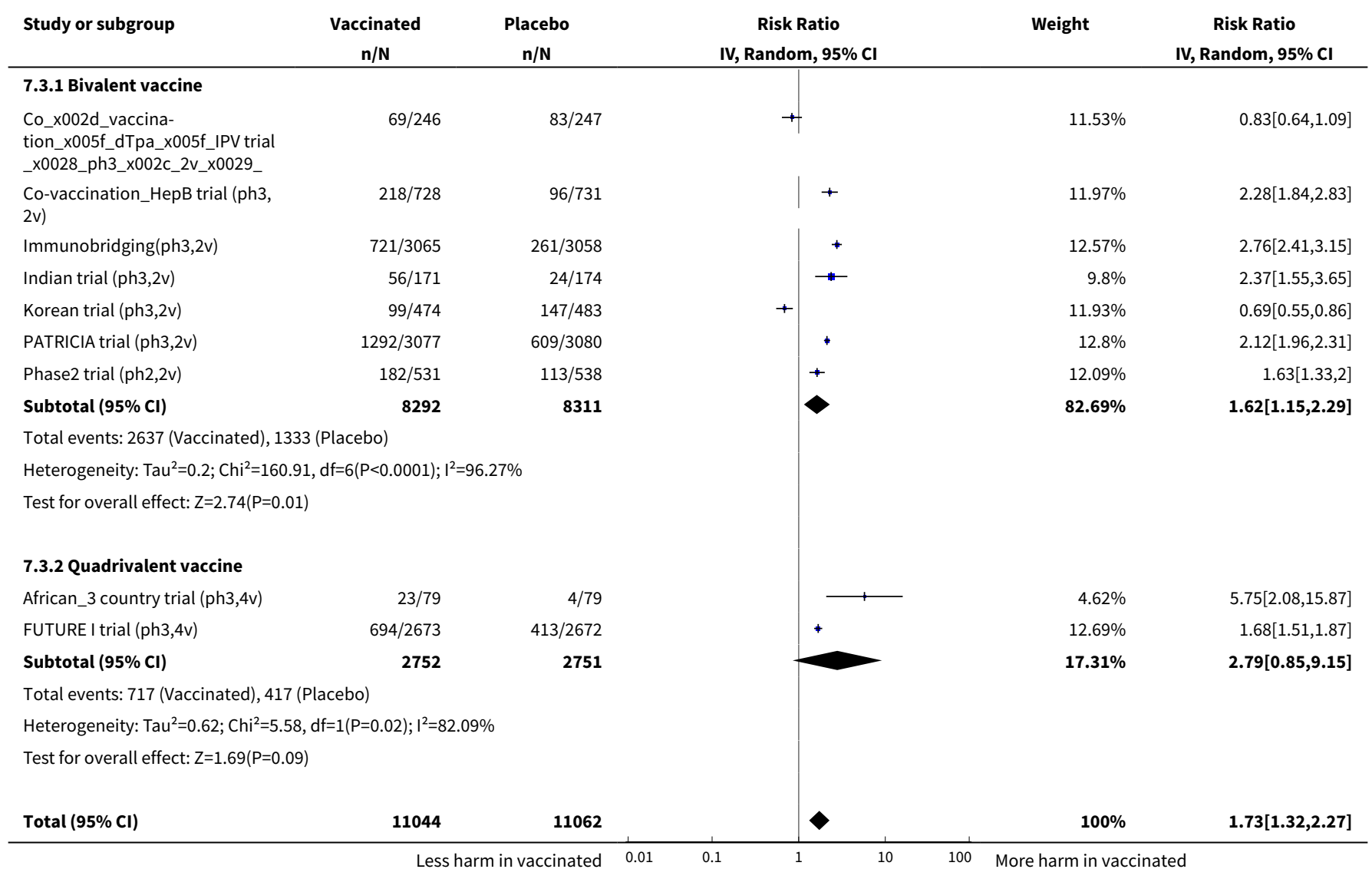




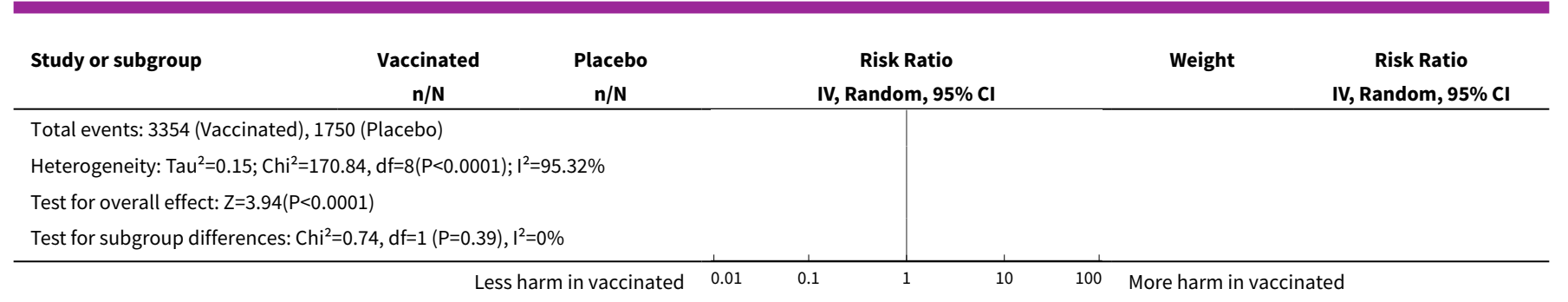

\section{Analysis 7.4. Comparison 7 Adverse events, Outcome 4 Redness at injection site.}

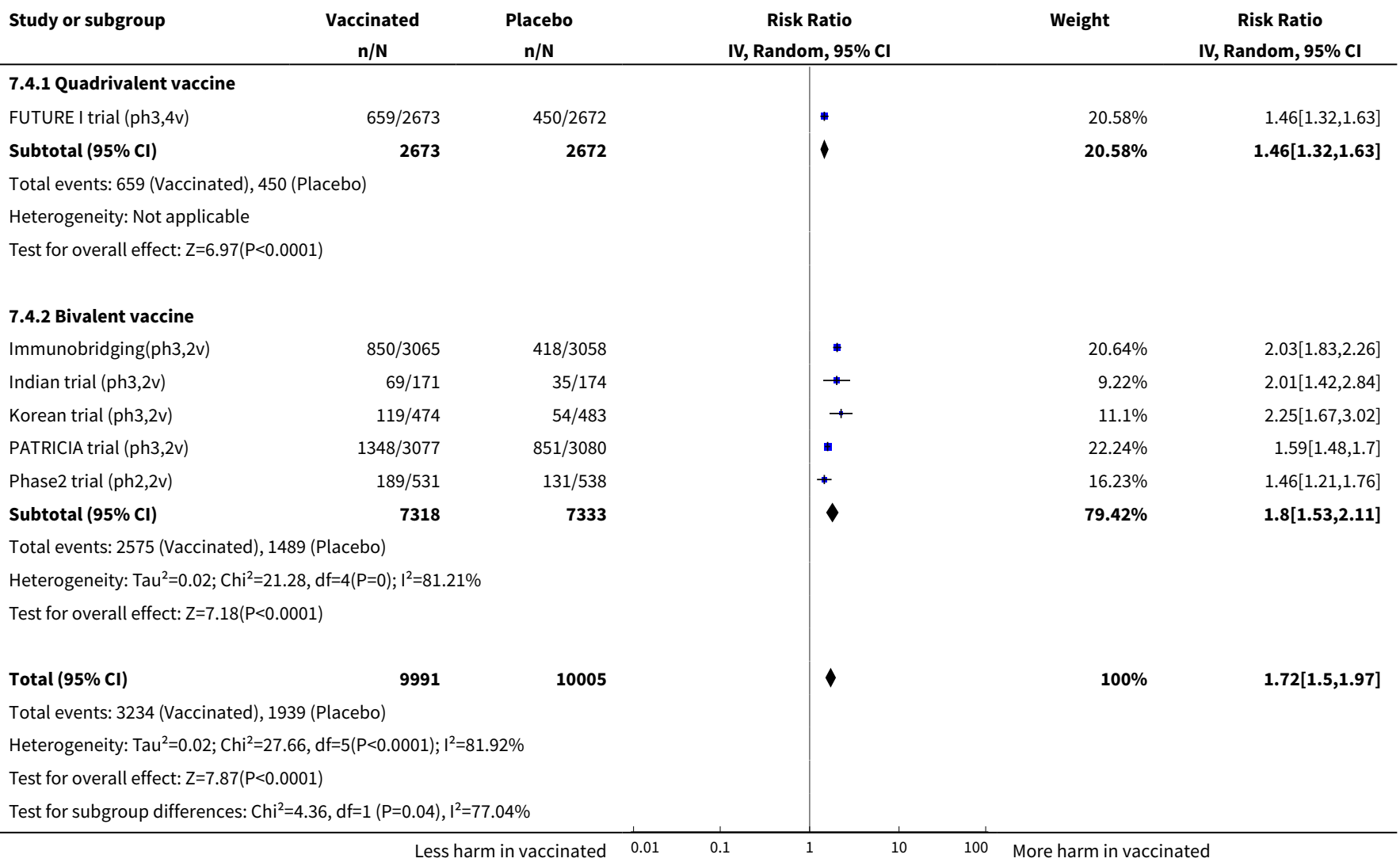

Analysis 7.5. Comparison 7 Adverse events, Outcome 5 Overall systemic event and general symptoms.

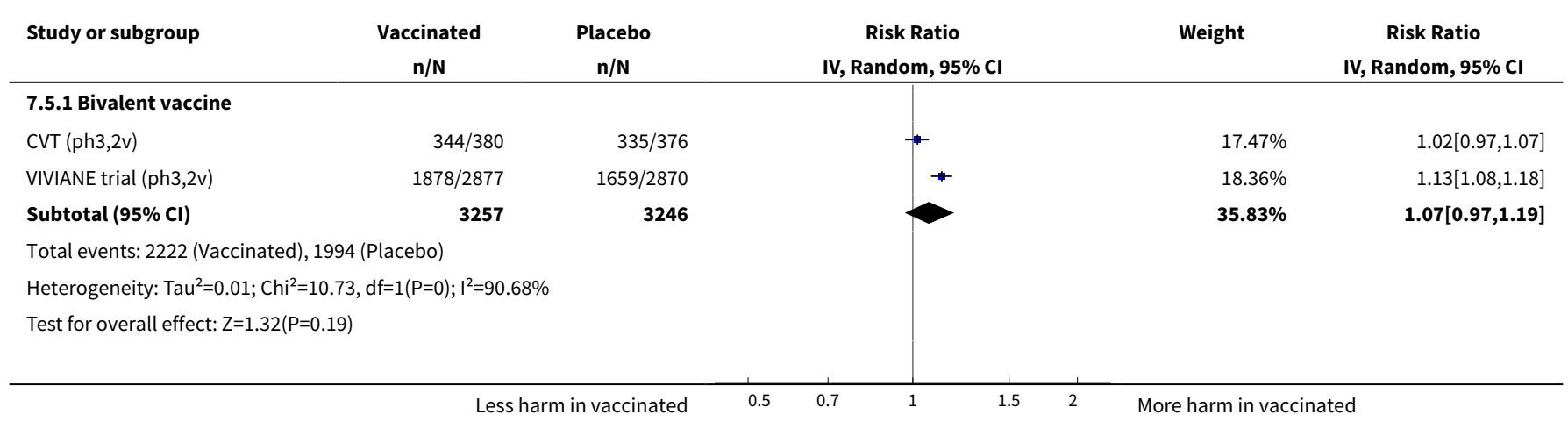




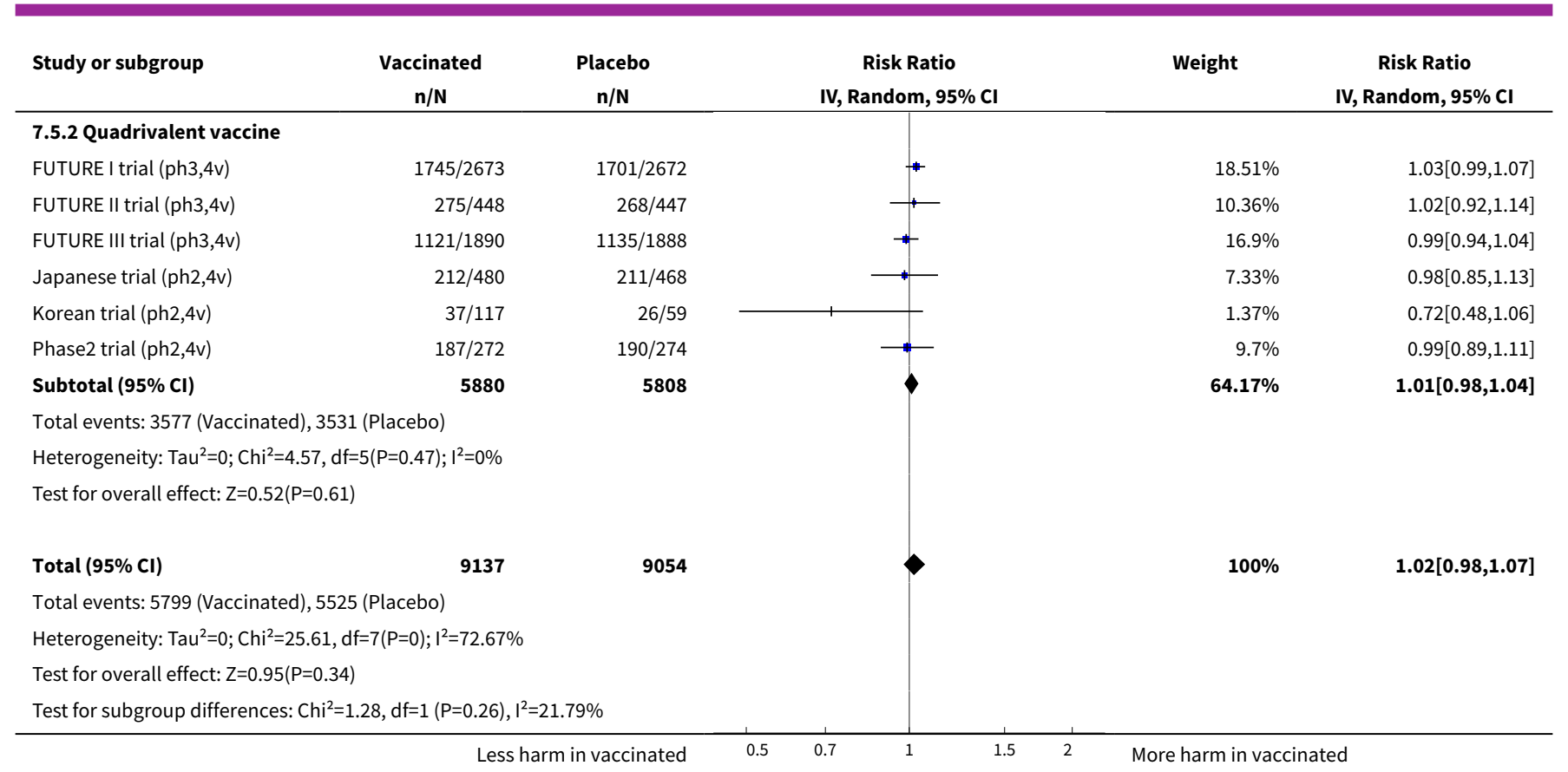

Analysis 7.6. Comparison 7 Adverse events, Outcome 6 Serious adverse events.

\begin{tabular}{|c|c|c|c|c|c|}
\hline Study or subgroup & $\begin{array}{c}\text { Vaccinated } \\
\mathrm{n} / \mathrm{N} \\
\end{array}$ & $\begin{array}{c}\text { Placebo } \\
\mathbf{n} / \mathbf{N}\end{array}$ & $\begin{array}{c}\text { Risk Ratio } \\
\text { IV, Random, 95\% CI }\end{array}$ & Weight & $\begin{array}{c}\text { Risk Ratio } \\
\text { IV, Random, } 95 \% \mathrm{CI}\end{array}$ \\
\hline \multicolumn{6}{|l|}{ 7.6.1 Monovalent vaccine } \\
\hline Phase2 trial (ph2,1v) & $19 / 1191$ & $20 / 1196$ & 1 & $1.08 \%$ & $0.95[0.51,1.78]$ \\
\hline Subtotal $(95 \% \mathrm{Cl})$ & 1191 & 1196 & & $1.08 \%$ & $0.95[0.51,1.78]$ \\
\hline \multicolumn{6}{|c|}{ Total events: 19 (Vaccinated), 20 (Placebo) } \\
\hline \multicolumn{6}{|l|}{ Heterogeneity: Not applicable } \\
\hline \multicolumn{6}{|c|}{ Test for overall effect: $Z=0.15(P=0.88)$} \\
\hline \multicolumn{6}{|l|}{ 7.6.2 Bivalent vaccine } \\
\hline African_2 country trial (ph3,2v) & $17 / 450$ & $14 / 226$ & $\rightarrow$ & $0.88 \%$ & $0.61[0.31,1.21]$ \\
\hline $\begin{array}{l}\text { Chinese trial }(\mathrm{ph} 3,2 \mathrm{v})_{-} \text {adoles- } \\
\text { cent }\end{array}$ & $5 / 374$ & $2 / 376$ & 1 & $0.16 \%$ & $2.51[0.49,12.87]$ \\
\hline Chinese trial (ph3,2v)_mid-adult & $3 / 606$ & $3 / 606$ & & $0.17 \%$ & $1[0.2,4.93]$ \\
\hline CVT (ph3,2v) & $912 / 3727$ & $891 / 3739$ & 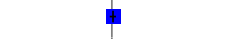 & $35.59 \%$ & $1.03[0.95,1.11]$ \\
\hline Hong Kong trial (ph3,2v) & $3 / 148$ & $1 / 146$ & & $0.08 \%$ & $2.96[0.31,28.12]$ \\
\hline Immunobridging(ph3,2v) & $24 / 1035$ & $23 / 1032$ & + & $1.3 \%$ & $1.04[0.59,1.83]$ \\
\hline Indian trial (ph3,2v) & $2 / 176$ & $4 / 178$ & - & $0.15 \%$ & $0.51[0.09,2.73]$ \\
\hline Japanese trial (ph2,2v) & $26 / 519$ & $34 / 521$ & & $1.69 \%$ & $0.77[0.47,1.26]$ \\
\hline Korean trial (ph3,2v) & $0 / 160$ & $1 / 161$ & & $0.04 \%$ & $0.34[0.01,8.17]$ \\
\hline Korean trial (ph3b,2v) & $2 / 149$ & $1 / 76$ & & $0.07 \%$ & $1.02[0.09,11.07]$ \\
\hline Malaysian trial (ph3,2v) & $5 / 135$ & $3 / 136$ & . & $0.21 \%$ & $1.68[0.41,6.89]$ \\
\hline PATRICIA trial (ph3,2v) & $835 / 9319$ & $829 / 9325$ & $\phi$ & $30.59 \%$ & $1.01[0.92,1.1]$ \\
\hline Phase2 trial (ph2,2v) & $22 / 560$ & $19 / 553$ & 1 & $1.15 \%$ & $1.14[0.63,2.09]$ \\
\hline VIVIANE trial (ph3,2v) & $291 / 2877$ & $269 / 2870$ & + & $14.01 \%$ & $1.08[0.92,1.26]$ \\
\hline Subtotal $(95 \% \mathrm{Cl})$ & 23261 & 22970 & 1 & $89.65 \%$ & $1.01[0.96,1.07]$ \\
\hline \multicolumn{6}{|c|}{ Total events: 2203 (Vaccinated), 2175 (Placebo) } \\
\hline
\end{tabular}




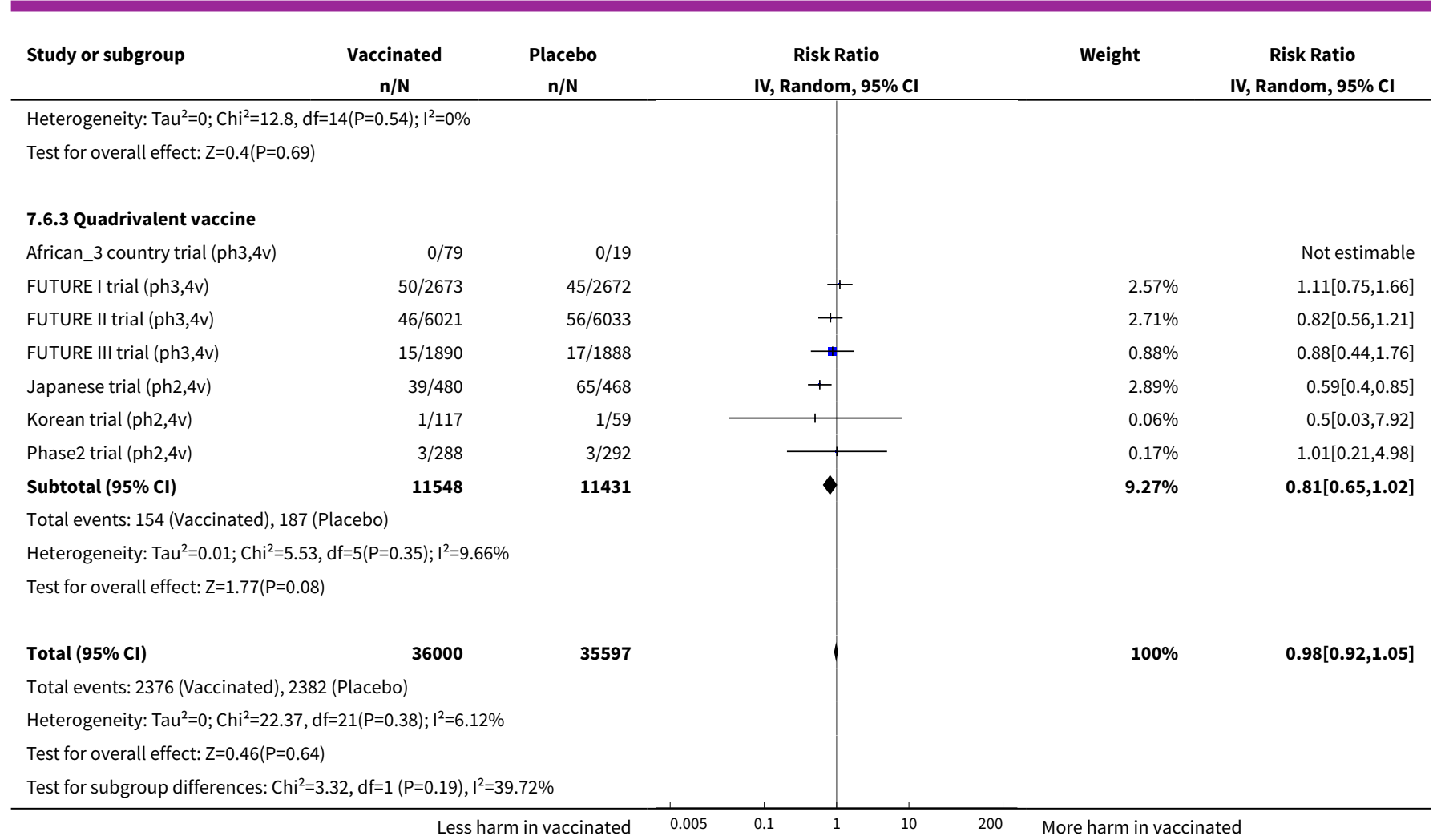

Analysis 7.7. Comparison 7 Adverse events, Outcome 7 Deaths.

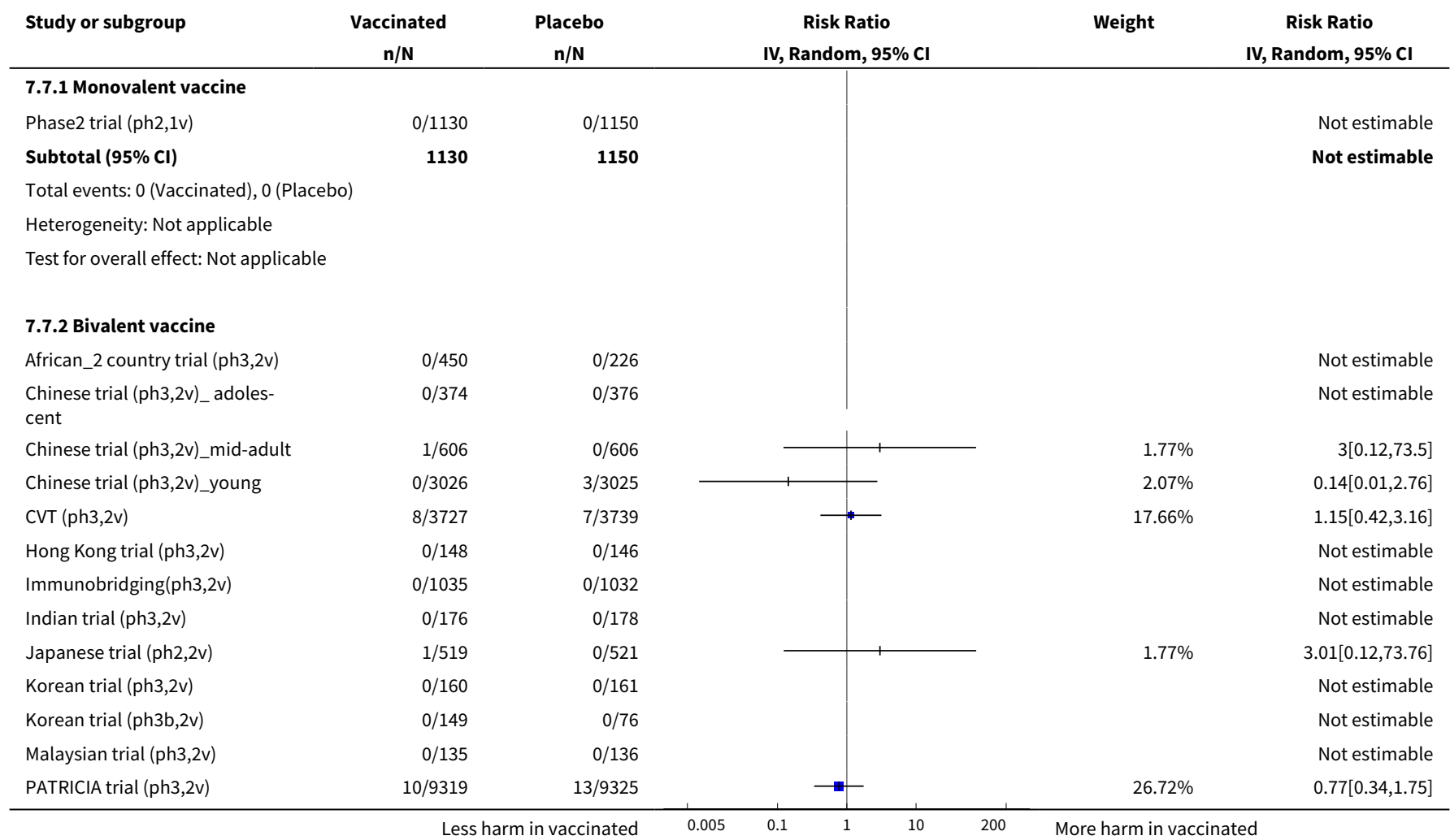




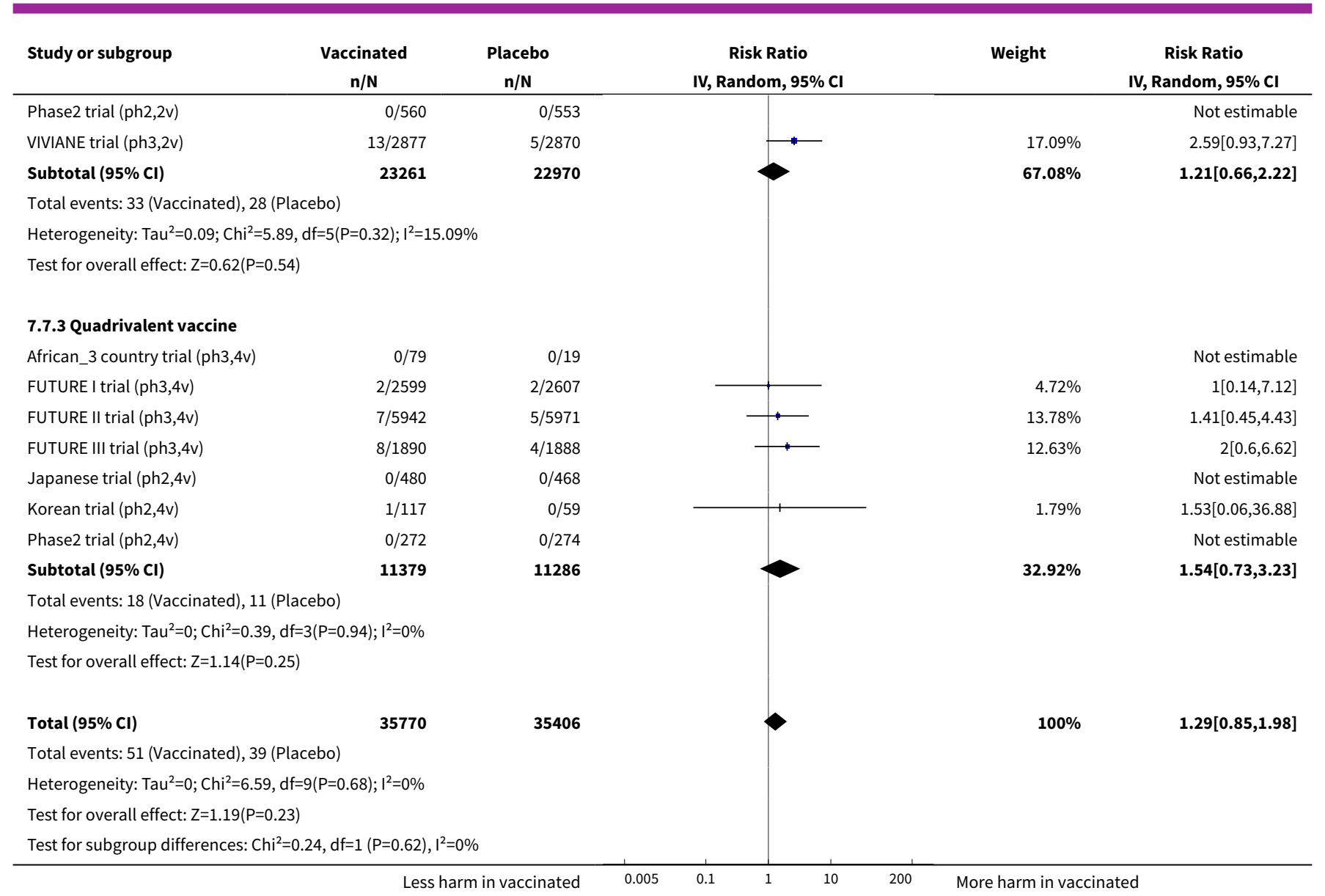

\section{Comparison 8. Pregnancy outcomes}

\begin{tabular}{|c|c|c|c|c|}
\hline Outcome or subgroup title & No. of studies & $\begin{array}{l}\text { No. of partici- } \\
\text { pants }\end{array}$ & Statistical method & Effect size \\
\hline 1 Normal infant & 8 & 8782 & Risk Ratio (IV, Random, 95\% CI) & $1.00[0.97,1.02]$ \\
\hline $\begin{array}{l}2 \text { Spontaneous abor- } \\
\text { tion/miscarriage }\end{array}$ & 9 & 8618 & Risk Ratio (IV, Random, 95\% CI) & $0.88[0.68,1.14]$ \\
\hline $\begin{array}{l}3 \text { Elective termination/in- } \\
\text { duced abortion }\end{array}$ & 9 & 10909 & Risk Ratio (IV, Random, 95\% CI) & $0.90[0.80,1.02]$ \\
\hline 4 Stillbirth & 6 & 8754 & Risk Ratio (IV, Random, 95\% CI) & $1.12[0.68,1.83]$ \\
\hline 5 Abnormal infant & 5 & 9252 & Risk Ratio (IV, Random, 95\% CI) & $1.22[0.88,1.69]$ \\
\hline
\end{tabular}


Analysis 8.1. Comparison 8 Pregnancy outcomes, Outcome 1 Normal infant.

\begin{tabular}{|c|c|c|c|c|c|}
\hline Study or subgroup & $\begin{array}{c}\text { Vaccinated } \\
\mathrm{n} / \mathrm{N} \\
\end{array}$ & $\begin{array}{c}\text { Placebo } \\
n / N\end{array}$ & $\begin{array}{c}\text { Risk Ratio } \\
\text { IV, Random, } 95 \% \text { CI }\end{array}$ & Weight & $\begin{array}{c}\text { Risk Ratio } \\
\text { IV, Random, 95\% CI }\end{array}$ \\
\hline African_2 country trial (ph3,2v) & $5 / 14$ & $5 / 10$ & & $0.07 \%$ & $0.71[0.28,1.82]$ \\
\hline Chinese trial (ph3,2v)_young & $106 / 188$ & $124 / 229$ & 1 & $2.12 \%$ & $1.04[0.88,1.24]$ \\
\hline FUTURE I trial (ph3,4v) & $411 / 618$ & $394 / 609$ & * & $9.66 \%$ & $1.03[0.95,1.11]$ \\
\hline FUTURE II trial (ph3,4v) & $855 / 1255$ & $871 / 1283$ & * & $22.3 \%$ & $1[0.95,1.06]$ \\
\hline FUTURE III trial (ph3,4v) & $83 / 123$ & $88 / 135$ & 1 & $2.1 \%$ & $1.04[0.87,1.23]$ \\
\hline PATRICIA \& CVT (ph3,2v) & $1401 / 1786$ & $1449 / 1813$ & + & $56.54 \%$ & $0.98[0.95,1.01]$ \\
\hline VIVIANE trial (ph3,2v) & $257 / 357$ & $250 / 358$ & 4 & $7.2 \%$ & $1.03[0.94,1.13]$ \\
\hline Total $(95 \% \mathrm{Cl})$ & 4343 & 4439 & l & $100 \%$ & $1[0.97,1.02]$ \\
\hline \multicolumn{6}{|c|}{ Total events: 3118 (Vaccinated), 3183 (Placebo) } \\
\hline \multicolumn{6}{|c|}{ Heterogeneity: $\mathrm{Tau}^{2}=0 ; \mathrm{Chi}^{2}=4.32, \mathrm{df}=7(\mathrm{P}=0.74) ; \mathrm{I}^{2}=0 \%$} \\
\hline
\end{tabular}

\section{Analysis 8.2. Comparison 8 Pregnancy outcomes, Outcome 2 Spontaneous abortion/miscarriage.}

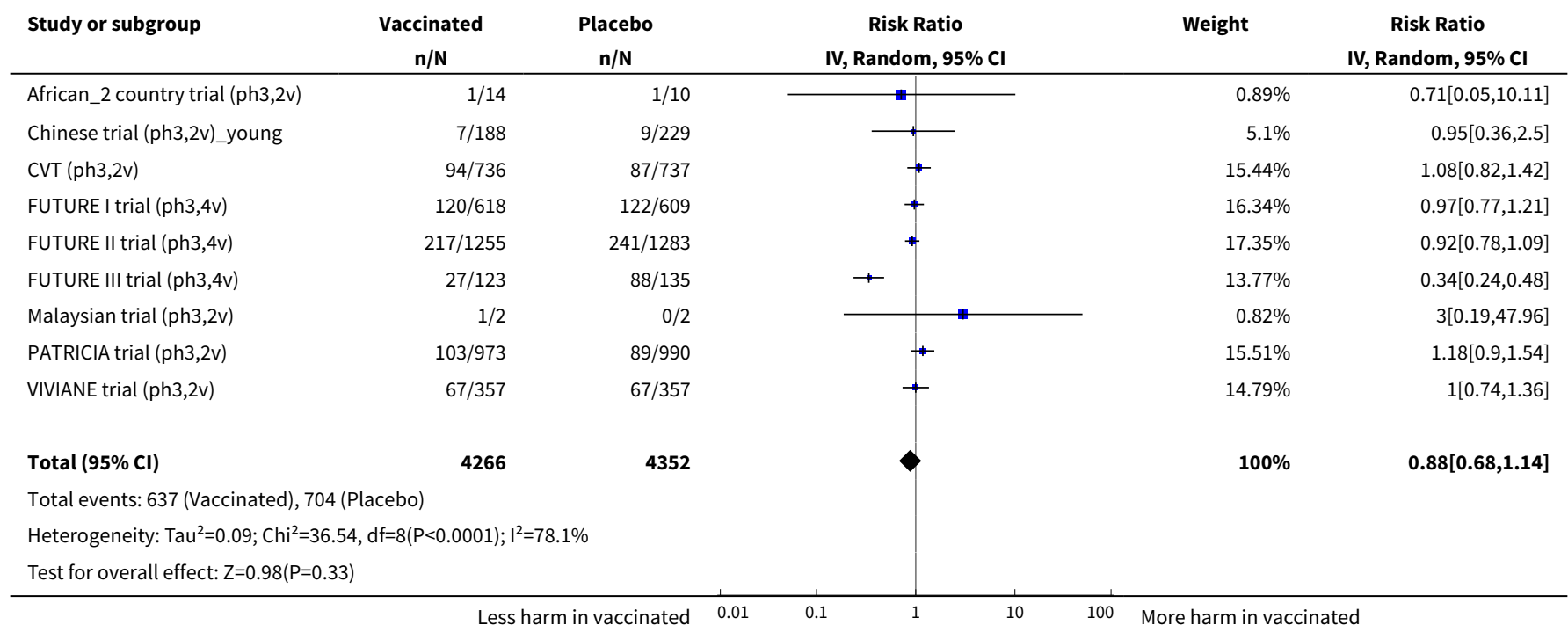

\section{Analysis 8.3. Comparison 8 Pregnancy outcomes, Outcome 3 Elective termination/induced abortion.}

\begin{tabular}{|c|c|c|c|c|c|}
\hline Study or subgroup & $\begin{array}{c}\text { Vaccinated } \\
n / N \\
\end{array}$ & $\begin{array}{c}\text { Placebo } \\
\mathrm{n} / \mathbf{N}\end{array}$ & $\begin{array}{c}\text { Risk Ratio } \\
\text { IV, Random, 95\% CI }\end{array}$ & Weight & $\begin{array}{c}\text { Risk Ratio } \\
\text { IV, Random, 95\% CI } \\
\end{array}$ \\
\hline African_2 country trial (ph3,2v) & $3 / 14$ & $3 / 10$ & $\longrightarrow-$ & $0.75 \%$ & $0.71[0.18,2.84]$ \\
\hline Chinese trial (ph3,2v)_mid-adult & $4 / 606$ & $1 / 606$ & 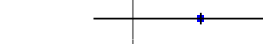 & $0.3 \%$ & $4[0.45,35.68]$ \\
\hline Chinese trial $(\mathrm{ph} 3,2 \mathrm{v})$ _young & $41 / 188$ & $65 / 229$ & $\rightarrow$ & $12.36 \%$ & $0.77[0.55,1.08]$ \\
\hline FUTURE I trial (ph3,4v) & $51 / 618$ & $55 / 609$ & $\rightarrow$ & $10.77 \%$ & $0.91[0.63,1.32]$ \\
\hline FUTURE II trial (ph3,4v) & $115 / 1255$ & $130 / 1283$ & 1 & $25.1 \%$ & $0.9[0.71,1.15]$ \\
\hline
\end{tabular}




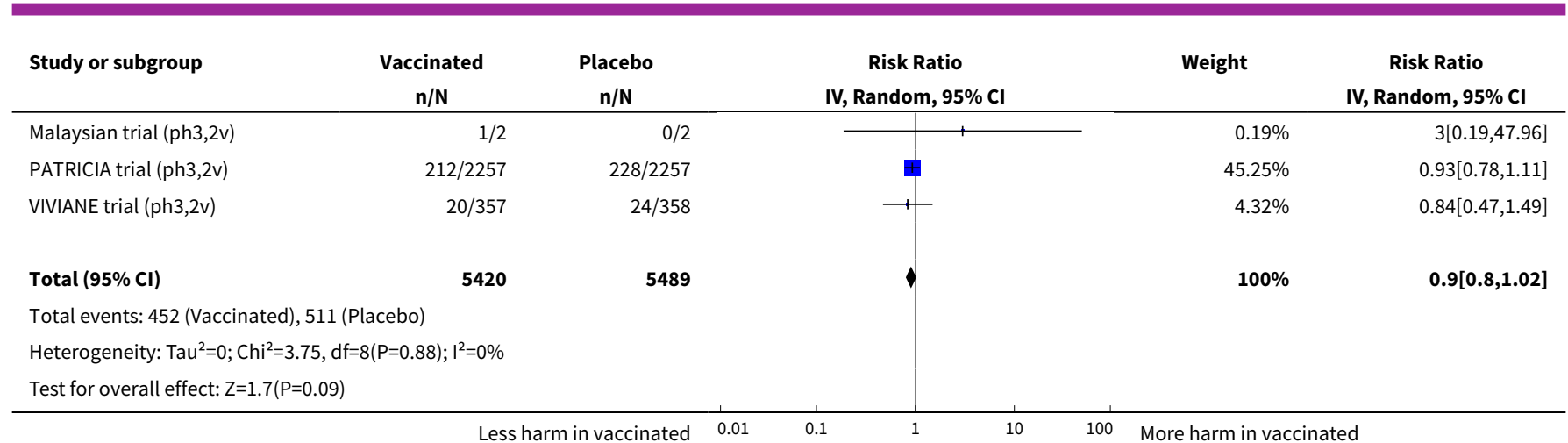

Analysis 8.4. Comparison 8 Pregnancy outcomes, Outcome 4 Stillbirth.

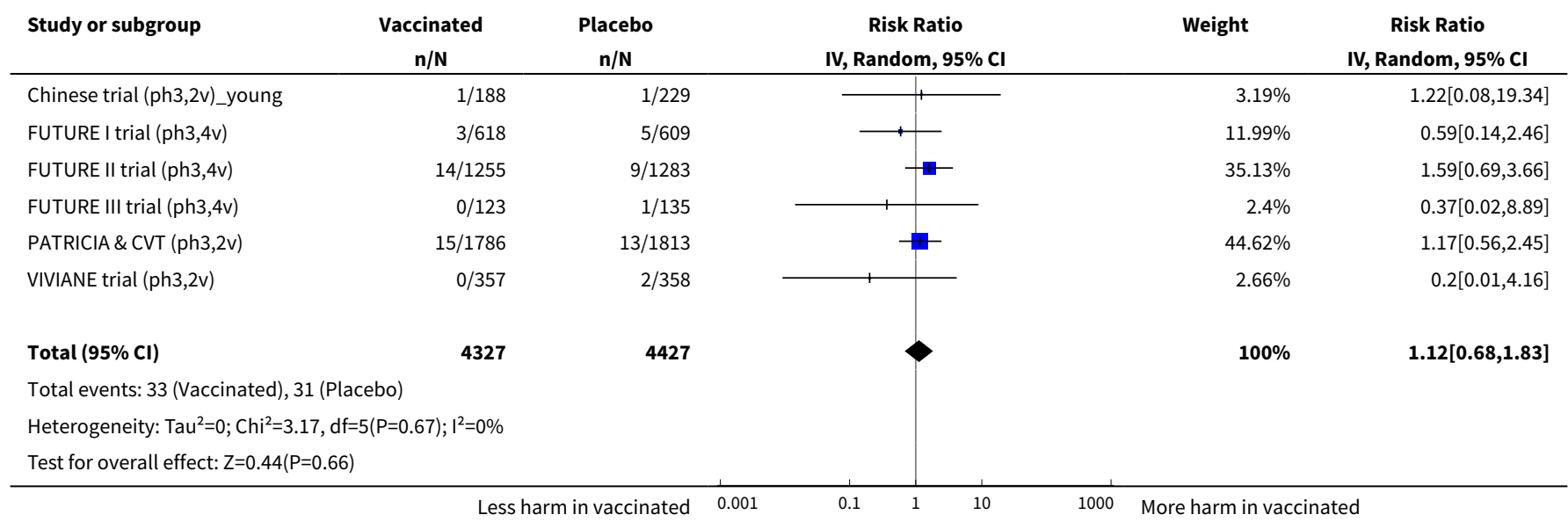

Analysis 8.5. Comparison 8 Pregnancy outcomes, Outcome 5 Abnormal infant.

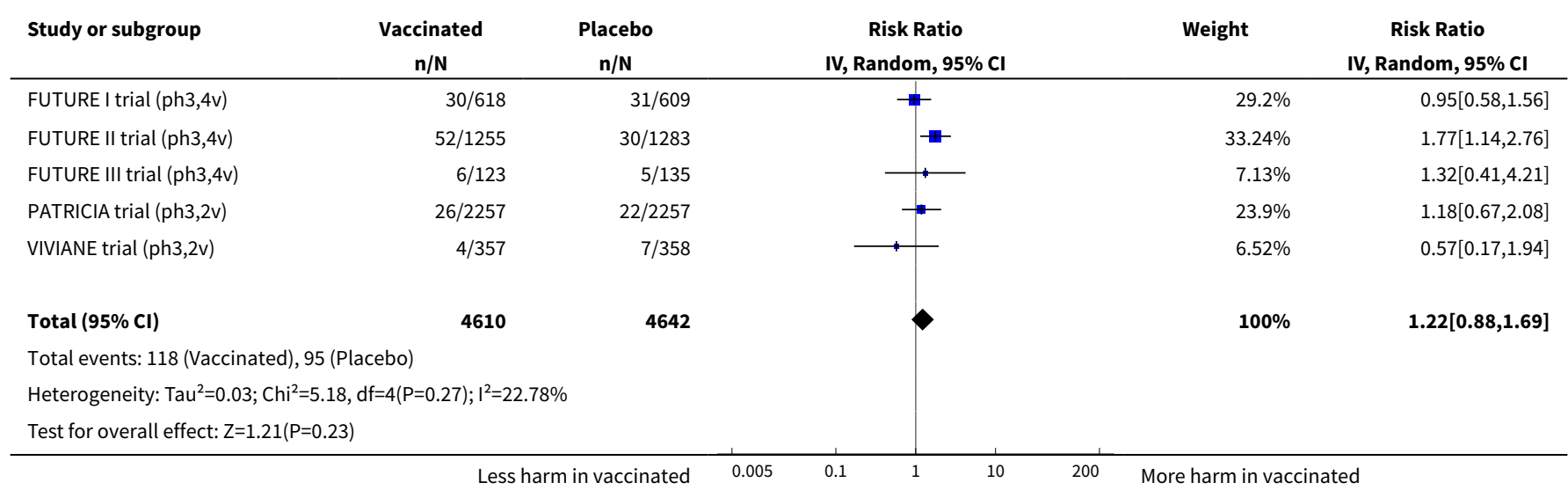

\section{ADDITIONAL TABLES}


Table 1. Listing of included trials

\begin{tabular}{|c|c|c|c|c|c|c|}
\hline Valency & Phase & $\begin{array}{l}\text { Number of } \\
\text { trials }\end{array}$ & Appelation & $\mathrm{N}$ & Outcomes & Main References \\
\hline Monovalent & II & 1 & Phase2 trial (ph2,1v) & 2392 & $\begin{array}{l}\text { Efficacy, safe- } \\
\text { ty }\end{array}$ & $\begin{array}{l}\text { Koutsky } 2002 \\
\text { Mao } 2006\end{array}$ \\
\hline & & & & & & Rowhani-Rahbar 2009 \\
\hline \multirow[t]{18}{*}{ Bivalent } & II & 2 & Japanese trial (ph2,2v) & 1040 & $\begin{array}{l}\text { Efficacy, safe- } \\
\text { ty }\end{array}$ & Konno 2010 \\
\hline & & & & & & Konno 2014 \\
\hline & & & Phase2 trial (ph2,2v) & 1113 & $\begin{array}{l}\text { Efficacy, safe- } \\
\text { ty }\end{array}$ & $\begin{array}{l}\text { Harper } 2004 \\
\text { Harper } 2006\end{array}$ \\
\hline & & & & & & $\begin{array}{l}\text { The GSK Study Group } \\
2009\end{array}$ \\
\hline & & & & & & De Carvalho 2010 \\
\hline & III & 16 & $\begin{array}{l}\text { African_2 country trial } \\
\text { (ph3,2v) }\end{array}$ & 676 & Safety & Sow 2013 \\
\hline & & & Chinese trial (ph3,2v)_young & 6051 & $\begin{array}{l}\text { Efficacy, safe- } \\
\text { ty }\end{array}$ & Zhu 2014 \\
\hline & & & $\begin{array}{l}\text { Chinese trial }(\mathrm{ph} 3,2 \mathrm{v})_{-} \text {ado- } \\
\text { lescent }\end{array}$ & 750 & Safety & Zhu 2014a \\
\hline & & & $\begin{array}{l}\text { Chinese trial (ph3,2v)_mid- } \\
\text { adult }\end{array}$ & 1212 & Safety & Zhu 2014a \\
\hline & & & $\begin{array}{l}\text { Co-vaccination_dTpa_IPV tri- } \\
\text { al (ph3,2v) }\end{array}$ & 494 & Safety & Garcia-Sicilia 2010 \\
\hline & & & $\begin{array}{l}\text { Co-vaccination_HAB trial } \\
(\mathrm{Ph} 3,2 \mathrm{v})\end{array}$ & 494 & Safety & Pedersen 2012 \\
\hline & & & $\begin{array}{l}\text { Co-vaccination_HepB trial } \\
\text { (ph3, 2v) }\end{array}$ & 541 & Safety & Schmeink 2011 \\
\hline & & & $\mathrm{CVT}(\mathrm{ph} 3,2 \mathrm{v})$ & 7466 & $\begin{array}{l}\text { Efficacy, safe- } \\
\text { ty }\end{array}$ & $\begin{array}{l}\text { Herrero } 2011 \\
\text { Kreimer } 2011\end{array}$ \\
\hline & & & Hong Kong trial (ph3,2v) & 294 & Safety & Ngan 2010 \\
\hline & & & Immunobridging(ph3,2v) & 2067 & Safety & Medina 2010 \\
\hline & & & Indian trial (ph3,2v) & 354 & Safety & Bhatla 2010 \\
\hline & & & Korean trial $(\mathrm{ph} 3,2 \mathrm{v})$ & 208 & Safety & Kim 2010 \\
\hline & & & Korean trial (ph3b,2v) & 321 & Safety & Kim 2011 \\
\hline
\end{tabular}


Table 1. Listing of included trials (Continued)

\begin{tabular}{llll} 
Malaysian trial (ph3,2v) & 271 & Safety & Lim 2014 \\
\hline PATRICIA trial (ph3,2v) & 18,644 & $\begin{array}{l}\text { Efficacy, safe- } \\
\text { ty }\end{array}$ & Paavonen 2007 \\
& & & Paavonen 2009
\end{tabular}

Szarewski 2011

Wheeler 2011

Lehtinen 2012

VIVIANE trial (ph3,2v) $5752 \quad$ Efficay, safety, $\quad$ Skinner 2014

Wheeler 2016

\begin{tabular}{|c|c|c|c|c|c|c|}
\hline \multirow{9}{*}{$\begin{array}{l}\text { Quadriva- } \\
\text { lent }\end{array}$} & II & 3 & Japanese trial (ph2,4v) & 1021 & Safety & Yoshikawa 2013 \\
\hline & & & Korean trial (ph2,4v) & 176 & Safety & Kang 2008 \\
\hline & & & Phase2 trial (ph2,4v) & 552 & $\begin{array}{l}\text { Efficacy, safe- } \\
\text { ty }\end{array}$ & Villa 2005 \\
\hline & & & & & & Villa 2006a \\
\hline & & & & & & Olsson 2009 \\
\hline & III & 4 & $\begin{array}{l}\text { African_3 country trial } \\
\text { (ph3,4v) }\end{array}$ & 98 & Safety & Mugo 2015 \\
\hline & & & FUTURE I trial (ph3,4v) & 5455 & $\begin{array}{l}\text { Efficacy, safe- } \\
\text { ty }\end{array}$ & Garland 2007 \\
\hline & & & FUTURE II trial (ph3,4v) & 12,167 & $\begin{array}{l}\text { Efficacy, safe- } \\
\text { ty }\end{array}$ & FUTURE-II 2007 \\
\hline & & & FUTURE III trial (ph3,4v) & 3819 & $\begin{array}{l}\text { Efficacy, safe- } \\
\text { ty }\end{array}$ & $\begin{array}{l}\text { Munoz } 2009 \\
\text { Castellsagué } 2011\end{array}$ \\
\hline Total & & 26 & & 73,428 & & \\
\hline
\end{tabular}




\begin{tabular}{|c|c|c|c|c|c|c|c|}
\hline \multirow[t]{2}{*}{ Outcomes and exposure subgroups } & \multicolumn{2}{|c|}{ Absolute risk / per 10,000 } & \multirow{2}{*}{$\begin{array}{l}\text { Relative risk } \\
(95 \% \mathrm{CI})\end{array}$} & \multirow{2}{*}{$\begin{array}{l}\text { Vaccine } \\
\text { efficacy } \\
(95 \% \mathrm{Cl})\end{array}$} & \multirow{2}{*}{$\begin{array}{l}\text { Risk differ- } \\
\text { ence/ per } \\
10,000 \\
(95 \% \mathrm{Cl})\end{array}$} & \multirow{2}{*}{$\begin{array}{l}\text { No of Partici- } \\
\text { pants } \\
\text { (studies) }\end{array}$} & \multirow{2}{*}{$\begin{array}{l}\text { Certainty of } \\
\text { evidence } \\
\text { (GRADE) }^{\star}\end{array}$} \\
\hline & Placebo & Vaccinated & & & & & \\
\hline \multicolumn{8}{|c|}{ 1. High-grade cervical lesions in women who were hrHPV DNA negative at baseline } \\
\hline $\begin{array}{l}\text { Analysis } 1.1 \mathrm{CIN} 2+\text { associated with HPV } 16 / 18 \text {, at } \\
\text { least } 1 \text { dose, age } 15-26 \text { years }\end{array}$ & 164 & 2 & $\begin{array}{l}\mathbf{0 . 0 1} \\
(0.00 \text { to } 0.05)\end{array}$ & $\begin{array}{l}\mathbf{9 9} \% \\
(95 \% \text { to } 100 \%)\end{array}$ & $\begin{array}{l}162 \\
\text { (157 to } 164)\end{array}$ & $\begin{array}{l}23,676 \\
\text { (3 studies) }\end{array}$ & $\begin{array}{l}\oplus \oplus \oplus \oplus \\
\text { high }\end{array}$ \\
\hline $\begin{array}{l}\text { Analysis } 1.2 \mathrm{CIN} 2+\text { associated with } \\
\text { HPV6/11/16/18, at least } 1 \text { dose, age } 15-26 \text { years }\end{array}$ & 197 & 2 & $\begin{array}{l}\mathbf{0 . 0 1} \\
(0.00 \text { to } 0.09)\end{array}$ & $\begin{array}{l}\mathbf{9 9} \% \\
(91 \% \text { to } 100 \%)\end{array}$ & $\begin{array}{l}195 \\
\text { (179 to } 197)\end{array}$ & $\begin{array}{l}9296 \\
\text { (1 study) }\end{array}$ & $\begin{array}{l}\oplus \oplus \oplus \ominus \\
\text { moderate }^{3}\end{array}$ \\
\hline $\begin{array}{l}\text { Analysis } 1.3 \mathrm{CIN} 3+\text { associated with HPV16/18, at } \\
\text { least } 1 \text { dose, age } 15-26 \text { years }\end{array}$ & 70 & $0^{*}$ & $\begin{array}{l}\mathbf{0 . 0 1} \\
(0.00 \text { to } 0.10)\end{array}$ & $\begin{array}{l}\mathbf{9 9} \% \\
(90 \% \text { to } 100 \%)\end{array}$ & $\begin{array}{l}70 \\
\text { (63 to } 70)\end{array}$ & $\begin{array}{l}20,214 \\
\text { (2 studies) }\end{array}$ & $\begin{array}{l}\oplus \oplus \oplus \oplus \\
\text { high }\end{array}$ \\
\hline $\begin{array}{l}\text { Analysis } 1.4 \mathrm{CIN} 3+\text { associated with } \\
\text { HPV6/11/16/18, at least } 1 \text { dose, age } 15-26 \text { years }\end{array}$ & 94 & $0^{*}$ & $\begin{array}{l}\mathbf{0 . 0 1} \\
(0.00 \text { to } 0.18)\end{array}$ & $\begin{array}{l}\mathbf{9 9} \% \\
(82 \% \text { to } 100 \%)\end{array}$ & $\begin{array}{l}94 \\
\text { (77 to } 94 \text { ) }\end{array}$ & $\begin{array}{l}9296 \\
\text { (1 study) }\end{array}$ & $\begin{array}{l}\oplus \oplus \oplus \ominus \\
\text { moderate }^{3}\end{array}$ \\
\hline $\begin{array}{l}\text { Analysis } 1.5 \text { AIS associated with HPV16/18, at } \\
\text { least } 1 \text { dose, age } 15-26 \text { years }\end{array}$ & 9 & $0^{*}$ & $\begin{array}{l}\mathbf{0 . 1 0} \\
(0.01 \text { to } 0.82)\end{array}$ & $\begin{array}{l}\mathbf{9 0 \%} \\
(18 \% \text { to } 99 \%)\end{array}$ & $\begin{array}{l}9 \\
\text { (2 to } 9)\end{array}$ & $\begin{array}{l}20,214 \\
\text { ( } 2 \text { studies) }\end{array}$ & $\begin{array}{l}\oplus \oplus \oplus \ominus \\
\text { moderate }\end{array}$ \\
\hline $\begin{array}{l}\text { Analysis } 1.6 \text { AIS associated with } \\
\text { HPV6/11/16/18m at least } 1 \text { dose, age 15-26 } \\
\text { years }\end{array}$ & 6 & $0^{*}$ & $\begin{array}{l}\mathbf{0 . 1 4} \\
(0.01 \text { to } 2.8)\end{array}$ & $\begin{array}{l}\mathbf{8 6 \%} \\
(-180 \% \text { to } \\
99 \%)\end{array}$ & $\begin{array}{l}6 \\
(-12 \text { to } 6)\end{array}$ & $\begin{array}{l}9296 \\
\text { (1 study) }\end{array}$ & $\begin{array}{l}\oplus \oplus \oplus \ominus \\
\text { moderate }^{3}\end{array}$ \\
\hline $\begin{array}{l}\text { Analysis 1.7.1 Any CIN2+ irrespective of HPV } \\
\text { types, at least } 1 \text { dose of the bivalent vaccine, } \\
\text { age } 15-26 \text { years }\end{array}$ & 285 & 94 & $\begin{array}{l}\mathbf{0 . 3 3} \\
(0.25 \text { to } 0.43)\end{array}$ & $\begin{array}{l}\mathbf{6 7 \%} \\
(57 \% \text { to } 75 \%)\end{array}$ & $\begin{array}{l}191 \\
\text { (163 to } 214)\end{array}$ & $\begin{array}{l}15,884 \\
\text { (4 studies) }\end{array}$ & $\begin{array}{l}\oplus \oplus \oplus \oplus \\
\text { high }\end{array}$ \\
\hline $\begin{array}{l}\text { Analysis 1.7.2 Any CIN2+ irrespective of HPV } \\
\text { types, at least } 1 \text { dose of the quadrivalent vac- } \\
\text { cine, age } 15-26 \text { years }\end{array}$ & 291 & 166 & $\begin{array}{l}\mathbf{0 . 5 7} \\
(0.44 \text { to } 0.76)\end{array}$ & $\begin{array}{l}43 \% \\
(24 \text { to } 56 \%)\end{array}$ & $\begin{array}{l}125 \\
\text { (70 to } 163)\end{array}$ & $\begin{array}{l}9296 \\
\text { (1 study) }\end{array}$ & $\begin{array}{l}\oplus \oplus \oplus \ominus \\
\text { moderate }^{3}\end{array}$ \\
\hline $\begin{array}{l}\text { Analysis 1.8.1 Any } \mathrm{CIN} 3+\text { irrespective of HPV } \\
\text { types, at least } 1 \text { dose of the bivalent vaccine, } \\
\text { age } 15-26 \text { years }\end{array}$ & 81 & 6 & $\begin{array}{l}\mathbf{0 . 0 8} \\
(0.03 \text { to } 0.23)\end{array}$ & $\begin{array}{l}\mathbf{9 2} \% \\
\text { (77\% to } 97 \% \text { ) }\end{array}$ & $\begin{array}{l}\mathbf{7 4} \\
\text { (62 to } 78 \text { ) }\end{array}$ & $\begin{array}{l}\text { 11,423 } \\
\text { (2 studies) }\end{array}$ & $\begin{array}{l}\oplus \oplus \oplus \oplus \\
\text { high }\end{array}$ \\
\hline
\end{tabular}




\begin{tabular}{|c|c|c|c|c|c|c|c|}
\hline $\begin{array}{l}\text { Analysis } 1.8 .2 \text { Any } \mathrm{CIN} 3+\text { irrespective of HPV } \\
\text { types, at least } 1 \text { dose of the quadrivalent vac- } \\
\text { cine, age } 15-26 \text { years }\end{array}$ & 143 & 77 & $\begin{array}{l}\mathbf{0 . 5 4} \\
(0.36 \text { to } 0.82)\end{array}$ & $\begin{array}{l}\mathbf{4 6 \%} \\
(17 \% \text { to } 64 \%)\end{array}$ & $\begin{array}{l}66 \\
(26 \text { to } 92)\end{array}$ & $\begin{array}{l}9296 \\
\text { (1 study) }\end{array}$ & $\begin{array}{l}\oplus \oplus \oplus \ominus \\
\text { moderate }\end{array}$ \\
\hline $\begin{array}{l}\text { Analysis } 1.9 \text { Any AIS irrespective of HPV types, } \\
\text { at least } 1 \text { dose }\end{array}$ & 10 & $0^{*}$ & $\begin{array}{l}\mathbf{0 . 1 0} \\
(0.01 \text { to } 0.76)\end{array}$ & $\begin{array}{l}90 \% \\
(24 \% \text { to } 99 \%)\end{array}$ & $\begin{array}{l}10 \\
(2 \text { to } 10)\end{array}$ & $\begin{array}{l}20,214 \\
\text { ( } 2 \text { studies) }\end{array}$ & $\begin{array}{l}\oplus \oplus \oplus \ominus \\
\text { moderate }^{4}\end{array}$ \\
\hline \multicolumn{8}{|c|}{ 2. High-grade cervical lesions in women who were HPV16/18 negative at baseline } \\
\hline $\begin{array}{l}\text { Analysis 2.1.1 CIN2+ associated with HPV16/18, } \\
3 \text { doses, age } 15-26 \text { years }\end{array}$ & 74 & 5 & $\begin{array}{l}\mathbf{0 . 0 7} \\
(0.03 \text { to } 0.15)\end{array}$ & $\begin{array}{l}\mathbf{9 3} \% \\
(85 \% \text { to } 97 \%)\end{array}$ & $\begin{array}{l}69 \\
(63 \text { to } 72)\end{array}$ & $\begin{array}{l}36,579 \\
\text { (6 studies) }\end{array}$ & $\begin{array}{l}\oplus \oplus \oplus \oplus \\
\text { high }\end{array}$ \\
\hline $\begin{array}{l}\text { Analysis 2.1.2 CIN2+ associated with HPV16/18, } \\
3 \text { doses, } 24-45 \text { years }\end{array}$ & 36 & 6 & $\begin{array}{l}\mathbf{0 . 1 6} \\
(0.04 \text { to } 0.74)\end{array}$ & $\begin{array}{l}\mathbf{8 4} \% \\
(26 \% \text { to } 96 \%)\end{array}$ & $\begin{array}{l}30 \\
(9 \text { to } 34)\end{array}$ & $\begin{array}{l}6797 \\
\text { (2 studies) }\end{array}$ & $\begin{array}{l}\oplus \oplus \oplus \ominus \\
\text { moderate }^{4}\end{array}$ \\
\hline $\begin{array}{l}\text { Analysis 2.2.1 CIN2+ associated with HPV16/18, } \\
\text { at least } 1 \text { dose, } 15-26 \text { years }\end{array}$ & 113 & 6 & $\begin{array}{l}\mathbf{0 . 0 5} \\
(0.03 \text { to } 0.10)\end{array}$ & $\begin{array}{l}\mathbf{9 5} \% \\
\text { (90\% to } 97 \%)\end{array}$ & $\begin{array}{l}107 \\
\text { (102 to } 110)\end{array}$ & $\begin{array}{l}34,478 \\
\text { (6 studies) }\end{array}$ & $\begin{array}{l}\oplus \oplus \oplus \oplus \\
\text { high }\end{array}$ \\
\hline $\begin{array}{l}\text { Analysis 2.2.2 CIN2+ associated with HPV16/18, } \\
\text { at least } 1 \text { dose, age } 24-45 \text { years }\end{array}$ & 45 & 14 & $\begin{array}{l}\mathbf{0 . 3 0} \\
(0.11 \text { to } 0.81)\end{array}$ & $\begin{array}{l}\mathbf{7 0} \% \\
(19 \% \text { to } 89 \%)\end{array}$ & $\begin{array}{l}32 \\
\text { (9 to } 40)\end{array}$ & $\begin{array}{l}7552 \\
\text { (2 studies) }\end{array}$ & $\begin{array}{l}\oplus \oplus \oplus \ominus \\
\text { moderate }^{4}\end{array}$ \\
\hline $\begin{array}{l}\text { Analysis 2.3.1 CIN2+ associated with HPV16/18, } \\
1 \text { or } 2 \text { doses, } 15-26 \text { years }{ }^{\star \star \star}\end{array}$ & 436 & 44 & $\begin{array}{l}\mathbf{0 . 1 0} \\
(0.04 \text { to } 0.26)\end{array}$ & $\begin{array}{l}\mathbf{9 0 \%} \\
(74 \% \text { to } 96 \%)\end{array}$ & $\begin{array}{l}392 \\
\text { (323 to } 418 \text { ) }\end{array}$ & $\begin{array}{l}2958 \\
\text { (5 studies) }\end{array}$ & $\begin{array}{l}\oplus \oplus \odot \odot \\
\text { low'\$ }\end{array}$ \\
\hline $\begin{array}{l}\text { Analysis } 2.3 .2 \text { CIN2+ associated with HPV16/18, } \\
1 \text { or } 2 \text { doses, age } 24-45 \text { years }{ }^{\star \star \star}\end{array}$ & 134 & 82 & $\begin{array}{l}\mathbf{0 . 6 1} \\
(0.14 \text { to } 2.67)\end{array}$ & $\begin{array}{l}\mathbf{3 9 \%} \\
(-167 \% \text { to } \\
86 \%)\end{array}$ & $\begin{array}{l}52 \\
(-2245 \text { to } 115)\end{array}$ & $\begin{array}{l}755 \\
\text { (2 studies) }\end{array}$ & $\begin{array}{l}\oplus \# \# \# \\
\text { very low } \mathbf{1 \$ , 4}\end{array}$ \\
\hline $\begin{array}{l}\text { Analysis } 2.4 \text { CIN2+ associated with } \\
\text { HPV6/11/16/18, } 3 \text { doses, age } 15-45 \text { years }\end{array}$ & 99 & 6 & $\begin{array}{l}\mathbf{0 . 0 6} \\
(0.01 \text { to } 0.61)\end{array}$ & $\begin{array}{l}\mathbf{9 4} \% \\
(39 \% \text { to } 99 \%)\end{array}$ & $\begin{array}{l}93 \\
\text { (39 to } 98)\end{array}$ & $\begin{array}{l}7664 \\
\text { (2 studies) }\end{array}$ & $\begin{array}{l}\oplus \oplus \oplus \ominus \\
\text { moderate }^{4}\end{array}$ \\
\hline $\begin{array}{l}\text { Analysis } 2.4 .1 \mathrm{CIN} 2+\text { associated with } \\
\text { HPV6/11/16/18, } 3 \text { doses, age } 15-26 \text { years }\end{array}$ & 142 & $0^{*}$ & $\begin{array}{l}0.02 \\
(0.00 \text { to } 0.25)\end{array}$ & $\begin{array}{l}98 \% \\
(75 \% \text { to } 100 \%)\end{array}$ & $\begin{array}{l}142 \\
\text { (93 to } 190)\end{array}$ & $\begin{array}{l}4499 \\
\text { (1 study) }\end{array}$ & $\begin{array}{l}\oplus \oplus \oplus \ominus \\
\text { moderate }^{3}\end{array}$ \\
\hline $\begin{array}{l}\text { Analysis } 2.4 .2 \mathrm{CIN} 2+\text { associated with } \\
\text { HPV6/11/16/18, } 3 \text { doses, age } 24-45 \text { years }\end{array}$ & 38 & 6 & 0.17 & $83 \%$ & 32 & 3165 & $\begin{array}{l}\oplus \oplus \ominus \ominus \\
\text { low }^{\mathbf{3}, 4}\end{array}$ \\
\hline
\end{tabular}




\begin{tabular}{|c|c|c|c|c|c|c|c|}
\hline & & & (0.02 to 1.39$)$ & (-39\% to $98 \%)$ & $(-1$ to 32$)$ & (1 study) & \\
\hline $\begin{array}{l}\text { Analysis 2.5.1 CIN2+ associated with } \\
\text { HPV6/11/16/18, at least } 1 \text { dose, age } 15-26 \text { years }\end{array}$ & 160 & $0^{*}$ & $\begin{array}{l}\mathbf{0 . 0 1} \\
(0.00 \text { to } 0.19)\end{array}$ & $\begin{array}{l}\mathbf{9 9} \% \\
(81 \% \text { to } 100 \%)\end{array}$ & $\begin{array}{l}160 \\
(130 \text { to } 159)\end{array}$ & $\begin{array}{l}5351 \\
\text { (1 study) }\end{array}$ & $\begin{array}{l}\oplus \oplus \oplus \ominus \\
\text { moderate }^{3}\end{array}$ \\
\hline $\begin{array}{l}\text { Analysis 2.5.2 CIN2+ associated with } \\
\text { HPV6/11/16/18, at least } 1 \text { dose, age } 24-45 \text { years }\end{array}$ & 44 & 16 & $\begin{array}{l}\mathbf{0 . 3 7} \\
(0.10 \text { to } 1.41)\end{array}$ & $\begin{array}{l}\mathbf{6 3} \% \\
(-41 \% \text { to } 90 \%)\end{array}$ & $\begin{array}{l}28 \\
\text { (-18 to } 40)\end{array}$ & $\begin{array}{l}3629 \\
\text { (1 study) }\end{array}$ & $\begin{array}{l}\oplus \oplus \oplus \ominus \\
\text { moderate } \mathbf{3 , 4}\end{array}$ \\
\hline $\begin{array}{l}\text { Analysis } 2.6 \text { CIN2+ associated with } \\
\text { HPV6/11/16/18, } 1 \text { or } 2 \text { doses, age } 15-45 \text { years }\end{array}$ & 199 & 48 & $\begin{array}{l}\mathbf{0 . 2 4} \\
(0.01 \text { to } 5)\end{array}$ & $\begin{array}{l}\mathbf{7 6 \%} \\
(-400 \% \text { to } \\
99 \%)\end{array}$ & $\begin{array}{l}\mathbf{1 5 1} \\
(-795 \text { to } 197)\end{array}$ & $\begin{array}{l}1316 \\
\text { (2 studies) }\end{array}$ & $\begin{array}{l}\oplus \odot \odot \ominus \\
\text { very low } \mathbf{1} \mathbf{\$}, \mathbf{4}\end{array}$ \\
\hline $\begin{array}{l}\text { Analysis } 2.6 .1 \text { CIN2+ associated with } \\
\text { HPV6/11/16/18, } 1 \text { or } 2 \text { doses, age } 15-26 \text { years }\end{array}$ & 258 & $0^{*}$ & $\begin{array}{l}0.04 \\
(0.00 \text { to } 0.74)\end{array}$ & $\begin{array}{l}96 \% \\
(26 \% \text { to } \\
100 \%))\end{array}$ & $\begin{array}{l}258 \\
\text { (108 to 409) }\end{array}$ & $\begin{array}{l}852 \\
\text { (1 study) }\end{array}$ & $\begin{array}{l}\oplus \odot \Theta \odot \\
\text { very } \\
\text { low } \mathbf{1} \mathbf{\$ , 3 , 4}\end{array}$ \\
\hline $\begin{array}{l}\text { Analysis } 2.6 .2 \text { CIN2+ associated with } \\
\text { HPV6/11/16/18, } 1 \text { or } 2 \text { doses, age } 24-45 \text { years }\end{array}$ & 88 & 85 & $\begin{array}{l}0.97 \\
(0.14 \text { to } 6.80)\end{array}$ & $\begin{array}{l}3 \% \\
(-580 \% \text { to } \\
86 \%)\end{array}$ & $\begin{array}{l}3 \\
(-165 \text { to } 171)\end{array}$ & $\begin{array}{l}464 \\
\text { (1 study) }\end{array}$ & $\begin{array}{l}\oplus \ominus \odot \odot \\
\text { very } \\
\text { low } \mathbf{1} \mathbf{\$ , 3 , 4}\end{array}$ \\
\hline $\begin{array}{l}\text { Analysis } 2.7 \text { CIN3+ associated with HPV16/18, } 3 \\
\text { doses, age } 15-26 \text { years }\end{array}$ & 40 & 3 & $\begin{array}{l}\mathbf{0 . 0 7} \\
(0.02 \text { to } 0.29)\end{array}$ & $\begin{array}{l}\mathbf{9 3} \% \\
(71 \% \text { to } 98 \%)\end{array}$ & $\begin{array}{l}37 \\
\text { (28 to } 39)\end{array}$ & $\begin{array}{l}29,720 \\
\text { (3 studies) }\end{array}$ & $\begin{array}{l}\oplus \oplus \oplus \oplus \\
\text { high }\end{array}$ \\
\hline $\begin{array}{l}\text { Analysis } 2.8 \mathrm{CIN} 3+\text { associated with HPV16/18, at } \\
\text { least } 1 \text { dose, age } 15-26 \text { years }\end{array}$ & 57 & 3 & $\begin{array}{l}\mathbf{0 . 0 5} \\
(0.02 \text { to } 0.14)\end{array}$ & $\begin{array}{l}\mathbf{9 5} \% \\
(86 \% \text { to } 98 \%)\end{array}$ & $\begin{array}{l}\mathbf{5 4} \\
\text { (49 to } 56)\end{array}$ & $\begin{array}{l}33,199 \\
\text { (3 studies) }\end{array}$ & $\begin{array}{l}\oplus \oplus \oplus \oplus \\
\text { high }\end{array}$ \\
\hline $\begin{array}{l}\text { Analysis } 2.9 \text { CIN3+ associated with HPV16/18, } 1 \\
\text { or } 2 \text { doses, age } 15-26 \text { years }\end{array}$ & 200 & 12 & $\begin{array}{l}\mathbf{0 . 0 6} \\
(0.01 \text { to } 0.24)\end{array}$ & $\begin{array}{l}\mathbf{9 4} \% \\
(26 \% \text { to } 100 \%)\end{array}$ & $\begin{array}{l}188 \\
\text { (152 to } 198)\end{array}$ & $\begin{array}{l}3479 \\
\text { (3 studies) }\end{array}$ & $\begin{array}{l}\oplus \oplus \ominus \ominus \\
\text { low'1\$ }\end{array}$ \\
\hline $\begin{array}{l}\text { Analysis } 2.10 \text { AIS+ associated with HPV16/18, } 3 \\
\text { doses, age } 15-26 \text { years }\end{array}$ & 8 & $0^{*}$ & $\begin{array}{l}\mathbf{0 . 1 2} \\
(0.02 \text { to } 0.70)\end{array}$ & $\begin{array}{l}\mathbf{8 8} \% \\
(36 \% \text { to } 99 \%)\end{array}$ & $\begin{array}{l}8 \\
(2 \text { to } 8)\end{array}$ & $\begin{array}{l}29,707 \\
\text { (3 studies) }\end{array}$ & $\begin{array}{l}\oplus \oplus \oplus \ominus \\
\text { moderate }^{4}\end{array}$ \\
\hline $\begin{array}{l}\text { Analysis } 2.11 \text { AIS+ associated with HPV16/18, at } \\
\text { least } 1 \text { dose, age } 15-26 \text { years }\end{array}$ & 12 & $0^{*}$ & $\begin{array}{l}\mathbf{0 . 0 9} \\
(0.01 \text { to } 0.72)\end{array}$ & $\begin{array}{l}\mathbf{8 1} \% \\
(28 \% \text { to } 99 \%)\end{array}$ & $\begin{array}{l}12 \\
\text { (3 to } 12)\end{array}$ & $\begin{array}{l}17,079 \\
\text { (2 studies) }\end{array}$ & $\begin{array}{l}\oplus \oplus \oplus \ominus \\
\text { moderate }^{4}\end{array}$ \\
\hline
\end{tabular}




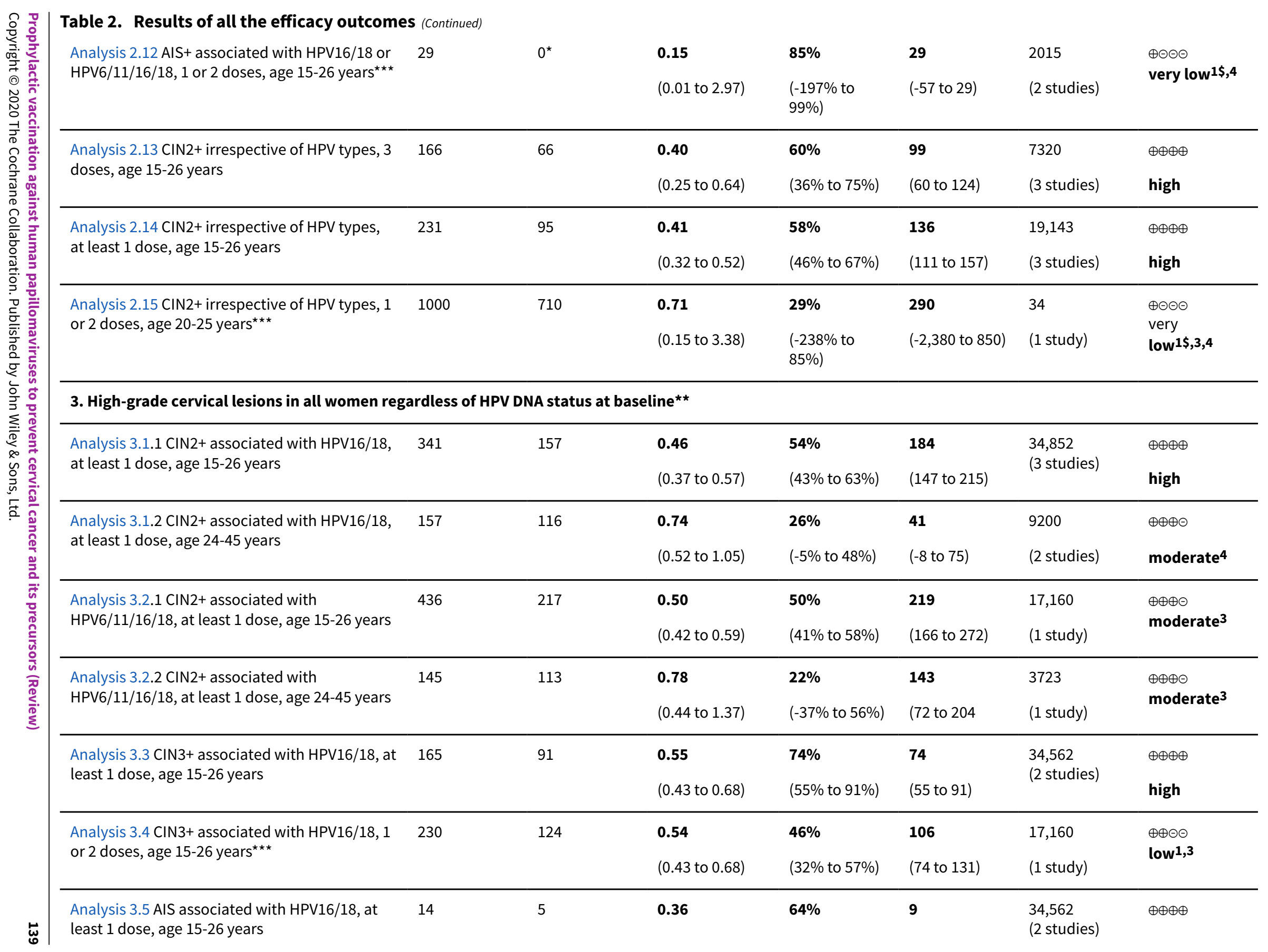




\begin{tabular}{|c|c|c|c|c|c|c|c|}
\hline & & & (0.17 to 0.78$)$ & $(22 \%$ to $83 \%)$ & (3 to 12 ) & & high \\
\hline $\begin{array}{l}\text { Analysis } 3.6 \text { AIS associated with HPV6/11/16/18, } \\
\text { at least } 1 \text { dose, age } 15-45 \text { years }\end{array}$ & 15 & 6 & $\begin{array}{l}\mathbf{0 . 4 0} \\
(0.16 \text { to } 0.98)\end{array}$ & $\begin{array}{l}\mathbf{6 0 \%} \\
(2 \% \text { to } 84 \%)\end{array}$ & $\begin{array}{l}9 \\
\text { (0 to } 13)\end{array}$ & $\begin{array}{l}20,830 \\
\text { (1 study) }\end{array}$ & $\begin{array}{l}\oplus \oplus \oplus \ominus \\
\text { moderate } 3,4\end{array}$ \\
\hline $\begin{array}{l}\text { Analysis 3.7.1 Any CIN2+ irrespective of HPV } \\
\text { types, at least } 1 \text { dose, age } 15-26 \text { years }\end{array}$ & 559 & 391 & $\begin{array}{l}\mathbf{0 . 7 0} \\
(0.58 \text { to } 0.85)\end{array}$ & $\begin{array}{l}\mathbf{3 0} \% \\
(15 \% \text { to } 42 \%)\end{array}$ & $\begin{array}{l}168 \\
\text { (84 to } 235)\end{array}$ & $\begin{array}{l}35,779 \\
\text { (4 studies) }\end{array}$ & $\begin{array}{l}\oplus \oplus \oplus \oplus \\
\text { high }\end{array}$ \\
\hline $\begin{array}{l}\text { Analysis } 3.72 \text { Any } \mathrm{CIN} 2+\text { irrespective of HPV } \\
\text { types, at least } 1 \text { dose, age } 24-45 \text { years }\end{array}$ & 342 & 356 & $\begin{array}{l}\mathbf{1 . 0 4} \\
(0.83 \text { to } 1.30)\end{array}$ & $\begin{array}{l}-4 \% \\
(-30 \% \text { to } 17 \%)\end{array}$ & $\begin{array}{l}-\mathbf{1 4} \\
(-103 \text { to } 58)\end{array}$ & $\begin{array}{l}9287 \\
\text { (2 studies) }\end{array}$ & $\begin{array}{l}\oplus \oplus \ominus \ominus \\
\text { moderate }^{4}\end{array}$ \\
\hline $\begin{array}{l}\text { Analysis } 3.8 \text { Any } \mathrm{CIN} 3+\text { irrespective of HPV } \\
\text { types, at least } 1 \text { dose, age } 18-26 \text { years, bivalent } \\
\text { vaccine }\end{array}$ & 188 & 103 & $\begin{array}{l}\mathbf{0 . 5 5} \\
(0.43 \text { to } 0.71)\end{array}$ & $\begin{array}{l}\mathbf{4 5 \%} \\
(29 \% \text { to } 57 \%)\end{array}$ & $\begin{array}{l}84 \\
(54 \text { to } 1107)\end{array}$ & $\begin{array}{l}18,329 \\
\text { (2 studies) }\end{array}$ & $\begin{array}{l}\oplus \oplus \oplus \oplus \\
\text { high }\end{array}$ \\
\hline $\begin{array}{l}\text { Analysis } 3.8 \text { Any } \mathrm{CIN} 3+\text { irrespective of HPV } \\
\text { types, at least } 1 \text { dose, age } 15-26 \text { years, quadri- } \\
\text { valent vaccine }\end{array}$ & 349 & 283 & $\begin{array}{l}\mathbf{0 . 8 1} \\
(0.69 \text { to } 0.96)\end{array}$ & $\begin{array}{l}19 \% \\
(4 \% \text { to } 31 \%)\end{array}$ & $\begin{array}{l}66 \\
\text { (14 to } 108)\end{array}$ & $\begin{array}{l}17,160 \\
\text { (1 study) }\end{array}$ & $\begin{array}{l}\oplus \oplus \oplus \ominus \\
\text { moderate }^{3}\end{array}$ \\
\hline $\begin{array}{l}\text { Analysis } 3.9 \text { Any AIS irrespective of HPV types, } \\
\text { at least } 1 \text { dose, age } 15-26 \text { years }\end{array}$ & 17 & 5 & $\begin{array}{l}\mathbf{0 . 3 2} \\
(0.15 \text { to } 0.67)\end{array}$ & $\begin{array}{l}\mathbf{6 8} \% \\
(33 \% \text { to } \\
0.85 \%)\end{array}$ & $\begin{array}{l}11 \\
(6 \text { to } 14)\end{array}$ & $\begin{array}{l}34,562 \\
\text { (2 studies) }\end{array}$ & $\begin{array}{l}\oplus \oplus \oplus \oplus \\
\text { high }\end{array}$ \\
\hline 4. HPV16/18 infection in women who were hrH & V DNA & at baseline & & & & & \\
\hline $\begin{array}{l}\text { Analysis } 4.1 \text { Incident HPV16/18 infection, } 3 \text { dos- } \\
\text { es, age } 18-26 \text { years }\end{array}$ & 2,457 & 147 & $\begin{array}{l}\mathbf{0 . 0 6} \\
(0.02 \text { to } 0.20)\end{array}$ & $\begin{array}{l}\mathbf{9 4 \%} \\
(80 \% \text { to } 98 \%)\end{array}$ & $\begin{array}{l}\mathbf{2 , 3 1 0} \\
(1,966 \text { to } \\
2,408)\end{array}$ & $\begin{array}{l}368 \\
\text { (1 study) }\end{array}$ & $\begin{array}{l}\oplus \oplus \oplus \ominus \\
\text { moderate }^{3}\end{array}$ \\
\hline $\begin{array}{l}\text { Analysis } 4.2 \text { Persistent HPV16/18 infection(6M), } \\
3 \text { doses, age } 15-26 \text { years }\end{array}$ & 971 & 29 & $\begin{array}{l}\mathbf{0 . 0 2} \\
(0.00 \text { to } 0.35)\end{array}$ & $\begin{array}{l}\mathbf{9 7} \% \\
(57 \% \text { to } 100 \%)\end{array}$ & $\begin{array}{l}942 \\
\text { (554 to } 971)\end{array}$ & $\begin{array}{l}368 \\
\text { (1 study) }\end{array}$ & $\begin{array}{l}\oplus \oplus \oplus \ominus \\
\text { moderate } 3\end{array}$ \\
\hline $\begin{array}{l}\text { Analysis } 4.3 \text { Persistent HPV16/18 infection( } 6 \mathrm{M}) \text {, } \\
\text { at least } 1 \text { dose, age } 18-25 \text { years }\end{array}$ & 96 & 7 & $\begin{array}{l}\mathbf{0 . 0 7} \\
(0.05 \text { to } 0.09)\end{array}$ & $\begin{array}{l}\mathbf{9 3} \% \\
(81 \% \text { to } 95 \%)\end{array}$ & $\begin{array}{l}90 \\
\text { (88 to } 91)\end{array}$ & $\begin{array}{l}10,826 \\
\text { (1 study) }\end{array}$ & $\begin{array}{l}\oplus \oplus \oplus \ominus \\
\text { moderate }\end{array}$ \\
\hline $\begin{array}{l}\text { Analysis 4.4 Persistent HPV16/18 infec- } \\
\text { tion(12M), } 3 \text { doses, age } 15-26 \text { years }\end{array}$ & 571 & 23 & $\begin{array}{l}\mathbf{0 . 0 4} \\
(0.00 \text { to } 0.73)\end{array}$ & $\begin{array}{l}\mathbf{9 6 \%} \\
(27 \% \text { to } 100 \%)\end{array}$ & $\begin{array}{l}\mathbf{5 4 9} \\
(154 \text { to } 571)\end{array}$ & $\begin{array}{l}368 \\
\text { (1 study) }\end{array}$ & $\begin{array}{l}\oplus \oplus \oplus \ominus \\
\text { moderate }\end{array}$ \\
\hline
\end{tabular}




\begin{tabular}{|c|c|c|c|c|c|c|c|}
\hline $\begin{array}{l}\text { Analysis } 4.5 \text { Persistent HPV16/18 infec- } \\
\text { tion(12M), at least } 1 \text { dose, age } 15-26 \text { years }\end{array}$ & 462 & 37 & $\begin{array}{l}\mathbf{0 . 0 8} \\
(0.05 \text { to } 0.12)\end{array}$ & $\begin{array}{l}\mathbf{9 2} \% \\
(88 \% \text { to } 95 \%)\end{array}$ & $\begin{array}{l}425 \\
\text { (406 to 439) }\end{array}$ & $\begin{array}{l}14,153 \\
\text { ( } 2 \text { studies) }\end{array}$ & $\begin{array}{l}\oplus \oplus \oplus \oplus \\
\text { high }\end{array}$ \\
\hline \multicolumn{8}{|c|}{ 5. HPV16/18 infection in women who were HPV16/18 negative at baseline } \\
\hline $\begin{array}{l}\text { Analysis } 5.1 \text { Incident HPV16/18 infection, } 3 \text { dos- } \\
\text { es, age } 15-26 \text { years }\end{array}$ & 474 & 81 & $\begin{array}{l}\mathbf{0 . 1 7} \\
(0.10 \text { to } 0.31)\end{array}$ & $\begin{array}{l}\mathbf{8 7} \% \\
\text { (78\% to } 92 \%)\end{array}$ & $\begin{array}{l}412 \\
\text { (369 to } 436 \text { ) }\end{array}$ & $\begin{array}{l}8,034 \\
\text { (4 studies) }\end{array}$ & $\begin{array}{l}\oplus \oplus \oplus \oplus \\
\text { high }\end{array}$ \\
\hline $\begin{array}{l}\text { Analysis } 5.2 \text { Incident HPV16/18 infection, at } \\
\text { least } 1 \text { dose, age } 15-26 \text { years }\end{array}$ & 1,326 & 305 & $\begin{array}{l}\mathbf{0 . 2 3} \\
(0.14 \text { to } 0.37)\end{array}$ & $\begin{array}{l}\mathbf{8 1} \% \\
(71 \% \text { to } 88 \%)\end{array}$ & $\begin{array}{l}\mathbf{1 , 0 7 4} \\
\text { (941 to } 1,167)\end{array}$ & $\begin{array}{l}23,872 \\
\text { (5 studies) }\end{array}$ & $\begin{array}{l}\oplus \oplus \oplus \oplus \\
\text { high }\end{array}$ \\
\hline $\begin{array}{l}\text { Analysis } 5.3 \text { Incident HPV16/18 infection, } 1 \text { or } 2 \\
\text { dose, age } 15-26 \text { years }^{\star \star \star}\end{array}$ & 2,568 & 1207 & $\begin{array}{l}\mathbf{0 . 4 7} \\
(0.26 \text { to } 0.84)\end{array}$ & $\begin{array}{l}\mathbf{7 4} \% \\
\text { (31\% to } 90 \%)\end{array}$ & $\begin{array}{l}\mathbf{1 , 9 0 1} \\
\text { (796 to } 2,311 \text { ) }\end{array}$ & $\begin{array}{l}331 \\
\text { (3 studies) }\end{array}$ & $\begin{array}{l}\oplus \oplus \oplus \ominus \\
\text { moderate }^{1}\end{array}$ \\
\hline $\begin{array}{l}\text { Analysis 5.4.1 Persistent HPV16/18 infection } \\
(6 \mathrm{M}), 3 \text { doses, age } 15-26 \text { years }\end{array}$ & 581 & 35 & $\begin{array}{l}\mathbf{0 . 0 6} \\
(0.05 \text { to } 0.08)\end{array}$ & $\begin{array}{l}\mathbf{9 4 \%} \\
\text { (91\% to } 95 \%)\end{array}$ & $\begin{array}{l}\mathbf{5 4 6} \\
\text { (534 to 552) }\end{array}$ & $\begin{array}{l}27,385 \\
\text { (6 studies) }\end{array}$ & $\begin{array}{l}\oplus \oplus \oplus \oplus \\
\text { high }\end{array}$ \\
\hline $\begin{array}{l}\text { Analysis 5.4.2 Persistent HPV16/18 infection } \\
(6 \mathrm{M}), 3 \text { doses, age } 24-45 \text { years }\end{array}$ & 350 & 38 & $\begin{array}{l}\mathbf{0 . 1 1} \\
(0.06 \text { to } 0.20)\end{array}$ & $\begin{array}{l}\mathbf{8 9} \% \\
(80 \% \text { to } 94 \%)\end{array}$ & $\begin{array}{l}311 \\
\text { (280 to } 329)\end{array}$ & $\begin{array}{l}6728 \\
\text { (2 studies) }\end{array}$ & $\begin{array}{l}\oplus \oplus \oplus \ominus \\
\text { moderate }\end{array}$ \\
\hline $\begin{array}{l}\text { Analysis 5.5.1 Persistent HPV16/18 infection } \\
(6 \mathrm{M}) \text {, at least } 1 \text { dose, age } 15-26 \text { years }\end{array}$ & 657 & 66 & $\begin{array}{l}\mathbf{0 . 1 0} \\
(0.08 \text { to } 0.13)\end{array}$ & $\begin{array}{l}\mathbf{9 0 \%} \\
(87 \% \text { to } 92 \%)\end{array}$ & $\begin{array}{l}591 \\
(572 \text { to } 605)\end{array}$ & $\begin{array}{l}22,803 \\
\text { (4 studies) }\end{array}$ & $\begin{array}{l}\oplus \oplus \oplus \oplus \\
\text { high }\end{array}$ \\
\hline $\begin{array}{l}\text { Analysis 5.5.2 Persistent HPV16/18 infection } \\
(6 \mathrm{M}) \text {, at least } 1 \text { dose, age } 24-45 \text { years }\end{array}$ & 441 & 75 & $\begin{array}{l}\mathbf{0 . 1 7} \\
(0.10 \text { to } 0.29)\end{array}$ & $\begin{array}{l}\mathbf{8 3} \% \\
\text { (71\% to } 90 \%)\end{array}$ & $\begin{array}{l}366 \\
\text { (313 to } 397 \text { ) }\end{array}$ & $\begin{array}{l}7520 \\
\text { (2 studies) }\end{array}$ & $\begin{array}{l}\oplus \oplus \oplus \oplus \\
\text { high }\end{array}$ \\
\hline $\begin{array}{l}\text { Analysis } 5.6 .1 \text { Persistent HPV16/18 infection } \\
(6 \mathrm{M}), 1 \text { or } 2 \text { doses, age } 15-26 \text { years }{ }^{\star \star \star}\end{array}$ & 996 & 119 & $\begin{array}{l}\mathbf{0 . 1 2} \\
(0.03 \text { to } 0.42)\end{array}$ & $\begin{array}{l}\mathbf{8 8} \% \\
(58 \% \text { to } 97 \%)\end{array}$ & $\begin{array}{l}876 \\
(577 \text { to } 966)\end{array}$ & $\begin{array}{l}437 \\
\text { (2 studies) }\end{array}$ & $\begin{array}{l}\oplus \oplus \ominus \ominus \\
\text { low } \mathbf{1 , 4}\end{array}$ \\
\hline $\begin{array}{l}\text { Analysis 5.6.2 Persistent HPV16/18 infection } \\
(6 \mathrm{M}), 1 \text { or } 2 \text { doses, age } 24-45 \text { years }{ }^{\star \star \star}\end{array}$ & 1,221 & 379 & $\begin{array}{l}\mathbf{0 . 3 1} \\
(0.18 \text { to } 0.54)\end{array}$ & $\begin{array}{l}\mathbf{6 9} \% \\
(46 \% \text { to } 82 \%)\end{array}$ & $\begin{array}{l}843 \\
\text { (562 to } 1002)\end{array}$ & $\begin{array}{l}792 \\
\text { (2 studies) }\end{array}$ & $\begin{array}{l}\oplus \oplus \oplus \ominus \\
\text { moderate }{ }^{1}\end{array}$ \\
\hline $\begin{array}{l}\text { Analysis } 5.7 \text { Persistent HPV6/11/16/18 infection } \\
(6 \mathrm{M}), 3 \text { doses }\end{array}$ & 518 & 62 & $\begin{array}{l}\mathbf{0 . 1 2} \\
(0.06 \text { to } 0.21)\end{array}$ & $\begin{array}{l}\mathbf{8 8} \% \\
\text { (79\% to } 94 \%)\end{array}$ & $\begin{array}{l}456 \\
\text { (409 to } 487)\end{array}$ & $\begin{array}{l}4008 \\
\text { (2 studies) }\end{array}$ & $\begin{array}{l}\oplus \oplus \oplus \oplus \\
\text { high }\end{array}$ \\
\hline
\end{tabular}

5. HPV16/18 infection in women who were HPV16/18 negative at baseline 


\begin{tabular}{|c|c|c|c|c|c|c|c|}
\hline $\begin{array}{l}\text { Analysis } 5.8 \text { Persistent HPV6/11/16/18 infection } \\
(6 \mathrm{M}) \text {, at least } 1 \text { dose }\end{array}$ & 907 & 118 & $\begin{array}{l}\mathbf{0 . 1 3} \\
(0.05 \text { to } 0.37)\end{array}$ & $\begin{array}{l}\mathbf{8 7 \%} \\
(63 \% \text { to } 95 \%)\end{array}$ & $\begin{array}{l}789 \\
\text { (571 to 862) }\end{array}$ & $\begin{array}{l}4129 \\
\text { (2 studies) }\end{array}$ & $\begin{array}{l}\oplus \oplus \oplus \oplus \\
\text { high }\end{array}$ \\
\hline $\begin{array}{l}\text { Analysis } 5.9 \text { Persistent HPV16/18 infection } \\
(12 \mathrm{M}), 3 \text { doses }\end{array}$ & 297 & 27 & $\begin{array}{l}\mathbf{0 . 0 9} \\
(0.06 \text { to } 0.13)\end{array}$ & $\begin{array}{l}\mathbf{9 1} \% \\
\text { (87\% to } 94 \%)\end{array}$ & $\begin{array}{l}270 \\
\text { (258 to } 279 \text { ) }\end{array}$ & $\begin{array}{l}22,267 \\
\text { (4 studies) }\end{array}$ & $\begin{array}{l}\oplus \oplus \oplus \oplus \\
\text { high }\end{array}$ \\
\hline $\begin{array}{l}\text { Analysis } 5.10 \text { Persistent HPV16/18 infection } \\
(12 \mathrm{M}) \text {, at least } 1 \text { dose }\end{array}$ & 365 & 58 & $\begin{array}{l}\mathbf{0 . 1 6} \\
(0.01 \text { to } 0.13)\end{array}$ & $\begin{array}{l}\mathbf{8 4} \% \\
\text { (87\% to } 99 \%)\end{array}$ & $\begin{array}{l}306 \\
\text { (292 to 361) }\end{array}$ & $\begin{array}{l}29,464 \\
\text { (5 studies) }\end{array}$ & $\begin{array}{l}\oplus \oplus \oplus \oplus \\
\text { high }\end{array}$ \\
\hline $\begin{array}{l}\text { Analysis } 5.11 \text { Persistent HPV16/18 infection } \\
(12 \mathrm{M}), 1 \text { or } 2 \text { doses }^{\star \star \star}\end{array}$ & 205 & 27 & $\begin{array}{l}\mathbf{0 . 1 3} \\
(0.06 \text { to } 0.33)\end{array}$ & $\begin{array}{l}87 \% \\
(67 \% \text { to } 94 \%)\end{array}$ & $\begin{array}{l}\mathbf{1 7 8} \\
\text { (137 to 193) }\end{array}$ & $\begin{array}{l}3912 \\
\text { (3 studies) }\end{array}$ & $\begin{array}{l}\oplus \oplus \oplus \odot \\
\text { moderate }^{1}\end{array}$ \\
\hline \multicolumn{8}{|c|}{ 6. HPV16/18 infection regardless of HPV DNA status at baseline ${ }^{\star \star}$} \\
\hline $\begin{array}{l}\text { Analysis } 6.1 \text { Incident HPV16/18 infection, at } \\
\text { least } 1 \text { dose, age } 15-26 \text { years }\end{array}$ & 807 & 194 & $\begin{array}{l}\mathbf{0 . 2 4} \\
(0.17 \text { to } 0.33)\end{array}$ & $\begin{array}{l}\mathbf{7 6} \% \\
(67 \% \text { to } 83 \%)\end{array}$ & $\begin{array}{l}\mathbf{6 1 3} \\
\text { (541 to } 670)\end{array}$ & $\begin{array}{l}4210 \\
\text { (1 study) }\end{array}$ & $\begin{array}{l}\oplus \oplus \oplus \ominus \\
\text { moderate }^{3}\end{array}$ \\
\hline $\begin{array}{l}\text { Analysis 6.2.1 Persistent HPV16/18 infection } \\
(6 \mathrm{M}) \text {, at least } 1 \text { dose, age } 15-26 \text { years }\end{array}$ & 1,359 & 598 & $\begin{array}{l}\mathbf{0 . 4 4} \\
(0.38 \text { to } 0.51)\end{array}$ & $\begin{array}{l}\mathbf{5 6} \% \\
(49 \% \text { to } 62 \%)\end{array}$ & $\begin{array}{l}\mathbf{7 6 1} \\
\text { (666 to 842) }\end{array}$ & $\begin{array}{l}25,199 \\
\text { (2 studies) }\end{array}$ & $\begin{array}{l}\oplus \oplus \oplus \oplus \\
\text { high }\end{array}$ \\
\hline $\begin{array}{l}\text { Analysis 6.2.2 Persistent HPV16/18 infection } \\
(6 \mathrm{M}) \text {, at least } 1 \text { dose, age } 24-45 \text { years }\end{array}$ & 642 & 366 & $\begin{array}{l}\mathbf{0 . 5 7} \\
(0.47 \text { to } 0.69)\end{array}$ & $\begin{array}{l}\mathbf{4 3} \% \\
(31 \% \text { to } 53 \%)\end{array}$ & $\begin{array}{l}276 \\
\text { (199 to 341) }\end{array}$ & $\begin{array}{l}8648 \\
\text { (2 studies) }\end{array}$ & $\begin{array}{l}\oplus \oplus \oplus \oplus \\
\text { high }\end{array}$ \\
\hline $\begin{array}{l}\text { Analysis } 6.3 \text { Persistent HPV6/11/16/18 infection } \\
(6 \mathrm{M}) \text {, at least } 1 \text { dose, age } 24-45 \text { years }\end{array}$ & 1,136 & 591 & $\begin{array}{l}\mathbf{0 . 5 2} \\
(0.42 \text { to } 0.65)\end{array}$ & $\begin{array}{l}\mathbf{4 8} \% \\
\text { (35\% to } 58 \%)\end{array}$ & $\begin{array}{l}\mathbf{5 4 5} \\
\text { (398 to 659) }\end{array}$ & $\begin{array}{l}3713 \\
\text { (1 study) }\end{array}$ & $\begin{array}{l}\oplus \oplus \oplus \ominus \\
\text { moderate }^{3}\end{array}$ \\
\hline $\begin{array}{l}\text { Analysis } 6.4 \text { Persistent HPV16/18 infection } \\
(12 \mathrm{M}) \text {, at least } 1 \text { dose, age } 15-26 \text { years }\end{array}$ & 861 & 396 & $\begin{array}{l}\mathbf{0 . 4 6} \\
(0.40 \text { to } 0.54)\end{array}$ & $\begin{array}{l}\mathbf{5 4} \% \\
(46 \% \text { to } 60 \%)\end{array}$ & $\begin{array}{l}465 \\
(396 \text { to } 516)\end{array}$ & $\begin{array}{l}24,785 \\
\text { (2 studies) }\end{array}$ & $\begin{array}{l}\oplus \oplus \oplus \oplus \\
\text { high }\end{array}$ \\
\hline
\end{tabular}

Cl: Confidence interval; RR: Risk Ratio;

GRADE Working Group grades of evidence

High quality: Further research is very unlikely to change our confidence in the estimate of effect. *The attribution of "high quality" depends on the following conditions: well-conducted randomised trials, with consistent findings, direct outcome, precise estimates (narrow confidence intervals), absence of reporting bias (Guyatt 2008).

Moderate quality: Further research is likely to have an important impact on our confidence in the estimate of effect and may change the estimate.

Low quality: Further research is very likely to have an important impact on our confidence in the estimate of effect and is likely to change the estimate. 
1 In case of study flaws as assessed by the Cochrane Collaboration's tool for assessing risk of bias in randomised trials (Higgins 2011b), not observed but calculated outcome;

2 Substantial heterogeneity defined as $\mathrm{I}^{2}>30 \%$, when multiple studies were available for the considered outcome;

When only one study was retrieved for the outcome;

4 Imprecision, when the width of the $95 \%$ confidence interval around RR $>0.60$

$0^{\star}$ When zero events occurred in the vaccine group a continuity correction was applied to compute the RR and its confidence interval. Nevertheless, in this case the absolute risks in the vaccine arms in Table 2 were computed considering an exact binomal distribution.

** Relative and absolute effects in women regardless of HPV DNA status at baseline (headings 3 and 6 ) must be interpreted with care since influenced by the prevalence of HPV infection at enrolment in the respective trials.

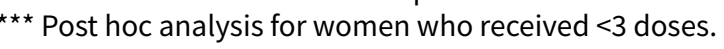

$\$$ For the precancer endpoints (CIN2/3 and AIS), a higher risk in the placebo arms was observed if $<3$ doses were received compared to those who received 3 doses Therefore the quality of evidence was downgraded to low or very low. 
Table 3. Number needed to vaccinate (NNV) to prevent one outcome event (in young women aged 15-26 years)

\begin{tabular}{|c|c|c|}
\hline \multirow[t]{2}{*}{ Outcome } & \multicolumn{2}{|c|}{ Initial HPV status at enrolment } \\
\hline & hrHPV negative & Regardless of HPV status \\
\hline $\begin{array}{l}\text { Lesions associated with } \\
\text { HPV16/18 }\end{array}$ & NNV $(95 \% \mathrm{Cl})$ & $\operatorname{NNV}(95 \% \mathrm{Cl})$ \\
\hline $\mathrm{CIN} 2+$ & 62 (61 to 64$)$ & 54 (46 to 68$)$ \\
\hline CIN3+ & 204 (149 to 333) & 135 (110 to 263$)$ \\
\hline AIS+ & 1111 (714 to 5000$)$ & 1111 (625 to 3333$)$ \\
\hline $\begin{array}{l}\text { Lesions irrespective of HPV } \\
\text { types }\end{array}$ & NNV $(95 \% \mathrm{Cl})$ & $\operatorname{NNV}(95 \% \mathrm{Cl})$ \\
\hline $\mathrm{CIN} 2+$ & 60 (50 to 76$)$ & 68 (52 to 97$)$ \\
\hline CIN3+ & 141 (106 to 208 ) & 133 (94 to 227$)$ \\
\hline AIS+ & $1000(556$ to 10,000$)$ & 833 (526 to 2000 ) \\
\hline
\end{tabular}

AIS: adenocarcinoma in situ, CIN: cervical intraepithelial neoplasia, CIN2+: CIN of degree II or worse, CIN3+: CIN of degree 3 or worse, hrHPV: high-risk human papillomavirus types, NNV: number needed to vaccinate.

Table 4. Results of all the safety outcomes (adverse events, pregnancy outcomes)

\begin{tabular}{|c|c|c|c|c|c|}
\hline \multirow[t]{2}{*}{ Outcomes } & \multicolumn{2}{|c|}{ Absolute risk/ per 10,000} & \multirow{2}{*}{$\begin{array}{l}\text { Relative effect } \\
(95 \% \mathrm{Cl})\end{array}$} & \multirow{2}{*}{$\begin{array}{l}\text { No of Participants } \\
\text { (studies) }\end{array}$} & \multirow{2}{*}{$\begin{array}{l}\text { Quality of the } \\
\text { evidence } \\
\text { (GRADE) }\end{array}$} \\
\hline & placebo & vaccinated & & & \\
\hline $\begin{array}{l}\text { Analysis } 7.1 \text { Overall local/injec- } \\
\text { tion site adverse events }\end{array}$ & 6847 & 8080 & $\begin{array}{l}\mathbf{1 . 1 8} \\
(1.16 \text { to } 1.20)\end{array}$ & $\begin{array}{l}18,113 \\
\text { (8 studies) }\end{array}$ & $\begin{array}{l}\oplus \oplus \oplus \ominus \\
\text { moderate }\end{array}$ \\
\hline $\begin{array}{l}\text { Analysis } 7.2 \text { Pain at injection } \\
\text { site }\end{array}$ & 6505 & 8782 & $\begin{array}{l}\mathbf{1 . 3 5} \\
\text { (1.23 to } 1.49)\end{array}$ & $\begin{array}{l}25,691 \\
\text { (13 studies) }\end{array}$ & $\begin{array}{l}\oplus \oplus \oplus \ominus \\
\text { moderate }^{2}\end{array}$ \\
\hline $\begin{array}{l}\text { Analysis } 7.3 \text { Swelling at injec- } \\
\text { tion site }\end{array}$ & 1582 & 2737 & $\begin{array}{l}\mathbf{1 . 7 3} \\
\text { (1.32 to } 2.27)\end{array}$ & $\begin{array}{l}22,106 \\
\text { (9 studies) }\end{array}$ & $\begin{array}{l}\oplus \oplus \oplus \ominus \\
\text { moderate }^{2}\end{array}$ \\
\hline $\begin{array}{l}\text { Analysis } 7.4 \text { Redness at injec- } \\
\text { tion site }\end{array}$ & 1938 & 3333 & $\begin{array}{l}\mathbf{1 . 7 2} \\
(1.50 \text { to } 1.97)\end{array}$ & $\begin{array}{l}19,996 \\
\text { (6 studies) }\end{array}$ & $\begin{array}{l}\oplus \oplus \oplus \ominus \\
\text { moderate }^{2}\end{array}$ \\
\hline $\begin{array}{l}\text { Analysis } 7.5 \text { Overall systematic } \\
\text { event and general symptoms }\end{array}$ & 6102 & 6224 & $\begin{array}{l}\mathbf{1 . 0 2} \\
\text { (0.98 to } 1.07)\end{array}$ & $\begin{array}{l}18,191 \\
\text { (8 studies) }\end{array}$ & $\begin{array}{l}\oplus \oplus \oplus \ominus \\
\text { moderate }^{2}\end{array}$ \\
\hline $\begin{array}{l}\text { Analysis } 7.6 \text { Serious adverse } \\
\text { events }\end{array}$ & 605 & 611 & $\begin{array}{l}\mathbf{1 . 0 1} \\
(0.95 \text { to } 1.07)\end{array}$ & $\begin{array}{l}6978 \\
\text { (21studies) }\end{array}$ & $\begin{array}{l}\oplus \oplus \oplus \oplus \\
\text { high }\end{array}$ \\
\hline Analysis 7.7 Deaths & 11 & 13 & 1.25 & 71,452 & $\oplus \oplus \# \#$ \\
\hline
\end{tabular}


Table 4. Results of all the safety outcomes (adverse events, pregnancy outcomes) (Continued)
(0.81 to 1.93)
(23 studies)

low $2,4, \dagger$

\begin{tabular}{|c|c|c|c|c|c|}
\hline Analysis 8.1 Normal infant & 7171 & 7171 & $\begin{array}{l}1.00 \\
(0.97 \text { to } 1.02)\end{array}$ & $\begin{array}{l}8782 \\
\text { (8 studies) }\end{array}$ & $\begin{array}{l}\oplus \oplus \oplus \oplus \\
\text { high }\end{array}$ \\
\hline $\begin{array}{l}\text { Analysis } 8.2 \text { Spontaneous abor- } \\
\text { tion/miscarriage }\end{array}$ & 1618 & 1424 & $\begin{array}{l}\mathbf{0 . 8 8} \\
(0.68 \text { to } 1.14)\end{array}$ & $\begin{array}{l}8618 \\
\text { (9 studies) }\end{array}$ & $\begin{array}{l}\oplus \oplus \oplus \oplus \\
\text { high }\end{array}$ \\
\hline $\begin{array}{l}\text { Analysis } 8.3 \text { Elective termina- } \\
\text { tion/induced abortion }\end{array}$ & 931 & 838 & $\begin{array}{l}\mathbf{0 . 9 0} \\
(0.80 \text { to } 1.02)\end{array}$ & $\begin{array}{l}10.909 \\
\text { (9 studies) }\end{array}$ & $\begin{array}{l}\oplus \oplus \oplus \oplus \\
\text { high }\end{array}$ \\
\hline Analysis 8.4 Stillbirth & 70 & 78 & $\begin{array}{l}1.12 \\
\text { (0.68 to } 1.83)\end{array}$ & $\begin{array}{l}8754 \\
\text { (6 studies) }\end{array}$ & $\begin{array}{l}\oplus \oplus \oplus \ominus^{4} \\
\text { moderate }\end{array}$ \\
\hline Analysis 8.5 Abnormal infant & 205 & 250 & $\begin{array}{l}1.22 \\
\text { (0.88 to } 1.69)\end{array}$ & $\begin{array}{l}9252 \\
\text { (5 studies) }\end{array}$ & $\begin{array}{l}\oplus \oplus \oplus \odot^{4} \\
\text { moderate }\end{array}$ \\
\hline
\end{tabular}

Cl: Confidence interval; RR: Risk Ratio

GRADE Working Group grades of evidence

High quality: Further research is very unlikely to change our confidence in the estimate of effect. *The attribution of "high quality" depends on the following conditions: well-conducted randomized trials, with consistent findings, direct outcome, precise estimates (narrow confidence intervals), absence of reporting bias (Guyatt 2008).

Moderate quality: Further research is likely to have an important impact on our confidence in the estimate of effect and may change the estimate.

Low quality: Further research is very likely to have an important impact on our confidence in the estimate of effect and is likely to change the estimate.

Very low quality: We are very uncertain about the estimate.

1 In case of study flaws as assessed by Cochrane's tool for assessing risk of bias in randomised trials (Higgins 2011b), not observed but calculated outcome

2 Substantial heterogeneity defined as $1^{2}>30 \%$, when multiple studies were available for the considered outcome

3 When only one study was retrieved for the outcome

${ }^{4}$ Imprecision, when the width of the $95 \%$ confidence interval around $\mathrm{RR}>0.60$

$\dagger$ inter-age group heterogeneity, absence of pattern in causes of deaths

Table 5. Deaths observed in the FUTURE III trial (quadrivalent vaccine, phase 3, women aged 24-45 years)

\begin{tabular}{lll} 
ID & Group & Death causes \\
\hline 1 & C & $\begin{array}{l}\text { Pulmonary thromboembolism with background of acute lymphoblastic } \\
\text { leukaemia }\end{array}$ \\
\hline 2 & V & Breast cancer \\
\hline 3 & V & Pulmonary tuberculosis \\
\hline 4 & V & Thyrotoxicosis \\
\hline 5 & V & Cerebral haemorrhage subsequent to hypertension \\
\hline 6 & V & Pericarditis on a background of lupus erythematosus
\end{tabular}

Prophylactic vaccination against human papillomaviruses to prevent cervical cancer and its precursors (Review)

Copyright ๑ 2020 The Cochrane Collaboration. Published by John Wiley \& Sons, Ltd. 
Table 5. Deaths observed in the FUTURE III trial (quadrivalent vaccine, phase 3, women aged 24-45 years) (Continued)

\begin{tabular}{lll}
7 & $\mathrm{~V}$ & Nasopharyngeal cancer with metastases to brain \\
\hline 8 & $\mathrm{~V}$ & Pulmonary embolism after intervention for uterine myoma
\end{tabular}

RR of deaths in vaccine vs placebo arm (7 over 1,890 vs 1 over 1888$)$ : RR = 6.99 (95\% Cl 0.86 to 56.78), 2-sided pexact $=0.070$.

The age at death varied between 29 and 45 years, seven of the deaths occurred in the Philippines and one in Columbia.

All participants received three doses of HPV vaccine or placebo except one who received only two doses of vaccine. The time interval between last dose and date at death ranged between 6 and 37 months.

Group:V = vaccinated against $\mathrm{HPV}, \mathrm{C}=$ control group.

Source: end-of-study analysis after a median follow-up of four years (Castellsagué 2011) and personal communication with Alfred Saah (MSD, 6/05/2016).

Table 6. Deaths observed in the VIVIANE trial (bivalent vaccine, phase 3 trial, women aged $>25$ years)

\begin{tabular}{|c|c|c|c|c|c|}
\hline Patient & Cause of death & Group & Age & Country & Source \\
\hline 1 & Breast cancer metastatic & V & 47 & Canada & 1 \\
\hline 2 & Suicide & V & 47 & Mexico & 1 \\
\hline 3 & $\begin{array}{l}\text { Lower respiratory tract infection and } \\
\text { sepsis }^{\star}\end{array}$ & C & 55 & Mexico & 1 \\
\hline 4 & Cervix cancer metastatic ${ }^{\star \star}$ & V & 45 & Mexico & 1 \\
\hline 5 & Interstitial lung disease & V & 41 & Mexico & 1 \\
\hline 6 & Breast cancer & 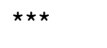 & 32 & Mexico & $1^{\star \star \star}$ \\
\hline 7 & Suicide & V & 41 & Mexico & 1 \\
\hline 8 & Cardiac valve disease and liver disorder ${ }^{\star}$ & $\mathrm{C}$ & 38 & Mexico & 1 \\
\hline 9 & $\begin{array}{l}\text { Drug hypersensitivity and acute renal } \\
\text { failure }^{\star}\end{array}$ & V & 46 & Peru & 1 \\
\hline 10 & Cardiorespiratory arrest & $\mathrm{C}$ & 44 & Phillipines & 1 \\
\hline 11 & Acute myocardial infarction & V & 31 & Phillipines & 1 \\
\hline 12 & $\begin{array}{l}\text { Multiple myeloma and pulmonary em- } \\
\text { bolism }^{\star}\end{array}$ & V & 50 & Phillipines & 1 \\
\hline 13 & Homicide & V & 32 & Phillipines & 1 \\
\hline 14 & Bronchopneumonia & $\mathrm{V}$ & 40 & Singapore & 1 \\
\hline 15 & Lung neoplasm malignant & $\mathrm{V}$ & 41 & Thailand & 1 \\
\hline 16 & Suicide & V & 28 & USA & 1 \\
\hline 17 & Glioblastoma multiforme & V & 45 & USA & 1 \\
\hline
\end{tabular}


Table 6. Deaths observed in the VIVIANE trial (bivalent vaccine, phase $\mathbf{3}$ trial, women aged $>25$ years) (Continued)

\begin{tabular}{llllll}
18 & Anaplastic astrocytoma & C & 43 & $\star \star \star \star$ & 2 \\
\hline 19 & Nasopharyngeal cancer & C & 41 & $\star \star \star \star$ & 2
\end{tabular}

\section{Remarks}

\begin{tabular}{ll}
\hline$*$ & Multiple death causes \\
\hline$* *$ & $\begin{array}{l}\text { This woman had normal cytology but was HPV-18 DNA-positive at study entry (May 2006). At the next scheduled cy- } \\
\text { tology testing at Month } 12 \text { (April 2007), the cytology finding was atypical squamous cells cannot exclude high-grade } \\
\text { squamous intraepithelial lesion. She was diagnosed with metastatic cervical cancer in May } 2007 \text { (approximately } 7 \\
\text { months after receiving the third dose of vaccine or control) and died in July } 2008\end{array}$ \\
\hline$* * * \quad \begin{array}{l}\text { One case of death due to breast cancer reported in the } 48 \text { month report (Skinner 2014) had to be excluded from the } \\
\text { analysis (Wheeler 2016). }\end{array}$ \\
$\begin{array}{l}\text { Two additional cases of death occurring in the control arm were reported in the 84-month report (Wheeler 2016). } \\
\text { The country for these two cases was not reported. }\end{array}$ \\
\hline
\end{tabular}

Source: 1) interim analysis after 48 months of follow-up (Skinner 2014); 2) report at 84 months of follow-up (Wheeler 2016)

The 84-month follow-up report revealed 13 deaths in the HPV arm $(\mathrm{N}=2877)$ versus $5(\mathrm{~N}=2870)$, with death causes allocated to the trial arms (vaccine versus placebo arm) the RR was $2.59(95 \% \mathrm{Cl} 0.93$ to 7.27$), 2$-sided $p_{\text {exact }}=0.0957$. No pattern was noticed which could indicate a causal role attributed to HPV vaccination.

Table 7. Trials for which vaccine efficacy is reported by smaller age subgroups

\begin{tabular}{lccc}
\hline Trial & Target age group & Age category & Reported age sub-groups \\
\hline Phase2 trial (ph2,1v) & $16-23$ & younger & none \\
\hline Phase2 trial (ph2,2v) & $15-25$ & younger & none \\
\hline Phase2 trial (ph2,4v) & $16-23$ & younger & none \\
\hline Japanese trial (ph2,2v) & $20-25$ & younger & none \\
\hline PATRICIA trial (ph3,2v) & $15-25,18-20,21-25$ \\
\hline CVT (ph3,2v) & $18-25$ & younger & $18-19,20-21,22-23,24-25$ \\
\hline VIVIANE trial (ph3,2v) & $26+$ & older & 26-35, 36-45, 46+ \\
\hline FUTURE I trial (ph3,4v) & $16-24$ & younger & none \\
\hline FUTURE II trial (ph3,4v) & $15-26$ & younger & none \\
\hline FUTURE III trial (ph3,4v) & $25-45$ & older & none \\
\hline
\end{tabular}

Table 8. Influence of age (PATRICIA trial)

\begin{tabular}{|c|c|c|c|c|c|c|}
\hline Outcome & Age & $\begin{array}{l}\text { Event/N } \\
\text { Vaccine }\end{array}$ & $\begin{array}{l}\text { Event/N } \\
\text { Placebo }\end{array}$ & $\begin{array}{l}\text { Relative risk } \\
(95 \% \mathrm{CI})\end{array}$ & $\begin{array}{l}\text { Vaccine efficacy } \\
\%(95 \% \mathrm{Cl})\end{array}$ & $\begin{array}{l}\text { P value for } \\
\text { linear ef- } \\
\text { fect of age }\end{array}$ \\
\hline
\end{tabular}


Table 8. Influence of age (PATRICIA trial) (Continued) In women with hrHPV DNA negative status at baseline

\begin{tabular}{|c|c|c|c|c|c|c|}
\hline \multirow{3}{*}{$\begin{array}{l}\text { CIN2+ asso- } \\
\text { ciated with } \\
\text { HPV16/18 }\end{array}$} & $15-17$ & $1 / 1997$ & $53 / 2022$ & $0.02(0.00$ to 0.14$)$ & $98 \%$ (86 to $100 \%)$ & \multirow[t]{3}{*}{0.995} \\
\hline & $18-20$ & $0 / 1096$ & $27 / 1144$ & 0.02 (0.00 to 0.32 ) & $98 \%$ (68 to $100 \%)$ & \\
\hline & $21-25$ & $0 / 2363$ & $17 / 2281$ & $0.03(0.00$ to 0.47$)$ & $97 \%$ (53 to $100 \%)$ & \\
\hline \multirow{3}{*}{$\begin{array}{l}\text { CIN2+ irre- } \\
\text { spective of } \\
\text { HPV types }\end{array}$} & $15-17$ & $34 / 1997$ & $101 / 2022$ & $0.34(0.23$ to 0.50$)$ & $66 \%$ (50 to $77 \%$ ) & \multirow[t]{3}{*}{0.355} \\
\hline & $18-20$ & $10 / 1096$ & $38 / 1144$ & $0.27(0.14$ to 0.55$)$ & $73 \%$ (45 to $86 \%$ ) & \\
\hline & $21-25$ & $17 / 2363$ & $33 / 2281$ & $0.50(0.28$ to 0.89$)$ & $50 \%(11$ to $72 \%)$ & \\
\hline \multirow{3}{*}{$\begin{array}{l}\text { CIN3+ asso- } \\
\text { ciated with } \\
\text { HPV16/18 }\end{array}$} & $15-17$ & 0/1997 & $14 / 2022$ & 0.04 (0.00 to 0.61$)$ & $96 \%$ (39 to100\%) & \multirow[t]{3}{*}{1.000} \\
\hline & $18-20$ & $0 / 1096$ & $8 / 1144$ & 0.07 (0.00 to 1.13$)$ & $93 \%(-13$ to $100 \%)$ & \\
\hline & $21-25$ & $0 / 2363$ & $5 / 2281$ & $0.10(0.00$ to 1.74$)$ & $90 \%(-74$ to $100 \%)$ & \\
\hline \multirow{3}{*}{$\begin{array}{l}\text { CIN3+ irre- } \\
\text { spective of } \\
\text { HPV types }\end{array}$} & $15-17$ & 2/1997 & $24 / 2022$ & $0.08(0.02$ to 0.36$)$ & $92 \%(64$ to $98 \%)$ & \multirow[t]{3}{*}{0.488} \\
\hline & $18-20$ & $1 / 1096$ & $11 / 1144$ & $0.09(0.01$ to 0.73$)$ & $91 \%(27$ to $99 \%)$ & \\
\hline & $21-25$ & $0 / 2363$ & $9 / 2281$ & $0.05(0.00$ to 0.92$)$ & $95 \%$ (8 to $100 \%)$ & \\
\hline \multirow{3}{*}{$\begin{array}{l}\text { Persistent } \\
\text { HPV16/18 in- } \\
\text { fection (6M) }\end{array}$} & $15-17$ & $14 / 1989$ & $303 / 2020$ & $0.05(0.03$ to 0.08$)$ & $95 \%$ (92 to $97 \%)$ & \multirow[t]{3}{*}{$0 . .042$} \\
\hline & $18-20$ & $9 / 1090$ & $110 / 1125$ & $0.08(0.04$ to 0.17$)$ & $92 \%(83$ to $96 \%)$ & \\
\hline & $21-25$ & $12 / 2338$ & $108 / 2249$ & 0.11 (0.06 to 0.19$)$ & $89 \%$ (81 to $94 \%$ ) & \\
\hline
\end{tabular}

Regardless of women's baseline HPV DNA status

\begin{tabular}{|c|c|c|c|c|c|c|}
\hline \multirow{3}{*}{$\begin{array}{l}\text { CIN2+ asso- } \\
\text { ciated with } \\
\text { HPV16/18 }\end{array}$} & $15-17$ & $21 / 2882$ & $100 / 2892$ & 0.21 (0.13 to 0.24$)$ & $79 \%$ (66 to $87 \%$ ) & 0.000 \\
\hline & $18-20$ & $23 / 1871$ & $66 / 1908$ & $0.36(0.22$ to 0.57$)$ & $64 \%$ (43 to $78 \%$ ) & \\
\hline & $21-25$ & $46 / 3929$ & $62 / 3898$ & $0.74(0.50$ to 1.08$)$ & $26 \%(-8$ to $50 \%)$ & \\
\hline \multirow{3}{*}{$\begin{array}{l}\text { CIN2+ irre- } \\
\text { spective of } \\
\text { HPV types }\end{array}$} & $15-17$ & $112 / 2882$ & $200 / 2892$ & $0.56(0.45$ to 0.70$)$ & $44 \%$ (30 to $55 \%$ ) & 0.006 \\
\hline & $18-20$ & $62 / 1871$ & $105 / 1908$ & $0.60(0.44$ to 0.82$)$ & $40 \%$ (18 to $56 \%$ ) & \\
\hline & $21-25$ & $113 / 3929$ & $123 / 3898$ & 0.91 (0.09 to 1.17$)$ & $9 \%(-17$ to $29 \%)$ & \\
\hline \multirow{3}{*}{$\begin{array}{l}\text { CIN3+ asso- } \\
\text { ciated with } \\
\text { HPV16/18 }\end{array}$} & $15-17$ & $7 / 2882$ & $36 / 2892$ & 0.20 (0.09 to 0.44$)$ & $80 \%$ (56 to $91 \%$ ) & 0.000 \\
\hline & $18-20$ & $13 / 1871$ & $30 / 1908$ & $0.44(0.23$ to 0.84$)$ & $56 \%$ (16 to $77 \%)$ & \\
\hline & $21-25$ & $31 / 3929$ & $28 / 3898$ & 1.10 (0.66 to 1.83$)$ & $-10 \%(-83$ to $34 \%)$ & \\
\hline \multirow{2}{*}{$\begin{array}{l}\text { CIN3+ irre- } \\
\text { spective of } \\
\text { HPV types }\end{array}$} & $15-17$ & $21 / 2882$ & $61 / 2892$ & 0.35 (0.21 to 0.57$)$ & $65 \%$ (43 to $79 \%$ ) & 0.008 \\
\hline & $18-20$ & $22 / 1871$ & $44 / 1908$ & 0.51 (0.31 to 0.85$)$ & $49 \%$ (15 to $69 \%$ ) & \\
\hline
\end{tabular}


Table 8. Influence of age (PATRICIA trial) (Continued)

\begin{tabular}{lccccc} 
& $21-25$ & $43 / 3929$ & $53 / 3898$ & $0.80(0.54$ to 1.20$)$ & $20 \%(-20$ to $46 \%)$ \\
\hline $\begin{array}{l}\text { Persistent } \\
\text { HPV16/18 in- } \\
\text { fection (6M) }\end{array}$ & $15-17$ & $167 / 2916$ & $588 / 2920$ & $0.28(0.24$ to 0.34$)$ & $72 \%(66$ to $76 \%)$ \\
\cline { 2 - 5 } & $21-25$ & $143 / 1925$ & $283 / 1961$ & $0.51(0.43$ to 0.62$)$ & $49 \%(38$ to $57 \%)$ \\
& $194 / 4009$ & $356 / 3979$ & $0.54(0.46$ to 0.64$)$ & $46 \%(36$ to $54 \%)$
\end{tabular}

Source: Lehtinen 2012.

CIN: cervical intraepithelial neoplasia, CIN2+: CIN of degree II or worse, CIN3+: CIN of degree 3 or worse, HPV: human papillomavirus types..

Table 9. Influence of age (CVT trial)

\begin{tabular}{|c|c|c|c|c|c|c|}
\hline Outcome & Age & Vaccine & Placebo & $\begin{array}{l}\text { Relative risk } \\
(95 \% \mathrm{CI})\end{array}$ & $\begin{array}{l}\text { Vaccine efficacy } \\
(95 \% \mathrm{Cl})\end{array}$ & $\begin{array}{l}P \text { value for } \\
\text { linear ef- } \\
\text { fect of age }\end{array}$ \\
\hline
\end{tabular}

\section{In women with HPV16/18 DNA negative status at baseline cohort}

\begin{tabular}{lccccc}
\hline $\begin{array}{l}\text { Persistent } \\
\text { HPV16/18 in- } \\
\text { fection (6M) }\end{array}$ & $18-19$ & $1 / 825$ & $51 / 870$ & $0.02(0.00$ to 0.10$)$ & 9.145 \\
\cline { 2 - 5 } & $20-21$ & $3 / 659$ & $36 / 649$ & $0.08(0.02$ to 0.24$)$ & $92 \%(76 \%$ to $98 \%)$ \\
\cline { 2 - 5 } & $22-23$ & $2 / 588$ & $36 / 625$ & $0.06(0.00$ to 0.20$)$ & $94 \%(80 \%$ to $100 \%)$ \\
\hline
\end{tabular}

\section{Regardless if women's baseline HPV DNA status}

\begin{tabular}{|c|c|c|c|c|c|c|}
\hline \multirow{4}{*}{$\begin{array}{l}\text { Persistent } \\
\text { HPV16/18 in- } \\
\text { fection (6M) }\end{array}$} & $18-19$ & $47 / 1193$ & $165 / 1,244$ & $0.30(0.21$ to 0.41$)$ & $70 \%$ (59\% to $79 \%)$ & 0.000 \\
\hline & $20-21$ & $64 / 946$ & $134 / 905$ & 0.46 (0.34 to 0.61$)$ & $54 \%$ (39\% to $66 \%)$ & \\
\hline & $22-23$ & $59 / 818$ & $112 / 848$ & 0.55 (0.40 to 0.75$)$ & $45 \%(25 \%$ to $60 \%)$ & \\
\hline & $24-25$ & $61 / 770$ & $75 / 742$ & $0.78(0.56$ to 1.99$)$ & $22 \%(-9.9$ to $44 \%)$ & \\
\hline
\end{tabular}

Source: Herrero 2011.

Table 10. Influence of age (VIVIANE trial)

\begin{tabular}{|c|c|c|c|c|c|c|}
\hline Outcome & Age & $\begin{array}{l}\text { Event/ } \\
\text { NVaccine }\end{array}$ & $\begin{array}{l}\text { Event/ } \\
\text { NPlacebo }\end{array}$ & $\begin{array}{l}\text { Relative risk } \\
(95 \% \mathrm{CI})\end{array}$ & $\begin{array}{l}\text { Vaccine efficacy } \\
(95 \% \mathrm{Cl})\end{array}$ & $\begin{array}{l}\text { P value for } \\
\text { linear ef- } \\
\text { fect of age }\end{array}$ \\
\hline \multicolumn{7}{|c|}{ In women with HPV16/18 DNA negative status at baseline cohort } \\
\hline \multirow{3}{*}{$\begin{array}{l}\text { Persistent } \\
\text { HPV16/18 infec- } \\
\text { tion (6M) }\end{array}$} & $26-35$ & $3 / 834$ & $22 / 800$ & 0.13 (0.04 to 0.44$)$ & $87 \%$ (56\% to $96 \%)$ & 0.532 \\
\hline & $36-45$ & $3 / 816$ & $12 / 809$ & 0.25 (0.07 to 0.88$)$ & $75 \%(12 \%$ to $93 \%)$ & \\
\hline & $46+$ & $0 / 219$ & $0 / 213$ & N.A. & N.A. & \\
\hline
\end{tabular}


Table 10. Influence of age (VIVIANE trial) (Continued)

\section{Regardless if women's baseline HPV DNA status}

\begin{tabular}{|c|c|c|c|c|c|c|}
\hline \multirow{3}{*}{$\begin{array}{l}\text { Persistent } \\
\text { HPV16/18 infec- } \\
\text { tion (6M) }\end{array}$} & $26-35$ & $48 / 1221$ & $78 / 1242$ & 0.63 (0.44 to 0.89 ) & $37 \%(11 \%$ to $56 \%)$ & \multirow[t]{3}{*}{0.177} \\
\hline & $36-45$ & $19 / 1244$ & $43 / 1228$ & $0.44(0.26$ to 0.74$)$ & $56 \%(26 \%$ to $74 \%)$ & \\
\hline & $46+$ & $4 / 300$ & $11 / 306$ & $0.37(0.12$ to 1.15$)$ & $63 \%(-15 \%$ to $88 \%)$ & \\
\hline
\end{tabular}

Source: Skinner 2014.

Table 11. Influence of the initial serological status on vaccine efficacy against cervical lesions associated with HPV16/18

\begin{tabular}{|c|c|c|c|c|c|}
\hline $\begin{array}{l}\text { Initial HPV } \\
\text { DNA/ status }\end{array}$ & $\begin{array}{l}\text { Serology } \\
\text { status }\end{array}$ & Vaccine & Placebo & $\begin{array}{l}\text { Relative Risk } \\
(95 \% \mathrm{Cl})\end{array}$ & $\begin{array}{l}\text { Relative Risk } \\
\text { ratio }\end{array}$ \\
\hline
\end{tabular}

FUTURE I trial (ph3,4v) (Garland 2007)*

\begin{tabular}{llllll}
\hline DNA(-) & Sero- & $0 / 2,241$ & $32 / 2258$ & 0.00 (0.02 to 0.26) & 15.93 \\
\cline { 2 - 5 } & Sero+ & $0 / 377$ & $2 / 379$ & $0.25(0.01$ to 5.20$)$ & 1.50 \\
\hline DNA(+) & Sero- & $27 / 232$ & $31 / 213$ & 0.80 (0.49 to 1.29$)$ & $1.20(0.80$ to 1.81$)$ \\
\cline { 2 - 5 } & Sero+ & $41 / 156$ & $30 / 137$ & $2007) * *$ \\
\hline
\end{tabular}

FUTURE II trial (ph3,4v) (FUTURE-II 2007)**

\begin{tabular}{|c|c|c|c|c|c|}
\hline \multirow[t]{2}{*}{ DNA(-) } & Sero- & $0 / 5,305$ & $28 / 5260$ & $0.02(0.00$ to 0.14$)$ & \multirow[t]{2}{*}{7.41} \\
\hline & Sero+ & $0 / 498$ & $4 / 524$ & 0.13 (0.01 to 2.43 ) & \\
\hline \multirow[t]{2}{*}{ DNA(+) } & Sero- & $33 / 423$ & $35 / 402$ & 0.90 (0.57 to 1.41$)$ & \multirow[t]{2}{*}{1.12} \\
\hline & Sero+ & $47 / 298$ & $52 / 332$ & 1.01 (0.70 to 1.45 & \\
\hline
\end{tabular}

PATRICIA trial (ph3,2v) (Paavonen 2009)**

\begin{tabular}{|c|c|c|c|c|c|}
\hline \multirow[t]{2}{*}{ DNA(-) } & Sero- & $5 / 8709$ & $92 / 8112$ & 0.05 (0.02 to 0.12$)$ & 6.16 \\
\hline & Sero+ & $3 / 1710$ & $10 / 1777$ & 0.31 (0.09 to 1.13$)$ & \\
\hline \multirow[t]{2}{*}{ DNA(+) } & Sero- & $20 / 309$ & $29 / 293$ & 0.65 (0.38 to 1.13$)$ & 1.70 \\
\hline & Sero+ & $53 / 333$ & $44 / 307$ & $1.11(0.77$ to 1.61$)$ & \\
\hline
\end{tabular}

\section{Pooled results for CIN2+ associated with HPV16/18}

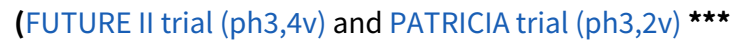

\begin{tabular}{llllll}
\hline DNA(-) & Sero- & $5 / 14,014$ & $120 / 13,372$ & 0.03 (0.02 to 0.09) & 5.85 \\
\cline { 2 - 5 } & Sero+ & $3 / 2205$ & $14 / 2301$ & $0.19(0.09$ t0 o.77) & $(0.53$ to 65.10$)$ \\
\hline
\end{tabular}


Table 11. Influence of the initial serological status on vaccine efficacy against cervical lesions associated with HPV16/18 (Continued)

\begin{tabular}{llllll} 
DNA(+) & Sero- & $53 / 679$ & $64 / 695$ & $0.79(0.60$ to 1.05 & 1.37 \\
\cline { 2 - 4 } & Sero+ & $100 / 531$ & $96 / 639$ & $1.10(0.88$ to 1.36$)$ & (0.97 to 1.93$)$
\end{tabular}

${ }^{\star}$ RR against HPV 6/11/16/18 related cervical lesions

** RR against HPV16/18 related CIN2+

*** Pooled only for FUTURE II and PATRIACIA, since, in the FUTURE I trial, the endpoints were cervical lesions and not CIN2+ associated with HPV16/18 


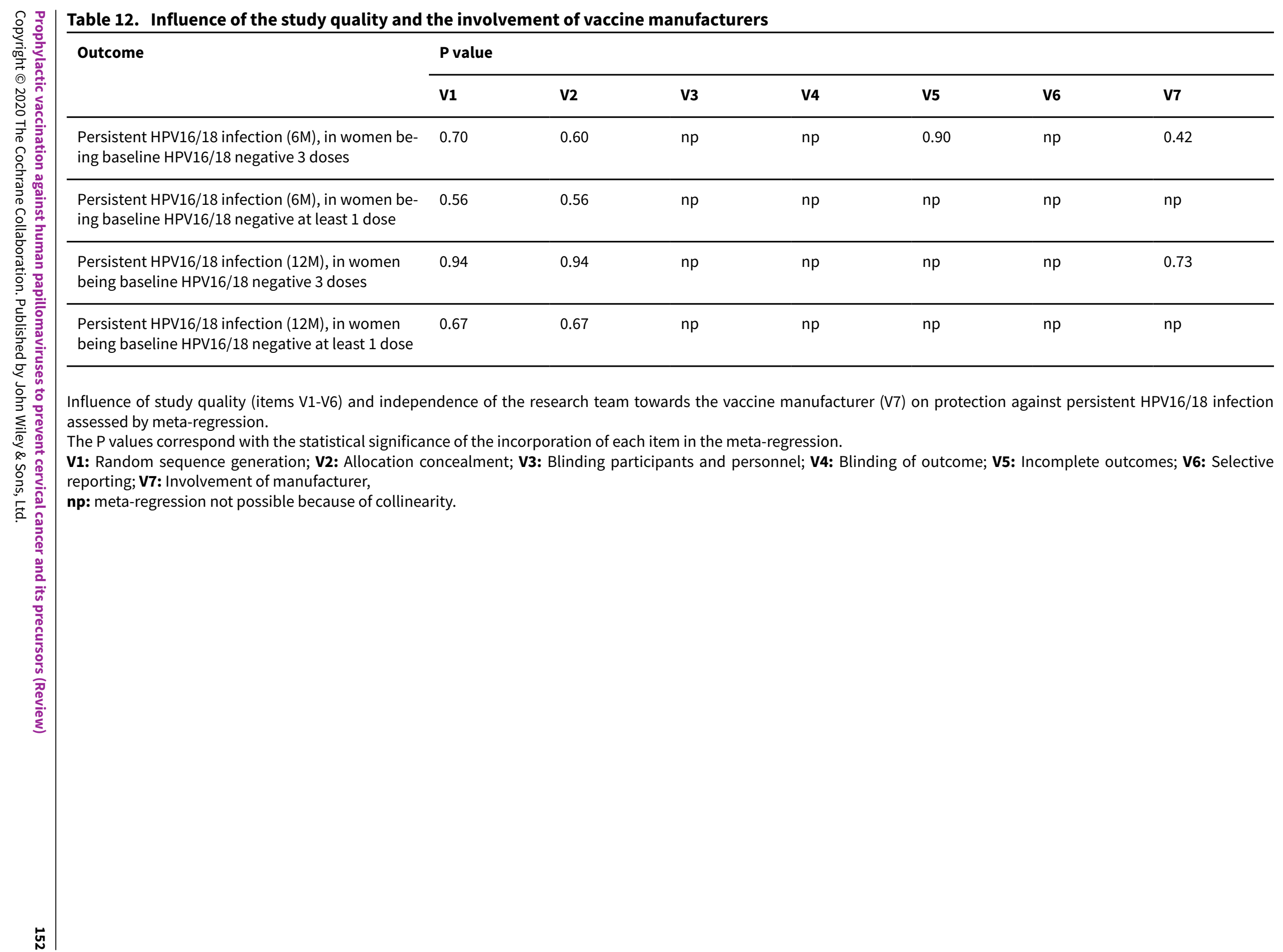


Table 13. Influence of the number of administered doses: one, two or three in two RCTs with four years of follow-up

\begin{tabular}{|c|c|c|c|c|c|}
\hline Outcome & No. of doses & $\begin{array}{l}\text { Vaccine } \\
\text { arm }\end{array}$ & $\begin{array}{l}\text { Placebo } \\
\text { arm }\end{array}$ & $\begin{array}{l}\text { Relative Risk } \\
(95 \% \mathrm{Cl})\end{array}$ & $\begin{array}{l}\text { P value for } \\
\text { linear } \\
\text { dose-effect } \\
\text { relation }\end{array}$ \\
\hline \multirow{2}{*}{$\begin{array}{l}\text { 12-month } \\
\text { persistent HPV16/18 }\end{array}$} & 3 & $84 / 11,104$ & $627 / 11,203$ & $0.135(0.108$ to 0.169$)$ & \multirow[t]{4}{*}{0.303} \\
\hline & 2 & $3 / 611$ & $26 / 574$ & $0.108(0.033$ to 0.356$)$ & \\
\hline \multirow{2}{*}{$\begin{array}{l}\text { infection } \\
\text { in women being } \\
\text { HPV16/18 negative at baseline }\end{array}$} & 1 & $1 / 292$ & $17 / 249$ & $0.050(0.007$ to 0.374$)$ & \\
\hline & & & & & \\
\hline \multirow{3}{*}{$\begin{array}{l}\text { 6-month } \\
\text { persistent HPV16/18 } \\
\text { infection }\end{array}$} & 3 & $114 / 11,104$ & $1000 / 11,209$ & $0.115(0.095$ to 0.139$)$ & \multirow[t]{5}{*}{0.269} \\
\hline & 2 & $4 / 611$ & $35 / 574$ & $0.107(0.038$ to 0.300$)$ & \\
\hline & 1 & $1 / 292$ & $24 / 250$ & $0.036(0.005$ to 0.261$)$ & \\
\hline \multirow{2}{*}{\multicolumn{5}{|c|}{$\begin{array}{l}\text { in women being } \\
\text { HPV16/18 negative at baseline }\end{array}$}} & \\
\hline & & & & & \\
\hline \multirow{3}{*}{$\begin{array}{l}\text { Incident HPV16/18 infection } \\
\text { in women being HPV16/18 }\end{array}$} & 3 & $529 / 11,110$ & $2172 / 11,217$ & $0.246(0.224$ to 0.269$)$ & \multirow[t]{3}{*}{0.337} \\
\hline & 2 & $22 / 611$ & $82 / 574$ & 0.252 (0.160 to 0.398$)$ & \\
\hline & 1 & $8 / 292$ & $45 / 251$ & $0.153(0.073$ to 0.318$)$ & \\
\hline \multirow{4}{*}{$\begin{array}{l}\text { 12-month } \\
\text { persistent HPV16/18 } \\
\text { infection } \\
\text { in women being } \\
\text { hrHPV negative at baseline }\end{array}$} & 3 & $27 / 6634$ & $351 / 6656$ & $0.077(0.052$ to 0.114$)$ & \multirow[t]{4}{*}{0.996} \\
\hline & 2 & $2 / 273$ & $12 / 276$ & $0.168(0.038$ to 0.746$)$ & \\
\hline & 1 & $0 / 138$ & $5 / 99$ & $0.071(0.004$ to 1.289$)$ & \\
\hline & & & & & \\
\hline \multirow{2}{*}{$\begin{array}{l}\text { 6-month } \\
\text { persistent HPV16/18 }\end{array}$} & 3 & $38 / 6634$ & $567 / 6660$ & 0.067 (0.049 to 0.093$)$ & \multirow[t]{4}{*}{0.809} \\
\hline & 2 & $2 / 273$ & $16 / 276$ & $0.126(0.029$ to 0.544$)$ & \\
\hline in women being & 1 & $0 / 138$ & $8 / 100$ & 0.045 (0.003 to 0.774$)$ & \\
\hline hrHPV negative at baseline & & & & & \\
\hline \multirow{3}{*}{$\begin{array}{l}\text { Incident HPV16/18 infection } \\
\text { in women being hrHPV } \\
\text { negative at baseline }\end{array}$} & 3 & $38 / 6634$ & $567 / 6660$ & $0.067(0.049$ to 0.093$)$ & \multirow[t]{3}{*}{0.809} \\
\hline & 2 & $2 / 273$ & $16 / 276$ & $0.126(0.029$ to 0.544$)$ & \\
\hline & 1 & $0 / 138$ & $8 / 100$ & $0.045(0.003$ to 0.774$)$ & \\
\hline
\end{tabular}

Source: PATRICIA \& CVT (ph3,2v) (Kreimer 2015). 
Table 14. Influence of the number of administered doses in the CVT trial (seven years of follow-up)

\begin{tabular}{|c|c|c|c|c|c|}
\hline Outcome & No. of doses & n events & $\mathbf{N}$ vaccinated & $\%(95 \% \mathrm{Cl})$ & $\begin{array}{l}P^{\star} \text { for differ- } \\
\text { ence with } 3 \text { dos- } \\
\text { es }\end{array}$ \\
\hline \multirow{2}{*}{$\begin{array}{l}\text { Cumulative } \\
\text { incidence }\end{array}$} & 3 & 88 & 2023 & 4.3 (3.5 to 5.3$)$ & - \\
\hline & 2 (at months $0 \& 6$ ) & 3 & 78 & $3.8(1.0$ to 10.1$)$ & 1.00 \\
\hline \multirow{2}{*}{$\begin{array}{l}\text { HPV16/18 } \\
\text { infections }\end{array}$} & 2 (at months $0 \& 1$ ) & 7 & 192 & 3.6 (1.6 to 7.1$)$ & 0.85 \\
\hline & 1 & 2 & 133 & $1.5(0.3$ to 4.9$)$ & 0.17 \\
\hline
\end{tabular}

Source: Safaeian 2018.

* two-sided exact test for difference between proportions.

Table 15. Influence of the number of administered doses: all three versus less than three doses

\begin{tabular}{|c|c|c|c|c|}
\hline Outcomes & $\begin{array}{l}\text { Age } \\
\text { Group } \\
\text { (years) }\end{array}$ & Studies & $\begin{array}{l}\text { RR if } 3 \text { doses } \\
(95 \% \mathrm{Cl})\end{array}$ & $\begin{array}{l}\text { RR if } 1-2 \text { doses } \\
(95 \% \mathrm{Cl})\end{array}$ \\
\hline \multirow[t]{2}{*}{$\begin{array}{l}\text { CIN2+ } \\
\text { due to } \\
\text { HPV16/18 }\end{array}$} & $15-26$ & $\begin{array}{l}5 \text { (FUTURE II trial (ph3,4v); Japanese trial (ph2,2v); } \\
\text { PATRICIA trial (ph3,2v); Phase2 trial (ph2,1v); Chi- } \\
\text { nese trial (ph3,2v)_young) }\end{array}$ & $\begin{array}{l}0.07(0.03 \text { to } \\
0.14)^{\star}\end{array}$ & $\begin{array}{l}0.10(0.04 \text { to } \\
0.26)^{\star}\end{array}$ \\
\hline & $24-45$ & 2 (FUTURE III trial (ph3,4v); VIVIANE trial (ph3,2v)) & $\begin{array}{l}0.14(0.03 \text { to } \\
0.79)^{\star}\end{array}$ & $\begin{array}{l}0.98 \text { ( } 0.20 \text { to } \\
4.83)\end{array}$ \\
\hline $\begin{array}{l}\text { CIN3+ } \\
\text { due to } \\
\text { HPV16/18 }\end{array}$ & $15-26$ & 1 (PATRICIA trial (ph3,2v)) & $\begin{array}{l}0.20(0.04 \text { to } \\
0.91)^{\star}\end{array}$ & $\begin{array}{l}0.04(0.01 \text { to } \\
0.74)^{\star}\end{array}$ \\
\hline $\begin{array}{l}\text { Incident } \\
\text { HPV16/18 infec- } \\
\text { tion }\end{array}$ & $15-26$ & $\begin{array}{l}3 \text { (Japanese trial (ph2,2v); Phase2 trial (ph2,1v);Chi- } \\
\text { nese trial (ph3,2v)_young) }\end{array}$ & $\begin{array}{l}0.20(0.10 \text { to } \\
0.41)^{\star}\end{array}$ & $\begin{array}{l}0.47(0.26 \text { to } \\
0.84)^{\star}\end{array}$ \\
\hline \multirow{2}{*}{$\begin{array}{l}\text { 6-month persis- } \\
\text { tent HPV16/18 } \\
\text { infection }\end{array}$} & $15-26$ & $\begin{array}{l}2 \text { (Japanese trial (ph2,2v);Chinese trial (ph3,2v)_y- } \\
\text { oung) }\end{array}$ & $\begin{array}{l}0.05(0.01 \text { to } \\
0.27)^{\star}\end{array}$ & $\begin{array}{l}0.12(0.03 \text { to } \\
0.42)^{\star}\end{array}$ \\
\hline & $24-45$ & 2 (FUTURE III trial (ph3,4v);VIVIANE trial (ph3,2v)) & $\begin{array}{l}0.15(0.09 \text { to } \\
0.27)^{\star}\end{array}$ & $\begin{array}{l}0.34(0.19 \text { to } \\
0.61)^{\star}\end{array}$ \\
\hline $\begin{array}{l}\text { 12-month } \\
\text { persistent } \\
\text { HPV16/18 infec- } \\
\text { tion }\end{array}$ & $15-26$ & $\begin{array}{l}3 \text { (Japanese trial (ph2,2v);CVT (ph3,2v); Chinese tri- } \\
\text { al (ph3,2v)_young) }\end{array}$ & $\begin{array}{l}0.09(0.05 \text { to } \\
0.19)^{\star}\end{array}$ & $\begin{array}{l}0.13(0.06 \text { to } \\
0.33)^{\star}\end{array}$ \\
\hline
\end{tabular}

*Vaccine efficacy in women being HPV16/18 DNA negative at enrolment and having received all three or less than three doses (computed from trials where per-protocol [all doses administered] and intention-to-treat analyses [at least one dose administered] are reported). 
Table 16. Influence of follow-up time

\begin{tabular}{|c|c|c|c|c|c|c|}
\hline Outcomes & Study & $\begin{array}{l}\text { Report } \\
\text { (duration of follow-up) }\end{array}$ & Vaccine & Placebo & $\begin{array}{l}\text { Relative Risk } \\
(95 \% \mathrm{CI})\end{array}$ & $\begin{array}{l}\text { P value for } \\
\text { linear dif- } \\
\text { ference } \\
\text { of fol- } \\
\text { low-up } \\
\text { time effect }\end{array}$ \\
\hline \multirow{10}{*}{$\begin{array}{l}\text { CIN2+ asso- } \\
\text { ciated with } \\
\text { HPV16/18 } \\
\text { in women be- } \\
\text { ing HPV neg- } \\
\text { ative at base- } \\
\text { line }\end{array}$} & \multirow[t]{6}{*}{ PATRICIA } & $\begin{array}{l}\text { Paavonen } 2007 \\
14.8 \text { moths }\end{array}$ & $2 / 7788$ & $21 / 7838$ & $0.096(0.007$ to 0.466$)$ & \multirow[t]{6}{*}{0.512} \\
\hline & & $\begin{array}{l}\text { Paavonen } 2009 \\
34.9 \text { months }\end{array}$ & $5 / 8040$ & $91 / 8080$ & $0.054(0.016$ to 0.137$)$ & \\
\hline & & Szarewski 2011 & $5 / 8079$ & $92 / 8112$ & 0.054 (0.016 to 0.137$)$ & \\
\hline & & 39.4 months & & & & \\
\hline & & Lehtinen 2011 & $5 / 7338$ & $97 / 7305$ & 0.051 (0.016 to 0.123$)$ & \\
\hline & & 43.7 months & & & & \\
\hline & \multirow[t]{4}{*}{ FUTURE } & $\begin{array}{l}\text { The FUTURE II study group } \\
2007\end{array}$ & $3 / 5865$ & $87 / 5836$ & 0.039 (0.011 to 0.109$)$ & \multirow[t]{4}{*}{0.994} \\
\hline & & 36 months & & & & \\
\hline & & Munoz 2010* & $0 / 4616$ & $89 / 4680$ & $0.006(0.000$ to 0.092$)$ & \\
\hline & & 43 months & & & & \\
\hline \multirow{6}{*}{$\begin{array}{l}\text { CIN2+ irre- } \\
\text { spective of } \\
\text { HPV types } \\
\text { regardless of } \\
\text { women's ini- } \\
\text { tial HPV DNA } \\
\text { status }\end{array}$} & \multirow[t]{2}{*}{ PATRICIA } & $\begin{array}{l}\text { Paavonen } 2009 \\
34.9 \text { months }\end{array}$ & $224 / 8667$ & $322 / 8682$ & $\begin{array}{l}0.696(0.579 \text { to } \\
0.8369)\end{array}$ & \multirow[t]{2}{*}{0.750} \\
\hline & & $\begin{array}{l}\text { Lehtinen } 2011 \\
43.7 \text { months }\end{array}$ & $287 / 8694$ & $428 / 8708$ & $0.669(0.574$ to 0.778$)$ & \\
\hline & \multirow[t]{4}{*}{ FUTURE } & $\begin{array}{l}\text { The FUTURE II study group } \\
2007\end{array}$ & $281 / 6087$ & $361 / 6080$ & $0.780(0.668$ to 0.905$)$ & \multirow[t]{4}{*}{0.665} \\
\hline & & 36 months & & & & \\
\hline & & Munoz 2010 & $421 / 8562$ & $520 / 8598$ & 0.807 (0.690 to 0.943$)$ & \\
\hline & & 43 months & & & & \\
\hline
\end{tabular}

Assessment of the influence of duration of follow-up on study outcomes using meta-regression. p-values correspond with the statistical significance of incorporating average follow-up time as a continuous variable.

Table 17. Influence of the number of sexual partners

\begin{tabular}{llll}
\hline $\begin{array}{l}\text { Number of sex part- } \\
\text { ners }\end{array}$ & Vaccine & Placebo & Relative Risk \\
& & $(95 \% \mathrm{Cl})$ & $\begin{array}{l}\text { P value of num- } \\
\text { ber of sexual } \\
\text { partners effect }\end{array}$ \\
\hline
\end{tabular}


Table 17. Influence of the number of sexual partners (Continued)

\section{In women being HPV16/18 DNA negative at baseline cohort}

\begin{tabular}{llll}
\hline Virgin & $1 / 566$ & $17 / 615$ & $0.064(0.003$ to 0.352$)$ \\
\hline 1 partner & $3 / 904$ & $27 / 915$ & $0.112(0.007$ to 0.335$)$ \\
\hline 2 partners & $1 / 544$ & $17 / 519$ & $0.056(0.003$ to 0.309$)$ \\
\hline $3+$ partners & $3 / 621$ & $28 / 628$ & $0.108(0.026$ to 0.321$)$ \\
\hline Regardless of women's baseline HPV DNA status & \\
\hline Virgin & $4 / 733$ & $21 / 819$ & $0.202(0.059$ to 0.551$)$ \\
\hline 1 partner & $40 / 1237$ & $83 / 1256$ & $0.489(0.333$ to 0.711$)$ \\
\hline 2 partners & $38 / 777$ & $81 / 753$ & $0.455(0.307$ to 0.665$)$ \\
\hline $3+$ partners & $71 / 940$ & $116 / 911$ & $0.593(0.440$ to 0.796$)$ \\
\hline
\end{tabular}

The influence of the number of lifetime sexual partners on vaccine efficacy was assessed by Poisson regression. The $P$ value corresponds with the likelihood ratio test comparing a Poisson model with and without inclusion of the sexual history with 3 possible categories. Source: CVT (ph3,2v) (Herrero 2011). 


\begin{tabular}{|c|c|c|c|c|c|c|c|}
\hline Outcomes & Study & $\begin{array}{l}\text { Number of } \\
\text { participants }\end{array}$ & Study size & Vaccine & Placebo & $\begin{array}{l}\text { Relative } \\
\text { Risk } \\
(95 \% \mathrm{Cl})\end{array}$ & $P$ value \\
\hline \multirow{6}{*}{$\begin{array}{l}\text { CIN2+ associated } \\
\text { with } \\
\text { HPV16/18 } \\
\text { in women being } \\
\text { HPV16/18 negative at } \\
\text { baseline }\end{array}$} & Phase2 trial (V1) & 2392 & $\mathrm{~S}$ & $0 / 126$ & $8 / 127$ & $\begin{array}{l}0.062^{\star} \\
(0.004 \text { to } 1.071)\end{array}$ & \multirow[t]{6}{*}{0.598} \\
\hline & Phase2 trial (V2) & 1113 & $\mathrm{~s}$ & $0 / 219$ & $3 / 212$ & $\begin{array}{l}0.161^{*} \\
(0.008 \text { to } 3.091)\end{array}$ & \\
\hline & Japanese trial (ph2,2v) & 1040 & $\mathrm{~S}$ & $0 / 422$ & $2 / 427$ & $\begin{array}{l}0.252^{\star} \\
(0.012 \text { to } 5.241)\end{array}$ & \\
\hline & PATRICIA trial $(p h 3,2 v)$ & 18,644 & $\mathrm{~L}$ & $5 / 8040$ & $91 / 8080$ & $\begin{array}{l}0.055 \\
\text { (0.022 to } 0.136)\end{array}$ & \\
\hline & FUTURE II trial (ph3, 4v) & 12,167 & L & $3 / 5865$ & $87 / 5863$ & $\begin{array}{l}0.034 \\
\text { (0.011 to } 0.109 \text { ) }\end{array}$ & \\
\hline & Chinese trial $(\mathrm{ph} 3,2 \mathrm{~V})$ & 6051 & $\mathrm{~L}$ & $0 / 2543$ & $4 / 2554$ & $\begin{array}{l}0.125 \\
\text { (0.001 to } 8.681 \text { ) }\end{array}$ & \\
\hline \multirow{3}{*}{$\begin{array}{l}\text { CIN2+ irrespective of } \\
\text { HPV types and } \\
\text { regardless of } \\
\text { women's } \\
\text { initial HPV DNA sta- } \\
\text { tus }\end{array}$} & FUTURE I/II trial (ph3,4v) & 17,622 & $\mathrm{~L}$ & $421 / 8562$ & $520 / 8598$ & $\begin{array}{l}0.813 \\
(0.718 \text { to } 0.921)\end{array}$ & \multirow[t]{3}{*}{0.703} \\
\hline & PATRICIA trial (ph3,2v) & 18,644 & L & $287 / 8694$ & $428 / 8708$ & $\begin{array}{l}0.672 \\
\text { (0.582 to } 0.778 \text { ) }\end{array}$ & \\
\hline & Phase2 trial ( $v 1)$ & 2392 & $\mathrm{~s}$ & $8 / 148$ & $12 / 142$ & $\begin{array}{l}0.640 \\
(0.269 \text { to } 1.568)\end{array}$ & \\
\hline
\end{tabular}

Assesment of the influence of the study size on the protection against CIN2+ associated with HPV16/18 according to study size $(\mathrm{S}=$ small, $<3000$ participants, $\mathrm{L}=$ large $>=3000$ participants) in women aged 15-26 years and received at least 1 dose.

* $P$ values correspond with the statistical significance of a meta-regression with vs without study size category. 
Table 19. Vaccine efficacy endpoints derived from phase 2 trials with longest follow-up time

\begin{tabular}{|c|c|c|c|c|}
\hline Analysis & Endpoint & Initial HPV status & Doses & Relative Risk \\
\hline \multicolumn{5}{|c|}{ Monovalent vaccine (Rowhani-Rahbar, 2009): 102 months of follow-up } \\
\hline 3.1 & CIN2+ associated with HPV16 & HPV16- & 3 & 0.00 \\
\hline 3.2 & CIN2+ associated with HPV16 & HPV16- & $>=1$ & 0.00 \\
\hline 3.3 & CIN2+ associated with HPV16 & HPV16- & $1-2$ & 0.00 \\
\hline 4.1 & Incident HPV16 infection & HPV16- & 3 & 0.05 \\
\hline 4.3 & Incident HPV16 infection & HPV16- & $>=1$ & 0.11 \\
\hline 4.3 & Incident HPV16 infection & HPV16- & $1-2$ & 0.25 \\
\hline 5.1 & CIN2+ associated with HPV16 & $\begin{array}{l}\text { regardless of HPV in- } \\
\text { fection }\end{array}$ & $>=1$ & 0.36 \\
\hline 5.3 & CIN2+ irrespective of HPV types & $\begin{array}{l}\text { regardless of HPV in- } \\
\text { fection }\end{array}$ & $>=1$ & 0.64 \\
\hline
\end{tabular}

Bivalent vaccine (De Calvaho, 2012): 88 months of follow-up

\begin{tabular}{lllll}
\hline 2.2 & 6 M persistent HPV16/18 infection & hrHPV & 3 & 0.00 \\
\hline 2.4 & 12 M persistent HPV16/18 infection & hrHPV- & 3 & 0.00 \\
\hline 3.2 & CIN2+ associated with HPV16/18 & HPV16/18- & $>=1$ & 0.00 \\
\hline
\end{tabular}

Quadrivalent vaccine (Villa, 2006): 60 months of follow-up

\begin{tabular}{lllll}
\hline 4.8 & Persistent HPV6/11/16/18 infection & HPV16/18- & 0.07 \\
\hline
\end{tabular}

Table 20. Cross-protective efficacy of the bivalent and quadrivalent vaccine

\begin{tabular}{|c|c|c|c|c|c|}
\hline \multirow[t]{2}{*}{ Trials } & \multirow[t]{2}{*}{ Ref } & \multirow[t]{2}{*}{ Endpoint } & \multicolumn{2}{|l|}{ Relative Risk (95\% CI) } & \multirow{2}{*}{$\begin{array}{l}\text { P value for } \\
\text { difference in } \\
\text { VE }\end{array}$} \\
\hline & & & Bivalent & Quadrivalent & \\
\hline $\begin{array}{l}\text { FUT I/II trials } \\
\text { (ph3,4v) }\end{array}$ & Malagon 2012 & $\begin{array}{l}\text { 6-month persistent HPV31 in- } \\
\text { fection }\end{array}$ & 0.229 (0.156 to 0.228$)$ & $\begin{array}{l}0.538(0.336 \text { to } \\
0.847)\end{array}$ & 0.003 \\
\hline $\begin{array}{l}\text { PATRICIA trial } \\
(\mathrm{ph} 3,2 \mathrm{v})\end{array}$ & & $\begin{array}{l}\text { 6-month persistent HPV45 in- } \\
\text { fection }\end{array}$ & 0.210 (0.106 to 0.387$)$ & $\begin{array}{l}0.922(0.507 \text { to } \\
1.670)\end{array}$ & 0.0003 \\
\hline $\begin{array}{l}\text { Phase2 trial } \\
\text { (ph2,2v) }\end{array}$ & & CIN2+ associated with HPV33 & 0.177 (0.053 to 0.466$)$ & $\begin{array}{l}0.760(0.328 \text { to } \\
1.712)\end{array}$ & 0.02 \\
\hline $\begin{array}{l}\text { Phase2 trial } \\
\text { (ph2,4v) }\end{array}$ & & CIN2+ associated with HPV45 & 0.000 (0.000 to 0.583$)$ & $\begin{array}{l}0.481(0.174 \text { to } \\
1.177)\end{array}$ & 0.04 \\
\hline
\end{tabular}


Table 20. Cross-protective efficacy of the bivalent and quadrivalent vaccine (Continued)

\begin{tabular}{llll} 
CVT (ph3,2v) & $\begin{array}{l}\text { Hildesheim } \\
2014\end{array}$ & $\begin{array}{l}\text { CIN2+ associated with other } \\
\text { hrHPV }\end{array}$ & 0.401 (0.192 to 0.793) \\
\hline $\begin{array}{l}\text { VIVIANE trial } \\
\text { (ph3,2v) }\end{array}$ & Skinner 2014 & $\begin{array}{l}\text { 6-month persistent HPV31 in- } \\
\text { fection }\end{array}$ & $0.209(0.041$ to 0.724) \\
\cline { 2 - 4 } & & $\begin{array}{l}\text { 6-month persistent HPV45 in- } \\
\text { fection }\end{array}$ & 0.221 (0.044 to 0.914)
\end{tabular}

Table 21. Relative risk ratio of adverse effects associated with the bivalent versus the quadrivalent vaccine, adjusted for age group and products administered in the control group

\begin{tabular}{|c|c|c|c|c|}
\hline \multirow[t]{2}{*}{ Adverse effect } & & \multirow{2}{*}{$\begin{array}{l}\text { Relative risk } \\
\text { Quadrivalent vs placebo }\end{array}$} & \multirow{2}{*}{$\begin{array}{l}\text { Relative risk ratio } \\
\text { Bivalent/Quadrivalent }\end{array}$} & \multirow[t]{2}{*}{ p value } \\
\hline & & & & \\
\hline 1 & $\begin{array}{l}\text { Overall adverse effects at in- } \\
\text { jection site }\end{array}$ & 1.19 (0.89 to 1.59$)$ & 1.69 (0.96 to 2.96$)$ & 0.061 \\
\hline 2 & Pain at injection site & $1.20(0.78$ to 1.85$)$ & $1.19(0.67$ to 2.12$)$ & 0.501 \\
\hline 3 & Swelling at injection site & 2.72 (0.77to 9.61) & 0.62 (0.16 to 2.41$)$ & 0.427 \\
\hline 4 & Redness at injection site & 1.46 (1.23 to 1.74$)$ & 1.08 (0.88 to 1.32$)$ & 0.307 \\
\hline 5 & Overall systemic events & 0.99 (0.91 to 1.07$)$ & $1.06(0.95$ to 1.19$)$ & 0.210 \\
\hline 6 & Serious adverse events & 0.94 (0.70 to 1.26$)$ & $1.08(0.80$ to 1.45$)$ & 0.583 \\
\hline 7 & Deaths & $1.18(0.25$ to 5.62$)$ & 0.84 (0.14 to 4.91$)$ & 0.775 \\
\hline
\end{tabular}

Relative risks of the quadrivalent vaccine versus placebo and the relative risk ratios were computed by meta-regression including vaccine, age group and type of product injected in the control group (aluminium adjuvants alone or other vaccine such as Hepatitis A vaccine) as covariate. The relative risk ratio reflects how much more an adverse effect is observed after vaccination with the bivalent versus the quadrivalent vaccine.

\section{APPENDICES}

\section{Appendix 1. List of abbreviations}

AGC: atypical glandular cells

AGUS: atypical glandular cells of undetermined significance

aHR: adjusted hazard raitio

AIS: adenocarcinoma in situ

ANSM: Agence nationale de sécurité du médicament et des produits de santé

ASC: atypical squamous cells (comprises ASC-US and ASC-H)

ASC-H: atypical squamous cells, HSIL cannot be ruled out

ASC-R: atypical squamous cells favouring a benign reactive process squamous cells of undetermined significance

ASC-US: atypical squamous cells of undetermined significance

ASCUS: atypical squamous cells of undetermined significance (comprises ASC-R, ASC-US and ASC-H)

ATP: according to protocol

CDC: Centre for Disease Control

CGCRG: Cochrange Gynaecologocal Cancer Review Group

CGIN: cervical glandular intraepithelial neoplasia 
CHMP: Committee for Medicinal Products for Human Use

$\mathrm{Cl}$ : (95\%) confidence interval

CIN: cervical Intra-epithelial neoplasia

CIS: carcinoma in situ

CISA: Clinical Immunization Safety Assessment

CNAMTS: Caisse nationale de l'assurance maladie des travailleurs salariés

CRPS: complex regional pain syndrome

CVT: Costa Rica Vaccination Trial

DNA: Desoxyribo-nucleic acid

EC: endocervical curettage

ECDC: European Centre for Disease Control

EMEA: European Medicines Agency

EPAR: European Public Assessment Reports

FDA: Food and Drugs Administration

FUTURE: Females United to Unilaterally Reduce Endo/Ectocervical Disease

GACVS: Global Advisory Committee on Vaccine Safety

GBS: Guillain-Baré syndrome

GRADE: Grading of Recommendations Assessment, Development and Evaluation

GSK: GlaxoSmithKline

HC: hybrid capture

HPV: human papillomavirus

HR: hazard ratio

hrHPV: high-risk HPV type

HSIL: high-grade squamous intraepithelial lesion

IARC: International Agency for Research on Cancer

ITT: intention-to-treat

IrHPV: low-risk HPV type

LSIL: low-grade squamous intraepithelial lesion

MCO: managed care organizations

MSD: Merck-Sharp \& Dome

MSM: men who have sex with men

MITT: modified intention-to-treat

NCBI: National Center for Biotechnology Information

$\mathrm{NCl}$ : National Cancer Institute

NNV: number needed to vaccinate

NRT: naive to the relevant HPV type

PATRICIA: PApiloma TRlal against Cancer In young Adults

PCR: polymerase chain reaction

POTS: postural orthostatic tachycardia syndrome

PP: per-protocol

$\mathrm{RCT}$ : randomised controlled trial

RD: risk difference

RR: risk ratio

TBS: The Bethesda System

TVC: total vaccinated cohort

UK: United Kingdom

USA: United States of America

VAERS: Vaccine Adverse Event Reporting System

VE: vaccine efficacy

VLP: virus-like particles

VSD: Vaccine Safety Datalink

WHO: World Health Organization

WSW: women who have sex with women

Appendix 2. Characteristics of prophylactic HPV vaccines 
(Continued)

\begin{tabular}{|c|c|c|c|}
\hline Manufacturer & $\begin{array}{l}\text { Merck, Sharp \& Dome (Merck } \\
\& \text { Co, Whitehouse Station, NJ, } \\
\text { USA) }\end{array}$ & $\begin{array}{l}\text { GlaxoSmithKline (GSK, Rixensart, } \\
\text { Belgium) }\end{array}$ & $\begin{array}{l}\text { Merck, Sharp \& Dome (Merck \& Co, } \\
\text { Whitehouse Station, NJ, USA) }\end{array}$ \\
\hline Antigens & 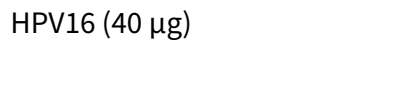 & $\begin{array}{l}\text { L1 VLPs of HPV16 }(20 \mu \mathrm{g}) \text { and } \\
\text { HPV18 }(20 \mu \mathrm{g})\end{array}$ & $\begin{array}{l}\text { L1 VLPs of HPV6 }(20 \mu \mathrm{g}), \text { HPV11 }(40 \\
\mu \mathrm{g}), \text { HPV16 }(40 \mu \mathrm{g}) \text { and HPV18 }(20 \mathrm{mg})\end{array}$ \\
\hline $\begin{array}{l}\text { Vaccination sched- } \\
\text { ule }\end{array}$ & $\begin{array}{l}3 \text { doses: at day } 1 \text {, month } 2 \text { and } \\
\text { month } 6\end{array}$ & $\begin{array}{l}3 \text { doses: at day } 1 \text {, month } 1 \text { and } \\
\text { month } 6\end{array}$ & $\begin{array}{l}3 \text { doses: at day } 1 \text {, month } 2 \text { and month } \\
6\end{array}$ \\
\hline Adjuvant & $\begin{array}{l}225 \mu \mathrm{g} \text { amorphous aluminium } \\
\text { hydroxyl-phosphate sulphate }\end{array}$ & $\begin{array}{l}\text { AS04: } 500 \mu \mathrm{g} \text { aluminium hy- } \\
\text { droxide, } 50 \mu \mathrm{g} \text { 3-deacylated } \\
\text { monophosphoryl lipid A (MPL) }\end{array}$ & $\begin{array}{l}225 \mu \mathrm{g} \text { amorphous aluminium hy- } \\
\text { droxyl-phosphate sulphate }\end{array}$ \\
\hline Trade name & Not commercialised & Cervarix & Gardasil, Silgard \\
\hline $\begin{array}{l}\text { Produced by re- } \\
\text { combinant tech- } \\
\text { nology using }\end{array}$ & $\begin{array}{l}\text { Saccharomyces cerevisae } \\
\text { (baker's yeast) }\end{array}$ & $\begin{array}{l}\text { Baculovirus in Trichoplusia in in- } \\
\text { sect cells }\end{array}$ & $\begin{array}{l}\text { Saccharomyces cerevisae (baker's } \\
\text { yeast) }\end{array}$ \\
\hline
\end{tabular}

Adapted from WHO 2009

VLP: virus-like particles

Nona-valent vaccine (Luxembourg 2015)

\begin{tabular}{ll}
\hline Manufacturer & Merck, Sharp \& Dome (Merck \& Co, Whitehouse Station, NJ, USA) \\
\hline Antigens & L1 VLPs of HPV6 (30 $\mu \mathrm{g})$, HPV11 $(40 \mu \mathrm{g}), \mathrm{HPV} 16(60 \mu \mathrm{g}), \mathrm{HPV} 18(40 \mathrm{mg})$, \\
& HPV31 $(20 \mu \mathrm{g}), \mathrm{HPV} 33(20 \mu \mathrm{g}), \mathrm{HPV} 45(20 \mu \mathrm{g}), \mathrm{HPV} 52(20 \mu \mathrm{g})$ \\
& and HPV58 $(20 \mu \mathrm{g})$. \\
\hline Vaccination schedule & 3 doses: at day 1, month 2 and month 6 \\
\hline Adjuvant & $500 \mu \mathrm{g}$ amorphous aluminium hydroxyl-phosphate sulphate \\
\hline Trade name & Gardasil-9 \\
\hline Produced by recombinant & Saccharomyces cerevisae (baker's yeast) \\
technology using &
\end{tabular}

Adapted from http://www.cdc.gov/mmwr/preview/mmwrhtml/rr6305a1.htm

VLP: virus-like particles

\section{Appendix 3. MEDLINE search strategy}

The following search strategy was used to retrieve references in MEDLINE (Ovid):

1) exp Papillomavirus Infections/

2) exp Papillomaviridae/ 
3) $\mathrm{HPV}^{\star} . \mathrm{mp}$.

4) human papillomavirus* ${ }^{\star} . \mathrm{mp}$.

5) human papilloma virus*.mp.

6) or/1-5

7) exp Papillomavirus Vaccines/

8) gardasil.mp.

9) cervarix.mp.

10) vaccin*.mp.

11) immuni*.mp.

12) or/7-11

13) 6 and 12

14) randomised controlled trial.pt.

15) controlled clinical trial.pt.

16) randomized.ab.

17) placebo.ab.

18) drug therapy.fs.

19) randomly.ab.

20) trial.ab.

21) groups.ab.

22) or/14-21

23) 13 and 22

24) (animals not (humans and animals)).sh.

25) 23 not 24

key:

$\mathrm{mp}=$ title, original title, abstract, name of substance word, subject heading word, unique identifier pt $=$ publication type

$a b=$ abstract

sh = subject heading

\section{Appendix 4. CENTRAL search strategy}

\#1 MeSH descriptor Papillomavirus Infections explode all trees \#2 MeSH descriptor Papillomaviridae explode all trees

\#3 $\left(\mathrm{HPV}^{*}\right)$

\#4 (human papillomavirus*)

\#5 (human papilloma virus*)

\#6 (\#1 OR \#2 OR \#3 OR \#4 OR \#5)

\#7 MeSH descriptor Papillomavirus Vaccines explode all trees

\#8 (gardasil)

\#9 (cervarix)

$\# 10\left(\right.$ vaccin $\left.^{\star}\right)$

$\# 11$ (immuni*)

\#12 (\#7 OR \#8 OR \#9 OR \#10 OR \#11)

\#13 (\#6 AND \#12)

\section{Appendix 5. Embase search strategy}

\section{Embase Ovid}

1 exp papillomavirus infection/

2 exp Papilloma virus/

$3 \mathrm{HPV}^{\star}$.mp.

4 human papillomavirus ${ }^{\star} . \mathrm{mp}$.

5 human papilloma virus*.mp.

6 or/1-5

7 exp Wart virus vaccine/

8 gardasil.mp.

9 cervarix.mp.

10 vaccin $^{\star} . \mathrm{mp}$.

11 immuni*.mp.

12 or/7-11

136 and 12

Prophylactic vaccination against human papillomaviruses to prevent cervical cancer and its precursors (Review) 
14 crossover procedure/

15 double blind procedure/

16 randomized controlled trial/

17 single blind procedure/

18 random*.mp.

19 factorial.mp.

20 crossover $^{\star} . \mathrm{mp}$.

21 cross over $^{\star} . \mathrm{mp}$.

22 cross-over ${ }^{\star} . \mathrm{mp}$

23 placebo*.mp.

24 (doubl $^{\star}$ adj blind $\left.{ }^{\star}\right) . \mathrm{mp}$.

25 (singl $^{\star}$ adj blind $\left.{ }^{\star}\right) . m p$.

26 assign $^{\star} . \mathrm{mp}$.

27 volunteer ${ }^{\star} . \mathrm{mp}$.

28 or/ $14-27$

2913 and 28

key:mp = title, abstract, subject headings, heading word, drug trade name, original title, device manufacturer, drug manufacturer 


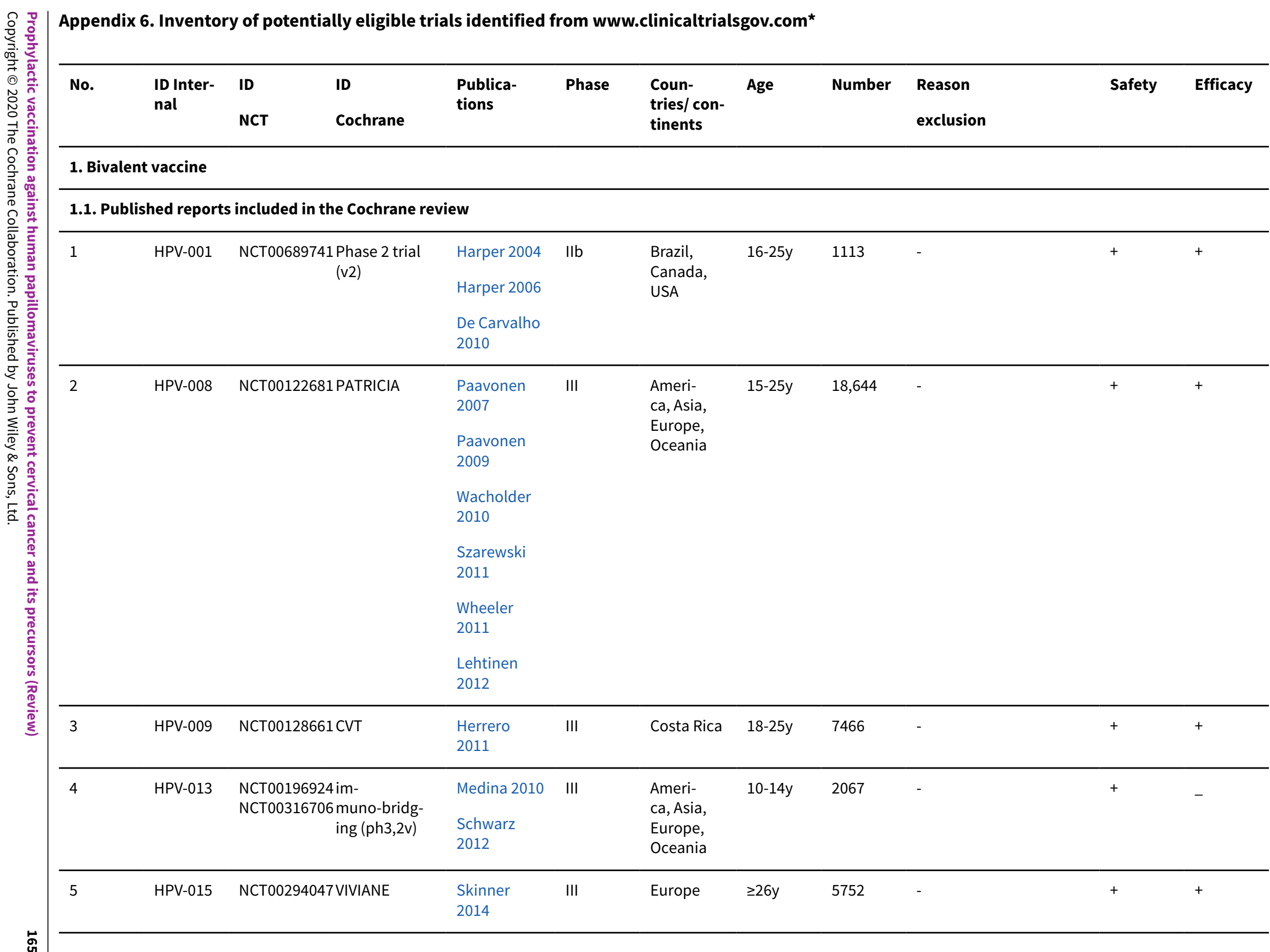




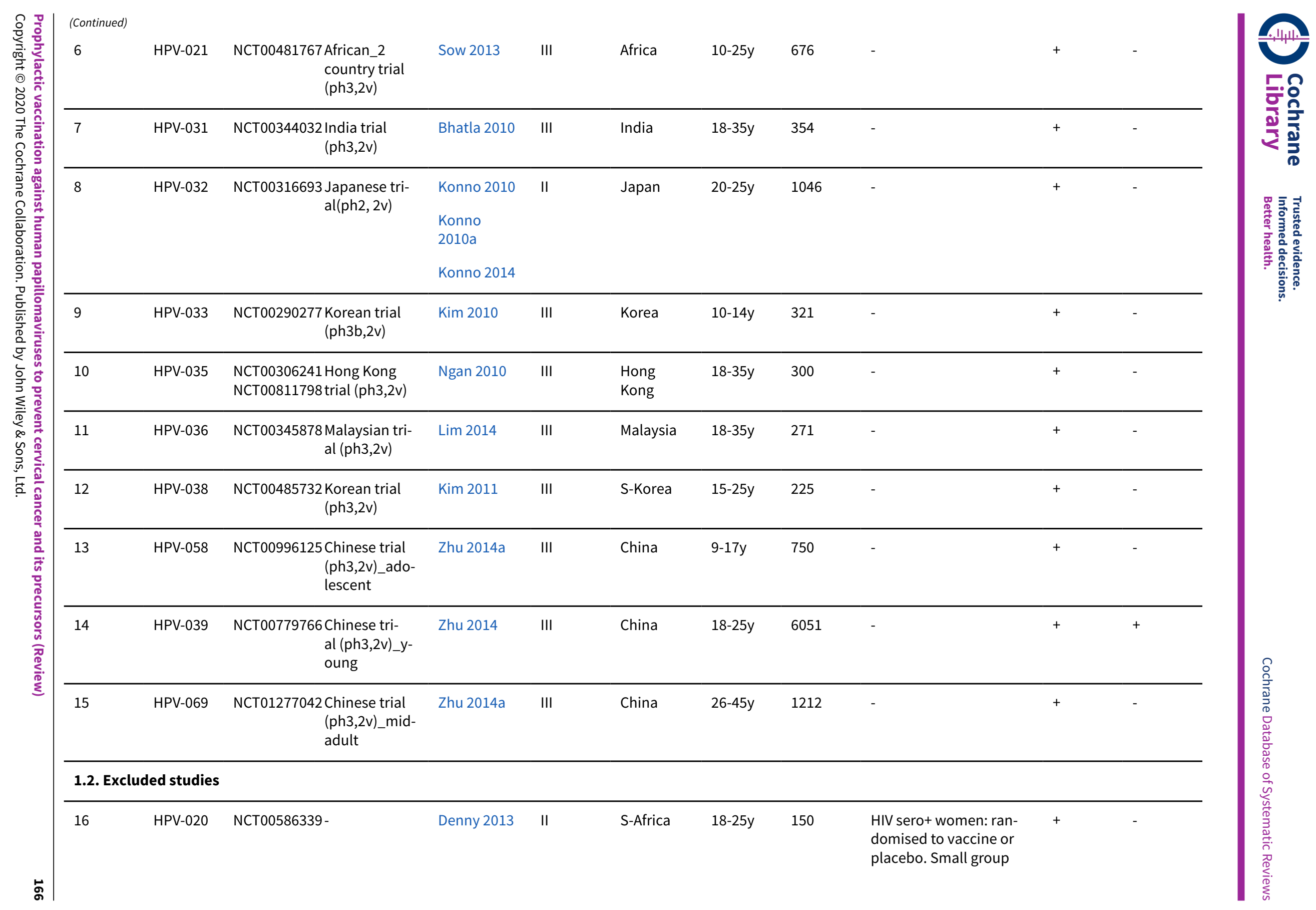




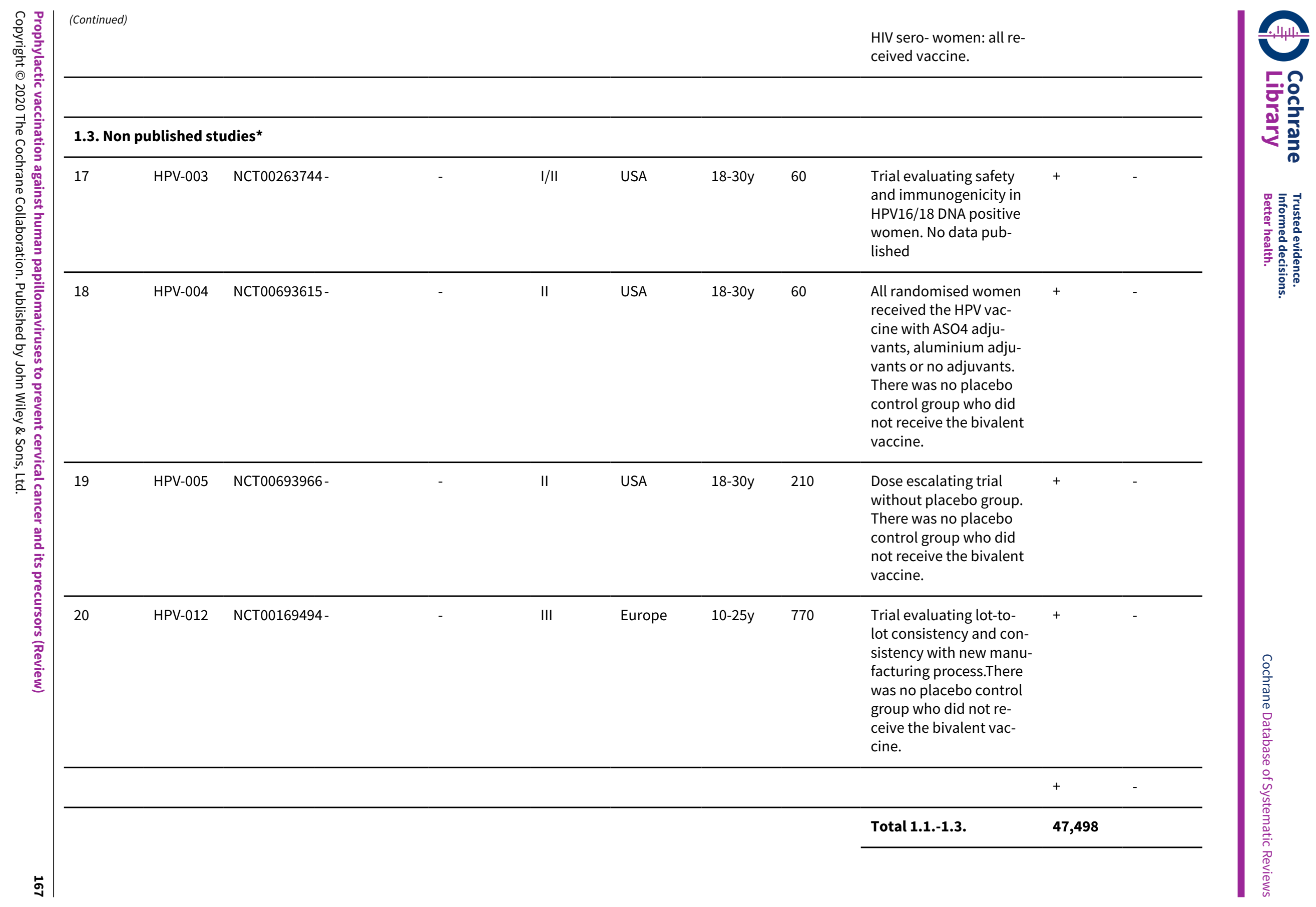




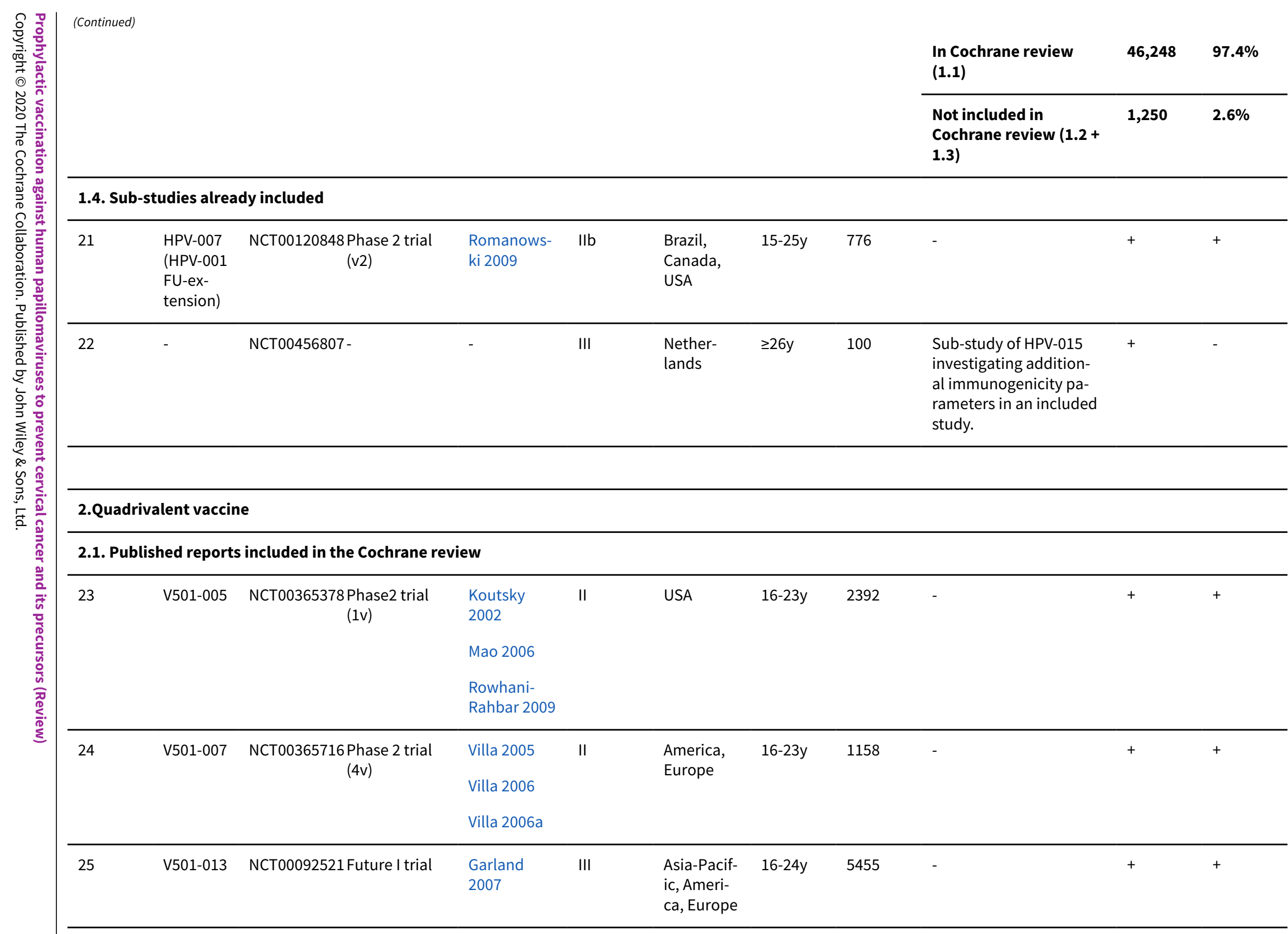




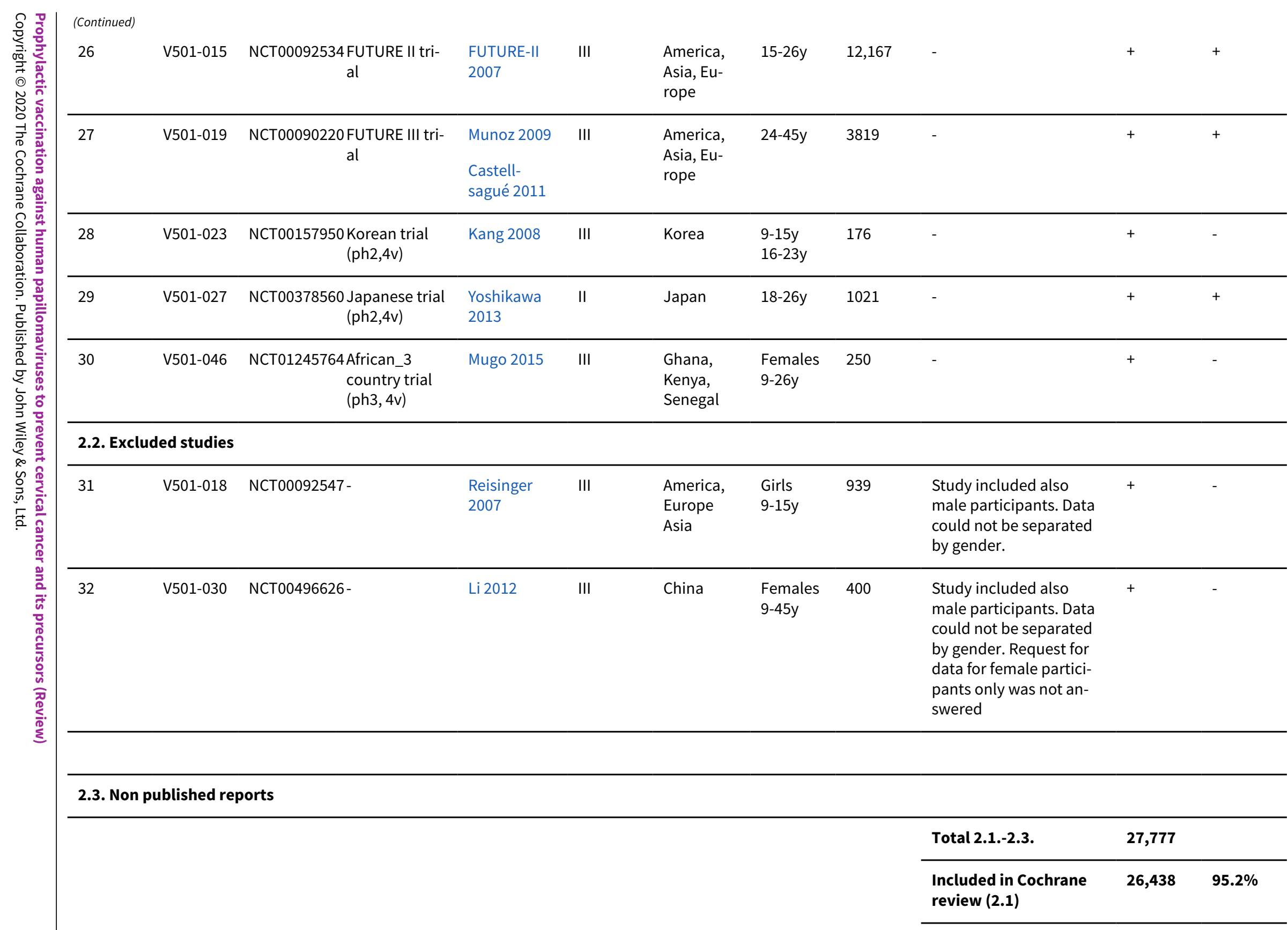




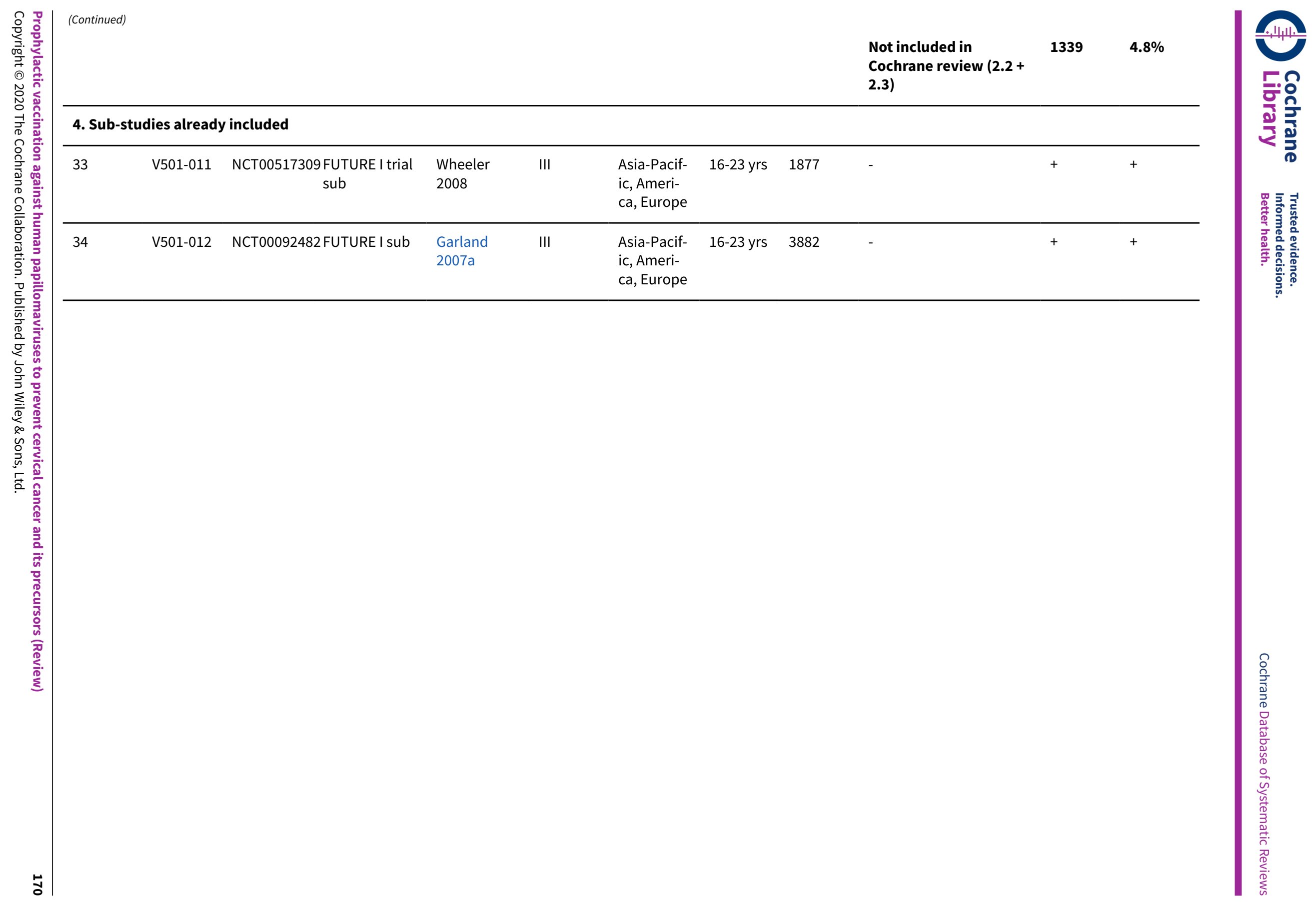


* Inventory of prophylactic HPV vaccination trials identified from https://clinicaltrials.gov/. Only randomised phase II-III trials documenting efficacy and/or safety of prophylactic HPV vaccines in female participants were included. When trials enrolled female and male participants, we tried to extract only data from the female participants.

* Unpublished trials with the bivalent vaccine:

\section{Appendix 7. Other characteristics of included studies I}

\begin{tabular}{|c|c|c|c|c|c|c|}
\hline Study & Location & $\begin{array}{l}\text { Recruit- } \\
\text { ment peri- } \\
\text { od }\end{array}$ & $\begin{array}{l}\text { Valency } \\
\text { of the vac- } \\
\text { cine }\end{array}$ & Placebo & Endpoints & Follow-up schedule \\
\hline $\begin{array}{l}\text { Phase2 trial } \\
\text { (ph2,1v) }\end{array}$ & US & $\begin{array}{l}\text { Oct98- } \\
\text { Nov99 }\end{array}$ & Monovalent & $\begin{array}{l}225 \mu \mathrm{g} \text { amor- } \\
\text { phous alumini- } \\
\text { um hydrox- } \\
\text { yl-phosphate } \\
\text { sulphate }\end{array}$ & $\begin{array}{l}\text { Persistent infection; } \\
\text { CIN 1,2 \& } 3 \text {; } \\
\text { Immunogenicity; Ad- } \\
\text { verse effects }\end{array}$ & $\begin{array}{l}7 \mathrm{M} \text {,every } 6 \mathrm{M} \text { until } 48 \mathrm{M} \text {; cy- } \\
\text { tology \& HPV DNA testing }\end{array}$ \\
\hline $\begin{array}{l}\text { Japan- } \\
\text { ese trial } \\
\text { (ph2,2v) }\end{array}$ & Japan & $\begin{array}{l}\text { Apr06- } \\
\text { Oct06 }\end{array}$ & Bivalent & $\begin{array}{l}\text { Hepatitis A Vac- } \\
\text { cine }\end{array}$ & $\begin{array}{l}\text { Persistent infection } \\
\text { (6 \&12 M); Cytological } \\
\text { abnormality and CIN; } \\
\text { Safety; } \\
\text { Immunogenicity }\end{array}$ & $\begin{array}{l}\text { Cytology and HPV DNA test } \\
\text { at M 0,6,12,18 and } 24 .\end{array}$ \\
\hline
\end{tabular}

\begin{tabular}{|c|c|c|c|c|c|c|}
\hline $\begin{array}{l}\text { Phase2 trial } \\
(\mathrm{ph} 2,2 \mathrm{v})\end{array}$ & $\begin{array}{l}\text { Brazil, } \\
\text { US, Canada }\end{array}$ & $\begin{array}{l}\text { Jul02- } \\
\text { Dec02 }\end{array}$ & Bivalent & $\begin{array}{l}500 \mu \text { g alumini- } \\
\text { um hydroxide }\end{array}$ & $\begin{array}{l}\text { Incident infection and } \\
\text { persistent infection( } 6 \\
\& 12 \mathrm{M}) ; \text { LSIL+, ASCUS } \\
\text { + and HSIL+; CIN1+, } \\
\text { CIN2+; }\end{array}$ & $\begin{array}{l}\text { Brush/spatula smears at } \\
6,12,18 \mathrm{M} \text { by provider; Cervi- } \\
\text { covaginal self-samples at } 0 \\
\& 6 \mathrm{M} \text {; subsequently every } 3 \\
\text { M until } 27 \mathrm{M}\end{array}$ \\
\hline & & & & & Immunogenecity; & \\
\hline & & & & & Safety \& tolerability & \\
\hline
\end{tabular}

\begin{tabular}{llllll}
\hline $\begin{array}{l}\text { African_2 } \\
\text { country tri- } \\
\text { al (ph3,2v) }\end{array}$ & Senegal & $\begin{array}{l}\text { Oct07- } \\
\text { Jul10 }\end{array}$ & Bivalent & $\begin{array}{l}500 \mu \text { galumini- } \\
\text { um hydroxide }\end{array}$ & $\begin{array}{l}\text { Adverse events; Im- } \\
\text { munogenicity }\end{array}$
\end{tabular}

\begin{tabular}{|c|c|c|c|c|c|c|}
\hline $\begin{array}{l}\text { Chinese } \\
\text { trial } \\
\text { (ph3,2v)_y- } \\
\text { oung }\end{array}$ & China & $\begin{array}{l}\text { Oct08- } \\
\text { Apr11 }\end{array}$ & Bivalent & $\begin{array}{l}50 \mu \mathrm{g} \text { MPL and } \\
500 \mu \mathrm{g} \text { alumini- } \\
\text { um hydroxide }\end{array}$ & $\begin{array}{l}\text { Incident infection and } \\
\text { persistent infection (6 } \\
\text { M \&12 M); } \\
\text { Adverse events; Im- } \\
\text { munogenicity }\end{array}$ & / \\
\hline $\begin{array}{l}\text { Chinese tri- } \\
\text { al (ph3,2v)_ } \\
\text { adolescent }\end{array}$ & China & $\begin{array}{l}\text { Oct09- } \\
\text { Nov12 }\end{array}$ & Bivalent & $\begin{array}{l}50 \mu \mathrm{g} M P L \text { and } \\
500 \mu \mathrm{g} \text { alumini- } \\
\text { um hydroxide }\end{array}$ & $\begin{array}{l}\text { Adverse events; Im- } \\
\text { munogenicity }\end{array}$ & / \\
\hline $\begin{array}{l}\text { Chinese } \\
\text { trial } \\
\text { (ph3,2v)_mid- } \\
\text { adult }\end{array}$ & China & $\begin{array}{l}\text { Jan11- } \\
\text { Oct14 }\end{array}$ & Bivalent & $\begin{array}{l}\text { Hepatitis B Vac- } \\
\text { cine }\end{array}$ & $\begin{array}{l}\text { Adverse events; Im- } \\
\text { munogenicity }\end{array}$ & I \\
\hline $\begin{array}{l}\text { Co-vacci- } \\
\text { nation_dT- } \\
\text { pa_IPV trial } \\
\text { (ph3,2v) }\end{array}$ & $\begin{array}{l}\text { France, } \\
\text { Germany } \\
\text { and Spain }\end{array}$ & $\begin{array}{l}\text { Feb07- } \\
\text { Mar08 }\end{array}$ & Bivalent & $\begin{array}{l}\text { Combined } \\
\text { Diphthe- } \\
\text { ria-Tetanus- } \\
\text { Acellular Per- } \\
\text { tussis-inac- }\end{array}$ & $\begin{array}{l}\text { Adverse events; Im- } \\
\text { munogenicity }\end{array}$ & I \\
\hline
\end{tabular}


(Continued)

tivated Po-

liovirus vac-

cine.

\begin{tabular}{|c|c|c|c|c|c|}
\hline $\begin{array}{l}\text { Co-vaccina- } \\
\text { tion_HAB } \\
\text { trial (Ph3, } \\
2 \mathrm{v})\end{array}$ & $\begin{array}{l}\text { Canada, } \\
\text { Denmark, } \\
\text { Hungary } \\
\text { and Swe- } \\
\text { den }\end{array}$ & $\begin{array}{l}\text { Dec07- } \\
\text { Dec08 }\end{array}$ & Bivalent & $\begin{array}{l}\text { GSK combined } \\
\text { hepatitis A and } \\
\text { B vaccine }\end{array}$ & $\begin{array}{l}\text { Adverse events; Im- } \\
\text { munogenicity }\end{array}$ \\
\hline
\end{tabular}

\begin{tabular}{|c|c|c|c|c|c|}
\hline $\begin{array}{l}\text { Co-vaccina- } \\
\text { tion_HepB } \\
\text { trial (ph3, }\end{array}$ & $\begin{array}{l}\text { Nederlands } \\
\text { and Swe- } \\
\text { den }\end{array}$ & $\begin{array}{l}\text { Apr08- } \\
\text { Jan10 }\end{array}$ & Bivalent & $\begin{array}{l}\text { Hepatitis B vac- } \\
\text { cine }\end{array}$ & $\begin{array}{l}\text { Adverse events; Im } \\
\text { munogenicity }\end{array}$ \\
\hline
\end{tabular}

$2 v)$

\begin{tabular}{|c|c|c|c|c|c|c|}
\hline $\begin{array}{l}\text { CVT } \\
\text { (ph3,2v) }\end{array}$ & Costa Rica & $\begin{array}{l}\text { Jun04- } \\
\text { Dec05 }\end{array}$ & Bivalent & $\begin{array}{l}\text { Hepatitis A Vac- } \\
\text { cine }\end{array}$ & $\begin{array}{l}\text { HPV16/18 persistent } \\
\text { infection ( } 6 \text { \&12 M); } \\
\text { Cross-protection; Ad- } \\
\text { verse events }\end{array}$ & $\begin{array}{l}\text { Cytology examinations } \\
\text { every } 12 \mathrm{M} \text {; } \\
\text { If LSIL or HPV+ASCUS, then } \\
\text { check for every } 6 \mathrm{M} \text {. }\end{array}$ \\
\hline
\end{tabular}

\begin{tabular}{llllll}
\hline $\begin{array}{l}\text { Hong } \\
\text { Kong trial } \\
(\mathrm{ph} 3,2 \mathrm{v})\end{array}$ & Hong Kong & $\begin{array}{l}\text { Mar06- } \\
\text { Jun07 }\end{array}$ & Bivalent & $\begin{array}{l}500 \mu \mathrm{g} \text { alumini- } \\
\text { um hydroxide }\end{array}$ & $\begin{array}{l}\text { Adverse events; Im- } \\
\text { munogenicity }\end{array}$
\end{tabular}

\begin{tabular}{|c|c|c|c|c|c|c|}
\hline $\begin{array}{l}\text { Immuno- } \\
\text { bridg- } \\
\text { ing(ph3,2v) }\end{array}$ & $\begin{array}{l}\text { Australia, } \\
\text { Colombia, } \\
\text { the Czech } \\
\text { Republic, } \\
\text { France, } \\
\text { Germany, } \\
\text { Honduras, } \\
\text { Korea, Nor- } \\
\text { way, Pana- } \\
\text { ma, Spain, } \\
\text { Sweden } \\
\text { and Taiwan }\end{array}$ & $\begin{array}{l}\text { Jun04- } \\
\text { Aug05 }\end{array}$ & Bivalent & $\begin{array}{l}\text { Hepatitis A Vac- } \\
\text { cine }\end{array}$ & $\begin{array}{l}\text { Adverse events; Im- } \\
\text { munogenicity }\end{array}$ & I \\
\hline $\begin{array}{l}\text { Indian trial } \\
(\mathrm{ph} 3,2 \mathrm{v})\end{array}$ & India & $\begin{array}{l}\text { Jul06- } \\
\text { Mar07 }\end{array}$ & Bivalent & $\begin{array}{l}500 \mu \mathrm{g} \text { alumini- } \\
\text { um hydroxide }\end{array}$ & $\begin{array}{l}\text { Adverse events; Im- } \\
\text { munogenicity }\end{array}$ & I \\
\hline $\begin{array}{l}\text { Korean trial } \\
\text { (ph3,2v) }\end{array}$ & Korea & $\begin{array}{l}\text { Nov05- } \\
\text { Aug06 }\end{array}$ & Bivalent & $\begin{array}{l}\text { Hepatitis A vac- } \\
\text { cine }\end{array}$ & $\begin{array}{l}\text { Adverse events; Im- } \\
\text { munogenicity }\end{array}$ & I \\
\hline $\begin{array}{l}\text { Korean trial } \\
\text { (ph3b,2v) }\end{array}$ & Korea & $\begin{array}{l}\text { Jun07- } \\
\text { Mar08 }\end{array}$ & Bivalent & $\begin{array}{l}500 \mu \mathrm{g} \text { alumini- } \\
\text { um hydroxide }\end{array}$ & $\begin{array}{l}\text { Adverse events; Im- } \\
\text { munogenicity }\end{array}$ & I \\
\hline $\begin{array}{l}\text { Malaysian } \\
\text { trial } \\
(\mathrm{ph} 3,2 \mathrm{v})\end{array}$ & Malaysia & $\begin{array}{l}\text { Sep06- } \\
\text { Dec07 }\end{array}$ & Bivalent & $\begin{array}{l}500 \mu \text { g alumini- } \\
\text { um hydroxide }\end{array}$ & $\begin{array}{l}\text { Adverse effects, } \\
\text { immunogenicity }\end{array}$ & I \\
\hline $\begin{array}{l}\text { PATRICIA } \\
\text { trial } \\
\text { (ph3,2v) }\end{array}$ & $\begin{array}{l}15 \text { coun- } \\
\text { tries in all } \\
\text { continents } \\
\text { but Africa }\end{array}$ & $\begin{array}{l}\text { May04- } \\
\text { Jun05 }\end{array}$ & Bivalent & $\begin{array}{l}\text { Hepatitis A Vac- } \\
\text { cine }\end{array}$ & $\begin{array}{l}\text { CIN2+; Persistent in- } \\
\text { fection ( } 6 \text { \&12 M); } \\
\text { CIN1+; } \\
\text { Immunogenecity; } \\
\text { Adverse events }\end{array}$ & $\begin{array}{l}\text { Cervical samples for HPV } \\
\text { genotyping, every } 6 \mathrm{M} \text {. Gy- } \\
\text { naecological and cytology } \\
\text { examinations every } 12 \mathrm{M} \text {. }\end{array}$ \\
\hline
\end{tabular}


(Continued)

\begin{tabular}{|c|c|c|c|c|c|}
\hline $\begin{array}{l}\text { VIVIANE tri- } \\
\text { al (ph3,2v) }\end{array}$ & $\begin{array}{l}12 \text { coun- } \\
\text { tries in all } \\
\text { continents } \\
\text { but Africa }\end{array}$ & $\begin{array}{l}\text { Feb06- } \\
\text { Dec10 }\end{array}$ & Bivalent & $\begin{array}{l}500 \mu \mathrm{g} \text { alumini- } \\
\text { um hydroxide }\end{array}$ & $\begin{array}{l}\text { Combined endpoints } \\
\text { of persistent infec- } \\
\text { tion ( } 6 \mathrm{M}) \text { or CIN1+; } \\
\text { Immunogenicity; Ad- } \\
\text { verse events }\end{array}$ \\
\hline
\end{tabular}

\begin{tabular}{|c|c|c|c|c|c|}
\hline $\begin{array}{l}\text { Japan- } \\
\text { ese trial } \\
\text { (ph2,4v) }\end{array}$ & Japan & $\begin{array}{l}\text { Not men- } \\
\text { tioned }\end{array}$ & $\begin{array}{l}\text { Quadriva- } \\
\text { lent }\end{array}$ & $\begin{array}{l}225 \mu g \text { amor- } \\
\text { phous alumini- } \\
\text { um hydrox- } \\
\text { yl-phosphate } \\
\text { sulphate }\end{array}$ & $\begin{array}{l}\text { Composite primary } \\
\text { endpoint of persistent } \\
\text { infection; cervical and } \\
\text { external genital dis- } \\
\text { ease }\end{array}$ \\
\hline
\end{tabular}

Cytology sample for HPV DNA testing every $6 \mathrm{M}$ and Pap smear every $12 \mathrm{M}$; If ASC-US+, then refer to colposcopy immediately.

Gynecological examination was done at day 1 and at months 7,12,18,24 and 30 . A ThinPrep Pap test and external genital and cervical swabs for PCR analysis of HPV were obtained from all participants at day 1 and at months 7,12,18,24 and 30 . Biopsy samples of external genital lesions identified during the study were taken and serum samples were obtained at day 1 and months 2,3,7,18 and 30 .

\begin{tabular}{llllll}
\hline $\begin{array}{l}\text { Korean trial } \\
(\mathrm{ph} 2,4 \mathrm{v})\end{array}$ & $\begin{array}{l}\text { South Ko- } \\
\text { rea }\end{array}$ & $\begin{array}{l}\text { Oct05- } \\
\text { May06 }\end{array}$ & $\begin{array}{l}\text { Quadriva- } \\
\text { lent }\end{array}$ & $\begin{array}{l}225 \mu \text { alumini- } \\
\text { um adjuvant of } \\
\text { safety compar- } \\
\text { isons } 0.5 \mathrm{~mL}\end{array}$ & $\begin{array}{l}\text { Adverse events; Im- } \\
\text { munogenicity }\end{array}$ \\
\end{tabular}

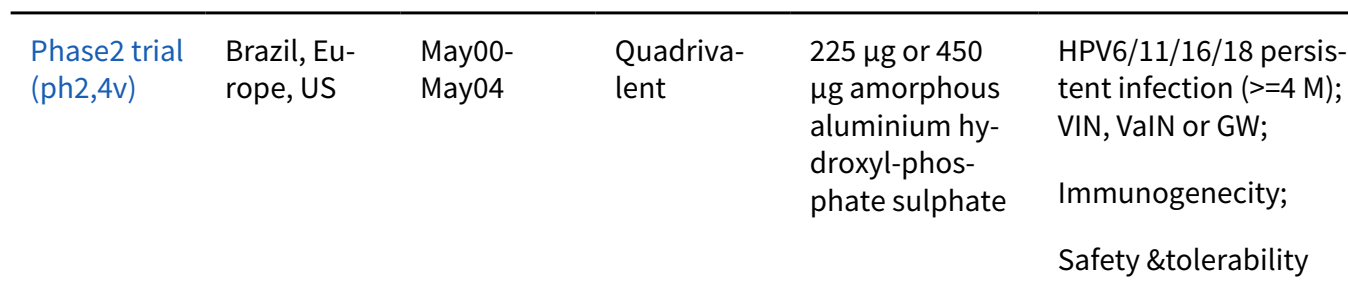

- Gynaecological examination at $0,7,12 \mathrm{M}$, subsequently every $6 \mathrm{M}$ until 36 M: - ThinPrep smear; swabs: cervix, vaginal, external genital for HPV PCR

- Biopsies from external genital lesions

- Serum at

$0,2,3,7,12,18,24,36 \mathrm{M}$

\begin{tabular}{|c|c|c|c|c|c|c|}
\hline $\begin{array}{l}\text { African_3 } \\
\text { country tri- } \\
\text { al (ph3,4v) }\end{array}$ & $\begin{array}{l}\text { Ghana, } \\
\text { Kenya, and } \\
\text { Senegal }\end{array}$ & I & $\begin{array}{l}\text { Quadriva- } \\
\text { lent }\end{array}$ & $\begin{array}{l}225 \mu g \text { amor- } \\
\text { phous alumini- } \\
\text { um hydrox- } \\
\text { yl-phosphate } \\
\text { sulphate }\end{array}$ & $\begin{array}{l}\text { Adverse events; Im- } \\
\text { munogenicity }\end{array}$ & I \\
\hline $\begin{array}{l}\text { FUTURE } \\
\text { I trial } \\
(\mathrm{ph} 3,4 \mathrm{v})\end{array}$ & $\begin{array}{l}16 \text { coun- } \\
\text { tries in } \\
\text { Asia-Pacif- } \\
\text { ic, North } \\
\text { America, } \\
\text { Latin Amer- } \\
\text { ica and Eu- } \\
\text { rope }\end{array}$ & $\begin{array}{l}\text { Dec01- } \\
\text { Aug08 }\end{array}$ & $\begin{array}{l}\text { Quadriva- } \\
\text { lent }\end{array}$ & $\begin{array}{l}225 \mu \mathrm{g} \text { amor- } \\
\text { phous alumini- } \\
\text { um hydrox- } \\
\text { yl-phosphate } \\
\text { sulphate }\end{array}$ & $\begin{array}{l}\text { CIN of any grade, AIS } \\
\text { or Cervical Cancer; } \\
\text { Incidence of GW, VIN } \\
\text { and VaIN; Adverse } \\
\text { events }\end{array}$ & $\begin{array}{l}\text { Gynecologic examination at } \\
\text { day } 1, M \text { M } 72,24,36 \text { and } 48 \text {; } \\
\text { Comprehensive anogenital } \\
\text { examination at day } 1, M 3, M \\
7,12,18,24,30,36 \text { and } 48 ; \\
\text { Day } 1, \text { M 7,12,18, 24, 30, 36, } \\
48 \text { ThinPrep Cytology; }\end{array}$ \\
\hline $\begin{array}{l}\text { FUTURE } \\
\text { II trial } \\
\text { (ph3,4v) }\end{array}$ & $\begin{array}{l}13 \text { coun- } \\
\text { tries in } \\
\text { Asia-Pacif- } \\
\text { ic, North } \\
\text { America, }\end{array}$ & $\begin{array}{l}\text { Jun02- } \\
\text { May03 }\end{array}$ & $\begin{array}{l}\text { Quadriva- } \\
\text { lent }\end{array}$ & $\begin{array}{l}225 \mu \mathrm{g} \text { amor- } \\
\text { phous alumini- } \\
\text { um hydrox- } \\
\text { yl-phosphate } \\
\text { sulphate }\end{array}$ & $\begin{array}{l}\text { CIN2, CIN3, AIS and } \\
\text { cervical cancer; Ad- } \\
\text { verse events }\end{array}$ & $\begin{array}{l}\text { Gynecologic examination, } \\
\text { comprehensive anogenital } \\
\text { examination and cytology } \\
\text { at day } 1 \text {, follow up at } M 7 \text {, } \\
12,24,36 \text { and } 48\end{array}$ \\
\hline
\end{tabular}


(Continued)

Latin Amer-

ica and Eu-

rope

\begin{tabular}{|c|c|c|c|c|c|c|}
\hline $\begin{array}{l}\text { FUTURE } \\
\text { III trial } \\
\text { (ph3,4v) }\end{array}$ & $\begin{array}{l}7 \text { countries } \\
\text { in all conti- } \\
\text { nents but } \\
\text { Africa }\end{array}$ & $\begin{array}{l}\text { Jun04- } \\
\text { Apr05 }\end{array}$ & $\begin{array}{l}\text { Quadriva- } \\
\text { lent }\end{array}$ & $\begin{array}{l}225 \mu \mathrm{g} \text { amor- } \\
\text { phous alumini- } \\
\text { um hydrox- } \\
\text { yl-phosphate } \\
\text { sulphate }\end{array}$ & $\begin{array}{l}\text { CIN1-3,VIN1-3, } \\
\text { ValN1-3,AIS, cervical, } \\
\text { vaginal and vulvar } \\
\text { cancer; Persistent in- } \\
\text { fection ( } 6 \text { M); Genital } \\
\text { wart }\end{array}$ & $\begin{array}{l}\text { Pelvic examination, inspec- } \\
\text { tion with loupe, labial, vul- } \\
\text { val, perineal, perianal,endo } \\
\& \text { ectocervical swabs at M } \\
0,7,12,18,24,30,36,42,48 \text {. }\end{array}$ \\
\hline
\end{tabular}

AIS: adenocarcinoma in situ;ASCUS: atypical squamous cells of undetermined significance; CIN: cervical intraepithelial neoplasia;GW: genital wart; HSIL: high-grade squamous intraepithelial lesion; LSIL: low-grade squamous intraepithelial lesion; M: month;PCR: polymerase chain reaction; VAIN: vaginal intra-epithelial neoplasia;VIN: vaginal intraepithelial neoplasia

\section{Appendix 8. Other characteristics of included studies II}

\begin{tabular}{lllll}
\hline Study & $\begin{array}{l}\text { HPV DNA detec- } \\
\text { tion methods }\end{array}$ & $\begin{array}{l}\text { HPV serology } \\
\text { method }\end{array}$ & Definition of per-protocol population & $\begin{array}{l}\text { Definition of inten- } \\
\text { tion-to- treat popula- } \\
\text { tion }\end{array}$
\end{tabular}

Phase2 trial PCR targeting L1, Competitive ra- Women who had 3 doses.

(ph2,1v) E6 and E7 genes dioimmunoas-
of HPV16. $\quad$ say (CLIA) to detect HPV16 antibodies (Merck Research Laboratories). Cutoff for sero+ 5.9 $\mathrm{mMU} / \mathrm{mL}$ At enrolment also an ELISA test was used. At $M$ $7,12,18,30,42$
Seronegative for HPV-16 and negative for HPV-16 DNA at day 0, and HPV-16 DNA negative at $M 7$. No sexual intercourse within 48 hours before day 0 and $M 7$ visit. No other non-study vaccine, no other drugs or involved in other studies.
Efficacy analysis including women with general protocol violations: had 3 doses, seronegative for HPV16 and negative for HPV16 DNA on day 0 and negative for HPV16 DNA at $\mathrm{M} 7$ and in any biopsy specimens obtained between day 0 and $M 7$.

\begin{tabular}{|c|c|c|c|c|}
\hline $\begin{array}{l}\text { Japanese trial } \\
(\mathrm{ph} 2,2 \mathrm{v})\end{array}$ & $\begin{array}{l}\text { SPF10 PCR (HPV } \\
\text { LiPA-version } 1 \text { ), } \\
\text { to identify } 14 \\
\text { hrHPV types (16, } \\
18,31,33,35, \\
39,45,51,52,56, \\
58,59,66,68) \text {. } \\
\text { If sample nega- } \\
\text { tive for HPV16 or } \\
18, \text { type-specif- } \\
\text { ic PCR for HPV16 } \\
\text { or } 18 \text { was per- } \\
\text { formed. }\end{array}$ & I & $\begin{array}{l}\text { Meet eligibility criteria, complied with pro- } \\
\text { tocol procedures, received } 3 \text { doses and } \\
\text { were DNA negative of corresponding HPV } \\
\text { types at M } 0 \text { and } 6 \text {, had efficacy endpoints } \\
\text { measures available, had no or low-grade } \\
\text { cytological abnormality at M0, and were } \\
\text { seronegative for the corresponding HPV } \\
\text { type at M } 0 \text {. }\end{array}$ & I \\
\hline $\begin{array}{l}\text { Phase2 trial } \\
\text { (ph2,2v) }\end{array}$ & $\begin{array}{l}\text { PCR SPF10 } \\
\text { primers } \\
\text { Typing with } \\
\text { DNA immunoen- } \\
\text { zyme assay (LiPA }\end{array}$ & $\begin{array}{l}\text { ELISA test using } \\
\text { HPV16 \& HPV18 } \\
\text { VLP as antigen. }\end{array}$ & $\begin{array}{l}\text { Women who have received the } 3 \text { scheduled } \\
\text { doses and complied with the protocol and } \\
\text { were not excluded. } \\
\text { - evaluation of safety: } 540 \text { versus } 541\end{array}$ & $\begin{array}{l}\text { Women who had re- } \\
\text { ceived at least one dose } \\
\text { of study vaccine or place- } \\
\text { bo in the initial efficacy } \\
\text { study, and who had any } \\
\text { data available for out- } \\
\text { come measurement in }\end{array}$ \\
\hline
\end{tabular}


(Continued)

[Innogenetics, Gent]

If LiPA+: type specific PCR: HPV16 (E6/7), HPV18 (L1)
- evaluation of efficacy: 366 versus 355 (initially seropositive, HPV DNA positive \& cytologically positive women are excluded)

- evaluation of immunogenicity: 384 vs 344: women with serology results at months 0,7 and 18 , who received all 3 doses, and did not become positive for HPV16/18 DNA during administration period.

$\begin{array}{ll}\text { African_2 coun- / } \quad \text { ELISA } & \text { ATP for immunogenicity, which } \\ \text { try trial (ph3,2v) } & \text { included evaluable participants meeting } \\ & \text { all eligibility criteria, } \\ & \text { complying with the procedures and inter- } \\ & \text { vals defined in the } \\ & \text { protocol (including receipt of the sched- } \\ & \text { uled number of doses), } \\ & \text { with no elimination criteria during the tri- } \\ & \text { al, for whom immunogenicity } \\ \text { data were available. }\end{array}$

Chinese trial PCR SPF10- ELISA ATP-E included women who were seroneg(ph3,2v)_young DEIA-LiPA25 version 1 test for HPV16,18, 31, 33, $35,39,45,51,52$, $56,58,59,66$ and 68. ative at $\mathrm{M} 0$ and DNA negative at $\mathrm{M} 0$ and 6 , received all 3 doses and had normal or low-grade cytology at baseline. the extended follow-up phase. For efficacy study, women who were HPV DNA negative for the specific HPV type at month 0 in the initial study also included.
TVC included all participants with at least one vaccine/placebo dose administration documented.

TVC-E included all vaccinated women for whom efficacy data were available and who had normal or low-grade cytology at baseline. Included women were seronegative at $\mathrm{M} 0$ and $\mathrm{HPV}$ negative at $\mathrm{M} 0$ and 6 .

$\begin{array}{lll}\begin{array}{l}\text { Chinese trial } \\ (\mathrm{ph} 3,2 \mathrm{v})_{-} \text {adoles- }\end{array} & \text { ELISA } & \begin{array}{l}\text { ATP for immunogenicity included women } \\ \text { who met eligibility criteria and were } \\ \text { cent }\end{array} \\ & \text { seronegative at M } 0 .\end{array}$

TVC included all participants with at least one vaccine/placebo dose administration documented.

\begin{tabular}{|c|c|c|c|}
\hline $\begin{array}{l}\text { Chinese trial } \\
\text { (ph3,2v)_mid- } \\
\text { adult }\end{array}$ & I & ELISA & $\begin{array}{l}\text { ATP for immunogenicity included women } \\
\text { who met eligibility criteria and were } \\
\text { seronegative at M } 0 \text {. }\end{array}$ \\
\hline
\end{tabular}

TVC included all participants with at least one vaccine/placebo dose administration documented.

\begin{tabular}{|c|c|c|c|}
\hline $\begin{array}{l}\text { Co-vaccina- } \\
\text { tion_dTpa_IPV } \\
\text { trial (ph3,2v) }\end{array}$ & I & ELISA & $\begin{array}{l}\text { ATP for immunogenicity included women } \\
\text { who met eligibility criteria and were } \\
\text { seronegative at } \mathrm{M} 0 \text {. }\end{array}$ \\
\hline
\end{tabular}

TVC included all participants with at least one vaccine/placebo dose administration documented.

\begin{tabular}{|c|c|c|c|}
\hline $\begin{array}{l}\text { Co-vaccina- } \\
\text { tion_HAB trial } \\
(\mathrm{Ph} 3,2 \mathrm{v})\end{array}$ & I & ELISA & $\begin{array}{l}\text { ATP for immunogenicity included women } \\
\text { who met eligibility criteria and were } \\
\text { seronegative at } \mathrm{M} 0 \text {. }\end{array}$ \\
\hline
\end{tabular}

TVC included all participants with at least one vaccine/placebo dose administration documented.

\begin{tabular}{|c|c|c|c|c|}
\hline $\begin{array}{l}\text { Co-vaccina- } \\
\text { tion_HepB trial } \\
(\mathrm{ph} 3,2 \mathrm{v})\end{array}$ & I & ELISA & $\begin{array}{l}\text { ATP for immunogenicity included women } \\
\text { who met eligibility criteria and were } \\
\text { seronegative at M } 0 \text {. }\end{array}$ & $\begin{array}{l}\text { TVC included all partic- } \\
\text { ipants with at least one } \\
\text { vaccine/placebo dose }\end{array}$ \\
\hline
\end{tabular}




\begin{tabular}{llll}
\hline CVT (ph3,2v) & $\begin{array}{l}\text { Broad-spectrum } \\
\text { PCR-based HPV }\end{array}$ & $\begin{array}{l}\text { ELISA used for } \\
\text { the detection }\end{array}$ & $\begin{array}{l}\text { ATP: no protocol violations, received all } 3 \\
\text { doses within protocol-defined period, had }\end{array}$ \\
& $\begin{array}{ll}\text { DNA test, use } \\
\text { SPF10 (HPV-Li- }\end{array}$ & $\begin{array}{l}\text { and quantifica- } \\
\text { tion of IgG anti- }\end{array}$ & $\begin{array}{l}\text { no biopsy/treatment before the 6-month } \\
\text { visit, and were HPV DNA-negative by PCR }\end{array}$ \\
& $\begin{array}{ll}\text { PA-version 1) to } \\
\text { ensure HPV16 }\end{array}$ & $\begin{array}{l}\text { bodies against } \\
\text { for the corresponding HPV type at enrol- }\end{array}$ & $\begin{array}{l}\text { ment and the 6-month visit. } \\
\text { and HPV18 infec- }\end{array}$ \\
& separately. &
\end{tabular}

ITT: All randomised women, regardless of compliance or enrolment infection.

\begin{tabular}{|c|c|c|c|}
\hline $\begin{array}{l}\text { Hong Kong trial } \\
\text { (ph3,2v) }\end{array}$ & I & $\begin{array}{l}\text { ELISA with cut- } \\
\text { off } 8 \text { EL.U/mL } \\
\text { for HPV16 and } 7 \\
\text { EL.U/mL for HPV } \\
18 .\end{array}$ & $\begin{array}{l}\text { ATP included participants who met eligibil- } \\
\text { ity criteria, complied with protocol-defined } \\
\text { procedures, and for whom post-vaccina- } \\
\text { tion assay results were available for anti- } \\
\text { bodies against at least one study vaccine } \\
\text { antigen. }\end{array}$ \\
\hline
\end{tabular}

\begin{tabular}{lll}
\hline Immunobridg- / ELISA \\
ing $(\mathrm{ph} 3,2 \mathrm{v})$
\end{tabular}

ATP:included participants who met all eligibility criteria, complied with study procedures, and had data available for antibodies against at least 1 antigen component of the bivalent vaccine.
TVC included participants who received at least one dose of the vaccine.

All participants who completed the study without considering protocol violation.

\begin{tabular}{ll}
\hline $\begin{array}{l}\text { Indian trial } \\
(\mathrm{ph} 3,2 \mathrm{v})\end{array}$ & ELISA with cut- \\
& off $8 \mathrm{EL} . \mathrm{U} / \mathrm{mL}$ \\
& for HPV 16 and 7 \\
& EL.U/mL for HPV \\
& 18.
\end{tabular}

ELISA

$\begin{array}{lll}\begin{array}{l}\text { Korean trial } \\ (p h 3,2 v)\end{array} & \text { ELISA }\end{array}$

ATP included all subjects meeting eligibility criteria, complying with the procedures defined in the protocol and for whom assay results were available fro antibodies against at least one study vaccine antigen component after vaccination.

ATP cohort including all participants meeting eligibility criteria, complying with the procedures defined in the protocol, and for whom assay results were available for antibodies against at least one study antigen component after vaccination.

$\begin{array}{ll}\begin{array}{l}\text { Korean trial } \\ (p h 3 b, 2 \mathrm{v})\end{array} & / \\ \end{array}$

ELISA

(ph3b,2v)

\section{ATP: included all eligible participants (those meeting all eligibility criteria, com- plying with protocol defined procedures,}

TVC included all subjects with at least one vaccine/placebo dose administration documented. without elimination criteria during the study) for whom immunogenicity data were available.

\begin{tabular}{ll}
\hline Malaysian trial $\quad /$ & ELISA with cut- \\
$(\mathrm{ph} 3,2 \mathrm{v})$ & off 8 EL.U/mL \\
& for HPV16 and \\
& $7 \mathrm{EL} . \mathrm{U} / \mathrm{mL}$ for \\
& $\mathrm{HPV} 18$.
\end{tabular}

ATP: all evaluable participants (those meeting all eligibility criteria, complying with protocol defined procedures, without elimination code during the study) for whom immunogenicity data were available.
TVC included all participants with at least one vaccine/placebo dose administration documented.
With at least one dose of vaccine administrated

\begin{tabular}{|c|c|c|c|c|}
\hline $\begin{array}{l}\text { PATRICIA trial } \\
\text { (ph3,2v) }\end{array}$ & $\begin{array}{l}\text { SPF10 PCR (HPV } \\
\text { LiPA-version } \\
\text { 1), to identify } \\
14 \text { hrHPV types } \\
(16,18,31,33,35, \\
39,45,51,52,56,\end{array}$ & $\begin{array}{l}\text { Serology } \\
\text { HPV16/18 (ELISA) } \\
\text { at M 0,7, } 24 \text { for } \\
\text { all and at M } 6,12 \text {, } \\
36,48 \text { at selected } \\
\text { sites. }\end{array}$ & $\begin{array}{l}\text { ATP-Efficacy: no protocol violations, re- } \\
\text { ceived } 3 \text { doses, NILM, ASC-US or LSIL } \\
\text { at baseline, evaluable for efficacy, case } \\
\text { counting after the } 3^{\text {rd }} \text { dose; }\end{array}$ & $\begin{array}{l}\text { TVC: at least } 1 \text { dose re- } \\
\text { ceived, baseline HPV/cy- } \\
\text { to exam and at least } 1 \mathrm{FU} \\
\text { examination (all HPV/cy- } \\
\text { to+ at baseline included). }\end{array}$ \\
\hline
\end{tabular}


(Continued)

$58,59,66,68)$. If multiple infection, causality was attributed to the type also present in a previous cervical sample.
ATP-Immunogenicity cohort: no protocol violations, received 3 doses, included in sites for study of immunogenicity.
TVC-E: idem but HSIL and unknown cyto at baseline excluded.

TVC-N: idem as TVC, but baseline NILM, hrHPV DNA- (14 types, M 0 \& $M 7$ ?) and sero- for HPV16/18 (M 0).

$\begin{array}{ll}\begin{array}{l}\text { VIVIANE trial } \\ \text { (ph3,2v) }\end{array} & \text { Broad-spec- } \\ & \text { trum PCR SPF10- } \\ & \text { DEIA-LiPA test } \\ & \text { for HPV16, } \\ & 18,31,33,35,39 \\ & 45,51,52,56,58, \\ & 59,66 \text { and } 68 / 73 . \\ & \text { Oncogenic HPV- } \\ & \text { positive women } \\ & \text { were tested by } \\ & \text { multiplex type- } \\ & \text { specific PCR and } \\ & \text { reverse hybridis- } \\ & \text { ation assay (MP- } \\ & \text { TS12) to detect } \\ & \text { HPV16,18, 31,33, } \\ & 35,45,52,58, \text { and } \\ & 59 .\end{array}$

ELISA used to de- ATP-E: no protocol violations, received all 3 tect antibody response against HPV16 and 18 at $6 \mathrm{M}$ intervals up to $24 \mathrm{M}$ and at $12 \mathrm{M}$ intervals thereafter. doses, data for efficacy endpoints available (baseline PCR or cytology sample and one further sample available); negative or lowgrade cytology at M 0 , no history of HPV disease; counting of events after 3 rd dose.
TVC: at least 1 dose; data available for efficacy endpoints; HSIL excluded; include participants of women with history of HPV disease (15\%); case counting after first dose; Endpoint assessed irrespective of baseline HPV DNA or serostatus;

TVC-E: all the same except that endpoint assessed in women DNA negative and seronegative for corresponding HPV type at month 0.

Japanese trial /
(ph2,4v)

Competitive immunoassay (cLIA, Luminex Crp, Austin,TX,US)
Per-protocol: women who were naive for the relevant HPV type at enrolment, remained free of infection with the same vaccine HPV type through completion of the vaccination regimen, had 3 doses, no protocol violations. Cases counting start form M 7.

Korean trial /

(ph2,4v)

/

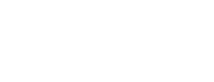

Competitive immunoassay (cLIA, Luminex Crp, Austin,TX,US
Per-protocol: received all 3-doses, meet all the eligibility of inclusion, complying with all the protocol procedures
With at least one dose of vaccine administrated

\begin{tabular}{lll}
\hline $\begin{array}{l}\text { Phase2 trial } \\
\text { (ph2,4v) }\end{array}$ & $\begin{array}{l}\text { Type specific } \\
\text { PCR for HPV6/11/ }\end{array}$ & $\begin{array}{l}\text { Competitive } \\
\text { immunoas- } \\
\text { 16/18. }\end{array}$ \\
& $\begin{array}{l}\text { say (cLIA, Lu- } \\
\text { minex Crp, }\end{array}$ \\
& HC2 triage for & Austin,TX,US) \\
& ASCUS cases. &
\end{tabular}

\begin{abstract}
Naïve for relevant HPV types at enrolment, still free of infection with HPV types 1 month after completion of vaccination regimen of 3 doses within 1 year, who did not violate protocol ( $\mathrm{N}=431$ for HPV6/11, 404 for HPV16, 456 for HPV18).
\end{abstract}

\section{l}

(n)
Cases are counted from $\mathrm{M} 7$.

\begin{tabular}{|c|c|c|c|c|}
\hline $\begin{array}{l}\text { African_3 coun- } \\
\text { try trial (ph3,4v) }\end{array}$ & I & $\begin{array}{l}\text { Competitive } \\
\text { immunoas- } \\
\text { say (cLIA, Lu- } \\
\text { minex Crp, } \\
\text { Austin,TX,US) }\end{array}$ & $\begin{array}{l}\text { ATP for immunogenicity included women } \\
\text { who met eligibility criteria and were } \\
\text { seronegative at M } 0 \text {. }\end{array}$ & $\begin{array}{l}\text { TVC included all partic- } \\
\text { ipants with at least one } \\
\text { vaccine/placebo dose } \\
\text { administration docu- } \\
\text { mented. }\end{array}$ \\
\hline $\begin{array}{l}\text { FUTURE I trial } \\
\text { (ph3,4v) }\end{array}$ & $\begin{array}{l}\text { Type specific } \\
\text { PCR for HPV6/11/ } \\
16 / 18 \text {. }\end{array}$ & $\begin{array}{l}\text { Competitive } \\
\text { immunoas- } \\
\text { say (cLIA, Lu- }\end{array}$ & $\begin{array}{l}\text { Per-protocol: received all } 3 \text { doses within } \\
12 \mathrm{M} \text {, seronegative and HPV DNA negative } \\
\text { for vaccine type from day } 1 \text { till } 1 \text { month af- }\end{array}$ & $\begin{array}{l}\text { ITT: included even if they } \\
\text { had infection or disease } \\
\text { associated with vaccine }\end{array}$ \\
\hline
\end{tabular}

Prophylactic vaccination against human papillomaviruses to prevent cervical cancer and its precursors (Review)

\section{Naïve to the relevant HPV type (S) at enrolment and had received at least one dose. Protocol violators were included.}


minex Crp, $\quad$ ter the $3^{\text {rd }}$ does, remained HPV negative; Austin,TX,US) no protocol violations, include even the first day cytology were abnormal type before vaccination; protocol violations were present; or results on cervical cytological examination at day 1 were abnormal.

\begin{tabular}{|c|c|c|c|c|}
\hline $\begin{array}{l}\text { FUTURE II trial } \\
\text { (ph3,4v) }\end{array}$ & $\begin{array}{l}\text { Type specific } \\
\text { PCR for HPV6/11/ } \\
\text { 16/18. }\end{array}$ & $\begin{array}{l}\text { Competitive } \\
\text { immunoas- } \\
\text { say (cLIA, Lu- } \\
\text { minex Crp, } \\
\text { Austin,TX,US) }\end{array}$ & $\begin{array}{l}\text { Per-protocol: received all } 3 \text { doses within } 12 \\
\text { M, seronegative and HPV DNA negative for } \\
\text { vaccine type understudy from day1 till M } 7 \text {. } \\
\text { Have } 1 \text { or more follow-up visit S after M } 7 \text {. } \\
\text { No protocol violations. }\end{array}$ & I \\
\hline $\begin{array}{l}\text { FUTURE III trial } \\
(\mathrm{ph} 3,4 \mathrm{v})\end{array}$ & $\begin{array}{l}\text { Type specific } \\
\text { multiplex PCR } \\
\text { for HPV6/11/ } \\
\text { 16/18 targeting } \\
\text { L1,E6,E7 genes. }\end{array}$ & $\begin{array}{l}\text { Competitive } \\
\text { immunoas- } \\
\text { say (cLIA, Lu- } \\
\text { minex Crp, } \\
\text { Austin,TX,US) at } \\
\text { month } 0,7,12 \text {, } \\
24,36,48 \text {. }\end{array}$ & $\begin{array}{l}\text { Seronegative for relevant type at day } 1 \text {, } \\
\text { PCR negative for that type in cervicovagi- } \\
\text { nal samples at day } 0 \text { and } M 7 \text {; all } 3 \text { doses } \\
\text { received within } 1 \text { year with } 1 \text { or more fol- } \\
\text { low-up visits after } 7 \mathrm{M} \text {. }\end{array}$ & $\begin{array}{l}\text { Women who received at } \\
\text { least one dose of vaccine } \\
\text { or placebo and had one } \\
\text { or more follow-up visits } \\
\text { after day } 1 \text {. Both proto- } \\
\text { col violators and those } \\
\text { with pre-existing HPV in- } \\
\text { fections were included in } \\
\text { ITT analyses. Cases were } \\
\text { counted starting at day } 1 .\end{array}$ \\
\hline
\end{tabular}

WHAT'S NEW

\begin{tabular}{lll}
\hline Date & Event & Description \\
\hline 10 March 2020 & Amended & Text added to published notes section. \\
\hline
\end{tabular}

\section{CONTRIBUTIONS OF AUTHORS}

Conception of the systematic review: M. Arbyn, L Markowitz, P. Martin-Hirsch.

Study design: M. Arbyn.

Writing of the protocol: M. Arbyn, A. Bryant, C. Simoens, L Markowitz.

Writing of the full review: M. Arbyn, L Xu, C Simeons.

Retrieval of references: M. Arbyn, L. Xu, C. Simoens.

Checking eligibility of references: M. Arbyn, L. Xu, C. Simoens.

Extraction of data: M. Arbyn, C. Simoens, L. Xu.

Statistical analysis: M. Arbyn, Lan Xu.

Critical review of the manuscript: P. Martin-Hirsch, C. Simoens, L. Markowitz.

\section{DECLARATIONS OF INTEREST}

MA: has received travel grants from MSD-Sanofi-Pasteur and GSK, (ceased in 2008).

$\mathrm{PM}-\mathrm{H}$ : travel grants received from GSK and MSD-Sanofi-Pasteur (ceased in 2008).

LX: no conflict of interest.

CS received travel grant from GSK (2007).

Authors of this review were assessed by the Cochrance Funding Arbiter Committee after Cochrane received correspondence and feedback on the published protocol. Current authors were approved by this committee based on stringent Cochrane conflict of interest guidelines. A unrestricted grant was provided by Sanofi-Pasteur-MSD to the University of Ghent who co-ordinated the SEHIB study (Surveillance of Effects of HPV Immunisation in Belgium). The grant was given in the framework of the EMA (European Medicine Agency) request to set up post-marketing surveillance of HPV vaccination effects in non-Nordic member states of the European Union. The Sciensano (employer of MA and LX, former name "Scientific Institute of Public Health") collaborated with the University of Ghent to conduct the SEHIB study. 


\section{SOURCES OF SUPPORT}

\section{Internal sources}

- Scientific Institute of Public Health (Brussels), Belgium.

Bibliographic support to obtain literature references, secretarial and logistic support in organising contacts and meetings with coauthors and to store and sort bibliographic references. references

\section{External sources}

- National Institute for Health Research, UK.

NHS Cochrane Programme Grant Scheme CPG-506 funding to the Gynaecological, Neuro-oncology and Orphan Cancer Group.

- European Cancer Network and the European Co-operation on development and implementation of Cancer screening and prevention Guidelines (ECCG), via the International Agency for Research on Cancer, Lyon), France.

Financial support received from the European Commission (DG SANCO, Luxembourg) for the production of guidelines for cervical cancer screening and HPV vaccination.

- Belgian Foundation Against Cancer (Brussels), Belgium.

Financial support to conduct methodological research on evaluation of emerging screening methods and to continue systematic reviews on cervical cancer prevention methods.

- IWT (Institute for the Promotion of Innovation by Science and Technology in Flanders, Brussels, project number 060081), Belgium.

Financial support to collect data for mathematical modelling of HPV infection (natural history, cost-effectiveness of HPV screening and vaccination).

- CoheaHr Network (Comparing Health Services Interventions for the Prevention of HPV-Related Cancer) (grant number 603019) funded by the 7th Framework Programme of DG Reasearch and Innovation, European Commission (Brussels), Belgium.

Financial support to update the Cochrane review (2015-18)

\section{DIFFERENCES BETWEEN PROTOCOLANDREVIEW}

The three following items, foreseen in the original protocol, were not addressed in the current version of the review and the reasons why are explained in the Discussion.

1. Immunogenicity of the vaccines

2. Request for non-published available data

3. Protection against high-grade cervical intra-epithelial neoplasia (CIN2 or worse) attributed to non-vaccine HPV types.

We were not able to conduct the latter analysis but the latter outcome was included indirectly in the outcome CIN2+ irrespective of HPV types.

The three points not assessed in the current review will be integrated in future updates of the review.

In the Cochrane protocol (developed when several trials were still ongoing), it was foreseen that websites of regulatory agencies like the US Food \& Drug Administration (FDA) and the European Medicine Agency (EMEA) would be consulted to obtain data on safety and efficacy effects. However, currently, nearly all end-of-study reports have been published in the peer-reviewed literature. We therefore did not need to consult these additional sources any more. For serious adverse events, death after vaccination and pregnancy outcomes, we consulted data posted on www.clinicaltrials.gov and http://www.gsk-clinicalstudyregister.com/ to obtain additional data on critical safety issues not available from the peer-reviewed sources. This has been incorporated into a sensitivity analysis (see Sensitivity analysis).

Assessment of the variation of vaccine efficacy by age group in more detail than the broad distinction younger or older than 25 years could not be done for most studies by lack of reported age-specific data. However, for the bivalent vaccine, an analysis by five-year age group could be performed.

Methods described in the protocol to handle continuous data were not used since immunogenicity was dropped from the review as an objective. Time-to-event data methods were not applied either, because of the abundance of dichotomous data reported at repeated time points and because of the rarity of presentation of results in longitudinal formats. Specific statistical methods to assess cluster-randomised trials were not required since all trials randomised enrolled participants at individual level.

In this Cochrane review, treatment effects were expressed as risk ratios (RR) and not as "vaccine efficacy" since the latter is not supported by Cochrane software. 
Sensitivity analyses excluding studies at moderate or high risk of bias were foreseen in the protocol. However, given the low risk of bias of all the trials reporting efficacy outcomes and the detailed subgroup analyses and meta-regression analyses assessing the impact of each separate item of the Cochrane tool for assessment of risk of bias, these sensitivity analyses were considered as superfluous.

We planned to distinguish adverse effects occurring in the period between zero to four weeks and more than four weeks after administration of vaccines. However, since this timing of observation of adverse events was not documented uniformly in the trials reports, this distinction could not be implemented in the review. No sensitivity analysis based on risk of bias was performed as described in the original protocol, as the studies were assessed to be at low risk of bias. Impact of influential factors, such as involvement of the vaccine manufacturers, were addressed sufficiently by meta-regression.

\section{NOTES}

Following the publication of a critical commentary of this review in July 2018 (https://ebm.bmj.com/content/23/5/165), its findings were subject to an investigation overseen by the then Editor in Chief of the Cochrane Library, Dr David Tovey. The outcome of this investigation was published online in September 2018 and can be found here: https://www.cochrane.org/news/cochraneseditor-chief-responds-bmj-ebm-article-criticizing-hpv-review. Since this time, a systematic review by the team of authors who wrote this commentary was published in March 2020: https://systematicreviewsjournal.biomedcentral.com/articles/10.1186/s13643-019-0983$\mathrm{y}$, with a related methods article (https://systematicreviewsjournal.biomedcentral.com/articles/10.1186/s13643-020-01300-1), and an accompanying commentary (https://systematicreviewsjournal.biomedcentral.com/articles/10.1186/s13643-020-01299-5).

In 2018 Cochrane made a public commitment to incorporate the findings of this assessment as an amendment of this review. In order to ensure that its numerical findings match with those presented in the original investigation of the review, this work is now being commissioned. Furthermore, in view of the continued importance of this vaccine, there is now an opportunity to look at the comparative effects of these vaccines and to incorporate evidence from multiple sources of data that are now available for these trials. This will be investigated as a separate Cochrane Review

\section{N DEX TERMS}

\section{Medical Subject Headings (MeSH)}

Cervical Intraepithelial Neoplasia [mortality] [ ${ }^{*}$ prevention \& control] [virology]; Human papillomavirus 16; Human papillomavirus 18; Papillomavirus Infections [complications] [mortality] [ ${ }^{\star}$ prevention \& control]; Papillomavirus Vaccines [ ${ }^{*}$ administration \& dosage] [adverse effects]; Precancerous Conditions [mortality] [ ${ }^{\star}$ prevention \& control] [virology]; Pregnancy Outcome; Randomized Controlled Trials as Topic; Uterine Cervical Neoplasms [mortality] [ ${ }^{*}$ prevention \& control] [virology]; Vaccination

\section{MeSH check words}

Adolescent; Adult; Female; Humans; Middle Aged; Pregnancy; Young Adult 